\title{
DEVELOPMENT OF A PLANNING ASSESSMENT TO REPURPOSE AND REDEVELOP UNDER-UTILIZED AND DETERIORATING TORONTO DISTRCIT SCHOOL BOARD SCHOOL PROPERTIES INTO COMMUNITY HUBS
}

\author{
By \\ Safiyyah Omar Saleh \\ BA, University of Toronto, 2016 \\ A Major Research Project \\ Presented to Ryerson University \\ In partial fulfillment of the requirements for the degree of \\ Master of Planning \\ In \\ Urban Development
}

Toronto, Ontario, Canada, 2018

(C) Safiyyah Omar Saleh 


\title{
DEVELOPMENT OF A PLANNING STRATEGY AND ASSESSMENT TO REPURPOSE AND REDEVELOP UNDER-UTILIZED AND DETERIORATING TORONTO DISTRCIT SCHOOL BOARD SCHOOL PROPERTIES INTO COMMUNITY HUBS
}

(C) Safiyyah Omar Saleh, 2018

\author{
Masters of Planning \\ In \\ Urban Development \\ Ryerson University
}

\begin{abstract}
:
One of the main issues faced by the Toronto District School Board (TDSB) is the poor structural condition of its aging school buildings. As of school year 2016/2017, the total renewal/repair backlog for all of TDSB schools has reached \$3.4 Billion, with approximately 103 TDSB schools operating at a $65 \%$ or lower utilization rate (TDSB, 2014f). There is an immense pressure on school boards, particularly the TDSB, to sell off schools that have been declared as surplus (Mangione \& Suen, 2015). However, the selling of school properties that have a high social and economic value is not a sustainable approach for the long run. Hence, there is a need for an effective and resilient planning strategy that will efficiently address the issues faced by the TDSB. As a result, this research will be recommending a land assessment tool that will efficiently repurpose and redevelop school properties, in critical condition, into community hubs and replace old and deteriorating TDBS schools with newer school facilities.
\end{abstract}

Key Words: TDSB; Surplus Schools; Community Hubs; Shrinking Cities; Public Assets; 


\section{$\underline{\text { Acknowledgements }}$}

I want to thank my supervisor, Professor Christopher De Sousa, for providing me with guidance and constructive criticism and feedback throughout the process of writing this MRP. I am also grateful to Ryerson University's School of Urban and Regional Planning faculty, staff, and students that have made my master's experience exciting and memorable.

I would also like to thank Daryl Sage, the CEO of Toronto Lands Corporation, for providing me with the data, documents, and guidance I needed to complete this research project.

Finally, I am grateful to the ongoing support of my parents and my entire family. Your encouragement and support have helped me persevere during my final year of Masters. 


\section{Table of Contents}

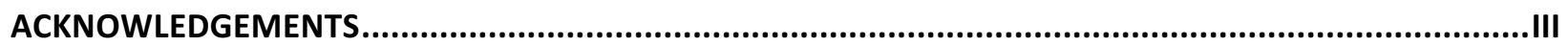

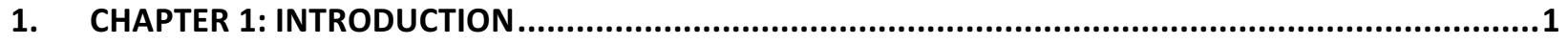

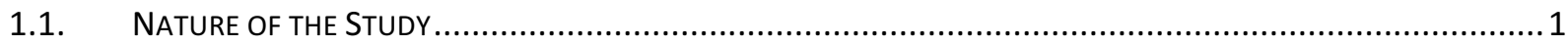

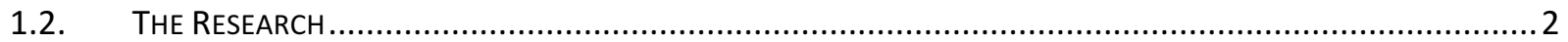

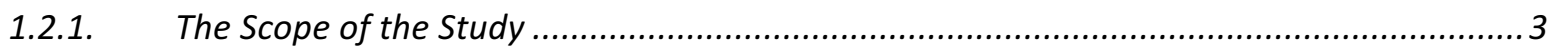

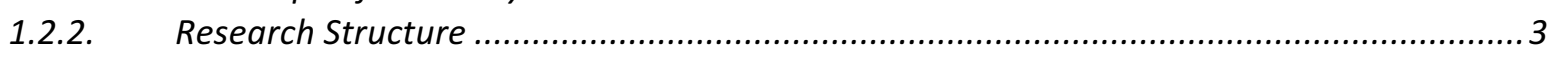

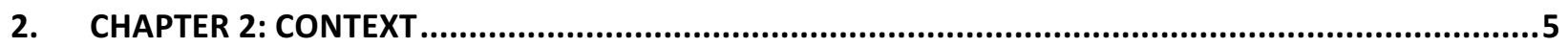

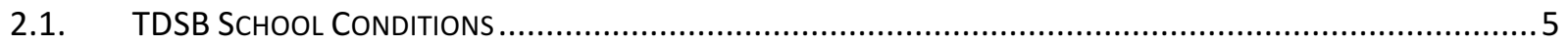

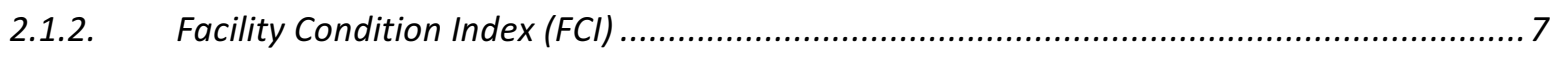

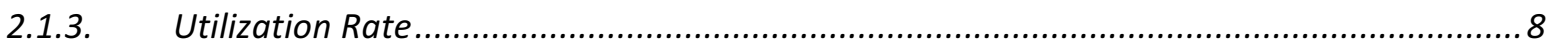

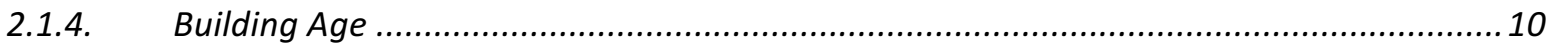

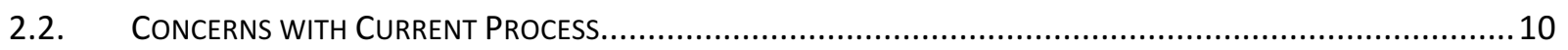

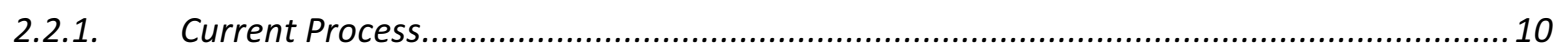

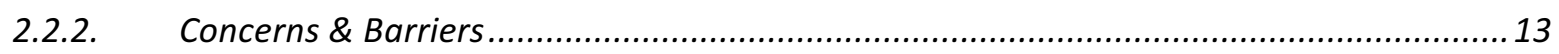

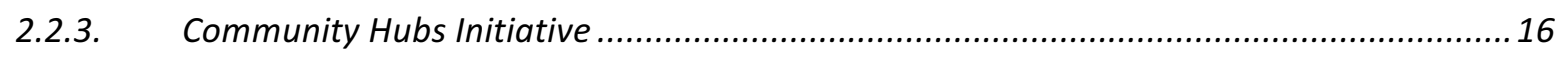

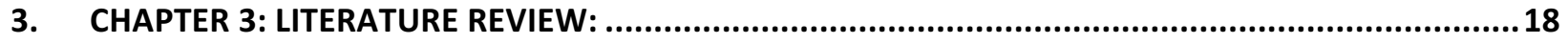

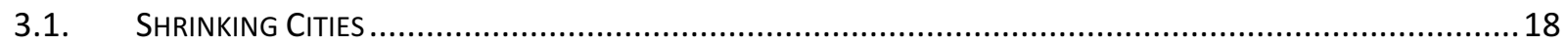

3.1.1. Declining Populations \& Under-utilized properties ................................................... 18

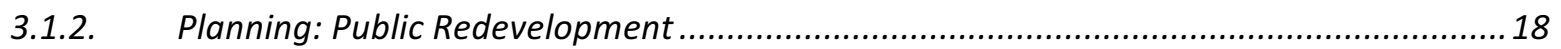

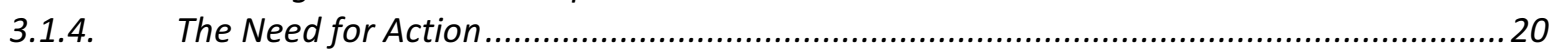

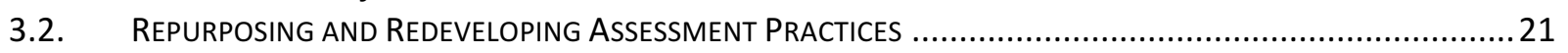

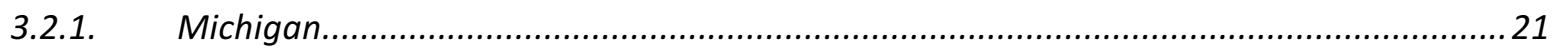

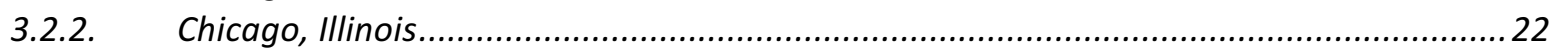

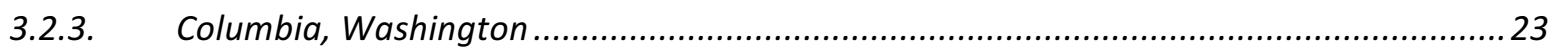

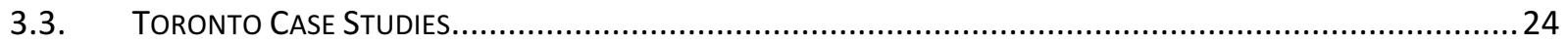

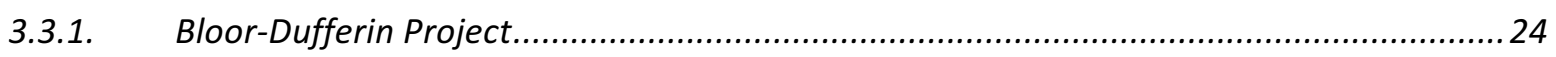

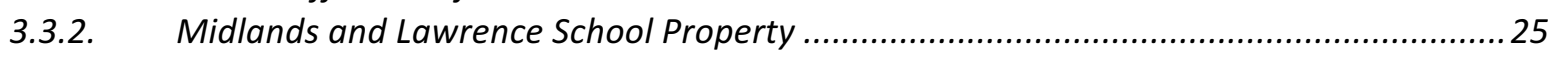

4. CHAPTER 4: PROPOSED METHODOLOGY FOR SURPLUS SCHOOL REPURPOSING ..........................27

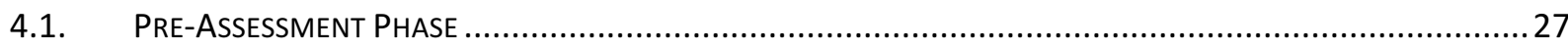

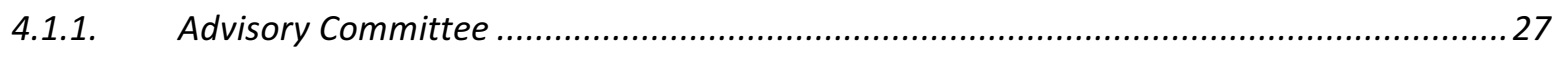

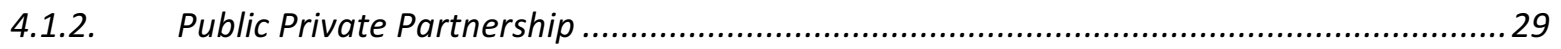

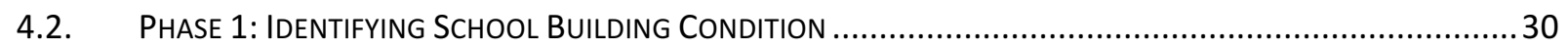

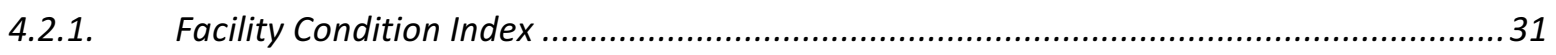

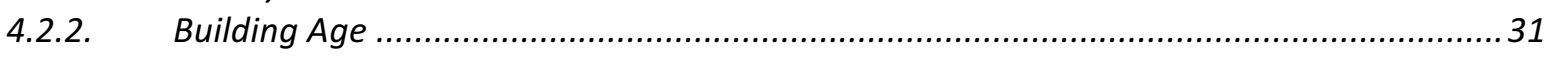

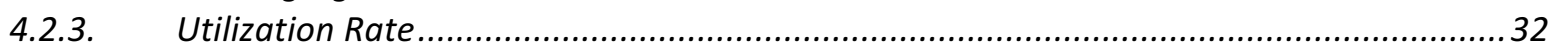

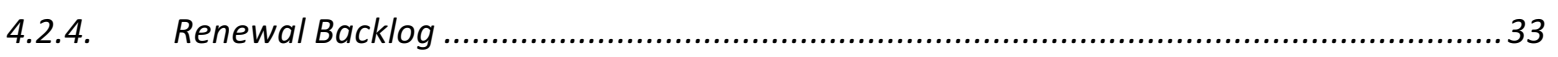

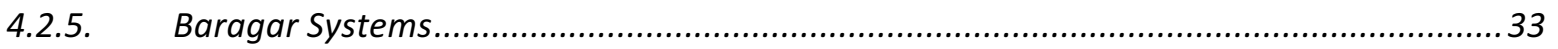

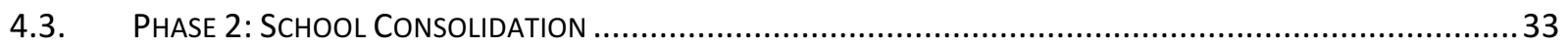

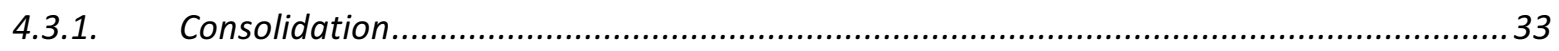

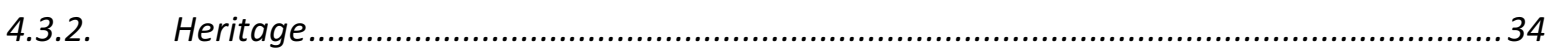

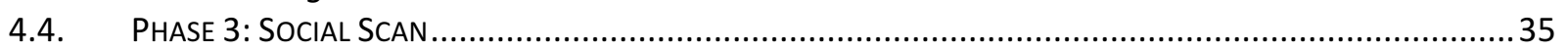

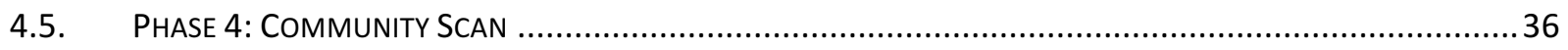

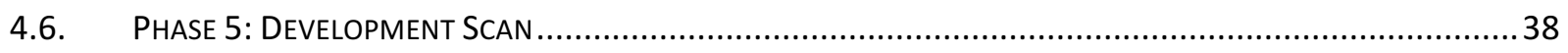


4.7. SUMMARY

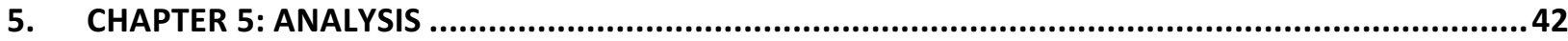

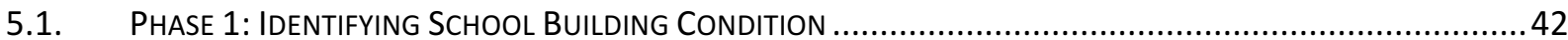

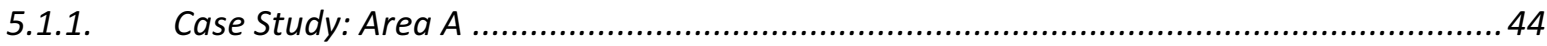

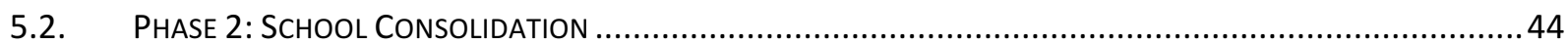

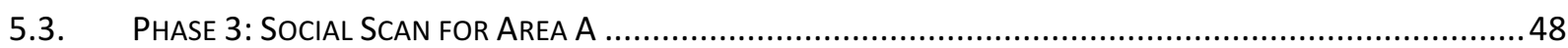

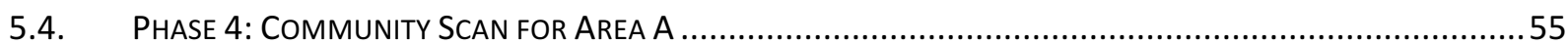

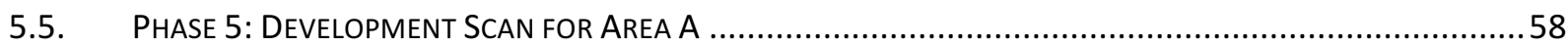

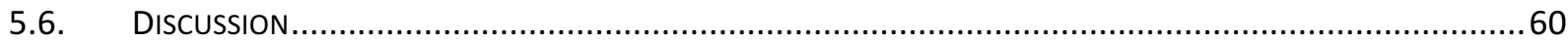

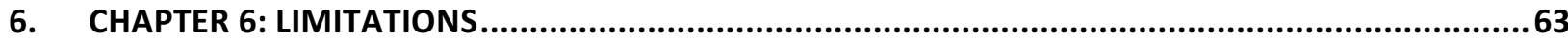

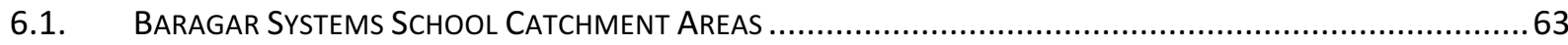

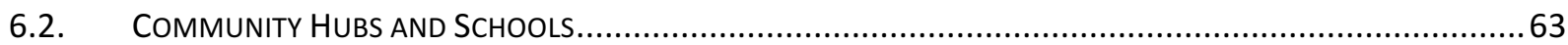

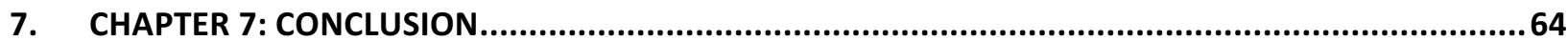

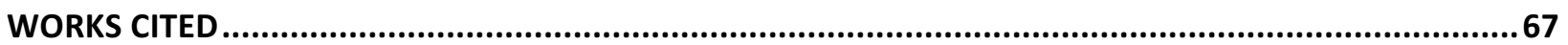

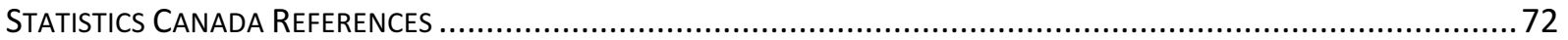

\section{List of Figures}

Figure 1: Annual Renewal Backlog and Project Expenditures.............................................. 6

Figure 2: TDSB Elementary Schools in Critical Condition, 2016-2017 ............................... 43

Figure 3: TDSB High School Catchment Areas, 2016-2017 ........................................... 43

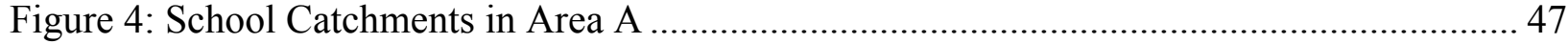

Figure 5: Census Tract Map for Area A ......................................................................... 48

Figure 6: Employment Concentrations Map........................................................................ 53

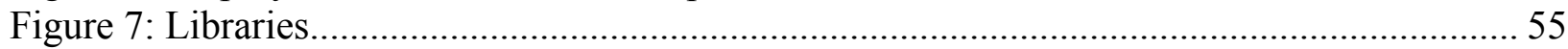

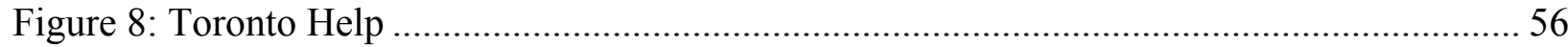

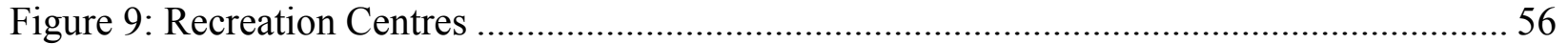

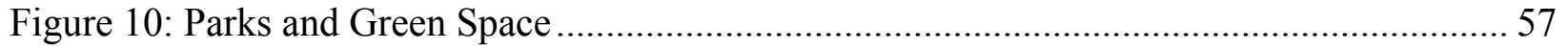

\section{List of Tables}

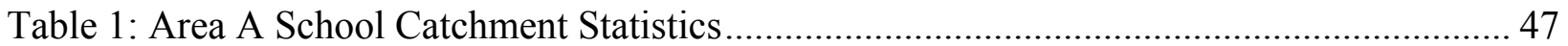

Table 2 Age Group populations in Area A ........................................................................... 49

Table 3: Income Levels in Area A ..................................................................................... 52

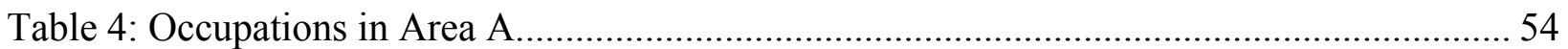

Appendix A - 2016-2017 TDSB Dataset

Appendix B - TDSB Policy Documents

Appendix C - Statistics Canada \& Other Datasets 



\section{Chapter 1: Introduction}

\subsection{Nature of the Study}

One of the main issues faced by the Toronto District School Board (TDSB) is the poor structural condition of its school buildings. As of school year 2016/2017, the total renewal backlog for all TDSB schools reached \$3.4 Billion. In addition, numerous TDSB schools are currently underutilized, with approximately 103 TDSB schools at a $60 \%$ or lower utilization rate. With a declining student enrollment rate, the TDSB receives less funding for operating costs (TDSB, 2014f). In terms of building age, the average age of a TDSB school building is 60 years of age, with 80 schools older than 90 years, and 7 of which were built before the 1900s.

Ontario's Education Minister, Liz Sandals, has stated that the province spends more than $\$ 1$ billion on under-utilized schools across all Ontario school boards. Considering that it is the largest school board in Canada, the TDSB has the majority of these under-utilized schools across the province. As a result, there is immense pressure on school boards, particularly the TDSB, to sell off schools that have been declared as surplus. (Mangione \& Suen, 2015). Trustees voiced their concerns that some of these under-utilized schools are not only used by students, but also used by the surrounding community for other purposes. And considering the prime real estate of these school properties, there is a concern that selling these properties without a deeper investigation of their true community value will result in a great loss for the neighbourhood in the long run (Brown \& Rushowy, 2015).

This issue faced by the TDSB is currently being experienced by cities on a much larger scale. Approximately 370 cities around the world are currently faced with an urban planning phenomenon known as the shrinking cities (Lee \& Sung, 2017). According to Hollander (2011), shrinking cities refers to urban areas experiencing a decline in their population. The negative impacts associated with shrinking cities is economic instability, declining labor and employment opportunities, and, most relevant to this research, the under-utilization and even vacancy of public buildings and properties. However, Hollander explains that situations like these truly show how resilient and versatile planning is when it comes to addressing these issues. Rather than attempting 
to reverse the issue, planning should be a flexible tool that is able to plan for the community and land use needs of a city with a smaller population.

And although Toronto's population is not in decline, its schools are experiencing a decline in student enrollments. Hence, there is a need for an effective and resilient planning strategy that will efficiently address the issues faced by the TDSB in terms of under-utilization and deteriorating school buildings. At the same time, this will provide an opportunity to present students, parents, and surrounding communities with a socially and economically suitable alternative to simply selling public asset properties for a maximum revenue. The "how" of this research will be discussed in further detail in section 1.2 of this chapter.

\subsection{The Research}

The purpose of the research is to develop a land assessment tool that will efficiently repurpose and redevelop surplus school lands into community needed infrastructure, services, and/or amenities and replace old and deteriorating TDSB schools with newer school facilities. The strategy will be presented in the form of a 5-step assessment process. Each step of the assessment will be informed by current land disposal practices from other jurisdictions, case studies, and from previous research.

The land disposal assessment considers factors that go beyond the physical school property itself. These factors include looking into the area demographics, existing services and amenities in the area, and conducting a planning due diligence of the school property and other properties surrounding it. By understanding these factors that go beyond the physical building or property, this allows for the TDSB, the City, students and parents, community members, and possibly the private sector to identify the highest and best use for this property that would maximize the benefits for each stakeholder group. 


\subsubsection{The Scope of the Study}

The main focus of this study will be on the Toronto District School Board (TDSB) within the City of Toronto, the largest school board in Canada and one of the largest in North America. The TDSB is currently operating approximately 588 schools across Toronto in order to accommodate more than 246,000 students and approximately 160,000 life-long learners in Adult and Continuing Education programs. In total, $12.5 \%$ of Ontario's student population attend TDSB schools. In other words, it is one of the largest school districts within Ontario. And with a large student body population to teach, the TDSB's mission is to enable and provide all of its students with tools, resources, and an environment that enables them to "reach high levels of achievement and to acquire knowledge, skills, and values they need to become responsible members of a democratic society" (TDSB, 2014c).

\subsubsection{Research Structure}

\section{Chapter 2: Context}

This section will begin by taking a deeper look into the issues that the TDSB is currently facing. This chapter will also provide a detailed overview of the current approaches the TDSB has taken to manage and deal with under-utilized, deteriorating, and aging school properties.

\section{Chapter 3: Literature Review}

This section will look at the general topic of under-utilized or vacant public properties/assets from an urban planning lens. It will begin by understanding the shrinking cities phenomenon and what role planning should and should not be playing to address these urban issues. The chapter will transition into exploring some of the practices being followed by other jurisdictions when it comes to assessing and disposing of public assets, more specifically surplus school properties.

\section{Chapter 4: Methodology}

As mentioned in section 1.2, this research will be proposing an assessment tool that will identify and prioritize school in critical condition, and that will the determine the highest and best use for the property. This chapter will introduce the Surplus School Land 5-step assessment tool. Each step or phase of the assessment will be broken down, explaining the specific factors that need to be considered and how each step is to be carried out. 


\section{Chapter 5: Analysis}

This chapter will apply the 5-step assessment (outlined in Chapter 5 of the report) to 4 school properties that have been identified to be in "critical condition" in phase 1 of the assessment. This will provide an example of how the assessment would perform if it were to be put into action.

\section{Chapter 6: Limitations}

This chapter will outline some of the limitations found in the approach of this research and in the assessment, itself.

\section{Chapter 7: Conclusion}

This chapter will provide concluding thoughts on the land current land disposal process and the proposed 5-step assessment tool. 


\section{Chapter 2: Context}

\subsection{TDSB School Conditions}

As mentioned earlier, one of the major issue currently faced by the TDSB is its deteriorating and aging school buildings. A quarter of TDSB schools are considered to be in critical conditions (Sachgau, 2016a). Currently, the TDSB is dealing with schools that have issues such as leaky roofs, old boilers, and broken AC units. It is important to note, however, that while schools are in deteriorating condition, this does not mean they are unsafe for students to attend. Anything that may be a threat to the students' safety, is addressed immediately.

However, this also does not mean that the root of the issue is being dealt with, rather the issue is patched up and kept "under control". A parent, quoted in a Toronto Star article, indicates that issues, such as leaky roofs, are being patched up and temporarily fixed, rather than replacing the roof altogether. Trustee for Etobicoke-Lakeshore, Pamela Gough, stated that due to limited funds, there is only so much that can be done in terms of fixing and replacing school building equipment and parts (Sachgau, 2016a). To further understand the extent of the problem, this section will be looking at the following four major categories: renewal backlog, the facility condition index (FCI), utilization rate, and building age.

\subsubsection{Renewal Backlog}

As of September 2017, the total value of repairs is approximately $\$ 3.7$ billion. This consists of 22,686 different repairs needed for 583 TDSB schools. And while this backlog does not reflect or represent the health or safety of the school, it is an issue that needs addressing nonetheless ${ }^{1}$. Robin Pilkey (2017), the Chair of the TDSB, states in a letter to the Provincial government that without adequate and consistent funding, the TDSB's renewal backlog is predicted to reach $\$ 5.6$ Billion in the year 2028 (TDSB, 2015a).

\footnotetext{
${ }^{1}$ http://www.tdsb.on.ca/About-Us/Accountability/Renewal-Needs-Backlog-and-Facility-Condition-Index/RenewalNeeds-Backlog
} 


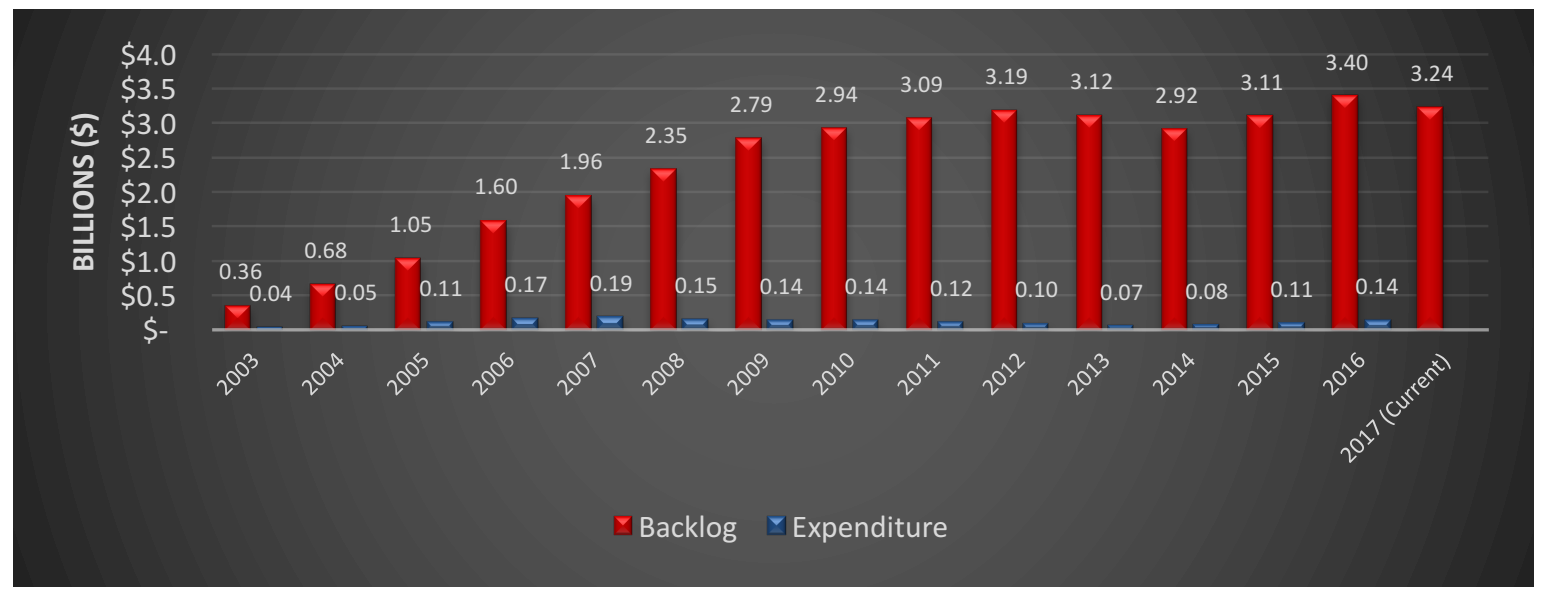

Figure 1: Annual Renewal Backlog and Project Expenditures (Source: Data provided by TDSB's Design, Construction \& Maintenance Department)

To put this into perspective, to build an elementary school (Grades JK to 8) it costs approximately $\$ 1,959.89$ per square metre, and with the average facility size of an elementary school within the City of Toronto being approximately 7064 square metres, this means it costs approximately $\$ 14$ million (more or less) to build one elementary school in Toronto. To build a secondary school (Grades 9 to 12), it costs approximately \$2,138.08 per square metre, and with the average facility size of a secondary school being approximately 7080 square meters, the cost of building one secondary school in Toronto is approximately $\$ 15$ million. Hence, with a current backlog of \$3.7 billion, the TDSB can build approximately 264 elementary schools or 246 secondary schools.

Referring to Figure 1 (above), the graph shows the renewal backlog for each school year (red) and the annual provincial funding allocated for project expenditures (blue). It is important to note that the province provides grants and funding specifically dedicated towards school renewal and repair projects. For school year 2016-2017, the TDSB received a total of \$261.5 million (Sekaly, 2016). However, due to the high volume of needed repairs and the short time period to complete these repairs (most work tends to be done over the summer when students are out of school), the TDSB was only able to spend \$140 million that school year (refer to Figure 1). This means that only 54\% of the allocated funding was spent that school year, and it only covered $4 \%$ of the $\$ 3.4$ billion backlog.

For school year 2017-2018, the province provided the board with a \$297 million grant. It is expected that $\$ 50$ million should be used to replace energy efficient building components. The 
remaining of the grant (\$247 million) should be used to replace/repair building components, with hopes of it reducing the FCI of school buildings (refer to section below for the definition of FCI $)^{1}$. This means that, if the funding were to be spent as indicated above, this will only reduce the overall renewal backlog by $8 \%$.

A Torontoist article suggests that the TDSB should explore different funding options such as increase in taxes, issuing bonds, or using educational development charges (EDCs) ${ }^{2}$. However, the bottom line many people and organizations are reaching is that there needs to be an increase in provincial grants and funding in order to reduce the backlog. And while the province's attempt to address growing repair backlogs for Ontario school boards was to increase funding to $\$ 1.1$ billion for 2016-2017 school year, it is not sufficient to solve the problem or significantly reduce the TDSB's \$3.7 billion backlog (Sachgau, 2016b). The need for funding is a fair request from school boards, however, it is also not a realistic or sustainable solution to address the TDSB's growing backlog, considering that there is only so much money that can go around each year to each school board in Ontario. This further proves that there is a need for a new and improved strategy that will reduce the TDSB's renewal backlog issue.

\subsubsection{Facility Condition Index (FCI)}

The facility condition index or FCI provides each school a rating based on its structural condition. The FCI is a percentage calculated by dividing the renewal backlog by the total cost of replacing/rebuilding a school $\left(\mathrm{FCI}=\right.$ Repair costs/ Rebuilding costs $\left.{ }^{3}\right)$. According to the Ministry of Education ranking system, schools with an FCI greater than $65 \%$ are better off being rebuilt, rather than spending money and resources on repairs. Schools with an FCI of $100 \%$ or greater means that repair costs are equal to or higher than the cost of rebuilding the school. The FCI is updated when the school's backlog is updated/adjusted, which occurs every 5 years (TDSB, 2014d). It is important to note that the FCI does not reflect the safety of the school building.

\footnotetext{
${ }^{2}$ https://torontoist.com/2016/09/the-tdsbs-repair-backlog-is-the-result-of-years-of-underfunding/

3 The total cost of rebuilding a school is determined by the total Asset Replacement Value (ARV)
} 
According to the 2016 TDSB dataset ${ }^{4}$, it has been identified that 98 elementary schools ${ }^{5}$ and 23 high schools ${ }^{6}$ had an FCI equal to or greater than 65\% (TDSB, 2014). In other words, the TDSB is currently spending funding and resources on repairs within schools that are identified and assessed to being unrepairable and are better off rebuilt from scratch. If these 121 schools were to be replaced with newer school facilities, this would potentially eliminate a grand total of $\$ 1.3$ billion from the renewal backlog (i.e. elementary schools $=\$ 752.9$ Million; high schools $=\$ 557.7$ Million).

\subsubsection{Utilization Rate}

\subsubsection{Under Capacity}

It is important to understand that even with high renewal backlogs, this does not necessarily mean that the TDSBs resources and schools are being utilized at full or over capacity. According to the 2016-2017 dataset, 129 TDSB schools (approx. 22\%) are currently operating at a utilization rate ${ }^{7}$ of $65 \%$ or lower. The pupil accommodation review indicates that schools that are operating at a utilization rate of $65 \%$ or lower and it is projected to remain so for the next 5 years, they are considered to be under-utilized and need to be assessed for potential closure (TDSB, 2014). A school in "good" standing in terms of enrollment should have a minimum utilization rate of $80 \%$ to $90 \%$ (TDSB, Long-term program, 2016b).

In 2015, the Ontario Education Minister, Liz Sandals, has requested from the TDSB to provide a list of schools that are considered to be under-utilized and to start the process of assessing these schools for potential closure. However, Brown and Rushowy (2015) explain that the province's measure cannot be applied on every school without considering additional factors. For example, Burnhamthorpe Collegiate Institute is operating at a 35\% enrollment rate, however, this calculation does not include the approximately 1,000 adult students going back to school to get high school credits because they are identified as "adults" in the system (Brown \& Rushowy, 2015). Even after

\footnotetext{
${ }^{4}$ The 2016-2017 TDSB dataset can also be found in Appendix $\boldsymbol{A}$.

${ }^{5}$ Toronto has many different types of elementary schools, with different grade ranges. Some elementary schools range from $\mathrm{Jk}$ to grade 5 , JK to grade 8 , JK to grade 4, etc. This particular statistic includes these schools. However, this does not include schools that go from grade 4 to 8 , grade 6 to 8 , or grades 1 to 5 .

6 These statistics includes schools that have grade ranges such as grade 9 to 12, grades 10 to 12, and grade 7 to 9 .

${ }^{7}$ Utilization Rate: School Enrollment or Full Time Enrollment (FTE) DIVIDED BY the Total School Capacity MULTIPLY BY 100 (TDSB, 2016a).
} 
providing a list of "under-utilized" schools, trustees warned against the ministry from taking any quick decisions solely based on the "utilization rate" calculation alone. Trustee Ausma Malik explained that some of the schools are being also used as community hubs, however under the ministry's formula, they appear to be "near-empty" (Brown \& Rushowy, 2015).

\subsubsection{Over Capacity}

On the other hand of the argument, there are a number of TDSB schools that are operating at overcapacity. According to the 2016-2017 dataset, 127 schools are currently operating at $100 \%$ capacity or greater (TDSB, 2014). According to an advocate group known as Fix Our Schools, explains that overcapacity schools operate extremely efficiently and they save the province a lot of money (Fix Our Schools, 2015). However, these schools are also not ideal learning environments for students. School staff are working all around the clock, spending time and energy on managing logistics rather than investing more time and energy into providing students with top quality learning experience. The group states that organizing an event such as a school assembly or a concert can be a mission impossible considering the large volume of students they need to coordinate and manage. And due to limited or no space at all, if a classroom is going through repairs, students, in some cases, must have their class in the hallway. In addition, caretakers and cleaning staff are working around the clock to ensure an overcapacity school is kept in good cleaning condition, and this can have a wear-and-tear effect on the school's facilities (Fix Our Schools, 2015).

The issue of over capacitated schools also stems from soaring condominium developments in certain parts of the City. There are several cases where new families are moving into a newly built development in an area and their child(ren) is(are) unable to register at the neighbourhood's local school due to overcapacity, and are bused to a further location. The TDSB has asked developers to insert a "warning clause" alerting potential buyers that their children may not be able to attend the local public school in their area (Otis, 2015). Hence, identifying and understanding the utilization rate of a school is necessary for the development of a new planning strategy or assessment, since these rates fluctuate each year for various reasons, which need to be taken into 
account. It is also important to consider enrollment or utilization rate projections for the next 5 years ${ }^{8}$.

\subsubsection{Building Age}

As mentioned earlier, the TDSB renewal backlog is currently at $\$ 3.4$ billion and continues to grow. A main contributor to the growth of the backlog is the aging TDSB school buildings. According to the TDSB (2014d) website, the average age of a TDSB school building is over 60 years of age, with 80 schools older than 90 years, 7 of which were built before the 1900's (TDSB, 2014a). This means that building components are also aging and require continuous repairs or complete replacement.

\subsection{Concerns with Current Process}

In order to understand the current land disposal of surplus school properties process and the concerns and barriers that surround this process, section 2.2 will be divided into two parts. The first part of the section (2.2.1. Current Process) will talk about who carries out the assessment, the factors considered within the assessment, and the provincial regulations in place to guide this assessment. The second part of this section (2.2.2. Concerns $\boldsymbol{\&}$ Barriers) will discuss the concerns and barriers that surround this very process. This will include concerns and issues expressed on the municipal level, the inability of the TDSB to access certain development revenue tools, and the current strategies in place to possibly address these issues (and why they may not be effective).

\subsubsection{Current Process}

i. $\quad$ Toronto Lands Corporation

Toronto Lands Corporation (TLC) is a wholly-owned subsidiary of the TDSB, and was incorporated on April of 2008. TLC manages the TDSB's leasing and selling portfolio for school properties that have been declared surplus. Their main objective is to maximize the income/revenue gathered on properties, whether that income comes from the sale of the property or from leasing it. Once the school property has been declared surplus by the TDSB, the property

${ }^{8}$ the decision for school closure or expansion is based on the utilization rate for the next 5 years. 
is passed on to TLC in order to begin the land disposition process outlined under O. Reg. 444/98 (TLC, 2014). As their mission statement suggests, TLC does not only aim to sell and lease surplus school properties. The corporation seeks to find new and innovative strategies to provide a quality education experience for TDSB students and ensure that the economic and social value of a school property is maximized through the management, redevelopment, and/or the sale of surplus school properties (TLC, 2014).

\section{ii. Pupil Accommodation Review}

The process of identifying the level of deterioration can be brief or thorough, depending on the property in question. In order for a TDSB school to be determined as surplus, the school must go through a Pupil Accommodation Review. A system or assessment such as this is applied to schools that are underutilized or closed schools. The process involves several steps including the establishing of a Pupil Accommodation Review Committee, presenting of an initial staff report, holding public consultations providing the opportunity for community participation, school staff engagement, and providing a final report (TDSB, 2009). Refer to Appendix B for the full Pupil Accommodation and Program Review document.

In certain scenarios, a shortened/ modified version of the Pupil Accommodation Review Process can be applied. The initial staff report must present a rationale for exempting the school from conducting the full-length assessment process ${ }^{9}$. The rationale must include one or more of the following factors and must meet the parameters listed below each factor (TDSB 2009):

1) Enrollment

a. An elementary school with an enrolment equal to or less than 150 students for the current school year, and is projected to continue to have low enrolment levels for the next 5 years

b. A secondary school with an enrolment equal to or less than 350 students for the current school year, and is projected to continue to have low enrolment levels for the next 5 years

\footnotetext{
${ }^{9}$ In this case, once the modified pupil accommodation review process is approved, a committee is not established.
} 
2) Utilization rate

a. A school with a utilization rate of $65 \%$ or lower, and projections show that it will remain low for the next five years

3) Facility condition

a. Schools repairs require large amounts of capital investments

b. When the facility condition index deems the school prohibitive to repair

4) Distance

a. Walking distance to the nearest public school to accommodate students from the school being considered for closure. Walking distance is based on the TDSB's Transportation of Students policy $\mathrm{P} 020^{10}$ :

- Walking distance for JK to grade 5: less than $1.6 \mathrm{~km}$

- Walking distance for grades 6 to 8 : less than $3.2 \mathrm{~km}$

- Walking distance for grades 9 to OAC: less than $4.8 \mathrm{~km}$

For full details of the TDSB Pupil Accommodation Review, refer to Appendix B.

\section{iii. $\quad$ O. Reg. 444/98 Disposition Real Property}

When a school is identified as surplus by the TDSB, it becomes the responsibility of Toronto Lands Corporation. The property goes through O. Reg. 444/98 Disposition Real Property, which is a 180day process that begins when a school property is declared as a surplus. In the first 90-days, other school boards, non-profit organizations, or the City are given priority to express interest in the surplus school property. After the first 90 days (i.e. 3 months) have passed, interested public agencies have another 90 days to present a proposal for the property stating their plans for the property. The property is either leased or sold at fair market price. If within the 180 days, no interest has been expressed from any of these agencies or an agreement has been reached with a public organization, the property is put on the open market for developers to purchase it. And while the funds collected from selling the land help support other schools in the district, there have been concerns raised about the closure and disposition of school properties.

${ }^{10} \mathrm{http://ppf.tdsb.on.ca/uploads/files/live/93/185.pdf}$ 


\subsubsection{Concerns \& Barriers}

i. $\quad$ Municipal Concerns with School Land Disposal

In a Staff Report, the City of Toronto expressed its concern with school closures happening due to ageing school building and changing student population. The report states that the City is not opposed to the sale of school properties, however it encourages the school board to consider the social and community value of the property. Some school properties hold a great value for the surrounding community and neighbourhood, that surpasses the physical economic value of the property. Losing these properties can have a negative impact on their well-being.

The discussion of school closures and the sale of school properties has become a great concern for the City and the community. As mentioned earlier in a 2016 Staff Report, the City of Toronto expressed their concern with the speed at which school properties are being sold at. They had stated that while there is a designated reserve fund for the purchasing of school properties, it is no longer able to continue to purchase these properties at fair market value with only $\$ 6$ million remaining in the fund. This is seen as an issue particularly for school properties that support public infrastructure and provide community services for the local neighbourhood. In addition, the moment these properties are taken out of public ownership, it becomes increasingly difficult for the public sector to utilize these properties for community use. The staff report provides recommendations in order to mitigate this issue. Their main recommendation is to have the Province of Ontario play an active role in coordinating the process of identifying and acquiring TDSB properties that "represent valuable public infrastructure". This infrastructure includes facilities that deliver provincially funded services, including mental health services, child and youth services, and community and social services.

However, holding on to a school identified as surplus is also not financially or socially viable. Even with the school shut down, it needs to be maintained and inspected regularly in order to ensure that it is not vandalized, destroyed, or broken into. And to ensure that there are no issues with the building utilities, such as electrical wiring potentially starting a fire or a water pipe bursting during the winter. Accidents and emergencies such as these become more likely for extremely old school that are in critical structural conditions. This is an additional cost the school board must cover for something that is not providing any value for the surrounding community, 
which could have been otherwise invested into other projects and programs. For example, the school board in Milwaukee in the US spent more than \$1 million annually maintaining its 27 surplus schools (DCG Strategies, n.d.). Hence, it is clear that balance needs to be established when dealing with surplus school properties, ensuring that these properties are not easily sold off and that taxpayer money is not being invested into a black hole.

\section{ii. $\quad$ Education Development Charges (EDCs)}

EDCs are a source of funding that school boards can receive by charging new development within close proximity to their schools. EDCs allows school boards to charge developers a percentage and allows them to purchase land to build more schools to accommodate the influx of new students into a particular area. Boards are not allowed to spend EDC funds on the building/construction of a new school or renovating an existing one. In the case of Toronto, the TDSB is not qualified to collect EDCs because its schools are not operating at full capacity (i.e. low student enrollment/utilization rate).

As mentioned earlier, certain parts of Toronto have experienced an increase in high-density, vertical residential development over the past decade. This has resulted in an influx of new residents entering Toronto neighbourhoods and it is projected that there will be 214,000 new residential units over the next 15 years, which has and will continue to cause pressure on TDSB schools. Currently, the TDSB has adopted accommodation strategies in order to manage the enrolment growth at some of its schools. These strategies include using portables, relocating new students to schools further away from their home schools, and the changing of school boundaries (TDSB, 2014e). However, these strategies are not enough to address the root of this issue. Councillor Mike Layton has expressed that students attending over-capacity schools in fast growing neighbourhoods should not be penalized just because schools in other parts of the city are operating under-capacity (Gordon, 2017).

Currently, the Toronto Catholic District School Board (TCDSB) is one of the school boards in Toronto that is able to tap into this source of funding. However, due to the spending restriction that EDCs place on school boards, it has not been a complete benefit for the TCDSB. A Toronto Star article explains that since EDCs can only be used to purchase land for new schools, this can 
be hard for school boards located in Toronto due to the limited amount of vacant land in in the City (Gordon, 2017).

As a result, the TDSB has been and continues to advocate for the Ontario Government to amend the EDC regulations under the Education Act, in order to allow the TDSB to use this tool and possibly use these funds to solve its ever-growing renewal backlog (TDSB, 2014e). Nonetheless, the TDSB is aware that the ability to access EDCs is not a permanent solution to address their renewal backlog and deteriorating school buildings issue. There is a need for a new and improved "funding strategy to reduce" the TDSB's "Renewal Needs Backlog and maintain and operate" its schools (TDSB, 2014e).

\section{iii. $\quad$ Better Schools, Brighter Futures Program}

In 2012, the TDSB had established a program called the Better Schools, Brighter Futures. The program was developed for the purpose of addressing the need for better and advanced programming, to address the declining student enrollment, the need to replace aging school building structures with new and innovative facilities, and the current pressures on both the operating and capital TDSB funding (TDSB, 2014f).

As mentioned earlier, the Toronto Lands cCorporation is a subsidiary of the TDSB and they manage the leased TDSB properties and are responsible of disposing properties that are declared surplus by the school board. The launch of this new program gained TLC the approval from the Ministry of Education that allows them to invest any funds collected from leasing or from disposing/selling of land into renovating existing schools or invested into building new school facilities that replace the older ones (TDSB, 2014f).

However, when considering the pace at which the TDSB's renewal backlog is growing, the long list of deteriorating and aging schools, and the selling of 60 plus TDSB properties, there is still a need for more work to be done. And while this program sets the goals and principles for how TDSB properties should be assessed, there is still no clear tactical plan that can put this program into motion. 
And while O. Reg. 444/98 provides a step by step process of school land disposition after it has been declared as surplus by the TDSB, it does not provide any guidance on how to select the ideal tenant or buyer for the land and ensure that the proposed development replaces previous public services and improves the overall well-being of the surrounding community and neighbourhood ${ }^{11}$. There is a need for an action plan that can be followed or referenced when a property is being considered for the leasing or the procurement and for redevelopment.

\subsubsection{Community Hubs Initiative}

In May of 2016, the Ontario government announced that surplus school properties can be considered for the placement of a community hubs or any other public services, while also ensuring that the school board receives "good economic value for the property". This initiative has been called the Community Hub Action Plan. Locating community hubs on surplus school properties is seen as ideal, because it is easily accessible to the surrounding neighbourhood and community (Carroll, Pasquino, Haile, 2016a). This is an example of a provincial initiative to address the issues some school boards are currently facing (including the TDSB), such as the declining public school enrollment, aging school buildings, and the ongoing increase of renewal/repair backlog (Carroll, Pasquino, Haile, 2016).

It is part of the Ontario government's initiative to foster collaborations between different government bodies and agencies to achieve cost efficient and effective delivery of services to diverse communities and neighbourhoods (Carroll, Pasquino, Haile, 2016b). The Community Hub Action Plan addresses and attempts to solve challenges relating to planning, integrated service delivery, and community infrastructure/ public properties. This allows organizations to address these issues without waiting for legislative or policy changes to happen (Carroll, Pasquino, Haile, 2016b).

The use of the term "community hub" for this initiative is brilliant and most ideal. The term "community hub" does not have a specific definition, model, or requirements. According to

\footnotetext{
${ }^{11}$ Note that the buyer is to be selected or negotiated after the land had been off the market for 90 days so that school boards and non-profit organizations are given a chance to put in an offer on the property.
} 
MPAC, community hubs are service delivery models, bringing a wide range of services and amenities to a neighbourhood to satisfy the needs and priorities of the surrounding community. These services and amenities can include affordable housing, recreational services, health clinics, legal aid, settlement and employment services, and child care facilities, just to name a few (MPAC, 2018). Hence, a community hub is what the surrounding community defines it to be. 


\section{Chapter 3: Literature Review:}

\subsection{Shrinking Cities}

\subsubsection{Declining Populations \& Under-utilized properties}

The issues the TDSB is currently facing in terms of declining student enrollment, low utilization rates, and aging and structurally deteriorating school buildings, are an example of a larger planning phenomenon that is occurring on a bigger scale for some cities around the world. This planning phenomenon is known as shrinking cities, which refers to the decline of population within a densely populated urban area (Hollander \& Nemeth, 2011). The impacts of shrinking cities are not limited to a declining population, but also include an economic transformation such as a decline in employment and tax revenue. Another major impact or sign of shrinking cities, and that is relevant to this research, is the aging and under-utilization or abandonment of public buildings and properties (Lee \& Sung, 2017).

And while Toronto is not necessarily facing a decline in its population, the TDSB is currently faced with this issue when it comes to student enrollment. As mentioned earlier, approximately $22 \%$ of TDSB schools are currently operating at a utilization rate of $65 \%$ or lower. In addition, a large proportion of these schools have significantly aged and have deteriorated in condition. These schools have proven to becoming an economic and social burden on the TDSB, which has been discussed in length in Chapter 2 of this research. However, in order to deal with these issues, one of the strategies adopted by the school board is the selling of these school properties. Between 2008 and 2015, 67 schools have been sold by Toronto Lands Corporation (Altus Group, 2016). And while the sale of these surplus school properties has generated a substantial amount of revenue for the school board, there is a concern that these prime real estate properties will be lost and will be difficult, if not impossible, to retrieve or find something similar to it in Toronto's limited land inventory.

\subsubsection{Planning: Public Redevelopment}

When attempting to understand the root reason for shrinking cities, there are several reasons for the causation of this planning phenomenon. This can include natural disasters, deindustrialization, suburbanization, globalization and the boom and bust of the economic cycle (Hollander \& Nemeth, 
2011). Nonetheless, regardless of what the reason maybe, what truly matters is how the issue is addressed and dealt with.

Hollander (2011) explores a new planning phenomenon called smart decline, which is "planning for less, fewer people, fewer buildings, (and) fewer land uses" (Pg. 131). In other words, planning should be prepared for "social emergencies" such as these and must have a contingency plan that allows the city to adjust to these changes and make the best out of them, rather than work to reverse this phenomenon. City officials and planners should ensure that the city experiences an "effective shrinkage", which means that the number of abandoned buildings and vacant lots are controlled and minimized, and that there is a plan in place to convert them into other land uses, such as parks, recreational, retail and/or residential uses (Hollander, 2011).

Hollander's (2011) article explains that the only option to addressing the issues caused by depopulation is to consider and rethink public redevelopment. The article states that the public redeveloping of a shrinking city aims to improve its overall economic conditions and it improves "the physical realm of an area" (Hollander, 2011, Pg. 130). However, public redevelopment may not be achievable for every single property due to limited resources and funds. As a result, with these demographic and urban changes and with limited funds, there is a need for a land use planning strategy and reforming of policies and regulations to address these issues in an efficient and cost-effective manner.

\subsubsection{Benefits of a Declining City}

With under-utilized or abandoned properties, there is an opportunity to enhance the overall quality and public health of communities and neighborhoods. For example, there is the opportunity to install or enhance the green infrastructure within the neighbourhood, that otherwise would not be possible due to limited space and/or funds (Schilling \& Logan, 2008).

An example of a city that has experienced the shrinking cities phenomenon is the city of Youngston, in the state of Ohio. As the city's population declined, the Master Plan was adjusted to adopt this change and to plan for a "better, smaller Youngstown". Rather than focusing on 
growing the city and attracting foot traffic, the updated plan aims to improve the quality of life for remaining residents (Hollander, 2011).

The repurposing and redevelopment of underutilized public properties will provide social and economic opportunities for the surrounding community. Some of these benefits or opportunities include the replacement of aging infrastructure and structures with brand new buildings, allowing the board or city to eliminate any repair costs associated with that particular structure. It also opens up the opportunity to brainstorm creative ideas to repurpose and redevelop the property, ensuring that this time around community needs and gaps in servicing and housing are met or addressed (BC Housing, n.d.). The BC Housing article also indicates that it provides the opportunity to build environmentally sustainable structures. According to the Advisory Committee for School Repurposing and Community Development in Chicago, if a vacant property is repurposed or redeveloped in an effective and efficient manner, these buildings or sites can provide "opportunity and positive change" to the surrounding communities (Milhouse, 2014, Pg. 8). With that being said, the benefits and opportunities that are a result of repurposing a vacant public property, can take a different form and can extend beyond the above list, depending on the nature and the surrounding environment of the property.

\subsubsection{The Need for Action}

Moreover, to summarize, the research on shrinking cities focuses on the benefits and value of this phenomenon and provides a general guideline of how planning should address issues such as declining populations and vacant public properties. However, similar to the TDSB's approach to addressing under-utilized deteriorating school buildings, there remains a missing discourse in regards to an action plan. And while Hollander (2011) states that a major strategy to addressing depopulation is rethinking public redevelopment, he does not provide any suggestions or recommendations on how this rethinking or redevelopment will look like and what is it trying to achieve exactly. Hence, there is a need for narrowed down recommendations, that can be customized for different geographical contexts, in which public officials can use as an action plan to act upon. 
This research paper attempts to fill in this gap and add further dimension and direction on this issue of shrinking populations (or student enrollments for that matter) and deteriorating public buildings (or schools). This research paper proposes and explores a land assessment tool that will allow public officials to efficiently identify, prioritize, analyze and repurpose under-utilized or vacant public properties. It is important to note that this recommended tool is not the ultimate and only tool that will address the shrinking cities phenomenon and the issues associated with it. However, it does provide a starting point for public officials that want to take action to address issues of declining populations and deteriorating public properties.

To further understand how this land assessment tool would look and function like, the next section will provide examples of cities and/or states using slightly different versions of this land assessment tool. These examples will also inform the structure and function of the assessment tool recommended/proposed for the TDSB in chapter 4 of this research.

\subsection{Repurposing and Redeveloping Assessment Practices}

The repurposing and revitalizing of deteriorating, under-utilized, and vacant public assets is not a new phenomenon. Different jurisdictions follow different strategies and processes that work best for their communities. As a result, this section will be looking into various approaches and systems that different school boards and municipal governments, from different jurisdictions, follow when it comes to determining which pubic properties are in poor conditions and are physically, economically, and socially better off being rebuilt and repurposed.

\subsubsection{Michigan}

In the state of Michigan, they have developed a guide for the repurposing of vacant and underutilized historic buildings. Similar to the TDSB, their guide allows public officials to develop a plan that will allow them to "identify, evaluate, market, and finance these buildings so they can...become valuable resources to the community" (Taylor, 2014, Pg. 2). Their process of repurposing vacant public assets begins by identifying and prioritizing buildings that are need of repurposing or redevelopment. This step of the assessment constitutes inspecting its architectural and structural integrity, including the roof, foundation, evaluating hazardous materials, safety features and accessibility infrastructure. This step is similar to identifying what the FCI is for the 
building at the TDSB. This will ensure that resources are invested efficiently and effectively into properties that are most in need.

However, unlike the TDSB, this assessment goes beyond the factors and parameters listed above by the TDSB. The community guide developed by the State of Michigan also explores whether the building is considered to be a "community anchor or local landmark" (Taylor, 2014). This step of the process considers the current social and community value of the property to the residents and neighbourhoods surrounding this property/building. This does not solely refer to the sentimental or nostalgic value that people tend to place on an old building or site they knew from a young age. It also refers to the public services this building provided the community and neighbourhood, such as a city hall, fire department, library, hospital or school (Taylor, 2014).

In some cases, public schools provide more than just educational services. They also provide space that is utilized by the community for various reasons. Understanding this value is important because it sets a precedent or a standard of what the future repurposing project should provide for the area at the very least. The process or guide also explores whether the repurposing or redevelopment of the site will result in a financial return for the community. The return can be in the shape of housing, needed public services, jobs, or even the revitalization/rebuilding of the existing building (Taylor, 2014).

\subsubsection{Chicago, Illinois}

In the state of Illinois, the Chicago Board of Education had requested from the Advisory Committee for School Repurposing and Community Development to develop a set of guiding principles and a proposed process for the repurposing/redeveloping of schools that have been identified as surplus by the school board. The document or guide developed by the Advisory Committee provides a guideline of "how to make the most efficient use of these properties in timely, financially viable ways that returned them to constructive use quickly" (Milhouse, 2014, pg 6).

The initial step of the process the advisory committee conducts a physical and financial assessment/ review for each property. To determine what kind of uses and development can be proposed for the property, the city of Chicago considers a list of principles and criteria when 
reviewing a development application/ proposal. Some of these criteria include ensuring that the proposers are able to carry out the project, the proposal project provides community support and benefits, the financial viability of the project, whether the proposal project complements the current character of the surrounding community, and ensuring there are employment opportunities during and after construction.

\subsubsection{Columbia, Washington}

In the state of Washington, in the district of Columbia, the evaluation process for a surplus school is similar to the above US examples. However, the District of Columbia implemented a point system rubric to score potential tenants and buyers that are interested in the surplus school property. Some of the criteria on this rubric include Financial Feasibility (25 points), Project Vision and Implementation Plan (20 points), Demonstration of how the respondent will meet identified needs and provide equitable service (15 points), and Community Engagement and Access (15 points) (DME, 2017). This allows the board to rank school tenants or buyers based on the services they will provide that will benefit the community the most. 


\subsection{Toronto Case Studies}

This section will present two Toronto case studies of schools declared surplus and the unique approach taken to repurpose and redevelop the properties to their highest and best use.

\subsubsection{Bloor-Dufferin Project}

A well-known project underway at the moment is the Bloor-Dufferin redevelopment project. Two large high schools, Bloor Collegiate Institute (includes the Alpha 2 Alternative school) and Kent Senior Public School, located on the corner of Bloor Street and Dufferin, were determined to be surplus school properties in 2016. According to the 2016-2017 TDSB dataset, Kent Senior Public School was built in 1909 , making it a 108 -year-old building with a student capacity of 794 . The building has an FCI of $160 \%$ and had a $\$ 16.1$ million repair backlog. The Kent Senior Public School is also designated as a historical building. As for Bloor Collegiate Institute, it is a 101-yearold school with a student capacity of 792 students. The school had an FCI of $77 \%$ and a renewal backlog of \$19.5 million (TDSB, 2014).

After going through the policies and regulations outlined in O. Reg. 444/98, the TDSB was seeking a proposal for the repurposing of the 7.3-hectare site. Once the successful proponent, Capital Developments and Metropia, was selected to redevelop the site, the public consultation process began. The final proposed project for the property was 2,219 residential units, retail space, office space, and community space. The development will also include a new public park and the creation of two new north-south streets. The 3-storey historic Kent school will be retained and restored and is proposed to be converted into a 30,000-square foot community hub, that will include space for a day care, on the main and basement floor, and office space on the second and third floors (Harrison, 2018).

Adjacent to the Bloor-Dufferin site is Brockton High School, located at the northeast corner of Croatia and Brock Street. On December 2016, the Government of Ontario announced that it will be providing \$20 million in funding for the construction of a new secondary school that will accommodate approximately 900 students. The funds provided by the Ontario government are 
expected to be spent on the construction of a new 30,000 square feet community hub, providing space for a child care centre and for community programming (Mirabelli, 2017).

This project is a perfect example of a public private partnership, between the city, the school board, the developer, and most importantly with the surrounding community. Understanding the needs of each of these stakeholder groups allowed for an informed negotiation resulted in benefits for each group. As part of the procurement of the site, the Toronto Lands Corporation ${ }^{12}$ negotiated with the developer to include a community hub. This is seen as an innovative and ground-breaking procurement process. From a financial standpoint alone, the redevelopment project also includes the replacement of two aging schools, which eliminates a total of $\$ 35.6$ millions of renewal backlog. The purchase price for the site was $\$ 121$ million, which can be reinvested into other TDSB school buildings/properties that are identified as a priority.

\subsubsection{Midlands and Lawrence School Property}

This project involves the consolidation of two high schools: Bendale Business and Technical Institute at 1555 Midland Avenue in Scarborough, and David and Mary Thomson Collegiate Institute at 2740 Lawrence Avenue East also in Scarborough. There is approximately $1 \mathrm{~km}$ distance between the two schools which equates to a 10-minute walk.

After the property had been declared as surplus and has gone through the disposal process outlined under O. Reg. 444/98, the Midland and Lawrence school property has been approved for a new project proposal. The TDSB is currently in the process of beginning the construction of a new school, a modern track and field facilities, and providing additional community walkways. In addition, the two separate parcels on the property, one at 7.6 acres and the other at 9 acres, have been sold for residential redevelopment (TLC, n.d.). This project is another example of a mixed used development.

If we look at the statistics for each school, it provides us with a better understanding of what the TDSB, students and parents gained from this agreement. For Bendale Business and Technical

12 an agency of the TDSB that manages school lands that have been declared surplus by the school board 
Institute, according to the 2016-2017 dataset, the school has an FCI of 70\% and a renewal backlog of $\$ 18.2$ million. The school had a capacity of 945 students however it was only being utilized at a rate of $43 \%$. Looking at the utilization rate projections for the next 5, 10, 15, and 20 years, the rate remains between $42 \%$ and $43 \%$. As for David and Mary Thomson Collegiate Institute, the high school has an FCI of 77\% and a backlog of $\$ 31.2$ million. The school had a capacity of 1623 students however it was only operating at a utilization rate of $60 \%$. According to the $5,10,15$, and 20 years student enrollment projection, the student enrollment utilization rate decreases and ranges between 52\% and 55\% (TDSB, 2014).

The new school facility will be accommodating students from both high schools, a total of 1500 students from grades 9 to 12 . Considering that it is only a 10-minute walk between the two schools, the consolidation of the two properties makes it viable for students from both high schools. The school has 4 floors, with classes dedicated from science labs, visual and media art rooms, construction technology, culinary art, and environmental sustainability. The construction of the school will be complete by September $2019^{13}$. With that being said, the community will not only gain a new state-of-the-art high school, it will also be able eliminate two schools in poor structural conditions and high FCIs, and eliminate a combined repair backlog of approximately $\$ 50$ million.

\footnotetext{
${ }^{13}$ For more information on the project, click on the following link: http://schoolweb.tdsb.on.ca/bendale/LawrenceMidland-Project
} 


\section{Chapter 4: Proposed Methodology for Surplus School Repurposing}

After exploring the various methodologies and approaches taken by various jurisdictions, chapter 4 will be presenting the proposed methodology for effectively and efficiently repurposing surplus school properties. The assessment process consists of $5 \mathrm{steps} / \mathrm{phases}$. The chapter will provide a detailed description of how each step/phase will be structured and carried out upon application.

\subsection{Pre-Assessment Phase}

Before diving into the land assessment tool, there is a pre-assessment phase that needs to be established. Section 4.1 will be discussing the need for an advisory committee with members of various skills and expertise. And it will also discuss the benefits of having a public private partnership when it comes to repurposing/redeveloping a public asset.

\subsubsection{Advisory Committee}

Similar to many of the land disposal processes mentioned in the previous chapter, including the TDSB, an advisory committee is needed in order to conduct a thorough assessment. Currently in Toronto, to carry out the Pupil Accommodation Review, a committee is formed to present an initial staff report on the property, hold public consultations for the community, school staff, and students, and providing a final report with recommendations on an action plan. The committee must include parent and guardian representatives from the school that is under review. However, it is not clear what kind of credentials these committee members have to conduct this process other than they are employees of the TDSB.

In the City of Chicago, in their School Repurposing and Community Development Guide, they state that it is necessary to have an advisory review and evaluation committee with members of skills and knowledge that will be an asset and benefit for the overall process (Milhouse, 2014). The six members on this particular advisory committee should each have expertise in one of the following categories:

- Community and Neighbourhood Planning

- Expertise should be in planning, zoning, and land use, ensuring that proposals for repurposing and redevelopment are "consistent with existing plans for their 
local areas, and are compatible with other neighborhood uses" (Milhouse, 2014, Pg. 14).

- Community Benefit

- This individual is to weigh in and identify the potential benefits (or lack of) the proposed project has for the surrounding area. The member is preferably from the neighbourhood development group or a community service provider.

- Job Creation

- This member would provide guidance and suggestions on potential job creation opportunities that can occur on the subject property. This includes job creation during and after the construction phase.

- Economic Development

○ This member provides input on tax generation and other potential economic benefits. The member can be from the chamber of commerce, a real estate consulting firm, or from the City's department of planning and development.

- Project Implementation

- A person, which can be a non-profit or for-profit developer, would provide their expertise on the feasibility of implementing a particular proposal on the subject site.

- Community Engagement

- This person can be a parent or a community representative providing input and perspective of a local resident.

Having each individual from the committee have at least one of these skills provides an informed discussion upon reviewing surplus school properties and reviewing future proposals for these sites. In addition, the Chicago advisory committee works closely with the Chicago Public School to develop criteria for proposals and to provide guidance on the community process (Milhouse, 2014, Pg. 14). 


\subsubsection{Public Private Partnership}

The purpose of Public Private Partnerships (PPP's) is to assist in the implementation and/or financing of a new project or business plan. This partnership allows for the costs and risks associated with a project, such as building public infrastructure or a public facility, to be shared among the public and private sector (ACEC, n.d.). In some cases, the public sector may not be able to deliver necessary services to the community in need within a timely matter due to limited resources and funds or is unable to pay for the maintenance of a service or facility. Hence, with a collaboration between the private and public sector, the City or anybody of government, can carry this service or project out without any delays and be assisted with the costs of operating and maintaining a property, facility, or service. With that being said, partnerships are not limited to the private sector. School boards are also encouraged to developing partnerships and collaborations with non-profit organizations. The Ontario Ministry of Education developed a guide that would promote more of this kind of collaboration.

\subsubsection{Non-Profit Partnerships}

To assist school boards in managing surplus school space, the province of Ontario has developed the Community Planning and Partnerships Guideline. This provides a framework for school boards that want to bring "a tenant or partner into a school or a portion of a school, on a cost recovery basis" (Ministry of Education, 2016, Pg. 5). These partnerships can include child care agencies, youth centres, settlement and health services, just to name a few (Ministry of Education, 2016).

It is the responsibility of all levels of government to maximize the use of vacant/surplus school space and to determine the highest and best use for these properties. Having partnerships between schools and non-profit organizations can strengthen the role of schools within a neighbourhood, providing surrounding communities access to programming and facilities that they would otherwise not be able to access easily. Hence, the guide was established to encourage school boards to collaborate with other school boards and community organizations (Ministry of Education, 2015). 
In terms of cost, the guide outlines that any operational and capital cost, such as administrative costs and property taxes, need to be charged to the partners. When it comes to new construction on the property, partners using or sharing the space are expected to pay and finance their share of construction as well. The guide states that cost details and expectations need to be made clear to future/potential partners (Ministry of Education, 2015).

\subsection{Phase 1: Identifying School Building Condition}

Similar to the cases mentioned above, the first step of this strategy is to identify schools that are considered to be in critical conditions. Similar to what is outlined in the Modified Pupil Accommodation Review, this research will be using the same standards to identify schools that are considered to be in poor conditions. These include: the FCI, Building Age, and Utilization Rate. This research will also be considering an additional factor and that is the renewal backlog ${ }^{14}$ for the school. While the FCI gives the committee an indication whether the school should be maintained or replaced, the renewal backlog places a price tag on the repairs and maintenance the school is in need of to be in "good condition". Note that the Modified Pupil Accommodation Review also considers the walking distance between two schools. The walking distance factor will be considered in the Second Phase: School Consolidation.

This phase can be used to prioritize which schools should be declared as surplus and/or for redevelopment. It is also important to note that the purpose of this phase is not to declare all properties in "critical condition" as surplus. Similar to the Bloor-Dufferin and Midland project, the objective of the assessment is to replace current school buildings, providing students with better school facilities, and an overall better learning experience. For each of the factors considered for

Phase 1 of the assessment, parameters have determined and a scoring has been applied for each parameter. These parameters will be entered into a software called Baragar Solutions Systems which is discussed further in section 4.1.7.

\footnotetext{
${ }^{14}$ As mentioned in the introduction, the renewal backlog is the said school's repair costs that have been accumulating over the years. In other words, any repair jobs that have not been completed in a certain school year, they get carried over to the next year and so on.
} 


\subsubsection{Facility Condition Index}

Based on the TDSB Capital Planning presentation (2015a) and the Accommodation and Program Review document (2016a), FCI is categorized and ranked in the following manner (blue columns):

\begin{tabular}{|l|l|l|}
\hline FCI & FCI Rating Range & Scoring \\
\hline Good & FCI $<10 \%$ & 5 \\
\hline Fair & $10 \%<$ FCI $<30 \%$ & 15 \\
\hline Poor & $30 \%<$ FCI $<65 \%$ & 30 \\
\hline Critical & $30 \%<$ FCI $<65 \%$ & 50 \\
\hline
\end{tabular}

The orange column is the scoring that was applied to each category in Baragar. The lower scoring refers to schools in good standing/good condition. The higher scoring refers to schools in worse conditions.

\subsubsection{Building Age}

The blue columns show how the variable was categorized and the orange columns shows the scoring for each category/group. The lower scoring refers to a newer/younger building and the high scoring refers to an older/aging building.

\begin{tabular}{|l|l|l|}
\hline Building Age & Building Age Range & Scoring \\
\hline Good & $4<\mathrm{BA}<30$ & 5 \\
\hline Fair & $30<\mathrm{BA}<60$ & 15 \\
\hline Poor & $60<\mathrm{BA}<90$ & 30 \\
\hline Critical & $90<\mathrm{BA}<126$ & 50 \\
\hline
\end{tabular}

Considering that majority of these are aging and are well above the 90 -year mark, it is important to check whether the school is also designated as a heritage building. However, that will be discussed in further detail in Phase 2 of the assessment. 


\subsubsection{Utilization Rate}

The utilization rate was categorized as shown below (blue columns):

\begin{tabular}{|l|l|l|}
\hline Utilization Rate & Utilization Rating Range & Scoring \\
\hline Critical & UR $<65 \%$ & 50 \\
\hline Poor & $65 \%<$ UR $<80 \%$ & 30 \\
\hline Fair & $80 \%<$ UR $<100 \%$ & 15 \\
\hline Good & $100 \%<$ UR & 5 \\
\hline
\end{tabular}

According to the Accommodation and Program Review, it focuses on schools with a utilization rate of $65 \%$ or lower (TDSB, 2016a). However, the document did not provide a category breakdown for schools with a utilization rate greater than $65 \%$. As a result, the rest of the values have been categorized based on my interpretation of the data. The orange column is the scoring applied to each category, with the lower scoring referring to a school with high utilization rate, and a lower scoring referring to a school with a high utilization rate or low vacancy rate.

\subsubsection{Enrollment Rate Projection}

The following step, is to evaluate the student enrollment projection for each school. While a school may be identified as "under-utilized" based on the high-level evaluation proposed above (i.e. utilization rate lower than $65 \%$ ), the following 5 years may indicate an increase in student enrollment projection and need for a school facility that will accommodate this increase. On the other hand, schools that may be identified as in "good standing" in terms of their enrollment rate (i.e. utilization rate greater than 65\%) may experience a decreasing student enrollment rate in the next five years.

There will also be a consideration for school properties with a utilization rate of $100 \%$ or greater. Over-populated schools tend to place pressure on its academic and extra-curricular programs. Hence, this evaluation will also look into the enrollment rate projections for these over-populated schools in order to understand if this high enrollment is due to certain circumstances that occur in one school year, or if this is an ongoing trend that needs to be addressed with a larger and newer school facility. This will be further explored in the school consolidation phase of this assessment. 


\subsubsection{Renewal Backlog}

\begin{tabular}{|l|l|l|}
\hline Renewal Backlog & Renewal Backlog Range & Scoring \\
\hline Good & $\mathrm{RB}<\$ 5 \mathrm{M}$ & 5 \\
\hline Fair & $\$ 5 \mathrm{M}<\mathrm{RB}<\$ 10 \mathrm{M}$ & 15 \\
\hline Poor & $\$ 10 \mathrm{M}<\mathrm{RB}<\$ 15 \mathrm{M}$ & 30 \\
\hline Critical & $\$ 15 \mathrm{M}<\mathrm{RB}$ & 50 \\
\hline
\end{tabular}

The renewal backlog variable was categorized as shown in the blue columns. The values were categorized/grouped in equal intervals. The lower scoring referred to a school with a low renewal backlog and the higher scoring referred to a school with a higher renewal backlog.

\subsubsection{Baragar Systems}

The factors, parameters, and scorings described above will be entered into a geographic software known as Baragar Systems. This software specifically geographically maps out school catchments and any statistics related to that school. It allows for the user to geographically analyze data associated with each school by conducting queries using different factors. It functions similar to ArcGIS software; however, only data related to schools can be entered and analyzed on this software. Section 5.1 will demonstrate how the software is use and what it is capable of producing.

\subsection{Phase 2: School Consolidation}

\subsubsection{Consolidation}

A major part of the process of repurposing/redeveloping a school site is understanding and being aware of other school properties within close proximity of the subject site. Before considering a property for repurposing/redeveloping, it is important to know what are the needs of schools surrounding the subject site, such as the need for additional space, the need for additional academic and extra-curricular programming, or the need for a completely new facility or building to replace an aging one. For example, in the state of Washington, in order to determine if a school is surplus, City Council must also consider the surrounding school area, including nearby enrollment, capacity, utilization rates, and academic programs (DME, 2017). By consolidating two aging schools, identified to be in "critical condition", the TDSB, students, and the surrounding 
community gain many benefits: The TDSB is able to eliminate two aging schools (or more), both with high FCIs and repair backlogs; Students gain a new building with up-to-date technology, equipment, and new program opportunities; And with the redevelopment of the site, there is an opportunity to provide additional services that would benefit and satisfy the needs of the surrounding community.

Once a school is identified as being in "critical condition" (i.e. identified in phase 1 of the research), this phase looks at surrounding school catchments and for the opportunity to consolidate with other schools. Schools that are within close proximity to one another and are also underutilized could be considered for consolidation, which means both schools would be combined onto one property, where a new school will be built. This will allow for the opportunity to build a completely new school building, providing students with a new school facility with advanced resources, technologies, and contemporary structural design. At the same time, the TDSB will be able to remove aging schools from its inventory and eliminating any repair backlog associated with each school. The selection of a suitable property for the purpose of combining/consolidating multiple schools will be based on factors such as location, land acreage, and walking distance to and from that location. The walking distance parameters are stated in Chapter 2, Section 2.2.1.2. Through this process of consolidation, this will result in having a vacant school property, which takes the research to phase 3 of the analysis.

\subsubsection{Heritage}

After identifying schools that are considered to be in poor conditions, it is important to identify which of these schools is declared as a heritage building. This will dictate whether the building can be completely replaced with a newer facility, or if the exterior walls and façade of the building are maintained while the insides are refurbished and upgraded. As observed from the Michigan repurposing guide in section 3.2.1, the state checks if the building on the subject property has been declared to being a historical/heritage building before considering it for potential redevelopment. 


\subsection{Phase 3: Social Scan}

The social scan will review and analyze demographic data of the population living in the area surrounding the school property. This will provide a better understanding of who is living in the area and what their needs are in the area. Knowing who lives in the surrounding community allows for an informed decision making process when it comes to deciding the type of resources and services that should be invested in the future. Understanding and analyzing demographic data provides insight into the types of businesses that could move into the area, if the area is in need of employment or language services, if there is a demand for after school programming or space for non-profit organizations and so on (French, 2014). From a planning perspective, demographic data shapes the neighbourhood's Master Plans and zoning by-laws and regulations (French, 2014).

This information will be based on an in-depth research of the Statistics Canada 2016 census data. As part of Phase 3 of the proposed assessment, school properties identified to being in poor condition (i.e. in Phase 1) will undergo an in-depth research and review of the following datasets:

- Age and population

- Household Structure (single couples, married couples with kids, no kids, etc.)

- Race \& language

- Income status

- Employment \& Unemployment Rate

- Occupations

- Tenure Type (Home ownership, rental, affordable housing, co-op housing, etc.) 


\subsection{Phase 4: Community Scan}

The community scan will analyse the available community/public services available and missing in the surrounding area. This type of assessment is conducted to collect data and analyse the community's problems and weaknesses, to identify the community's needs, to identify the services, resources and assets the community currently owns and has access to. This phase will also analyse and identify what services the subject school property provided to the community. This phase will ask questions such as:

- Does the school run extra-curricular activities after school hours?

- Do community groups use the school building after school hours?

- What existing services and amenities does the area have within walking distance? Examples include (but not limited to)

○ Community Centre

- Local and diverse businesses

- Public and Green space

○ Grocery stores

- Language and immigration centre

- Employment centre and services

- Medical clinics and/or hospitals

- Child care services

- Local restaurants and dining spots

- Emergency services (ambulance, police department, fire department)

- If not accessible within walking distance, are these services and amenities accessible via public transportation?

It is important to have this phase come after Phase 3: Social scan in order to see if current services in the area are truly catering to the needs of the current neighbourhood population. For example, if majority of the population have children and are working parents, it may be reasonable to consider bringing in child care services or provide after school programming that provides homework/academic assistance for the students. As a result, the questions asked and investigated in this phase may shift and change depending on the school property. 
It is important to note that using geographical data that locates the existing services within the area (i.e. secondary data) is not necessarily sufficient to truly capture the quality of these services. In order to truly understand the current quality and effectiveness of these services, an in-depth investigation needs to be done on a qualitative scale. In other words, based on a report by the Ontario Centre of Excellence for Child and Youth Mental Health, it suggests to conduct the community assessment and collect data from multiple groups living or providing services (private or public) within the area. These groups include health and human providers, government officials, community activists, businesses, and residents, just to name a few. The report suggests various methods such as looking into existing data, conducting surveys, interviews, consultations, observations, inventories, and so on (Ontario, 2013).

Hence, this scan can be considered to be a "preliminary" scan to provide the committee a highlevel idea of the area's strengths, weaknesses, needs, and wants. The results accumulated from the community scan can assist in guiding upcoming public consultations, allowing for a slightly more narrowed down discussion on what the issues the area and its residents may be facing. Once, it has been established that further information is needed to determine the true issues the area is facing and its needs, an internal or external team can be appointed to conduct an in-depth community assessment. 


\subsection{Phase 5: Development Scan}

In this stage of the analysis, a full planning due diligence review of the land will take place. Conducting a development scan or a planning due diligence of the property will identify the provincial and municipal regulations and policies that apply to the site (Halinski \& Grover, 2010). The development scan will also identify zoning by-laws that dictate the permitted uses on the land, maximum/minimum density, size and height of the building, building setbacks, required parking spaces, just to name a few (Taylor, 2014). Nonetheless, understanding the overall provincial and municipal planning and zoning regulations and policies, allows the TDSB and the City to be aware of what kind of development could potentially go on the land.

It also provides a heads-ups for the school board and the City of some of the zoning by-law amendments the developer will need to go through in order to gain approval for a certain use, to increase height/density, to deal with setback restrictions (Halinski \& Grover, 2010). This sets realistic expectations for the proposed project timeline, potentially saving time and reducing risk for everyone.

In the City of Toronto, the following searches are included in the conducting a planning due diligence (Halinski \& Grover, 2010):

1. Provincial Policy Statement

2. Provincial Plans
a. Oak Ridges Moraine Conservation Plan
b. Niagara Escarpment Plan
c. Greenbelt Plan
d. Greater Golden Horseshoe Growth Management Plan
e. Parkway Belt West Plan

3. Official Plans
a. Official Plan
b. Secondary Plan(s)

4. Zoning By-laws 
5. Committee of Adjustment Decisions (Variances)

6. Ontario Municipal Board Decisions

7. Heritage Act Designation or Listing

8. Conservation Authority Regulation

9. Municipal Ravine and Tree By-Laws

10. Municipal Demolition of Rental Housing Controls

11. Statutory Agreements Registered on Title

12. Legal Non-Conforming Status Enquiry

As mentioned earlier, the process and procedure followed for each phase may be slightly different depending on the property in question. Hence, these searches provide a head start or guidance of how to apply or conduct the development scan or planning due diligence for the property, but it does not necessarily mean that these are the only questions that must be asked (Halinski \& Grover, 2010). 


\subsection{Summary}

The proposed tool attempts to effectively and efficiently repurpose and redevelop surplus school properties. The table below summarizes the proposed tool and the purpose of each step in the assessment.

\begin{tabular}{|l|l|}
\hline Pre-assessment Phase & $\begin{array}{l}\text { Establish advisory committee and potential private } \\
\text { or non-profit partnerships }\end{array}$ \\
\hline Phase 1: Identifying School Building Conditions & $\begin{array}{l}\text { This phase will identify schools that are considered } \\
\text { to be in critical condition. The factors that will be } \\
\text { considered to identify schools in critical conditions } \\
\text { are the FCI, building age, utilization rate, and } \\
\text { renewal backlog. The purpose of this phase is to } \\
\text { establish a priority list of schools that should be } \\
\text { catered to right away. }\end{array}$ \\
\hline Phase 2: School Consolidation & $\begin{array}{l}\text { Once a list of schools in "critical condition" have } \\
\text { been complied, this phase looks at surrounding } \\
\text { school catchments and for the opportunity to } \\
\text { consolidate with other schools. The selection of a } \\
\text { suitable property for the purpose of } \\
\text { combining/consolidating multiple schools will be } \\
\text { based on factors such as location, land acreage, and } \\
\text { walking distance to and from that location. In } \\
\text { addition, in this phase, we will also identify if the } \\
\text { school property is designated as a heritage } \\
\text { building. }\end{array}$ \\
\hline
\end{tabular}




\begin{tabular}{|c|c|}
\hline Phase 3: Social Scan & $\begin{array}{l}\text { In phase } 3 \text { of the assessment, demographic area for } \\
\text { the communities and neighbourhoods surrounding } \\
\text { the school will be reviewed and analyzed. Data } \\
\text { collected for this phase will include age, } \\
\text { population, household structure, immigration, } \\
\text { language, income status, employment, and tenure } \\
\text { type. This phase will provide the advisory } \\
\text { committee a clear idea of who lives in the area and } \\
\text { what their social and economic needs may be. }\end{array}$ \\
\hline Phase 4: Community Scan & $\begin{array}{l}\text { The community scan will analyse the available } \\
\text { community/public services available and missing } \\
\text { in the surrounding area. This phase will also } \\
\text { analyse the role the school site in the area played } \\
\text { for the community by identifying what services the } \\
\text { school provided to the community. This phase will } \\
\text { ask questions such as: Does the school run extra- } \\
\text { curricular activities after school hours? Do } \\
\text { community groups use the school building after } \\
\text { school hours? What existing services and amenities } \\
\text { does the area have within walking distance? } \\
\text { Examples include (but not limited to) Community } \\
\text { Centres, libraries, Public and Green space and } \\
\text { Grocery stores. }\end{array}$ \\
\hline Phase 5: Development Scan & $\begin{array}{l}\text { In this stage of the assessment, a full planning due } \\
\text { diligence review of the school property will take } \\
\text { place. The development scan will identify zoning } \\
\text { by-laws that dictate the permitted uses on the land, } \\
\text { maximum/minimum density, size and height of the } \\
\text { building, building setbacks, required parking } \\
\text { spaces, just to name a few (Taylor, 2014). }\end{array}$ \\
\hline
\end{tabular}




\section{Chapter 5: Analysis}

To grasp an idea of how this methodology or 5-step assessment would look like if applied in a real-world setting, this chapter will be applying the assessment to 4 adjacent school catchments. Note that this assessment will not investigate or explore every single dataset, service, amenity, and zoning policy related to this area. The purpose of this assessment is to provide an example of what it would look like if it were to be carried out in real life.

\subsection{Phase 1: Identifying School Building Condition}

The factors and parameters outlined in phase 1 (section 4.3), were entered into Baragar Systems software. Using Baragar allows the user to conduct phase 1 for all schools at once, rather than entering in the data separately for each school. However, for the rest of the assessment, each property will need be assessed on a school to school basis. Hence, for the purposes of this study, this research will only focus on a cluster of adjacent school properties.

Once the factors, parameters, and scores were overlapped, they resulted with the map in Figure 2 and $\mathbf{3}$ (below). Figure $\mathbf{2}$ shows a map of TDSB elementary schools and Figure 3 shows a map of TDSB high schools. For each map, the schools have been categorized into 4 different quartiles. The colour associated with each school catchment area indicates the amount of points that were assigned for that school, indicates the condition of the school. Schools in relatively "good condition" are in deep dark red and received a scoring between 20 and 65 points. Schools in relatively "fair condition" are in bright red and received a scoring between 65 and 80 points. Schools that are in relatively "poor condition" are in orange and received a scoring between 80 and 100 points. And schools that are in relatively "critical condition" are in bright yellow and received a scoring between 100 and 200 points. In other words, the greater amount of points, the worse the school's condition is.

Since this type of assessment is unique of its kind, there are no parameters or thresholds that indicates what constitutes to being in good, fair, poor, and critical condition. As a result, I divided the final results into equal quartiles (i.e. each colour group has an equal number of schools). 
Figure 2: TDSB Elementary Schools in Critical Condition, 2016-2017 (Below)

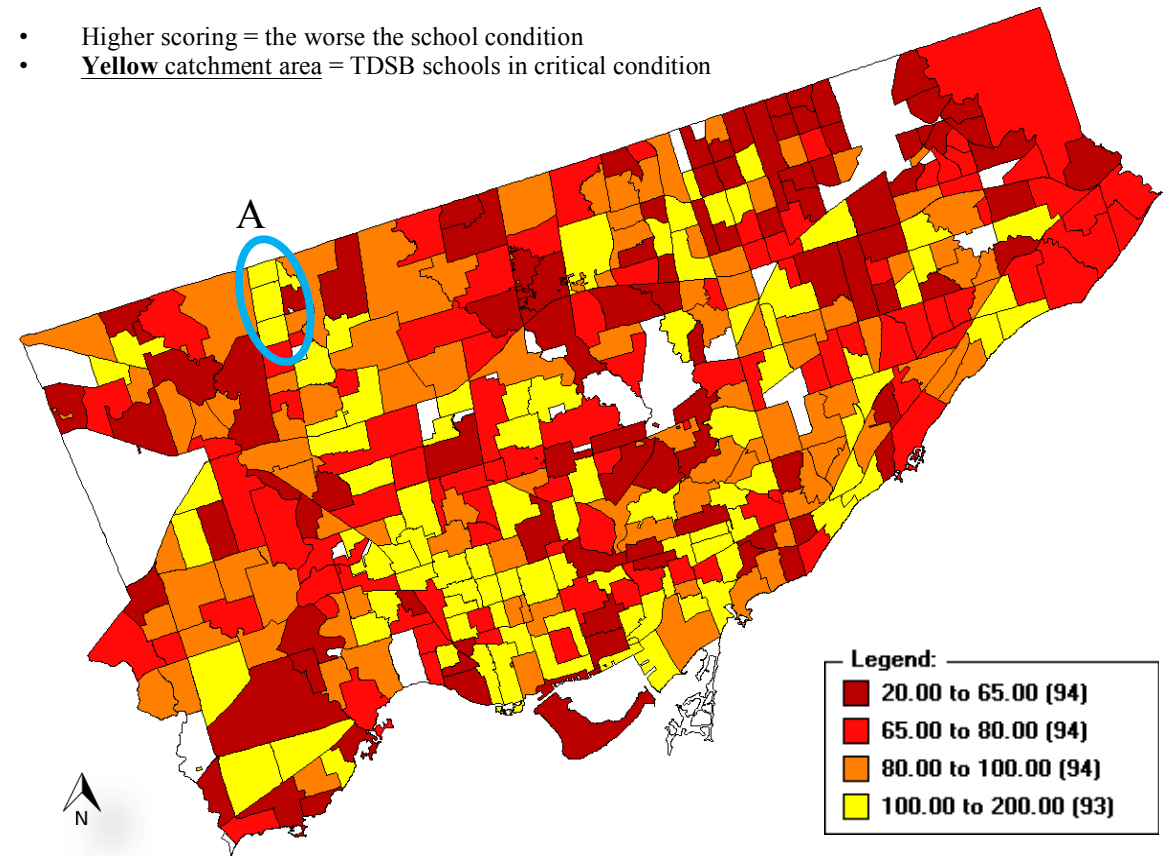

Figure 3: TDSB High School Catchment Areas, 2016-2017 (Below)

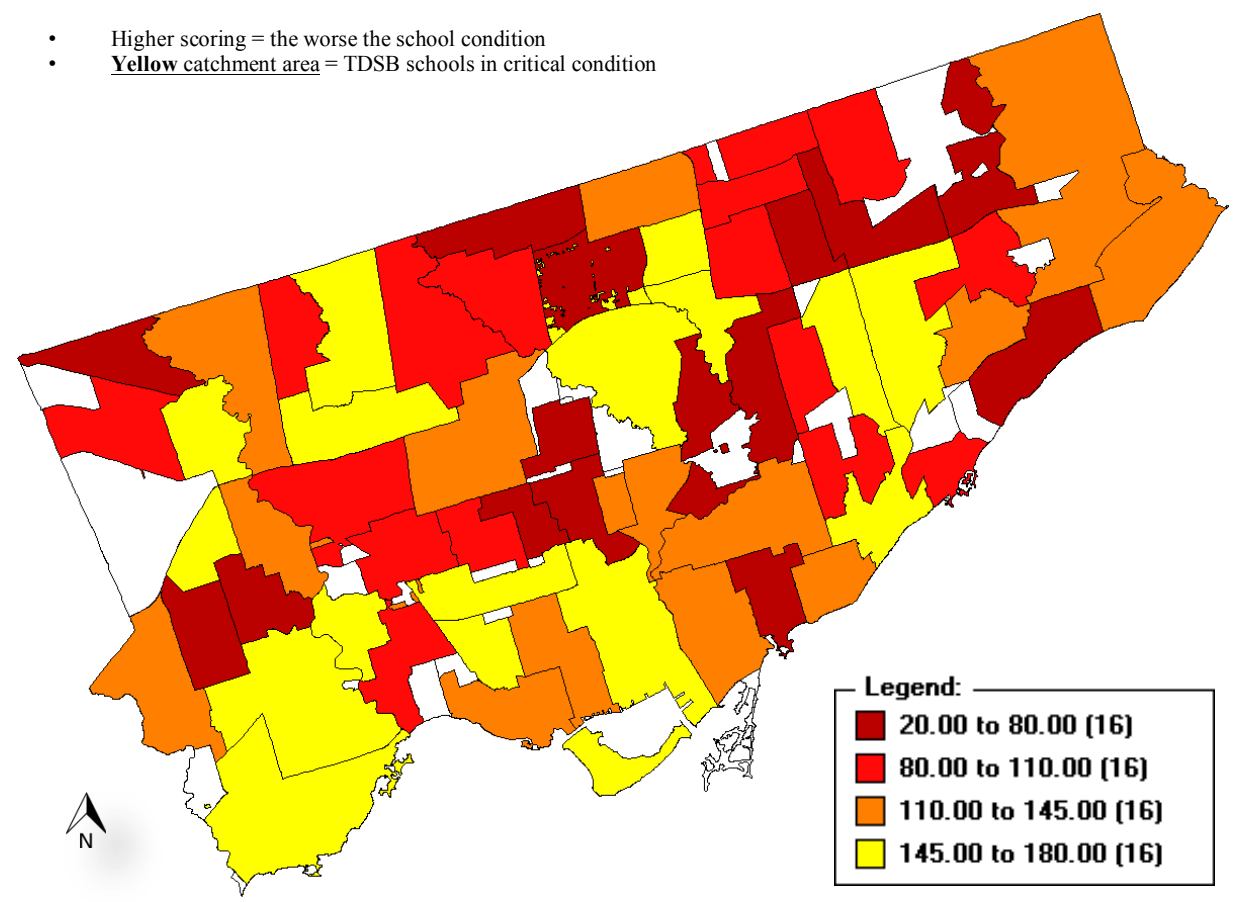




\subsubsection{Case Study: Area A}

As mentioned earlier, phase 1 can be applied to all properties. However, for phases 2 to 5 , the assessment will be applied to specific school catchments. For this example, the assessment will be applied to school catchments in Area A that have been identified to be in "critical condition" (i.e. yellow catchments: schools with 100 to 200 points), which is circled in blue on Figure 2. The schools within the circled area and that will be considered for this example are the following:

1) Blacksmith Public School

2) Shoreham Public Sports \& Wellness Academy

3) Gosford Public School

4) Firgrove Public School

To see where these schools are exactly located, refer to the map in Figure 4 (below).

\subsection{Phase 2: School Consolidation}

The first part of phase $\mathbf{2}$ is to determine if the schools, that have been identified to be in "critical condition", can be possibly consolidated onto one property. The second part of phase 2 is to identify if the school is designated as a historical or heritage building. Figure 4 (below) provides a geographical reference of where these schools are located relative to one another and the distance between each school. Table 1 (below) provides the following statistics for each school: grade range, land acreage, renewal backlog, FCI, current capacity, utilization rate, and building age.

Before analyzing the statistics into depth, I will be identifying some obvious facts about these 4 properties. In terms of FCI, all 4 properties are well above the $65 \%$ threshold, which means no matter which school is consolidated, it will be a benefit for the TDSB and its students. None of the properties have a heritage designation, which makes sense considering that they are not too old in terms of age.

The first factor that will be considered is the distance between each school. As established earlier, walking distance for an elementary school is anything less than $1.6 \mathrm{~km}$. Schools 1,2 and 3 are within walking distance of one another. However, school number 4 is too far for students coming from schools 1 and 2, hence it may not be a suitable option for consolidation. It is important to 
note that with consolidation, the distance between some of the students' homes and the new consolidated school will increase, hence, it no longer is considered to be "walking distance". However, upon determining which schools are to be consolidated, the shortest distance between the schools should be selected or preferred.

In terms of enrollment, schools 1 and 2 are both below the $65 \%$ utilization rate threshold. Also, when considering the enrollment/utilization rate projections for the next 5 years, school 1 is projected to decrease down from $63 \%$ to $56 \%$ and school 2 utilization rate is projected to decrease down from $51 \%$ to $44 \%$. And while school 3 is well above the utilization rate threshold (80\%), it is projected that in the next 5 years it will decrease to $71 \%$. Hence, building a new school that accommodates the area's needs and accommodates two or more schools onto one property may be a suitable option to consider.

There are different consolidation options that can be considered for Area A school catchments. It is important to note that the consolidation options below are solely based on numerical data. Once we go through phases 3 to 4 , this decision may change. The each consolidation options are described as follows:

\section{Consolidation Option \# 1}

Schools 1 and 2 can be consolidated on either property (since both almost have the same size of land). The school will have a capacity approximately between 500 to 600 students and this will eliminate a total renewal backlog of approximately \$14 million. Simultaneously, schools 3 and 4 can be consolidated onto one property as well. The new school will have a capacity approximately 750 to 800 students and will eliminate a total backlog of approximately $\$ 17.4$ million. If this option of consolidation is implemented, this will allow for the TDSB to eliminate 4 deteriorating schools (with a high FCI) and eliminate a total backlog of \$31 million.

\section{Consolidation Option \# 2}

School 1, 2 and 3 can be consolidated onto school property 3 which has 6 acres of land. Since school 3 is located in the centre, between schools 1 and 2 , it is seen as the most suitable for this 
option of consolidation. This will mean the new school will have a capacity of approximately 800 students. By building a new school, the TDSB will be able to eliminate 3 schools, with a total backlog of $\$ 21$ million, each with an FCI ranging between $70 \%$ and $90 \%$. This will potentially free up two school properties, with a combined land acreage total of approximately 12 acres, opening up the opportunity for repurposing and/or redevelopment.

\section{Consolidation Option \# 3}

Schools 1 and 3 can be consolidated onto one school property. This will eliminate approximately $\$ 13$ millions of renewal backlog. And this will free up approximately 6 acres of land.

\section{Consolidation Option \# 4}

Schools 2 and 3 can be consolidated one property. This will eliminate approximately $\$ 16$ millions of renewal backlog. And this will free up approximately 5 to 6 acres of land. 
Figure 4: School Catchments in Area A

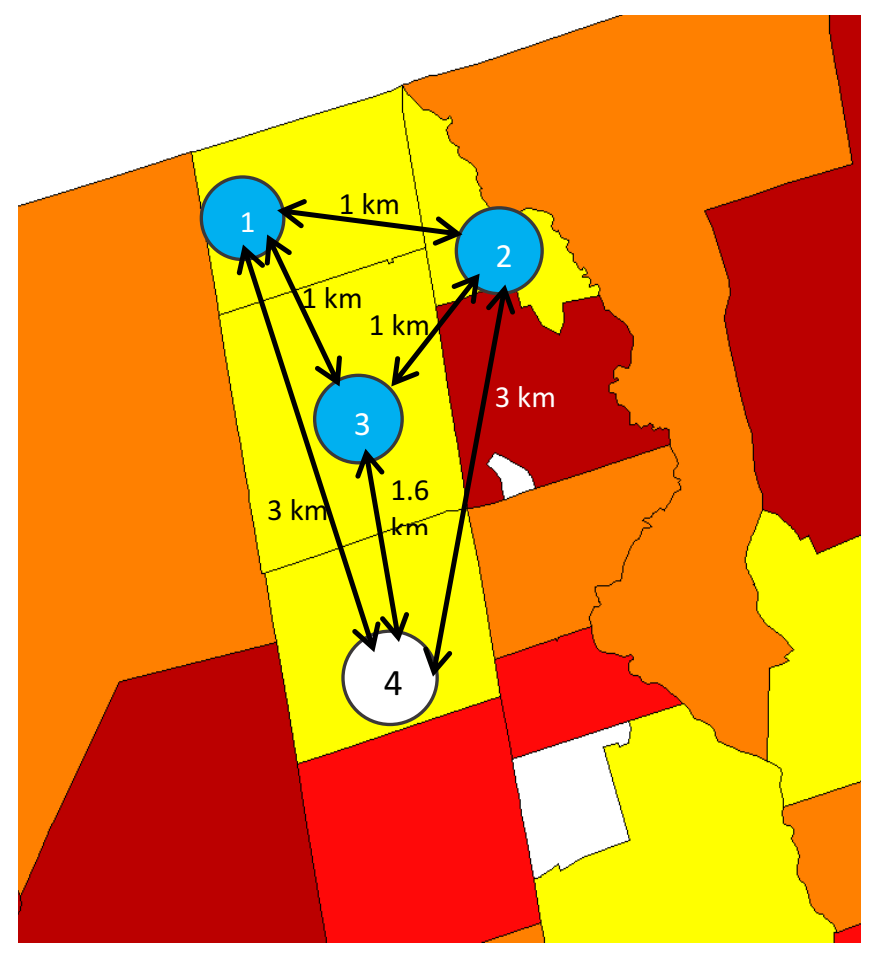

Table 1: Area A School Catchment Statistics (below) (The data in this table are extracted from the 2016-2017 TDSB dataset in Appendix A)

\begin{tabular}{|c|c|c|c|c|c|c|c|c|}
\hline School & $\begin{array}{l}\text { Grade } \\
\text { Range }\end{array}$ & $\begin{array}{l}\text { Land } \\
\text { Acreage }\end{array}$ & $\begin{array}{l}\text { Renewal } \\
\text { Backlog }\end{array}$ & FCI & $\begin{array}{l}\text { Current } \\
\text { Capacity }\end{array}$ & $\begin{array}{l}\text { Enrollment (Utilization } \\
\text { Rate) }\end{array}$ & $\begin{array}{l}\text { Building } \\
\text { Age }\end{array}$ & $\begin{array}{l}\text { Heritage } \\
\text { Designation }\end{array}$ \\
\hline $\begin{array}{l}1 \\
\text { Blacksmith PS }\end{array}$ & JK-5 & 6.03 & $\$ 5.2 \mathrm{M}$ & $72 \%$ & 326 & $207(63 \%)$ & 50 & No \\
\hline $\begin{array}{l}2 \\
\text { Shoreham } \\
\text { Public Sports } \\
\& \quad \text { Wellness } \\
\text { Academy }\end{array}$ & JK-5 & 5.93 & $\$ 8.5 \mathrm{M}$ & $72 \%$ & 524 & $266(51 \%)$ & 49 & No \\
\hline $\begin{array}{l}3 \\
\text { Gosford PS }\end{array}$ & JK-5 & 6 & $\$ 7.3 \mathrm{M}$ & $93 \%$ & 349 & $279(80 \%)$ & 54 & No \\
\hline $\begin{array}{l}4 \\
\text { Firgrove PS }\end{array}$ & JK-5 & 8.01 & $\$ 10.1 \mathrm{M}$ & $72 \%$ & 643 & $432(67 \%)$ & 47 & No \\
\hline
\end{tabular}




\subsection{Phase 3: Social Scan for Area A}

To conduct a social scan, demographic data for the communities surrounding the area will be analyzed. The demographic data will be based on the census tract in which the 4 schools listed above are located in. The census tracts that will be analyzed for Area $A$ are 312.04, 312.05, 315.03, 316.01, 316.03, 316.04, 316.05, and 316.06 (Statistics Canada, 2016a). Figure 5 (below) provides a map of the census tracts that will be analyzed for this phase.

Figure 5: Census Tract Map for Area $A^{15}$

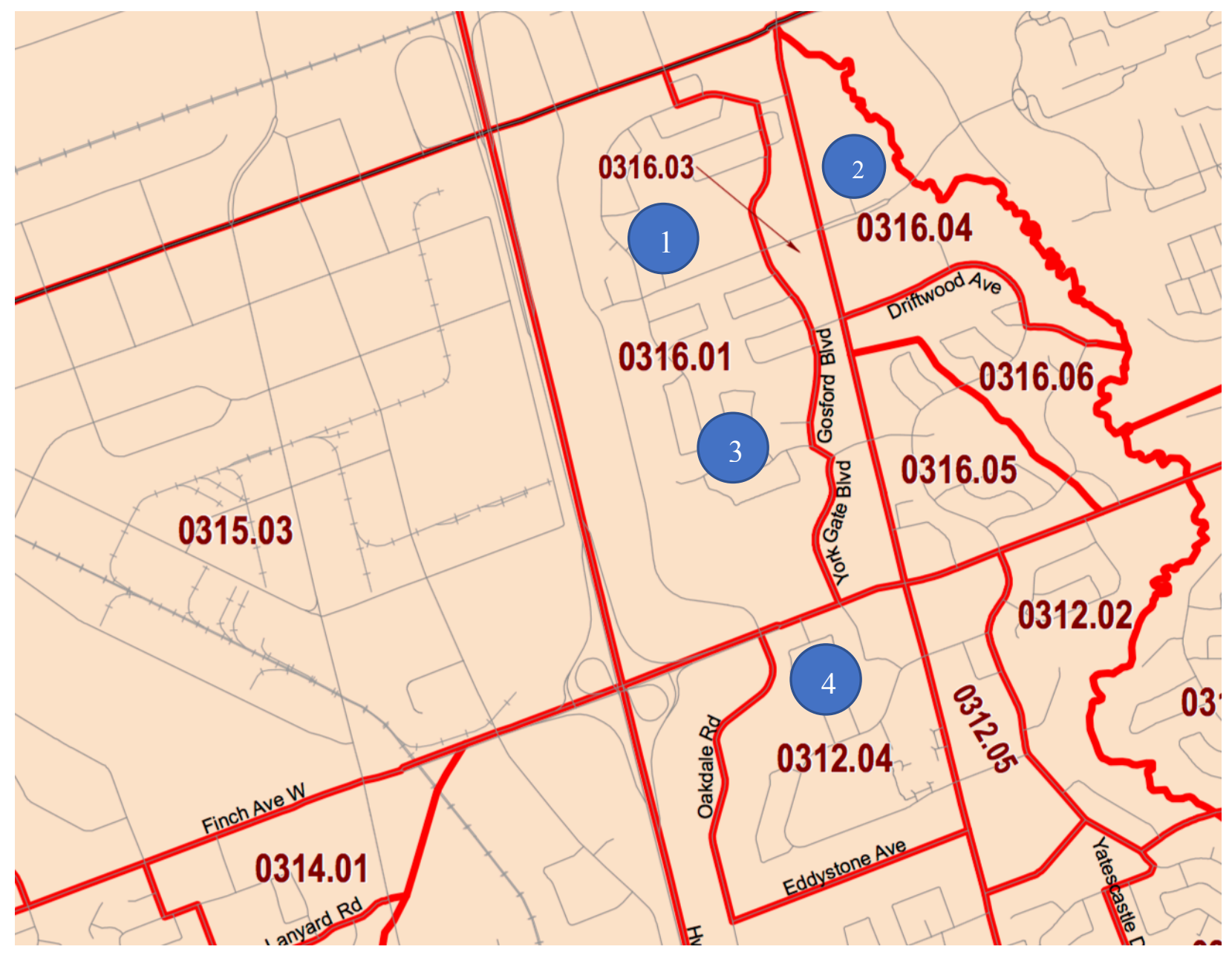

15

Statistics Canada. (2016a). Census Tract by CMA 535. Retrieved from: http://www12.statcan.gc.ca/census-recensement/2016/geo/mapcarte/ref/ct/files-fichiers/2016-92146-535-04.pdf 


\section{Age and population}

Table 2 below shows the overall percentage for each age group for all census tracts combined ${ }^{16}$. The total population of the area (i.e. combining population from each census tract) is 36,830 and the average age for the area is 37 years old. Looking across all age groups (except for age cohort 65 and older), they all have close percentages ranging between $5 \%$ and $8 \%$. Ages 5 to 14 years old make up $14 \%$ of the area population, which is the min age group for elementary schools in the area. The working age group (i.e. 15 to 64 year olds) are the majority, consisting of $66 \%$ of the area population. The senior group (i.e. 65 years and older) make up $13 \%$ of the area ${ }^{16}$.

Table 2 Age Group populations in Area A

\begin{tabular}{|l|l|}
\hline Age Cohort & Percentage \\
\hline $\mathbf{0}$ to 4 years & $7 \%$ \\
\hline $\mathbf{5}$ to 9 years & $7 \%$ \\
\hline $\mathbf{1 0}$ to 14 years & $7 \%$ \\
\hline $\mathbf{1 5}$ to 19 years & $7 \%$ \\
\hline $\mathbf{2 0}$ to 24 years & $8 \%$ \\
\hline $\mathbf{2 5}$ to 29 years & $7 \%$ \\
\hline $\mathbf{3 0}$ to 34 years & $7 \%$ \\
\hline $\mathbf{3 5}$ to 39 years & $6 \%$ \\
\hline $\mathbf{4 0}$ to 44 years & $6 \%$ \\
\hline $\mathbf{4 5}$ to 49 years & $7 \%$ \\
\hline $\mathbf{5 0}$ to 54 years & $7 \%$ \\
\hline $\mathbf{5 5}$ to 59 years & $6 \%$ \\
\hline $\mathbf{6 0}$ to 64 years & $5 \%$ \\
\hline $\mathbf{6 5}$ years and over & $13 \%$ \\
\hline
\end{tabular}

${ }^{16}$ Statistics Canada. (May 2017). 2016 Census of Population- Age and Sex. http://www12.statcan.gc.ca/censusrecensement/2016/dp-pd/dt-td/Rpeng.cfm? TABID $=2 \& \mathrm{LANG}=\mathrm{E} \& \mathrm{~A}=\mathrm{R} \& \mathrm{APATH}=3 \& \mathrm{DETAIL}=0 \& \mathrm{DIM}=0 \& \mathrm{FL}=\mathrm{A} \& \mathrm{FREE}=0 \& \mathrm{GC}=53503$ $12.04 \& \mathrm{GL}=-$ $1 \& \mathrm{GID}=1244052 \& \mathrm{GK}=8 \& \mathrm{GRP}=1 \& \mathrm{O}=\mathrm{D} \& \mathrm{PID}=109527 \& \mathrm{PRID}=10 \& \mathrm{PTYPE}=109445 \& \mathrm{~S}=0 \& \mathrm{SHOWAL}$ $\underline{\mathrm{L}}=0 \& \mathrm{SUB}=0 \& \mathrm{Temporal}=2016 \& \mathrm{THEME}=115 \& \mathrm{VID}=0 \& \mathrm{VNAMEE}=\& \mathrm{VNAMEF}=\& \mathrm{D} 1=0 \& \mathrm{D} 2=0 \& \mathrm{D} 3=$ $\underline{0 \& \mathrm{D} 4=0 \& \mathrm{D} 5=0 \& \mathrm{D} 6=0}$ 


\section{Household Structure}

This section looks at the general family structure in the area and it is based on the 2016 Census of Population Family Characteristics of Adults dataset. This dataset indicates that $49 \%$ of adults are married or are in a common-law relationship. Of those married couples, 32\% do not have children and $68 \%$ have children. Single parent households (i.e. lone parent) make up $18 \%$ of the population. Single adults or adults that are not living with a family member or non-family member, make up $20 \%$ of the area's population ${ }^{17}$. It is clear that Area A predominantly consists of married couples with one or more children. This means that any future services, amenities, and or development should appeal to a growing family household.

\section{Immigration}

This section presents statistics about the percentage of people living in Area A that are identified as immigrants or non-immigrants. The immigration and language data is based on the 2011 National Housing Survey Income and Housing Dataset. According to the dataset, $63 \%$ of the area consists of individuals identified as immigrants and 37\% are identified as non-immigrants. In terms of the mother tongue language, $53 \%$ of the Area A's mother tongue language is English and $46 \%$ has a non-official language as their mother tongue (i.e. not English). Only 1\% of the area speaks French as their mother tongue language. Considering that the majority of Area A's population are immigrants and almost half of the area's population speak a language other than English, there needs to be consideration for amenities and services that cater to their needs. These services and amenities can include ESL classes, employment services, and cultural centres, just to name a few.

\footnotetext{
${ }^{17}$ Statistics Canada. (Aug 2017). 2016 Census of Population - Families, households, and martial status. http://www12.statcan.gc.ca/census-recensement/2016/dp-pd/dt-td/Rpeng.cfm?TABID=1\&LANG=E\&A=R\&APATH=3\&DETAIL =0\&DIM=0\&FL=A\&FREE=0\&GC=5350312.04\&GL =$\overline{1} \& \mathrm{GID}=1244052 \& \mathrm{GK}=8 \& \mathrm{GRP}=1 \& \mathrm{O}=\mathrm{D} \& \mathrm{PID}=109645 \& \mathrm{PRID}=10 \& \mathrm{PTYPE}=109445 \& \mathrm{~S}=0$ \&SHOWALL $=0 \& \mathrm{SU}$ $\underline{\mathrm{B}}=0 \&$ Temporal $=2016 \& \mathrm{THEME}=117 \& \mathrm{VID}=0 \& \mathrm{VNAMEE}=\& \mathrm{VNAMEF}=\& \mathrm{D} 1=0 \& \mathrm{D} 2=0 \& \mathrm{D} 3=0 \& \mathrm{D} 4=0 \& \mathrm{D} 5=0 \&$ $\underline{\mathrm{D} 6=0}$
} 


\section{Income status}

Income levels for the area are based on the 2011 National Household Survey (NHS) Income and Housing dataset since 2016 is not available ${ }^{18}$. The data shown in Table 3 (below) is based on "After-tax income" of private households in 2010. According to Statistics Canada, the household income is the sum of the after-tax incomes of all members of that household. A private household refers to a person or a group of people who live in the same private dwelling and do not have a usual place of residence elsewhere in Canada.

Referring to Table 3 (below), the income level with the highest percentage of residents are $\$ 20,000$ to $\$ 29,999$ and $\$ 30,000$ to $\$ 39,999$, both levels at $15 \%$. The second highest is $\$ 60,000$ to $\$ 79,999$ at $14 \%$. The third highest is $\$ 40,000$ to $\$ 49,999$ at $13 \%$. It is clear that there is a greater percentage of residents that fall below or close to the poverty line. For reference, according to the Low Income Cut Offs (1992 base) After Tax chart (refer to the table in Appendix C), for the year 2008 and for urban areas with a population of 500,000 and over, a one-person household would be considered below the poverty line at an income of $\$ 18,373$ or lower. For 2 to 4 person households, the cut off would be approximately between $\$ 22,000$ and $\$ 35,000$. And households for 5 to 7 persons (or more) have a cut off of approximately between $\$ 40,000$ and $\$ 48,000$ (Statistics Canada, 2015).

\footnotetext{
18 Statistics Canada. (Sept 2013). 2011 National Household Survey (NHS) - Income and Housing. http://www12.statcan.gc.ca/datasets/Indexeng.cfm?Temporal $=2013 \&$ Theme $=98 \& \mathrm{VNAMEE}=\& \mathrm{GA}=8 \& \mathrm{~S}=0$
} 


\begin{tabular}{|l|l|}
\hline Income & Percentages \\
\hline Under $\mathbf{\$ 5 , 0 0 0}$ & $3 \%$ \\
\hline $\mathbf{\$ 5 , 0 0 0}$ to $\$ \mathbf{9 , 9 9 9}$ & $2 \%$ \\
\hline $\mathbf{\$ 1 0 , 0 0 0}$ to $\$ \mathbf{1 4 , 9 9 9}$ & $5 \%$ \\
\hline $\mathbf{\$ 1 5 , 0 0 0}$ to $\mathbf{\$ 1 9 , 9 9 9}$ & $8 \%$ \\
\hline $\mathbf{\$ 2 0 , 0 0 0}$ to $\mathbf{\$ 2 9 , 9 9 9}$ & $15 \%$ \\
\hline $\mathbf{\$ 3 0 , 0 0 0}$ to $\mathbf{\$ 3 9 , 9 9 9}$ & $15 \%$ \\
\hline $\mathbf{\$ 4 0 , 0 0 0}$ to $\mathbf{\$ 4 9 , 9 9 9}$ & $13 \%$ \\
\hline $\mathbf{\$ 5 0 , 0 0 0}$ to $\mathbf{\$ 5 9 , 9 9 9}$ & $10 \%$ \\
\hline $\mathbf{\$ 6 0 , 0 0 0}$ to $\mathbf{\$ 7 9 , 9 9 9}$ & $14 \%$ \\
\hline $\mathbf{\$ 8 0 , 0 0 0}$ to $\mathbf{\$ 9 9 , 9 9 9}$ & $\mathbf{7} \%$ \\
\hline $\mathbf{\$ 1 0 0 , 0 0 0}$ and over & $8 \%$ \\
\hline
\end{tabular}

\section{Employment \& Unemployment Rate}

To further understand the income levels observed in the Income Status section, this analysis will also be analyzing the employment trends occurring in Area A and the surrounding environment. Starting at a larger scale, jobs in North York, which is where all 4 schools are located in, make up $2.6 \%$ of jobs in the City overall. According to the Toronto Employment Survey for 2017, 8 in 10 jobs in North York Centre are office jobs, which are approximately 33,000 office jobs (Toronto, 2018). However, the North York Centre saw an overall decrease of in the office, retail and manufacturing sectors. On the other hand, the centre has experienced a growth and increase in the service, institutional, and community and entertainment industries (Toronto, 2018).

On a more focused scale, based on the 2011 National Household Survey Labour and Housing dataset, Area A has an employment rate of $48 \%$ and unemployment rate of $13.7 \%{ }^{19}$. While the unemployment rate for Area $\mathrm{A}$ is low, however the employment rate is not that high. An

19 Statistics Canada. (Sept 2013). 2011 National Household Survey (NHS) - Income and Housing. http://www12.statcan.gc.ca/datasets/Indexeng.cfm?Temporal $=2013 \&$ Theme $=98 \&$ VNAMEE $=\& \mathrm{GA}=8 \& \mathrm{~S}=0$ 
assumption for this current state can be identified by observing the employment activity in the area shown on the Employment Concentrations map in Figure 6 (below). The map shows that Area A is located directly to the right-hand side of a "Core Employment Area". The map shows that majority of the activity and employment is clustered mostly west of Highway 400. Hence, the census tracts used to collect this data would not be able to capture the employment activity occurring west of Area A.

Figure 6: Employment Concentrations Map (Toronto, 2018)

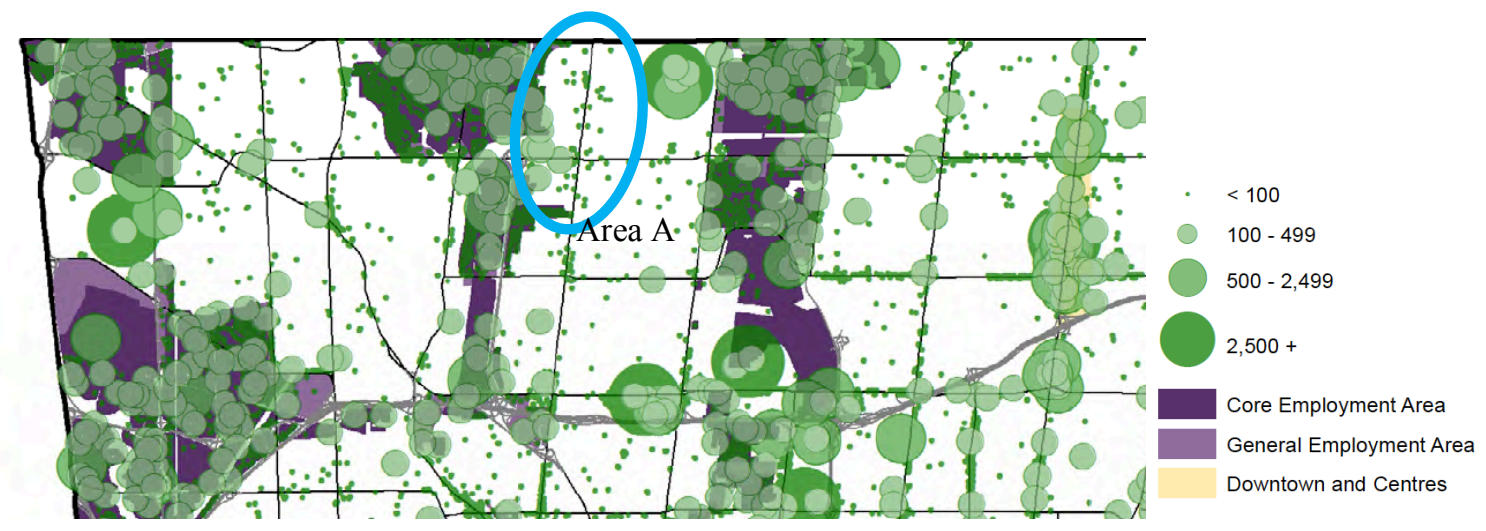

\section{Occupations}

Table 4 (below) provides a list of occupation industries that residents in Area A work in. This data is based on the 2011 National Housing Survey labour and housing data set ${ }^{19}$. The occupation or job sector with the highest percentage is sales and service with 28\%. The second highest is trades; transport and equipment operators; and related occupations, with $20 \%$ and the third highest job sector is the manufacturing and utilities sector with $18 \%$. While the manufacturing industry in the North York Centre has decreased in 2017, overall it is clear that it remains a predominant employment industry for Area A. 


\begin{tabular}{|l|l|}
\hline Occupations & Percentages \\
\hline Management occupations & $5 \%$ \\
\hline Business, finance and administration occupations & $14 \%$ \\
\hline Natural and applied sciences and related occupations & $3 \%$ \\
\hline Health occupations & $5 \%$ \\
\hline $\begin{array}{l}\text { Occupations in education, law and social, community and government } \\
\text { services }\end{array}$ & $6 \%$ \\
\hline Occupations in art, culture, recreation and sport & $1 \%$ \\
\hline Sales and service occupations & $28 \%$ \\
\hline Trades, transport and equipment operators and related occupations & $20 \%$ \\
\hline Natural resources, agriculture and related production occupations & $1 \%$ \\
\hline Occupations in manufacturing and utilities & $18 \%$ \\
\hline
\end{tabular}

\section{Tenure Type}

The tenure data will be providing data on the percentage of residents that own or rent their homes. According to the 2011 National Housing Survey labour and housing dataset, 36\% of Area A residents own their homes and $64 \%$ rent their homes ${ }^{20}$. It is clear that there is large market for rental housing in the area. In addition, it is important to consider how much of the rental housing consists of affordable or subsidized housing. According to Statistics Canada, the definition of "affordable or subsidized housing" can include rent geared income units, social housing, public housing, government-assisted housing, non-profit housing, rent supplements and housing allowances (Statistics Canada, 2011). In the case of Area A, 31\% of residents live in subsidized housing. Hence, in addition to the fact that there is a demand for rental housing, any future development should also consider some form of social housing.

${ }^{20}$ Statistics Canada. (Sept 2013). 2011 National Household Survey (NHS) - Income and Housing. http://www12.statcan.gc.ca/datasets/Indexeng.cfm? Temporal $=2013 \&$ Theme $=98 \&$ VNAMEE $=\& \mathrm{GA}=8 \& \mathrm{~S}=0$ 


\subsection{Phase 4: Community Scan for Area A}

This scan will observe services and amenities within close proximity to each of these schools. The data for this scan have been compiled from Toronto services map and google maps.

i. Libraries

Referring to Figure 7 (below), there is only one library that is accessible to all four schools. The library is within $1.4 \mathrm{~km}$ from Firgrove, $1.6 \mathrm{~km}$ from Gosford, $1.7 \mathrm{~km}$ from Shoreham, and $2.5 \mathrm{~km}$ from Blacksmith.

Figure 7: Libraries (Toronto Maps v2, n.d.)

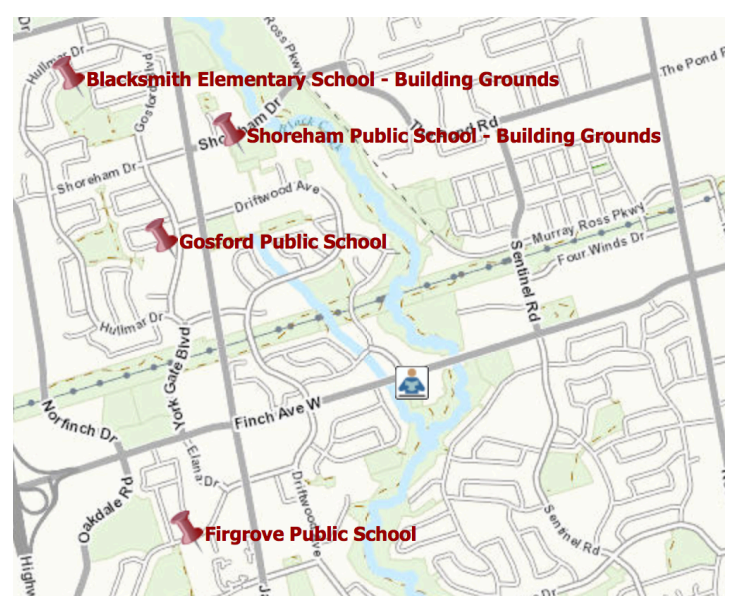

ii. Social Services

In terms of social services, Area A has high accessibility to these types of services. These services range from child care services, pharmacies, chiropractors, Academic upgrading services, to immigration and refugee services. These social services appear as green circles on Figure 8 below. While these green circles are not labelled, upon clicking on each one, I noticed that all four schools had access to a child care centre, however all academic and immigration centres and services were clustered closer to Firgrove Public School. 
Figure 8: Toronto Help (Toronto Maps v2, n.d.)

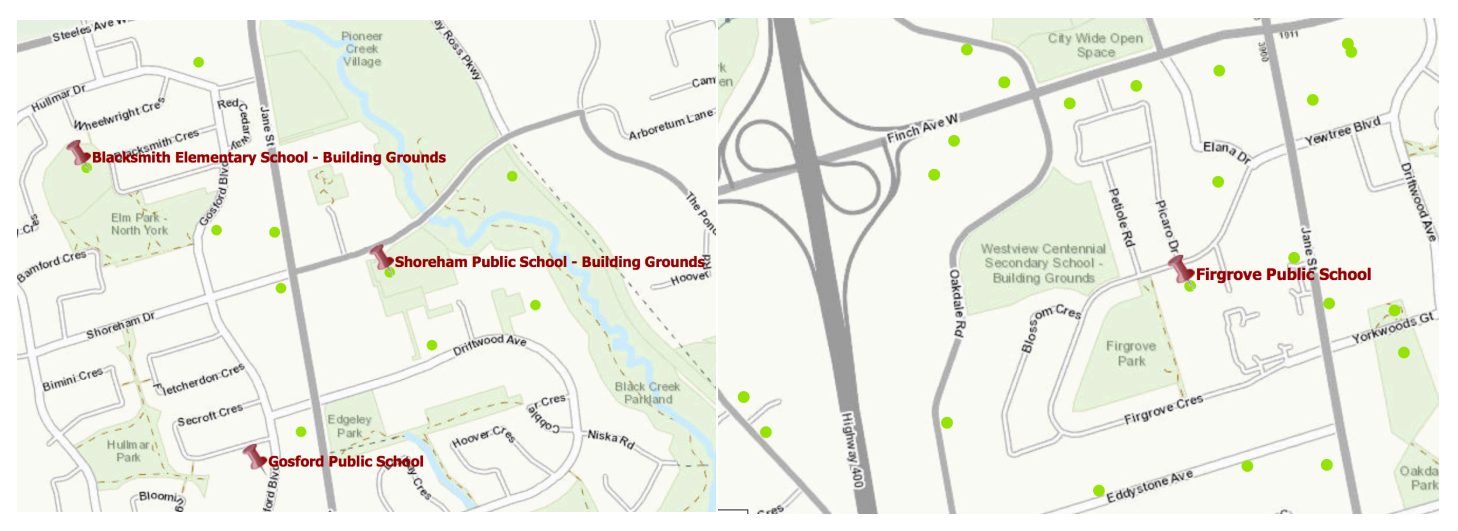

iii. Recreation/ Community Centres

Referring to Figure 9 (below), it is clear that all four schools have great access to recreational and community centres.

Figure 9: Recreation Centres (Toronto Maps v2, n.d.)

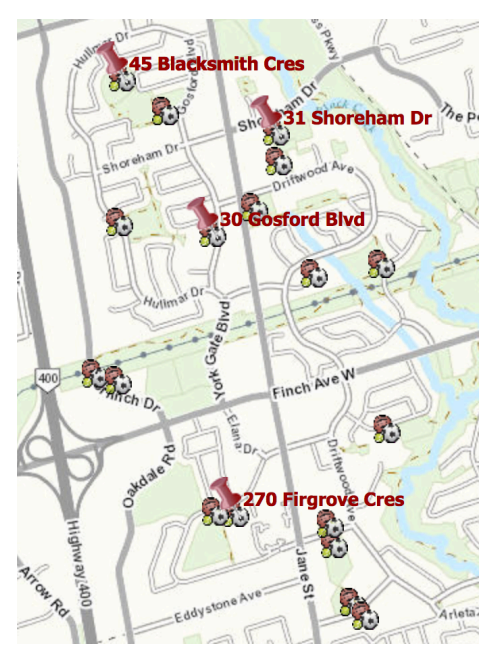


iv. Parks and Green Space

Referring to Figure 10 below, it is clear that the area's residents have great access to parks and green space.

Figure 10: Parks and Green Space (Google Maps)

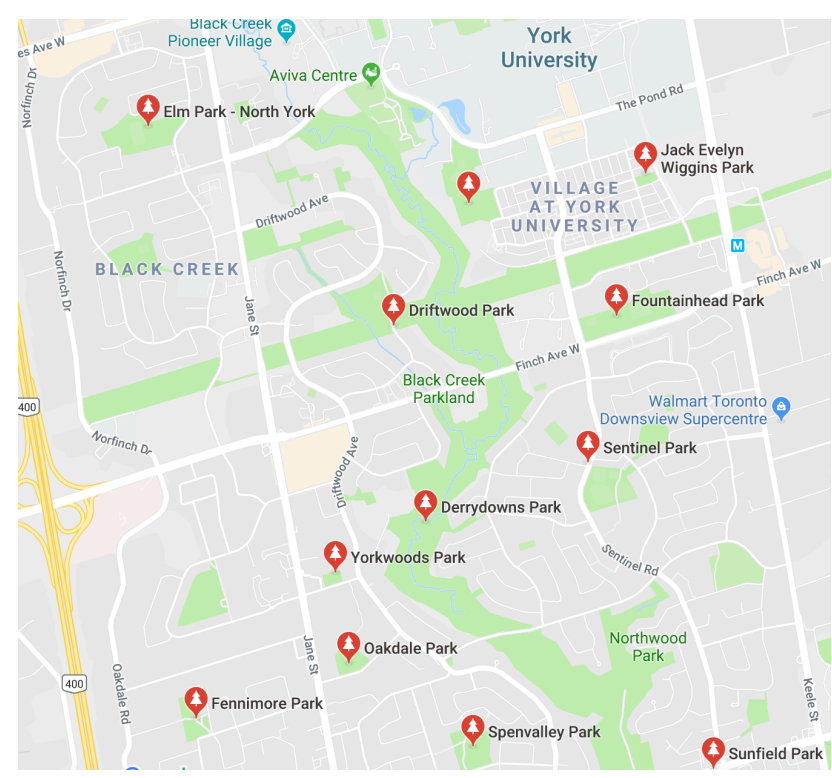

v. Transportation

The major transit service available in the area is bus services. As suspected, these school properties do not have any form of public transit service (i.e. buses) stopping right in front of them. Students commuting to these properties will need to walk from the bus stop to their school. Bus service that runs along Jane Street (north-south) is bus 35 Jane. The 35 bus runs from Jane subway station to York University. This bus also stops at Pioneer Village subway station. Along Finch Avenue (west-east) bus 36 Finch West, and it runs from Finch subway station to the Woodbine Race Tracks. This bus also stops at Finch West subway station (TTC, 2018). 


\subsection{Phase 5: Development Scan for Area A}

The development scan will provide a planning due-diligence for Area A. This particular scan will provide a high-level development scan and will not go into a detailed due-diligence analysis. However, upon applying this tool in a real-life scenario, it is highly recommended to conduct a full due diligence analysis in order to support future decisions relating to the subject property.

\section{i. $\quad$ Toronto Official Plan}

Referring to Toronto's Official Plan Map 13 Land Use Plan, all four school properties are located within neighbourhood designated areas. The City of Toronto Official Plan describes neighborhoods as a residential area with a wide range of lower scale buildings such as detached houses, semi-detached houses, duplexes, triplexes, and townhouses. There are also apartment buildings that are no higher than 4 storeys. Neighborhoods can also have parks, small scale recreational and cultural facilities, small scale retail, service and office use. You can also find low scale local institutions, such as schools, places of worship, libraries, community centres, day nurseries, nursing homes, etc.

When it comes to developing on a or near a neighborhood designated property, it is important to maintain the physical character of the neighborhood. New development must respect, preserve, and establish the current physical patterns in the neighbourhood. Any physical changes to the neighborhood must be sensitive, gradual, and fit the current physical fabric and character of the area. It must be noted that no changes will be made through rezoning, minor variance, consent or other public action that do not match the general character of the neighbourhood. The zoning bylaw will contain numerical standards and regulations that will ensure that new development will be compatible with the physical character of a neighbourhood.

\section{ii. $\quad$ Zoning by-law}

For all four properties, they have all been zoned under the Former City of North York By-law No. 7625 (OMB, 1953). According the zoning code map, the schools are zoned as Multiple Family Dwelling Third Density Zone or RM3. Under this zoning they permit semi-detached, duplex, converted, double duplex, and apartment houses. The height of any residential use must not go beyond 3 storeys. This designation also permits uses listed under R1, R2, and R3 zones. These 
uses include recreational uses such as parks, playgrounds, golf courses and includes institutional uses such as schools, Sunday schools, or libraries. The maximum height permitted for any use built on these school properties is 3 storeys.

\section{iii. Surrounding Zoning}

It is also important to note that while the school properties are zoned by the former by-law, the surrounding properties are zoned by the City of Toronto Zoning By-law 569-2013. All 4 of the schools are mostly surround by residential neighbourhoods, ranging from single-detach, townhouses, and apartment buildings. In addition, there is a substantial amount of parkland surrounding these areas. To further understand the surrounding environment, this part of the assessment will be exploring the various zoning designations that currently surround all four school properties. Listed below are the main zoning uses that surround the four schools along with a brief description of the permitted uses for each zoning:

\section{a. Residential Detached Zone- RD}

In a Residential Detached Zone or RD zone, it permits dwelling units and parks. It also permits other uses but under certain conditions. Some of these conditional uses include a day nursery, community centre, group home, or library. The maximum permitted height is 10 metres (3 storeys).

\section{b. Residential Multiple Dwelling Zone - RM}

In a Residential Multiple Dwelling Zone or RM zone, the permitted uses are the same as an RD zone, however the permitted height is 12 metres (3 to 4 storeys).

\section{c. Residential Townhouse Zone - RT}

In a Residential Townhouse Zone or RT zone, the permitted uses are the same as an RD and RM zones, however the permitted height is 24 metres ( 7 storeys).

\section{d. Residential Apartment Commercial - RAC}

In a Residential Apartment Commercial Zone or RAC zone, the permitted uses are the same as an $\mathrm{RD}, \mathrm{RM}$, and RT zones, however the permitted height is 10 metres ( 3 storeys). 


\section{e. Open Space - Recreation Zone - OR}

In an Open Space- Recreational Zone, it permits uses such as art galleries, community centres, day nurseries, parks, and libraries. The maximum permitted height on an OR zone is 15 metres (4 to 5 storeys).

\section{f. Open Space - Natural Zone - ON}

In an Open Space- Natural Zone, it permits uses such as agricultural use, parks, police station, public utility, and transportation use. The maximum permitted height on an ON zone is 15 metres (4 to 5 storeys).

\subsection{Discussion}

Based on this preliminary overview of the area surrounding Blacksmith Public School, Shoreham Public Sport and Wellness Academy, Gosford Public School and Firgrove Public School, the following conclusions and recommendations have been complied:

In terms of available services and amenities, the four schools or residents of Area A have access to many public services including recreational/community centres, schools, parks, and child care centres. However, the community scan did raise some red flags and concerns in regards to certain services. As mentioned in section 5.3, majority of academic and immigration services were clustered closer to the Firgrove Public School property. Considering that $63 \%$ of the area's population are identified as immigrants and $46 \%$ of residents speak a non-official language (i.e. not English) as their mother language, there is a need for academic, ESL, and/or immigration services closer to the other three school properties. The area is also in need of more libraries. As mentioned in section 5.3, there is only one library servicing the area, and it is within $1.4 \mathrm{~km}$ of Firgrove, $1.6 \mathrm{~km}$ of Gosford, $1.7 \mathrm{~km}$ of Shoreham, and $2.5 \mathrm{~km}$ of Blacksmith. And while three of

the schools are within reasonable distance, having another library closer to Blacksmith and Shoreham would service the area well.

In terms of development, there are several points that need to be taken into consideration. While the zoning designation for these school properties allow for residential uses, the designated height and density make it harder to build a high density residential project like an apartment building 
that goes higher than 3 storeys. And while small scale housing can be provided such as townhouses or single detached that will complement the current uses in the surrounding area (e.g. single-detach homes, townhouses, apartment buildings), it may not be the highest and best use for the area. As a result, it is recommended that any future development to occur onto these properties should be developed into a community hub that focuses on providing social, cultural, and employment services for the area.

As mentioned in section $5.3,30 \%$ of the area's population fall within the $\$ 20,000$ to $\$ 40,000$ income bracket, and the area has an employment rate of $48 \%$. Hence, with the potential of redeveloping these properties, there is an opportunity to bring in employment opportunities for the area. This can be done by dedicating space on the properties for commercial use, such as convenient stores, medical clinics, and coffee shops. In addition, considering that the highest percentage of the area's population works in the sale and servicing sector, these commercial uses will be in demand by the area's residents.

To conclude this assessment, after considering all the area's demographic data, available services and amenities, and land and zoning uses, one can make an informed decision upon choosing the best consolidation options, which were presented in section 5.2 of this chapter. Considering that the Shoreham Public Sport and Wellness Academy and the Firgrove Public School property are surrounded by RAC zones (i.e. Residential Apartment Commercial zones) there is a high possibility for the school board to rebuild bigger school buildings that will accommodate students from different schools.

In addition, the other two school properties, Blacksmith and Firgrove Public schools are the two properties that are the farthest from existing services and amenities. Hence, there is an opportunity to build a community hub (as described in the paragraph above iii. Development Opportunities) that will complement the existing character and the needs of the surrounding neighbourhoods, and that can remain within the zoning regulations in terms of permitted uses, height, and density. Hence, it is recommended that Blacksmith Public School is consolidated with Shoreham Public Sport and Wellness Academy onto the Shoreham school property and that Firgrove Public School is consolidated with Gosford Public School onto the Gosford school property. In addition, 
consolidating all four of the schools (i.e. two on each property) will allow for the elimination of approximately \$31 million in renewal backlog and replacing four old, deteriorating schools with newer facilities. 


\section{Chapter 6: Limitations}

\subsection{Baragar Systems School Catchment Areas}

Baragar does not account for all the schools within the TDSB district. It excludes alternative schools, schools with specific or special programming, adult schools, and so on. These types of schools are not included into Baragar because they do not have a catchment boundary. Students from all over the City can apply and attend these schools, once they are accepted. It also does not include administrative buildings or schools that have closed down.

\subsection{Community Hubs and Schools}

While this research encourages a collaboration between the TDSB, the City, the public, and/or the private sector, it is important to note that the TDSB is not responsible of running or managing anything but TDSB schools. Any other uses on the property will need to be managed by the City and/or the responsible organization. In addition, it must be made clear that community hubs cannot be implemented or imposed by the TDSB onto the proponent. This will need to be the City's responsibility to negotiate with the proponent, as community services and facilities are implemented and managed by the City. Hence, while the TDSB can provide insight and a preliminary analysis of how to develop the school property to its highest and best use, once the property is sold and is no longer under the TDSB jurisdiction, it has no control over what is done on the property after that point. 


\section{Chapter 7: Conclusion}

This research paper explores and analyzes one of the major issues faced by the TDSB and that is the deteriorating conditions of its school buildings. The TDSB's renewal backlog has reached an all-time high of $\$ 3.4$ billion and has 103 schools (18\% of school inventory) that operate at a $65 \%$ or lower utilization rate (TDSB, 2014). In addition, TDSB school buildings are aging, with approximately 80 school older than 90 years and 7 schools older than 100 years. To address this issue, under-utilized and deteriorating properties have been declared surplus by the TDSB in order to be sold or leased to other school boards, non-profit organizations, or private investors/developers (Mangione \& Suen, 2015). However, the selling of properties with high social and economic value can be a great loss for the school board and for the public, and may not be a sustainable approach for the long run (Brown \& Rushowy, 2015).

To further understand these issues from a planning perspective, the research dives into the urban planning phenomenon known as Shrinking Cities. According to Hollander (2011), shrinking cities refers to urban areas that are experiencing a decline in their population (Hollander, 2011). One of the main impacts associated with shrinking cities is the under-utilization or even the vacancy of public buildings and properties, a similar dilemma that the TDSB currently faces. However, Hollander (2011) explains that the role planning should play in a situation like this is to adapt to these new changes and to adjust its strategies and policies in order to accommodate a smaller population. Similarly, the TDSB is in need of a new strategy that will efficiently address these issues and allow the TDSB to adapt to these new changes.

Hence, this research recommends and proposes a new tool/assessment that will efficiently repurpose and redevelop surplus school lands into community needed infrastructure, services, and/or amenities and replace old and deteriorating TDBS schools with newer school facilities. The assessment consists of the 5 steps/phases. The pre-assessment phase includes establishing an advisory committee that will overlook this assessment and includes identifying potential partnerships, non-profit and/or private, that the TDSB can establish before the site development. 


\section{Phase 1: Identifying School Building Conditions}

This phase will identify schools that are considered to be in critical condition. The factors that will be considered to identify schools in critical conditions are the FCI, building age, utilization rate, and renewal backlog. The purpose of this phase is to establish a priority list of schools that should be catered to right away.

\section{Phase 2: School Consolidation}

Once a list of schools in "critical condition" have been complied, this phase looks at surrounding school catchments and for the opportunity to consolidate with other schools. The selection of a suitable property for the purpose of combining/consolidating multiple schools will be based on factors such as location, land acreage, and walking distance to and from that location. In addition, in this phase, we will also identify if the school property is designated as a heritage building.

\section{Phase 3: Social Scan}

In phase 3 of the assessment, demographic area for the communities and neighbourhoods surrounding the school will be reviewed and analyzed. Data collected for this phase will include age, population, household structure, immigration, language, income status, employment, and tenure type. This phase will provide the advisory committee a clear idea of who lives in the area and what their social and economic needs may be

\section{Phase 4: Community Scan}

The community scan will analyse the available community/public services available and missing in the surrounding area. This phase will also analyse the role the school site in the area played for the community by identifying what services the school provided to the community. This phase will ask questions such as: Does the school run extra-curricular activities after school hours? Do community groups use the school building after school hours? What existing services and amenities does the area have within walking distance? Examples include (but not limited to) Community Centres, libraries, Public and Green space and Grocery stores. 


\section{Phase 5: Development Scan}

In this stage of the assessment, a full planning due diligence review of the school property will take place. The development scan will identify zoning by-laws that dictate the permitted uses on the land, maximum/minimum density, size and height of the building, building setbacks, required parking spaces, just to name a few (Taylor, 2014).

To further test this proposed tool, the paper applies this assessment to 4 TDSB school properties that have been identified to being in "critical condition" in phase 1. While the assessment in this paper was conducted at a high level, it was able to provide insight on alternative ways these school properties can be repurposed and enhanced in terms of use. 


\section{$\underline{\text { Works Cited }}$}

ACEC. (n.d.). Understanding Public Private Partnerships in Canada. https://www.acec.ca/files/resources/acec P3 report v3.pdf

Altus Group. (Sept 2016). Economic and Fiscal Benefits of Toronto Lands Corporation Site Dispositions. http://www.torontolandscorp.com/wp-content/uploads/2017/12/Economic-and-Fiscal-Benefits-TLCFINAL-r.pdf

Baxter, E. (1947). What about surplus property for schools? NASSP Bulletin, 31(149), 49-53. doi:10.1177/019263654703114906

BC Housing. (n.d.). Redevelopment Process and Principles. Retrieved from: https://www.bchousing.org/partner$\underline{\text { services/asset-management-redevelopment/redevelopment-process-princlples }}$

Carroll, B.M., Pasquino, N.G., Webnesh, H. (May, 2016a). Dispositions of Surplus Properties by School BoardsHow New Rules Benefit Community Hubs and Impact Others. Borden Ladner Gervais (BLG). http://blg.com/en/News-And-Publications/Publication_4508

Carroll, B.M., Pasquino, N.G., Haile, W. (April, 2016b). Introducing to Ontario’s Community Hubs Strategic Framework and Action Plan. Borden Ladner Gervais (BLG). http://blg.com/en/News-AndPublications/Publication 4484

City of Toronto. (Mar 2016). Staff Report: Steps to Respond to the Toronto Distric School Board Disposition of Surplus Properties under Ontario Regulation 444/98. Retrieved from: https://www.toronto.ca/legdocs/mmis/2016/cc/bgrd/backgroundfile-91634.pdf

Cote, A., \& Tam, H. (2013). Affordable Housing in Ontario: Mobilizing Private Capital in an Era of Public Constraint. Toronto: The Institute on Municipal Finance and Governance. https://munkschool.utoronto.ca/imfg/uploads/238/1409affordablehousingproofr2.pdf

DCG Strategies. (n.d.) What to do with an abandoned old school building. Retrieved from: https://dcgstrategies.com/blog/dcg-blog/what-to-do-with-an-abandoned-old-school-building/ 
DME. (June, 2017). School Reuse Process.

https://dme.dc.gov/sites/default/files/dc/sites/dme/publication/attachments/School\%20Reuse \%20Process \%206.6.2017.pdf

Fix Our Schools. (April, 2015). What about TDSB schools that are over-capacity?. Fix Our Schools. http://fixourschools.ca/2015/04/21/what-about-tdsb-schools-that-are-over-utilized/

French, Dr. C. (Nov 2014). Why Demographic Data matters. Community Planning New Hampshire. Retrieved from: https://extension.unh.edu/resources/files/Resource004765 Rep6784.pdf

Gordon, A. (Nov, 2017). Toronto board wants developer levies used to fix aging schools. Retrieved from: https://www.thestar.com/yourtoronto/education/2017/11/10/toronto-board-wants-developer-levies-usedto-fix-aging-schools.html

Halinski, T. and Grover, A. (Oct 2010). How Land Use Planning Law Affects Your Transaction. Aird \& Berlis. http://www.airdberlis.com/docs/default-source/articles/how-land-use-planning-law-affects-your$\underline{\text { transaction.pdf?sfvrsn }=2}$

Hollander, J. B. (2011). Can a city successfully shrink? evidence from survey data on neighborhood quality. Urban Affairs Review, 47(1), 129-141. doi:10.1177/1078087410379099

Hollander, J. B., \& Németh, J. (2011). The bounds of smart decline: A foundational theory for planning shrinking cities. Housing Policy Debate, 21(3), 349-367. doi:10.1080/10511482.2011.585164

Lee, J., \& Sung, J. (2017). Conflicts of interest and change in original intent: A case study of vacant and abandoned homes repurposed as community gardens in a shrinking city, daegu, south korea. Sustainability, 9(11), 2140. doi:10.3390/su9112140

Mangione, K. \& Suen, F.Y. (Feb 2015). Toronto has the most underutilized schools across Ontario. CTV News Toronto. https://toronto.ctvnews.ca/toronto-has-the-most-underutilized-schools-across-ontario-1.2211549

Milhouse, W. (Feb 2014). Report of the Advisory Committee for School Repurposing and Community Development. Retrieved from: https://www.cityofchicago.org/content/dam/city/depts/mayor/Press\%20Room/Press\%20Releases/2014/Ja nuary/02.07.14CPSREPORT.pdf 
Ministry of Education. (Mar 2015). Community Planning and Partnerships Guideline.

http://www.edu.gov.on.ca/eng/funding/1516/2015B9appenBEN.pdf

Ministry of Education. (2016). Guide to Pupil Accomdation Reviews. Queen's Printer for Ontario.

http://www.edu.gov.on.ca/eng/policyfunding/GuidePupilReviews.pdf

Mirabelli, J. (Sept 2017). Details Emerge for Major Redevelopment at Bloor and Dufferin. Urban Toronto.

http://urbantoronto.ca/news/2017/09/details-emerge-major-redevelopment-bloor-dufferin

Moskalyk, A. (September, 2008). The Role of Public-Private Partnerships in Funding Social Housing in Canada. http://rcrpp.org/documents/50550_EN.pdf

MPAC. (2018). Community Hubs - Assessment Guide.

https://www.mpac.ca/PropertyTypes/PropertyAssessmentProcedures/CommunityHubsAssessmentGuide

OMB. (1953). Township of North York By-law No. 7625. Toronto Archives.

https://www.toronto.ca/legdocs/pre1998bylaws/north\%20york\%20-\%20township\%20of/7625.pdf

Ontario Centre of Excellence for Child and Youth Mental Health. (July 2013). Evidence In-Sight: Conducting a community needs assessment.

http://www.excellenceforchildandyouth.ca/sites/default/files/resource/EIS_Community Needs_Assessme $\underline{\text { nt.pdf }}$

Ontario, Government of. (January, 2017). Education Act Ontario Regulation 444/98 Disposition of Surplus Real Property. https://www.ontario.ca/laws/regulation/980444

Pilkey, R. (2017). Toronto District School Board 2017 Ontario Budget Submission. TDSB.

https://www.google.ca/url? sa=t\&rct=j\&q=\&esrc=s\&source=web\&cd=1\&cad=rja\&uact=8\&ved=0ahUKE wiLrL-

ckqrVAhXGNT4KHRDmAlcQFggmMAA\&url=http $\% 3 \mathrm{~A} \% 2 \mathrm{~F} \% 2 \mathrm{Fwww}$.tdsb.on.ca $\% 2 \mathrm{FPortals} \% 2 \mathrm{~F} 0 \% 2$

FAboutUs\%2Fdocs\%2FChairPilkey2017OntarioBudgetSubmission.FINAL.pdf\&usg=AFQjCNHvv29KE fYA95x-Uba76NDS7xx3qQ

Ross, S., \& Alphonso, C. (Mar 2017). Ontario School Boards will be forced to pay developer-level prices for surplus properties. The Globe and Mail. Retreived from: 
https://www.theglobeandmail.com/news/national/education/ontario-school-boards-to-vie-withdevelopers-for-surplus-properties/article29909455/

Sachgau, O. (2016a). Quarter of TDSB schools in critical condition: Report; auditor general says at least \$1.4B needed per year to maintain aging infrastructure. The Toronto Star (Toronto, Ontario)

Sachgau, O. (2016b). Toronto schools face \$3.4 billion repair backlog. Toronto Star. Retrieved from: https://www.thestar.com/yourtoronto/education/2016/08/22/toronto-schools-faces-34-billion-repairbacklog.html

Schilling, J., \& Logan, J. (2008). Greening the rust belt: A green infrastructure model for right sizing america's shrinking cities. Journal of the American Planning Association, 74(4), 451-466. doi:10.1080/01944360802354956

Sekaly, G.F. (July, 2016). New Renewal Funding to Keep Schools in a State of Good Repair. Ministry of Education, Ontario.

https://www.google.ca/url?sa=t\&rct=j\&q=\&esrc=s\&source=web\&cd=1\&cad=rja\&uact=8\&ved=0ahUKE wi8peSlov_VAhUJ44MKHQdwAQAQFggmMAA\&url=https\%3A\%2F\%2Fefis.fma.csc.gov.on.ca\%2Ffa ab\%2FMemos\%2FB2016\%2FB13 EN_AODA.pdf\&usg=AFQjCNGPCvCJQOiKuSVu41Xtx2iMYCN7 pA

Sharam, A., McShane, I., Bryant, L., \& De Silva, A. (2016). The barriers to re-purposing not-for-profit real property assets. Property Management, 34(3), 247-261. doi:10.1108/PM-09-2015-0046

Taylor, N. (April 2014). A Community Guide to Repurposing Vacant and Underutilized Historic Buildings. State Hisotric Preservation Office; Michigan State Housing Development Authority; Michigan Historic Preservation Network. Retrieved from: https://www.mhpn.org/wpcontent/uploads/2012/08/A Community Guide to Repurposing Vacant and Underutilized Historic B uildings_FINAL.pdf

TDSB. (2014). School Data (abbreviated Version). TDSB System Inventory. http://www.tdsb.on.ca/AboutUs/StrategyPlanning/LongTermProgramandAccommodationStrategy.aspx

TDSB. (2014a). Renewal Needs Backlog and Facility Condition Index. http://www.tdsb.on.ca/aboutus/accountability/renewalneedsbacklogandfacilityconditionindex.aspx 
TDSB. (June, 2014b). Capital Facts: Building Strong and Vibrant School Communities.

www.tdsb.on.ca/Portals/0/AboutUs/Budget/CapitalFacts.pdf

TDSB. (2014c). Our Mission. http://www.tdsb.on.ca/AboutUs.aspx

TDSB. (2014d). Facility Condition Index (FCI).

http://www.tdsb.on.ca/aboutus/accountability/renewalneedsbacklogandfacilityconditionindex/facilitycond $\underline{\text { itionindex.aspx }}$

TDSB. (2014e). Education Development Charges. Retrieved from: http://www.tdsb.on.ca/About-

Us/Accountability/Renewal-Needs-Backlog-and-Facility-Condition-Index/Education-Development-

Charges

TDSB. (2014f). What is Better Schools Brighter Futures. Retrieved from: http://www.tdsb.on.ca/About-

Us/Strategy-Planning/What-is-Better-Schools-Brighter-Futures

TDSB. (2015a). TDSB Capital Planning: Thoughts on Infrastructure Financing- Presentation to Trustees.

http://www.tdsb.on.ca/Portals/ward11/TDSB\%20Capital\%20Planning\%20-

\%20Thoughts\%20on\%20Infrastructure\%20Financing\%20Jan\%202015.pdf

TDSB. (Jan, 2016a). Ward Council Meeting-Space Forum: Capacity and Utilization.

http:/www.tdsb.on.ca/Portals/ward9/docs/P20160113-CapacityAndUtilization-

WithFloorPlansShowingCapacity.pdf

TDSB. (June 2016b). Long-Term Program and Accommodation Strategy 2016-2025. Retrieved from:

http://www.tdsb.on.ca/Portals/ward11/160615\%20Long\%20Term\%20App\%20A\%20Part\%20A\%20Smal 1.pdf

TLC. (2016). Bloor/Dufferin (Redevelopment Project).

http://www.torontolandscorp.com/tlc.php/property/bloordufferin-redevelopment-project/

TLC. (2014). Corporate History. http://www.torontolandscorp.com/tlc.php/corporate-history/

TLC. (n.d.). Lawrence/Midland (Redevelopment Project).

http://www.torontolandscorp.com/tlc.php/property/lawrencemidland-redevelopment-project/\#prettyPhoto 
Toronto. (Feb 2018). Toronto Employment Survey 2017. https://www.toronto.ca/wpcontent/uploads/2018/03/95b8-Toronto-Employment-Survey-2017-Bulletin.pdf

Toronto Maps v2. (n.d.). http://map.toronto.ca/maps/map.jsp?app=TorontoMaps v2

TTC. (2018). TTC Systems Map. Retrieved from: http://www.ttc.ca/PDF/Maps/TTC SystemMap.pdf

\section{Statistics Canada References}

Statistics Canada. (Sept 2013). 2011 National Household Survey (NHS) - Income and Housing.

http://www12.statcan.gc.ca/datasets/Index-

eng.cfm?Temporal $=2013 \&$ Theme $=98 \& \mathrm{VNAMEE}=\& \mathrm{GA}=8 \& \mathrm{~S}=0$

Statistics Canada. (Nov 2015). Table 2: Low Income Cut-offs (1992 base) after tax.

http://www.statcan.gc.ca/pub/75f0002m/2009002/tbl/tbl-2-eng.htm

Statistics Canada. (2016). Subsidized Housing: Part A: Short Definition. http://www12.statcan.gc.ca/nhsenm/2011/ref/dict/dwelling-logements017-eng.cfm

Statistics Canada. (2016a). Census Tract by CMA 535. Retrieved from: http://www12.statcan.gc.ca/censusrecensement/2016/geo/map-carte/ref/ct/files-fichiers/2016-92146-535-04.pdf

Statistics Canada. (May 2017). 2016 Census of Population- Age and Sex. http://www12.statcan.gc.ca/censusrecensement/2016/dp-pd/dt-td/Rpeng.cfm?TABID $=2 \& L A N G=E \& A=R \& A P A T H=3 \& D E T A I L=0 \& D I M=0 \& F L=A \& F R E E=0 \& G C=53503$ $\underline{12.04 \& \mathrm{GL}=-}$

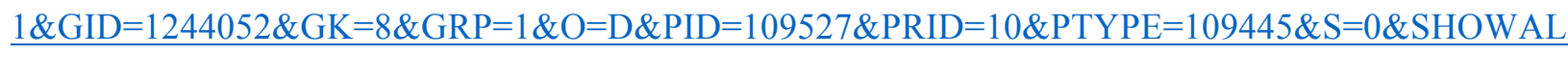

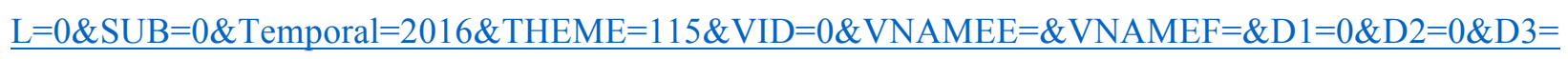
$\underline{0 \& \mathrm{D} 4=0 \& \mathrm{D} 5=0 \& \mathrm{D} 6=0}$

Statistics Canada. (Aug 2017). 2016 Census of Population - Families, households, and martial status. http://www12.statcan.gc.ca/census-recensement/2016/dp-pd/dt-td/Rpeng.cfm?TABID $=1 \& L A N G=E \& A=R \& A P A T H=3 \& D E T A I L=0 \& D I M=0 \& F L=A \& F R E E=0 \& G$ $\underline{\mathrm{C}=5350312.04 \& \mathrm{GL}=-}$

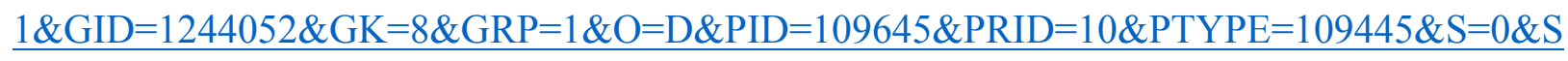


$\underline{\mathrm{HOWALL}}=0 \& \mathrm{SUB}=0 \&$ Temporal $=2016 \& \mathrm{THEME}=117 \& \mathrm{VID}=0 \& \mathrm{VNAMEE}=\& \mathrm{VNAMEF}=\&$ $\underline{\mathrm{D} 1}=0 \& \mathrm{D} 2=0 \& \mathrm{D} 3=0 \& \mathrm{D} 4=0 \& \mathrm{D} 5=0 \& \mathrm{D} 6=0$ 


\section{Appendix A: 2016-2017 TDSB Dataset}




\section{TAB 10}

School Data

(abbreviated version) 
Long-Term Program and Accommodation Strategy 2017-2026

Blank Page 


\section{School Data}

\section{(abbreviated data)}

The projections were calculated in the 2015-2016 school year for the period 2016-2017 to 2036-2037. They do not reflect recent enrolment trends that emerged in the fall of 2015 or Board decisions on grade range, boundaries, and program locations made in the 2016-2017 school year. Enrolment projections are revised each spring and are made available in the following year. 


\begin{tabular}{|c|c|c|c|c|c|c|c|c|c|c|c|c|c|c|c|c|c|c|c|c|c|c|}
\hline \multirow[b]{2}{*}{ School Name } & \multicolumn{8}{|c|}{ General Information } & \multicolumn{14}{|c|}{ Facility Information } \\
\hline & Panel & $\begin{array}{l}\text { Grade } \\
\text { Range }\end{array}$ & $\begin{array}{c}\text { Semestered } \\
\text { (Sec) }\end{array}$ & Ward & Trustee & $\begin{array}{c}\text { Superintendent } \\
\text { of Education }\end{array}$ & 2017 LOI & $\begin{array}{c}2017-18 \\
\text { Opt } \\
\text { Attend } \\
\text { Reg/FI/EF }\end{array}$ & $\begin{array}{l}\text { Site Size } \\
\text { (Acres) }\end{array}$ & $\begin{array}{c}\text { Facility Size } \\
\text { (Sq. Ft.) }\end{array}$ & $\begin{array}{c}\text { Current } \\
\text { Backlog (M) }\end{array}$ & $\begin{array}{l}\text { Port. } \\
\text { (Instruc) }\end{array}$ & \begin{tabular}{|c|}
$\begin{array}{c}\text { Port. (Non- } \\
\text { Instruc) }\end{array}$ \\
\end{tabular} & $\begin{array}{c}\text { Current FCI } \\
\text { (EDU) }\end{array}$ & $\begin{array}{c}\text { Eco } \\
\text { Schools }\end{array}$ & Pool & $\begin{array}{l}\text { Elem. } \\
\text { D\&T } \\
\text { Shops }\end{array}$ & $\begin{array}{l}\text { Barrier } \\
\text { Free / } \\
\text { Access }\end{array}$ & Elevator & \begin{tabular}{|l|} 
Build \\
Date
\end{tabular} & $\begin{array}{c}\text { Building } \\
\text { Age }\end{array}$ & $\begin{array}{l}\text { T.O Comm } \\
\text { Centre }\end{array}$ \\
\hline Adam Beck Junior Public School & $\mathrm{E}$ & JK-6 & & 16 & $\begin{array}{l}\text { Sheila Cary- } \\
\text { Meagher }\end{array}$ & John Chasty & 438 & $\begin{array}{c}\text { Closed } \\
\text { Closed } \\
\text { N/A }\end{array}$ & 2.97 & 51,843 & $\$ 3.5$ & 2 & & $36 \%$ & Gold & & & & & 1926 & 91 & Cat. 1 \\
\hline Africentric Alternative School & E & JK-8 & & 5 & Alexandra Lulka & Curtis Ennis & 29 & \begin{tabular}{|c|} 
Limited \\
N/A \\
N/A
\end{tabular} & & & & 2 & & & & & & & & & & \\
\hline Agincourt Junior Public School & $\mathrm{E}$ & $\mathrm{JK}-6$ & & 21 & Abdul Hai Patel & Andrew Howard & 321 & $\begin{array}{c}\text { Closed } \\
\text { N/A } \\
\text { N/A }\end{array}$ & 2.79 & 18,099 & $\$ 1.5$ & 3 & & $29 \%$ & Bronze & & & & & 1914 & 103 & \\
\hline Agnes Macphail Public School & E & $\mathrm{JK}-8$ & & 21 & Abdul Hai Patel & $\begin{array}{l}\text { Jacqueline } \\
\text { Spence }\end{array}$ & 310 & $\begin{array}{c}\text { Closed } \\
\text { N/A } \\
\text { N/A }\end{array}$ & 4.99 & 40,151 & $\$ 1.7$ & 1 & & $22 \%$ & \begin{tabular}{|l|} 
Platinum \\
\end{tabular} & & & & & 1981 & 36 & \\
\hline Albion Heights Junior Middle School & $\mathrm{E}$ & $\mathrm{JK}-8$ & & 1 & Avtar Minhas & Annie Appleby & 157 & $\begin{array}{c}\text { Closed } \\
\text { N/A } \\
\text { N/A }\end{array}$ & 6.13 & 56,683 & $\$ 6.4$ & & & $60 \%$ & & & & & & 1971 & 46 & \\
\hline $\begin{array}{l}\text { Alexander Muir/Gladstone Ave Junior } \\
\text { and Senior Public School }\end{array}$ & $\mathrm{E}$ & JK-8 & & 9 & Marit Stiles & $\begin{array}{l}\text { Jane Phillips- } \\
\text { Long }\end{array}$ & 201 & \begin{tabular}{c|} 
Limited \\
N/A \\
N/A
\end{tabular} & 4.67 & 119,906 & $\$ 18.6$ & & & $105 \%$ & Gold & & Yes & & & 1924 & 93 & \\
\hline Alexander Stirling Public School & $\mathrm{E}$ & $\mathrm{JK}-8$ & & 21 & Abdul Hai Patel & Nadira Persaud & 137 & $\begin{array}{c}\text { Closed } \\
\text { Limited } \\
\text { N/A }\end{array}$ & 4.99 & 64,696 & $\$ 4.1$ & & & $35 \%$ & Bronze & & & & No & 1984 & 33 & \\
\hline Alexmuir Junior Public School & E & JK-6 & & 21 & Abdul Hai Patel & $\begin{array}{l}\text { Jacqueline } \\
\text { Spence }\end{array}$ & 216 & $\begin{array}{c}\text { Limited } \\
\text { N/A } \\
\text { N/A }\end{array}$ & 4.99 & 58,942 & $\$ 4.5$ & & & $41 \%$ & & & & & & 1975 & 42 & \\
\hline Allenby Junior Public School & $\mathrm{E}$ & $\mathrm{JK}-6$ & & 8 & Jennifer Arp & Vicky Branco & 467 & $\begin{array}{c}\text { Closed } \\
\text { Closed } \\
\text { N/A }\end{array}$ & 3.43 & 85,850 & $\$ 4.3$ & & & $32 \%$ & \begin{tabular}{|l|} 
Platinum \\
\end{tabular} & TLC & & A1 & & 1927 & 90 & \\
\hline ALPHA Alternative Junior School & E & $\mathrm{JK}-6$ & & 10 & Ausma Malik & $\begin{array}{l}\text { Mary Jane } \\
\text { McNamara }\end{array}$ & 284 & $\begin{array}{c}\text { Limited } \\
\text { N/A } \\
\text { N/A }\end{array}$ & 0.62 & 19,148 & $\$ 2.7$ & & & $49 \%$ & & & & & & 1926 & 91 & \\
\hline Alvin Curling Public School & E & $\mathrm{JK}-8$ & & 21 & Abdul Hai Patel & Nadira Persaud & 375 & $\begin{array}{c}\text { Limited } \\
\text { N/A } \\
\text { N/A }\end{array}$ & & 69,250 & 0 & & & $0 \%$ & Gold & & & A1 & & 2013 & 4 & \\
\hline Amesbury Middle School & E & 6-8 & & 6 & Chris Tonks & $\begin{array}{l}\text { Angela Nardi- } \\
\text { Addesa }\end{array}$ & 32 & \begin{tabular}{c|} 
Limited \\
N/A \\
N/A
\end{tabular} & 12.01 & 86,230 & $\$ 12.5$ & & & $105 \%$ & & & & & & 1958 & 59 & \\
\hline Ancaster Public School & $\mathrm{E}$ & JK-5 & & 5 & Alexandra Lulka & Linda Curtis & 131 & \begin{tabular}{c|} 
Limited \\
N/A \\
N/A
\end{tabular} & 2.99 & 19,978 & $\$ 3.8$ & & & $71 \%$ & Gold & & & & & 1956 & 61 & \\
\hline $\begin{array}{l}\text { Annette Street Junior and Senior } \\
\text { Public School }\end{array}$ & $\mathrm{E}$ & JK-8 & & 7 & Robin Pilkey & Tracy Hayhurst & 392 & $\begin{array}{c}\text { Closed } \\
\text { N/A } \\
\text { N/A }\end{array}$ & 3.76 & 105,960 & $\$ 14.5$ & & & $87 \%$ & Gold & & Yes & & & 1910 & 107 & Cat. 4 \\
\hline Anson Park Public School & $\mathrm{E}$ & $\mathrm{JK}-8$ & & 18 & Parthi Kandavel & $\begin{array}{l}\text { Jacqueline } \\
\text { Spence }\end{array}$ & 193 & $\begin{array}{c}\text { Closed } \\
\text { N/A } \\
\text { N/A }\end{array}$ & 6.23 & 20,889 & $\$ 1.4$ & 4 & & $32 \%$ & Gold & & & & & 1958 & 59 & \\
\hline Anson S Taylor Junior Public School & E & $\mathrm{JK}-6$ & & 21 & Abdul Hai Patel & $\begin{array}{l}\text { Jacqueline } \\
\text { Spence }\end{array}$ & 295 & $\begin{array}{c}\text { Closed } \\
\text { N/A } \\
\text { N/A }\end{array}$ & 4.99 & 31,103 & $\$ 2.2$ & & & $38 \%$ & & & & & & 1979 & 38 & \\
\hline Arbor Glen Public School & $\mathrm{E}$ & JK-5 & & 12 & Alexander Brown & Elizabeth Addo & 414 & $\begin{array}{c}\text { Closed } \\
\text { N/A } \\
\text { N/A }\end{array}$ & 6 & 37,588 & $\$ 2.1$ & & & $33 \%$ & & & & & & 1975 & 42 & \\
\hline Armour Heights Public School & E & JK-6 & & 8 & Jennifer Arp & Vicky Branco & 449 & $\begin{array}{c}\text { Closed } \\
\text { N/A } \\
\text { N/A }\end{array}$ & 7.17 & 38,083 & $\$ 3.2$ & 1 & 1 & $45 \%$ & & & & & & 1946 & 71 & \\
\hline
\end{tabular}




\begin{tabular}{|c|c|c|c|c|c|c|c|c|c|c|c|c|c|c|c|c|c|c|}
\hline \multirow{2}{*}{ School Name } & \multicolumn{2}{|c|}{ Participation Rates } & \multicolumn{16}{|c|}{2016 Enrolment and Projections FTE, Surplus Seats = OTG Revised - Projection FTE, Utilizations = Projection/OTG Revised } \\
\hline & $\begin{array}{c}\text { TOSB 2013-14 } \\
\text { TDSB Participation } \\
\text { Rate }\end{array}$ & $\begin{array}{c}\text { TOSB 2013-14 } \\
\text { Home school } \\
\text { Particication Rate }\end{array}$ & $\begin{array}{c}\text { Revised } \\
\text { Capacity } \\
2016\end{array}$ & $\begin{array}{c}\text { HC } \\
\text { (Oct. 2016) }\end{array}$ & $\begin{array}{c}\text { FTE } \\
\text { (Oct. 2016) }\end{array}$ & $\begin{array}{l}2016 \\
\text { Utilization } \\
\text { Rate }\end{array}$ & $\begin{array}{c}2021 \\
\text { Projected }\end{array}$ & $\begin{array}{l}2021 \\
\text { Surplus } \\
\text { Seats }\end{array}$ & $\begin{array}{c}2021 \\
\text { Utilization } \\
\text { Rate }\end{array}$ & $\begin{array}{c}2026 \\
\text { Projected }\end{array}$ & $\begin{array}{l}2026 \\
\text { Surplus } \\
\text { Seats }\end{array}$ & $\begin{array}{l}2026 \\
\text { Utilization } \\
\text { Rate }\end{array}$ & $\begin{array}{c}2031 \\
\text { Projected }\end{array}$ & $\begin{array}{l}2031 \\
\text { Surplus } \\
\text { Seats }\end{array}$ & $\begin{array}{c}2031 \\
\text { Utilization } \\
\text { Rate }\end{array}$ & $\begin{array}{c}2036 \\
\text { Projected }\end{array}$ & $\begin{array}{l}2036 \\
\text { Surplus } \\
\text { Seats }\end{array}$ & $\begin{array}{l}2036 \\
\text { Utilization } \\
\text { Rate }\end{array}$ \\
\hline Adam Beck Junior Public School & $85.2 \%$ & $76.0 \%$ & 458 & 501 & 501.0 & $109 \%$ & 544 & -86 & $119 \%$ & 558 & -100 & $122 \%$ & 558 & -100 & $122 \%$ & 558 & -100 & $122 \%$ \\
\hline Africentric Alternative School & & & 216 & 101 & 101.0 & $47 \%$ & 128 & 88 & $59 \%$ & 145 & 71 & $67 \%$ & 145 & 71 & $67 \%$ & 145 & 71 & $67 \%$ \\
\hline Agincourt Junior Public School & 93.1\% & $75.8 \%$ & 153 & 224 & 224.0 & $146 \%$ & 216 & -63 & $141 \%$ & 236 & -83 & $154 \%$ & 238 & -85 & $156 \%$ & 238 & -85 & $156 \%$ \\
\hline Agnes Macphail Public School & $74.3 \%$ & $65.8 \%$ & 314 & 308 & 308.0 & $98 \%$ & 380 & -66 & $121 \%$ & 417 & -103 & $133 \%$ & 418 & -104 & $133 \%$ & 418 & -104 & $133 \%$ \\
\hline Albion Heights Junior Middle School & $74.2 \%$ & $63.5 \%$ & 509 & 444 & 444.0 & $87 \%$ & 366 & 143 & $72 \%$ & 322 & 187 & $63 \%$ & 323 & 186 & $63 \%$ & 323 & 186 & $63 \%$ \\
\hline $\begin{array}{l}\text { Alexander Muir/Gladstone Ave Junior } \\
\text { and Senior Public School }\end{array}$ & $69.7 \%$ & $29.9 \%$ & 522 & 416 & 416.0 & $80 \%$ & 557 & -35 & $107 \%$ & 567 & -45 & $109 \%$ & 560 & -38 & $107 \%$ & 560 & -38 & $107 \%$ \\
\hline Alexander Stirling Public School & 73.1\% & $66.0 \%$ & 583 & 396 & 396.0 & $68 \%$ & 396 & 187 & $68 \%$ & 384 & 199 & $66 \%$ & 381 & 202 & $65 \%$ & 381 & 202 & $65 \%$ \\
\hline Alexmuir Junior Public School & $84.4 \%$ & 74.3\% & 516 & 391 & 391.0 & $76 \%$ & 395 & 121 & $77 \%$ & 396 & 120 & $77 \%$ & 396 & 120 & $77 \%$ & 396 & 120 & $77 \%$ \\
\hline Allenby Junior Public School & $88.7 \%$ & $81.3 \%$ & 776 & 818 & 818.0 & $105 \%$ & 784 & -8 & $101 \%$ & 802 & -26 & $103 \%$ & 807 & -31 & $104 \%$ & 807 & -31 & $104 \%$ \\
\hline ALPHA Alternative Junior School & & & 95 & 83 & 83.0 & $87 \%$ & 89 & 6 & $94 \%$ & 86 & 9 & $91 \%$ & 86 & 9 & $91 \%$ & 86 & 9 & $91 \%$ \\
\hline Alvin Curling Public School & $64.7 \%$ & $55.8 \%$ & 702 & 499 & 499.0 & $71 \%$ & 461 & 241 & $66 \%$ & 375 & 327 & $53 \%$ & 371 & 331 & $53 \%$ & 371 & 331 & $53 \%$ \\
\hline Amesbury Middle School & $41.9 \%$ & $28.1 \%$ & 563 & 286 & 286.0 & $51 \%$ & 320 & 243 & $57 \%$ & 299 & 264 & $53 \%$ & 300 & 263 & $53 \%$ & 304 & 259 & $54 \%$ \\
\hline Ancaster Public School & $26.7 \%$ & $22.2 \%$ & 148 & 123 & 123.0 & $83 \%$ & 120 & 28 & $81 \%$ & 120 & 28 & $81 \%$ & 128 & 20 & $86 \%$ & 135 & 13 & $91 \%$ \\
\hline $\begin{array}{l}\text { Annette Street Junior and Senior } \\
\text { Public School }\end{array}$ & $79.4 \%$ & $44.8 \%$ & 568 & 502 & 502.0 & $88 \%$ & 526 & 42 & $93 \%$ & 534 & 34 & $94 \%$ & 530 & 38 & $93 \%$ & 531 & 37 & $93 \%$ \\
\hline Anson Park Public School & $81.1 \%$ & $63.8 \%$ & 159 & 247 & 247.0 & $155 \%$ & 278 & -119 & $175 \%$ & 301 & -142 & $189 \%$ & 303 & -144 & $191 \%$ & 303 & -144 & $191 \%$ \\
\hline Anson S Taylor Junior Public School & $59.1 \%$ & $50.7 \%$ & 271 & 217 & 216.5 & $80 \%$ & 206 & 65 & $76 \%$ & 195 & 76 & $72 \%$ & 196 & 75 & $72 \%$ & 195 & 76 & $72 \%$ \\
\hline Arbor Glen Public School & $96.2 \%$ & $81.5 \%$ & 300 & 291 & 291.0 & $97 \%$ & 314 & -14 & $105 \%$ & 315 & -15 & $105 \%$ & 315 & -15 & $105 \%$ & 315 & -15 & $105 \%$ \\
\hline Armour Heights Public School & $62.9 \%$ & $42.2 \%$ & 303 & 304 & 304.0 & $100 \%$ & 295 & 8 & $97 \%$ & 304 & -1 & $100 \%$ & 310 & -7 & $102 \%$ & 323 & -20 & $107 \%$ \\
\hline
\end{tabular}




\begin{tabular}{|c|c|c|c|c|c|c|c|c|c|c|c|c|c|c|c|c|c|c|c|c|c|c|}
\hline \multirow[b]{2}{*}{ School Name } & \multicolumn{8}{|c|}{ General Information } & \multicolumn{14}{|c|}{ Facility Information } \\
\hline & Panel & $\begin{array}{l}\text { Grade } \\
\text { Range }\end{array}$ & $\begin{array}{c}\text { Semestered } \\
(\mathrm{Sec})\end{array}$ & Ward & Trustee & $\begin{array}{l}\text { Superintendent } \\
\text { of Education }\end{array}$ & 2017 LOI & $\begin{array}{c}2017-18 \\
\mathrm{Opt} \\
\text { Attend } \\
\mathrm{Reg} / \mathrm{F} / \mathrm{EF}\end{array}$ & $\begin{array}{l}\text { Site Size } \\
\text { (Acres) }\end{array}$ & $\begin{array}{c}\text { Facility Size } \\
\text { (Sq. Ft.) }\end{array}$ & $\mid \begin{array}{c}\text { Current } \\
\text { Backlog (M) }\end{array}$ & \begin{tabular}{|l} 
Port. \\
(Instruc)
\end{tabular} & $\begin{array}{l}\text { Port. (Non- } \\
\text { Instruc) }\end{array}$ & $\begin{array}{c}\text { Current FCI } \\
\text { (EDU) }\end{array}$ & $\begin{array}{l}\text { Eco } \\
\text { Schools }\end{array}$ & Pool & $\begin{array}{l}\text { Elem. } \\
\text { D\&T } \\
\text { Shops }\end{array}$ & $\begin{array}{l}\text { Barrier } \\
\text { Free / } \\
\text { Access }\end{array}$ & Elevator & $\begin{array}{l}\text { Build } \\
\text { Date }\end{array}$ & $\begin{array}{c}\text { Building } \\
\text { Age }\end{array}$ & $\begin{array}{l}\text { T.O Comm } \\
\text { Centre }\end{array}$ \\
\hline $\begin{array}{l}\text { Avondale Elementary Alternative } \\
\text { School }\end{array}$ & E & JK-8 & & 12 & Alexander Brown & Linda Curtis & 385 & \begin{tabular}{|c|} 
Limited \\
N/A \\
N/A \\
\end{tabular} & 5.41 & 35,887 & & & & & & & & & & 1949 & 68 & \\
\hline Avondale Public School & E & JK-8 & & 12 & Alexander Brown & Linda Curtis & 347 & $\begin{array}{c}\text { Closed } \\
\text { N/A } \\
\text { N/A }\end{array}$ & & & & 7 & & & & & & & & 1949 & 68 & \\
\hline Bala Avenue Community School & $\mathrm{E}$ & JK-5 & & 6 & Chris Tonks & $\begin{array}{l}\text { Angela Nardi- } \\
\text { Addesa }\end{array}$ & 20 & $\begin{array}{c}\text { Limited } \\
\text { N/A } \\
\text { N/A }\end{array}$ & 2.87 & 41,803 & $\$ 2.5$ & & & $31 \%$ & & & & & & 1913 & 104 & \\
\hline Balmy Beach Community School & E & JK-6 & & 16 & $\begin{array}{l}\text { Sheila Cary- } \\
\text { Meagher }\end{array}$ & John Chasty & 451 & $\begin{array}{c}\text { Closed } \\
\text { N/A } \\
\text { N/A }\end{array}$ & 2.27 & 47,030 & $\$ 1.7$ & & 1 & $21 \%$ & & & & & & 1975 & 42 & Cat. 1 \\
\hline Banting and Best Public School & E & JK-8 & & 21 & Abdul Hai Patel & $\begin{array}{l}\text { Jacqueline } \\
\text { Spence }\end{array}$ & 242 & $\begin{array}{c}\text { Closed } \\
\text { N/A } \\
\text { N/A }\end{array}$ & 4.99 & 41,039 & $\$ 1.7$ & 3 & 2 & $23 \%$ & Gold & & & & Yes & 1989 & 28 & \\
\hline Baycrest Public School & E & JK-5 & & 8 & Jennifer Arp & Leila Girdhar-Hill & 109 & \begin{tabular}{c|} 
Limited \\
N/A \\
N/A
\end{tabular} & 5.39 & 22,206 & $\$ 5.3$ & & & $100 \%$ & & & & & & 1953 & 64 & \\
\hline Bayview Middle School & E & 6-8 & & 12 & Alexander Brown & Linda Curtis & 371 & $\begin{array}{c}\text { Closed } \\
\text { N/A } \\
\text { N/A }\end{array}$ & 6.3 & 83,582 & $\$ 11.2$ & & & $\mathbf{9 8 \%}$ & Bronze & & Yes & & & 1958 & 59 & \\
\hline Beaches Alternative Junior School & E & JK-6 & & 16 & $\begin{array}{l}\text { Sheila Cary- } \\
\text { Meagher }\end{array}$ & John Chasty & 354 & $\begin{array}{c}\text { Limited } \\
\text { N/A } \\
\text { N/A }\end{array}$ & & & & & & & & & & & & & & \\
\hline $\begin{array}{l}\text { Beaumonde Heights Junior Middle } \\
\text { School }\end{array}$ & $\mathrm{E}$ & JK-8 & & 1 & Avtar Minhas & Glenford Duffus & 192 & $\begin{array}{c}\text { Closed } \\
\text { N/A } \\
\text { N/A }\end{array}$ & 6.62 & 66,328 & $\$ 1.8$ & 2 & 1 & $16 \%$ & Gold & & & & & 1960 & 57 & \\
\hline Bedford Park Public School & E & JK-8 & & 13 & Gerri Gershon & Kathleen Garner & 466 & $\begin{array}{c}\text { Closed } \\
\text { N/A } \\
\text { N/A }\end{array}$ & 3.98 & 92,965 & $\$ 11.7$ & 1 & & $84 \%$ & & City & & & & 1910 & 107 & Cat. 1 \\
\hline Bellmere Junior Public School & E & JK-6 & & 19 & David Smith & Shirley Chan & 277 & $\begin{array}{c}\text { Closed } \\
\text { N/A } \\
\text { N/A }\end{array}$ & 5.61 & 40,357 & $\$ 6$. & 3 & & $72 \%$ & Platinum & & & & & 1964 & 53 & \\
\hline Ben Heppner Vocal Music Academy & E & $4-8$ & & 22 & Jerry Chadwick & Nadira Persaud & 86 & \begin{tabular}{c|} 
Limited \\
N/A \\
N/A
\end{tabular} & & & & & & & & & & & & & & \\
\hline Bendale Junior Public School & E & JK-6 & & 19 & David Smith & Shirley Chan & 236 & $\begin{array}{c}\text { Closed } \\
\text { N/A } \\
\text { N/A }\end{array}$ & 6 & 34,982 & $\$ 2.3$ & 4 & & $30 \%$ & Gold & & & & & 1957 & 60 & \\
\hline $\begin{array}{l}\text { Bennington Heights Elementary } \\
\text { School }\end{array}$ & $\mathrm{E}$ & JK-6 & & 13 & Gerri Gershon & Leila Girdhar-Hill & 450 & $\begin{array}{c}\text { Closed } \\
\text { N/A } \\
\text { N/A }\end{array}$ & 6.37 & 25,806 & $\$ 3.3$ & 7 & & $74 \%$ & & & & & & 1950 & 67 & \\
\hline Berner Trail Junior Public School & E & JK-6 & & 21 & Abdul Hai Patel & Andrew Howard & 199 & $\begin{array}{c}\text { Limited } \\
\text { N/A } \\
\text { N/A }\end{array}$ & 5.07 & 60,874 & $\$ 1.8$ & & & $19 \%$ & Gold & & & & & 1973 & 44 & Cat. 3 \\
\hline $\begin{array}{l}\text { Bessborough Drive Elementary and } \\
\text { Middle School }\end{array}$ & E & JK-8 & & 13 & Gerri Gershon & Leila Girdhar-Hill & 453 & $\begin{array}{c}\text { Closed } \\
\text { N/A } \\
\text { N/A }\end{array}$ & 3.6 & 56,748 & $\$ 1.5$ & 3 & & $17 \%$ & & & & & & 1923 & 94 & \\
\hline Beverley Heights Middle School & E & 6-8 & & 5 & Alexandra Lulka & Elizabeth Addo & 25 & $\begin{array}{l}\text { Limited } \\
\text { Limited } \\
\text { Limited }\end{array}$ & 7.91 & 73,540 & $\$ 6.2$ & & & $64 \%$ & & & Yes & & No & 1957 & 60 & \\
\hline Beverley School & E & JK-8 & & 10 & Ausma Malik & $\begin{array}{l}\text { Mary Jane } \\
\text { McNamara }\end{array}$ & 194 & $\begin{array}{c}\text { Limited } \\
\text { N/A } \\
\text { N/A }\end{array}$ & 1.14 & 50,870 & $\$ 1.8$ & & & $27 \%$ & & TDSB & & & Yes & 1950 & 67 & \\
\hline
\end{tabular}




\begin{tabular}{|c|c|c|c|c|c|c|c|c|c|c|c|c|c|c|c|c|c|c|}
\hline & Participat & tion Rates & & & & & Enrolment a & Projectio & FTE, Surplus & eats $=$ OTG R & ised - Proj & tion FTE, Uti & tions $=$ Proj & ion/OTG & vised & & & \\
\hline School Name & $\begin{array}{c}\text { TDSB 2013-14 } \\
\text { TDSB Participation } \\
\text { Rate }\end{array}$ & $\begin{array}{c}\text { TDSB 2013-14 } \\
\text { Home School } \\
\text { Participation Rate }\end{array}$ & $\begin{array}{c}\text { Revised } \\
\text { Capacity } \\
2016\end{array}$ & $\begin{array}{c}\text { HC } \\
\text { (Oct. 2016) }\end{array}$ & $\begin{array}{c}\text { FTE } \\
\text { (Oct. 2016) }\end{array}$ & $\begin{array}{c}2016 \\
\text { Utilization } \\
\text { Rate }\end{array}$ & $\begin{array}{c}2021 \\
\text { Projected }\end{array}$ & $\begin{array}{c}2021 \\
\text { Surplus } \\
\text { Seats }\end{array}$ & $\begin{array}{c}2021 \\
\text { Utilization } \\
\text { Rate }\end{array}$ & $\begin{array}{c}2026 \\
\text { Projected }\end{array}$ & $\begin{array}{c}2026 \\
\text { Surplus } \\
\text { Seats }\end{array}$ & $\begin{array}{c}2026 \\
\text { Utilization } \\
\text { Rate }\end{array}$ & $\begin{array}{c}2031 \\
\text { Projected }\end{array}$ & $\begin{array}{l}2031 \\
\text { Surplus } \\
\text { Seats }\end{array}$ & $\begin{array}{c}2031 \\
\text { Utilization } \\
\text { Rate }\end{array}$ & $\begin{array}{c}2036 \\
\text { Projected }\end{array}$ & $\begin{array}{l}2036 \\
\text { Surplus } \\
\text { Seats }\end{array}$ & $\begin{array}{c}2036 \\
\text { Utilization } \\
\text { Rate }\end{array}$ \\
\hline $\begin{array}{l}\text { Avondale Elementary Alternative } \\
\text { School }\end{array}$ & & & 138 & 139 & 139.0 & $101 \%$ & 135 & 3 & $98 \%$ & 135 & 3 & $98 \%$ & 135 & 3 & $98 \%$ & 135 & 3 & $98 \%$ \\
\hline Avondale Public School & $63.5 \%$ & $41.9 \%$ & 138 & 327 & 327.0 & $237 \%$ & 696 & -558 & $504 \%$ & 693 & -555 & $502 \%$ & 682 & -544 & $494 \%$ & 684 & -546 & $496 \%$ \\
\hline Bala Avenue Community School & $77.4 \%$ & $57.2 \%$ & 294 & 249 & 249.0 & $85 \%$ & 214 & 80 & $73 \%$ & 209 & 85 & $71 \%$ & 211 & 83 & $72 \%$ & 220 & 74 & $75 \%$ \\
\hline Balmy Beach Community School & $85.2 \%$ & $75.8 \%$ & 398 & 409 & 409.0 & $103 \%$ & 398 & 0 & $100 \%$ & 403 & -5 & $101 \%$ & 405 & -7 & $102 \%$ & 405 & -7 & $102 \%$ \\
\hline Banting and Best Public School & $74.2 \%$ & $67.1 \%$ & 363 & 385 & 385.0 & $106 \%$ & 365 & -2 & $101 \%$ & 346 & 17 & $95 \%$ & 337 & 26 & $93 \%$ & 337 & 26 & $93 \%$ \\
\hline Baycrest Public School & $29.0 \%$ & $11.9 \%$ & 176 & 129 & 129.0 & $73 \%$ & 141 & 35 & $80 \%$ & 132 & 44 & $75 \%$ & 132 & 44 & $75 \%$ & 132 & 44 & $75 \%$ \\
\hline Bayview Middle School & $73.1 \%$ & $52.5 \%$ & 341 & 397 & 397.0 & $116 \%$ & 419 & -78 & $123 \%$ & 490 & -149 & $144 \%$ & 485 & -144 & $142 \%$ & 485 & -144 & $142 \%$ \\
\hline Beaches Alternative Junior School & & & 92 & 71 & 71.0 & $77 \%$ & 74 & 18 & $80 \%$ & 75 & 17 & $82 \%$ & 75 & 17 & $82 \%$ & 75 & 17 & $82 \%$ \\
\hline $\begin{array}{l}\text { Beaumonde Heights Junior Middle } \\
\text { School }\end{array}$ & $56.7 \%$ & $48.4 \%$ & 620 & 629 & 629.0 & $101 \%$ & 555 & 65 & $90 \%$ & 536 & 84 & $86 \%$ & 545 & 75 & $88 \%$ & 545 & 75 & $88 \%$ \\
\hline Bedford Park Public School & $76.1 \%$ & $57.3 \%$ & 786 & 756 & 755.5 & $96 \%$ & 653 & 133 & $83 \%$ & 605 & 181 & $77 \%$ & 601 & 185 & $76 \%$ & 601 & 185 & $76 \%$ \\
\hline Bellmere Junior Public School & $81.3 \%$ & $73.2 \%$ & 386 & 399 & 399.0 & $103 \%$ & 386 & 0 & $100 \%$ & 397 & -11 & $103 \%$ & 408 & -22 & $106 \%$ & 408 & -22 & $106 \%$ \\
\hline Ben Heppner Vocal Music Academy & & & 92 & 66 & 66.0 & $72 \%$ & 78 & 14 & $85 \%$ & 78 & 14 & $85 \%$ & 78 & 14 & $85 \%$ & 78 & 14 & $85 \%$ \\
\hline Bendale Junior Public School & $65.9 \%$ & $56.1 \%$ & 376 & 405 & 405.0 & $108 \%$ & 539 & -163 & $143 \%$ & 552 & -176 & $147 \%$ & 552 & -176 & $147 \%$ & 552 & -176 & $147 \%$ \\
\hline $\begin{array}{l}\text { Bennington Heights Elementary } \\
\text { School }\end{array}$ & $88.1 \%$ & $76.1 \%$ & 179 & 184 & 184.0 & $103 \%$ & 198 & -19 & $111 \%$ & 241 & -62 & $135 \%$ & 240 & -61 & $134 \%$ & 240 & -61 & $134 \%$ \\
\hline Berner Trail Junior Public School & $80.9 \%$ & $74.0 \%$ & 461 & 295 & 295.0 & $64 \%$ & 253 & 208 & $55 \%$ & 252 & 209 & $55 \%$ & 252 & 209 & $55 \%$ & 252 & 209 & $55 \%$ \\
\hline $\begin{array}{l}\text { Bessborough Drive Elementary and } \\
\text { Middle School }\end{array}$ & $79.9 \%$ & $65.0 \%$ & 459 & 505 & 505.0 & $110 \%$ & 480 & -21 & $105 \%$ & 444 & 15 & $97 \%$ & 439 & 20 & $96 \%$ & 437 & 22 & $95 \%$ \\
\hline Beverley Heights Middle School & $44.5 \%$ & $33.9 \%$ & 588 & 406 & 406.0 & $69 \%$ & 425 & 163 & $72 \%$ & 401 & 187 & $68 \%$ & 404 & 184 & $69 \%$ & 404 & 184 & $69 \%$ \\
\hline Beverley School & & & 126 & 91 & 91.0 & $72 \%$ & 83 & 43 & $66 \%$ & 85 & 41 & $67 \%$ & 85 & 41 & $67 \%$ & 85 & 41 & $67 \%$ \\
\hline
\end{tabular}




\begin{tabular}{|c|c|c|c|c|c|c|c|c|c|c|c|c|c|c|c|c|c|c|c|c|c|c|}
\hline \multirow[b]{2}{*}{ School Name } & \multicolumn{8}{|c|}{ General Information } & \multicolumn{14}{|c|}{ Facility Information } \\
\hline & Panel & $\begin{array}{l}\text { Grade } \\
\text { Range }\end{array}$ & $\begin{array}{c}\text { Semestered } \\
\text { (Sec) }\end{array}$ & Ward & Trustee & $\begin{array}{c}\text { Superintendent } \\
\text { of Education }\end{array}$ & 2017 LOI & $\begin{array}{c}2017-18 \\
\text { Opt } \\
\text { Attend } \\
\text { Reg/F//FF }\end{array}$ & $\begin{array}{l}\text { Site Size } \\
\text { (Acres) }\end{array}$ & \begin{tabular}{|} 
Facility Size \\
(Sq. Ft.)
\end{tabular} & $\begin{array}{c}\text { Current } \\
\text { Backlog (M) }\end{array}$ & $\begin{array}{l}\text { Port. } \\
\text { (Instruc) }\end{array}$ & $\begin{array}{c}\text { Port. (Non- } \\
\text { Instruc) }\end{array}$ & $\begin{array}{c}\text { Current FCI } \\
\text { (EDU) }\end{array}$ & $\begin{array}{l}\text { Eco } \\
\text { Schools }\end{array}$ & Pool & $\begin{array}{l}\text { Elem. } \\
\text { D\&\& } \\
\text { Shops }\end{array}$ & $\begin{array}{l}\text { Barrier } \\
\text { Free / } \\
\text { Access }\end{array}$ & Elevator & $\begin{array}{l}\text { Build } \\
\text { Date }\end{array}$ & $\begin{array}{c}\text { Building } \\
\text { Age }\end{array}$ & $\begin{array}{c}\text { T.O Comm } \\
\text { Centre }\end{array}$ \\
\hline Beverly Glen Junior Public School & $\mathrm{E}$ & $\mathrm{JK}-6$ & & 20 & Manna Wong & Beth Veale & 246 & \begin{tabular}{|c|} 
Closed \\
Limited \\
N/A
\end{tabular} & 4.99 & 52,470 & $\$ 2.4$ & & & $23 \%$ & Gold & & & & & 1971 & 46 & \\
\hline Birch Cliff Heights Public School & E & JK-8 & & 18 & Parthi Kandavel & Peter Chang & 204 & $\begin{array}{c}\text { Closed } \\
\text { N/A } \\
\text { N/A }\end{array}$ & 4.89 & 43,043 & $\$ 5.9$ & & & $79 \%$ & & & & & & 1922 & 95 & \\
\hline Birch Cliff Public School & $\mathrm{E}$ & JK-8 & & 18 & Parthi Kandavel & Peter Chang & 350 & $\begin{array}{c}\text { Limited } \\
\text { Limited } \\
\text { N/A }\end{array}$ & 7.19 & 62,998 & $\$ 7.9$ & & & $81 \%$ & Gold & & & & & 1916 & 101 & \\
\hline Blacksmith Public School & E & JK-5 & & 4 & Tiffany Ford & Lynn Strangway & 35 & $\begin{array}{c}\text { Limited } \\
\text { N/A } \\
\text { N/A }\end{array}$ & 6.03 & 40,556 & $\$ 5.2$ & & & $72 \%$ & Gold & & & & & 1967 & 50 & \\
\hline Blake Street Junior Public School & $\mathrm{E}$ & $\mathrm{JK}-6$ & & 15 & Jennifer Story & $\begin{array}{l}\text { Mary Jane } \\
\text { McNamara }\end{array}$ & 96 & $\begin{array}{c}\text { Limited } \\
\text { Limited } \\
\text { N/A }\end{array}$ & 3.83 & 69,995 & $\$ 1.7$ & & & $10 \%$ & Gold & & & & & 1971 & 46 & \\
\hline Blantyre Public School & E & JK-8 & & 18 & Parthi Kandavel & Peter Chang & 377 & $\begin{array}{c}\text { Closed } \\
\text { N/A } \\
\text { N/A }\end{array}$ & 3.9 & 47,930 & $\$ 2.1$ & 1 & & $29 \%$ & Gold & & & A1 & Yes & 1993 & 24 & \\
\hline Blaydon Public School & $\mathrm{E}$ & JK-5 & & 5 & Alexandra Lulka & Linda Curtis & 55 & $\begin{array}{c}\text { Limited } \\
\text { N/A } \\
\text { Limited }\end{array}$ & 5.29 & 27,211 & $\$ 3.5$ & 1 & & $59 \%$ & Silver & & & & & 1955 & 62 & \\
\hline Bliss Carman Senior Public School & E & 7-8 & & 18 & Parthi Kandavel & $\begin{array}{l}\text { Kerry-Lynn } \\
\text { Stadnyk }\end{array}$ & 66 & $\begin{array}{c}\text { Limited } \\
\text { N/A } \\
\text { N/A }\end{array}$ & 8.06 & 54,056 & $\$ 4$. & & & $47 \%$ & Gold & & & & & 1973 & 44 & \\
\hline Bloordale Middle School & $\mathrm{E}$ & 6-8 & & 2 & Chris Glover & Glenford Duffus & 325 & $\begin{array}{c}\text { Limited } \\
\text { N/A } \\
\text { N/A }\end{array}$ & 8.1 & 70,346 & $\$ 3.7$ & & & $39 \%$ & Gold & & Yes & & & 1960 & 57 & Cat. 1 \\
\hline Bloorlea Middle School & E & 6-8 & & 3 & Pamela Gough & Tracy Hayhurst & 223 & $\begin{array}{c}\text { Limited } \\
N / A \\
\text { Limited }\end{array}$ & 9.49 & 40,408 & $\$ 2.3$ & & & $29 \%$ & Silver & & Yes & & & 1957 & 60 & \\
\hline Blythwood Junior Public School & E & $\mathrm{JK}-6$ & & 13 & Gerri Gershon & Leila Girdhar-Hill & 470 & $\begin{array}{c}\text { Limited } \\
\text { N/A } \\
\text { N/A }\end{array}$ & 5.31 & 40,933 & $\$ 5$. & & & $63 \%$ & Silver & & & & & 1932 & 85 & \\
\hline $\begin{array}{l}\text { Bowmore Road Junior and Senior } \\
\text { Public School }\end{array}$ & $\mathrm{E}$ & JK-8 & & 16 & $\begin{array}{l}\text { Sheila Cary- } \\
\text { Meagher }\end{array}$ & John Chasty & 370 & \begin{tabular}{|c|} 
Closed \\
Closed \\
Limited
\end{tabular} & 3.58 & 156,466 & $\$ 9.5$ & & & $52 \%$ & Gold & City & & & & 1922 & 95 & Cat. 3 \\
\hline Boys Leadership Academy & $\mathrm{E}$ & 4-8 & & 1 & Avtar Minhas & Glenford Duffus & 28 & \begin{tabular}{c|} 
Limited \\
N/A \\
N/A
\end{tabular} & & & & & & & & & & & & & & \\
\hline Braeburn Junior School & $\mathrm{E}$ & JK-5 & & 1 & Avtar Minhas & Glenford Duffus & 14 & $\begin{array}{c}\text { Limited } \\
\text { N/A } \\
\text { N/A }\end{array}$ & 4.99 & 32,841 & $\$ 3.7$ & & 3 & $49 \%$ & & & & & & 1968 & 49 & \\
\hline Brian Public School & $\mathrm{E}$ & $\mathrm{JK}-6$ & & 17 & Ken Lister & Beth Veale & 324 & $\begin{array}{l}\text { Closed } \\
\text { Closed } \\
\text { N/A }\end{array}$ & 5.93 & 35,048 & $\$ 5.4$ & 6 & & $75 \%$ & Gold & & & & No & 1956 & 61 & \\
\hline Briarcrest Junior School & E & JK-5 & & 2 & Chris Glover & Glenford Duffus & 274 & $\begin{array}{c}\text { Limited } \\
N / A \\
\text { Limited }\end{array}$ & 5.66 & 33,487 & $\$ 2$. & 1 & & $28 \%$ & & & & & & 1958 & 59 & \\
\hline Bridlewood Junior Public School & $\mathrm{E}$ & $\mathrm{JK}-6$ & & 20 & Manna Wong & $\begin{array}{l}\text { Louie } \\
\text { Papathanasakis }\end{array}$ & 379 & $\begin{array}{c}\text { Closed } \\
\text { N/A } \\
\text { N/A }\end{array}$ & 5.63 & 32,365 & $\$ 1.6$ & & & $28 \%$ & Gold & & & & & 1963 & 54 & \\
\hline $\begin{array}{l}\text { Brimwood Boulevard Junior Public } \\
\text { School }\end{array}$ & E & JK-6 & & 21 & Abdul Hai Patel & $\begin{array}{l}\text { Jacqueline } \\
\text { Spence }\end{array}$ & 270 & $\begin{array}{c}\text { Closed } \\
\text { Limited } \\
\text { N/A }\end{array}$ & 3.58 & 34,887 & $\$ 4.1$ & 2 & 2 & $56 \%$ & & & & & & 1974 & 43 & \\
\hline
\end{tabular}




\begin{tabular}{|c|c|c|c|c|c|c|c|c|c|c|c|c|c|c|c|c|c|c|}
\hline & Participa & tion Rates & & & & & Enrolment a & Projectio & FTE, Surplus & ats = OTG R & ised - Proj & tion FTE, Utili & tions $=$ Proj & ion/OTG & vised & & & \\
\hline School Name & $\begin{array}{c}\text { TDSB } 2013-14 \\
\text { TDSB Participation } \\
\text { Rate }\end{array}$ & $\begin{array}{c}\text { TDSB 2013-14 } \\
\text { Home School } \\
\text { Particication Rate }\end{array}$ & $\begin{array}{c}\text { Revised } \\
\text { Capacity } \\
2016\end{array}$ & $\begin{array}{c}\text { HC } \\
\text { (Oct. 2016) }\end{array}$ & $\begin{array}{c}\text { FTE } \\
\text { (Oct. 2016) }\end{array}$ & $\begin{array}{l}2016 \\
\text { Utilization } \\
\text { Rate }\end{array}$ & $\begin{array}{c}2021 \\
\text { Projected }\end{array}$ & $\begin{array}{c}2021 \\
\text { Surplus } \\
\text { Seats }\end{array}$ & $\begin{array}{c}2021 \\
\text { Utilization } \\
\text { Rate }\end{array}$ & $\begin{array}{l}2026 \\
\text { Projected }\end{array}$ & $\begin{array}{c}2026 \\
\text { Surplus } \\
\text { Seats }\end{array}$ & $\begin{array}{l}2026 \\
\text { Utilization } \\
\text { Rate }\end{array}$ & $\begin{array}{c}2031 \\
\text { Projected }\end{array}$ & $\begin{array}{c}2031 \\
\text { Surplus } \\
\text { Seats }\end{array}$ & $\begin{array}{c}2031 \\
\text { Utilization } \\
\text { Rate }\end{array}$ & $\begin{array}{c}2036 \\
\text { Projected }\end{array}$ & $\begin{array}{c}2036 \\
\text { Surplus } \\
\text { Seats }\end{array}$ & $\begin{array}{l}2036 \\
\text { Utilization } \\
\text { Rate }\end{array}$ \\
\hline Beverly Glen Junior Public School & $73.7 \%$ & $67.3 \%$ & 527 & 485 & 485.0 & $92 \%$ & 472 & 55 & $90 \%$ & 469 & 58 & $89 \%$ & 463 & 64 & $88 \%$ & 463 & 64 & $88 \%$ \\
\hline Birch Cliff Heights Public School & $77.6 \%$ & $62.6 \%$ & 309 & 273 & 272.0 & $88 \%$ & 299 & 10 & $97 \%$ & 316 & -7 & $102 \%$ & 314 & -5 & $102 \%$ & 314 & -5 & $102 \%$ \\
\hline Birch Cliff Public School & $74.8 \%$ & $55.8 \%$ & 438 & 377 & 378.0 & $86 \%$ & 528 & -90 & $121 \%$ & 556 & -118 & $127 \%$ & 557 & -119 & $127 \%$ & 557 & -119 & $127 \%$ \\
\hline Blacksmith Public School & $46.6 \%$ & $40.4 \%$ & 326 & 207 & 207.0 & $63 \%$ & 183 & 143 & $56 \%$ & 183 & 143 & $56 \%$ & 183 & 143 & $56 \%$ & 183 & 143 & $56 \%$ \\
\hline Blake Street Junior Public School & $75.2 \%$ & $33.0 \%$ & 573 & 324 & 324.0 & $57 \%$ & 549 & 24 & $96 \%$ & 555 & 18 & $97 \%$ & 555 & 18 & $97 \%$ & 555 & 18 & $97 \%$ \\
\hline Blantyre Public School & $76.5 \%$ & $59.4 \%$ & 323 & 291 & 291.0 & $90 \%$ & 403 & -80 & $125 \%$ & 454 & -131 & $141 \%$ & 436 & -113 & $135 \%$ & 436 & -113 & $135 \%$ \\
\hline Blaydon Public School & $60.8 \%$ & $53.0 \%$ & 174 & 181 & 181.0 & $104 \%$ & 187 & -13 & $107 \%$ & 192 & -18 & $110 \%$ & 192 & -18 & $110 \%$ & 192 & -18 & $110 \%$ \\
\hline Bliss Carman Senior Public School & $70.6 \%$ & $47.4 \%$ & 412 & 297 & 297.0 & $72 \%$ & 275 & 137 & $67 \%$ & 242 & 170 & $59 \%$ & 229 & 183 & $56 \%$ & 229 & 183 & $56 \%$ \\
\hline Bloordale Middle School & $59.9 \%$ & $39.6 \%$ & 544 & 322 & 322.0 & $59 \%$ & 270 & 274 & $50 \%$ & 264 & 280 & $49 \%$ & 257 & 287 & $47 \%$ & 257 & 287 & $47 \%$ \\
\hline Bloorlea Middle School & $47.0 \%$ & $35.2 \%$ & 306 & 256 & 256.0 & $84 \%$ & 376 & -70 & $123 \%$ & 348 & -42 & $114 \%$ & 331 & -25 & $108 \%$ & 331 & -25 & $108 \%$ \\
\hline Blythwood Junior Public School & $77.0 \%$ & $61.5 \%$ & 369 & 398 & 398.0 & $108 \%$ & 440 & -71 & $119 \%$ & 493 & -124 & $134 \%$ & 487 & -118 & $132 \%$ & 487 & -118 & $132 \%$ \\
\hline $\begin{array}{l}\text { Bowmore Road Junior and Senior } \\
\text { Public School }\end{array}$ & $78.7 \%$ & $54.9 \%$ & 970 & 884 & 884.0 & $91 \%$ & 1,050 & -80 & $108 \%$ & 1,073 & -103 & $111 \%$ & 1,076 & -106 & $111 \%$ & 1,076 & -106 & $111 \%$ \\
\hline Boys Leadership Academy & & & 115 & 104 & 104.0 & $\mathbf{9 0 \%}$ & 112 & 3 & $97 \%$ & 112 & 3 & $97 \%$ & 112 & 3 & $97 \%$ & 112 & 3 & $97 \%$ \\
\hline Braeburn Junior School & $56.3 \%$ & $34.5 \%$ & 366 & 168 & 168.0 & $46 \%$ & 162 & 204 & $44 \%$ & 161 & 205 & $44 \%$ & 161 & 205 & $44 \%$ & 161 & 205 & $44 \%$ \\
\hline Brian Public School & $87.8 \%$ & $81.4 \%$ & 358 & 435 & 435.0 & $122 \%$ & 354 & 4 & $99 \%$ & 357 & 1 & $100 \%$ & 357 & 1 & $100 \%$ & 357 & 1 & $100 \%$ \\
\hline Briarcrest Junior School & $65.6 \%$ & $56.0 \%$ & 309 & 286 & 286.0 & $93 \%$ & 285 & 24 & $92 \%$ & 287 & 22 & $93 \%$ & 287 & 22 & $93 \%$ & 287 & 22 & $93 \%$ \\
\hline Bridlewood Junior Public School & $97.9 \%$ & $84.5 \%$ & 261 & 238 & 238.0 & $91 \%$ & 303 & -42 & $116 \%$ & 315 & -54 & $121 \%$ & 315 & -54 & $121 \%$ & 315 & -54 & $121 \%$ \\
\hline $\begin{array}{l}\text { Brimwood Boulevard Junior Public } \\
\text { School }\end{array}$ & $79.6 \%$ & $74.7 \%$ & 424 & 387 & 387.0 & $91 \%$ & 385 & 39 & $91 \%$ & 377 & 47 & $89 \%$ & 378 & 46 & $89 \%$ & 378 & 46 & $89 \%$ \\
\hline
\end{tabular}




\begin{tabular}{|c|c|c|c|c|c|c|c|c|c|c|c|c|c|c|c|c|c|c|c|c|c|c|}
\hline \multirow[b]{2}{*}{ School Name } & \multicolumn{8}{|c|}{ General Information } & \multicolumn{14}{|c|}{ Facility Information } \\
\hline & Panel & $\begin{array}{l}\text { Grade } \\
\text { Range }\end{array}$ & $\begin{array}{c}\text { Semestered } \\
\text { (Sec) }\end{array}$ & Ward & Trustee & $\begin{array}{l}\text { Superintendent } \\
\text { of Education }\end{array}$ & 2017 LOI & $\begin{array}{c}2017-18 \\
\text { Opt } \\
\text { Attend } \\
\text { Reg/FI/EF }\end{array}$ & $\begin{array}{l}\text { Site Size } \\
\text { (Acres) }\end{array}$ & $\begin{array}{l}\text { Facility Size } \\
\text { (Sq. Ft.) }\end{array}$ & $\mid \begin{array}{c}\text { Current } \\
\text { Backlog (M) }\end{array}$ & $\begin{array}{l}\text { Port. } \\
\text { (Instruc) }\end{array}$ & $\begin{array}{c}\begin{array}{c}\text { Port. (Non- } \\
\text { Instruc) }\end{array} \\
\end{array}$ & $\begin{array}{c}\text { Current FCI } \\
\text { (EDU) }\end{array}$ & $\begin{array}{c}\text { Eco } \\
\text { Schools }\end{array}$ & Pool & $\begin{array}{l}\text { Elem. } \\
\text { D\&T } \\
\text { Shops }\end{array}$ & $\begin{array}{l}\text { Barrier } \\
\text { Free / } \\
\text { Access }\end{array}$ & Elevator & $\begin{array}{l}\text { Build } \\
\text { Date }\end{array}$ & $\begin{array}{c}\text { Building } \\
\text { Age }\end{array}$ & $\begin{array}{l}\text { T.o Comm } \\
\text { Centre }\end{array}$ \\
\hline Broadacres Junior School & $\mathrm{E}$ & JK-5 & & 2 & Chris Glover & Annie Appleby & 294 & $\begin{array}{c}\text { Limited } \\
\text { Limited } \\
\text { N/A }\end{array}$ & 6.25 & 40,150 & $\$ 2.4$ & & & $25 \%$ & & & & B1 & & 1959 & 58 & \\
\hline Broadlands Public School & E & SK-6 & & 17 & Ken Lister & Curtis Ennis & 264 & $\begin{array}{c}\text { N/A } \\
\text { Closed } \\
\text { N/A }\end{array}$ & 5.76 & 38,266 & $\$ 1.9$ & 7 & & $22 \%$ & & & & & & 1959 & 58 & \\
\hline Brock Public School & $\mathrm{E}$ & JK-8 & & 9 & Marit Stiles & $\begin{array}{l}\text { Jane Phillips- } \\
\text { Long }\end{array}$ & 228 & $\begin{array}{c}\text { Limited } \\
\text { N/A } \\
\text { N/A }\end{array}$ & 1.75 & 58,636 & $\$ 2.3$ & & & $28 \%$ & & & & & & 1914 & 103 & \\
\hline Brookhaven Public School & E & JK-5 & & 6 & Chris Tonks & $\begin{array}{l}\text { Angela Nardi- } \\
\text { Addesa }\end{array}$ & 47 & $\begin{array}{c}\text { Limited } \\
\text { N/A } \\
\text { N/A }\end{array}$ & 7.66 & 63,600 & $\$ 2.5$ & & & $22 \%$ & Gold & & & A1 & Yes & 1992 & 25 & \\
\hline $\begin{array}{l}\text { Brookmill Boulevard Junior Public } \\
\text { School }\end{array}$ & $\mathrm{E}$ & JK-6 & & 20 & Manna Wong & Beth Veale & 318 & $\begin{array}{c}\text { Limited } \\
\text { N/A } \\
\text { N/A }\end{array}$ & 4.99 & 34,887 & $\$ 1.3$ & & & $17 \%$ & Silver & & & & & 1974 & 43 & \\
\hline Brookside Public School & E & JK-8 & & 21 & Abdul Hai Patel & Andrew Howard & 367 & $\begin{array}{c}\text { Closed } \\
\text { N/A } \\
\text { N/A }\end{array}$ & 6.03 & 62,950 & $\$ .1$ & 3 & & $0 \%$ & Gold & & Yes & A1 & Yes & 2007 & 10 & \\
\hline Brookview Middle School & $\mathrm{E}$ & 6-8 & & 4 & Tiffany Ford & Curtis Ennis & 16 & $\begin{array}{c}\text { Limited } \\
\text { N/A } \\
\text { N/A }\end{array}$ & 9.96 & 94,196 & $\$ 2.8$ & & & $28 \%$ & Bronze & & & & & 1967 & 50 & \\
\hline Brown Junior Public School & E & JK-6 & & 11 & Shelley Laskin & Ian Allison & 443 & $\begin{array}{c}\text { Closed } \\
\text { Closed } \\
\text { N/A }\end{array}$ & 2.67 & 69,359 & $\$ 2.7$ & & & $22 \%$ & Silver & TLC & & & & 1973 & 44 & Cat. 1 \\
\hline Bruce Public School & $\mathrm{E}$ & JK-8 & & 15 & Jennifer Story & John Chasty & 170 & $\begin{array}{c}\text { Limited } \\
\text { N/A } \\
\text { N/A }\end{array}$ & 1.98 & 49,921 & $\$ 3.5$ & & & $40 \%$ & Silver & & & & & 1922 & 95 & \\
\hline Buchanan Public School & E & JK-8 & & 19 & David Smith & Nadira Persaud & 188 & $\begin{array}{c}\text { Limited } \\
\text { N/A } \\
\text { N/A }\end{array}$ & 7.76 & 43,293 & $\$ 2.4$ & & & $28 \%$ & Gold & & Yes & & & 1953 & 64 & \\
\hline Burrows Hall Junior Public School & E & $\mathrm{JK}-6$ & & 21 & Abdul Hai Patel & Andrew Howard & 217 & $\begin{array}{c}\text { Limited } \\
\text { N/A } \\
\text { N/A }\end{array}$ & 4.99 & 34,288 & $\$ 4.2$ & & 1 & $59 \%$ & & & & & & 1975 & 42 & \\
\hline C D Farquharson Junior Public School & E & $\mathrm{JK}-6$ & & 21 & Abdul Hai Patel & Andrew Howard & 226 & $\begin{array}{c}\text { Closed } \\
\text { N/A } \\
\text { N/A }\end{array}$ & 7.78 & 46,332 & $\$ 6.9$ & & & $79 \%$ & Gold & & & & & 1953 & 64 & \\
\hline C R Marchant Middle School & $\mathrm{E}$ & 6-8 & & 6 & Chris Tonks & $\begin{array}{l}\text { Angela Nardi- } \\
\text { Addesa }\end{array}$ & 51 & \begin{tabular}{c|} 
Limited \\
N/A \\
N/A
\end{tabular} & 2.59 & 71,053 & $\$ 2.8$ & & & $28 \%$ & & & Yes & & & 1948 & 69 & \\
\hline Calico Public School & $\mathrm{E}$ & JK-5 & & 5 & Alexandra Lulka & Elizabeth Addo & 40 & $\begin{array}{c}\text { Limited } \\
\text { N/A } \\
\text { N/A }\end{array}$ & 4.69 & 59,740 & $\$ 8.7$ & & & $64 \%$ & Silver & & & & & 1958 & 59 & \\
\hline Cameron Public School & $\mathrm{E}$ & JK-5 & & 12 & Alexander Brown & Elizabeth Addo & 428 & $\begin{array}{c}\text { Closed } \\
\text { N/A } \\
\text { N/A }\end{array}$ & 4.1 & 35,446 & $\$ 1.9$ & & & $25 \%$ & Gold & & & & & 1949 & 68 & \\
\hline $\begin{array}{l}\text { Carleton Village Junior and Senior } \\
\text { Sports and Wellness Academy }\end{array}$ & E & JK-8 & & 9 & Marit Stiles & Sandra Tondat & 73 & $\begin{array}{c}\text { Limited } \\
\text { Limited } \\
\text { N/A }\end{array}$ & 1.98 & 100,803 & $\$ 9.6$ & & & $69 \%$ & Silver & TLC & Yes & & & 1961 & 56 & \\
\hline Cassandra Public School & $\mathrm{E}$ & JK-5 & & 17 & Ken Lister & Curtis Ennis & 273 & $\begin{array}{c}\text { Closed } \\
\text { N/A } \\
\text { Closed }\end{array}$ & 6.33 & 36,081 & $\$ 1.9$ & & & $27 \%$ & Platinum & & & & & 1964 & 53 & \\
\hline Cedar Drive Junior Public School & E & JK-6 & & 18 & Parthi Kandavel & $\begin{array}{l}\text { Kerry-Lynn } \\
\text { Stadnyk }\end{array}$ & 50 & $\begin{array}{c}\text { Closed } \\
\text { N/A } \\
\text { N/A }\end{array}$ & 4.99 & 96,849 & $\$ 8.2$ & 5 & 2 & $50 \%$ & Bronze & & & & Yes & 1970 & 47 & \\
\hline
\end{tabular}




\begin{tabular}{|c|c|c|c|c|c|c|c|c|c|c|c|c|c|c|c|c|c|c|}
\hline Board & Participa & tion Rates & & & & & Enrolment a & Projectio & FTE, Surplus & ats = OTG R & ised-Proje & tion FTE, Utili & tions $=$ Proj & ion/OTG $\mathrm{R}$ & vised & & & \\
\hline School Name & $\begin{array}{c}\text { TDSB 2013-14 } \\
\text { TDSB Participation } \\
\text { Rate }\end{array}$ & $\begin{array}{c}\text { TDSB 2013-14 } \\
\text { Home School } \\
\text { Participation Rate }\end{array}$ & $\begin{array}{c}\text { Revised } \\
\text { Capacity } \\
2016\end{array}$ & $\begin{array}{c}\text { HC } \\
\text { (Oct. 2016) }\end{array}$ & \begin{tabular}{|c|} 
FTE \\
(Oct. 2016)
\end{tabular} & $\begin{array}{c}2016 \\
\text { Utilization } \\
\text { Rate }\end{array}$ & $\begin{array}{c}2021 \\
\text { Projected }\end{array}$ & $\begin{array}{l}2021 \\
\text { Surplus } \\
\text { Seats }\end{array}$ & $\begin{array}{c}2021 \\
\text { Utilization } \\
\text { Rate }\end{array}$ & $\begin{array}{c}2026 \\
\text { Projected }\end{array}$ & $\begin{array}{l}2026 \\
\text { Surplus } \\
\text { Seats }\end{array}$ & $\begin{array}{c}2026 \\
\text { Utilization } \\
\text { Rate }\end{array}$ & $\begin{array}{c}2031 \\
\text { Projected }\end{array}$ & $\begin{array}{l}2031 \\
\text { Surplus } \\
\text { Seats }\end{array}$ & $\begin{array}{c}2031 \\
\text { Utilization } \\
\text { Rate }\end{array}$ & $\begin{array}{c}2036 \\
\text { Projected }\end{array}$ & $\begin{array}{l}2036 \\
\text { Surplus } \\
\text { Seats }\end{array}$ & $\begin{array}{c}2036 \\
\text { Utilization } \\
\text { Rate }\end{array}$ \\
\hline Broadacres Junior School & $59.7 \%$ & $45.3 \%$ & 464 & 354 & 354.0 & $76 \%$ & 354 & 110 & $76 \%$ & 351 & 113 & $76 \%$ & 351 & 113 & $76 \%$ & 351 & 113 & $76 \%$ \\
\hline Broadlands Public School & & & 444 & 583 & 583.0 & $131 \%$ & 615 & -171 & $139 \%$ & 611 & -167 & $138 \%$ & 611 & -167 & $138 \%$ & 611 & -167 & $138 \%$ \\
\hline Brock Public School & $49.6 \%$ & $25.0 \%$ & 364 & 275 & 275.0 & $76 \%$ & 306 & 58 & $84 \%$ & 292 & 72 & $80 \%$ & 293 & 71 & $80 \%$ & 293 & 71 & $80 \%$ \\
\hline Brookhaven Public School & $51.4 \%$ & $39.8 \%$ & 614 & 413 & 413.0 & $67 \%$ & 472 & 142 & $77 \%$ & 453 & 161 & $74 \%$ & 453 & 161 & $74 \%$ & 453 & 161 & $74 \%$ \\
\hline $\begin{array}{l}\text { Brookmill Boulevard Junior Public } \\
\text { School }\end{array}$ & $73.8 \%$ & $63.9 \%$ & 305 & 258 & 258.0 & $85 \%$ & 248 & 57 & $81 \%$ & 237 & 68 & $78 \%$ & 236 & 69 & $77 \%$ & 236 & 69 & $77 \%$ \\
\hline Brookside Public School & $74.6 \%$ & $68.9 \%$ & 743 & 772 & 773.0 & $104 \%$ & 695 & 48 & $94 \%$ & 650 & 93 & $87 \%$ & 654 & 89 & $88 \%$ & 654 & 89 & $88 \%$ \\
\hline Brookview Middle School & $56.9 \%$ & $45.3 \%$ & 499 & 408 & 408.0 & $82 \%$ & 333 & 166 & $67 \%$ & 302 & 197 & $61 \%$ & 308 & 191 & $62 \%$ & 308 & 191 & $62 \%$ \\
\hline Brown Junior Public School & $79.6 \%$ & $68.4 \%$ & 601 & 614 & 613.0 & $102 \%$ & 646 & -45 & $107 \%$ & 656 & -55 & $109 \%$ & 653 & -52 & $109 \%$ & 653 & -52 & $109 \%$ \\
\hline Bruce Public School & $91.6 \%$ & $44.3 \%$ & 332 & 217 & 217.0 & $65 \%$ & 261 & 71 & $79 \%$ & 266 & 66 & $80 \%$ & 269 & 63 & $81 \%$ & 269 & 63 & $81 \%$ \\
\hline Buchanan Public School & $64.8 \%$ & $51.7 \%$ & 439 & 309 & 309.0 & $70 \%$ & 248 & 191 & $56 \%$ & 239 & 200 & $54 \%$ & 235 & 204 & $54 \%$ & 235 & 204 & $54 \%$ \\
\hline Burrows Hall Junior Public School & $59.0 \%$ & $50.4 \%$ & 329 & 218 & 218.0 & $66 \%$ & 299 & 30 & $91 \%$ & 312 & 17 & $95 \%$ & 312 & 17 & $95 \%$ & 312 & 17 & $95 \%$ \\
\hline C D Farquharson Junior Public School & $81.1 \%$ & $73.6 \%$ & 408 & 371 & 371.0 & $91 \%$ & 259 & 149 & $63 \%$ & 257 & 151 & $63 \%$ & 257 & 151 & $63 \%$ & 257 & 151 & $63 \%$ \\
\hline C R Marchant Middle School & $69.0 \%$ & $56.0 \%$ & 488 & 394 & 394.0 & $81 \%$ & 414 & 74 & $85 \%$ & 365 & 123 & $75 \%$ & 347 & 141 & $71 \%$ & 351 & 137 & $72 \%$ \\
\hline Calico Public School & $44.6 \%$ & $30.4 \%$ & 524 & 269 & 269.0 & $51 \%$ & 250 & 274 & $48 \%$ & 266 & 258 & $51 \%$ & 266 & 258 & $51 \%$ & 266 & 258 & $51 \%$ \\
\hline Cameron Public School & $60.7 \%$ & $42.1 \%$ & 320 & 325 & 325.0 & $102 \%$ & 339 & -19 & $106 \%$ & 334 & -14 & $104 \%$ & 333 & -13 & $104 \%$ & 333 & -13 & $104 \%$ \\
\hline $\begin{array}{l}\text { Carleton Village Junior and Senior } \\
\text { Sports and Wellness Academy }\end{array}$ & $43.5 \%$ & $25.8 \%$ & 616 & 308 & 308.0 & $50 \%$ & 392 & 224 & $64 \%$ & 441 & 175 & $72 \%$ & 444 & 172 & $72 \%$ & 444 & 172 & $72 \%$ \\
\hline Cassandra Public School & $71.1 \%$ & $32.7 \%$ & 288 & 266 & 266.0 & $92 \%$ & 241 & 47 & $84 \%$ & 235 & 53 & $82 \%$ & 235 & 53 & $82 \%$ & 235 & 53 & $82 \%$ \\
\hline Cedar Drive Junior Public School & $80.9 \%$ & $69.2 \%$ & 830 & 723 & 723.0 & $87 \%$ & 653 & 177 & $79 \%$ & 631 & 199 & $76 \%$ & 631 & 199 & $76 \%$ & 631 & 199 & $76 \%$ \\
\hline
\end{tabular}




\begin{tabular}{|c|c|c|c|c|c|c|c|c|c|c|c|c|c|c|c|c|c|c|c|c|c|c|}
\hline \multirow[b]{2}{*}{ School Name } & \multicolumn{8}{|c|}{ General Information } & \multicolumn{14}{|c|}{ Facility Information } \\
\hline & Panel & $\begin{array}{l}\text { Grade } \\
\text { Range }\end{array}$ & $\begin{array}{c}\text { Semestered } \\
(\mathrm{sec})\end{array}$ & Ward & Trustee & $\begin{array}{l}\text { Superintendent } \\
\text { of Education }\end{array}$ & 2017 LOI & $\begin{array}{c}\text { 2017-18 } \\
\text { Opt } \\
\text { Attend } \\
\text { Reg/F/EF }\end{array}$ & $\begin{array}{l}\text { Site Size } \\
\text { (Acres) }\end{array}$ & $\begin{array}{c}\text { Facility Size } \\
\text { (Sq. Ft.) }\end{array}$ & $\begin{array}{c}\text { Current } \\
\text { Backlog (M) }\end{array}$ & $\begin{array}{c}\text { Port. } \\
\text { (Instruc) }\end{array}$ & $\begin{array}{c}\text { Port. (Non- } \\
\text { Instruc) }\end{array}$ & $\begin{array}{c}\text { Current FCI } \\
\text { (EDU) }\end{array}$ & $\begin{array}{c}\text { Eco } \\
\text { Schools }\end{array}$ & Pool & $\begin{array}{l}\text { Elem. } \\
\text { D\&T } \\
\text { Shops }\end{array}$ & $\begin{array}{l}\text { Barrier } \\
\text { Free / } \\
\text { Access }\end{array}$ & Elevator & $\begin{array}{l}\text { Build } \\
\text { Date }\end{array}$ & $\begin{array}{c}\text { Building } \\
\text { Age }\end{array}$ & $\begin{array}{l}\text { T.O Comm } \\
\text { Centre }\end{array}$ \\
\hline Cedarbrook Public School & $\mathrm{E}$ & JK-8 & & 19 & David Smith & Anne Seymour & 140 & $\begin{array}{c}\text { Closed } \\
\text { N/A } \\
\text { Limited }\end{array}$ & 5.19 & 48,047 & $\$ 7$. & & & $65 \%$ & & & Yes & & & 1957 & 60 & \\
\hline Cedarvale Community School & E & JK-8 & & 11 & Shelley Laskin & Leila Girdhar-Hill & 460 & $\begin{array}{c}\text { Closed } \\
\text { N/A } \\
\text { N/A }\end{array}$ & 4.03 & 42,497 & $\$ 3.3$ & 1 & & $41 \%$ & Platinum & & & & & 1950 & 67 & \\
\hline Centennial Road Junior Public School & $\mathrm{E}$ & JK-6 & & 22 & Jerry Chadwick & $\begin{array}{l}\text { Kerry-Lynn } \\
\text { Stadnyk }\end{array}$ & 396 & $\begin{array}{c}\text { Closed } \\
\text { N/A } \\
\text { Closed }\end{array}$ & 6 & 25,704 & $\$ 2.7$ & 3 & & $47 \%$ & Gold & & & & & 1945 & 72 & \\
\hline Chalkfarm Public School & E & JK-5 & & 4 & Tiffany Ford & Lynn Strangway & 26 & $\begin{array}{c}\text { Closed } \\
\text { N/A } \\
\text { N/A }\end{array}$ & 4.15 & 28,976 & $\$ 1.3$ & 1 & 3 & $21 \%$ & & & & & & 1958 & 59 & \\
\hline Charles E Webster Public School & $\mathrm{E}$ & JK-8 & & 6 & Chris Tonks & Vicky Branco & 19 & $\begin{array}{l}\text { Limited } \\
\text { N/A } \\
\text { N/A }\end{array}$ & 5.29 & 60,094 & $\$ 2.2$ & & & $19 \%$ & Silver & & & & Yes & 1950 & 67 & \\
\hline Charles G Fraser Junior Public School & E & JK-6 & & 10 & Ausma Malik & Mike Gallagher & 191 & $\begin{array}{c}\text { Limited } \\
\text { N/A } \\
\text { N/A }\end{array}$ & 2.1 & 79,251 & $\$ 6.3$ & & & $58 \%$ & Gold & & & & & 1910 & 107 & \\
\hline Charles Gordon Senior Public School & $\mathrm{E}$ & $7-8$ & & 19 & David Smith & Shirley Chan & 108 & $\begin{array}{c}\text { Limited } \\
\text { N/A } \\
\text { Limited }\end{array}$ & 2.2 & 70,828 & $\$ 4.5$ & & & $40 \%$ & Silver & & Yes & A1 & & 1971 & 46 & \\
\hline Charles H Best Middle School & $\mathrm{E}$ & $5-8$ & & 5 & Alexandra Lulka & Elizabeth Addo & 150 & $\begin{array}{c}\text { Limited } \\
\text { N/A } \\
\text { N/A }\end{array}$ & 8.75 & 81,365 & $\$ 3.4$ & & & $33 \%$ & Bronze & & Yes & & & 1959 & 58 & \\
\hline Charlottetown Junior Public School & $\mathrm{E}$ & JK-6 & & 22 & Jerry Chadwick & $\begin{array}{l}\text { Kerry-Lynn } \\
\text { Stadnyk }\end{array}$ & 430 & $\begin{array}{l}\text { Closed } \\
\text { N/A } \\
\text { N/A }\end{array}$ & 5.81 & 44,125 & $\$ 3.5$ & & & $36 \%$ & & & & & & 1968 & 49 & \\
\hline Chartland Junior Public School & E & JK-6 & & 21 & Abdul Hai Patel & $\begin{array}{l}\text { Jacqueline } \\
\text { Spence }\end{array}$ & 283 & $\begin{array}{c}\text { Closed } \\
\text { N/A } \\
\text { N/A }\end{array}$ & 5.98 & 44,339 & $\$ 2.5$ & & & $22 \%$ & Silver & & & & & 1967 & 50 & \\
\hline Cherokee Public School & E & JK-6 & & 17 & Ken Lister & Beth Veale & 332 & $\begin{array}{c}\text { Limited } \\
\text { N/A } \\
\text { Limited }\end{array}$ & 6 & 26,781 & $\$ 2.4$ & & & $40 \%$ & & & & & & 1975 & 42 & \\
\hline Chester Elementary School & E & JK-5 & & 15 & Jennifer Story & Lucy Giannotta & 195 & $\begin{array}{c}\text { Limited } \\
\text { N/A } \\
\text { Limited }\end{array}$ & 3.26 & 68,906 & $\$ 9.6$ & & & $87 \%$ & Gold & & & & & 1959 & 58 & \\
\hline Chester Le Junior Public School & $\mathrm{E}$ & JK-6 & & 20 & Manna Wong & Beth Veale & 68 & $\begin{array}{c}\text { Limited } \\
\text { N/A } \\
\text { N/A }\end{array}$ & 4.99 & 47,361 & $\$ 6.6$ & & & $96 \%$ & Bronze & & & & & 1974 & 43 & \\
\hline Chief Dan George Public School & E & JK-8 & & 22 & Jerry Chadwick & Nadira Persaud & 176 & $\begin{array}{c}\text { Limited } \\
\text { Closed } \\
\text { N/A }\end{array}$ & 4.99 & 41,231 & $\$ 4.1$ & 1 & 1 & $57 \%$ & & & & & Yes & 1984 & 33 & \\
\hline Chine Drive Public School & $\mathrm{E}$ & JK-8 & & 18 & Parthi Kandavel & $\begin{array}{l}\text { Jacqueline } \\
\text { Spence }\end{array}$ & 411 & $\begin{array}{c}\text { Closed } \\
\text { N/A } \\
\text { N/A }\end{array}$ & 5.07 & 13,600 & $\$ .9$ & 4 & & $27 \%$ & Platinum & & & & & 1956 & 61 & \\
\hline Church Street Junior Public School & $\mathrm{E}$ & JK-6 & & 14 & Chris Moise & Mike Gallagher & 282 & $\begin{array}{c}\text { Closed } \\
\text { N/A } \\
\text { N/A }\end{array}$ & 1.95 & 51,900 & $\$ 6.9$ & & & $59 \%$ & & & & A1 & Yes & 1956 & 61 & \\
\hline Churchill Heights Public School & E & JK-8 & & 19 & David Smith & Shirley Chan & 234 & $\begin{array}{c}\text { Closed } \\
\text { N/A } \\
\text { N/A }\end{array}$ & 6.97 & 42,299 & $\$ 6.8$ & & & $67 \%$ & Platinum & & & & & 1956 & 61 & \\
\hline Churchill Public School & E & JK-5 & & 12 & Alexander Brown & Elizabeth Addo & 420 & $\begin{array}{l}\text { Closed } \\
\text { N/A } \\
\text { N/A }\end{array}$ & 4.13 & 55,334 & $\$ 1.3$ & & & $22 \%$ & & & & & & 1946 & 71 & \\
\hline
\end{tabular}




\begin{tabular}{|c|c|c|c|c|c|c|c|c|c|c|c|c|c|c|c|c|c|c|}
\hline & Participa & tion Rates & & & & & Enrolment a & Projectio & FTE, Surplus & eats $=$ OTG R & ised - Proj & tion FTE, Utili & itions $=$ Proj & ion/OTG I & vised & & & \\
\hline School Name & $\begin{array}{c}\text { TDSB 2013-14 } \\
\text { TDSB Participation } \\
\text { Rate }\end{array}$ & $\begin{array}{c}\text { TDSB } 2013-14 \\
\text { Home Schol } \\
\text { Participation Rate }\end{array}$ & $\begin{array}{c}\text { Revised } \\
\text { Capacity } \\
2016\end{array}$ & $\begin{array}{c}\text { HC } \\
\text { (Oct. 2016) }\end{array}$ & $\begin{array}{c}\text { FTE } \\
\text { (Oct. 2016) }\end{array}$ & $\begin{array}{c}2016 \\
\text { Utilization } \\
\text { Rate }\end{array}$ & $\begin{array}{c}2021 \\
\text { Projected }\end{array}$ & $\begin{array}{c}2021 \\
\text { Surplus } \\
\text { Seats }\end{array}$ & $\begin{array}{c}2021 \\
\text { Utilization } \\
\text { Rate }\end{array}$ & $\begin{array}{c}2026 \\
\text { Projected }\end{array}$ & $\begin{array}{c}2026 \\
\text { Surplus } \\
\text { Seats }\end{array}$ & $\begin{array}{c}2026 \\
\text { Utilization } \\
\text { Rate }\end{array}$ & $\begin{array}{c}2031 \\
\text { Projected }\end{array}$ & $\begin{array}{l}2031 \\
\text { Surplus } \\
\text { Seats }\end{array}$ & $\begin{array}{c}2031 \\
\text { Utilization } \\
\text { Rate }\end{array}$ & $\begin{array}{c}2036 \\
\text { Projected }\end{array}$ & $\begin{array}{l}2036 \\
\text { Surplus } \\
\text { Seats }\end{array}$ & $\begin{array}{c}2036 \\
\text { Utilization } \\
\text { Rate }\end{array}$ \\
\hline Cedarbrook Public School & $63.0 \%$ & $53.8 \%$ & 516 & 510 & 510.0 & $99 \%$ & 489 & 27 & $95 \%$ & 489 & 27 & $95 \%$ & 493 & 23 & $96 \%$ & 493 & 23 & $96 \%$ \\
\hline Cedarvale Community School & $63.2 \%$ & $55.8 \%$ & 383 & 402 & 402.0 & $105 \%$ & 394 & -11 & $103 \%$ & 376 & 7 & $98 \%$ & 373 & 10 & $97 \%$ & 373 & 10 & $97 \%$ \\
\hline Centennial Road Junior Public School & $57.9 \%$ & $50.3 \%$ & 222 & 251 & 251.0 & $113 \%$ & 245 & -23 & $110 \%$ & 261 & -39 & $118 \%$ & 261 & -39 & $118 \%$ & 261 & -39 & $118 \%$ \\
\hline Chalkfarm Public School & $64.5 \%$ & $50.2 \%$ & 254 & 195 & 194.0 & $76 \%$ & 196 & 58 & $77 \%$ & 221 & 33 & $87 \%$ & 221 & 33 & $87 \%$ & 221 & 33 & $87 \%$ \\
\hline Charles E Webster Public School & $58.4 \%$ & $38.5 \%$ & 600 & 426 & 426.0 & $71 \%$ & 359 & 241 & $60 \%$ & 346 & 254 & $58 \%$ & 352 & 248 & $59 \%$ & 360 & 240 & $60 \%$ \\
\hline Charles G Fraser Junior Public School & $72.6 \%$ & $30.5 \%$ & 400 & 241 & 241.0 & $60 \%$ & 262 & 138 & $66 \%$ & 255 & 145 & $64 \%$ & 253 & 147 & $63 \%$ & 253 & 147 & $63 \%$ \\
\hline Charles Gordon Senior Public School & $62.3 \%$ & $51.9 \%$ & 528 & 355 & 355.0 & $67 \%$ & 390 & 138 & $74 \%$ & 332 & 196 & $63 \%$ & 332 & 196 & $63 \%$ & 332 & 196 & $63 \%$ \\
\hline Charles H Best Middle School & $52.3 \%$ & $38.5 \%$ & 480 & 310 & 310.0 & $65 \%$ & 274 & 206 & $57 \%$ & 279 & 201 & $58 \%$ & 282 & 198 & $59 \%$ & 282 & 198 & $59 \%$ \\
\hline Charlottetown Junior Public School & $69.0 \%$ & $59.4 \%$ & 475 & 431 & 431.0 & $91 \%$ & 371 & 104 & $78 \%$ & 379 & 96 & $80 \%$ & 371 & 104 & $78 \%$ & 367 & 108 & $77 \%$ \\
\hline Chartland Junior Public School & $91.9 \%$ & $80.9 \%$ & 248 & 219 & 219.0 & $88 \%$ & 227 & 21 & $92 \%$ & 219 & 29 & $88 \%$ & 221 & 27 & $89 \%$ & 221 & 27 & $89 \%$ \\
\hline Cherokee Public School & $78.5 \%$ & $65.9 \%$ & 257 & 203 & 203.0 & $79 \%$ & 196 & 61 & $76 \%$ & 198 & 59 & $77 \%$ & 198 & 59 & $77 \%$ & 198 & 59 & $77 \%$ \\
\hline Chester Elementary School & $68.3 \%$ & $48.9 \%$ & 540 & 445 & 445.0 & $82 \%$ & 409 & 131 & $76 \%$ & 410 & 130 & $76 \%$ & 410 & 130 & $76 \%$ & 410 & 130 & $76 \%$ \\
\hline Chester Le Junior Public School & $57.8 \%$ & $44.5 \%$ & 246 & 146 & 146.0 & $59 \%$ & 121 & 125 & $49 \%$ & 121 & 125 & $49 \%$ & 120 & 126 & $49 \%$ & 120 & 126 & $49 \%$ \\
\hline Chief Dan George Public School & $58.7 \%$ & $44.4 \%$ & 383 & 347 & 347.0 & $91 \%$ & 294 & 89 & $77 \%$ & 275 & 108 & $72 \%$ & 275 & 108 & $72 \%$ & 275 & 108 & $72 \%$ \\
\hline Chine Drive Public School & $95.1 \%$ & $83.7 \%$ & 118 & 156 & 156.0 & $132 \%$ & 138 & -20 & $117 \%$ & 143 & -25 & $121 \%$ & 143 & -25 & $121 \%$ & 143 & -25 & $121 \%$ \\
\hline Church Street Junior Public School & $76.6 \%$ & $53.0 \%$ & 421 & 437 & 437.0 & $104 \%$ & 533 & -112 & $127 \%$ & 540 & -119 & $128 \%$ & 539 & -118 & $128 \%$ & 539 & -118 & $128 \%$ \\
\hline Churchill Heights Public School & $78.4 \%$ & $71.6 \%$ & 495 & 404 & 404.0 & $82 \%$ & 339 & 156 & $68 \%$ & 343 & 152 & $69 \%$ & 344 & 151 & $69 \%$ & 344 & 151 & $69 \%$ \\
\hline Churchill Public School & $60.8 \%$ & $46.5 \%$ & 508 & 434 & 433.5 & $85 \%$ & 447 & 61 & $88 \%$ & 458 & 50 & $90 \%$ & 457 & 51 & $90 \%$ & 457 & 51 & $90 \%$ \\
\hline
\end{tabular}




\begin{tabular}{|c|c|c|c|c|c|c|c|c|c|c|c|c|c|c|c|c|c|c|c|c|c|c|}
\hline \multirow[b]{2}{*}{ School Name } & \multicolumn{8}{|c|}{ General Information } & \multicolumn{14}{|c|}{ Facility Information } \\
\hline & Panel & $\begin{array}{l}\text { Grade } \\
\text { Range }\end{array}$ & $\begin{array}{c}\text { Semestered } \\
\text { (Sec) }\end{array}$ & Ward & Trustee & $\begin{array}{l}\text { Superintendent } \\
\text { of Education }\end{array}$ & 2017 LOI & $\begin{array}{c}2017-18 \\
\text { Opt } \\
\text { Attend } \\
\text { Reg/FI/EF }\end{array}$ & $\begin{array}{l}\text { Site Size } \\
\text { (Acres) }\end{array}$ & $\begin{array}{c}\text { Facility Size } \\
\text { (Sq. Ft.) }\end{array}$ & $\begin{array}{c}\text { Current } \\
\text { Backlog (M) }\end{array}$ & \begin{tabular}{|c} 
Port. \\
(Instruc)
\end{tabular} & $\begin{array}{l}\text { Port. (Non- } \\
\text { Instruc) }\end{array}$ & $\begin{array}{c}\text { Current FCI } \\
\text { (EDU) }\end{array}$ & $\begin{array}{l}\text { Eco } \\
\text { Schools }\end{array}$ & Pool & $\begin{array}{l}\text { Elem. } \\
\text { D\&T } \\
\text { Shops }\end{array}$ & $\begin{array}{l}\text { Barrier } \\
\text { Free / } \\
\text { Access }\end{array}$ & Elevator & $\begin{array}{l}\text { Build } \\
\text { Date }\end{array}$ & $\begin{array}{c}\text { Building } \\
\text { Age }\end{array}$ & $\begin{array}{l}\text { T.O Comm } \\
\text { Centre }\end{array}$ \\
\hline City View Alternative Senior School & E & $7-8$ & & 9 & Marit Stiles & $\begin{array}{l}\text { Jane Phillips- } \\
\text { Long }\end{array}$ & 340 & \begin{tabular}{|c|} 
Limited \\
N/A \\
N/A \\
\end{tabular} & & & & & & & & & & & & 1961 & 56 & \\
\hline Claireville Junior School & E & JK-5 & & 1 & Avtar Minhas & Glenford Duffus & 123 & $\begin{array}{c}\text { Closed } \\
\text { N/A } \\
\text { N/A }\end{array}$ & 6 & 39,073 & $\$ 5.4$ & 1 & 4 & $72 \%$ & & & & & & 1967 & 50 & \\
\hline Clairlea Public School & $\mathrm{E}$ & $\mathrm{JK}-8$ & & 18 & Parthi Kandavel & Peter Chang & 181 & $\begin{array}{l}\text { Closed } \\
\text { Closed } \\
\text { N/A }\end{array}$ & 5.29 & 45,951 & $\$ 5.2$ & 3 & & $45 \%$ & & & Yes & & & 1951 & 66 & \\
\hline Claude Watson School for the Arts & E & $4-8$ & & 12 & Alexander Brown & Linda Curtis & 389 & $\begin{array}{c}\text { Limited } \\
\text { N/A } \\
\text { N/A }\end{array}$ & 1.86 & 65,833 & $\$ .1$ & & & $1 \%$ & Platinum & & & & & 2006 & 11 & \\
\hline Cliffside Public School & E & JK-8 & & 18 & Parthi Kandavel & Peter Chang & 63 & $\begin{array}{c}\text { Limited } \\
\text { N/A } \\
\text { Limited }\end{array}$ & 4.23 & 23,076 & $\$ 2.4$ & & & $43 \%$ & Gold & & & & & 1951 & 66 & \\
\hline Cliffwood Public School & E & JK-5 & & 12 & Alexander Brown & Elizabeth Addo & 369 & $\begin{array}{l}\text { Closed } \\
\text { Closed } \\
\text { Closed }\end{array}$ & 7.86 & 42,636 & $\$ 1$. & 2 & & $10 \%$ & Gold & & & & & 1973 & 44 & \\
\hline Clinton Street Junior Public School & E & JK-6 & & 10 & Ausma Malik & Mike Gallagher & 315 & $\begin{array}{c}\text { Limited } \\
\text { N/A } \\
\text { N/A }\end{array}$ & 3.29 & 69,044 & $\$ 6.9$ & & & $55 \%$ & Gold & & & & & 1964 & 53 & \\
\hline Cordella Junior Public School & E & JK-6 & & 6 & Chris Tonks & Vicky Branco & 15 & $\begin{array}{c}\text { Limited } \\
\text { N/A } \\
\text { N/A }\end{array}$ & 3.29 & 28,586 & $\$ 3.7$ & & & $62 \%$ & & & & & & 1960 & 57 & \\
\hline Cornell Junior Public School & $\mathrm{E}$ & $\mathrm{JK}-6$ & & 22 & Jerry Chadwick & $\begin{array}{l}\text { Kerry-Lynn } \\
\text { Stadnyk }\end{array}$ & 120 & $\begin{array}{c}\text { Closed } \\
\text { N/A } \\
\text { N/A }\end{array}$ & 5.51 & 87,314 & $\$ 7.2$ & & 2 & $49 \%$ & Silver & & & A1 & Yes & 1959 & 58 & \\
\hline Corvette Junior Public School & E & JK-6 & & 18 & Parthi Kandavel & Peter Chang & 85 & $\begin{array}{c}\text { Closed } \\
\text { Limited } \\
\text { N/A }\end{array}$ & 8.28 & 66,221 & $\$ 4.8$ & & & $38 \%$ & & & & & & 1953 & 64 & \\
\hline Cosburn Middle School & E & 6-8 & & 15 & Jennifer Story & Lucy Giannotta & 313 & $\begin{array}{l}\text { Closed } \\
\text { Closed } \\
\text { Closed }\end{array}$ & 3.35 & 95,220 & $\$ 7.6$ & & & $66 \%$ & Platinum & & Yes & & Yes & 1949 & 68 & \\
\hline Cottingham Junior Public School & E & JK-6 & & 11 & Shelley Laskin & Ian Allison & 452 & $\begin{array}{c}\text { Limited } \\
\text { N/A } \\
\text { N/A }\end{array}$ & 2.64 & 14,420 & $\$ 2.4$ & 1 & 1 & $62 \%$ & & & & & & 1955 & 62 & \\
\hline Courcelette Public School & E & JK-8 & & 18 & Parthi Kandavel & Peter Chang & 458 & $\begin{array}{c}\text { Closed } \\
\text { N/A } \\
\text { N/A }\end{array}$ & 2.03 & 21,272 & $\$ 3.4$ & 4 & & $61 \%$ & Bronze & & & & & 1950 & 67 & \\
\hline Crescent Town Elementary School & E & $\mathrm{JK}-4$ & & 16 & $\begin{array}{l}\text { Sheila Cary- } \\
\text { Meagher }\end{array}$ & Lucy Giannotta & 203 & $\begin{array}{c}\text { Limited } \\
\text { N/A } \\
\text { N/A }\end{array}$ & 3.89 & 67,924 & $\$ 4.3$ & & & $25 \%$ & Gold & & & A1 & Yes & 1973 & 44 & \\
\hline Cresthaven Public School & E & JK-5 & & 12 & Alexander Brown & Elizabeth Addo & 174 & $\begin{array}{c}\text { Limited } \\
\text { N/A } \\
\text { N/A }\end{array}$ & 4.99 & 41,528 & $\$ 2.8$ & & & $37 \%$ & Platinum & & & & & 1963 & 54 & \\
\hline Crestview Public School & E & JK-6 & & 17 & Ken Lister & Audley Salmon & 300 & $\begin{array}{c}\text { Limited } \\
\text { N/A } \\
\text { N/A }\end{array}$ & 6.99 & 49,105 & $\$ 4.9$ & & & $44 \%$ & & & & & & 1973 & 44 & \\
\hline Cummer Valley Middle School & E & 6-8 & & 12 & Alexander Brown & Linda Curtis & 363 & $\begin{array}{c}\text { Limited } \\
\text { N/A } \\
\text { N/A }\end{array}$ & 10.4 & 82,000 & $\$ 6.8$ & & & $57 \%$ & Gold & & Yes & & & 1959 & 58 & \\
\hline D A Morrison Middle School & E & $6-8$ & & 16 & $\begin{array}{l}\text { Sheila Cary- } \\
\text { Meagher }\end{array}$ & Lucy Giannotta & 124 & $\begin{array}{c}\text { Limited } \\
\text { Limited } \\
\text { N/A }\end{array}$ & 4.87 & 114,147 & $\$ 8.8$ & & & $72 \%$ & & City & Yes & & & 1977 & 40 & \\
\hline
\end{tabular}




\begin{tabular}{|c|c|c|c|c|c|c|c|c|c|c|c|c|c|c|c|c|c|c|}
\hline & Participa & tion Rates & & & & & Enrolment a & Projection & FTE, Surplus & ats = OTG R & ised - Proje & tion FTE, Utili & itions $=$ Proj & ion/OTG R & vised & & & \\
\hline School Name & $\begin{array}{c}\text { TDSB 2013-14 } \\
\text { TDSB Participation } \\
\text { Rate }\end{array}$ & $\begin{array}{c}\text { TOSB 2013-14 } \\
\text { Home school } \\
\text { Participation Rate }\end{array}$ & $\begin{array}{c}\text { Revised } \\
\text { Capacity } \\
2016\end{array}$ & $\begin{array}{c}\text { HC } \\
\text { (Oct. 2016) }\end{array}$ & $\begin{array}{c}\text { FTE } \\
\text { (Oct. 2016) }\end{array}$ & $\begin{array}{c}2016 \\
\text { Utilization } \\
\text { Rate }\end{array}$ & $\begin{array}{c}2021 \\
\text { Projected }\end{array}$ & $\begin{array}{l}2021 \\
\text { Surplus } \\
\text { Seats }\end{array}$ & $\begin{array}{c}2021 \\
\text { Utilization } \\
\text { Rate }\end{array}$ & $\begin{array}{c}2026 \\
\text { Projected }\end{array}$ & $\begin{array}{l}2026 \\
\text { Surplus } \\
\text { Seats }\end{array}$ & $\begin{array}{c}2026 \\
\text { Utilization } \\
\text { Rate }\end{array}$ & $\begin{array}{c}2031 \\
\text { Projected }\end{array}$ & $\begin{array}{l}2031 \\
\text { Surplus } \\
\text { Seats }\end{array}$ & $\begin{array}{c}2031 \\
\text { Utilization } \\
\text { Rate }\end{array}$ & $\begin{array}{c}2036 \\
\text { Projected }\end{array}$ & $\begin{array}{l}2036 \\
\text { Surplus } \\
\text { Seats }\end{array}$ & $\begin{array}{l}2036 \\
\text { Utilization } \\
\text { Rate }\end{array}$ \\
\hline City View Alternative Senior School & & & 92 & 56 & 56.0 & $61 \%$ & 60 & 32 & $65 \%$ & 60 & 32 & $65 \%$ & 60 & 32 & $65 \%$ & 60 & 32 & $65 \%$ \\
\hline Claireville Junior School & $73.2 \%$ & $58.8 \%$ & 358 & 305 & 305.0 & $85 \%$ & 275 & 83 & $77 \%$ & 297 & 61 & $83 \%$ & 297 & 61 & $83 \%$ & 297 & 61 & $83 \%$ \\
\hline Clairlea Public School & $64.1 \%$ & $53.7 \%$ & 573 & 592 & 592.0 & $103 \%$ & 705 & -132 & $123 \%$ & 767 & -194 & $134 \%$ & 732 & -159 & $128 \%$ & 725 & -152 & $127 \%$ \\
\hline Claude Watson School for the Arts & & & 354 & 300 & 300.0 & $85 \%$ & 300 & 54 & $85 \%$ & 300 & 54 & $85 \%$ & 300 & 54 & $85 \%$ & 300 & 54 & $85 \%$ \\
\hline Cliffside Public School & $70.2 \%$ & $43.8 \%$ & 222 & 128 & 128.0 & $58 \%$ & 217 & 5 & $\mathbf{9 8 \%}$ & 225 & -3 & $101 \%$ & 224 & -2 & $101 \%$ & 224 & -2 & $101 \%$ \\
\hline Cliffwood Public School & $79.9 \%$ & $71.3 \%$ & 435 & 383 & 383.0 & $88 \%$ & 372 & 63 & $86 \%$ & 359 & 76 & $83 \%$ & 359 & 76 & $83 \%$ & 359 & 76 & $83 \%$ \\
\hline Clinton Street Junior Public School & $89.7 \%$ & $69.5 \%$ & 560 & 396 & 396.0 & $71 \%$ & 435 & 125 & $78 \%$ & 431 & 129 & $77 \%$ & 431 & 129 & $77 \%$ & 431 & 129 & $77 \%$ \\
\hline Cordella Junior Public School & $34.6 \%$ & $21.4 \%$ & 191 & 134 & 134.0 & $70 \%$ & 119 & 72 & $62 \%$ & 129 & 62 & $68 \%$ & 133 & 58 & $70 \%$ & 141 & 50 & $74 \%$ \\
\hline Cornell Junior Public School & $89.3 \%$ & $83.0 \%$ & 896 & 746 & 746.0 & $83 \%$ & 635 & 261 & $71 \%$ & 598 & 298 & $67 \%$ & 598 & 298 & $67 \%$ & 598 & 298 & $67 \%$ \\
\hline Corvette Junior Public School & $48.5 \%$ & $37.8 \%$ & 625 & 550 & 550.0 & $88 \%$ & 655 & -30 & $105 \%$ & 665 & -40 & $106 \%$ & 666 & -41 & $107 \%$ & 666 & -41 & $107 \%$ \\
\hline Cosburn Middle School & $104.9 \%$ & $90.4 \%$ & 643 & 672 & 671.0 & $104 \%$ & 645 & -2 & $100 \%$ & 649 & -6 & $101 \%$ & 634 & 9 & $99 \%$ & 634 & 9 & $99 \%$ \\
\hline Cottingham Junior Public School & $86.7 \%$ & $62.2 \%$ & 104 & 150 & 150.0 & $144 \%$ & 156 & -52 & $150 \%$ & 151 & -47 & $145 \%$ & 151 & -47 & $145 \%$ & 151 & -47 & $145 \%$ \\
\hline Courcelette Public School & $99.1 \%$ & $90.6 \%$ & 196 & 326 & 326.0 & $166 \%$ & 318 & -122 & $162 \%$ & 321 & -125 & $164 \%$ & 321 & -125 & $164 \%$ & 321 & -125 & $164 \%$ \\
\hline Crescent Town Elementary School & $85.8 \%$ & $80.1 \%$ & 792 & 632 & 632.0 & $80 \%$ & 592 & 200 & $75 \%$ & 586 & 206 & $74 \%$ & 586 & 206 & $74 \%$ & 586 & 206 & $74 \%$ \\
\hline Cresthaven Public School & $66.6 \%$ & $45.1 \%$ & 318 & 199 & 199.0 & $63 \%$ & 270 & 48 & $85 \%$ & 274 & 44 & $86 \%$ & 274 & 44 & $86 \%$ & 274 & 44 & $86 \%$ \\
\hline Crestview Public School & $67.9 \%$ & $52.8 \%$ & 513 & 407 & 407.0 & $79 \%$ & 447 & 66 & $87 \%$ & 448 & 65 & $87 \%$ & 442 & 71 & $86 \%$ & 442 & 71 & $86 \%$ \\
\hline Cummer Valley Middle School & $81.6 \%$ & $62.9 \%$ & 630 & 476 & 476.0 & $76 \%$ & 408 & 222 & $65 \%$ & 452 & 178 & $72 \%$ & 431 & 199 & $68 \%$ & 431 & 199 & $68 \%$ \\
\hline D A Morrison Middle School & $76.7 \%$ & $49.5 \%$ & 589 & 310 & 310.0 & $53 \%$ & 421 & 168 & $71 \%$ & 499 & 90 & $85 \%$ & 499 & 90 & $85 \%$ & 499 & 90 & $85 \%$ \\
\hline
\end{tabular}




\begin{tabular}{|c|c|c|c|c|c|c|c|c|c|c|c|c|c|c|c|c|c|c|c|c|c|c|}
\hline \multirow[b]{2}{*}{ School Name } & \multicolumn{8}{|c|}{ General Information } & \multicolumn{14}{|c|}{ Facility Information } \\
\hline & Panel & $\begin{array}{l}\text { Grade } \\
\text { Range }\end{array}$ & $\begin{array}{c}\text { Semestered } \\
\text { (Sec) }\end{array}$ & Ward & Trustee & $\begin{array}{l}\text { Superintendent } \\
\text { of Education }\end{array}$ & 2017 LOI & $\begin{array}{c}2017-18 \\
\text { Opt } \\
\text { Attend } \\
\mathrm{Reg} / \mathrm{F} / \mathrm{FF}\end{array}$ & $\begin{array}{l}\text { Site Size } \\
\text { (Acres) }\end{array}$ & $\begin{array}{c}\text { Facility Size } \\
\text { (Sq. Ft.) }\end{array}$ & $\begin{array}{c}\text { Current } \\
\text { Backlog (M) }\end{array}$ & $\begin{array}{c}\text { Port. } \\
\text { (Instruc) }\end{array}$ & $\begin{array}{c}\text { Port. (Non- } \\
\text { Instruc) }\end{array}$ & $\begin{array}{c}\text { Current FCI } \\
\text { (EDU) }\end{array}$ & $\begin{array}{c}\text { Eco } \\
\text { Schools }\end{array}$ & Pool & $\begin{array}{l}\text { Elem. } \\
\text { D\&T } \\
\text { Shops }\end{array}$ & $\begin{array}{c}\text { Barrier } \\
\text { Free / } \\
\text { Access }\end{array}$ & Elevator & $\begin{array}{l}\text { Build } \\
\text { Date }\end{array}$ & $\begin{array}{c}\text { Building } \\
\text { Age }\end{array}$ & $\begin{array}{l}\text { T.O Comm } \\
\text { Centre }\end{array}$ \\
\hline da Vinci School & $\mathrm{E}$ & $\mathrm{JK}-6$ & & 10 & Ausma Malik & $\begin{array}{l}\text { Mary Jane } \\
\text { McNamara }\end{array}$ & 327 & \begin{tabular}{|c|} 
Limited \\
N/A \\
N/A \\
\end{tabular} & & & & & & & Gold & & & & & & & \\
\hline Dallington Public School & E & JK-6 & & 17 & Ken Lister & Audley Salmon & 316 & $\begin{array}{c}\text { Closed } \\
\text { Closed } \\
\text { N/A }\end{array}$ & 6 & 57,372 & $\$ 3.5$ & 1 & & $30 \%$ & Gold & & & & & 1963 & 54 & \\
\hline Danforth Gardens Public School & $\mathrm{E}$ & JK-8 & & 18 & Parthi Kandavel & Peter Chang & 198 & $\begin{array}{c}\text { Closed } \\
\text { N/A } \\
\text { N/A }\end{array}$ & 5.73 & 46,398 & $\$ 5.1$ & 2 & & $46 \%$ & Gold & & & & & 1956 & 61 & \\
\hline David Hornell Junior School & E & JK-5 & & 3 & Pamela Gough & Sandra Tondat & 227 & $\begin{array}{c}\text { Closed } \\
\text { N/A } \\
\text { N/A }\end{array}$ & 4.15 & 24,757 & \$2. & 3 & & $36 \%$ & & & & & & 1960 & 57 & \\
\hline David Lewis Public School & $\mathrm{E}$ & $\mathrm{JK}-8$ & & 20 & Manna Wong & \begin{tabular}{|l} 
Louie \\
Papathanasakis
\end{tabular} & 292 & $\begin{array}{c}\text { Closed } \\
\text { N/A } \\
\text { N/A }\end{array}$ & 4.99 & 39,896 & $\$ 2.7$ & 5 & & $36 \%$ & Gold & & & & & 1989 & 28 & \\
\hline Davisville Junior Public School & E & $\mathrm{JK}-6$ & & 11 & Shelley Laskin & Ian Allison & 320 & $\begin{array}{c}\text { Closed } \\
\text { Closed } \\
\text { N/A }\end{array}$ & 3.81 & 85,068 & $\$ 5.4$ & & & $33 \%$ & Platinum & & & & & 1960 & 57 & \\
\hline Daystrom Public School & $\mathrm{E}$ & JK-5 & & 4 & Tiffany Ford & Audley Salmon & 105 & $\begin{array}{c}\text { Limited } \\
\text { N/A } \\
\text { N/A }\end{array}$ & 4.94 & 67,932 & $\$ 9.4$ & & & $65 \%$ & & & & & & 1959 & 58 & \\
\hline $\begin{array}{l}\text { Deer Park Junior and Senior Public } \\
\text { School }\end{array}$ & E & JK-8 & & 11 & Shelley Laskin & Ian Allison & 425 & $\begin{array}{c}\text { Closed } \\
\text { N/A } \\
\text { N/A }\end{array}$ & 4.92 & 93,724 & $\$ 12$. & & & $115 \%$ & & TLC & Yes & A1 & Yes & 1959 & 58 & \\
\hline Delta Alternative Senior School & $\mathrm{E}$ & $7-8$ & & 10 & Ausma Malik & Mike Gallagher & 391 & \begin{tabular}{c|} 
Limited \\
N/A \\
N/A
\end{tabular} & & & & & & & & & & & & & & \\
\hline Denlow Public School & E & JK-6 & & 13 & Gerri Gershon & Kathleen Garner & 459 & $\begin{array}{c}\text { Closed } \\
\text { N/A } \\
\text { N/A }\end{array}$ & 8.1 & 48,277 & $\$ 5.9$ & 2 & 1 & $67 \%$ & Gold & & & & & 1968 & 49 & \\
\hline Dennis Avenue Community School & E & JK-5 & & 6 & Chris Tonks & Vicky Branco & 53 & $\begin{array}{c}\text { Limited } \\
\text { N/A } \\
\text { N/A }\end{array}$ & 2.3 & 36,132 & $\$ 2.2$ & & & $29 \%$ & Gold & & & & & 1891 & 126 & \\
\hline Derrydown Public School & E & JK-5 & & 4 & Tiffany Ford & Audley Salmon & 72 & $\begin{array}{c}\text { Closed } \\
\text { Limited } \\
\text { N/A }\end{array}$ & 6 & 44,283 & $\$ 1.8$ & 1 & 2 & $19 \%$ & Bronze & & & & & 1964 & 53 & \\
\hline Dewson Street Junior Public School & $\mathrm{E}$ & $\mathrm{JK}-6$ & & 10 & Ausma Malik & Mike Gallagher & 351 & $\begin{array}{c}\text { Limited } \\
\text { Limited } \\
\text { N/A }\end{array}$ & 3.31 & 60,191 & $\$ 8.3$ & & & $65 \%$ & & & & & & 1966 & 51 & \\
\hline Diefenbaker Elementary School & $\mathrm{E}$ & JK-5 & & 15 & Jennifer Story & Lucy Giannotta & 412 & $\begin{array}{c}\text { Closed } \\
\text { N/A } \\
\text { Closed }\end{array}$ & 2.9 & 40,540 & $\$ 3.8$ & & & $50 \%$ & Gold & & & & & 1956 & 61 & \\
\hline Dixon Grove Junior Middle School & $\mathrm{E}$ & $\mathrm{JK}-8$ & & 2 & Chris Glover & $\begin{array}{l}\text { Angela Nardi- } \\
\text { Addesa }\end{array}$ & 78 & $\begin{array}{c}\text { Limited } \\
\text { N/A } \\
\text { N/A }\end{array}$ & 8.15 & 90,837 & $\$ 12.7$ & & & $73 \%$ & Gold & & & & No & 1960 & 57 & \\
\hline Don Mills Middle School & E & 6-8 & & 17 & Ken Lister & Beth Veale & 238 & $\begin{array}{c}\text { Limited } \\
\text { N/A } \\
\text { N/A }\end{array}$ & 7.78 & 78,696 & $\$ 2.9$ & & & $36 \%$ & Silver & & & & Yes & 1960 & 57 & \\
\hline Don Valley Middle School & $\mathrm{E}$ & $7-8$ & & 17 & Ken Lister & Audley Salmon & 304 & $\begin{array}{c}\text { Limited } \\
\text { Limited } \\
\text { N/A }\end{array}$ & 10.01 & 77,350 & $\$ 5.9$ & & & $50 \%$ & Silver & & Yes & & & 1968 & 49 & \\
\hline $\begin{array}{l}\text { Donview Middle Health and Wellness } \\
\text { Academy }\end{array}$ & E & 6-8 & & 17 & Ken Lister & Beth Veale & 213 & $\begin{array}{c}\text { Limited } \\
\text { N/A } \\
\text { N/A }\end{array}$ & 6.4 & 95,455 & $\$ 2.7$ & & & $20 \%$ & & & Yes & & & 1964 & 53 & \\
\hline
\end{tabular}




\begin{tabular}{|c|c|c|c|c|c|c|c|c|c|c|c|c|c|c|c|c|c|c|}
\hline Board & Participa & tion Rates & & & & & Enrolment a & Projectio & FTE, Surplus & ats = OTG R & ised-Proje & tion FTE, Utili & tions $=$ Proj & ion/OTG $\mathrm{R}$ & vised & & & \\
\hline School Name & $\begin{array}{c}\text { TDSB 2013-14 } \\
\text { TDSB Participation } \\
\text { Rate }\end{array}$ & $\begin{array}{c}\text { TDSB 2013-14 } \\
\text { Home School } \\
\text { Participation Rate }\end{array}$ & $\begin{array}{c}\text { Revised } \\
\text { Capacity } \\
2016\end{array}$ & $\begin{array}{c}\text { HC } \\
\text { (Oct. 2016) }\end{array}$ & \begin{tabular}{|c|} 
FTE \\
(Oct. 2016)
\end{tabular} & $\begin{array}{c}2016 \\
\text { Utilization } \\
\text { Rate }\end{array}$ & $\begin{array}{c}2021 \\
\text { Projected }\end{array}$ & $\begin{array}{l}2021 \\
\text { Surplus } \\
\text { Seats }\end{array}$ & $\begin{array}{c}2021 \\
\text { Utilization } \\
\text { Rate }\end{array}$ & $\begin{array}{c}2026 \\
\text { Projected }\end{array}$ & $\begin{array}{l}2026 \\
\text { Surplus } \\
\text { Seats }\end{array}$ & $\begin{array}{c}2026 \\
\text { Utilization } \\
\text { Rate }\end{array}$ & $\begin{array}{c}2031 \\
\text { Projected }\end{array}$ & $\begin{array}{l}2031 \\
\text { Surplus } \\
\text { Seats }\end{array}$ & $\begin{array}{c}2031 \\
\text { Utilization } \\
\text { Rate }\end{array}$ & $\begin{array}{c}2036 \\
\text { Projected }\end{array}$ & $\begin{array}{l}2036 \\
\text { Surplus } \\
\text { Seats }\end{array}$ & $\begin{array}{c}2036 \\
\text { Utilization } \\
\text { Rate }\end{array}$ \\
\hline da Vinci School & & & 92 & 79 & 79.0 & $86 \%$ & 87 & 5 & $95 \%$ & 87 & 5 & $95 \%$ & 87 & 5 & $95 \%$ & 87 & 5 & $95 \%$ \\
\hline Dallington Public School & $57.8 \%$ & $48.7 \%$ & 628 & 555 & 555.0 & $88 \%$ & 547 & 81 & $87 \%$ & 553 & 75 & $88 \%$ & 551 & 77 & $88 \%$ & 551 & 77 & $88 \%$ \\
\hline Danforth Gardens Public School & $72.2 \%$ & $54.2 \%$ & 576 & 507 & 507.0 & $88 \%$ & 451 & 125 & $78 \%$ & 464 & 112 & $81 \%$ & 473 & 103 & $82 \%$ & 473 & 103 & $82 \%$ \\
\hline David Hornell Junior School & $78.5 \%$ & $54.1 \%$ & 199 & 227 & 227.0 & $114 \%$ & 241 & -42 & $121 \%$ & 246 & -47 & $124 \%$ & 246 & -47 & $124 \%$ & 246 & -47 & $124 \%$ \\
\hline David Lewis Public School & $95.8 \%$ & $91.2 \%$ & 337 & 462 & 462.0 & $137 \%$ & 451 & -114 & $134 \%$ & 427 & -90 & $127 \%$ & 427 & -90 & $127 \%$ & 427 & -90 & $127 \%$ \\
\hline Davisville Junior Public School & $76.9 \%$ & $61.4 \%$ & 469 & 561 & 561.0 & $120 \%$ & 628 & -159 & $134 \%$ & 632 & -163 & $135 \%$ & 632 & -163 & $135 \%$ & 632 & -163 & $135 \%$ \\
\hline Daystrom Public School & $66.6 \%$ & $60.6 \%$ & 647 & 460 & 460.0 & $71 \%$ & 553 & 94 & $85 \%$ & 539 & 108 & $83 \%$ & 540 & 107 & $83 \%$ & 540 & 107 & $83 \%$ \\
\hline $\begin{array}{l}\text { Deer Park Junior and Senior Public } \\
\text { School }\end{array}$ & $65.4 \%$ & $50.1 \%$ & 508 & 479 & 479.0 & $94 \%$ & 699 & -191 & $138 \%$ & 737 & -229 & $145 \%$ & 751 & -243 & $148 \%$ & 754 & -246 & $148 \%$ \\
\hline Delta Alternative Senior School & & & 115 & 60 & 60.0 & $52 \%$ & 61 & 54 & $53 \%$ & 61 & 54 & $53 \%$ & 61 & 54 & $53 \%$ & 61 & 54 & $53 \%$ \\
\hline Denlow Public School & $90.0 \%$ & $79.0 \%$ & 442 & 392 & 392.0 & $89 \%$ & 316 & 126 & $71 \%$ & 308 & 134 & $70 \%$ & 303 & 139 & $69 \%$ & 303 & 139 & $69 \%$ \\
\hline Dennis Avenue Community School & $58.0 \%$ & $35.6 \%$ & 268 & 136 & 136.0 & $51 \%$ & 118 & 150 & $44 \%$ & 117 & 151 & $44 \%$ & 117 & 151 & $44 \%$ & 117 & 151 & $44 \%$ \\
\hline Derrydown Public School & $66.0 \%$ & $61.2 \%$ & 533 & 455 & 455.0 & $85 \%$ & 498 & 35 & $93 \%$ & 489 & 44 & $92 \%$ & 489 & 44 & $92 \%$ & 489 & 44 & $92 \%$ \\
\hline Dewson Street Junior Public School & $84.8 \%$ & $50.0 \%$ & 603 & 488 & 488.0 & $81 \%$ & 478 & 125 & $79 \%$ & 476 & 127 & $79 \%$ & 472 & 131 & $78 \%$ & 472 & 131 & $78 \%$ \\
\hline Diefenbaker Elementary School & $105.0 \%$ & $95.5 \%$ & 424 & 402 & 402.0 & $95 \%$ & 380 & 44 & $90 \%$ & 374 & 50 & $88 \%$ & 374 & 50 & $88 \%$ & 374 & 50 & $88 \%$ \\
\hline Dixon Grove Junior Middle School & $48.7 \%$ & $36.9 \%$ & 868 & 656 & 656.0 & $76 \%$ & 603 & 265 & $69 \%$ & 554 & 314 & $64 \%$ & 565 & 303 & $65 \%$ & 565 & 303 & $65 \%$ \\
\hline Don Mills Middle School & $54.6 \%$ & $17.9 \%$ & 441 & 373 & 373.0 & $85 \%$ & 392 & 49 & $89 \%$ & 343 & 98 & $78 \%$ & 346 & 95 & $78 \%$ & 346 & 95 & $78 \%$ \\
\hline Don Valley Middle School & $63.2 \%$ & $33.5 \%$ & 441 & 136 & 136.0 & $31 \%$ & 370 & 71 & $84 \%$ & 400 & 41 & $91 \%$ & 383 & 58 & $87 \%$ & 384 & 57 & $87 \%$ \\
\hline $\begin{array}{l}\text { Donview Middle Health and Wellness } \\
\text { Academy }\end{array}$ & $62.6 \%$ & $46.3 \%$ & 637 & 379 & 379.0 & $59 \%$ & 386 & 251 & $61 \%$ & 329 & 308 & $52 \%$ & 325 & 312 & $51 \%$ & 322 & 315 & $51 \%$ \\
\hline
\end{tabular}




\begin{tabular}{|c|c|c|c|c|c|c|c|c|c|c|c|c|c|c|c|c|c|c|c|c|c|c|}
\hline \multirow[b]{2}{*}{ School Name } & \multicolumn{8}{|c|}{ General Information } & \multicolumn{14}{|c|}{ Facility Information } \\
\hline & Panel & $\begin{array}{l}\text { Grade } \\
\text { Range }\end{array}$ & $\begin{array}{c}\text { Semestered } \\
\text { (Sec) }\end{array}$ & Ward & Trustee & $\begin{array}{l}\text { Superintendent } \\
\text { of Education }\end{array}$ & 2017 LOI & $\begin{array}{c}2017-18 \\
\text { Opt } \\
\text { Attend } \\
\text { Reg/FI/EF }\end{array}$ & $\begin{array}{l}\text { Site Size } \\
\text { (Acres) }\end{array}$ & $\begin{array}{l}\text { Facility Size } \\
\text { (Sq. Ft.) }\end{array}$ & $\mid \begin{array}{c}\text { Current } \\
\text { Backlog (M) }\end{array}$ & $\begin{array}{l}\text { Port. } \\
\text { (Instruc) }\end{array}$ & $\begin{array}{c}\text { Port. (Non- } \\
\text { Instruc) }\end{array}$ & $\begin{array}{c}\text { Current FCI } \\
\text { (EDU) }\end{array}$ & $\begin{array}{c}\text { Eco } \\
\text { Schools }\end{array}$ & Pool & $\begin{array}{l}\text { Elem. } \\
\text { D\&T } \\
\text { Shops }\end{array}$ & $\begin{array}{l}\text { Barrier } \\
\text { Free / } \\
\text { Access }\end{array}$ & Elevator & $\begin{array}{l}\text { Build } \\
\text { Date }\end{array}$ & $\begin{array}{c}\text { Building } \\
\text { Age }\end{array}$ & $\begin{array}{l}\text { T.o Comm } \\
\text { Centre }\end{array}$ \\
\hline Donwood Park Public School & $\mathrm{E}$ & $\mathrm{JK}-8$ & & 19 & David Smith & Shirley Chan & 121 & $\begin{array}{c}\text { Closed } \\
\text { N/A } \\
\text { N/A }\end{array}$ & 8.97 & 92,722 & $\$ 7.4$ & 1 & 1 & $34 \%$ & \begin{tabular}{|l|} 
Platinum \\
\end{tabular} & & & & & 1957 & 60 & \\
\hline Dorset Park Public School & E & JK-8 & & 19 & David Smith & Anne Seymour & 135 & $\begin{array}{c}\text { Limited } \\
\text { N/A } \\
\text { N/A }\end{array}$ & 8.01 & 37,635 & $\$ 2$. & & & $24 \%$ & & & & & & 1956 & 61 & \\
\hline Dovercourt Public School & $\mathrm{E}$ & JK-8 & & 9 & Marit Stiles & $\begin{array}{l}\text { Jane Phillips- } \\
\text { Long }\end{array}$ & 225 & $\begin{array}{c}\text { Limited } \\
\text { N/A } \\
\text { N/A }\end{array}$ & 3.34 & 68,916 & $\$ 9.3$ & & & $101 \%$ & \begin{tabular}{|l|} 
Platinum \\
\end{tabular} & & & & & 1916 & 101 & \\
\hline Downsview Public School & E & JK-5 & & 5 & Alexandra Lulka & Linda Curtis & 97 & $\begin{array}{c}\text { Closed } \\
\text { N/A } \\
\text { N/A }\end{array}$ & 4.92 & 24,144 & $\$ 3.9$ & 1 & & $69 \%$ & & & & & & 1947 & 70 & \\
\hline Downtown Alternative School & $\mathrm{E}$ & JK-6 & & 14 & Chris Moise & $\begin{array}{l}\text { Jane Phillips- } \\
\text { Long }\end{array}$ & 262 & $\begin{array}{c}\text { Limited } \\
\text { N/A } \\
\text { N/A }\end{array}$ & 1.19 & 26,029 & $\$ .7$ & & & $13 \%$ & & & & & & 1979 & 38 & \\
\hline $\begin{array}{l}\text { Downtown Vocal Music Academy of } \\
\text { Toronto }\end{array}$ & E & 4-8 & & 10 & Ausma Malik & Mike Gallagher & 143 & \begin{tabular}{c|} 
Limited \\
N/A \\
N/A
\end{tabular} & & & & & & & & & & & & & & \\
\hline $\begin{array}{l}\text { Dr Marion Hilliard Senior Public } \\
\text { School }\end{array}$ & $\mathrm{E}$ & $7-8$ & & 21 & Abdul Hai Patel & Andrew Howard & 179 & $\begin{array}{c}\text { Closed } \\
\text { N/A } \\
\text { N/A }\end{array}$ & 7.02 & 65,523 & $\$ 3$. & & & $38 \%$ & & & & & & 1978 & 39 & \\
\hline Driftwood Public School & E & JK-5 & & 4 & Tiffany Ford & Lynn Strangway & 6 & $\begin{array}{c}\text { Closed } \\
\text { N/A } \\
\text { N/A }\end{array}$ & 6 & 53,960 & $\$ 2.2$ & 4 & & $21 \%$ & & & & & & 1964 & 53 & \\
\hline $\begin{array}{l}\text { Dublin Heights Elementary and } \\
\text { Middle School }\end{array}$ & $\mathrm{E}$ & JK-8 & & 5 & Alexandra Lulka & Linda Curtis & 353 & $\begin{array}{c}\text { Limited } \\
\text { N/A } \\
\text { N/A }\end{array}$ & 9.09 & 94,075 & $\$ 5.6$ & & & $37 \%$ & Gold & & & A1 & Yes & 1957 & 60 & \\
\hline $\begin{array}{l}\text { Duke of Connaught Junior and Senior } \\
\text { Public School }\end{array}$ & E & JK-8 & & 16 & $\begin{array}{l}\text { Sheila Cary- } \\
\text { Meagher }\end{array}$ & $\begin{array}{l}\text { Mary Jane } \\
\text { McNamara }\end{array}$ & 260 & $\begin{array}{l}\text { Limited } \\
\text { Limited } \\
\text { Limited }\end{array}$ & 6.35 & 169,393 & $\$ 6.8$ & & & $36 \%$ & \begin{tabular}{|l|} 
Platinum \\
\end{tabular} & City & & & & 1912 & 105 & Cat. 3 \\
\hline Dundas Junior Public School & E & JK-5 & & 15 & Jennifer Story & John Chasty & 106 & $\begin{array}{l}\text { Limited } \\
\text { Limited } \\
\text { Limited }\end{array}$ & 4.61 & 82,946 & $\$ 3.3$ & & & $20 \%$ & & & & & & 1917 & 100 & \\
\hline Dunlace Public School & E & $\mathrm{JK}-6$ & & 13 & Gerri Gershon & Kathleen Garner & 419 & $\begin{array}{l}\text { Closed } \\
\text { Closed } \\
\text { Closed }\end{array}$ & 5.34 & 38,826 & $\$ 1.7$ & 3 & & $21 \%$ & Platinum & & & & & 1962 & 55 & \\
\hline $\begin{array}{l}\text { Earl Beatty Junior and Senior Public } \\
\text { School }\end{array}$ & $\mathrm{E}$ & JK-8 & & 16 & $\begin{array}{l}\text { Sheila Cary- } \\
\text { Meagher }\end{array}$ & $\begin{array}{l}\text { Mary Jane } \\
\text { McNamara }\end{array}$ & 382 & $\begin{array}{c}\text { Closed } \\
\text { Limited } \\
\text { N/A }\end{array}$ & 2.32 & 92,676 & $\$ 9.6$ & & & $76 \%$ & & City & & & & 1924 & 93 & Cat. 1 \\
\hline Earl Grey Senior Public School & $\mathrm{E}$ & $7-8$ & & 15 & Jennifer Story & $\begin{array}{l}\text { Mary Jane } \\
\text { McNamara }\end{array}$ & 374 & $\begin{array}{l}\text { Closed } \\
\text { Closed } \\
\text { Closed }\end{array}$ & 3.43 & 92,400 & $\$ 11.1$ & & & $110 \%$ & Bronze & TLC & Yes & & & 1960 & 57 & \\
\hline Earl Haig Public School & $\mathrm{E}$ & $\mathrm{JK}-8$ & & 16 & $\begin{array}{l}\text { Sheila Cary- } \\
\text { Meagher }\end{array}$ & $\begin{array}{l}\text { Mary Jane } \\
\text { McNamara }\end{array}$ & 301 & $\begin{array}{l}\text { Closed } \\
\text { Closed } \\
\text { N/A }\end{array}$ & 2.82 & 68,137 & $\$ 6.3$ & & & $57 \%$ & Platinum & & & & & 1921 & 96 & \\
\hline East Alternative School of Toronto & E & $7-8$ & & 15 & Jennifer Story & $\begin{array}{l}\text { Mary Jane } \\
\text { McNamara }\end{array}$ & 393 & $\begin{array}{c}\text { Limited } \\
\text { N/A } \\
\text { N/A }\end{array}$ & & & $\$ 1.1$ & & & $22 \%$ & Gold & & & & & & & \\
\hline Eastview Public School & $\mathrm{E}$ & $\mathrm{JK}-8$ & & 22 & Jerry Chadwick & $\begin{array}{l}\text { Kerry-Lynn } \\
\text { Stadnyk }\end{array}$ & 21 & $\begin{array}{c}\text { Limited } \\
\text { N/A } \\
\text { N/A }\end{array}$ & 6.5 & 55,737 & $\$ 7.4$ & & & $70 \%$ & & & & & & 1953 & 64 & \\
\hline Eatonville Junior School & E & JK-5 & & 2 & Chris Glover & Glenford Duffus & 208 & $\begin{array}{c}\text { Limited } \\
\text { N/A } \\
\text { N/A }\end{array}$ & 6 & 33,476 & $\$ 3.8$ & & & $53 \%$ & Gold & & & & & 1955 & 62 & \\
\hline
\end{tabular}




\begin{tabular}{|c|c|c|c|c|c|c|c|c|c|c|c|c|c|c|c|c|c|c|}
\hline & Participa & ition Rates & & & & & Enrolment a & Projectio & FTE, Surplus & eats $=$ OTG R & ised - Proj & tion FTE, Utili & itions $=$ Proj & ion/OTG R & vised & & & \\
\hline School Name & $\begin{array}{c}\text { TSSB 2013-14 } \\
\text { TDSB Participation } \\
\text { Rate }\end{array}$ & $\begin{array}{c}\text { TDSB 2013-14 } \\
\text { Home school } \\
\text { Participation Rate }\end{array}$ & $\begin{array}{c}\text { Revised } \\
\text { Capacity } \\
2016\end{array}$ & $\begin{array}{c}\text { HC } \\
\text { (Oct. 2016) }\end{array}$ & $\begin{array}{c}\text { FTE } \\
\text { (Oct. 2016) }\end{array}$ & $\begin{array}{c}2016 \\
\text { Utilization } \\
\text { Rate }\end{array}$ & $\begin{array}{c}2021 \\
\text { Projected }\end{array}$ & $\begin{array}{l}2021 \\
\text { Surplus } \\
\text { Seats }\end{array}$ & $\begin{array}{c}2021 \\
\text { Utilization } \\
\text { Rate }\end{array}$ & $\begin{array}{c}2026 \\
\text { Projected }\end{array}$ & $\begin{array}{l}2026 \\
\text { Surplus } \\
\text { Seats }\end{array}$ & $\begin{array}{c}2026 \\
\text { Utilization } \\
\text { Rate }\end{array}$ & $\begin{array}{c}2031 \\
\text { Projected }\end{array}$ & $\begin{array}{l}2031 \\
\text { Surplus } \\
\text { Seats }\end{array}$ & $\begin{array}{c}2031 \\
\text { Utilization } \\
\text { Rate }\end{array}$ & $\begin{array}{c}2036 \\
\text { Projected }\end{array}$ & $\begin{array}{l}2036 \\
\text { Surplus } \\
\text { Seats }\end{array}$ & $\begin{array}{c}2036 \\
\text { Utilization } \\
\text { Rate }\end{array}$ \\
\hline Donwood Park Public School & $76.1 \%$ & $71.2 \%$ & 925 & 716 & 716.0 & $77 \%$ & 703 & -273 & $76 \%$ & 661 & -231 & $71 \%$ & 651 & -221 & $70 \%$ & 651 & -221 & $70 \%$ \\
\hline Dorset Park Public School & $46.3 \%$ & $36.1 \%$ & 337 & 237 & 237.0 & $70 \%$ & 226 & 111 & $67 \%$ & 239 & 98 & $71 \%$ & 244 & 93 & $72 \%$ & 245 & 92 & $73 \%$ \\
\hline Dovercourt Public School & $63.4 \%$ & $40.5 \%$ & 470 & 366 & 366.0 & $78 \%$ & 447 & 23 & $95 \%$ & 409 & 61 & $87 \%$ & 410 & 60 & $87 \%$ & 410 & 60 & $87 \%$ \\
\hline Downsview Public School & $43.4 \%$ & $35.2 \%$ & 208 & 182 & 182.0 & $88 \%$ & 199 & 9 & $96 \%$ & 193 & 15 & $93 \%$ & 192 & 16 & $92 \%$ & 192 & 16 & $92 \%$ \\
\hline Downtown Alternative School & & & 202 & 114 & 114.0 & $56 \%$ & 136 & 66 & $67 \%$ & 136 & 66 & $67 \%$ & 136 & 66 & $67 \%$ & 136 & 66 & $67 \%$ \\
\hline $\begin{array}{l}\text { Downtown Vocal Music Academy of } \\
\text { Toronto }\end{array}$ & & & 115 & 50 & 50.0 & $43 \%$ & 68 & 47 & $59 \%$ & 68 & 47 & $59 \%$ & 68 & 47 & $59 \%$ & 68 & 47 & $59 \%$ \\
\hline $\begin{array}{l}\text { Dr Marion Hilliard Senior Public } \\
\text { school }\end{array}$ & $57.7 \%$ & $45.3 \%$ & 375 & 317 & 317.0 & $85 \%$ & 255 & 120 & $68 \%$ & 221 & 154 & $59 \%$ & 217 & 158 & $58 \%$ & 217 & 158 & $58 \%$ \\
\hline Driftwood Public School & $65.8 \%$ & $49.3 \%$ & 460 & 465 & 465.0 & $101 \%$ & 402 & 58 & $87 \%$ & 406 & 54 & $88 \%$ & 406 & 54 & $88 \%$ & 406 & 54 & $88 \%$ \\
\hline $\begin{array}{l}\text { Dublin Heights Elementary and } \\
\text { Middle School }\end{array}$ & $45.0 \%$ & $31.4 \%$ & 718 & 710 & 710.0 & $\mathbf{9 9 \%}$ & 854 & -136 & $119 \%$ & 822 & -104 & $114 \%$ & 810 & -92 & $113 \%$ & 810 & -92 & $113 \%$ \\
\hline $\begin{array}{l}\text { Duke of Connaught Junior and Senior } \\
\text { Public School }\end{array}$ & $81.5 \%$ & $53.3 \%$ & 1016 & 858 & 858.0 & $84 \%$ & 956 & 60 & $94 \%$ & 931 & 85 & $92 \%$ & 929 & 87 & $91 \%$ & 929 & 87 & $91 \%$ \\
\hline Dundas Junior Public School & $84.1 \%$ & $57.3 \%$ & 651 & 344 & 344.0 & $53 \%$ & 881 & -230 & $135 \%$ & 856 & -205 & $131 \%$ & 856 & -205 & $131 \%$ & 856 & -205 & $131 \%$ \\
\hline Dunlace Public School & $76.8 \%$ & $67.0 \%$ & 387 & 446 & 446.0 & $115 \%$ & 418 & -31 & $108 \%$ & 394 & -7 & $102 \%$ & 384 & 3 & $99 \%$ & 384 & 3 & $99 \%$ \\
\hline $\begin{array}{l}\text { Earl Beatty Junior and Senior Public } \\
\text { school }\end{array}$ & $70.8 \%$ & $44.2 \%$ & 600 & 444 & 444.0 & $74 \%$ & 471 & 129 & $79 \%$ & 462 & 138 & $77 \%$ & 462 & 138 & $77 \%$ & 462 & 138 & $77 \%$ \\
\hline Earl Grey Senior Public School & $97.9 \%$ & $72.6 \%$ & 499 & 422 & 422.0 & $85 \%$ & 513 & -14 & $103 \%$ & 551 & -52 & $110 \%$ & 553 & -54 & $111 \%$ & 553 & -54 & $111 \%$ \\
\hline Earl Haig Public School & $75.5 \%$ & $48.4 \%$ & 576 & 558 & 558.0 & $97 \%$ & 617 & -41 & $107 \%$ & 603 & -27 & $105 \%$ & 607 & -31 & $105 \%$ & 607 & -31 & $105 \%$ \\
\hline East Alternative School of Toronto & & & 138 & 65 & 65.0 & $47 \%$ & 67 & 71 & $49 \%$ & 67 & 71 & $49 \%$ & 67 & 71 & $49 \%$ & 67 & 71 & $49 \%$ \\
\hline Eastview Public School & $66.4 \%$ & $48.2 \%$ & 530 & 404 & 404.0 & $76 \%$ & 419 & 111 & $79 \%$ & 392 & 138 & $74 \%$ & 391 & 139 & $74 \%$ & 391 & 139 & $74 \%$ \\
\hline Eatonville Junior School & $60.1 \%$ & $47.4 \%$ & 306 & 234 & 234.0 & $76 \%$ & 253 & 53 & $83 \%$ & 243 & 63 & $79 \%$ & 242 & 64 & $79 \%$ & 242 & 64 & $79 \%$ \\
\hline
\end{tabular}




\begin{tabular}{|c|c|c|c|c|c|c|c|c|c|c|c|c|c|c|c|c|c|c|c|c|c|c|}
\hline \multirow[b]{2}{*}{ School Name } & \multicolumn{8}{|c|}{ General Information } & \multicolumn{14}{|c|}{ Facility Information } \\
\hline & Panel & $\begin{array}{l}\text { Grade } \\
\text { Range }\end{array}$ & $\begin{array}{c}\text { Semestered } \\
\text { (Sec) }\end{array}$ & Ward & Trustee & $\begin{array}{l}\text { Superintendent } \\
\text { of Education }\end{array}$ & 2017 LOI & $\begin{array}{c}2017-18 \\
\mathrm{Opt} \\
\text { Attend } \\
\mathrm{Reg} / \mathrm{F} / \mathrm{EF}\end{array}$ & $\begin{array}{l}\text { Site Size } \\
\text { (Acres) }\end{array}$ & $\begin{array}{c}\text { Facility Size } \\
\text { (Sq. Ft.) }\end{array}$ & $\begin{array}{c}\text { Current } \\
\text { Backlog (M) }\end{array}$ & \begin{tabular}{|l} 
Port. \\
(Instruc)
\end{tabular} & \begin{tabular}{|c|} 
Port. (Non- \\
Instruc)
\end{tabular} & $\begin{array}{c}\text { Current FCI } \\
\text { (EDU) }\end{array}$ & $\begin{array}{c}\text { Eco } \\
\text { Schools }\end{array}$ & Pool & $\begin{array}{l}\text { Elem. } \\
\text { D\&T } \\
\text { Shops }\end{array}$ & $\begin{array}{l}\text { Barrier } \\
\text { Free / } \\
\text { Access }\end{array}$ & Elevator & $\begin{array}{l}\text { Build } \\
\text { Date }\end{array}$ & $\begin{array}{c}\text { Building } \\
\text { Age }\end{array}$ & $\begin{array}{l}\text { T.O Comm } \\
\text { Centre }\end{array}$ \\
\hline Edgewood Public School & E & JK-8 & & 19 & David Smith & Shirley Chan & 142 & \begin{tabular}{|c|} 
Limited \\
N/A \\
N/A \\
\end{tabular} & 5.51 & 38,130 & $\$ 1.8$ & & & $23 \%$ & & & Yes & & & 1959 & 58 & \\
\hline Eglinton Junior Public School & E & JK-6 & & 11 & Shelley Laskin & Ian Allison & 373 & $\begin{array}{c}\text { Closed } \\
\text { N/A } \\
\text { Closed }\end{array}$ & 1.61 & 64,007 & $\$ .2$ & & & $2 \%$ & Gold & & & A1 & Yes & 1998 & 19 & \\
\hline Elia Middle School & $\mathrm{E}$ & 6-8 & & 4 & Tiffany Ford & Audley Salmon & 80 & $\begin{array}{c}\text { Limited } \\
\text { N/A } \\
\text { N/A }\end{array}$ & 9.71 & 85,368 & $\$ 5.9$ & & & $56 \%$ & Bronze & & & & & 1963 & 54 & \\
\hline Elizabeth Simcoe Junior Public School & $\mathrm{E}$ & $\mathrm{JK}-6$ & & 22 & Jerry Chadwick & $\begin{array}{l}\text { Kerry-Lynn } \\
\text { Stadnyk }\end{array}$ & 336 & $\begin{array}{c}\text { Limited } \\
\text { N/A } \\
\text { N/A }\end{array}$ & 5.98 & 45,116 & $\$ 4.5$ & & & $58 \%$ & Gold & & & & & 1963 & 54 & \\
\hline Elkhorn Public School & E & JK-5 & & 12 & Alexander Brown & Linda Curtis & 387 & $\begin{array}{c}\text { Closed } \\
\text { N/A } \\
\text { N/A }\end{array}$ & 5.31 & 35,812 & $\$ 6.3$ & 5 & & $81 \%$ & & & & & & 1956 & 61 & \\
\hline Ellesmere-Statton Public School & E & JK-8 & & 19 & David Smith & Anne Seymour & 164 & \begin{tabular}{c|} 
Limited \\
N/A \\
N/A
\end{tabular} & 8.75 & 94,357 & $\$ 11.4$ & & & $76 \%$ & & & Yes & & & 1951 & 66 & \\
\hline Elmbank Junior Middle Academy & E & JK-8 & & 1 & Avtar Minhas & Annie Appleby & 57 & $\begin{array}{c}\text { Limited } \\
\text { N/A } \\
\text { N/A }\end{array}$ & 9.59 & 98,921 & $\$ 8.9$ & & & $60 \%$ & & & & & & 1967 & 50 & \\
\hline Elmlea Junior School & E & JK-5 & & 1 & Avtar Minhas & Glenford Duffus & 79 & $\begin{array}{c}\text { Closed } \\
\text { Closed } \\
\text { N/A }\end{array}$ & 6.5 & 42,173 & $\$ 2.3$ & 1 & 4 & $22 \%$ & & & & & & 1953 & 64 & \\
\hline Emily Carr Public School & $\mathrm{E}$ & JK-8 & & 21 & Abdul Hai Patel & Nadira Persaud & 177 & $\begin{array}{c}\text { Limited } \\
N / A \\
\text { Limited }\end{array}$ & 5.81 & 51,521 & $\$ 4.9$ & & & $50 \%$ & & & & A1 & Yes & 1981 & 36 & \\
\hline Equinox Holistic Alternative School & E & JK-8 & & 15 & Jennifer Story & John Chasty & 388 & $\begin{array}{c}\text { Limited } \\
\text { N/A } \\
\text { N/A }\end{array}$ & & & & & & & & & & & & & & \\
\hline Ernest Public School & E & JK-6 & & 17 & Ken Lister & Beth Veale & 348 & $\begin{array}{c}\text { Limited } \\
\text { Limited } \\
\text { N/A }\end{array}$ & 5.96 & 46,716 & $\$ 2.9$ & & & $32 \%$ & Platinum & & & & & 1969 & 48 & \\
\hline Essex Junior and Senior Public School & E & JK-8 & & 10 & Ausma Malik & Mike Gallagher & 215 & \begin{tabular}{c|} 
Limited \\
N/A \\
N/A
\end{tabular} & 3.34 & 83,917 & $\$ 12.4$ & & & $77 \%$ & Platinum & & Yes & A1 & Yes & 1956 & 61 & \\
\hline Étienne Brûlé Junior School & E & JK-5 & & 3 & Pamela Gough & Tracy Hayhurst & 263 & $\begin{array}{c}\text { Limited } \\
\text { N/A } \\
\text { N/A }\end{array}$ & 1.61 & 26,792 & $\$ 1.9$ & & & $35 \%$ & & & & & & 1957 & 60 & \\
\hline F H Miller Junior Public School & E & $\mathrm{JK}-6$ & & 9 & Marit Stiles & Sandra Tondat & 180 & $\begin{array}{c}\text { Limited } \\
\text { N/A } \\
\text { N/A }\end{array}$ & 1.73 & 32,905 & $\$ 1$. & & & $16 \%$ & & & & & & 1925 & 92 & \\
\hline $\begin{array}{l}\text { Fairbank Memorial Community } \\
\text { School }\end{array}$ & E & JK-5 & & 9 & Marit Stiles & Sandra Tondat & 92 & $\begin{array}{c}\text { Limited } \\
\text { N/A } \\
\text { N/A }\end{array}$ & 2.22 & 56,921 & $\$ 8.2$ & & & $67 \%$ & & & & & & 1920 & 97 & \\
\hline Fairbank Public School & E & JK-8 & & 8 & Jennifer Arp & Leila Girdhar-Hill & 119 & $\begin{array}{c}\text { Limited } \\
\text { N/A } \\
\text { N/A }\end{array}$ & 3.06 & 51,518 & $\$ 2.7$ & & & $36 \%$ & & & Yes & & & 1963 & 54 & \\
\hline Fairglen Junior Public School & E & JK-6 & & 20 & Manna Wong & $\begin{array}{l}\text { Louie } \\
\text { Papathanasakis }\end{array}$ & 231 & $\begin{array}{c}\text { Limited } \\
\text { N/A } \\
\text { N/A }\end{array}$ & 6.23 & 36,147 & $\$ 1.3$ & & & $16 \%$ & & & & & & 1966 & 51 & \\
\hline Fairmount Public School & E & JK-8 & & 18 & Parthi Kandavel & $\begin{array}{l}\text { Jacqueline } \\
\text { Spence }\end{array}$ & 359 & $\begin{array}{c}\text { Closed } \\
\text { N/A } \\
\text { N/A }\end{array}$ & 7.57 & 39,035 & $\$ 3.8$ & 2 & & $46 \%$ & Gold & & & & & 1951 & 66 & \\
\hline
\end{tabular}




\begin{tabular}{|c|c|c|c|c|c|c|c|c|c|c|c|c|c|c|c|c|c|c|}
\hline & Participa & tion Rates & & & & & Enrolment a & Projection & FTE, Surplus & ats = OTG R & ised - Proje & ion FTE, Util & itions $=$ Proj & ion/OTG I & vised & & & \\
\hline School Name & $\begin{array}{c}\text { TDSB 2013-14 } \\
\text { TDSB Participation } \\
\text { Rate }\end{array}$ & $\begin{array}{c}\text { TOSB 2013-14 } \\
\text { Home school } \\
\text { Participation Rate }\end{array}$ & $\begin{array}{c}\text { Revised } \\
\text { Capacity } \\
2016\end{array}$ & $\begin{array}{c}\text { HC } \\
\text { (Oct. 2016) }\end{array}$ & \begin{tabular}{|c|} 
FTE \\
(Oct. 2016)
\end{tabular} & $\begin{array}{c}2016 \\
\text { Utilization } \\
\text { Rate }\end{array}$ & $\begin{array}{c}2021 \\
\text { Projected }\end{array}$ & $\begin{array}{l}2021 \\
\text { Surplus } \\
\text { Seats }\end{array}$ & $\begin{array}{c}2021 \\
\text { Utilization } \\
\text { Rate }\end{array}$ & $\begin{array}{l}2026 \\
\text { Projected }\end{array}$ & $\begin{array}{l}2026 \\
\text { Surplus } \\
\text { Seats }\end{array}$ & $\begin{array}{l}2026 \\
\text { Utilization } \\
\text { Rate }\end{array}$ & $\begin{array}{c}2031 \\
\text { Projected }\end{array}$ & $\begin{array}{l}2031 \\
\text { Surplus } \\
\text { Seats }\end{array}$ & $\begin{array}{c}2031 \\
\text { Utilization } \\
\text { Rate }\end{array}$ & $\begin{array}{l}2036 \\
\text { Projected }\end{array}$ & $\begin{array}{l}2036 \\
\text { Surplus } \\
\text { Seats }\end{array}$ & $\begin{array}{c}2036 \\
\text { Utilization } \\
\text { Rate }\end{array}$ \\
\hline Edgewood Public School & $73.1 \%$ & $44.7 \%$ & 355 & 227 & 227.0 & $64 \%$ & 381 & -26 & $107 \%$ & 389 & -34 & $110 \%$ & 391 & -36 & $110 \%$ & 391 & -36 & $110 \%$ \\
\hline Eglinton Junior Public School & $74.9 \%$ & $45.7 \%$ & 507 & 567 & 567.0 & $112 \%$ & 540 & -33 & $107 \%$ & 554 & -47 & $109 \%$ & 559 & -52 & $110 \%$ & 559 & -52 & $110 \%$ \\
\hline Elia Middle School & $61.4 \%$ & $49.0 \%$ & 551 & 381 & 381.0 & $69 \%$ & 317 & 234 & $58 \%$ & 309 & 242 & $56 \%$ & 318 & 233 & $58 \%$ & 318 & 233 & $58 \%$ \\
\hline Elizabeth Simcoe Junior Public School & $85.6 \%$ & $76.9 \%$ & 369 & 262 & 262.0 & $71 \%$ & 250 & 119 & $68 \%$ & 250 & 119 & $68 \%$ & 255 & 114 & $69 \%$ & 255 & 114 & $69 \%$ \\
\hline Elkhorn Public School & $78.7 \%$ & $62.9 \%$ & 358 & 410 & 410.0 & $115 \%$ & 461 & -103 & $129 \%$ & 479 & -121 & $134 \%$ & 479 & -121 & $134 \%$ & 479 & -121 & $134 \%$ \\
\hline Ellesmere-Statton Public School & $70.7 \%$ & $60.5 \%$ & 784 & 622 & 621.5 & $79 \%$ & 574 & 210 & $73 \%$ & 500 & 284 & $64 \%$ & 501 & 283 & $64 \%$ & 501 & 283 & $64 \%$ \\
\hline Elmbank Junior Middle Academy & $32.3 \%$ & $25.5 \%$ & 679 & 348 & 348.0 & $51 \%$ & 485 & 194 & $71 \%$ & 594 & 85 & $87 \%$ & 598 & 81 & $88 \%$ & 598 & 81 & $88 \%$ \\
\hline Elmlea Junior School & $61.1 \%$ & $42.2 \%$ & 481 & 423 & 423.0 & $88 \%$ & 368 & 113 & $77 \%$ & 378 & 103 & $79 \%$ & 378 & 103 & $79 \%$ & 378 & 103 & $79 \%$ \\
\hline Emily Carr Public School & $63.9 \%$ & $54.3 \%$ & 475 & 318 & 318.0 & $67 \%$ & 274 & 201 & $58 \%$ & 277 & 198 & $58 \%$ & 280 & 195 & $59 \%$ & 280 & 195 & $59 \%$ \\
\hline Equinox Holistic Alternative School & & & 210 & 189 & 189.0 & $90 \%$ & 169 & 41 & $80 \%$ & 169 & 41 & $80 \%$ & 169 & 41 & $80 \%$ & 169 & 41 & $80 \%$ \\
\hline Ernest Public School & $66.1 \%$ & $47.9 \%$ & 452 & 143 & 143.0 & $32 \%$ & 131 & 321 & $29 \%$ & 136 & 316 & $30 \%$ & 136 & 316 & $30 \%$ & 136 & 316 & $30 \%$ \\
\hline Essex Junior and Senior Public School & $74.8 \%$ & $27.8 \%$ & 457 & 276 & 276.0 & $60 \%$ & 256 & 201 & $56 \%$ & 252 & 205 & $55 \%$ & 250 & 207 & $55 \%$ & 250 & 207 & $55 \%$ \\
\hline Étienne Brûlé Junior School & $49.7 \%$ & $34.8 \%$ & 205 & 179 & 179.0 & $87 \%$ & 239 & -34 & $117 \%$ & 222 & -17 & $108 \%$ & 222 & -17 & $108 \%$ & 222 & -17 & $108 \%$ \\
\hline F H Miller Junior Public School & $35.8 \%$ & $23.6 \%$ & 269 & 115 & 115.0 & $43 \%$ & 93 & 176 & $35 \%$ & 96 & 173 & $36 \%$ & 96 & 173 & $36 \%$ & 96 & 173 & $36 \%$ \\
\hline $\begin{array}{l}\text { Fairbank Memorial Community } \\
\text { School }\end{array}$ & $32.9 \%$ & $18.1 \%$ & 486 & 163 & 160.3 & $33 \%$ & 141 & 345 & $29 \%$ & 144 & 342 & $30 \%$ & 144 & 342 & $30 \%$ & 144 & 342 & $30 \%$ \\
\hline Fairbank Public School & $32.9 \%$ & $14.2 \%$ & 314 & 258 & 258.0 & $82 \%$ & 289 & 25 & $92 \%$ & 284 & 30 & $90 \%$ & 283 & 31 & $90 \%$ & 283 & 31 & $90 \%$ \\
\hline Fairglen Junior Public School & $62.7 \%$ & $51.0 \%$ & 383 & 306 & 306.0 & $80 \%$ & 295 & 88 & $77 \%$ & 292 & 91 & $76 \%$ & 292 & 91 & $76 \%$ & 292 & 91 & $76 \%$ \\
\hline Fairmount Public School & $81.6 \%$ & $73.7 \%$ & 386 & 435 & 435.0 & $113 \%$ & 384 & 2 & $99 \%$ & 369 & 17 & $96 \%$ & 369 & 17 & $96 \%$ & 369 & 17 & $96 \%$ \\
\hline
\end{tabular}




\begin{tabular}{|c|c|c|c|c|c|c|c|c|c|c|c|c|c|c|c|c|c|c|c|c|c|c|}
\hline \multirow[b]{2}{*}{ School Name } & \multicolumn{8}{|c|}{ General Information } & \multicolumn{14}{|c|}{ Facility Information } \\
\hline & Panel & $\begin{array}{l}\text { Grade } \\
\text { Range }\end{array}$ & $\begin{array}{c}\text { Semestered } \\
\text { (Sec) }\end{array}$ & Ward & Trustee & $\begin{array}{l}\text { Superintendent } \\
\text { of Education }\end{array}$ & 2017 LOI & $\begin{array}{c}2017-18 \\
\text { Opt } \\
\text { Attend } \\
\text { Reg/F//EF }\end{array}$ & $\begin{array}{l}\text { Site Size } \\
\text { (Acres) }\end{array}$ & $\begin{array}{l}\text { Facility Size } \\
\text { (Sq. Ft.) }\end{array}$ & $\begin{array}{c}\text { Current } \\
\text { Backlog (M) }\end{array}$ & $\begin{array}{l}\text { Port. } \\
\text { (Instruc) }\end{array}$ & $\begin{array}{c}\text { Port. (Non- } \\
\text { Instruc) }\end{array}$ & $\begin{array}{c}\text { Current FCI } \\
\text { (EDU) }\end{array}$ & $\begin{array}{l}\text { Eco } \\
\text { Schools }\end{array}$ & Pool & $\begin{array}{l}\text { Elem. } \\
\text { D\&T } \\
\text { Shops }\end{array}$ & $\begin{array}{l}\text { Barrier } \\
\text { Free / } \\
\text { Access }\end{array}$ & Elevator & $\begin{array}{l}\text { Build } \\
\text { Date }\end{array}$ & $\begin{array}{c}\text { Building } \\
\text { Age }\end{array}$ & $\begin{array}{l}\text { T.O Comm } \\
\text { Centre }\end{array}$ \\
\hline $\begin{array}{l}\text { Faywood Arts-Based Curriculum } \\
\text { School }\end{array}$ & $\mathrm{E}$ & JK-8 & & 5 & Alexandra Lulka & Linda Curtis & 229 & \begin{tabular}{|c|} 
Limited \\
N/A \\
N/A \\
\end{tabular} & 6 & 55,155 & $\$ 5.2$ & 2 & & $43 \%$ & & & & & & 1950 & 67 & \\
\hline Fenside Public School & E & JK-5 & & 17 & Ken Lister & Curtis Ennis & 185 & \begin{tabular}{|c|} 
Limited \\
N/A \\
N/A
\end{tabular} & 5.09 & 48,072 & $\$ 3.8$ & & & $40 \%$ & Gold & & & & & 1960 & 57 & \\
\hline $\begin{array}{l}\text { Fern Avenue Junior and Senior Public } \\
\text { School }\end{array}$ & $\mathrm{E}$ & JK-8 & & 7 & Robin Pilkey & $\begin{array}{l}\text { Jane Phillips- } \\
\text { Long }\end{array}$ & 410 & $\begin{array}{l}\text { Closed } \\
\text { Closed } \\
\text { Closed }\end{array}$ & 2.69 & 95,486 & $\$ 9$. & & & $66 \%$ & Gold & TLC & & & & 1894 & 123 & \\
\hline Finch Public School & $\mathrm{E}$ & JK-5 & & 12 & Alexander Brown & Linda Curtis & 296 & $\begin{array}{l}\text { Closed } \\
\text { N/A } \\
\text { N/A }\end{array}$ & 5.09 & 34,295 & $\$ 1.1$ & 3 & 1 & $13 \%$ & Gold & & & & & 1950 & 67 & \\
\hline Firgrove Public School & $\mathrm{E}$ & JK-5 & & 4 & Tiffany Ford & Lynn Strangway & 4 & $\begin{array}{c}\text { Limited } \\
\text { N/A } \\
\text { N/A }\end{array}$ & 8.01 & 69,535 & $\$ 10.1$ & & & $72 \%$ & Silver & & & & & 1970 & 47 & \\
\hline $\begin{array}{l}\text { First Nations Junior and Senior School } \\
\text { of Toronto }\end{array}$ & $\mathrm{E}$ & JK-8 & & 15 & Jennifer Story & John Chasty & 54 & $\begin{array}{c}\text { Limited } \\
\text { N/A } \\
\text { N/A }\end{array}$ & & & & & & & Gold & & & & & & & \\
\hline Fisherville Senior Public School & $\mathrm{E}$ & $7-8$ & & 5 & Alexandra Lulka & Elizabeth Addo & 230 & \begin{tabular}{|c|} 
Limited \\
N/A \\
N/A \\
\end{tabular} & 7.26 & 89,912 & $\$ 3.7$ & & & $28 \%$ & & & Yes & & & 1965 & 52 & \\
\hline Fleming Public School & E & JK-8 & & 21 & Abdul Hai Patel & Nadira Persaud & 212 & \begin{tabular}{|c|} 
Limited \\
N/A \\
N/A
\end{tabular} & 5.51 & 45,039 & $\$ 1.7$ & & 1 & $22 \%$ & & & & A1 & Yes & 1991 & 26 & \\
\hline Flemington Public School & $\mathrm{E}$ & JK-5 & & 8 & Jennifer Arp & Leila Girdhar-Hill & 3 & \begin{tabular}{|c|} 
Limited \\
N/A \\
N/A
\end{tabular} & 6.08 & 65,381 & $\$ 5.1$ & & & $38 \%$ & Gold & & & & & 1957 & 60 & \\
\hline $\begin{array}{l}\text { Forest Hill Junior and Senior Public } \\
\text { School }\end{array}$ & E & JK-8 & & 11 & Shelley Laskin & Leila Girdhar-Hill & 434 & $\begin{array}{l}\text { Closed } \\
\text { N/A } \\
\text { Closed }\end{array}$ & 6.18 & 112,705 & $\$ 15.8$ & & & $\mathbf{9 8 \%}$ & Gold & & Yes & & & 1925 & 92 & \\
\hline Forest Manor Public School & $\mathrm{E}$ & JK-6 & & 17 & Ken Lister & Audley Salmon & 269 & $\begin{array}{l}\text { Closed } \\
\text { N/A } \\
\text { N/A }\end{array}$ & 8.01 & 65,313 & $\$ 2.1$ & 4 & 2 & $15 \%$ & Platinum & & & & & 1967 & 50 & \\
\hline Frankland Community School & E & JK-6 & & 15 & Jennifer Story & $\begin{array}{l}\text { Mary Jane } \\
\text { McNamara }\end{array}$ & 440 & $\begin{array}{c}\text { Closed } \\
\text { N/A } \\
\text { N/A }\end{array}$ & 3.95 & 55,380 & $\$ 2.5$ & & 4 & $33 \%$ & & City & & & Yes & 1979 & 38 & Cat. 1 \\
\hline $\begin{array}{l}\text { Fraser Mustard Early Learning } \\
\text { Academy }\end{array}$ & $\mathrm{E}$ & JK-SK & & 13 & Gerri Gershon & Ian Allison & 169 & $\begin{array}{c}\text { Closed } \\
\text { N/A } \\
\text { N/A }\end{array}$ & 2.75 & 85,965 & 0 & & & $0 \%$ & Silver & & & A1 & & 2013 & 4 & \\
\hline Galloway Road Public School & $\mathrm{E}$ & JK-8 & & 22 & Jerry Chadwick & Anne Seymour & 23 & \begin{tabular}{|c|} 
Limited \\
N/A \\
N/A
\end{tabular} & 7.39 & 26,187 & $\$ 2.5$ & & 1 & $42 \%$ & & & & & & 1956 & 61 & \\
\hline Garden Avenue Junior Public School & $\mathrm{E}$ & JK-6 & & 7 & Robin Pilkey & $\begin{array}{l}\text { Jane Phillips- } \\
\text { Long }\end{array}$ & 407 & $\begin{array}{l}\text { Closed } \\
\text { N/A } \\
\text { N/A }\end{array}$ & 1.73 & 25,421 & $\$ 3.5$ & & & $48 \%$ & & & & & & 1956 & 61 & \\
\hline Gateway Public School & E & JK-6 & & 13 & Gerri Gershon & Ian Allison & 130 & $\begin{array}{l}\text { Closed } \\
\text { N/A } \\
\text { N/A }\end{array}$ & 9.44 & 102,366 & $\$ 2.1$ & 2 & 2 & $12 \%$ & Platinum & & & & Yes & 1967 & 50 & \\
\hline General Brock Public School & $\mathrm{E}$ & JK-8 & & 18 & Parthi Kandavel & Peter Chang & 205 & $\begin{array}{l}\text { Closed } \\
\text { N/A } \\
\text { N/A }\end{array}$ & 8.01 & 42,498 & $\$ 3.3$ & 1 & & $33 \%$ & & & & & & 1955 & 62 & \\
\hline General Crerar Public School & E & JK-8 & & 19 & David Smith & Anne Seymour & 110 & $\mid \begin{array}{c}\text { Closed } \\
\text { Limited } \\
\text { N/A }\end{array}$ & 7.51 & 28,464 & $\$ 2.5$ & 1 & & $32 \%$ & Gold & & & & & 1954 & 63 & \\
\hline
\end{tabular}




\begin{tabular}{|c|c|c|c|c|c|c|c|c|c|c|c|c|c|c|c|c|c|c|}
\hline & Participa & tion Rates & & & & & Enrolment a & Projectio & FTE, Surplus & eats $=$ OTG R & ised - Proj & tion FTE, Util & tions $=$ Proj & tion/OTG F & vised & & & \\
\hline School Name & $\begin{array}{c}\text { TDSB 2013-14 } \\
\text { TDSB Participation } \\
\text { Rate }\end{array}$ & $\begin{array}{c}\text { TDSB 2013-14 } \\
\text { Home School } \\
\text { Participation Rate }\end{array}$ & $\begin{array}{c}\text { Revised } \\
\text { Capacity } \\
2016\end{array}$ & $\begin{array}{c}\text { HC } \\
\text { (Oct. 2016) }\end{array}$ & $\begin{array}{c}\text { FTE } \\
\text { (Oct. 2016) }\end{array}$ & $\begin{array}{c}2016 \\
\text { Utilization } \\
\text { Rate }\end{array}$ & $\begin{array}{c}2021 \\
\text { Projected }\end{array}$ & $\begin{array}{l}2021 \\
\text { Surplus } \\
\text { Seats }\end{array}$ & $\begin{array}{c}2021 \\
\text { Utilization } \\
\text { Rate }\end{array}$ & $\begin{array}{c}2026 \\
\text { Projected }\end{array}$ & $\begin{array}{l}2026 \\
\text { Surplus } \\
\text { Seats }\end{array}$ & $\begin{array}{c}2026 \\
\text { Utilization } \\
\text { Rate }\end{array}$ & $\begin{array}{c}2031 \\
\text { Projected }\end{array}$ & $\begin{array}{l}2031 \\
\text { Surplus } \\
\text { Seats }\end{array}$ & $\begin{array}{c}2031 \\
\text { Utilization } \\
\text { Rate }\end{array}$ & $\begin{array}{c}2036 \\
\text { Projected }\end{array}$ & $\begin{array}{l}2036 \\
\text { Surplus } \\
\text { Seats }\end{array}$ & $\begin{array}{c}2036 \\
\text { Utilization } \\
\text { Rate }\end{array}$ \\
\hline $\begin{array}{l}\text { Faywood Arts-Based Curriculum } \\
\text { School }\end{array}$ & $31.7 \%$ & $21.5 \%$ & 440 & 465 & 465.0 & $106 \%$ & 480 & -40 & $109 \%$ & 471 & -31 & $107 \%$ & 468 & -28 & $106 \%$ & 468 & -28 & $106 \%$ \\
\hline Fenside Public School & $71.9 \%$ & $51.2 \%$ & 410 & 296 & 296.0 & $72 \%$ & 304 & 106 & $74 \%$ & 295 & 115 & $72 \%$ & 295 & 115 & $72 \%$ & 295 & 115 & $72 \%$ \\
\hline $\begin{array}{l}\text { Fern Avenue Junior and Senior Public } \\
\text { School }\end{array}$ & $82.8 \%$ & $55.4 \%$ & 771 & 672 & 672.0 & $87 \%$ & 680 & 91 & $88 \%$ & 646 & 125 & $84 \%$ & 653 & 118 & $85 \%$ & 653 & 118 & $85 \%$ \\
\hline Finch Public School & $81.9 \%$ & $42.5 \%$ & 387 & 324 & 324.0 & $84 \%$ & 313 & 74 & $81 \%$ & 314 & 73 & $81 \%$ & 317 & 70 & $82 \%$ & 317 & 70 & $82 \%$ \\
\hline Firgrove Public School & $69.5 \%$ & $58.8 \%$ & 643 & 432 & 430.0 & $67 \%$ & 335 & 308 & $52 \%$ & 334 & 309 & $52 \%$ & 334 & 309 & $52 \%$ & 334 & 309 & $52 \%$ \\
\hline $\begin{array}{l}\text { First Nations Junior and Senior School } \\
\text { of Toronto }\end{array}$ & & & 216 & 119 & 126.0 & $58 \%$ & 111 & 105 & $51 \%$ & 111 & 105 & $51 \%$ & 111 & 105 & $51 \%$ & 111 & 105 & $51 \%$ \\
\hline Fisherville Senior Public School & $57.7 \%$ & $41.1 \%$ & 447 & 184 & 184.0 & $41 \%$ & 156 & 291 & $35 \%$ & 150 & 297 & $34 \%$ & 144 & 303 & $32 \%$ & 144 & 303 & $32 \%$ \\
\hline Fleming Public School & $80.0 \%$ & $66.1 \%$ & 372 & 270 & 270.0 & $73 \%$ & 229 & 143 & $62 \%$ & 228 & 144 & $61 \%$ & 230 & 142 & $62 \%$ & 230 & 142 & $62 \%$ \\
\hline Flemington Public School & $69.6 \%$ & $49.2 \%$ & 632 & 235 & 235.0 & $37 \%$ & 285 & 347 & $45 \%$ & 303 & 329 & $48 \%$ & 303 & 329 & $48 \%$ & 303 & 329 & $48 \%$ \\
\hline $\begin{array}{l}\text { Forest Hill Junior and Senior Public } \\
\text { School }\end{array}$ & $50.4 \%$ & 41.1\% & 774 & 795 & 795.0 & $103 \%$ & 895 & -121 & $116 \%$ & 904 & -130 & $117 \%$ & 906 & -132 & $117 \%$ & 906 & -132 & $117 \%$ \\
\hline Forest Manor Public School & $84.0 \%$ & $74.0 \%$ & 704 & 736 & 736.0 & $105 \%$ & 732 & -28 & $104 \%$ & 737 & -33 & $105 \%$ & 738 & -34 & $105 \%$ & 738 & -34 & $105 \%$ \\
\hline Frankland Community School & $104.1 \%$ & $79.2 \%$ & 340 & 370 & 370.0 & $109 \%$ & 349 & -9 & $103 \%$ & 355 & -15 & $104 \%$ & 355 & -15 & $104 \%$ & 355 & -15 & $104 \%$ \\
\hline $\begin{array}{l}\text { Fraser Mustard Early Learning } \\
\text { Academy }\end{array}$ & $78.3 \%$ & $75.7 \%$ & 685 & 663 & 663.0 & $97 \%$ & 584 & 101 & $85 \%$ & 579 & 106 & $85 \%$ & 579 & 106 & $85 \%$ & 579 & 106 & $85 \%$ \\
\hline Galloway Road Public School & $62.0 \%$ & $40.7 \%$ & 231 & 190 & 190.0 & $82 \%$ & 194 & 37 & $84 \%$ & 204 & 27 & $88 \%$ & 205 & 26 & $89 \%$ & 205 & 26 & $89 \%$ \\
\hline Garden Avenue Junior Public School & $81.3 \%$ & $58.4 \%$ & 294 & 290 & 289.0 & $98 \%$ & 242 & 52 & $82 \%$ & 227 & 67 & $77 \%$ & 227 & 67 & $77 \%$ & 227 & 67 & $77 \%$ \\
\hline Gateway Public School & $69.3 \%$ & $57.4 \%$ & 965 & 884 & 884.0 & $92 \%$ & 795 & 170 & $82 \%$ & 791 & 174 & $82 \%$ & 791 & 174 & $82 \%$ & 791 & 174 & $82 \%$ \\
\hline General Brock Public School & $61.1 \%$ & $53.2 \%$ & 496 & 437 & 437.0 & $88 \%$ & 578 & -82 & $117 \%$ & 596 & -100 & $120 \%$ & 594 & -98 & $120 \%$ & 594 & -98 & $120 \%$ \\
\hline General Crerar Public School & $45.7 \%$ & $40.2 \%$ & 359 & 354 & 354.0 & $99 \%$ & 359 & 0 & $100 \%$ & 359 & 0 & $100 \%$ & 359 & 0 & $100 \%$ & 359 & 0 & $100 \%$ \\
\hline
\end{tabular}




\begin{tabular}{|c|c|c|c|c|c|c|c|c|c|c|c|c|c|c|c|c|c|c|c|c|c|c|}
\hline \multirow[b]{2}{*}{ School Name } & \multicolumn{8}{|c|}{ General Information } & \multicolumn{14}{|c|}{ Facility Information } \\
\hline & Panel & $\begin{array}{l}\text { Grade } \\
\text { Range }\end{array}$ & $\begin{array}{c}\text { Semestered } \\
\text { (Sec) }\end{array}$ & Ward & Trustee & $\begin{array}{l}\text { Superintendent } \\
\text { of Education }\end{array}$ & 2017 LOI & $\begin{array}{c}2017-18 \\
\mathrm{Opt} \\
\text { Attend } \\
\mathrm{Reg} / \mathrm{F} / \mathrm{EF}\end{array}$ & $\begin{array}{l}\text { Site Size } \\
\text { (Acres) }\end{array}$ & $\begin{array}{c}\text { Facility Size } \\
\text { (Sq. Ft.) }\end{array}$ & $\begin{array}{c}\text { Current } \\
\text { Backlog (M) }\end{array}$ & \begin{tabular}{|c} 
Port. \\
(Instruc)
\end{tabular} & $\begin{array}{c}\text { Port. (Non- } \\
\text { Instruc) }\end{array}$ & $\begin{array}{c}\text { Current FCI } \\
\text { (EDU) }\end{array}$ & $\begin{array}{l}\text { Eco } \\
\text { Schools }\end{array}$ & Pool & $\begin{array}{l}\text { Elem. } \\
\text { D\&T } \\
\text { Shops }\end{array}$ & $\begin{array}{l}\text { Barrier } \\
\text { Free / } \\
\text { Access }\end{array}$ & Elevator & $\begin{array}{l}\text { Build } \\
\text { Date }\end{array}$ & $\begin{array}{c}\text { Building } \\
\text { Age }\end{array}$ & $\begin{array}{l}\text { T.O Comm } \\
\text { Centre }\end{array}$ \\
\hline General Mercer Junior Public School & E & JK-6 & & 9 & Marit Stiles & Sandra Tondat & 83 & \begin{tabular}{|c|} 
Limited \\
N/A \\
N/A \\
\end{tabular} & 4.32 & 70,648 & $\$ 5.4$ & & & $50 \%$ & & & & & & 1923 & 94 & \\
\hline George Anderson Public School & E & JK-5 & & 6 & Chris Tonks & $\begin{array}{l}\text { Angela Nardi- } \\
\text { Addesa }\end{array}$ & 129 & $\begin{array}{c}\text { Limited } \\
\text { N/A } \\
\text { N/A }\end{array}$ & 4.69 & 23,003 & $\$ 4$. & & & $68 \%$ & Platinum & & & & & 1955 & 62 & \\
\hline George B Little Public School & $\mathrm{E}$ & JK-8 & & 22 & Jerry Chadwick & Nadira Persaud & 152 & $\begin{array}{c}\text { Limited } \\
\text { N/A } \\
\text { N/A }\end{array}$ & 6.89 & 54,013 & $\$ 5.6$ & & & $53 \%$ & Gold & & & & & 1956 & 61 & \\
\hline George P Mackie Junior Public School & $\mathrm{E}$ & $\mathrm{JK}-6$ & & 18 & Parthi Kandavel & $\begin{array}{l}\text { Kerry-Lynn } \\
\text { Stadnyk }\end{array}$ & 247 & $\begin{array}{c}\text { Limited } \\
\text { N/A } \\
\text { N/A }\end{array}$ & 4.99 & 21,066 & $\$ 2$. & & & $41 \%$ & Platinum & & & & & 1953 & 64 & \\
\hline George Peck Public School & E & JK-8 & & 19 & David Smith & Nadira Persaud & 136 & $\begin{array}{c}\text { Limited } \\
\text { N/A } \\
\text { N/A }\end{array}$ & 4.79 & 39,285 & $\$ 5.4$ & & & $84 \%$ & & & & & No & 1955 & 62 & \\
\hline George R Gauld Junior School & E & JK-5 & & 3 & Pamela Gough & Sandra Tondat & 239 & $\begin{array}{c}\text { Limited } \\
\text { N/A } \\
\text { N/A }\end{array}$ & 3.8 & 23,283 & $\$ 3.6$ & 1 & 2 & $67 \%$ & Gold & & & & & 1922 & 95 & \\
\hline George Syme Community School & E & JK-5 & & 6 & Chris Tonks & Vicky Branco & 41 & $\begin{array}{c}\text { Limited } \\
\text { Limited } \\
\text { N/A }\end{array}$ & 4.6 & 78,341 & $\$ 2.4$ & & & $19 \%$ & & & & & & 1972 & 45 & \\
\hline George Webster Elementary School & E & JK-8 & & 16 & $\begin{array}{l}\text { Sheila Cary- } \\
\text { Meagher }\end{array}$ & Lucy Giannotta & 102 & $\begin{array}{c}\text { Closed } \\
\text { N/A } \\
\text { N/A }\end{array}$ & 6.19 & 51,188 & $\$ 6.4$ & 16 & 3 & $86 \%$ & Gold & & & & & 1954 & 63 & \\
\hline Givins/Shaw Junior Public School & $\mathrm{E}$ & $\mathrm{JK}-6$ & & 10 & Ausma Malik & Mike Gallagher & 376 & $\begin{array}{c}\text { Closed } \\
\text { N/A } \\
\text { N/A }\end{array}$ & 3.42 & 41,782 & $\$ 7.1$ & & & $91 \%$ & & & & & & 1956 & 61 & \\
\hline Glamorgan Junior Public School & E & JK-6 & & 20 & Manna Wong & Lynn Strangway & 172 & $\begin{array}{c}\text { Closed } \\
\text { N/A } \\
\text { N/A }\end{array}$ & 6 & 74,915 & $\$ 6.4$ & 1 & 1 & $48 \%$ & & & & A1 & Yes & 1972 & 45 & \\
\hline Gledhill Junior Public School & E & JK-6 & & 16 & $\begin{array}{l}\text { Sheila Cary- } \\
\text { Meagher }\end{array}$ & $\begin{array}{l}\text { Mary Jane } \\
\text { McNamara }\end{array}$ & 256 & $\begin{array}{c}\text { Closed } \\
\text { Limited } \\
\text { N/A }\end{array}$ & 4.37 & 79,573 & $\$ 5.1$ & & 1 & $45 \%$ & Silver & & & & & 1915 & 102 & \\
\hline Glen Ames Senior Public School & E & $7-8$ & & 16 & $\begin{array}{l}\text { Sheila Cary- } \\
\text { Meagher }\end{array}$ & John Chasty & 437 & $\begin{array}{c}\text { Closed } \\
\text { Limited } \\
\text { Limited }\end{array}$ & 3.05 & 95,898 & $\$ 3.5$ & 3 & & $54 \%$ & & City & & & & 1959 & 58 & Cat. 4 \\
\hline Glen Park Public School & E & JK-6 & & 8 & Jennifer Arp & Leila Girdhar-Hill & 224 & $\begin{array}{c}\text { Limited } \\
\text { Limited } \\
\mathrm{N} / \mathrm{A}\end{array}$ & 8.03 & 63,774 & $\$ 2.1$ & & & $20 \%$ & Silver & & & A1 & Yes & 1998 & 19 & \\
\hline Glen Ravine Junior Public School & E & $\mathrm{JK}-6$ & & 19 & David Smith & Shirley Chan & 65 & $\begin{array}{c}\text { Closed } \\
\text { N/A } \\
\text { N/A }\end{array}$ & 5.49 & 34,915 & $\$ 6.5$ & & & $80 \%$ & Silver & & & & & 1956 & 61 & \\
\hline Glenview Senior Public School & E & $7-8$ & & 8 & Jennifer Arp & Vicky Branco & 457 & $\begin{array}{l}\text { Closed } \\
\text { Closed } \\
\text { Closed }\end{array}$ & 8.1 & 94,927 & $\$ 4.1$ & & & $39 \%$ & Platinum & TLC & Yes & B1 & Yes & 1965 & 52 & \\
\hline Golf Road Junior Public School & E & JK-6 & & 19 & David Smith & Anne Seymour & 162 & $\begin{array}{c}\text { Closed } \\
\text { N/A } \\
\text { N/A }\end{array}$ & 4.99 & 40,447 & $\$ 1.4$ & & 1 & $17 \%$ & Platinum & & & & & 1952 & 65 & \\
\hline Gordon A Brown Middle School & E & 6-8 & & 16 & $\begin{array}{l}\text { Sheila Cary- } \\
\text { Meagher }\end{array}$ & Lucy Giannotta & 189 & $\begin{array}{c}\text { Limited } \\
\text { N/A } \\
\text { Limited }\end{array}$ & 4.5 & 79,866 & $\$ 4.5$ & 4 & & $50 \%$ & & City & & & & 1950 & 67 & \\
\hline Gosford Public School & E & JK-5 & & 4 & Tiffany Ford & Lynn Strangway & 18 & $\begin{array}{c}\text { Limited } \\
\text { N/A } \\
\text { N/A }\end{array}$ & 6 & 42,367 & $\$ 7.3$ & & & $\mathbf{9 3} \%$ & & & & & & 1963 & 54 & \\
\hline
\end{tabular}




\begin{tabular}{|c|c|c|c|c|c|c|c|c|c|c|c|c|c|c|c|c|c|c|}
\hline & Participa & tion Rates & & & & & Enrolment a & Projectio & FTE, Surplus & eats $=$ OTG R & ised - Proj & tion FTE, Utili & itions $=$ Proj & ion/OTG I & vised & & & \\
\hline School Name & $\begin{array}{c}\text { TDSB 2013-14 } \\
\text { TDSB Participation } \\
\text { Rate }\end{array}$ & $\begin{array}{c}\text { TDSB } 2013-14 \\
\text { Home Schol } \\
\text { Participation Rate }\end{array}$ & $\begin{array}{c}\text { Revised } \\
\text { Capacity } \\
2016\end{array}$ & $\begin{array}{c}\text { HC } \\
\text { (Oct. 2016) }\end{array}$ & $\begin{array}{c}\text { FTE } \\
\text { (Oct. 2016) }\end{array}$ & $\begin{array}{c}2016 \\
\text { Utilization } \\
\text { Rate }\end{array}$ & $\begin{array}{c}2021 \\
\text { Projected }\end{array}$ & $\begin{array}{c}2021 \\
\text { Surplus } \\
\text { Seats }\end{array}$ & $\begin{array}{c}2021 \\
\text { Utilization } \\
\text { Rate }\end{array}$ & $\begin{array}{c}2026 \\
\text { Projected }\end{array}$ & $\begin{array}{c}2026 \\
\text { Surplus } \\
\text { Seats }\end{array}$ & $\begin{array}{c}2026 \\
\text { Utilization } \\
\text { Rate }\end{array}$ & $\begin{array}{c}2031 \\
\text { Projected }\end{array}$ & $\begin{array}{l}2031 \\
\text { Surplus } \\
\text { Seats }\end{array}$ & $\begin{array}{c}2031 \\
\text { Utilization } \\
\text { Rate }\end{array}$ & $\begin{array}{c}2036 \\
\text { Projected }\end{array}$ & $\begin{array}{l}2036 \\
\text { Surplus } \\
\text { Seats }\end{array}$ & $\begin{array}{c}2036 \\
\text { Utilization } \\
\text { Rate }\end{array}$ \\
\hline General Mercer Junior Public School & $50.6 \%$ & $24.4 \%$ & 438 & 202 & 202.0 & $46 \%$ & 218 & 220 & $50 \%$ & 206 & 232 & $47 \%$ & 206 & 232 & $47 \%$ & 206 & 232 & $47 \%$ \\
\hline George Anderson Public School & $51.0 \%$ & $39.8 \%$ & 202 & 200 & 200.0 & $99 \%$ & 213 & -11 & $105 \%$ & 207 & -5 & $102 \%$ & 219 & -17 & $108 \%$ & 226 & -24 & $112 \%$ \\
\hline George B Little Public School & $68.2 \%$ & $57.3 \%$ & 522 & 432 & 432.0 & $83 \%$ & 353 & 169 & $68 \%$ & 312 & 210 & $60 \%$ & 308 & 214 & $59 \%$ & 303 & 219 & $58 \%$ \\
\hline George P Mackie Junior Public School & $62.7 \%$ & $48.8 \%$ & 176 & 139 & 139.0 & $79 \%$ & 167 & 9 & $95 \%$ & 182 & -6 & $103 \%$ & 181 & -5 & $103 \%$ & 181 & -5 & $103 \%$ \\
\hline George Peck Public School & $64.1 \%$ & $51.3 \%$ & 307 & 239 & 239.0 & $78 \%$ & 228 & 79 & $74 \%$ & 211 & 96 & $69 \%$ & 215 & 92 & $70 \%$ & 215 & 92 & $70 \%$ \\
\hline George R Gauld Junior School & $46.3 \%$ & $33.3 \%$ & 179 & 150 & 150.0 & $84 \%$ & 187 & -8 & $104 \%$ & 179 & 0 & $100 \%$ & 179 & 0 & $100 \%$ & 179 & 0 & $100 \%$ \\
\hline George Syme Community School & $66.0 \%$ & $52.0 \%$ & 626 & 392 & 392.0 & $63 \%$ & 433 & 193 & $69 \%$ & 433 & 193 & $69 \%$ & 433 & 193 & $69 \%$ & 433 & 193 & $69 \%$ \\
\hline George Webster Elementary School & $73.4 \%$ & $55.4 \%$ & 299 & 696 & 696.0 & $233 \%$ & 572 & -273 & $191 \%$ & 562 & -263 & $188 \%$ & 561 & -262 & $188 \%$ & 561 & -262 & $188 \%$ \\
\hline Givins/Shaw Junior Public School & $72.7 \%$ & $46.9 \%$ & 349 & 335 & 335.0 & $96 \%$ & 333 & 16 & $95 \%$ & 312 & 37 & $89 \%$ & 312 & 37 & $89 \%$ & 312 & 37 & $89 \%$ \\
\hline Glamorgan Junior Public School & $71.4 \%$ & $62.6 \%$ & 656 & 559 & 559.0 & $85 \%$ & 480 & 176 & $73 \%$ & 454 & 202 & $69 \%$ & 454 & 202 & $69 \%$ & 454 & 202 & $69 \%$ \\
\hline Gledhill Junior Public School & $69.5 \%$ & $54.0 \%$ & 562 & 521 & 521.0 & $93 \%$ & 498 & 64 & $89 \%$ & 501 & 61 & $89 \%$ & 501 & 61 & $89 \%$ & 501 & 61 & $89 \%$ \\
\hline Glen Ames Senior Public School & $90.5 \%$ & $70.9 \%$ & 354 & 471 & 471.0 & $133 \%$ & 463 & -109 & $131 \%$ & 473 & -119 & $134 \%$ & 472 & -118 & $133 \%$ & 472 & -118 & $133 \%$ \\
\hline Glen Park Public School & $22.6 \%$ & $15.3 \%$ & 525 & 487 & 487.0 & $93 \%$ & 505 & 20 & $96 \%$ & 514 & 11 & $98 \%$ & 513 & 12 & $98 \%$ & 513 & 12 & $98 \%$ \\
\hline Glen Ravine Junior Public School & $57.4 \%$ & $49.0 \%$ & 337 & 302 & 302.0 & $90 \%$ & 262 & 75 & $78 \%$ & 255 & 82 & $76 \%$ & 255 & 82 & $76 \%$ & 255 & 82 & $76 \%$ \\
\hline Glenview Senior Public School & $82.8 \%$ & $70.7 \%$ & 619 & 737 & 737.0 & $119 \%$ & 772 & -153 & $125 \%$ & 746 & -127 & $121 \%$ & 764 & -145 & $123 \%$ & 763 & -144 & $123 \%$ \\
\hline Golf Road Junior Public School & $70.1 \%$ & $63.0 \%$ & 376 & 316 & 316.0 & $84 \%$ & 287 & 89 & $76 \%$ & 305 & 71 & $81 \%$ & 305 & 71 & $81 \%$ & 305 & 71 & $81 \%$ \\
\hline Gordon A Brown Middle School & $82.5 \%$ & $54.7 \%$ & 418 & 359 & 359.0 & $86 \%$ & 357 & 61 & $85 \%$ & 359 & 59 & $86 \%$ & 357 & 61 & $85 \%$ & 357 & 61 & $85 \%$ \\
\hline Gosford Public School & $63.5 \%$ & $50.0 \%$ & 349 & 279 & 279.0 & $80 \%$ & 249 & 100 & $71 \%$ & 252 & 97 & $72 \%$ & 252 & 97 & $72 \%$ & 252 & 97 & $72 \%$ \\
\hline
\end{tabular}




\begin{tabular}{|c|c|c|c|c|c|c|c|c|c|c|c|c|c|c|c|c|c|c|c|c|c|c|}
\hline \multirow[b]{2}{*}{ School Name } & \multicolumn{8}{|c|}{ General Information } & \multicolumn{14}{|c|}{ Facility Information } \\
\hline & Panel & $\begin{array}{l}\text { Grade } \\
\text { Range }\end{array}$ & $\begin{array}{c}\text { Semestered } \\
\text { (Sec) }\end{array}$ & Ward & Trustee & $\begin{array}{l}\text { Superintendent } \\
\text { of Education }\end{array}$ & 2017 LOI & $\begin{array}{c}2017-18 \\
\text { Opt } \\
\text { Attend } \\
\text { Reg/FI/EF }\end{array}$ & $\begin{array}{l}\text { Site Size } \\
\text { (Acres) }\end{array}$ & $\begin{array}{c}\text { Facility Size } \\
\text { (Sq. Ft.) }\end{array}$ & $\mid \begin{array}{c}\text { Current } \\
\text { Backlog (M) }\end{array}$ & $\begin{array}{l}\text { Port. } \\
\text { (Instruc) }\end{array}$ & $\begin{array}{c}\begin{array}{c}\text { Port. (Non- } \\
\text { Instruc) }\end{array} \\
\end{array}$ & $\begin{array}{c}\text { Current FCI } \\
\text { (EDU) }\end{array}$ & $\begin{array}{c}\text { Eco } \\
\text { Schools }\end{array}$ & Pool & $\begin{array}{l}\text { Elem. } \\
\text { D\&T } \\
\text { Shops }\end{array}$ & $\begin{array}{l}\text { Barrier } \\
\text { Free / } \\
\text { Access }\end{array}$ & Elevator & $\begin{array}{l}\text { Build } \\
\text { Date }\end{array}$ & $\begin{array}{c}\text { Building } \\
\text { Age }\end{array}$ & $\begin{array}{l}\text { T.o Comm } \\
\text { Centre }\end{array}$ \\
\hline Gracedale Public School & $\mathrm{E}$ & JK-5 & & 4 & Tiffany Ford & Audley Salmon & 147 & $\begin{array}{c}\text { Closed } \\
\text { N/A } \\
\text { N/A }\end{array}$ & 5.02 & 72,399 & $\$ 8.1$ & & 1 & $54 \%$ & Platinum & & & & & 1964 & 53 & \\
\hline Gracefield Public School & E & JK-5 & & 6 & Chris Tonks & $\begin{array}{l}\text { Angela Nardi- } \\
\text { Addesa }\end{array}$ & 146 & $\begin{array}{c}\text { Closed } \\
\text { N/A } \\
\text { N/A }\end{array}$ & 5.58 & 23,509 & $\$ 2.5$ & 2 & 1 & $43 \%$ & & & & & & 1955 & 62 & \\
\hline Greenholme Junior Middle School & $\mathrm{E}$ & JK-8 & & 1 & Avtar Minhas & Annie Appleby & 13 & $\begin{array}{c}\text { Limited } \\
N / A \\
\text { Limited }\end{array}$ & 11.07 & 73,529 & $\$ 3.6$ & & & $24 \%$ & Silver & & & & & 1966 & 51 & \\
\hline Greenland Public School & E & JK-5 & & 17 & Ken Lister & Beth Veale & 326 & $\begin{array}{c}\text { Closed } \\
\text { N/A } \\
\text { N/A }\end{array}$ & 7.54 & 28,315 & \$3. & & 1 & $56 \%$ & \begin{tabular}{|l|} 
Platinum \\
\end{tabular} & & & & & 1955 & 62 & \\
\hline Grenoble Public School & $\mathrm{E}$ & $\mathrm{JK}-6$ & & 13 & Gerri Gershon & Ian Allison & 76 & $\begin{array}{c}\text { Closed } \\
\text { N/A } \\
\text { Closed }\end{array}$ & 4.5 & 66,209 & $\$ 9.5$ & 13 & 3 & $68 \%$ & & & & A1 & Yes & 1960 & 57 & \\
\hline Grey Owl Junior Public School & E & $\mathrm{JK}-6$ & & 21 & Abdul Hai Patel & Andrew Howard & 70 & $\begin{array}{c}\text { Limited } \\
\text { N/A } \\
\text { N/A }\end{array}$ & 4.99 & 32,645 & $\$ 1.3$ & & & $18 \%$ & & & & & & 1975 & 42 & \\
\hline Guildwood Junior Public School & $\mathrm{E}$ & JK-6 & & 22 & Jerry Chadwick & $\begin{array}{l}\text { Kerry-Lynn } \\
\text { Stadnyk }\end{array}$ & 167 & $\begin{array}{c}\text { Limited } \\
\text { N/A } \\
\text { N/A }\end{array}$ & 6.03 & 29,900 & $\$ 4.3$ & & & $72 \%$ & Platinum & & & & & 1958 & 59 & \\
\hline Gulfstream Public School & E & JK-8 & & 4 & Tiffany Ford & Audley Salmon & 100 & $\begin{array}{c}\text { Closed } \\
\text { N/A } \\
\text { N/A }\end{array}$ & 5.31 & 59,762 & $\$ 3.9$ & 1 & & $33 \%$ & & & & & & 1958 & 59 & \\
\hline H A Halbert Junior Public School & $\mathrm{E}$ & $\mathrm{JK}-6$ & & 18 & Parthi Kandavel & $\begin{array}{l}\text { Jacqueline } \\
\text { Spence }\end{array}$ & 149 & $\begin{array}{c}\text { Limited } \\
\text { N/A } \\
\text { N/A }\end{array}$ & 4.3 & 31,608 & $\$ 2.9$ & & & $48 \%$ & \begin{tabular}{|l|} 
Platinum \\
\end{tabular} & & & A1 & No & 1950 & 67 & \\
\hline H J Alexander Community School & E & JK-5 & & 6 & Chris Tonks & $\begin{array}{l}\text { Angela Nardi- } \\
\text { Addesa }\end{array}$ & 8 & $\begin{array}{c}\text { Closed } \\
\text { N/A } \\
\text { N/A }\end{array}$ & 3.14 & 87,468 & $\$ 3.8$ & 6 & & $33 \%$ & Bronze & & & A1 & & 1995 & 22 & \\
\hline Harrison Public School & E & $\mathrm{JK}-6$ & & 13 & Gerri Gershon & Kathleen Garner & 431 & $\begin{array}{c}\text { Closed } \\
\text { N/A } \\
\text { N/A }\end{array}$ & 4.99 & 21,883 & $\$ 2.9$ & 2 & & $54 \%$ & Gold & & & & & 1951 & 66 & \\
\hline Harwood Public School & E & JK-8 & & 6 & Chris Tonks & Vicky Branco & 103 & $\begin{array}{c}\text { Limited } \\
\text { N/A } \\
\text { N/A }\end{array}$ & 3.01 & 33,424 & $\$ 5.3$ & & & $68 \%$ & & & & & & 1926 & 91 & \\
\hline $\begin{array}{l}\text { Hawthorne II Bilingual Alternative } \\
\text { Junior School }\end{array}$ & $\mathrm{E}$ & $\mathrm{JK}-6$ & & 10 & Ausma Malik & Mike Gallagher & 355 & $\begin{array}{c}\text { Limited } \\
\text { N/A } \\
\text { Limited }\end{array}$ & & & & & & & Platinum & & & & Yes & & & \\
\hline Heather Heights Junior Public School & $\mathrm{E}$ & $\mathrm{JK}-6$ & & 22 & Jerry Chadwick & Nadira Persaud & 126 & $\begin{array}{c}\text { Limited } \\
\text { N/A } \\
\text { N/A }\end{array}$ & 5.29 & 48,975 & $\$ 1.9$ & & & $19 \%$ & & & & & & 1959 & 58 & \\
\hline Henry Hudson Senior Public School & $\mathrm{E}$ & $7-8$ & & 22 & Jerry Chadwick & Nadira Persaud & 139 & $\begin{array}{c}\text { Limited } \\
\text { N/A } \\
\text { Limited }\end{array}$ & 4.6 & 78,235 & $\$ 4.8$ & & & $44 \%$ & & & Yes & & Yes & 1972 & 45 & \\
\hline Henry Kelsey Senior Public School & E & $7-8$ & & 21 & Abdul Hai Patel & $\begin{array}{l}\text { Jacqueline } \\
\text { Spence }\end{array}$ & 276 & $\begin{array}{c}\text { Limited } \\
\text { N/A } \\
\text { N/A }\end{array}$ & 4.03 & 72,614 & $\$ 7.8$ & & & $69 \%$ & Silver & & Yes & & & 1971 & 46 & \\
\hline Heritage Park Public School & $\mathrm{E}$ & $\mathrm{JK}-8$ & & 21 & Abdul Hai Patel & Andrew Howard & 235 & $\begin{array}{c}\text { Limited } \\
\text { N/A } \\
\text { N/A }\end{array}$ & 5.04 & 55,013 & $\$ 1.5$ & & & $16 \%$ & Platinum & & Yes & A1 & Yes & 1994 & 23 & \\
\hline High Park Alternative Junior School & E & JK-8 & & 7 & Robin Pilkey & Tracy Hayhurst & 399 & \begin{tabular}{c|} 
Limited \\
N/A \\
N/A
\end{tabular} & & & & & & & Gold & & & & & & & \\
\hline
\end{tabular}




\begin{tabular}{|c|c|c|c|c|c|c|c|c|c|c|c|c|c|c|c|c|c|c|}
\hline & Participa & tion Rates & & & & & Enrolment a & Projectio & FTE, Surplus & eats $=$ OTG R & ised - Proj & tion FTE, Utili & itions $=$ Proj & ion/OTG I & vised & & & \\
\hline School Name & $\begin{array}{c}\text { TDSB 2013-14 } \\
\text { TDSB Participation } \\
\text { Rate }\end{array}$ & $\begin{array}{c}\text { TOSB 2013-14 } \\
\text { Home school } \\
\text { Participation Rate }\end{array}$ & $\begin{array}{c}\text { Revised } \\
\text { Capacity } \\
2016\end{array}$ & $\begin{array}{c}\text { HC } \\
\text { (Oct. 2016) }\end{array}$ & $\begin{array}{c}\text { FTE } \\
\text { (Oct. 2016) }\end{array}$ & $\begin{array}{c}2016 \\
\text { Utilization } \\
\text { Rate }\end{array}$ & $\begin{array}{c}2021 \\
\text { Projected }\end{array}$ & $\begin{array}{l}2021 \\
\text { Surplus } \\
\text { Seats }\end{array}$ & $\begin{array}{c}2021 \\
\text { Utilization } \\
\text { Rate }\end{array}$ & $\begin{array}{c}2026 \\
\text { Projected }\end{array}$ & $\begin{array}{c}2026 \\
\text { Surplus } \\
\text { Seats }\end{array}$ & $\begin{array}{c}2026 \\
\text { Utilization } \\
\text { Rate }\end{array}$ & $\begin{array}{c}2031 \\
\text { Projected }\end{array}$ & $\begin{array}{l}2031 \\
\text { Surplus } \\
\text { Seats }\end{array}$ & $\begin{array}{c}2031 \\
\text { Utilization } \\
\text { Rate }\end{array}$ & $\begin{array}{c}2036 \\
\text { Projected }\end{array}$ & $\begin{array}{l}2036 \\
\text { Surplus } \\
\text { Seats }\end{array}$ & $\begin{array}{c}2036 \\
\text { Utilization } \\
\text { Rate }\end{array}$ \\
\hline Gracedale Public School & $58.7 \%$ & $53.0 \%$ & 737 & 670 & 670.0 & $91 \%$ & 620 & 117 & $84 \%$ & 620 & 117 & $84 \%$ & 620 & 117 & $84 \%$ & 620 & 117 & $84 \%$ \\
\hline Gracefield Public School & $36.5 \%$ & $26.4 \%$ & 208 & 211 & 211.0 & $101 \%$ & 272 & -64 & $131 \%$ & 277 & -69 & $133 \%$ & 284 & -76 & $137 \%$ & 291 & -83 & $140 \%$ \\
\hline Greenholme Junior Middle School & $68.0 \%$ & $47.2 \%$ & 742 & 325 & 325.0 & $44 \%$ & 277 & 465 & $37 \%$ & 255 & 487 & $34 \%$ & 256 & 486 & $35 \%$ & 256 & 486 & $35 \%$ \\
\hline Greenland Public School & & & 222 & 162 & 162.0 & $73 \%$ & 249 & -27 & $112 \%$ & 235 & -13 & $106 \%$ & 234 & -12 & $105 \%$ & 234 & -12 & $105 \%$ \\
\hline Grenoble Public School & $80.3 \%$ & $71.1 \%$ & 758 & 904 & 904.0 & $119 \%$ & 833 & -75 & $110 \%$ & 838 & -80 & $111 \%$ & 839 & -81 & $111 \%$ & 839 & -81 & $111 \%$ \\
\hline Grey OwI Junior Public School & $67.7 \%$ & $50.7 \%$ & 332 & 253 & 253.0 & $76 \%$ & 233 & 99 & $70 \%$ & 240 & 92 & $72 \%$ & 240 & 92 & $72 \%$ & 240 & 92 & $72 \%$ \\
\hline Guildwood Junior Public School & $67.1 \%$ & $50.7 \%$ & 242 & 116 & 116.0 & $48 \%$ & 106 & 136 & $44 \%$ & 122 & 120 & $50 \%$ & 127 & 115 & $52 \%$ & 127 & 115 & $52 \%$ \\
\hline Gulfstream Public School & $41.8 \%$ & $35.7 \%$ & 541 & 575 & 575.0 & $106 \%$ & 572 & -31 & $106 \%$ & 568 & -27 & $105 \%$ & 560 & -19 & $104 \%$ & 560 & -19 & $104 \%$ \\
\hline H A Halbert Junior Public School & $63.5 \%$ & $42.6 \%$ & 272 & 235 & 235.0 & $86 \%$ & 224 & 48 & $82 \%$ & 204 & 68 & $75 \%$ & 200 & 72 & $74 \%$ & 202 & 70 & $74 \%$ \\
\hline H J Alexander Community School & $76.0 \%$ & $66.3 \%$ & 579 & 589 & 589.0 & $102 \%$ & 600 & -21 & $104 \%$ & 587 & -8 & $101 \%$ & 586 & -7 & $101 \%$ & 586 & -7 & $101 \%$ \\
\hline Harrison Public School & $80.9 \%$ & $75.0 \%$ & 179 & 207 & 207.0 & $116 \%$ & 211 & -32 & $118 \%$ & 225 & -46 & $126 \%$ & 226 & -47 & $126 \%$ & 226 & -47 & $126 \%$ \\
\hline Harwood Public School & $56.1 \%$ & $25.9 \%$ & 337 & 155 & 155.0 & $46 \%$ & 115 & 222 & $34 \%$ & 108 & 229 & $32 \%$ & 109 & 228 & $32 \%$ & 109 & 228 & $32 \%$ \\
\hline $\begin{array}{l}\text { Hawthorne II Bilingual Alternative } \\
\text { Junior School }\end{array}$ & & & 219 & 200 & 200.0 & $91 \%$ & 204 & 15 & $93 \%$ & 203 & 16 & $93 \%$ & 203 & 16 & $93 \%$ & 203 & 16 & $93 \%$ \\
\hline Heather Heights Junior Public School & $67.0 \%$ & $55.9 \%$ & 310 & 166 & 166.0 & $54 \%$ & 157 & 153 & $51 \%$ & 154 & 156 & $50 \%$ & 154 & 156 & $50 \%$ & 154 & 156 & $50 \%$ \\
\hline Henry Hudson Senior Public School & $76.4 \%$ & $65.2 \%$ & 506 & 310 & 310.0 & $61 \%$ & 265 & 241 & $52 \%$ & 234 & 272 & $46 \%$ & 236 & 270 & $47 \%$ & 236 & 270 & $47 \%$ \\
\hline Henry Kelsey Senior Public School & $75.7 \%$ & $53.9 \%$ & 547 & 297 & 297.0 & $54 \%$ & 339 & 208 & $62 \%$ & 326 & 221 & $60 \%$ & 322 & 225 & $59 \%$ & 322 & 225 & $59 \%$ \\
\hline Heritage Park Public School & $71.7 \%$ & $65.2 \%$ & 438 & 307 & 307.0 & $70 \%$ & 315 & 123 & $72 \%$ & 290 & 148 & $66 \%$ & 289 & 149 & $66 \%$ & 289 & 149 & $66 \%$ \\
\hline High Park Alternative Junior School & & & 190 & 149 & 149.0 & $78 \%$ & 160 & 30 & $84 \%$ & 160 & 30 & $84 \%$ & 160 & 30 & $84 \%$ & 160 & 30 & $84 \%$ \\
\hline
\end{tabular}




\begin{tabular}{|c|c|c|c|c|c|c|c|c|c|c|c|c|c|c|c|c|c|c|c|c|c|c|}
\hline \multirow[b]{2}{*}{ School Name } & \multicolumn{8}{|c|}{ General Information } & \multicolumn{14}{|c|}{ Facility Information } \\
\hline & Panel & $\begin{array}{l}\text { Grade } \\
\text { Range }\end{array}$ & $\begin{array}{c}\text { Semestered } \\
(\mathrm{Sec})\end{array}$ & Ward & Trustee & $\begin{array}{l}\text { Superintendent } \\
\text { of Education }\end{array}$ & 2017 LOI & $\begin{array}{c}2017-18 \\
\mathrm{Opt} \\
\text { Attend } \\
\mathrm{Reg} / \mathrm{F} / \mathrm{EF}\end{array}$ & $\begin{array}{l}\text { Site Size } \\
\text { (Acres) }\end{array}$ & $\begin{array}{c}\text { Facility Size } \\
\text { (Sq. Ft.) }\end{array}$ & $\mid \begin{array}{c}\text { Current } \\
\text { Backlog (M) }\end{array}$ & $\begin{array}{l}\text { Port. } \\
\text { (Instruc) }\end{array}$ & \begin{tabular}{|c|} 
Port. (Non- \\
Instruc)
\end{tabular} & $\begin{array}{c}\text { Current FCI } \\
\text { (EDU) }\end{array}$ & $\begin{array}{c}\text { Eco } \\
\text { Schools }\end{array}$ & Pool & $\begin{array}{l}\text { Elem. } \\
\text { D\&T } \\
\text { Shops }\end{array}$ & $\begin{array}{l}\text { Barrier } \\
\text { Free / } \\
\text { Access }\end{array}$ & Elevator & $\begin{array}{l}\text { Build } \\
\text { Date }\end{array}$ & $\begin{array}{c}\text { Building } \\
\text { Age }\end{array}$ & $\begin{array}{c}\text { T.O Comm } \\
\text { Centre }\end{array}$ \\
\hline Highcastle Public School & E & JK-8 & & 22 & Jerry Chadwick & Nadira Persaud & 200 & $\begin{array}{c}\text { Closed } \\
\text { N/A } \\
\text { N/A }\end{array}$ & 5.83 & 38,846 & $\$ 8.1$ & & & $114 \%$ & & & & & & 1966 & 51 & \\
\hline Highfield Junior School & E & JK-5 & & 1 & Avtar Minhas & Glenford Duffus & 158 & $\begin{array}{c}\text { Limited } \\
\text { N/A } \\
\text { N/A }\end{array}$ & 6 & 100,312 & $\$ 11.7$ & & 3 & $59 \%$ & Platinum & & & A1 & & 1964 & 53 & \\
\hline Highland Creek Public School & $\mathrm{E}$ & JK-8 & & 22 & Jerry Chadwick & Nadira Persaud & 266 & $\begin{array}{c}\text { Limited } \\
\text { N/A } \\
\text { N/A }\end{array}$ & 4.97 & 31,479 & $\$ 2.8$ & & & $44 \%$ & & & & & & 1908 & 109 & \\
\hline Highland Heights Junior Public School & E & $\mathrm{JK}-6$ & & 20 & Manna Wong & Lynn Strangway & 43 & $\begin{array}{c}\text { Limited } \\
\text { N/A } \\
\text { N/A }\end{array}$ & 6 & 50,276 & $\$ 6.1$ & & & $51 \%$ & & & & & & 1967 & 50 & \\
\hline Highland Middle School & E & 6-8 & & 12 & Alexander Brown & Elizabeth Addo & 357 & $\begin{array}{c}\text { Limited } \\
\text { N/A } \\
\text { N/A }\end{array}$ & 8.06 & 78,405 & $\$ 2.8$ & & & $24 \%$ & & & Yes & & & 1975 & 42 & \\
\hline Highview Public School & E & JK-5 & & 5 & Alexandra Lulka & Linda Curtis & 38 & \begin{tabular}{c|} 
Limited \\
N/A \\
N/A
\end{tabular} & 4.1 & 27,179 & $\$ 5.2$ & & & $91 \%$ & Gold & & & & & 1956 & 61 & \\
\hline Hillcrest Community School & E & JK-6 & & 11 & Shelley Laskin & Kathleen Garner & 402 & $\begin{array}{c}\text { Closed } \\
\text { N/A } \\
\text { N/A }\end{array}$ & 2.37 & 69,818 & $\$ 2.8$ & & & $31 \%$ & Silver & City & & & & 1905 & 112 & Cat. 1 \\
\hline Hillmount Public School & E & JK-5 & & 12 & Alexander Brown & Elizabeth Addo & 378 & $\begin{array}{c}\text { Limited } \\
\text { N/A } \\
\text { N/A }\end{array}$ & 7.17 & 34,273 & $\$ 5.2$ & & & $88 \%$ & & & & & & 1970 & 47 & \\
\hline Hilltop Middle School & $\mathrm{E}$ & 6-8 & & 2 & Chris Glover & Annie Appleby & 141 & $\begin{array}{l}\text { Closed } \\
\text { Closed } \\
\text { N/A }\end{array}$ & 6.3 & 88,773 & $\$ 6.8$ & & & $55 \%$ & Gold & & Yes & & & 1958 & 59 & Cat. 1 \\
\hline Hodgson Senior Public School & E & 7-8 & & 11 & Shelley Laskin & Ian Allison & 432 & $\begin{array}{l}\text { Closed } \\
\text { N/A } \\
\text { Closed }\end{array}$ & 4.18 & 64,796 & $\$ 3.5$ & & & $43 \%$ & Bronze & & Yes & & & 1914 & 103 & \\
\hline Hollycrest Middle School & E & 6-8 & & 2 & Chris Glover & Glenford Duffus & 372 & $\begin{array}{c}\text { Limited } \\
\text { N/A } \\
\text { N/A }\end{array}$ & 16.75 & 60,346 & $\$ 7.8$ & & & $88 \%$ & & & Yes & & & 1966 & 51 & Cat. 1 \\
\hline Hollywood Public School & E & JK-5 & & 12 & Alexander Brown & Linda Curtis & 366 & $\begin{array}{c}\text { Closed } \\
\text { Limited } \\
\text { N/A }\end{array}$ & 6.7 & 31,269 & $\$ 1.3$ & 4 & & $21 \%$ & Platinum & & & & & 1949 & 68 & \\
\hline Horizon Alternative Senior School & E & $7-8$ & & 10 & Ausma Malik & Mike Gallagher & 413 & $\begin{array}{c}\text { Limited } \\
\text { N/A } \\
\text { N/A }\end{array}$ & & & & & & & & & & & & & & \\
\hline Howard Junior Public School & E & $\mathrm{JK}-6$ & & 7 & Robin Pilkey & $\begin{array}{l}\text { Jane Phillips- } \\
\text { Long }\end{array}$ & 423 & $\begin{array}{c}\text { Limited } \\
\text { Closed } \\
\text { N/A }\end{array}$ & 2.87 & 57,894 & $\$ 6.5$ & & & $58 \%$ & Gold & & & & & 1970 & 47 & \\
\hline Humber Summit Middle School & E & 6-8 & & 4 & Tiffany Ford & Audley Salmon & 95 & $\begin{array}{c}\text { Limited } \\
\text { N/A } \\
\text { N/A }\end{array}$ & 7.31 & 82,506 & $\$ 4.4$ & & 2 & $38 \%$ & & & & A1 & Yes & 1968 & 49 & \\
\hline $\begin{array}{l}\text { Humber Valley Village Junior Middle } \\
\text { School }\end{array}$ & E & JK-8 & & 2 & Chris Glover & Annie Appleby & 456 & $\begin{array}{c}\text { Limited } \\
\text { N/A } \\
\text { N/A }\end{array}$ & 8.18 & 58,330 & $\$ 2.5$ & & & $27 \%$ & & & & & & 1952 & 65 & \\
\hline Humbercrest Public School & E & JK-8 & & 7 & Robin Pilkey & Tracy Hayhurst & 403 & $\begin{array}{c}\text { Closed } \\
\text { Closed } \\
\text { N/A }\end{array}$ & 2.47 & 83,160 & $\$ 3.3$ & & & $23 \%$ & Gold & & & & & 1915 & 102 & \\
\hline $\begin{array}{l}\text { Humberwood Downs Junior Middle } \\
\text { Academy }\end{array}$ & E & JK-8 & & 1 & Avtar Minhas & Annie Appleby & 243 & $\begin{array}{c}\text { Limited } \\
\text { N/A } \\
\text { N/A }\end{array}$ & 11.49 & 118,000 & $\$ .4$ & & & $1 \%$ & & & Yes & A1 & & 1997 & 20 & \\
\hline
\end{tabular}




\begin{tabular}{|c|c|c|c|c|c|c|c|c|c|c|c|c|c|c|c|c|c|c|}
\hline & Participa & ition Rates & & & & & Enrolment a & Projection & FTE, Surplus & eats = OTG R & ised - Proj & tion FTE, Util & itions $=$ Proj & ion/OTG R & vised & & & \\
\hline School Name & $\begin{array}{c}\text { TDSB 2013-14 } \\
\text { TDSB Participation } \\
\text { Rate }\end{array}$ & $\begin{array}{c}\text { TDSB 2013-14 } \\
\text { Home school } \\
\text { Participation Rate }\end{array}$ & $\begin{array}{c}\text { Revised } \\
\text { Capacity } \\
2016\end{array}$ & $\begin{array}{c}\text { HC } \\
\text { (Oct. 2016) }\end{array}$ & $\begin{array}{c}\text { FTE } \\
\text { (Oct. 2016) }\end{array}$ & $\begin{array}{c}2016 \\
\text { Utilization } \\
\text { Rate }\end{array}$ & $\begin{array}{c}2021 \\
\text { Projected }\end{array}$ & $\begin{array}{l}2021 \\
\text { Surplus } \\
\text { Seats }\end{array}$ & $\begin{array}{c}2021 \\
\text { Utilization } \\
\text { Rate }\end{array}$ & $\begin{array}{c}2026 \\
\text { Projected }\end{array}$ & $\begin{array}{c}2026 \\
\text { Surplus } \\
\text { Seats }\end{array}$ & $\begin{array}{c}2026 \\
\text { Utilization } \\
\text { Rate }\end{array}$ & $\begin{array}{c}2031 \\
\text { Projected }\end{array}$ & $\begin{array}{l}2031 \\
\text { Surplus } \\
\text { Seats }\end{array}$ & $\begin{array}{c}2031 \\
\text { Utilization } \\
\text { Rate }\end{array}$ & $\begin{array}{c}2036 \\
\text { Projected }\end{array}$ & $\begin{array}{l}2036 \\
\text { Surplus } \\
\text { Seats }\end{array}$ & $\begin{array}{c}2036 \\
\text { Utilization } \\
\text { Rate }\end{array}$ \\
\hline Highcastle Public School & $50.7 \%$ & $40.6 \%$ & 323 & 331 & 330.0 & $102 \%$ & 345 & -22 & $107 \%$ & 364 & -41 & $113 \%$ & 366 & -43 & $113 \%$ & 366 & -43 & $113 \%$ \\
\hline Highfield Junior School & $61.1 \%$ & $55.5 \%$ & 1003 & 694 & 694.0 & $69 \%$ & 610 & 393 & $61 \%$ & 615 & 388 & $61 \%$ & 615 & 388 & $61 \%$ & 615 & 388 & $61 \%$ \\
\hline Highland Creek Public School & $64.4 \%$ & $44.8 \%$ & 260 & 146 & 146.0 & $56 \%$ & 124 & 136 & $48 \%$ & 114 & 146 & $44 \%$ & 115 & 145 & $44 \%$ & 115 & 145 & $44 \%$ \\
\hline Highland Heights Junior Public School & $58.4 \%$ & $37.9 \%$ & 262 & 161 & 161.0 & $61 \%$ & 208 & 54 & $79 \%$ & 205 & 57 & $78 \%$ & 205 & 57 & $78 \%$ & 205 & 57 & $78 \%$ \\
\hline Highland Middle School & $84.3 \%$ & $60.4 \%$ & 458 & 389 & 389.0 & $85 \%$ & 431 & 27 & $94 \%$ & 442 & 16 & $97 \%$ & 436 & 22 & $95 \%$ & 436 & 22 & $95 \%$ \\
\hline Highview Public School & $94.1 \%$ & $64.8 \%$ & 194 & 164 & 164.0 & $85 \%$ & 164 & 30 & $85 \%$ & 163 & 31 & $84 \%$ & 163 & 31 & $84 \%$ & 163 & 31 & $84 \%$ \\
\hline Hillcrest Community School & $73.0 \%$ & $54.2 \%$ & 424 & 368 & 368.0 & $87 \%$ & 362 & 62 & $85 \%$ & 389 & 35 & $92 \%$ & 389 & 35 & $92 \%$ & 389 & 35 & $92 \%$ \\
\hline Hillmount Public School & $109.2 \%$ & $97.7 \%$ & 246 & 235 & 235.0 & $96 \%$ & 237 & 9 & $96 \%$ & 245 & 1 & $100 \%$ & 245 & 1 & $100 \%$ & 245 & 1 & $100 \%$ \\
\hline Hilltop Middle School & $36.9 \%$ & $29.4 \%$ & 614 & 547 & 547.0 & $89 \%$ & 455 & 159 & $74 \%$ & 428 & 186 & $70 \%$ & 432 & 182 & $70 \%$ & 432 & 182 & $70 \%$ \\
\hline Hodgson Senior Public School & $73.9 \%$ & $50.8 \%$ & 367 & 336 & 336.0 & $92 \%$ & 691 & -324 & $188 \%$ & 714 & -347 & $195 \%$ & 718 & -351 & $196 \%$ & 718 & -351 & $196 \%$ \\
\hline Hollycrest Middle School & $64.7 \%$ & $52.4 \%$ & 404 & 422 & 422.0 & $104 \%$ & 377 & 27 & $93 \%$ & 377 & 27 & $93 \%$ & 377 & 27 & $93 \%$ & 377 & 27 & $93 \%$ \\
\hline Hollywood Public School & $66.6 \%$ & $44.8 \%$ & 303 & 411 & 411.0 & $136 \%$ & 308 & -5 & $102 \%$ & 308 & -5 & $102 \%$ & 308 & -5 & $102 \%$ & 308 & -5 & $102 \%$ \\
\hline Horizon Alternative Senior School & & & 92 & 72 & 72.0 & $78 \%$ & 71 & 21 & $77 \%$ & 71 & 21 & $77 \%$ & 71 & 21 & $77 \%$ & 71 & 21 & $77 \%$ \\
\hline Howard Junior Public School & $84.1 \%$ & $72.7 \%$ & 577 & 549 & 549.0 & $95 \%$ & 508 & 69 & $88 \%$ & 509 & 68 & $88 \%$ & 509 & 68 & $88 \%$ & 509 & 68 & $88 \%$ \\
\hline Humber Summit Middle School & $57.3 \%$ & $51.2 \%$ & 588 & 479 & 479.0 & $81 \%$ & 439 & 149 & $75 \%$ & 393 & 195 & $67 \%$ & 400 & 188 & $68 \%$ & 400 & 188 & $68 \%$ \\
\hline $\begin{array}{l}\text { Humber Valley Village Junior Middle } \\
\text { School }\end{array}$ & $65.3 \%$ & $52.2 \%$ & 475 & 351 & 351.0 & $74 \%$ & 423 & 52 & $89 \%$ & 445 & 30 & $94 \%$ & 456 & 19 & $96 \%$ & 456 & 19 & $96 \%$ \\
\hline Humbercrest Public School & $74.2 \%$ & $61.1 \%$ & 819 & 717 & 717.0 & $88 \%$ & 694 & 125 & $85 \%$ & 690 & 129 & $84 \%$ & 683 & 136 & $83 \%$ & 683 & 136 & $83 \%$ \\
\hline $\begin{array}{l}\text { Humberwood Downs Junior Middle } \\
\text { Academy }\end{array}$ & $92.6 \%$ & $86.6 \%$ & 1048 & 889 & 889.0 & $85 \%$ & 781 & 267 & $75 \%$ & 744 & 304 & $71 \%$ & 760 & 288 & $73 \%$ & 760 & 288 & $73 \%$ \\
\hline
\end{tabular}




\begin{tabular}{|c|c|c|c|c|c|c|c|c|c|c|c|c|c|c|c|c|c|c|c|c|c|c|}
\hline \multirow[b]{2}{*}{ School Name } & \multicolumn{8}{|c|}{ General Information } & \multicolumn{14}{|c|}{ Facility Information } \\
\hline & Panel & $\begin{array}{l}\text { Grade } \\
\text { Range }\end{array}$ & $\begin{array}{c}\text { Semestered } \\
(\mathrm{Sec})\end{array}$ & Ward & Trustee & $\begin{array}{c}\text { Superintendent } \\
\text { of Education }\end{array}$ & 2017 LOI & $\begin{array}{c}2017-18 \\
\text { Opt } \\
\text { Attend } \\
\text { Reg/FI/EF }\end{array}$ & $\begin{array}{l}\text { Site Size } \\
\text { (Acres) }\end{array}$ & $\begin{array}{c}\text { Facility Size } \\
\text { (Sq. Ft.) }\end{array}$ & $\mid \begin{array}{c}\text { Current } \\
\text { Backlog (M) }\end{array}$ & $\begin{array}{l}\text { Port. } \\
\text { (Instruc) }\end{array}$ & $\begin{array}{c}\text { Port. (Non- } \\
\text { Instruc) }\end{array}$ & $\begin{array}{c}\text { Current FCI } \\
\text { (EDU) }\end{array}$ & $\begin{array}{l}\text { Eco } \\
\text { Schools }\end{array}$ & Pool & $\begin{array}{l}\text { Elem. } \\
\text { D\&T } \\
\text { Shops }\end{array}$ & $\begin{array}{l}\text { Barrier } \\
\text { Free / } \\
\text { Access }\end{array}$ & Elevator & $\begin{array}{l}\text { Build } \\
\text { Date }\end{array}$ & $\begin{array}{c}\text { Building } \\
\text { Age }\end{array}$ & $\begin{array}{c}\text { T.o Comm } \\
\text { Centre }\end{array}$ \\
\hline Humewood Community School & $\mathrm{E}$ & $\mathrm{JK}-8$ & & 11 & Shelley Laskin & Kathleen Garner & 409 & $\begin{array}{c}\text { Closed } \\
\text { Closed } \\
\text { N/A }\end{array}$ & 3.83 & 64,676 & $\$ 4.4$ & & & $39 \%$ & Gold & & & A1 & Yes & 1972 & 45 & \\
\hline Hunter's Glen Junior Public School & E & JK-6 & & 19 & David Smith & Shirley Chan & 153 & $\begin{array}{c}\text { Closed } \\
\text { N/A } \\
\text { Limited } \\
\end{array}$ & 5.88 & 39,361 & $\$ 2.3$ & 1 & & $27 \%$ & Platinum & & & & & 1956 & 61 & \\
\hline Huron Street Junior Public School & $\mathrm{E}$ & $\mathrm{JK}-6$ & & 10 & Ausma Malik & Mike Gallagher & 345 & $\begin{array}{c}\text { Limited } \\
\text { N/A } \\
\text { N/A }\end{array}$ & 2.55 & 52,030 & $\$ 8.5$ & & & $78 \%$ & Platinum & & & & & 1914 & 103 & \\
\hline $\begin{array}{l}\text { Indian Road Crescent Junior Public } \\
\text { School }\end{array}$ & E & $\mathrm{JK}-6$ & & 7 & Robin Pilkey & Tracy Hayhurst & 383 & $\begin{array}{c}\text { Closed } \\
\text { N/A } \\
\text { N/A }\end{array}$ & 2.77 & 41,160 & $\$ 1$. & & 2 & $13 \%$ & & & & & & 1964 & 53 & \\
\hline $\begin{array}{l}\text { Inglewood Heights Junior Public } \\
\text { School }\end{array}$ & $\mathrm{E}$ & $\mathrm{JK}-6$ & & 20 & Manna Wong & Lynn Strangway & 245 & $\begin{array}{c}\text { Closed } \\
\text { N/A } \\
\text { N/A }\end{array}$ & 3.78 & 27,766 & $\$ 2.8$ & 2 & & $45 \%$ & Gold & & & & & 1956 & 61 & \\
\hline Ionview Public School & E & JK-8 & & 19 & David Smith & Anne Seymour & 117 & $\begin{array}{c}\text { Closed } \\
\text { N/A } \\
\text { N/A }\end{array}$ & 6 & 38,988 & $\$ 4.4$ & 1 & & $47 \%$ & Platinum & & & & & 1952 & 65 & \\
\hline Iroquois Junior Public School & $\mathrm{E}$ & JK-6 & & 21 & Abdul Hai Patel & $\begin{array}{l}\text { Jacqueline } \\
\text { Spence }\end{array}$ & 237 & $\begin{array}{c}\text { Limited } \\
\text { N/A } \\
\text { N/A }\end{array}$ & 6 & 44,385 & $\$ 3.7$ & & & $48 \%$ & & & & & & 1969 & 48 & \\
\hline Island Public/Natural Science School & E & JK-6 & & 14 & Chris Moise & $\begin{array}{l}\text { Jane Phillips- } \\
\text { Long }\end{array}$ & 401 & $\begin{array}{c}\text { Closed } \\
\text { N/A } \\
\text { N/A }\end{array}$ & 3.68 & 57,281 & $\$ 2.6$ & & & $44 \%$ & Gold & & & A1 & No & 1999 & 18 & \\
\hline Islington Junior Middle School & $\mathrm{E}$ & JK-8 & & 3 & Pamela Gough & Tracy Hayhurst & 159 & $\begin{array}{c}\text { Limited } \\
\text { Limited } \\
\text { N/A }\end{array}$ & 6.5 & 79,149 & $\$ 7.2$ & & & $58 \%$ & Gold & & Yes & & & 1974 & 43 & Cat. 1 \\
\hline J B Tyrrell Senior Public School & E & 7-8 & & 20 & Manna Wong & $\begin{array}{l}\text { Louie } \\
\text { Papathanasakis }\end{array}$ & 305 & $\begin{array}{c}\text { Closed } \\
\text { N/A } \\
\text { Limited }\end{array}$ & 4.05 & 75,735 & $\$ 2.7$ & 2 & & $31 \%$ & Gold & & Yes & & & 1972 & 45 & \\
\hline J G Workman Public School & E & $\mathrm{JK}-8$ & & 18 & Parthi Kandavel & Peter Chang & 77 & $\begin{array}{c}\text { Limited } \\
\text { N/A } \\
\text { N/A }\end{array}$ & 5.91 & 28,333 & $\$ 4.7$ & & & $77 \%$ & Platinum & & & & & 1948 & 69 & \\
\hline J R Wilcox Community School & E & JK-8 & & 11 & Shelley Laskin & Kathleen Garner & 144 & $\begin{array}{c}\text { Limited } \\
\text { N/A } \\
\text { Limited }\end{array}$ & 2.87 & 56,600 & $\$ 1.5$ & & & $16 \%$ & & & & & & 1920 & 97 & \\
\hline Jack Miner Senior Public School & $\mathrm{E}$ & $7-8$ & & 22 & Jerry Chadwick & $\begin{array}{l}\text { Kerry-Lynn } \\
\text { Stadnyk }\end{array}$ & 344 & \begin{tabular}{c|} 
Limited \\
N/A \\
N/A
\end{tabular} & 11.29 & 63,163 & $\$ 2.3$ & & & $30 \%$ & & & Yes & & & 1971 & 46 & \\
\hline Jackman Avenue Junior Public School & $\mathrm{E}$ & $\mathrm{JK}-6$ & & 15 & Jennifer Story & $\begin{array}{l}\text { Mary Jane } \\
\text { McNamara }\end{array}$ & 445 & $\begin{array}{c}\text { Closed } \\
\text { Closed } \\
\text { N/A }\end{array}$ & 3.71 & 61,350 & $\$ 3.6$ & 3 & & $29 \%$ & Platinum & & & & & 1961 & 56 & \\
\hline $\begin{array}{l}\text { James S Bell Junior Middle Sports and } \\
\text { Wellness Academy }\end{array}$ & $\mathrm{E}$ & $\mathrm{JK}-8$ & & 3 & Pamela Gough & Sandra Tondat & 280 & $\begin{array}{c}\text { Limited } \\
\text { N/A } \\
\text { N/A }\end{array}$ & 4.2 & 74,917 & $\$ 6.3$ & & & $55 \%$ & Gold & & Yes & & & 1966 & 51 & Cat. 1 \\
\hline $\begin{array}{l}\text { Jean Augustine Girls' Leadership } \\
\text { Academy }\end{array}$ & $\mathrm{E}$ & 4-8 & & 20 & Manna Wong & Lynn Strangway & & $\begin{array}{c}\text { Limited } \\
\text { N/A } \\
\text { N/A }\end{array}$ & & & & & & & & & & & & & & \\
\hline $\begin{array}{l}\text { Jesse Ketchum Junior and Senior } \\
\text { Public School }\end{array}$ & $\mathrm{E}$ & $\mathrm{JK}-8$ & & 14 & Chris Moise & Mike Gallagher & 289 & $\begin{array}{c}\text { Limited } \\
\text { N/A } \\
\text { N/A }\end{array}$ & 5.39 & 96,606 & $\$ 15.5$ & 1 & & $121 \%$ & & & Yes & & & 1915 & 102 & \\
\hline John A Leslie Public School & E & JK-8 & & 18 & Parthi Kandavel & $\begin{array}{l}\text { Jacqueline } \\
\text { Spence }\end{array}$ & 138 & $\begin{array}{c}\text { Closed } \\
\text { N/A } \\
\text { Limited }\end{array}$ & 7.09 & 51,633 & $\$ 3.5$ & 4 & & $36 \%$ & Gold & & & & & 1923 & 94 & \\
\hline
\end{tabular}




\begin{tabular}{|c|c|c|c|c|c|c|c|c|c|c|c|c|c|c|c|c|c|c|}
\hline & Participa & tion Rates & & & & & Enrolment a & Projectio & FTE, Surplus & eats $=$ OTG R & ised - Proj & tion FTE, Utili & itions $=$ Proj & ion/OTG I & vised & & & \\
\hline School Name & $\begin{array}{c}\text { TSSB 2013-14 } \\
\text { TDSB Participation } \\
\text { Rate }\end{array}$ & $\begin{array}{c}\text { TOSB 2013-14 } \\
\text { Home school } \\
\text { Participation Rate }\end{array}$ & $\begin{array}{c}\text { Revised } \\
\text { Capacity } \\
2016\end{array}$ & $\begin{array}{c}\text { HC } \\
\text { (Oct. 2016) }\end{array}$ & $\begin{array}{c}\text { FTE } \\
\text { (Oct. 2016) }\end{array}$ & $\begin{array}{c}2016 \\
\text { Utilization } \\
\text { Rate }\end{array}$ & $\begin{array}{c}2021 \\
\text { Projected }\end{array}$ & $\begin{array}{c}2021 \\
\text { Surplus } \\
\text { Seats }\end{array}$ & $\begin{array}{c}2021 \\
\text { Utilization } \\
\text { Rate }\end{array}$ & $\begin{array}{c}2026 \\
\text { Projected }\end{array}$ & $\begin{array}{c}2026 \\
\text { Surplus } \\
\text { Seats }\end{array}$ & $\begin{array}{c}2026 \\
\text { Utilization } \\
\text { Rate }\end{array}$ & $\begin{array}{c}2031 \\
\text { Projected }\end{array}$ & $\begin{array}{l}2031 \\
\text { Surplus } \\
\text { Seats }\end{array}$ & $\begin{array}{c}2031 \\
\text { Utilization } \\
\text { Rate }\end{array}$ & $\begin{array}{c}2036 \\
\text { Projected }\end{array}$ & $\begin{array}{l}2036 \\
\text { Surplus } \\
\text { Seats }\end{array}$ & $\begin{array}{c}2036 \\
\text { Utilization } \\
\text { Rate }\end{array}$ \\
\hline Humewood Community School & $68.0 \%$ & $51.0 \%$ & 588 & 571 & 571.0 & $97 \%$ & 629 & -41 & $107 \%$ & 614 & -26 & $104 \%$ & 613 & -25 & $104 \%$ & 613 & -25 & $104 \%$ \\
\hline Hunter's Glen Junior Public School & $84.2 \%$ & 74.1\% & 395 & 377 & 376.5 & $95 \%$ & 474 & -79 & $120 \%$ & 481 & -86 & $122 \%$ & 481 & -86 & $122 \%$ & 481 & -86 & $122 \%$ \\
\hline Huron Street Junior Public School & $75.3 \%$ & $49.3 \%$ & 444 & 408 & 408.0 & $92 \%$ & 423 & 21 & $95 \%$ & 441 & 3 & $\mathbf{9 9 \%}$ & 441 & 3 & $\mathbf{9 9 \%}$ & 441 & 3 & $\mathbf{9 9 \%}$ \\
\hline $\begin{array}{l}\text { Indian Road Crescent Junior Public } \\
\text { School }\end{array}$ & $73.3 \%$ & $43.6 \%$ & 364 & 336 & 336.0 & $92 \%$ & 290 & 74 & $80 \%$ & 298 & 66 & $82 \%$ & 302 & 62 & $83 \%$ & 302 & 62 & $83 \%$ \\
\hline $\begin{array}{l}\text { Inglewood Heights Junior Public } \\
\text { School }\end{array}$ & $66.7 \%$ & $58.3 \%$ & 240 & 229 & 229.0 & $95 \%$ & 384 & -144 & $160 \%$ & 404 & -164 & $168 \%$ & 420 & -180 & $175 \%$ & 426 & -186 & $178 \%$ \\
\hline Ionview Public School & $63.2 \%$ & $53.8 \%$ & 428 & 391 & 391.0 & $91 \%$ & 385 & 43 & $90 \%$ & 393 & 35 & $92 \%$ & 399 & 29 & $93 \%$ & 399 & 29 & $93 \%$ \\
\hline Iroquois Junior Public School & $83.1 \%$ & $70.2 \%$ & 357 & 305 & 305.0 & $85 \%$ & 252 & 105 & $71 \%$ & 235 & 122 & $66 \%$ & 236 & 121 & $66 \%$ & 236 & 121 & $66 \%$ \\
\hline Island Public/Natural Science School & 99.1\% & 93.1\% & 176 & 240 & 240.0 & $136 \%$ & 261 & -85 & $148 \%$ & 248 & -72 & $141 \%$ & 248 & -72 & $141 \%$ & 248 & -72 & $141 \%$ \\
\hline Islington Junior Middle School & $55.8 \%$ & $40.3 \%$ & 635 & 490 & 490.0 & $77 \%$ & 568 & 67 & $89 \%$ & 571 & 64 & $90 \%$ & 574 & 61 & $90 \%$ & 574 & 61 & $90 \%$ \\
\hline J B Tyrrell Senior Public School & $74.4 \%$ & $63.8 \%$ & 423 & 393 & 393.0 & $93 \%$ & 373 & 50 & $88 \%$ & 323 & 100 & $76 \%$ & 324 & 99 & $77 \%$ & 324 & 99 & $77 \%$ \\
\hline J G Workman Public School & $83.3 \%$ & $73.0 \%$ & 228 & 217 & 217.0 & $95 \%$ & 161 & 67 & $71 \%$ & 151 & 77 & $66 \%$ & 156 & 72 & $68 \%$ & 156 & 72 & $68 \%$ \\
\hline J R Wilcox Community School & $45.0 \%$ & $22.6 \%$ & 434 & 311 & 311.0 & $72 \%$ & 329 & 105 & $76 \%$ & 314 & 120 & $72 \%$ & 310 & 124 & $71 \%$ & 310 & 124 & $71 \%$ \\
\hline Jack Miner Senior Public School & $86.2 \%$ & $81.4 \%$ & 354 & 171 & 171.0 & $48 \%$ & 165 & 189 & $47 \%$ & 164 & 190 & $46 \%$ & 164 & 190 & $46 \%$ & 168 & 186 & $47 \%$ \\
\hline Jackman Avenue Junior Public School & $99.8 \%$ & $94.2 \%$ & 660 & 653 & 653.0 & $99 \%$ & 666 & -6 & $101 \%$ & 666 & -6 & $101 \%$ & 666 & -6 & $101 \%$ & 666 & -6 & $101 \%$ \\
\hline $\begin{array}{l}\text { James S Bell Junior Middle Sports and } \\
\text { Wellness Academy }\end{array}$ & $63.8 \%$ & $41.9 \%$ & 565 & 467 & 467.0 & $83 \%$ & 510 & 55 & $90 \%$ & 519 & 46 & $92 \%$ & 492 & 73 & $87 \%$ & 492 & 73 & $87 \%$ \\
\hline $\begin{array}{l}\text { Jean Augustine Girls' Leadership } \\
\text { Academy }\end{array}$ & & & 138 & 5 & 5.0 & $4 \%$ & 20 & 118 & $14 \%$ & 20 & 118 & $14 \%$ & 20 & 118 & $14 \%$ & 20 & 118 & $14 \%$ \\
\hline $\begin{array}{l}\text { Jesse Ketchum Junior and Senior } \\
\text { Public School }\end{array}$ & $66.7 \%$ & $40.2 \%$ & 603 & 452 & 452.0 & $75 \%$ & 505 & 98 & $84 \%$ & 499 & 104 & $83 \%$ & 500 & 103 & $83 \%$ & 500 & 103 & $83 \%$ \\
\hline John A Leslie Public School & $72.3 \%$ & $54.2 \%$ & 504 & 543 & 543.0 & $108 \%$ & 460 & 44 & $91 \%$ & 412 & 92 & $82 \%$ & 406 & 98 & $81 \%$ & 406 & 98 & $81 \%$ \\
\hline
\end{tabular}




\begin{tabular}{|c|c|c|c|c|c|c|c|c|c|c|c|c|c|c|c|c|c|c|c|c|c|c|}
\hline \multirow[b]{2}{*}{ School Name } & \multicolumn{8}{|c|}{ General Information } & \multicolumn{14}{|c|}{ Facility Information } \\
\hline & Panel & $\begin{array}{l}\text { Grade } \\
\text { Range }\end{array}$ & $\begin{array}{c}\text { Semestered } \\
(\mathrm{sec})\end{array}$ & Ward & Trustee & $\begin{array}{l}\text { Superintendent } \\
\text { of Education }\end{array}$ & 2017 LOI & $\begin{array}{c}\text { 2017-18 } \\
\text { Opt } \\
\text { Attend } \\
\text { Reg/F/EF }\end{array}$ & $\begin{array}{l}\text { Site Size } \\
\text { (Acres) }\end{array}$ & $\begin{array}{l}\text { Facility Size } \\
\text { (Sq. Ft.) }\end{array}$ & $\begin{array}{c}\text { Current } \\
\text { Backlog (M) }\end{array}$ & $\begin{array}{c}\text { Port. } \\
\text { (Instruc) }\end{array}$ & $\begin{array}{c}\text { Port. (Non- } \\
\text { Instruc) }\end{array}$ & $\begin{array}{c}\text { Current FCI } \\
\text { (EDU) }\end{array}$ & $\begin{array}{c}\text { Eco } \\
\text { Schools }\end{array}$ & Pool & $\begin{array}{l}\text { Elem. } \\
\text { D\&T } \\
\text { Shops }\end{array}$ & $\begin{array}{l}\text { Barrier } \\
\text { Free / } \\
\text { Access }\end{array}$ & Elevator & $\begin{array}{l}\text { Build } \\
\text { Date }\end{array}$ & $\begin{array}{c}\text { Building } \\
\text { Age }\end{array}$ & $\begin{array}{l}\text { T.O Comm } \\
\text { Centre }\end{array}$ \\
\hline John Buchan Senior Public School & $\mathrm{E}$ & $7-8$ & & 20 & Manna Wong & Lynn Strangway & 175 & $\begin{array}{c}\text { Limited } \\
\text { N/A } \\
\text { N/A }\end{array}$ & 6 & 71,847 & $\$ 1.8$ & & & $20 \%$ & & & & & No & 1971 & 46 & \\
\hline John D Parker Junior School & E & JK-5 & & 1 & Avtar Minhas & Glenford Duffus & 187 & $\begin{array}{c}\text { Closed } \\
\text { N/A } \\
\text { N/A }\end{array}$ & 4.99 & 47,362 & $\$ 1.4$ & 1 & 2 & $12 \%$ & Silver & & & & & 1974 & 43 & \\
\hline John English Junior Middle School & $\mathrm{E}$ & JK-8 & & 3 & Pamela Gough & Sandra Tondat & 328 & $\begin{array}{l}\text { Limited } \\
\text { Closed } \\
\text { Closed }\end{array}$ & 6.05 & 150,424 & $\$ 20.5$ & & & $100 \%$ & Gold & & Yes & & & 1924 & 93 & Cat. 1 \\
\hline John Fisher Junior Public School & E & SK-6 & & 13 & Gerri Gershon & Leila Girdhar-Hill & 448 & $\begin{array}{c}\text { N/A } \\
\text { Closed } \\
\text { N/A }\end{array}$ & 2.74 & 62,450 & $\$ 2$. & & & $18 \%$ & & & & & & 1887 & 130 & \\
\hline John G Althouse Middle School & $\mathrm{E}$ & 6-8 & & 2 & Chris Glover & Annie Appleby & 417 & \begin{tabular}{|c} 
Limited \\
N/A \\
N/A
\end{tabular} & 8.5 & 64,261 & $\$ 7.8$ & 1 & 1 & $73 \%$ & Gold & & Yes & & & 1957 & 60 & Cat. 1 \\
\hline John G Diefenbaker Public School & E & JK-8 & & 22 & Jerry Chadwick & Nadira Persaud & 207 & \begin{tabular}{|c} 
Limited \\
N/A \\
N/A
\end{tabular} & 4.99 & 43,008 & $\$ 1.8$ & & & $23 \%$ & & & & & & 1980 & 37 & \\
\hline John McCrae Public School & $\mathrm{E}$ & JK-8 & & 19 & David Smith & Anne Seymour & 94 & $\begin{array}{c}\text { Limited } \\
\text { N/A } \\
\text { N/A }\end{array}$ & 2.94 & 93,649 & $\$ 7.2$ & & & $65 \%$ & & & & & & 1969 & 48 & \\
\hline $\begin{array}{l}\text { John Ross Robertson Junior Public } \\
\text { School }\end{array}$ & $\mathrm{E}$ & JK-6 & & 8 & Jennifer Arp & Vicky Branco & 468 & $\begin{array}{c}\text { Closed } \\
\text { Closed } \\
\text { N/A }\end{array}$ & 4.92 & 70,212 & $\$ 5.2$ & 2 & & $47 \%$ & Gold & & & A1 & Yes & 1919 & 98 & \\
\hline John Wanless Junior Public School & $\mathrm{E}$ & JK-6 & & 8 & Jennifer Arp & Vicky Branco & 461 & $\begin{array}{c}\text { Closed } \\
\text { N/A } \\
\text { N/A }\end{array}$ & 2.84 & 92,277 & $\$ 9.7$ & & & $70 \%$ & & & & A1 & & 1926 & 91 & \\
\hline Joseph Brant Public School & E & JK-8 & & 22 & Jerry Chadwick & $\begin{array}{l}\text { Kerry-Lynn } \\
\text { Stadnyk }\end{array}$ & 58 & \begin{tabular}{|c} 
Limited \\
N/A \\
N/A
\end{tabular} & 7.42 & 91,868 & $\$ 4.7$ & & & $31 \%$ & & & Yes & & & 1971 & 46 & \\
\hline Joseph Howe Senior Public School & E & $7-8$ & & 22 & Jerry Chadwick & $\begin{array}{l}\text { Kerry-Lynn } \\
\text { Stadnyk }\end{array}$ & 406 & $\begin{array}{l}\text { Closed } \\
\text { N/A } \\
\text { Limited }\end{array}$ & 8.01 & 50,863 & $\$ 2.3$ & 2 & & $31 \%$ & Gold & & Yes & A1 & Yes & 1978 & 39 & \\
\hline Joyce Public School & E & JK-6 & & 8 & Jennifer Arp & Leila Girdhar-Hill & 112 & \begin{tabular}{|c|} 
Limited \\
N/A \\
N/A
\end{tabular} & 4.92 & 35,457 & $\$ 4.2$ & & 1 & $55 \%$ & Gold & & & & & 1961 & 56 & \\
\hline Karen Kain School of the Arts & $\mathrm{E}$ & 6-8 & & 3 & Pamela Gough & Tracy Hayhurst & 416 & $\begin{array}{c}\text { Limited } \\
\text { N/A } \\
\text { N/A }\end{array}$ & 3.41 & 17,191 & $\$ .4$ & & & $8 \%$ & Platinum & & & & & 1952 & 65 & \\
\hline Keele Street Public School & E & JK-8 & & 7 & Robin Pilkey & Sandra Tondat & 361 & $\begin{array}{c}\text { Closed } \\
\text { N/A } \\
\text { N/A }\end{array}$ & 3.19 & 66,729 & $\$ 3.3$ & & & $35 \%$ & Gold & TLC & & & & 1978 & 39 & Cat. 1 \\
\hline Keelesdale Junior Public School & $\mathrm{E}$ & JK-6 & & 6 & Chris Tonks & Vicky Branco & 33 & \begin{tabular}{|c} 
Limited \\
N/A \\
N/A
\end{tabular} & 4.15 & 36,613 & $\$ 2.2$ & & & $31 \%$ & & & & & & 1960 & 57 & \\
\hline Kennedy Public School & $\mathrm{E}$ & JK-8 & & 20 & Manna Wong & $\begin{array}{l}\text { Louie } \\
\text { Papathanasakis }\end{array}$ & 259 & $\begin{array}{c}\text { Closed } \\
\text { N/A } \\
\text { N/A }\end{array}$ & 4.99 & 58,718 & $\$ 1.3$ & 2 & & $12 \%$ & Gold & & & & & 1987 & 30 & \\
\hline Kensington Community School & E & JK-6 & & 10 & Ausma Malik & Mike Gallagher & 186 & $\begin{array}{c}\text { Limited } \\
\text { N/A } \\
\text { N/A }\end{array}$ & 2.1 & 67,121 & $\$ 2.7$ & & & $23 \%$ & & TLC & & A1 & & 1972 & 45 & \\
\hline Kew Beach Junior Public School & E & JK-6 & & 16 & $\begin{array}{l}\text { Sheila Cary- } \\
\text { Meagher }\end{array}$ & John Chasty & 447 & $\begin{array}{l}\text { Closed } \\
\text { N/A } \\
\text { N/A }\end{array}$ & 3.21 & 51,809 & $\$ 7.1$ & 3 & 1 & $81 \%$ & Silver & & & & & 1962 & 55 & \\
\hline
\end{tabular}




\begin{tabular}{|c|c|c|c|c|c|c|c|c|c|c|c|c|c|c|c|c|c|c|}
\hline & Participa & tion Rates & & & & & Enrolment a & Projectio & FTE, Surplus & eats $=$ OTG R & ised - Proj & tion FTE, Util & itions $=$ Proj & ion/OTG R & vised & & & \\
\hline School Name & $\begin{array}{c}\text { TDSB 2013-14 } \\
\text { TDSB Participation } \\
\text { Rate }\end{array}$ & $\begin{array}{c}\text { TOSB 2013-14 } \\
\text { Home school } \\
\text { Participation Rate }\end{array}$ & $\begin{array}{c}\text { Revised } \\
\text { Capacity } \\
2016\end{array}$ & $\begin{array}{c}\text { HC } \\
\text { (Oct. 2016) }\end{array}$ & $\begin{array}{c}\text { FTE } \\
\text { (Oct. 2016) }\end{array}$ & $\begin{array}{c}2016 \\
\text { Utilization } \\
\text { Rate }\end{array}$ & $\begin{array}{c}2021 \\
\text { Projected }\end{array}$ & $\begin{array}{l}2021 \\
\text { Surplus } \\
\text { Seats }\end{array}$ & $\begin{array}{c}2021 \\
\text { Utilization } \\
\text { Rate }\end{array}$ & $\begin{array}{c}2026 \\
\text { Projected }\end{array}$ & $\begin{array}{c}2026 \\
\text { Surplus } \\
\text { Seats }\end{array}$ & $\begin{array}{c}2026 \\
\text { Utilization } \\
\text { Rate }\end{array}$ & $\begin{array}{c}2031 \\
\text { Projected }\end{array}$ & $\begin{array}{l}2031 \\
\text { Surplus } \\
\text { Seats }\end{array}$ & $\begin{array}{c}2031 \\
\text { Utilization } \\
\text { Rate }\end{array}$ & $\begin{array}{c}2036 \\
\text { Projected }\end{array}$ & $\begin{array}{l}2036 \\
\text { Surplus } \\
\text { Seats }\end{array}$ & $\begin{array}{c}2036 \\
\text { Utilization } \\
\text { Rate }\end{array}$ \\
\hline John Buchan Senior Public School & $56.4 \%$ & $36.8 \%$ & 464 & 232 & 232.0 & $50 \%$ & 216 & 248 & $47 \%$ & 216 & 248 & $47 \%$ & 210 & 254 & $45 \%$ & 212 & 252 & $46 \%$ \\
\hline John D Parker Junior School & $76.2 \%$ & $66.8 \%$ & 612 & 541 & 541.0 & $88 \%$ & 454 & 158 & $74 \%$ & 479 & 133 & $78 \%$ & 479 & 133 & $78 \%$ & 479 & 133 & $78 \%$ \\
\hline John English Junior Middle School & $69.0 \%$ & $57.3 \%$ & 860 & 901 & 901.0 & $105 \%$ & 891 & -31 & $104 \%$ & 871 & -11 & $101 \%$ & 862 & -2 & $100 \%$ & 862 & -2 & $100 \%$ \\
\hline John Fisher Junior Public School & & & 521 & 490 & 490.0 & $94 \%$ & 499 & 22 & $96 \%$ & 476 & 45 & $91 \%$ & 476 & 45 & $91 \%$ & 476 & 45 & $91 \%$ \\
\hline John G Althouse Middle School & $74.3 \%$ & $63.4 \%$ & 527 & 530 & 530.0 & $101 \%$ & 570 & -43 & $108 \%$ & 572 & -45 & $109 \%$ & 558 & -31 & $106 \%$ & 558 & -31 & $106 \%$ \\
\hline John G Diefenbaker Public School & $58.5 \%$ & 47.1\% & 369 & 239 & 239.0 & $65 \%$ & 323 & 46 & $88 \%$ & 335 & 34 & $91 \%$ & 336 & 33 & $91 \%$ & 336 & 33 & $91 \%$ \\
\hline John McCrae Public School & $50.6 \%$ & $38.8 \%$ & 763 & 642 & 642.0 & $84 \%$ & 585 & 178 & $77 \%$ & 585 & 178 & $77 \%$ & 584 & 179 & $77 \%$ & 584 & 179 & $77 \%$ \\
\hline $\begin{array}{l}\text { John Ross Robertson Junior Public } \\
\text { School }\end{array}$ & $80.8 \%$ & $72.5 \%$ & 550 & 593 & 593.0 & $108 \%$ & 498 & 52 & $91 \%$ & 497 & 53 & $90 \%$ & 507 & 43 & $92 \%$ & 516 & 34 & $94 \%$ \\
\hline John Wanless Junior Public School & $88.7 \%$ & $72.5 \%$ & 743 & 721 & 721.0 & $97 \%$ & 684 & 59 & $92 \%$ & 699 & 44 & $94 \%$ & 698 & 45 & $94 \%$ & 698 & 45 & $94 \%$ \\
\hline Joseph Brant Public School & $69.8 \%$ & $52.1 \%$ & 759 & 557 & 557.0 & $73 \%$ & 473 & 286 & $62 \%$ & 460 & 299 & $61 \%$ & 459 & 300 & $60 \%$ & 459 & 300 & $60 \%$ \\
\hline Joseph Howe Senior Public School & $76.0 \%$ & $67.7 \%$ & 331 & 321 & 321.0 & $\mathbf{9 7 \%}$ & 338 & -7 & $102 \%$ & 339 & -8 & $102 \%$ & 339 & -8 & $102 \%$ & 337 & -6 & $102 \%$ \\
\hline Joyce Public School & $61.0 \%$ & $53.4 \%$ & 340 & 284 & 284.0 & $84 \%$ & 250 & 90 & $74 \%$ & 231 & 109 & $68 \%$ & 240 & 100 & $71 \%$ & 243 & 97 & $71 \%$ \\
\hline Karen Kain School of the Arts & & & 176 & 177 & 177.0 & $101 \%$ & 175 & 1 & $99 \%$ & 175 & 1 & $99 \%$ & 175 & 1 & $99 \%$ & 175 & 1 & $99 \%$ \\
\hline Keele Street Public School & $80.5 \%$ & $48.3 \%$ & 521 & 491 & 491.0 & $94 \%$ & 668 & -147 & $128 \%$ & 741 & -220 & $142 \%$ & 733 & -212 & $141 \%$ & 733 & -212 & $141 \%$ \\
\hline Keelesdale Junior Public School & $45.9 \%$ & $30.7 \%$ & 300 & 149 & 149.0 & $50 \%$ & 153 & 147 & $51 \%$ & 156 & 144 & $52 \%$ & 156 & 144 & $52 \%$ & 156 & 144 & $52 \%$ \\
\hline Kennedy Public School & $87.8 \%$ & $83.0 \%$ & 559 & 620 & 620.0 & $111 \%$ & 639 & -80 & $114 \%$ & 691 & -132 & $124 \%$ & 703 & -144 & $126 \%$ & 703 & -144 & $126 \%$ \\
\hline Kensington Community School & $72.7 \%$ & $33.3 \%$ & 249 & 147 & 147.0 & $59 \%$ & 180 & 69 & $72 \%$ & 188 & 61 & $76 \%$ & 188 & 61 & $76 \%$ & 188 & 61 & $76 \%$ \\
\hline Kew Beach Junior Public School & $87.2 \%$ & $77.3 \%$ & 412 & 523 & 523.0 & $127 \%$ & 471 & -59 & $114 \%$ & 482 & -70 & $117 \%$ & 482 & -70 & $117 \%$ & 482 & -70 & $117 \%$ \\
\hline
\end{tabular}




\begin{tabular}{|c|c|c|c|c|c|c|c|c|c|c|c|c|c|c|c|c|c|c|c|c|c|c|}
\hline \multirow[b]{2}{*}{ School Name } & \multicolumn{8}{|c|}{ General Information } & \multicolumn{14}{|c|}{ Facility Information } \\
\hline & Panel & $\begin{array}{l}\text { Grade } \\
\text { Range }\end{array}$ & $\begin{array}{c}\text { Semestered } \\
\text { (Sec) }\end{array}$ & Ward & Trustee & $\begin{array}{c}\text { Superintendent } \\
\text { of Education }\end{array}$ & 2017 LOI & $\begin{array}{c}2017-18 \\
\text { Opt } \\
\text { Attend } \\
\text { Reg/FI/EF }\end{array}$ & $\begin{array}{l}\text { Site Size } \\
\text { (Acres) }\end{array}$ & \begin{tabular}{|} 
Facility Size \\
(Sq. Ft.)
\end{tabular} & $\begin{array}{c}\text { Current } \\
\text { Backlog (M) }\end{array}$ & $\begin{array}{l}\text { Port. } \\
\text { (Instruc) }\end{array}$ & $\begin{array}{c}\text { Port. (Non- } \\
\text { Instruc) }\end{array}$ & $\begin{array}{c}\text { Current FCI } \\
\text { (EDU) }\end{array}$ & \begin{tabular}{|c} 
Eco \\
Schools
\end{tabular} & Pool & $\begin{array}{l}\text { Elem. } \\
\text { D\&T } \\
\text { Shops }\end{array}$ & $\begin{array}{l}\text { Barrier } \\
\text { Free / } \\
\text { Access }\end{array}$ & Elevator & $\begin{array}{l}\text { Build } \\
\text { Date }\end{array}$ & $\begin{array}{c}\text { Building } \\
\text { Age }\end{array}$ & $\begin{array}{c}\text { T.o Comm } \\
\text { Centre }\end{array}$ \\
\hline Kimberley Junior Public School & $\mathrm{E}$ & JK-6 & & 16 & $\begin{array}{l}\text { Sheila Cary- } \\
\text { Meagher }\end{array}$ & John Chasty & 381 & $\begin{array}{c}\text { Closed } \\
\text { N/A } \\
\text { N/A }\end{array}$ & 2.94 & 40,174 & $\$ 4.6$ & 3 & & $51 \%$ & & & & & & 1964 & 53 & \\
\hline $\begin{array}{l}\text { King Edward Junior and Senior Public } \\
\text { School }\end{array}$ & E & JK-8 & & 10 & Ausma Malik & Mike Gallagher & 322 & $\begin{array}{l}\text { Closed } \\
\text { Closed } \\
\text { N/A }\end{array}$ & 3.41 & 69,374 & $\$ 9.6$ & & & $82 \%$ & & & Yes & & & 1958 & 59 & \\
\hline King George Junior Public School & $\mathrm{E}$ & $\mathrm{JK}-6$ & & 7 & Robin Pilkey & Tracy Hayhurst & 398 & $\begin{array}{c}\text { Closed } \\
\text { N/A } \\
\text { N/A }\end{array}$ & 1.61 & 28,677 & $\$ 4.1$ & & & $70 \%$ & Silver & & & & & 1964 & 53 & \\
\hline Kingslake Public School & E & $\mathrm{JK}-6$ & & 17 & Ken Lister & Audley Salmon & 244 & $\begin{array}{c}\text { Limited } \\
\text { N/A } \\
\text { N/A }\end{array}$ & 6 & 42,464 & $\$ 7.3$ & & & $75 \%$ & Gold & & & & & 1964 & 53 & \\
\hline Kingsview Village Junior School & $\mathrm{E}$ & JK-5 & & 1 & Avtar Minhas & Glenford Duffus & 24 & $\begin{array}{c}\text { Closed } \\
\text { N/A } \\
\text { N/A }\end{array}$ & 6.99 & 71,725 & $\$ 6.4$ & 3 & 6 & $52 \%$ & Platinum & & & A1 & Yes & 1956 & 61 & Cat. 1 \\
\hline Knob Hill Public School & E & JK-8 & & 19 & David Smith & Shirley Chan & 69 & $\begin{array}{c}\text { Closed } \\
\text { N/A } \\
\text { N/A }\end{array}$ & 5.29 & 55,043 & $\$ 4.3$ & & & $36 \%$ & Gold & & & & & 1955 & 62 & \\
\hline Lamberton Public School & $\mathrm{E}$ & JK-5 & & 4 & Tiffany Ford & Audley Salmon & 115 & $\begin{array}{c}\text { Limited } \\
\text { N/A } \\
\text { N/A }\end{array}$ & 6.03 & 37,556 & $\$ 6.3$ & & & $78 \%$ & & & & & & 1965 & 52 & \\
\hline Lambton Park Community School & E & $\mathrm{JK}-6$ & & 6 & Chris Tonks & Vicky Branco & 27 & $\begin{array}{c}\text { Limited } \\
\text { N/A } \\
\text { N/A }\end{array}$ & 1.53 & 49,513 & $\$ 1.4$ & & & $16 \%$ & Platinum & & & A1 & & 1993 & 24 & \\
\hline $\begin{array}{l}\text { Lambton-Kingsway Junior Middle } \\
\text { School }\end{array}$ & $\mathrm{E}$ & JK-8 & & 3 & Pamela Gough & Tracy Hayhurst & 469 & $\begin{array}{c}\text { Closed } \\
\text { N/A } \\
\text { N/A }\end{array}$ & 3.39 & 75,585 & $\$ 2.3$ & & 1 & $17 \%$ & & & & A1 & & 1993 & 24 & \\
\hline Lanor Junior Middle School & E & JK-8 & & 3 & Pamela Gough & Sandra Tondat & 311 & $\begin{array}{c}\text { Limited } \\
\text { N/A } \\
\text { N/A }\end{array}$ & 6.5 & 41,000 & $\$ 3.1$ & & & $38 \%$ & & & & & & 1952 & 65 & \\
\hline Lawrence Heights Middle School & E & 6-8 & & 8 & Jennifer Arp & Leila Girdhar-Hill & 11 & $\begin{array}{c}\text { Limited } \\
\text { N/A } \\
\text { N/A }\end{array}$ & 5.02 & 67,027 & $\$ 4.9$ & & & $54 \%$ & & & Yes & & & 1957 & 60 & \\
\hline $\begin{array}{l}\text { Ledbury Park Elementary and Middle } \\
\text { School }\end{array}$ & $\mathrm{E}$ & JK-8 & & 8 & Jennifer Arp & Leila Girdhar-Hill & 433 & $\begin{array}{c}\text { Closed } \\
\text { N/A } \\
\text { N/A }\end{array}$ & 4.99 & 67,566 & $\$ 2.7$ & & & $27 \%$ & Gold & & Yes & & & 1949 & 68 & \\
\hline Lescon Public School & $\mathrm{E}$ & $\mathrm{JK}-6$ & & 17 & Ken Lister & Audley Salmon & 335 & \begin{tabular}{c|} 
Limited \\
N/A \\
N/A
\end{tabular} & 6 & 54,272 & $\$ 3.7$ & & & $36 \%$ & Silver & & & & & 1964 & 53 & \\
\hline Leslieville Junior Public School & $\mathrm{E}$ & $\mathrm{JK}-6$ & & 15 & Jennifer Story & $\begin{array}{l}\text { Mary Jane } \\
\text { McNamara }\end{array}$ & 190 & $\begin{array}{c}\text { Closed } \\
\text { N/A } \\
\text { N/A }\end{array}$ & 2.82 & 49,388 & $\$ 6.6$ & & & $68 \%$ & Gold & & & & & 1961 & 56 & \\
\hline Lester B Pearson Elementary School & $\mathrm{E}$ & SK-8 & & 12 & Alexander Brown & $\begin{array}{l}\text { Louie } \\
\text { Papathanasakis }\end{array}$ & 352 & $\begin{array}{c}\mathrm{N} / \mathrm{A} \\
\text { Closed } \\
\mathrm{N} / \mathrm{A}\end{array}$ & 4.6 & 48,470 & $\$ 6.7$ & 6 & & $73 \%$ & Platinum & & & & & 1963 & 54 & \\
\hline Lillian Public School & E & JK-5 & & 12 & Alexander Brown & \begin{tabular}{|l} 
Louie \\
Papathanasakis
\end{tabular} & 341 & $\begin{array}{c}\text { Closed } \\
\text { N/A } \\
\text { N/A }\end{array}$ & 7.17 & 40,634 & $\$ 2.6$ & & & $31 \%$ & Platinum & & & & & 1948 & 69 & \\
\hline $\begin{array}{l}\text { Lord Dufferin Junior and Senior Public } \\
\text { School }\end{array}$ & $\mathrm{E}$ & $\mathrm{JK}-8$ & & 14 & Chris Moise & $\begin{array}{l}\text { Jane Phillips- } \\
\text { Long }\end{array}$ & 10 & $\begin{array}{c}\text { Limited } \\
\text { N/A } \\
\text { N/A }\end{array}$ & 3.71 & 85,804 & $\$ .5$ & & & $3 \%$ & & & & A1 & Yes & 1999 & 18 & \\
\hline $\begin{array}{l}\text { Lord Lansdowne Junior and Senior } \\
\text { Public School }\end{array}$ & E & SK-8 & & 10 & Ausma Malik & $\begin{array}{l}\text { Mary Jane } \\
\text { McNamara }\end{array}$ & 302 & $\begin{array}{c}\text { N/A } \\
\text { Limited } \\
\text { Limited }\end{array}$ & 2.35 & 72,610 & $\$ 4.1$ & & & $35 \%$ & Gold & & & & & 1960 & 57 & \\
\hline
\end{tabular}




\begin{tabular}{|c|c|c|c|c|c|c|c|c|c|c|c|c|c|c|c|c|c|c|}
\hline & Participa & ition Rates & & & & & Enrolment a & Projection & FTE, Surplus & eats $=$ OTG R & ised - Proj & tion FTE, Util & itions $=$ Proj & ion/OTG R & vised & & & \\
\hline School Name & $\begin{array}{c}\text { TSSB 2013-14 } \\
\text { TDSB Participation } \\
\text { Rate }\end{array}$ & $\begin{array}{c}\text { TDSB 2013-14 } \\
\text { Home school } \\
\text { Participation Rate }\end{array}$ & $\begin{array}{c}\text { Revised } \\
\text { Capacity } \\
2016\end{array}$ & $\begin{array}{c}\text { HC } \\
\text { (Oct. 2016) }\end{array}$ & $\begin{array}{c}\text { FTE } \\
\text { (Oct. 2016) }\end{array}$ & $\begin{array}{c}2016 \\
\text { Utilization } \\
\text { Rate }\end{array}$ & $\begin{array}{c}2021 \\
\text { Projected }\end{array}$ & $\begin{array}{l}2021 \\
\text { Surplus } \\
\text { Seats }\end{array}$ & $\begin{array}{c}2021 \\
\text { Utilization } \\
\text { Rate }\end{array}$ & $\begin{array}{c}2026 \\
\text { Projected }\end{array}$ & $\begin{array}{c}2026 \\
\text { Surplus } \\
\text { Seats }\end{array}$ & $\begin{array}{c}2026 \\
\text { Utilization } \\
\text { Rate }\end{array}$ & $\begin{array}{c}2031 \\
\text { Projected }\end{array}$ & $\begin{array}{l}2031 \\
\text { Surplus } \\
\text { Seats }\end{array}$ & $\begin{array}{c}2031 \\
\text { Utilization } \\
\text { Rate }\end{array}$ & $\begin{array}{c}2036 \\
\text { Projected }\end{array}$ & $\begin{array}{l}2036 \\
\text { Surplus } \\
\text { Seats }\end{array}$ & $\begin{array}{c}2036 \\
\text { Utilization } \\
\text { Rate }\end{array}$ \\
\hline Kimberley Junior Public School & $65.8 \%$ & $46.3 \%$ & 245 & 271 & 271.0 & $111 \%$ & 222 & 23 & $91 \%$ & 221 & 24 & $90 \%$ & 220 & 25 & $90 \%$ & 220 & 25 & $90 \%$ \\
\hline $\begin{array}{l}\text { King Edward Junior and Senior Public } \\
\text { School }\end{array}$ & $86.4 \%$ & $49.1 \%$ & 610 & 545 & 545.0 & $89 \%$ & 660 & -50 & $108 \%$ & 691 & -81 & $113 \%$ & 689 & -79 & $113 \%$ & 688 & -78 & $113 \%$ \\
\hline King George Junior Public School & $73.6 \%$ & $36.1 \%$ & 231 & 193 & 193.0 & $84 \%$ & 183 & 48 & $79 \%$ & 175 & 56 & $76 \%$ & 175 & 56 & $76 \%$ & 175 & 56 & $76 \%$ \\
\hline Kingslake Public School & $69.1 \%$ & $54.4 \%$ & 404 & 237 & 237.0 & $59 \%$ & 245 & 159 & $61 \%$ & 250 & 154 & $62 \%$ & 252 & 152 & $62 \%$ & 252 & 152 & $62 \%$ \\
\hline Kingsview Village Junior School & $56.6 \%$ & $48.8 \%$ & 653 & 524 & 524.0 & $80 \%$ & 473 & 180 & $72 \%$ & 480 & 173 & $74 \%$ & 480 & 173 & $74 \%$ & 480 & 173 & $74 \%$ \\
\hline Knob Hill Public School & $102.4 \%$ & $81.5 \%$ & 589 & 461 & 461.0 & $78 \%$ & 350 & 239 & $59 \%$ & 345 & 244 & $59 \%$ & 341 & 248 & $58 \%$ & 341 & 248 & $58 \%$ \\
\hline Lamberton Public School & $54.9 \%$ & $41.8 \%$ & 366 & 307 & 307.0 & $84 \%$ & 253 & 113 & $69 \%$ & 247 & 119 & $67 \%$ & 247 & 119 & $67 \%$ & 247 & 119 & $67 \%$ \\
\hline Lambton Park Community School & $36.5 \%$ & $18.9 \%$ & 336 & 137 & 137.0 & $41 \%$ & 125 & 211 & $37 \%$ & 122 & 214 & $36 \%$ & 122 & 214 & $36 \%$ & 122 & 214 & $36 \%$ \\
\hline $\begin{array}{l}\text { Lambton-Kingsway Junior Middle } \\
\text { School }\end{array}$ & $80.7 \%$ & $70.9 \%$ & 701 & 619 & 619.0 & $88 \%$ & 582 & 119 & $83 \%$ & 571 & 130 & $81 \%$ & 573 & 128 & $82 \%$ & 573 & 128 & $82 \%$ \\
\hline Lanor Junior Middle School & $60.6 \%$ & $34.8 \%$ & 406 & 233 & 233.0 & $57 \%$ & 276 & 130 & $68 \%$ & 285 & 121 & $70 \%$ & 280 & 126 & $69 \%$ & 280 & 126 & $69 \%$ \\
\hline Lawrence Heights Middle School & $44.8 \%$ & $23.8 \%$ & 430 & 194 & 194.0 & $45 \%$ & 168 & 262 & $39 \%$ & 161 & 269 & $37 \%$ & 151 & 279 & $35 \%$ & 151 & 279 & $35 \%$ \\
\hline $\begin{array}{l}\text { Ledbury Park Elementary and Middle } \\
\text { School }\end{array}$ & $27.7 \%$ & $15.3 \%$ & 508 & 511 & 509.0 & $100 \%$ & 565 & -57 & $111 \%$ & 580 & -72 & $114 \%$ & 578 & -70 & $114 \%$ & 578 & -70 & $114 \%$ \\
\hline Lescon Public School & $87.6 \%$ & $45.8 \%$ & 521 & 220 & 220.0 & $42 \%$ & 208 & 313 & $40 \%$ & 205 & 316 & $39 \%$ & 205 & 316 & $39 \%$ & 205 & 316 & $39 \%$ \\
\hline Leslieville Junior Public School & $80.4 \%$ & $48.5 \%$ & 439 & 366 & 366.0 & $83 \%$ & 334 & 105 & $76 \%$ & 330 & 109 & $75 \%$ & 330 & 109 & $75 \%$ & 330 & 109 & $75 \%$ \\
\hline Lester B Pearson Elementary School & & & 429 & 519 & 519.0 & $121 \%$ & 488 & -59 & $114 \%$ & 494 & -65 & $115 \%$ & 494 & -65 & $115 \%$ & 494 & -65 & $115 \%$ \\
\hline Lillian Public School & $59.3 \%$ & $44.6 \%$ & 407 & 323 & 323.0 & $79 \%$ & 328 & 79 & $81 \%$ & 340 & 67 & $84 \%$ & 333 & 74 & $82 \%$ & 333 & 74 & $82 \%$ \\
\hline $\begin{array}{l}\text { Lord Dufferin Junior and Senior Public } \\
\text { School }\end{array}$ & $73.2 \%$ & $43.6 \%$ & 692 & 503 & 502.5 & $73 \%$ & 401 & 291 & $58 \%$ & 360 & 332 & $52 \%$ & 349 & 343 & $50 \%$ & 349 & 343 & $50 \%$ \\
\hline $\begin{array}{l}\text { Lord Lansdowne Junior and Senior } \\
\text { Public School }\end{array}$ & $70.7 \%$ & $36.9 \%$ & 476 & 265 & 265.0 & $56 \%$ & 414 & 62 & $87 \%$ & 440 & 36 & $92 \%$ & 440 & 36 & $92 \%$ & 440 & 36 & $92 \%$ \\
\hline
\end{tabular}




\begin{tabular}{|c|c|c|c|c|c|c|c|c|c|c|c|c|c|c|c|c|c|c|c|c|c|c|}
\hline \multirow[b]{2}{*}{ School Name } & \multicolumn{8}{|c|}{ General Information } & \multicolumn{14}{|c|}{ Facility Information } \\
\hline & Panel & $\begin{array}{l}\text { Grade } \\
\text { Range }\end{array}$ & $\begin{array}{c}\text { Semestered } \\
(\mathrm{Sec})\end{array}$ & Ward & Trustee & $\begin{array}{l}\text { Superintendent } \\
\text { of Education }\end{array}$ & 2017 LOI & $\begin{array}{c}2017-18 \\
\mathrm{Opt} \\
\text { Attend } \\
\mathrm{Reg} / \mathrm{F} / \mathrm{EF}\end{array}$ & $\begin{array}{l}\text { Site Size } \\
\text { (Acres) }\end{array}$ & $\begin{array}{c}\text { Facility Size } \\
\text { (Sq. Ft.) }\end{array}$ & $\mid \begin{array}{c}\text { Current } \\
\text { Backlog (M) }\end{array}$ & \begin{tabular}{|l} 
Port. \\
(Instruc)
\end{tabular} & \begin{tabular}{|c|} 
Port. (Non- \\
Instruc)
\end{tabular} & $\begin{array}{c}\text { Current FCI } \\
\text { (EDU) }\end{array}$ & $\begin{array}{c}\text { Eco } \\
\text { Schools }\end{array}$ & Pool & $\begin{array}{l}\text { Elem. } \\
\text { D\&T } \\
\text { Shops }\end{array}$ & $\begin{array}{l}\text { Barrier } \\
\text { Free / } \\
\text { Access }\end{array}$ & Elevator & $\begin{array}{l}\text { Build } \\
\text { Date }\end{array}$ & $\begin{array}{c}\text { Building } \\
\text { Age }\end{array}$ & $\begin{array}{l}\text { T.O Comm } \\
\text { Centre }\end{array}$ \\
\hline Lord Roberts Junior Public School & E & JK-6 & & 19 & David Smith & Shirley Chan & 218 & \begin{tabular}{c|} 
Closed \\
N/A \\
N/A
\end{tabular} & 4.35 & 41,712 & $\$ 6.1$ & & & $60 \%$ & & & & & & 1958 & 59 & \\
\hline $\begin{array}{l}\text { Lucy Maud Montgomery Public } \\
\text { School }\end{array}$ & E & JK-8 & & 21 & Abdul Hai Patel & Andrew Howard & 209 & $\begin{array}{c}\text { Limited } \\
\text { N/A } \\
\text { N/A }\end{array}$ & 4.99 & 47,149 & $\$ 1.6$ & & & $22 \%$ & & & & & Yes & 1990 & 27 & \\
\hline Lucy McCormick Senior School & $\mathrm{E}$ & $7-8$ & & 7 & Robin Pilkey & Tracy Hayhurst & 62 & $\begin{array}{c}\text { Limited } \\
\text { N/A } \\
\text { N/A }\end{array}$ & 1.24 & 41,642 & $\$ 1.1$ & & & $23 \%$ & & & Yes & & Yes & 1975 & 42 & \\
\hline Lynngate Junior Public School & E & JK-6 & & 20 & Manna Wong & Lynn Strangway & 268 & $\begin{array}{c}\text { Limited } \\
\text { N/A } \\
\text { N/A }\end{array}$ & 4.55 & 34,216 & $\$ 3.7$ & & & $60 \%$ & Silver & & & & & 1959 & 58 & \\
\hline $\begin{array}{l}\text { Lynnwood Heights Junior Public } \\
\text { School }\end{array}$ & $\mathrm{E}$ & JK-6 & & 20 & Manna Wong & $\begin{array}{l}\text { Louie } \\
\text { Papathanasakis }\end{array}$ & 145 & $\begin{array}{c}\text { Closed } \\
\text { N/A } \\
\text { N/A }\end{array}$ & 4.99 & 23,619 & $\$ 1.1$ & 1 & & $20 \%$ & Gold & & & & & 1956 & 61 & \\
\hline Macklin Public School & E & JK-8 & & 21 & Abdul Hai Patel & $\begin{array}{l}\text { Jacqueline } \\
\text { Spence }\end{array}$ & 250 & $\begin{array}{c}\text { Closed } \\
\text { N/A } \\
\text { N/A }\end{array}$ & 4.99 & 55,384 & $\$ 1.7$ & & & $15 \%$ & Bronze & & & B1 & & 1987 & 30 & \\
\hline Malvern Junior Public School & E & JK-6 & & 21 & Abdul Hai Patel & Andrew Howard & 255 & $\begin{array}{c}\text { Closed } \\
\text { N/A } \\
\text { N/A }\end{array}$ & 4.99 & 31,433 & $\$ 3.1$ & 4 & & $40 \%$ & Gold & & & & & 1975 & 42 & \\
\hline Manhattan Park Junior Public School & E & JK-6 & & 19 & David Smith & Nadira Persaud & 168 & $\begin{array}{c}\text { Limited } \\
\text { N/A } \\
\text { N/A }\end{array}$ & 2 & 20,189 & $\$ 3.1$ & & & $72 \%$ & & & & & & 1956 & 61 & \\
\hline Maple Leaf Public School & $\mathrm{E}$ & $\mathrm{JK}-8$ & & 6 & Chris Tonks & $\begin{array}{l}\text { Angela Nardi- } \\
\text { Addesa }\end{array}$ & 7 & $\begin{array}{c}\text { Limited } \\
\text { N/A } \\
\text { N/A }\end{array}$ & 6.15 & 56,662 & $\$ 4.7$ & & & $54 \%$ & & & & & & 1912 & 105 & \\
\hline $\begin{array}{l}\text { Market Lane Junior and Senior Public } \\
\text { School }\end{array}$ & E & JK-8 & & 14 & Chris Moise & $\begin{array}{l}\text { Jane Phillips- } \\
\text { Long }\end{array}$ & 84 & $\begin{array}{c}\text { Closed } \\
\text { N/A } \\
\text { N/A }\end{array}$ & 2.35 & 83,423 & $\$ 2.2$ & & & $22 \%$ & & & & & Yes & 1992 & 25 & \\
\hline Mary Shadd Public School & E & JK-8 & & 21 & Abdul Hai Patel & Andrew Howard & 240 & $\begin{array}{c}\text { Limited } \\
\text { N/A } \\
\text { N/A }\end{array}$ & 4.99 & 60,270 & $\$ 1.3$ & & & $13 \%$ & & & & & Yes & 1987 & 30 & \\
\hline Maryvale Public School & E & JK-8 & & 19 & David Smith & Nadira Persaud & 132 & \begin{tabular}{c|} 
Limited \\
N/A \\
N/A
\end{tabular} & 10.01 & 40,232 & $\$ 5.3$ & & 1 & $60 \%$ & & & & & & 1954 & 63 & \\
\hline Mason Road Junior Public School & E & JK-6 & & 18 & Parthi Kandavel & $\begin{array}{l}\text { Jacqueline } \\
\text { Spence }\end{array}$ & 36 & $\begin{array}{c}\text { Limited } \\
\text { N/A } \\
\text { N/A }\end{array}$ & 5.86 & 50,805 & $\$ 3.6$ & & & $33 \%$ & Gold & & & & & 1956 & 61 & \\
\hline Maurice Cody Junior Public School & E & $\mathrm{JK}-6$ & & 11 & Shelley Laskin & Ian Allison & 464 & $\begin{array}{c}\text { Closed } \\
\text { N/A } \\
\text { N/A }\end{array}$ & 2.89 & 75,148 & $\$ 9.8$ & & & $66 \%$ & & & & & & 1928 & 89 & Cat. 1 \\
\hline McKee Public School & E & JK-5 & & 12 & Alexander Brown & Linda Curtis & 368 & $\begin{array}{c}\text { Closed } \\
\text { N/A } \\
\text { N/A }\end{array}$ & 3.78 & 70,501 & $\$ 3.1$ & 4 & & $23 \%$ & Platinum & & & A1 & Yes & 1998 & 19 & \\
\hline McMurrich Junior Public School & E & JK-6 & & 11 & Shelley Laskin & Kathleen Garner & 308 & $\begin{array}{c}\text { Limited } \\
\text { N/A } \\
\text { N/A }\end{array}$ & 2.91 & 82,501 & $\$ 9.2$ & & & $75 \%$ & & & & & & 1910 & 107 & \\
\hline Meadowvale Public School & E & JK-8 & & 22 & Jerry Chadwick & Nadira Persaud & 364 & $\begin{array}{c}\text { Closed } \\
\text { N/A } \\
\text { N/A }\end{array}$ & 7.88 & 29,526 & $\$ 3.8$ & 1 & 1 & $62 \%$ & Gold & & & & & 1953 & 64 & \\
\hline Melody Village Junior School & E & JK-5 & & 1 & Avtar Minhas & Annie Appleby & 154 & $\begin{array}{c}\text { Limited } \\
\text { Limited } \\
\text { N/A }\end{array}$ & 6.03 & 60,246 & $\$ 8.8$ & & & $\mathbf{9 8 \%}$ & & & & & & 1971 & 46 & \\
\hline
\end{tabular}




\begin{tabular}{|c|c|c|c|c|c|c|c|c|c|c|c|c|c|c|c|c|c|c|}
\hline & Participa & tion Rates & & & & & Enrolment a & Projectio & FTE, Surplus & eats $=$ OTG R & ised - Proj & tion FTE, Util & tions $=$ Proj & tion/OTG $\mathrm{F}$ & vised & & & \\
\hline School Name & $\begin{array}{c}\text { TDSB 2013-14 } \\
\text { TDSB Participation } \\
\text { Rate }\end{array}$ & $\begin{array}{c}\text { TDSB 2013-14 } \\
\text { Home School } \\
\text { Participation Rate }\end{array}$ & $\begin{array}{c}\text { Revised } \\
\text { Capacity } \\
2016\end{array}$ & $\begin{array}{c}\text { HC } \\
\text { (Oct. 2016) }\end{array}$ & $\begin{array}{c}\text { FTE } \\
\text { (Oct. 2016) }\end{array}$ & $\begin{array}{c}2016 \\
\text { Utilization } \\
\text { Rate }\end{array}$ & $\begin{array}{c}2021 \\
\text { Projected }\end{array}$ & $\begin{array}{l}2021 \\
\text { Surplus } \\
\text { Seats }\end{array}$ & $\begin{array}{c}2021 \\
\text { Utilization } \\
\text { Rate }\end{array}$ & $\begin{array}{c}2026 \\
\text { Projected }\end{array}$ & $\begin{array}{c}2026 \\
\text { Surplus } \\
\text { Seats }\end{array}$ & $\begin{array}{c}2026 \\
\text { Utilization } \\
\text { Rate }\end{array}$ & $\begin{array}{c}2031 \\
\text { Projected }\end{array}$ & $\begin{array}{l}2031 \\
\text { Surplus } \\
\text { Seats }\end{array}$ & $\begin{array}{c}2031 \\
\text { Utilization } \\
\text { Rate }\end{array}$ & $\begin{array}{c}2036 \\
\text { Projected }\end{array}$ & $\begin{array}{l}2036 \\
\text { Surplus } \\
\text { Seats }\end{array}$ & $\begin{array}{c}2036 \\
\text { Utilization } \\
\text { Rate }\end{array}$ \\
\hline Lord Roberts Junior Public School & $66.8 \%$ & $56.3 \%$ & 467 & 407 & 407.0 & $87 \%$ & 307 & 160 & $66 \%$ & 273 & 194 & $58 \%$ & 273 & 194 & $58 \%$ & 273 & 194 & $58 \%$ \\
\hline $\begin{array}{l}\text { Lucy Maud Montgomery Public } \\
\text { School }\end{array}$ & $60.4 \%$ & $51.0 \%$ & 355 & 214 & 213.5 & $60 \%$ & 197 & 158 & $55 \%$ & 183 & 172 & $52 \%$ & 186 & 169 & $52 \%$ & 186 & 169 & $52 \%$ \\
\hline Lucy McCormick Senior School & & & 171 & 76 & 76.0 & $44 \%$ & 65 & 106 & $38 \%$ & 65 & 106 & $38 \%$ & 65 & 106 & $38 \%$ & 65 & 106 & $38 \%$ \\
\hline Lynngate Junior Public School & $71.4 \%$ & $58.8 \%$ & 241 & 168 & 168.0 & $70 \%$ & 163 & 78 & $68 \%$ & 154 & 87 & $64 \%$ & 154 & 87 & $64 \%$ & 154 & 87 & $64 \%$ \\
\hline $\begin{array}{l}\text { Lynnwood Heights Junior Public } \\
\text { School }\end{array}$ & $82.2 \%$ & $70.1 \%$ & 142 & 136 & 136.0 & $96 \%$ & 225 & -83 & $158 \%$ & 215 & -73 & $151 \%$ & 214 & -72 & $151 \%$ & 214 & -72 & $151 \%$ \\
\hline Macklin Public School & $72.0 \%$ & $62.2 \%$ & 599 & 514 & 514.0 & $86 \%$ & 528 & 71 & $88 \%$ & 552 & 47 & $92 \%$ & 552 & 47 & $92 \%$ & 552 & 47 & $92 \%$ \\
\hline Malvern Junior Public School & $57.8 \%$ & $52.1 \%$ & 338 & 409 & 409.0 & $121 \%$ & 371 & -33 & $110 \%$ & 376 & -38 & $111 \%$ & 376 & -38 & $111 \%$ & 376 & -38 & $111 \%$ \\
\hline Manhattan Park Junior Public School & $66.7 \%$ & $51.5 \%$ & 145 & 114 & 114.0 & $79 \%$ & 123 & 22 & $85 \%$ & 129 & 16 & $89 \%$ & 132 & 13 & $91 \%$ & 132 & 13 & $91 \%$ \\
\hline Maple Leaf Public School & $35.3 \%$ & $28.1 \%$ & 388 & 235 & 235.0 & $61 \%$ & 225 & 163 & $58 \%$ & 216 & 172 & $56 \%$ & 216 & 172 & $56 \%$ & 216 & 172 & $56 \%$ \\
\hline $\begin{array}{l}\text { Market Lane Junior and Senior Public } \\
\text { School }\end{array}$ & $52.9 \%$ & $33.2 \%$ & 427 & 324 & 324.0 & $76 \%$ & 354 & 73 & $83 \%$ & 345 & 82 & $81 \%$ & 340 & 87 & $80 \%$ & 340 & 87 & $80 \%$ \\
\hline Mary Shadd Public School & $71.1 \%$ & $62.9 \%$ & 536 & 465 & 465.0 & $87 \%$ & 406 & 130 & $76 \%$ & 407 & 129 & $76 \%$ & 410 & 126 & $76 \%$ & 410 & 126 & $76 \%$ \\
\hline Maryvale Public School & $70.8 \%$ & $58.1 \%$ & 382 & 269 & 269.0 & $70 \%$ & 273 & 109 & $71 \%$ & 283 & 99 & $74 \%$ & 289 & 93 & $76 \%$ & 289 & 93 & $76 \%$ \\
\hline Mason Road Junior Public School & $69.7 \%$ & $54.1 \%$ & 542 & 379 & 379.0 & $70 \%$ & 295 & 247 & $54 \%$ & 271 & 271 & $50 \%$ & 271 & 271 & $50 \%$ & 271 & 271 & $50 \%$ \\
\hline Maurice Cody Junior Public School & $88.0 \%$ & $77.4 \%$ & 686 & 668 & 668.0 & $97 \%$ & 657 & 29 & $96 \%$ & 650 & 36 & $95 \%$ & 648 & 38 & $94 \%$ & 648 & 38 & $94 \%$ \\
\hline McKee Public School & $75.2 \%$ & $64.5 \%$ & 711 & 767 & 767.0 & $108 \%$ & 747 & -36 & $105 \%$ & 711 & 0 & $100 \%$ & 711 & 0 & $100 \%$ & 711 & 0 & $100 \%$ \\
\hline McMurrich Junior Public School & $72.5 \%$ & $40.6 \%$ & 601 & 512 & 512.0 & $85 \%$ & 512 & 89 & $85 \%$ & 518 & 83 & $86 \%$ & 517 & 84 & $86 \%$ & 517 & 84 & $86 \%$ \\
\hline Meadowvale Public School & $55.4 \%$ & $41.8 \%$ & 280 & 258 & 258.0 & $92 \%$ & 204 & 76 & $73 \%$ & 177 & 103 & $63 \%$ & 177 & 103 & $63 \%$ & 177 & 103 & $63 \%$ \\
\hline Melody Village Junior School & $67.6 \%$ & $49.4 \%$ & 505 & 224 & 224.0 & $44 \%$ & 275 & 230 & $54 \%$ & 319 & 186 & $63 \%$ & 322 & 183 & $64 \%$ & 322 & 183 & $64 \%$ \\
\hline
\end{tabular}




\begin{tabular}{|c|c|c|c|c|c|c|c|c|c|c|c|c|c|c|c|c|c|c|c|c|c|c|}
\hline \multirow[b]{2}{*}{ School Name } & \multicolumn{8}{|c|}{ General Information } & \multicolumn{14}{|c|}{ Facility Information } \\
\hline & Panel & $\begin{array}{l}\text { Grade } \\
\text { Range }\end{array}$ & $\begin{array}{c}\text { Semestered } \\
\text { (Sec) }\end{array}$ & Ward & Trustee & $\begin{array}{c}\text { Superintendent } \\
\text { of Education }\end{array}$ & 2017 LOI & $\begin{array}{c}2017-18 \\
\text { Opt } \\
\text { Attend } \\
\text { Reg/FI/EF }\end{array}$ & $\begin{array}{l}\text { Site Size } \\
\text { (Acres) }\end{array}$ & \begin{tabular}{|} 
Facility Size \\
(Sq. Ft.)
\end{tabular} & $\begin{array}{c}\text { Current } \\
\text { Backlog (M) }\end{array}$ & $\begin{array}{l}\text { Port. } \\
\text { (Instruc) }\end{array}$ & \begin{tabular}{|c|}
$\begin{array}{c}\text { Port. (Non- } \\
\text { Instruc) }\end{array}$ \\
\end{tabular} & $\begin{array}{c}\text { Current FCI } \\
\text { (EDU) }\end{array}$ & $\begin{array}{l}\text { Eco } \\
\text { Schools }\end{array}$ & Pool & $\begin{array}{l}\text { Elem. } \\
\text { D\&T } \\
\text { Shops }\end{array}$ & $\begin{array}{l}\text { Barrier } \\
\text { Free / } \\
\text { Access }\end{array}$ & Elevator & \begin{tabular}{|l|} 
Build \\
Date
\end{tabular} & $\begin{array}{c}\text { Building } \\
\text { Age }\end{array}$ & $\begin{array}{l}\text { T.O Comm } \\
\text { Centre }\end{array}$ \\
\hline Military Trail Public School & $\mathrm{E}$ & $\mathrm{JK}-8$ & & 22 & Jerry Chadwick & Nadira Persaud & 46 & \begin{tabular}{|c|} 
Limited \\
N/A \\
N/A \\
\end{tabular} & 5.81 & 76,287 & $\$ 3.7$ & & & $26 \%$ & & & & & Yes & 1970 & 47 & \\
\hline Mill Valley Junior School & E & JK-5 & & 2 & Chris Glover & Glenford Duffus & 338 & $\begin{array}{c}\text { Limited } \\
\text { N/A } \\
\text { N/A }\end{array}$ & 6.03 & 31,797 & $\$ 1.1$ & & & $20 \%$ & Gold & & & & & 1970 & 47 & \\
\hline Milliken Public School & $\mathrm{E}$ & JK-8 & & 21 & Abdul Hai Patel & $\begin{array}{l}\text { Jacqueline } \\
\text { Spence }\end{array}$ & 303 & $\begin{array}{c}\text { Closed } \\
\text { N/A } \\
\text { N/A }\end{array}$ & 5.02 & 38,589 & $\$ 1.3$ & 2 & & $20 \%$ & Platinum & & & & & 1983 & 34 & \\
\hline Millwood Junior School & E & JK-5 & & 2 & Chris Glover & Glenford Duffus & 404 & $\begin{array}{c}\text { Limited } \\
\text { Closed } \\
\text { N/A }\end{array}$ & 6.18 & 42,109 & $\$ 6.6$ & 1 & 1 & $66 \%$ & Gold & & & & & 1962 & 55 & \\
\hline Milne Valley Middle School & $\mathrm{E}$ & 6-8 & & 17 & Ken Lister & Curtis Ennis & 221 & $\begin{array}{c}\text { Limited } \\
\text { N/A } \\
\text { Limited }\end{array}$ & 7.91 & 93,819 & $\$ 10.3$ & & & $80 \%$ & & & Yes & & & 1963 & 54 & \\
\hline Montrose Junior Public School & E & $\mathrm{JK}-6$ & & 10 & Ausma Malik & Mike Gallagher & 358 & $\begin{array}{c}\text { Limited } \\
\text { N/A } \\
\text { N/A }\end{array}$ & 2.37 & 56,301 & $\$ 8.9$ & & & $\mathbf{9 0 \%}$ & & & & & & 1961 & 56 & \\
\hline Morrish Public School & $\mathrm{E}$ & $\mathrm{JK}-8$ & & 22 & Jerry Chadwick & Nadira Persaud & 265 & $\begin{array}{c}\text { Closed } \\
\text { N/A } \\
\text { N/A }\end{array}$ & 4.99 & 46,656 & $\$ .7$ & & & $9 \%$ & Bronze & & & A1 & & 1990 & 27 & \\
\hline Morse Street Junior Public School & E & JK-6 & & 15 & Jennifer Story & John Chasty & 290 & $\begin{array}{c}\text { Closed } \\
\text { Limited } \\
\text { N/A }\end{array}$ & 2.77 & 59,896 & $\$ 8$. & & & $71 \%$ & Gold & & & A1 & & 1970 & 47 & \\
\hline Mountview Alternative Junior School & $\mathrm{E}$ & $\mathrm{JK}-6$ & & 7 & Robin Pilkey & Sandra Tondat & 427 & $\begin{array}{c}\text { Limited } \\
\text { N/A } \\
\text { N/A }\end{array}$ & & & & & & & Gold & & & & & & & \\
\hline Muirhead Public School & E & $\mathrm{JK}-6$ & & 17 & Ken Lister & Beth Veale & 279 & $\begin{array}{c}\text { Limited } \\
\text { N/A } \\
\text { N/A }\end{array}$ & 6 & 34,649 & $\$ 6.6$ & & & $85 \%$ & & & & & & 1967 & 50 & \\
\hline Nelson Mandela Park Public School & E & $\mathrm{JK}-8$ & & 14 & Chris Moise & $\begin{array}{l}\text { Jane Phillips- } \\
\text { Long }\end{array}$ & 22 & $\begin{array}{c}\text { Limited } \\
\text { N/A } \\
\text { N/A }\end{array}$ & 3.55 & 10,704 & $\$ 2.3$ & & & $14 \%$ & Gold & & & & & 1915 & 102 & \\
\hline Niagara Street Junior Public School & E & JK-6 & & 10 & Ausma Malik & $\begin{array}{l}\text { Mary Jane } \\
\text { McNamara }\end{array}$ & 258 & $\begin{array}{c}\text { Limited } \\
\text { N/A } \\
\text { N/A }\end{array}$ & 0.86 & 55,881 & $\$ 1.8$ & & & $27 \%$ & & & & & Yes & 1914 & 103 & \\
\hline Norman Cook Junior Public School & $\mathrm{E}$ & JK-6 & & 18 & Parthi Kandavel & Peter Chang & 81 & \begin{tabular}{c|} 
Limited \\
N/A \\
N/A
\end{tabular} & 6.1 & 31,450 & $\$ 4.1$ & & 1 & $72 \%$ & & & & & & 1951 & 66 & \\
\hline Norman Ingram Public School & $\mathrm{E}$ & JK-5 & & 13 & Gerri Gershon & Kathleen Garner & 408 & $\begin{array}{c}\text { Closed } \\
\text { N/A } \\
\text { N/A }\end{array}$ & 9.56 & 28,256 & $\$ 3.7$ & 1 & & $62 \%$ & Platinum & & & & & 1953 & 64 & \\
\hline Norseman Junior Middle School & $\mathrm{E}$ & $\mathrm{JK}-8$ & & 3 & Pamela Gough & Tracy Hayhurst & 435 & $\begin{array}{c}\text { Closed } \\
\text { N/A } \\
\text { N/A }\end{array}$ & 6.13 & 69,470 & $\$ 1.7$ & 10 & & $20 \%$ & Gold & & & & & 1952 & 65 & \\
\hline North Agincourt Junior Public School & E & $\mathrm{JK}-6$ & & 21 & Abdul Hai Patel & Andrew Howard & 288 & $\begin{array}{l}\text { Closed } \\
\text { Closed } \\
\text { Closed }\end{array}$ & 6.47 & 31,028 & $\$ 2$. & 3 & & $27 \%$ & Gold & & & & & 1956 & 61 & \\
\hline North Bendale Junior Public School & $\mathrm{E}$ & $\mathrm{JK}-6$ & & 19 & David Smith & Shirley Chan & 196 & $\begin{array}{c}\text { Limited } \\
\text { N/A } \\
\text { N/A }\end{array}$ & 6.3 & 28,038 & $\$ 3.2$ & & & $60 \%$ & & & & & & 1959 & 58 & Cat. 1 \\
\hline $\begin{array}{l}\text { North Bridlewood Junior Public } \\
\text { School }\end{array}$ & E & JK-6 & & 20 & Manna Wong & $\begin{array}{l}\text { Louie } \\
\text { Papathanasakis }\end{array}$ & 309 & $\begin{array}{c}\text { Limited } \\
\text { N/A } \\
\text { N/A }\end{array}$ & 6 & 39,101 & $\$ 3.1$ & & & $34 \%$ & Silver & & & & & 1966 & 51 & \\
\hline
\end{tabular}




\begin{tabular}{|c|c|c|c|c|c|c|c|c|c|c|c|c|c|c|c|c|c|c|}
\hline Board & Participa & ition Rates & & & & & Enrolment a & Projectio & FTE, Surplus & ats = OTG R & ised-Proje & tion FTE, Utili & tions $=$ Proj & ion/OTG I & vised & & & \\
\hline School Name & $\begin{array}{c}\text { TDSB 2013-14 } \\
\text { TDSB Participation } \\
\text { Rate }\end{array}$ & $\begin{array}{c}\text { TDSB 2013-14 } \\
\text { Home School } \\
\text { Participation Rate }\end{array}$ & $\begin{array}{c}\text { Revised } \\
\text { Capacity } \\
2016\end{array}$ & $\begin{array}{c}\text { HC } \\
\text { (Oct. 2016) }\end{array}$ & $\begin{array}{c}\text { FTE } \\
\text { (Oct. 2016) }\end{array}$ & $\begin{array}{c}2016 \\
\text { Utilization } \\
\text { Rate }\end{array}$ & $\begin{array}{c}2021 \\
\text { Projected }\end{array}$ & $\begin{array}{l}2021 \\
\text { Surplus } \\
\text { Seats }\end{array}$ & $\begin{array}{c}2021 \\
\text { Utilization } \\
\text { Rate }\end{array}$ & $\begin{array}{c}2026 \\
\text { Projected }\end{array}$ & $\begin{array}{l}2026 \\
\text { Surplus } \\
\text { Seats }\end{array}$ & $\begin{array}{c}2026 \\
\text { Utilization } \\
\text { Rate }\end{array}$ & $\begin{array}{c}2031 \\
\text { Projected }\end{array}$ & $\begin{array}{c}2031 \\
\text { Surplus } \\
\text { Seats }\end{array}$ & $\begin{array}{c}2031 \\
\text { Utilization } \\
\text { Rate }\end{array}$ & $\begin{array}{c}2036 \\
\text { Projected }\end{array}$ & $\begin{array}{l}2036 \\
\text { Surplus } \\
\text { Seats }\end{array}$ & $\begin{array}{c}2036 \\
\text { Utilization } \\
\text { Rate }\end{array}$ \\
\hline Military Trail Public School & $80.9 \%$ & $68.0 \%$ & 683 & 477 & 477.0 & $70 \%$ & 390 & 293 & $57 \%$ & 348 & 335 & $51 \%$ & 352 & 331 & $52 \%$ & 352 & 331 & $52 \%$ \\
\hline Mill Valley Junior School & $59.8 \%$ & $45.8 \%$ & 245 & 169 & 169.0 & $69 \%$ & 156 & 89 & $64 \%$ & 159 & 86 & $65 \%$ & 159 & 86 & $65 \%$ & 159 & 86 & $65 \%$ \\
\hline Milliken Public School & $78.4 \%$ & $72.7 \%$ & 254 & 291 & 291.0 & $115 \%$ & 303 & -49 & $119 \%$ & 300 & -46 & $118 \%$ & 306 & -52 & $120 \%$ & 306 & -52 & $120 \%$ \\
\hline Millwood Junior School & $65.9 \%$ & $61.9 \%$ & 522 & 453 & 453.0 & $87 \%$ & 444 & 78 & $85 \%$ & 449 & 73 & $86 \%$ & 449 & 73 & $86 \%$ & 449 & 73 & $86 \%$ \\
\hline Milne Valley Middle School & $70.0 \%$ & $43.7 \%$ & 671 & 545 & 545.0 & $81 \%$ & 484 & 187 & $72 \%$ & 469 & 202 & $70 \%$ & 465 & 206 & $69 \%$ & 468 & 203 & $70 \%$ \\
\hline Montrose Junior Public School & $82.8 \%$ & $41.4 \%$ & 263 & 148 & 148.0 & $56 \%$ & 161 & 102 & $61 \%$ & 171 & 92 & $65 \%$ & 171 & 92 & $65 \%$ & 171 & 92 & $65 \%$ \\
\hline Morrish Public School & $69.4 \%$ & $54.8 \%$ & 364 & 310 & 310.0 & $85 \%$ & 290 & 74 & $80 \%$ & 301 & 63 & $83 \%$ & 290 & 74 & $80 \%$ & 281 & 83 & $77 \%$ \\
\hline Morse Street Junior Public School & $80.5 \%$ & $55.0 \%$ & 505 & 481 & 480.4 & $95 \%$ & 611 & -106 & $121 \%$ & 594 & -89 & $118 \%$ & 593 & -88 & $117 \%$ & 593 & -88 & $117 \%$ \\
\hline Mountview Alternative Junior School & & & 115 & 101 & 101.0 & $88 \%$ & 103 & 12 & $90 \%$ & 105 & 10 & $91 \%$ & 105 & 10 & $91 \%$ & 105 & 10 & $91 \%$ \\
\hline Muirhead Public School & $64.6 \%$ & $46.5 \%$ & 292 & 194 & 194.0 & $66 \%$ & 203 & 89 & $70 \%$ & 182 & 110 & $62 \%$ & 182 & 110 & $62 \%$ & 182 & 110 & $62 \%$ \\
\hline Nelson Mandela Park Public School & $67.3 \%$ & $45.8 \%$ & 778 & 328 & 328.0 & $42 \%$ & 577 & 201 & $74 \%$ & 583 & 195 & $75 \%$ & 585 & 193 & $75 \%$ & 585 & 193 & $75 \%$ \\
\hline Niagara Street Junior Public School & $61.3 \%$ & $32.0 \%$ & 251 & 225 & 224.0 & $89 \%$ & 278 & -27 & $111 \%$ & 271 & -20 & $108 \%$ & 272 & -21 & $108 \%$ & 272 & -21 & $108 \%$ \\
\hline Norman Cook Junior Public School & $68.2 \%$ & $55.6 \%$ & 217 & 147 & 147.0 & $68 \%$ & 183 & 34 & $84 \%$ & 191 & 26 & $88 \%$ & 191 & 26 & $88 \%$ & 191 & 26 & $88 \%$ \\
\hline Norman Ingram Public School & & & 225 & 216 & 216.0 & $96 \%$ & 330 & -105 & $147 \%$ & 328 & -103 & $146 \%$ & 328 & -103 & $146 \%$ & 328 & -103 & $146 \%$ \\
\hline Norseman Junior Middle School & $53.5 \%$ & $41.9 \%$ & 507 & 749 & 749.0 & $148 \%$ & 959 & -452 & $189 \%$ & 942 & -435 & $186 \%$ & 924 & -417 & $182 \%$ & 924 & -417 & $182 \%$ \\
\hline North Agincourt Junior Public School & $75.5 \%$ & $65.8 \%$ & 317 & 351 & 351.0 & $111 \%$ & 387 & -70 & $122 \%$ & 404 & -87 & $127 \%$ & 404 & -87 & $127 \%$ & 404 & -87 & $127 \%$ \\
\hline North Bendale Junior Public School & $55.6 \%$ & $47.2 \%$ & 203 & 163 & 163.0 & $80 \%$ & 161 & 42 & $79 \%$ & 176 & 27 & $87 \%$ & 176 & 27 & $87 \%$ & 176 & 27 & $87 \%$ \\
\hline $\begin{array}{l}\text { North Bridlewood Junior Public } \\
\text { School }\end{array}$ & $62.6 \%$ & $47.5 \%$ & 413 & 230 & 231.0 & $56 \%$ & 194 & 219 & $47 \%$ & 198 & 215 & $48 \%$ & 197 & 216 & $48 \%$ & 197 & 216 & $48 \%$ \\
\hline
\end{tabular}




\begin{tabular}{|c|c|c|c|c|c|c|c|c|c|c|c|c|c|c|c|c|c|c|c|c|c|c|}
\hline \multirow[b]{2}{*}{ School Name } & \multicolumn{8}{|c|}{ General Information } & \multicolumn{14}{|c|}{ Facility Information } \\
\hline & Panel & $\begin{array}{l}\text { Grade } \\
\text { Range }\end{array}$ & $\begin{array}{c}\text { Semestered } \\
(\mathrm{Sec})\end{array}$ & Ward & Trustee & $\begin{array}{c}\text { Superintendent } \\
\text { of Education }\end{array}$ & 2017 LOI & $\begin{array}{c}2017-18 \\
\text { Opt } \\
\text { Attend } \\
\text { Reg/FI/EF }\end{array}$ & $\begin{array}{l}\text { Site Size } \\
\text { (Acres) }\end{array}$ & $\begin{array}{c}\text { Facility Size } \\
\text { (Sq. Ft.) }\end{array}$ & $\mid \begin{array}{c}\text { Current } \\
\text { Backlog (M) }\end{array}$ & $\begin{array}{l}\text { Port. } \\
\text { (Instruc) }\end{array}$ & $\begin{array}{l}\text { Port. (Non- } \\
\text { Instruc) }\end{array}$ & $\begin{array}{c}\text { Current FCI } \\
\text { (EDU) }\end{array}$ & $\begin{array}{c}\text { Eco } \\
\text { Schools }\end{array}$ & Pool & $\begin{array}{l}\text { Elem. } \\
\text { D\&T } \\
\text { Shops }\end{array}$ & $\begin{array}{l}\text { Barrier } \\
\text { Free / } \\
\text { Access }\end{array}$ & Elevator & $\begin{array}{l}\text { Build } \\
\text { Date }\end{array}$ & $\begin{array}{c}\text { Building } \\
\text { Age }\end{array}$ & $\begin{array}{c}\text { T.o Comm } \\
\text { Centre }\end{array}$ \\
\hline North Kipling Junior Middle School & $\mathrm{E}$ & $\mathrm{JK}-8$ & & 1 & Avtar Minhas & Glenford Duffus & 101 & $\begin{array}{c}\text { Closed } \\
\text { N/A } \\
\text { N/A }\end{array}$ & 10.01 & 96,833 & $\$ .7$ & & & $5 \%$ & & & Yes & & Yes & 1999 & 18 & Cat. 2 \\
\hline $\begin{array}{l}\text { North Preparatory Junior Public } \\
\text { School }\end{array}$ & E & $\mathrm{JK}-6$ & & 8 & Jennifer Arp & Vicky Branco & 349 & $\begin{array}{c}\text { Closed } \\
\text { N/A } \\
\text { N/A }\end{array}$ & 2.3 & 34,803 & $\$ 2.1$ & 1 & 1 & $36 \%$ & Gold & & & & & 1936 & 81 & \\
\hline $\begin{array}{l}\text { Northlea Elementary and Middle } \\
\text { School }\end{array}$ & $\mathrm{E}$ & JK-8 & & 13 & Gerri Gershon & Leila Girdhar-Hill & 424 & $\begin{array}{l}\text { Closed } \\
\text { Closed } \\
\text { N/A }\end{array}$ & 4.6 & 92,183 & $\$ 1.3$ & 1 & 1 & $8 \%$ & Silver & & & & No & 1943 & 74 & \\
\hline Norway Junior Public School & E & JK-6 & & 16 & $\begin{array}{l}\text { Sheila Cary- } \\
\text { Meagher }\end{array}$ & John Chasty & 405 & $\begin{array}{c}\text { Closed } \\
\text { N/A } \\
\text { N/A }\end{array}$ & 4.67 & 37,148 & $\$ 4.2$ & 1 & & $57 \%$ & & & & & & 1976 & 41 & \\
\hline Oakdale Park Middle School & $\mathrm{E}$ & 6-8 & & 5 & Alexandra Lulka & Curtis Ennis & 12 & $\begin{array}{c}\text { Limited } \\
\text { N/A } \\
\text { N/A }\end{array}$ & 6.99 & 90,256 & $\$ 4.3$ & & 2 & $39 \%$ & & & & & No & 1964 & 53 & \\
\hline Oakridge Junior Public School & E & JK-4 & & 18 & Parthi Kandavel & Peter Chang & 116 & $\begin{array}{c}\text { Closed } \\
\text { N/A } \\
\text { N/A }\end{array}$ & 3.58 & 75,999 & $\$ 9.1$ & 3 & & $67 \%$ & \begin{tabular}{|l|} 
Platinum \\
\end{tabular} & & & & Yes & 1966 & 51 & \\
\hline O'Connor Public School & $\mathrm{E}$ & JK-5 & & 17 & Ken Lister & Curtis Ennis & 67 & $\begin{array}{c}\text { Limited } \\
\text { N/A } \\
\text { N/A }\end{array}$ & 4.97 & 33,250 & $\$ 6.3$ & & & $102 \%$ & & & & & & 1967 & 50 & \\
\hline Ogden Junior Public School & E & JK-6 & & 10 & Ausma Malik & $\begin{array}{l}\text { Mary Jane } \\
\text { McNamara }\end{array}$ & 166 & $\begin{array}{c}\text { Limited } \\
\text { N/A } \\
\text { N/A }\end{array}$ & 1.78 & 28,309 & $\$ 2.5$ & & & $39 \%$ & & & & & & 1956 & 61 & \\
\hline Orde Street Public School & $\mathrm{E}$ & JK-7 & & 10 & Ausma Malik & $\begin{array}{l}\text { Mary Jane } \\
\text { McNamara }\end{array}$ & 306 & $\begin{array}{c}\text { Limited } \\
\text { N/A } \\
\text { N/A }\end{array}$ & 1.26 & 58,622 & $\$ 9$. & & & $96 \%$ & & & & & & 1914 & 103 & \\
\hline Oriole Park Junior Public School & E & $\mathrm{JK}-6$ & & 11 & Shelley Laskin & Ian Allison & 462 & $\begin{array}{c}\text { Closed } \\
\text { N/A } \\
\text { N/A }\end{array}$ & 3.14 & 34,396 & $\$ 5$. & 2 & & $85 \%$ & Gold & & & & & 1929 & 88 & \\
\hline $\begin{array}{l}\text { Ossington/Old Orchard Junior Public } \\
\text { School }\end{array}$ & $\mathrm{E}$ & JK-6 & & 10 & Ausma Malik & Mike Gallagher & 337 & $\begin{array}{c}\text { Limited } \\
\text { N/A } \\
\text { N/A }\end{array}$ & 4.65 & 50,460 & $\$ 4.7$ & 1 & & $71 \%$ & & & & & & 1959 & 58 & \\
\hline Owen Public School & E & $\mathrm{JK}-6$ & & 13 & Gerri Gershon & Kathleen Garner & 421 & $\begin{array}{c}\text { Closed } \\
\text { Closed } \\
\text { N/A }\end{array}$ & 4.99 & 63,215 & $\$ 1$. & 6 & & $10 \%$ & Platinum & & & & No & 1993 & 24 & \\
\hline $\begin{array}{l}\text { Palmerston Avenue Junior Public } \\
\text { School (including Annex) }\end{array}$ & $\mathrm{E}$ & $\mathrm{JK}-6$ & & 10 & Ausma Malik & Mike Gallagher & 380 & $\begin{array}{c}\text { Limited } \\
\text { Closed } \\
\text { N/A }\end{array}$ & 3.06 & 64,566 & $\$ 9.2$ & & & $73 \%$ & & & & & & 1915 & 102 & \\
\hline Pape Avenue Junior Public School & $\mathrm{E}$ & $\mathrm{JK}-6$ & & 15 & Jennifer Story & $\begin{array}{l}\text { Mary Jane } \\
\text { McNamara }\end{array}$ & 330 & $\begin{array}{c}\text { Limited } \\
\text { N/A } \\
\text { N/A }\end{array}$ & 2.67 & 69,296 & $\$ 7.4$ & & & $63 \%$ & & & & & & 1898 & 119 & \\
\hline Park Lane Public School & $\mathrm{E}$ & $\mathrm{JK}-8$ & & 13 & Gerri Gershon & Leila Girdhar-Hill & 160 & \begin{tabular}{c|} 
Limited \\
N/A \\
N/A
\end{tabular} & 5.71 & 20,979 & $\$ 2.9$ & & & $198 \%$ & Platinum & & & A1 & No & 1968 & 49 & \\
\hline Park Lawn Junior Middle School & E & JK-8 & & 3 & Pamela Gough & Tracy Hayhurst & 365 & $\begin{array}{c}\text { Closed } \\
\text { N/A } \\
\text { N/A }\end{array}$ & 6.4 & 51,407 & $\$ 4.8$ & & & $49 \%$ & Gold & & Yes & A1 & No & 1952 & 65 & Cat. 5 \\
\hline $\begin{array}{l}\text { Parkdale Junior and Senior Public } \\
\text { School }\end{array}$ & $\mathrm{E}$ & $\mathrm{JK}-8$ & & 7 & Robin Pilkey & $\begin{array}{l}\text { Jane Phillips- } \\
\text { Long }\end{array}$ & 118 & $\begin{array}{c}\text { Limited } \\
\text { Limited } \\
\text { N/A }\end{array}$ & 3.56 & 148,780 & $\$ 4.9$ & & & $28 \%$ & Platinum & City & Yes & A1 & Yes & 1993 & 24 & Cat. 2 \\
\hline Parkfield Junior School & $\mathrm{E}$ & JK-5 & & 2 & Chris Glover & $\begin{array}{l}\text { Angela Nardi- } \\
\text { Addesa }\end{array}$ & 64 & $\begin{array}{c}\text { Limited } \\
\text { N/A } \\
\text { N/A }\end{array}$ & 6 & 43,174 & $\$ 3.9$ & & & $41 \%$ & Platinum & & & & & 1965 & 52 & \\
\hline
\end{tabular}




\begin{tabular}{|c|c|c|c|c|c|c|c|c|c|c|c|c|c|c|c|c|c|c|}
\hline & Participa & tion Rates & & & & & Enrolment a & Projectio & FTE, Surplus & eats $=$ OTG R & ised - Proj & tion FTE, Utili & itions $=$ Proj & ion/OTG R & vised & & & \\
\hline School Name & $\begin{array}{c}\text { TDSB 2013-14 } \\
\text { TDSB Participation } \\
\text { Rate }\end{array}$ & $\begin{array}{c}\text { TOSB 2013-14 } \\
\text { Home school } \\
\text { Participation Rate }\end{array}$ & $\begin{array}{c}\text { Revised } \\
\text { Capacity } \\
2016\end{array}$ & $\begin{array}{c}\text { HC } \\
\text { (Oct. 2016) }\end{array}$ & $\begin{array}{c}\text { FTE } \\
\text { (Oct. 2016) }\end{array}$ & $\begin{array}{c}2016 \\
\text { Utilization } \\
\text { Rate }\end{array}$ & $\begin{array}{c}2021 \\
\text { Projected }\end{array}$ & $\begin{array}{c}2021 \\
\text { Surplus } \\
\text { Seats }\end{array}$ & $\begin{array}{c}2021 \\
\text { Utilization } \\
\text { Rate }\end{array}$ & $\begin{array}{c}2026 \\
\text { Projected }\end{array}$ & $\begin{array}{c}2026 \\
\text { Surplus } \\
\text { Seats }\end{array}$ & $\begin{array}{c}2026 \\
\text { Utilization } \\
\text { Rate }\end{array}$ & $\begin{array}{c}2031 \\
\text { Projected }\end{array}$ & $\begin{array}{l}2031 \\
\text { Surplus } \\
\text { Seats }\end{array}$ & $\begin{array}{c}2031 \\
\text { Utilization } \\
\text { Rate }\end{array}$ & $\begin{array}{c}2036 \\
\text { Projected }\end{array}$ & $\begin{array}{l}2036 \\
\text { Surplus } \\
\text { Seats }\end{array}$ & $\begin{array}{c}2036 \\
\text { Utilization } \\
\text { Rate }\end{array}$ \\
\hline North Kipling Junior Middle School & $68.8 \%$ & $60.0 \%$ & 803 & 744 & 743.0 & $93 \%$ & 540 & 263 & $67 \%$ & 520 & 283 & $65 \%$ & 531 & 272 & $66 \%$ & 531 & 272 & $66 \%$ \\
\hline $\begin{array}{l}\text { North Preparatory Junior Public } \\
\text { School }\end{array}$ & $62.9 \%$ & $41.0 \%$ & 222 & 198 & 198.0 & $89 \%$ & 217 & 5 & $\mathbf{9 8} \%$ & 194 & 28 & $87 \%$ & 191 & 31 & $86 \%$ & 191 & 31 & $86 \%$ \\
\hline $\begin{array}{l}\text { Northlea Elementary and Middle } \\
\text { School }\end{array}$ & $90.2 \%$ & $84.2 \%$ & 776 & 748 & 748.0 & $96 \%$ & 684 & 92 & $88 \%$ & 661 & 115 & $85 \%$ & 661 & 115 & $85 \%$ & 661 & 115 & $85 \%$ \\
\hline Norway Junior Public School & $90.2 \%$ & $60.8 \%$ & 294 & 302 & 299.8 & $102 \%$ & 362 & -68 & $123 \%$ & 385 & -91 & $131 \%$ & 385 & -91 & $131 \%$ & 385 & -91 & $131 \%$ \\
\hline Oakdale Park Middle School & $59.0 \%$ & $47.4 \%$ & 649 & 432 & 432.0 & $67 \%$ & 387 & 262 & $60 \%$ & 299 & 350 & $46 \%$ & 309 & 340 & $48 \%$ & 309 & 340 & $48 \%$ \\
\hline Oakridge Junior Public School & $81.7 \%$ & $74.2 \%$ & 703 & 664 & 664.0 & $94 \%$ & 632 & 71 & $90 \%$ & 636 & 67 & $\mathbf{9 0 \%}$ & 636 & 67 & $90 \%$ & 636 & 67 & $90 \%$ \\
\hline O'Connor Public School & $61.7 \%$ & $43.9 \%$ & 254 & 166 & 166.0 & $65 \%$ & 148 & 106 & $58 \%$ & 147 & 107 & $58 \%$ & 147 & 107 & $58 \%$ & 147 & 107 & $58 \%$ \\
\hline Ogden Junior Public School & $46.3 \%$ & $28.0 \%$ & 242 & 202 & 202.0 & $83 \%$ & 261 & -19 & $108 \%$ & 258 & -16 & $107 \%$ & 258 & -16 & $107 \%$ & 258 & -16 & $107 \%$ \\
\hline Orde Street Public School & $69.0 \%$ & $49.2 \%$ & 448 & 403 & 402.0 & $90 \%$ & 319 & 129 & $71 \%$ & 305 & 143 & $68 \%$ & 305 & 143 & $68 \%$ & 305 & 143 & $68 \%$ \\
\hline Oriole Park Junior Public School & $73.5 \%$ & $60.6 \%$ & 242 & 307 & 307.0 & $127 \%$ & 253 & -11 & $105 \%$ & 229 & 13 & $95 \%$ & 229 & 13 & $95 \%$ & 229 & 13 & $95 \%$ \\
\hline $\begin{array}{l}\text { Ossington/Old Orchard Junior Public } \\
\text { school }\end{array}$ & $76.5 \%$ & $45.9 \%$ & 260 & 298 & 298.0 & $115 \%$ & 277 & -17 & $107 \%$ & 265 & -5 & $102 \%$ & 265 & -5 & $102 \%$ & 265 & -5 & $102 \%$ \\
\hline Owen Public School & $69.0 \%$ & $63.0 \%$ & 559 & 631 & 631.0 & $113 \%$ & 631 & -72 & $113 \%$ & 638 & -79 & $114 \%$ & 638 & -79 & $114 \%$ & 638 & -79 & $114 \%$ \\
\hline $\begin{array}{l}\text { Palmerston Avenue Junior Public } \\
\text { School (including Annex) }\end{array}$ & 99.1\% & $87.9 \%$ & 400 & 465 & 465.0 & $116 \%$ & 397 & 3 & $99 \%$ & 387 & 13 & $97 \%$ & 387 & 13 & $97 \%$ & 387 & 13 & $97 \%$ \\
\hline Pape Avenue Junior Public School & $104.8 \%$ & $79.2 \%$ & 459 & 385 & 385.0 & $84 \%$ & 444 & 15 & $97 \%$ & 450 & 9 & $\mathbf{9 8} \%$ & 451 & 8 & $\mathbf{9 8 \%}$ & 451 & 8 & $\mathbf{9 8} \%$ \\
\hline Park Lane Public School & & & 54 & 58 & 58.0 & $107 \%$ & 63 & -9 & $117 \%$ & 65 & -11 & $120 \%$ & 65 & -11 & $120 \%$ & 65 & -11 & $120 \%$ \\
\hline Park Lawn Junior Middle School & $68.7 \%$ & $52.3 \%$ & 499 & 481 & 481.0 & $96 \%$ & 563 & -64 & $113 \%$ & 562 & -63 & $113 \%$ & 549 & -50 & $110 \%$ & 549 & -50 & $110 \%$ \\
\hline $\begin{array}{l}\text { Parkdale Junior and Senior Public } \\
\text { School }\end{array}$ & $92.0 \%$ & $68.2 \%$ & 774 & 531 & 531.0 & $69 \%$ & 652 & 122 & $84 \%$ & 663 & 111 & $86 \%$ & 645 & 129 & $83 \%$ & 645 & 129 & $83 \%$ \\
\hline Parkfield Junior School & $53.3 \%$ & $42.9 \%$ & 416 & 275 & 275.0 & $66 \%$ & 279 & 137 & $67 \%$ & 305 & 111 & $73 \%$ & 305 & 111 & $73 \%$ & 305 & 111 & $73 \%$ \\
\hline
\end{tabular}




\begin{tabular}{|c|c|c|c|c|c|c|c|c|c|c|c|c|c|c|c|c|c|c|c|c|c|c|}
\hline \multirow[b]{2}{*}{ School Name } & \multicolumn{8}{|c|}{ General Information } & \multicolumn{14}{|c|}{ Facility Information } \\
\hline & Panel & $\begin{array}{l}\text { Grade } \\
\text { Range }\end{array}$ & $\begin{array}{c}\text { Semestered } \\
\text { (Sec) }\end{array}$ & Ward & Trustee & $\begin{array}{l}\text { Superintendent } \\
\text { of Education }\end{array}$ & 2017 LOI & $\begin{array}{c}2017-18 \\
\mathrm{Opt} \\
\text { Attend } \\
\mathrm{Reg} / \mathrm{F} / \mathrm{EF}\end{array}$ & $\begin{array}{l}\text { Site Size } \\
\text { (Acres) }\end{array}$ & $\begin{array}{c}\text { Facility Size } \\
\text { (Sq. Ft.) }\end{array}$ & $\begin{array}{c}\text { Current } \\
\text { Backlog (M) }\end{array}$ & \begin{tabular}{|c} 
Port. \\
(Instruc)
\end{tabular} & $\begin{array}{c}\text { Port. (Non- } \\
\text { Instruc) }\end{array}$ & $\begin{array}{c}\text { Current FCI } \\
\text { (EDU) }\end{array}$ & $\begin{array}{l}\text { Eco } \\
\text { Schools }\end{array}$ & Pool & $\begin{array}{l}\text { Elem. } \\
\text { D\&T } \\
\text { Shops }\end{array}$ & $\begin{array}{l}\text { Barrier } \\
\text { Free / } \\
\text { Access }\end{array}$ & Elevator & $\begin{array}{l}\text { Build } \\
\text { Date }\end{array}$ & $\begin{array}{c}\text { Building } \\
\text { Age }\end{array}$ & $\begin{array}{l}\text { T.O Comm } \\
\text { Centre }\end{array}$ \\
\hline Parkside Elementary School & E & JK-5 & & 16 & $\begin{array}{l}\text { Sheila Cary- } \\
\text { Meagher }\end{array}$ & Lucy Giannotta & 171 & \begin{tabular}{|c|} 
Limited \\
N/A \\
N/A \\
\end{tabular} & 4.89 & 33,426 & $\$ 5$. & & & $89 \%$ & Bronze & & & & & 1953 & 64 & \\
\hline Pauline Johnson Junior Public School & E & JK-6 & & 20 & Manna Wong & Lynn Strangway & 114 & $\begin{array}{c}\text { Limited } \\
\text { N/A } \\
\text { N/A }\end{array}$ & 4 & 47,133 & $\$ 4.2$ & & & $44 \%$ & & & & & & 1969 & 48 & \\
\hline Pauline Junior Public School & $\mathrm{E}$ & $\mathrm{JK}-6$ & & 9 & Marit Stiles & $\begin{array}{l}\text { Jane Phillips- } \\
\text { Long }\end{array}$ & 241 & $\begin{array}{c}\text { Limited } \\
\text { N/A } \\
\text { N/A }\end{array}$ & 2.92 & 83,055 & $\$ 16.4$ & & & $85 \%$ & & & & & & 1912 & 105 & \\
\hline Pelmo Park Public School & E & JK-5 & & 6 & Chris Tonks & $\begin{array}{l}\text { Angela Nardi- } \\
\text { Addesa }\end{array}$ & 31 & \begin{tabular}{c|} 
Closed \\
N/A \\
N/A
\end{tabular} & 8.6 & 29,375 & $\$ 3.7$ & 2 & & $49 \%$ & & & & & & 1950 & 67 & \\
\hline Percy Williams Junior Public School & E & JK-6 & & 21 & Abdul Hai Patel & $\begin{array}{l}\text { Jacqueline } \\
\text { Spence }\end{array}$ & 291 & $\begin{array}{c}\text { Limited } \\
\text { N/A } \\
\text { N/A }\end{array}$ & 4.99 & 49,208 & $\$ 1.7$ & & 1 & $21 \%$ & & & & B1 & & 1983 & 34 & \\
\hline Perth Avenue Junior Public School & E & JK-6 & & 9 & Marit Stiles & $\begin{array}{l}\text { Jane Phillips- } \\
\text { Long }\end{array}$ & 249 & $\begin{array}{c}\text { Closed } \\
\text { Closed } \\
\text { N/A }\end{array}$ & 3.36 & 57,194 & $\$ 9.6$ & & & $57 \%$ & & & & & & 1964 & 53 & \\
\hline Pierre Laporte Middle School & E & 6-8 & & 5 & Alexandra Lulka & Linda Curtis & 75 & $\begin{array}{c}\text { Limited } \\
\text { N/A } \\
\text { N/A }\end{array}$ & 8.82 & 80,508 & $\$ 8.2$ & & & $101 \%$ & Gold & & Yes & & & 1970 & 47 & \\
\hline Pineway Public School & E & JK-5 & & 12 & Alexander Brown & Elizabeth Addo & 113 & $\begin{array}{c}\text { Limited } \\
\text { N/A } \\
\text { N/A }\end{array}$ & 6.13 & 44,197 & $\$ 2.5$ & & & $29 \%$ & Gold & & & & & 1967 & 50 & \\
\hline Pleasant Public School & $\mathrm{E}$ & $\mathrm{JK}-6$ & & 12 & Alexander Brown & \begin{tabular}{|l} 
Louie \\
Papathanasakis
\end{tabular} & 275 & $\begin{array}{c}\text { Closed } \\
\text { N/A } \\
\text { Limited }\end{array}$ & 3.98 & 39,404 & $\$ 4.7$ & 2 & & $53 \%$ & Silver & & & & & 1959 & 58 & \\
\hline Poplar Road Junior Public School & E & JK-6 & & 22 & Jerry Chadwick & $\begin{array}{l}\text { Kerry-Lynn } \\
\text { Stadnyk }\end{array}$ & 211 & $\begin{array}{c}\text { Limited } \\
\text { N/A } \\
\text { Limited }\end{array}$ & 6.87 & 33,821 & $\$ 6.5$ & & & $105 \%$ & & & & & & 1959 & 58 & \\
\hline Port Royal Public School & E & JK-8 & & 21 & Abdul Hai Patel & $\begin{array}{l}\text { Jacqueline } \\
\text { Spence }\end{array}$ & 219 & $\begin{array}{c}\text { Closed } \\
\text { N/A } \\
\text { N/A }\end{array}$ & 5.02 & 54,328 & $\$ .9$ & 1 & & $11 \%$ & Silver & & & A1 & Yes & 1993 & 24 & \\
\hline Portage Trail Community School & E & JK-8 & & 6 & Chris Tonks & $\begin{array}{l}\text { Angela Nardi- } \\
\text { Addesa }\end{array}$ & 34 & \begin{tabular}{c|} 
Limited \\
N/A \\
N/A
\end{tabular} & 5.91 & 109,767 & $\$ 1.2$ & & & $6 \%$ & & & & & Yes & 1999 & 18 & \\
\hline Presteign Heights Elementary School & E & JK-5 & & 16 & $\begin{array}{l}\text { Sheila Cary- } \\
\text { Meagher }\end{array}$ & Lucy Giannotta & 394 & $\begin{array}{c}\text { Closed } \\
\text { N/A } \\
\text { Closed }\end{array}$ & 4.88 & 23,335 & $\$ 4.4$ & 3 & & $77 \%$ & Platinum & & & & & 1951 & 66 & \\
\hline Princess Margaret Junior School & E & JK-5 & & 2 & Chris Glover & Annie Appleby & 286 & $\begin{array}{c}\text { Limited } \\
\text { N/A } \\
\text { N/A }\end{array}$ & 6.45 & 41,377 & $\$ 5$. & & & $66 \%$ & Gold & & & & & 1959 & 58 & \\
\hline Queen Alexandra Middle School & E & $6-8$ & & 15 & Jennifer Story & John Chasty & 90 & $\begin{array}{c}\text { Limited } \\
N / A \\
\text { Limited }\end{array}$ & 5 & 97,575 & $\$ 5.6$ & & & $37 \%$ & & TLC & & & & 1955 & 62 & \\
\hline Queen Victoria Public School & E & JK-8 & & 7 & Robin Pilkey & $\begin{array}{l}\text { Jane Phillips- } \\
\text { Long }\end{array}$ & 151 & $\begin{array}{c}\text { Limited } \\
\text { N/A } \\
\text { N/A }\end{array}$ & 3.58 & 115,629 & $\$ 1.3$ & & & $6 \%$ & & & & & Yes & 1999 & 18 & \\
\hline Quest Alternative Senior School & E & $7-8$ & & 15 & Jennifer Story & $\begin{array}{l}\text { Mary Jane } \\
\text { McNamara }\end{array}$ & 441 & $\begin{array}{c}\text { Limited } \\
\text { N/A } \\
\text { N/A }\end{array}$ & & & & & & & & & & & & & & \\
\hline R H McGregor Elementary School & E & JK-5 & & 15 & Jennifer Story & Lucy Giannotta & 415 & $\begin{array}{l}\text { Closed } \\
\text { Closed } \\
\text { N/A }\end{array}$ & 3.94 & 76,766 & $\$ 3.6$ & 4 & & $27 \%$ & & & & & & 1972 & 45 & \\
\hline
\end{tabular}




\begin{tabular}{|c|c|c|c|c|c|c|c|c|c|c|c|c|c|c|c|c|c|c|}
\hline & Participa & tion Rates & & & & & Enrolment a & Projectio & FTE, Surplus & eats $=$ OTG R & ised - Proj & tion FTE, Utili & itions $=$ Proj & ion/OTG R & vised & & & \\
\hline School Name & $\begin{array}{c}\text { TDSB 2013-14 } \\
\text { TDSB Participation } \\
\text { Rate }\end{array}$ & $\begin{array}{c}\text { TDSB } 2013-14 \\
\text { Home Schol } \\
\text { Participation Rate }\end{array}$ & $\begin{array}{c}\text { Revised } \\
\text { Capacity } \\
2016\end{array}$ & $\begin{array}{c}\text { HC } \\
\text { (Oct. 2016) }\end{array}$ & $\begin{array}{c}\text { FTE } \\
\text { (Oct. 2016) }\end{array}$ & $\begin{array}{c}2016 \\
\text { Utilization } \\
\text { Rate }\end{array}$ & $\begin{array}{c}2021 \\
\text { Projected }\end{array}$ & $\begin{array}{c}2021 \\
\text { Surplus } \\
\text { Seats }\end{array}$ & $\begin{array}{c}2021 \\
\text { Utilization } \\
\text { Rate }\end{array}$ & $\begin{array}{c}2026 \\
\text { Projected }\end{array}$ & $\begin{array}{c}2026 \\
\text { Surplus } \\
\text { Seats }\end{array}$ & $\begin{array}{c}2026 \\
\text { Utilization } \\
\text { Rate }\end{array}$ & $\begin{array}{c}2031 \\
\text { Projected }\end{array}$ & $\begin{array}{l}2031 \\
\text { Surplus } \\
\text { Seats }\end{array}$ & $\begin{array}{c}2031 \\
\text { Utilization } \\
\text { Rate }\end{array}$ & $\begin{array}{c}2036 \\
\text { Projected }\end{array}$ & $\begin{array}{l}2036 \\
\text { Surplus } \\
\text { Seats }\end{array}$ & $\begin{array}{c}2036 \\
\text { Utilization } \\
\text { Rate }\end{array}$ \\
\hline Parkside Elementary School & $63.8 \%$ & $36.9 \%$ & 189 & 158 & 158.0 & $84 \%$ & 174 & 15 & $92 \%$ & 182 & 7 & $96 \%$ & 182 & 7 & $96 \%$ & 182 & 7 & $96 \%$ \\
\hline Pauline Johnson Junior Public School & $59.5 \%$ & $49.7 \%$ & 452 & 289 & 289.0 & $64 \%$ & 365 & 87 & $81 \%$ & 389 & 63 & $86 \%$ & 389 & 63 & $86 \%$ & 389 & 63 & $86 \%$ \\
\hline Pauline Junior Public School & $65.2 \%$ & 43.1\% & 378 & 278 & 278.0 & $74 \%$ & 220 & 158 & $58 \%$ & 227 & 151 & $60 \%$ & 227 & 151 & $60 \%$ & 227 & 151 & $60 \%$ \\
\hline Pelmo Park Public School & $48.8 \%$ & $34.7 \%$ & 286 & 247 & 247.0 & $86 \%$ & 270 & 16 & $94 \%$ & 274 & 12 & $96 \%$ & 274 & 12 & $96 \%$ & 274 & 12 & $96 \%$ \\
\hline Percy Williams Junior Public School & $72.5 \%$ & $65.3 \%$ & 396 & 319 & 319.0 & $81 \%$ & 276 & 120 & $70 \%$ & 273 & 123 & $69 \%$ & 273 & 123 & $69 \%$ & 273 & 123 & $69 \%$ \\
\hline Perth Avenue Junior Public School & $54.7 \%$ & $30.0 \%$ & 297 & 326 & 326.0 & $110 \%$ & 377 & -80 & $127 \%$ & 373 & -76 & $126 \%$ & 374 & -77 & $126 \%$ & 374 & -77 & $126 \%$ \\
\hline Pierre Laporte Middle School & $43.1 \%$ & $34.8 \%$ & 444 & 401 & 401.0 & $90 \%$ & 395 & 49 & $89 \%$ & 358 & 86 & $81 \%$ & 358 & 86 & $81 \%$ & 359 & 85 & $81 \%$ \\
\hline Pineway Public School & $76.6 \%$ & $57.1 \%$ & 297 & 128 & 128.0 & $43 \%$ & 164 & 133 & $55 \%$ & 179 & 118 & $60 \%$ & 179 & 118 & $60 \%$ & 179 & 118 & $60 \%$ \\
\hline Pleasant Public School & $60.9 \%$ & $52.3 \%$ & 418 & 425 & 425.0 & $102 \%$ & 442 & -24 & $106 \%$ & 445 & -27 & $106 \%$ & 442 & -24 & $106 \%$ & 442 & -24 & $106 \%$ \\
\hline Poplar Road Junior Public School & $81.3 \%$ & $71.9 \%$ & 275 & 212 & 212.0 & $77 \%$ & 225 & 50 & $82 \%$ & 232 & 43 & $84 \%$ & 232 & 43 & $84 \%$ & 232 & 43 & $84 \%$ \\
\hline Port Royal Public School & $88.8 \%$ & $82.8 \%$ & 392 & 376 & 376.0 & $96 \%$ & 473 & -81 & $121 \%$ & 476 & -84 & $121 \%$ & 474 & -82 & $121 \%$ & 474 & -82 & $121 \%$ \\
\hline Portage Trail Community School & $63.5 \%$ & $51.2 \%$ & 896 & 611 & 611.0 & $68 \%$ & 554 & 342 & $62 \%$ & 528 & 368 & $59 \%$ & 523 & 373 & $58 \%$ & 523 & 373 & $58 \%$ \\
\hline Presteign Heights Elementary School & $104.7 \%$ & $89.7 \%$ & 173 & 224 & 224.0 & $129 \%$ & 217 & -44 & $125 \%$ & 220 & -47 & $127 \%$ & 220 & -47 & $127 \%$ & 220 & -47 & $127 \%$ \\
\hline Princess Margaret Junior School & $64.0 \%$ & $45.8 \%$ & 392 & 334 & 334.0 & $85 \%$ & 362 & 30 & $92 \%$ & 357 & 35 & $91 \%$ & 357 & 35 & $91 \%$ & 357 & 35 & $91 \%$ \\
\hline Queen Alexandra Middle School & $86.4 \%$ & $51.6 \%$ & 462 & 277 & 277.0 & $60 \%$ & 424 & 38 & $92 \%$ & 482 & -20 & $104 \%$ & 477 & -15 & $103 \%$ & 478 & -16 & $103 \%$ \\
\hline Queen Victoria Public School & $79.8 \%$ & $61.5 \%$ & 1046 & 806 & 806.0 & $77 \%$ & 804 & 242 & $77 \%$ & 882 & 164 & $84 \%$ & 897 & 149 & $86 \%$ & 897 & 149 & $86 \%$ \\
\hline Quest Alternative Senior School & & & 69 & 69 & 69.0 & $100 \%$ & 67 & 2 & $97 \%$ & 67 & 2 & $97 \%$ & 67 & 2 & $97 \%$ & 67 & 2 & $97 \%$ \\
\hline R H McGregor Elementary School & $87.8 \%$ & $73.5 \%$ & 767 & 778 & 778.0 & $101 \%$ & 835 & -68 & $109 \%$ & 803 & -36 & $105 \%$ & 801 & -34 & $104 \%$ & 801 & -34 & $104 \%$ \\
\hline
\end{tabular}




\begin{tabular}{|c|c|c|c|c|c|c|c|c|c|c|c|c|c|c|c|c|c|c|c|c|c|c|}
\hline \multirow[b]{2}{*}{ School Name } & \multicolumn{8}{|c|}{ General Information } & \multicolumn{14}{|c|}{ Facility Information } \\
\hline & Panel & $\begin{array}{l}\text { Grade } \\
\text { Range }\end{array}$ & $\begin{array}{c}\text { Semestered } \\
(\mathrm{sec})\end{array}$ & Ward & Trustee & $\begin{array}{l}\text { Superintendent } \\
\text { of Education }\end{array}$ & 2017 LOI & $\begin{array}{c}\text { 2017-18 } \\
\text { Opt } \\
\text { Attend } \\
\text { Reg/F//EF }\end{array}$ & $\begin{array}{l}\text { Site Size } \\
\text { (Acres) }\end{array}$ & $\begin{array}{l}\text { Facility Size } \\
\text { (Sq. Ft.) }\end{array}$ & $\begin{array}{c}\text { Current } \\
\text { Backlog (M) }\end{array}$ & $\begin{array}{c}\text { Port. } \\
\text { (Instruc) }\end{array}$ & $\begin{array}{c}\text { Port. (Non- } \\
\text { Instruc) }\end{array}$ & $\begin{array}{c}\text { Current FCI } \\
\text { (EDU) }\end{array}$ & $\begin{array}{c}\text { Eco } \\
\text { Schools }\end{array}$ & Pool & $\begin{array}{l}\text { Elem. } \\
\text { D\&T } \\
\text { Shops }\end{array}$ & $\begin{array}{l}\text { Barrier } \\
\text { Free / } \\
\text { Access }\end{array}$ & Elevator & $\begin{array}{l}\text { Build } \\
\text { Date }\end{array}$ & $\begin{array}{c}\text { Building } \\
\text { Age }\end{array}$ & $\begin{array}{l}\text { T.O Comm } \\
\text { Centre }\end{array}$ \\
\hline $\begin{array}{l}\text { R J Lang Elementary and Middle } \\
\text { School }\end{array}$ & $\mathrm{E}$ & JK-8 & & 12 & Alexander Brown & $\begin{array}{l}\text { Louie } \\
\text { Papathanasakis }\end{array}$ & 297 & $\begin{array}{c}\text { Closed } \\
\text { N/A } \\
\text { N/A }\end{array}$ & 10.08 & 78,502 & $\$ 2.9$ & 1 & & $27 \%$ & Gold & & Yes & & & 1960 & 57 & \\
\hline Ranchdale Public School & E & JK-5 & & 17 & Ken Lister & Curtis Ennis & 214 & $\begin{array}{c}\text { Limited } \\
\text { N/A } \\
\text { N/A }\end{array}$ & 8.03 & 51,226 & $\$ 3.1$ & & & $37 \%$ & & & & & & 1968 & 49 & \\
\hline Rawlinson Community School & $\mathrm{E}$ & JK-8 & & 9 & Marit Stiles & Sandra Tondat & 178 & $\begin{array}{l}\text { Limited } \\
\text { Limited } \\
\text { Limited }\end{array}$ & 5.86 & 108,612 & $\$ 5$. & & & $29 \%$ & & & & & & 1920 & 97 & \\
\hline Regal Road Junior Public School & E & $J K-6$ & & 9 & Marit Stiles & Sandra Tondat & 334 & $\begin{array}{l}\text { Closed } \\
\text { Closed } \\
\text { N/A }\end{array}$ & 3.48 & 79,644 & $\$ 5.9$ & & & $56 \%$ & Gold & & & & & 1913 & 104 & \\
\hline Regent Heights Public School & $\mathrm{E}$ & JK-8 & & 18 & Parthi Kandavel & Peter Chang & 220 & $\begin{array}{l}\text { Closed } \\
\text { N/A } \\
\text { N/A }\end{array}$ & 4.55 & 40,312 & $\$ 3$. & 6 & & $30 \%$ & & & & & & 1945 & 72 & \\
\hline $\begin{array}{l}\text { Rene Gordon Health and Wellness } \\
\text { Academy }\end{array}$ & E & JK-5 & & 17 & Ken Lister & Beth Veale & 210 & $\begin{array}{c}\text { Limited } \\
\text { N/A } \\
\text { Limited }\end{array}$ & 5.66 & 44,175 & $\$ 1.7$ & & & $19 \%$ & & & & & & 1963 & 54 & \\
\hline Rippleton Public School & $\mathrm{E}$ & JK-6 & & 13 & Gerri Gershon & Kathleen Garner & 397 & $\begin{array}{c}\text { Closed } \\
\text { N/A } \\
\text { N/A }\end{array}$ & 8.9 & 32,798 & $\$ 2.4$ & 1 & & $30 \%$ & & & & & & 1955 & 62 & \\
\hline Rivercrest Junior School & E & JK-5 & & 1 & Avtar Minhas & Annie Appleby & 222 & $\begin{array}{c}\text { Limited } \\
\text { N/A } \\
\text { N/A }\end{array}$ & 6.2 & 31,323 & $\$ 4.3$ & & & $60 \%$ & & & & B1 & & 1956 & 61 & \\
\hline Robert Service Senior Public School & $\mathrm{E}$ & $7-8$ & & 18 & Parthi Kandavel & Peter Chang & 98 & $\begin{array}{l}\text { Limited } \\
\text { N/A } \\
\text { N/A }\end{array}$ & 4.97 & 53,765 & $\$ 2.9$ & & & $45 \%$ & Silver & & Yes & & & 1972 & 45 & \\
\hline Rockcliffe Middle School & E & 6-8 & & 6 & Chris Tonks & Vicky Branco & 45 & \begin{tabular}{|c} 
Limited \\
$N / A$ \\
Limited
\end{tabular} & 14.63 & 69,321 & $\$ 8.5$ & & & $91 \%$ & & & Yes & & & 1966 & 51 & \\
\hline Rockford Public School & E & JK-6 & & 5 & Alexandra Lulka & Elizabeth Addo & 267 & $\begin{array}{l}\text { Closed } \\
\text { Closed } \\
\text { N/A }\end{array}$ & 6.08 & 70,343 & $\$ 9.4$ & & & $64 \%$ & & & & & & 1962 & 55 & \\
\hline Roden Public School & E & JK-8 & & 15 & Jennifer Story & John Chasty & 248 & $\begin{array}{l}\text { Limited } \\
\text { N/A } \\
\text { N/A }\end{array}$ & 3.46 & 80,595 & $\$ 4.3$ & & & $25 \%$ & & & & & & 1970 & 47 & \\
\hline Rolph Road Elementary School & $\mathrm{E}$ & $J K-6$ & & 13 & Gerri Gershon & Leila Girdhar-Hill & 463 & $\begin{array}{c}\text { Closed } \\
\text { N/A } \\
\text { N/A }\end{array}$ & 4.6 & 35,802 & $\$ 1.8$ & 4 & & $25 \%$ & Gold & & & & & 1939 & 78 & \\
\hline Rose Avenue Junior Public School & E & JK-6 & & 14 & Chris Moise & John Chasty & 99 & $\begin{array}{c}\text { Closed } \\
\text { N/A } \\
\text { N/A }\end{array}$ & 2.97 & 89,757 & $\$ 7.3$ & 2 & & $44 \%$ & Platinum & & & & Yes & 1921 & 96 & \\
\hline Rosedale Junior Public School & $\mathrm{E}$ & JK-6 & & 14 & Chris Moise & Mike Gallagher & 454 & $\begin{array}{l}\text { Closed } \\
\text { N/A } \\
\text { N/A }\end{array}$ & 2.52 & 21,072 & $\$ 3.5$ & 3 & & $63 \%$ & & & & & & 1957 & 60 & \\
\hline Roselands Junior Public School & E & JK-6 & & 6 & Chris Tonks & Vicky Branco & 48 & \begin{tabular}{|c} 
Limited \\
N/A \\
Limited
\end{tabular} & 12.9 & 45,824 & $\$ 2.8$ & & & $28 \%$ & Gold & & & & & 1955 & 62 & \\
\hline Rosethorn Junior School & E & JK-5 & & 2 & Chris Glover & Annie Appleby & 436 & \begin{tabular}{|c} 
Limited \\
Closed \\
N/A
\end{tabular} & 6.33 & 26,372 & $\$ 2.7$ & 5 & 1 & $39 \%$ & & & & & & 1952 & 65 & \\
\hline Rouge Valley Public School & E & JK-8 & & 22 & Jerry Chadwick & $\begin{array}{l}\text { Kerry-Lynn } \\
\text { Stadnyk }\end{array}$ & 317 & $\begin{array}{l}\text { Limited } \\
\text { N/A } \\
\text { N/A }\end{array}$ & 4.99 & 47,786 & $\$ 1.2$ & & & $15 \%$ & & & & & & 1991 & 26 & \\
\hline
\end{tabular}




\begin{tabular}{|c|c|c|c|c|c|c|c|c|c|c|c|c|c|c|c|c|c|c|}
\hline & Participa & tion Rates & & & & & Enrolment a & Projectio & FTE, Surplus & eats $=$ OTG R & ised - Proj & tion FTE, Util & tions $=$ Proj & tion/OTG F & vised & & & \\
\hline School Name & $\begin{array}{c}\text { TDSB 2013-14 } \\
\text { TDSB Participation } \\
\text { Rate }\end{array}$ & $\begin{array}{c}\text { TDSB 2013-14 } \\
\text { Home School } \\
\text { Participation Rate }\end{array}$ & $\begin{array}{c}\text { Revised } \\
\text { Capacity } \\
2016\end{array}$ & $\begin{array}{c}\text { HC } \\
\text { (Oct. 2016) }\end{array}$ & $\begin{array}{c}\text { FTE } \\
\text { (Oct. 2016) }\end{array}$ & $\begin{array}{c}2016 \\
\text { Utilization } \\
\text { Rate }\end{array}$ & $\begin{array}{c}2021 \\
\text { Projected }\end{array}$ & $\begin{array}{l}2021 \\
\text { Surplus } \\
\text { Seats }\end{array}$ & $\begin{array}{c}2021 \\
\text { Utilization } \\
\text { Rate }\end{array}$ & $\begin{array}{c}2026 \\
\text { Projected }\end{array}$ & $\begin{array}{c}2026 \\
\text { Surplus } \\
\text { Seats }\end{array}$ & $\begin{array}{c}2026 \\
\text { Utilization } \\
\text { Rate }\end{array}$ & $\begin{array}{c}2031 \\
\text { Projected }\end{array}$ & $\begin{array}{l}2031 \\
\text { Surplus } \\
\text { Seats }\end{array}$ & $\begin{array}{c}2031 \\
\text { Utilization } \\
\text { Rate }\end{array}$ & $\begin{array}{c}2036 \\
\text { Projected }\end{array}$ & $\begin{array}{l}2036 \\
\text { Surplus } \\
\text { Seats }\end{array}$ & $\begin{array}{c}2036 \\
\text { Utilization } \\
\text { Rate }\end{array}$ \\
\hline $\begin{array}{l}\text { R J Lang Elementary and Middle } \\
\text { School }\end{array}$ & $54.8 \%$ & $40.9 \%$ & 542 & 495 & 495.0 & $91 \%$ & 472 & 70 & $87 \%$ & 435 & 107 & $80 \%$ & 430 & 112 & $79 \%$ & 430 & 112 & $79 \%$ \\
\hline Ranchdale Public School & $62.9 \%$ & $34.1 \%$ & 370 & 265 & 264.5 & $71 \%$ & 232 & 138 & $63 \%$ & 234 & 136 & $63 \%$ & 234 & 136 & $63 \%$ & 234 & 136 & $63 \%$ \\
\hline Rawlinson Community School & $49.0 \%$ & $29.1 \%$ & 865 & 752 & 752.0 & $87 \%$ & 807 & 58 & $93 \%$ & 810 & 55 & $94 \%$ & 811 & 54 & $94 \%$ & 811 & 54 & $94 \%$ \\
\hline Regal Road Junior Public School & $59.3 \%$ & $42.7 \%$ & 637 & 573 & 573.0 & $90 \%$ & 715 & -78 & $112 \%$ & 667 & -30 & $105 \%$ & 667 & -30 & $105 \%$ & 667 & -30 & $105 \%$ \\
\hline Regent Heights Public School & $71.8 \%$ & $58.7 \%$ & 484 & 544 & 544.0 & $112 \%$ & 587 & -103 & $121 \%$ & 583 & -99 & $120 \%$ & 590 & -106 & $122 \%$ & 590 & -106 & $122 \%$ \\
\hline $\begin{array}{l}\text { Rene Gordon Health and Wellness } \\
\text { Academy }\end{array}$ & $68.5 \%$ & $50.7 \%$ & 398 & 249 & 249.0 & $63 \%$ & 206 & 192 & $52 \%$ & 205 & 193 & $52 \%$ & 205 & 193 & $52 \%$ & 205 & 193 & $52 \%$ \\
\hline Rippleton Public School & $76.7 \%$ & $59.1 \%$ & 346 & 322 & 322.0 & $93 \%$ & 345 & 1 & $100 \%$ & 347 & -1 & $100 \%$ & 348 & -2 & $101 \%$ & 348 & -2 & $101 \%$ \\
\hline Rivercrest Junior School & $47.6 \%$ & $39.6 \%$ & 277 & 234 & 234.0 & $84 \%$ & 236 & 41 & $85 \%$ & 236 & 41 & $85 \%$ & 236 & 41 & $85 \%$ & 236 & 41 & $85 \%$ \\
\hline Robert Service Senior Public School & $56.9 \%$ & $41.8 \%$ & 294 & 170 & 170.0 & $58 \%$ & 160 & 134 & $54 \%$ & 162 & 132 & $55 \%$ & 169 & 125 & $57 \%$ & 169 & 125 & $57 \%$ \\
\hline Rockcliffe Middle School & $48.0 \%$ & $37.1 \%$ & 476 & 296 & 296.0 & $62 \%$ & 336 & 140 & $71 \%$ & 339 & 137 & $71 \%$ & 343 & 133 & $72 \%$ & 343 & 133 & $72 \%$ \\
\hline Rockford Public School & $58.7 \%$ & $48.5 \%$ & 709 & 672 & 672.0 & $95 \%$ & 688 & 21 & $97 \%$ & 690 & 19 & $97 \%$ & 688 & 21 & $97 \%$ & 688 & 21 & $97 \%$ \\
\hline Roden Public School & $78.0 \%$ & $44.7 \%$ & 551 & 401 & 401.0 & $73 \%$ & 440 & 111 & $80 \%$ & 426 & 125 & $77 \%$ & 428 & 123 & $78 \%$ & 428 & 123 & $78 \%$ \\
\hline Rolph Road Elementary School & $74.0 \%$ & $63.6 \%$ & 317 & 390 & 390.0 & $123 \%$ & 385 & -68 & $121 \%$ & 389 & -72 & $123 \%$ & 389 & -72 & $123 \%$ & 389 & -72 & $123 \%$ \\
\hline Rose Avenue Junior Public School & $62.3 \%$ & $52.2 \%$ & 758 & 639 & 639.0 & $84 \%$ & 706 & 52 & $93 \%$ & 682 & 76 & $90 \%$ & 680 & 78 & $90 \%$ & 680 & 78 & $90 \%$ \\
\hline Rosedale Junior Public School & $58.4 \%$ & $50.0 \%$ & 225 & 262 & 262.0 & $116 \%$ & 265 & -40 & $118 \%$ & 242 & -17 & $108 \%$ & 242 & -17 & $108 \%$ & 242 & -17 & $108 \%$ \\
\hline Roselands Junior Public School & $70.9 \%$ & $42.2 \%$ & 341 & 334 & 334.0 & $\mathbf{9 8} \%$ & 407 & -66 & $119 \%$ & 422 & -81 & $124 \%$ & 422 & -81 & $124 \%$ & 422 & -81 & $124 \%$ \\
\hline Rosethorn Junior School & $60.1 \%$ & $54.8 \%$ & 366 & 413 & 413.0 & $113 \%$ & 453 & -87 & $124 \%$ & 462 & -96 & $126 \%$ & 462 & -96 & $126 \%$ & 462 & -96 & $126 \%$ \\
\hline Rouge Valley Public School & $63.1 \%$ & $55.9 \%$ & 338 & 259 & 259.0 & $77 \%$ & 290 & 48 & $86 \%$ & 308 & 30 & $91 \%$ & 314 & 24 & $93 \%$ & 314 & 24 & $93 \%$ \\
\hline
\end{tabular}




\begin{tabular}{|c|c|c|c|c|c|c|c|c|c|c|c|c|c|c|c|c|c|c|c|c|c|c|}
\hline \multirow[b]{2}{*}{ School Name } & \multicolumn{8}{|c|}{ General Information } & \multicolumn{14}{|c|}{ Facility Information } \\
\hline & Panel & $\begin{array}{l}\text { Grade } \\
\text { Range }\end{array}$ & $\begin{array}{c}\text { Semestered } \\
(\mathrm{Sec})\end{array}$ & Ward & Trustee & $\begin{array}{l}\text { Superintendent } \\
\text { of Education }\end{array}$ & 2017 LOI & $\begin{array}{c}2017-18 \\
\mathrm{Opt} \\
\text { Attend } \\
\mathrm{Reg} / \mathrm{F} / \mathrm{EF}\end{array}$ & $\begin{array}{l}\text { Site Size } \\
\text { (Acres) }\end{array}$ & $\begin{array}{c}\text { Facility Size } \\
\text { (Sq. Ft.) }\end{array}$ & $\mid \begin{array}{c}\text { Current } \\
\text { Backlog (M) }\end{array}$ & \begin{tabular}{|l} 
Port. \\
(Instruc)
\end{tabular} & $\begin{array}{l}\text { Port. (Non- } \\
\text { Instruc) }\end{array}$ & $\begin{array}{c}\text { Current FCI } \\
\text { (EDU) }\end{array}$ & $\begin{array}{c}\text { Eco } \\
\text { Schools }\end{array}$ & Pool & $\begin{array}{l}\text { Elem. } \\
\text { D\&T } \\
\text { Shops }\end{array}$ & $\begin{array}{l}\text { Barrier } \\
\text { Free / } \\
\text { Access }\end{array}$ & Elevator & $\begin{array}{l}\text { Build } \\
\text { Date }\end{array}$ & $\begin{array}{c}\text { Building } \\
\text { Age }\end{array}$ & $\begin{array}{l}\text { T.O Comm } \\
\text { Centre }\end{array}$ \\
\hline Roywood Public School & E & JK-5 & & 17 & Ken Lister & Curtis Ennis & 165 & \begin{tabular}{|c|} 
Limited \\
N/A \\
N/A \\
\end{tabular} & 5.58 & 31,388 & $\$ 4.6$ & & & $65 \%$ & & & & & & 1963 & 54 & \\
\hline $\begin{array}{l}\text { Runnymede Junior and Senior Public } \\
\text { School }\end{array}$ & E & JK-8 & & 7 & Robin Pilkey & Sandra Tondat & 455 & $\begin{array}{c}\text { Closed } \\
\text { Closed } \\
\text { N/A }\end{array}$ & 4.45 & 115,346 & $\$ 6.4$ & 2 & 1 & $37 \%$ & Gold & & & & & 1915 & 102 & \\
\hline Ryerson Community School & $\mathrm{E}$ & $\mathrm{JK}-8$ & & 10 & Ausma Malik & Mike Gallagher & 125 & $\begin{array}{c}\text { Limited } \\
\text { N/A } \\
\text { N/A }\end{array}$ & 4.94 & 102,656 & $\$ 17.5$ & & & $107 \%$ & & & Yes & & & 1914 & 103 & \\
\hline Samuel Hearne Middle School & E & $5-8$ & & 18 & Parthi Kandavel & Peter Chang & 104 & \begin{tabular}{c|} 
Closed \\
N/A \\
N/A
\end{tabular} & 2.84 & 58,574 & $\$ 6.2$ & & & $73 \%$ & Silver & & Yes & & & 1973 & 44 & Cat. 3 \\
\hline Scarborough Village Public School & E & JK-8 & & 19 & David Smith & Anne Seymour & 74 & $\begin{array}{c}\text { Limited } \\
\text { N/A } \\
\text { N/A }\end{array}$ & 2.8 & 48,807 & $\$ 4.2$ & & & $65 \%$ & & & & & Yes & 1998 & 19 & \\
\hline Second Street Junior Middle School & E & JK-8 & & 3 & Pamela Gough & Sandra Tondat & 161 & $\begin{array}{l}\text { Limited } \\
\text { Limited } \\
\text { Limited }\end{array}$ & 4.42 & 93,937 & $\$ 2.2$ & & & $16 \%$ & Gold & & Yes & A1 & Yes & 1949 & 68 & \\
\hline Secord Elementary School & E & JK-5 & & 16 & $\begin{array}{l}\text { Sheila Cary- } \\
\text { Meagher }\end{array}$ & Lucy Giannotta & 91 & $\begin{array}{c}\text { Limited } \\
\text { N/A } \\
\text { N/A }\end{array}$ & 4.66 & 75,474 & $\$ 5.2$ & 12 & 2 & $42 \%$ & Gold & & & & & 1964 & 53 & Cat. 2 \\
\hline Selwyn Elementary School & E & JK-5 & & 16 & $\begin{array}{l}\text { Sheila Cary- } \\
\text { Meagher }\end{array}$ & Lucy Giannotta & 184 & $\begin{array}{c}\text { Closed } \\
\text { N/A } \\
\text { N/A }\end{array}$ & 1.5 & 31,583 & $\$ 4.3$ & & & $75 \%$ & & & & & & 1957 & 60 & \\
\hline Seneca Hill Public School & $\mathrm{E}$ & $\mathrm{JK}-6$ & & 17 & Ken Lister & Audley Salmon & 360 & $\begin{array}{c}\text { Limited } \\
\text { N/A } \\
\text { N/A }\end{array}$ & 6 & 35,629 & $\$ 1.4$ & 1 & & $20 \%$ & Gold & & & & & 1968 & 49 & \\
\hline Seneca School & E & JK-8 & & 2 & Chris Glover & Annie Appleby & 52 & $\begin{array}{c}\text { Limited } \\
\text { N/A } \\
\text { N/A }\end{array}$ & 3.34 & 23,799 & $\$ 2.9$ & & & $62 \%$ & & & & A1 & No & 1966 & 51 & \\
\hline Seventh Street Junior School & E & JK-5 & & 3 & Pamela Gough & Sandra Tondat & 253 & $\begin{array}{c}\text { Limited } \\
\text { N/A } \\
\text { N/A }\end{array}$ & 2.5 & 41,775 & $\$ 1.5$ & & & $24 \%$ & & & & & & 1989 & 28 & \\
\hline Shaughnessy Public School & E & JK-8 & & 17 & Ken Lister & Beth Veale & 298 & \begin{tabular}{c|} 
Limited \\
N/A \\
N/A
\end{tabular} & 5.26 & 37,685 & $\$ 2.1$ & & & $34 \%$ & Platinum & & & & & 1965 & 52 & \\
\hline Sheppard Public School & E & JK-5 & & 5 & Alexandra Lulka & Curtis Ennis & 42 & $\begin{array}{c}\text { Limited } \\
\text { N/A } \\
\text { N/A }\end{array}$ & 6.2 & 65,564 & $\$ 7.5$ & & & $59 \%$ & & & & & & 1957 & 60 & \\
\hline Shirley Street Junior Public School & E & $\mathrm{JK}-6$ & & 9 & Marit Stiles & $\begin{array}{l}\text { Jane Phillips- } \\
\text { Long }\end{array}$ & 261 & $\begin{array}{c}\text { Limited } \\
\text { N/A } \\
\text { N/A }\end{array}$ & 2.55 & 43,683 & $\$ 2.4$ & & & $26 \%$ & & & & & & 1961 & 56 & \\
\hline $\begin{array}{l}\text { Shoreham Public Sports \& Wellness } \\
\text { Academy }\end{array}$ & E & JK-5 & & 4 & Tiffany Ford & Lynn Strangway & 2 & $\begin{array}{c}\text { Limited } \\
\text { N/A } \\
\text { N/A }\end{array}$ & 5.93 & 64,670 & $\$ 8.5$ & & & $72 \%$ & Platinum & & & & & 1968 & 49 & \\
\hline Silver Springs Public School & E & JK-8 & & 20 & Manna Wong & Beth Veale & 155 & $\begin{array}{c}\text { Limited } \\
\text { N/A } \\
\text { N/A }\end{array}$ & 5.21 & 51,461 & $\$ 7.9$ & 1 & 1 & $94 \%$ & & & & & Yes & 1975 & 42 & \\
\hline Silverthorn Community School & E & JK-8 & & 6 & Chris Tonks & Vicky Branco & 61 & $\begin{array}{c}\text { Limited } \\
\text { N/A } \\
\text { N/A }\end{array}$ & 4.68 & 80,786 & $\$ 8.2$ & & & $68 \%$ & & & Yes & & Yes & 1969 & 48 & \\
\hline Sir Adam Beck Junior School & E & JK-5 & & 3 & Pamela Gough & Sandra Tondat & 384 & $\begin{array}{c}\text { Limited } \\
\text { Closed } \\
\text { N/A }\end{array}$ & 9.86 & 54,692 & $\$ .6$ & & & $6 \%$ & & & & B1 & & 1999 & 18 & Cat. 2 \\
\hline
\end{tabular}




\begin{tabular}{|c|c|c|c|c|c|c|c|c|c|c|c|c|c|c|c|c|c|c|}
\hline & Participa & tion Rates & & & & & Enrolment a & Projectio & FTE, Surplus & eats $=$ OTG R & ised - Proj & tion FTE, Utili & itions $=$ Proj & ion/OTG I & vised & & & \\
\hline School Name & $\begin{array}{c}\text { TDSB 2013-14 } \\
\text { TDSB Participation } \\
\text { Rate }\end{array}$ & $\begin{array}{c}\text { TDSB } 2013-14 \\
\text { Home Schol } \\
\text { Participation Rate }\end{array}$ & $\begin{array}{c}\text { Revised } \\
\text { Capacity } \\
2016\end{array}$ & $\begin{array}{c}\text { HC } \\
\text { (Oct. 2016) }\end{array}$ & $\begin{array}{c}\text { FTE } \\
\text { (Oct. 2016) }\end{array}$ & $\begin{array}{c}2016 \\
\text { Utilization } \\
\text { Rate }\end{array}$ & $\begin{array}{c}2021 \\
\text { Projected }\end{array}$ & $\begin{array}{c}2021 \\
\text { Surplus } \\
\text { Seats }\end{array}$ & $\begin{array}{c}2021 \\
\text { Utilization } \\
\text { Rate }\end{array}$ & $\begin{array}{c}2026 \\
\text { Projected }\end{array}$ & $\begin{array}{c}2026 \\
\text { Surplus } \\
\text { Seats }\end{array}$ & $\begin{array}{c}2026 \\
\text { Utilization } \\
\text { Rate }\end{array}$ & $\begin{array}{c}2031 \\
\text { Projected }\end{array}$ & $\begin{array}{l}2031 \\
\text { Surplus } \\
\text { Seats }\end{array}$ & $\begin{array}{c}2031 \\
\text { Utilization } \\
\text { Rate }\end{array}$ & $\begin{array}{c}2036 \\
\text { Projected }\end{array}$ & $\begin{array}{l}2036 \\
\text { Surplus } \\
\text { Seats }\end{array}$ & $\begin{array}{c}2036 \\
\text { Utilization } \\
\text { Rate }\end{array}$ \\
\hline Roywood Public School & $61.8 \%$ & $40.0 \%$ & 214 & 208 & 208.0 & $97 \%$ & 162 & 52 & $76 \%$ & 153 & 61 & $71 \%$ & 147 & 67 & $69 \%$ & 147 & 67 & $69 \%$ \\
\hline $\begin{array}{l}\text { Runnymede Junior and Senior Public } \\
\text { School }\end{array}$ & $85.8 \%$ & $79.2 \%$ & 1011 & 1,003 & $1,003.0$ & $99 \%$ & 1,006 & 5 & $100 \%$ & 957 & 54 & $95 \%$ & 976 & 35 & $97 \%$ & 976 & 35 & $97 \%$ \\
\hline Ryerson Community School & $75.8 \%$ & $31.7 \%$ & 602 & 356 & 356.0 & $59 \%$ & 396 & 206 & $66 \%$ & 400 & 202 & $66 \%$ & 389 & 213 & $65 \%$ & 389 & 213 & $65 \%$ \\
\hline Samuel Hearne Middle School & $78.5 \%$ & $72.1 \%$ & 423 & 402 & 402.0 & $95 \%$ & 358 & 65 & $85 \%$ & 371 & 52 & $88 \%$ & 373 & 50 & $88 \%$ & 373 & 50 & $88 \%$ \\
\hline Scarborough Village Public School & & & 239 & 215 & 215.0 & $90 \%$ & 222 & 17 & $93 \%$ & 214 & 25 & $90 \%$ & 214 & 25 & $90 \%$ & 214 & 25 & $90 \%$ \\
\hline Second Street Junior Middle School & $66.1 \%$ & $34.2 \%$ & 708 & 336 & 336.0 & $47 \%$ & 609 & 99 & $86 \%$ & 668 & 40 & $94 \%$ & 658 & 50 & $93 \%$ & 658 & 50 & $93 \%$ \\
\hline Secord Elementary School & $83.4 \%$ & $59.7 \%$ & 591 & 635 & 635.0 & $107 \%$ & 708 & -117 & $120 \%$ & 718 & -127 & $121 \%$ & 719 & -128 & $122 \%$ & 719 & -128 & $122 \%$ \\
\hline Selwyn Elementary School & $85.6 \%$ & $70.8 \%$ & 254 & 246 & 246.0 & $\mathbf{9 7 \%}$ & 252 & 2 & $99 \%$ & 247 & 7 & $97 \%$ & 247 & 7 & $97 \%$ & 247 & 7 & $97 \%$ \\
\hline Seneca Hill Public School & $119.8 \%$ & $109.6 \%$ & 277 & 277 & 277.0 & $100 \%$ & 251 & 26 & $91 \%$ & 237 & 40 & $86 \%$ & 237 & 40 & $86 \%$ & 237 & 40 & $86 \%$ \\
\hline Seneca School & & & 120 & 82 & 82.0 & $68 \%$ & 80 & 40 & $67 \%$ & 80 & 40 & $67 \%$ & 80 & 40 & $67 \%$ & 80 & 40 & $67 \%$ \\
\hline Seventh Street Junior School & $76.0 \%$ & $44.0 \%$ & 234 & 213 & 213.0 & $91 \%$ & 219 & 15 & $94 \%$ & 225 & 9 & $96 \%$ & 225 & 9 & $96 \%$ & 225 & 9 & $96 \%$ \\
\hline Shaughnessy Public School & $59.3 \%$ & $38.6 \%$ & 266 & 234 & 234.0 & $88 \%$ & 231 & 35 & $87 \%$ & 220 & 46 & $83 \%$ & 217 & 49 & $82 \%$ & 217 & 49 & $82 \%$ \\
\hline Sheppard Public School & $48.2 \%$ & $31.5 \%$ & 287 & 186 & 186.0 & $65 \%$ & 183 & 104 & $64 \%$ & 189 & 98 & $66 \%$ & 189 & 98 & $66 \%$ & 189 & 98 & $66 \%$ \\
\hline Shirley Street Junior Public School & $73.9 \%$ & $38.2 \%$ & 188 & 185 & 185.0 & $98 \%$ & 187 & 1 & $99 \%$ & 192 & -4 & $102 \%$ & 196 & -8 & $104 \%$ & 196 & -8 & $104 \%$ \\
\hline $\begin{array}{l}\text { Shoreham Public Sports \& Wellness } \\
\text { Academy }\end{array}$ & $68.9 \%$ & $58.2 \%$ & 524 & 266 & 266.0 & $51 \%$ & 228 & 296 & $44 \%$ & 232 & 292 & $44 \%$ & 232 & 292 & $44 \%$ & 232 & 292 & $44 \%$ \\
\hline Silver Springs Public School & $63.3 \%$ & $57.0 \%$ & 438 & 341 & 341.0 & $78 \%$ & 313 & 125 & $71 \%$ & 320 & 118 & $73 \%$ & 314 & 124 & $72 \%$ & 314 & 124 & $72 \%$ \\
\hline Silverthorn Community School & $39.4 \%$ & $26.9 \%$ & 547 & 303 & 303.0 & $55 \%$ & 281 & 266 & $51 \%$ & 280 & 267 & $51 \%$ & 279 & 268 & $51 \%$ & 279 & 268 & $51 \%$ \\
\hline Sir Adam Beck Junior School & $75.6 \%$ & $68.2 \%$ & 533 & 448 & 448.0 & $84 \%$ & 461 & 72 & $86 \%$ & 476 & 57 & $89 \%$ & 476 & 57 & $89 \%$ & 476 & 57 & $89 \%$ \\
\hline
\end{tabular}




\begin{tabular}{|c|c|c|c|c|c|c|c|c|c|c|c|c|c|c|c|c|c|c|c|c|c|c|}
\hline \multirow[b]{2}{*}{ School Name } & \multicolumn{8}{|c|}{ General Information } & \multicolumn{14}{|c|}{ Facility Information } \\
\hline & Panel & $\begin{array}{l}\text { Grade } \\
\text { Range }\end{array}$ & $\begin{array}{c}\text { Semestered } \\
\text { (Sec) }\end{array}$ & Ward & Trustee & $\begin{array}{c}\text { Superintendent } \\
\text { of Education }\end{array}$ & 2017 LOI & $\begin{array}{c}2017-18 \\
\text { Opt } \\
\text { Attend } \\
\text { Reg/FI/EF }\end{array}$ & $\begin{array}{l}\text { Site Size } \\
\text { (Acres) }\end{array}$ & $\begin{array}{l}\text { Facility Size } \\
\text { (Sq. Ft.) }\end{array}$ & $\mid \begin{array}{c}\text { Current } \\
\text { Backlog (M) }\end{array}$ & $\begin{array}{l}\text { Port. } \\
\text { (Instruc) }\end{array}$ & $\begin{array}{c}\text { Port. (Non- } \\
\text { Instruc) }\end{array}$ & $\begin{array}{c}\text { Current FCI } \\
\text { (EDU) }\end{array}$ & $\begin{array}{c}\text { Eco } \\
\text { Schools }\end{array}$ & Pool & $\begin{array}{l}\text { Elem. } \\
\text { D\&T } \\
\text { Shops }\end{array}$ & $\begin{array}{l}\text { Barrier } \\
\text { Free / } \\
\text { Access }\end{array}$ & Elevator & $\begin{array}{l}\text { Build } \\
\text { Date }\end{array}$ & $\begin{array}{c}\text { Building } \\
\text { Age }\end{array}$ & $\begin{array}{c}\text { T.O Comm } \\
\text { Centre }\end{array}$ \\
\hline $\begin{array}{l}\text { Sir Alexander Mackenzie Senior } \\
\text { Public School }\end{array}$ & $\mathrm{E}$ & $7-8$ & & 21 & Abdul Hai Patel & Andrew Howard & 278 & $\begin{array}{l}\text { Closed } \\
\text { Closed } \\
\text { Closed }\end{array}$ & 9.41 & 49,363 & $\$ 6.8$ & 1 & & $87 \%$ & Silver & & Yes & & & 1971 & 46 & \\
\hline $\begin{array}{l}\text { Sir Ernest MacMillan Senior Public } \\
\text { School }\end{array}$ & E & $7-8$ & & 20 & Manna Wong & Beth Veale & 272 & $\begin{array}{l}\text { Limited } \\
\text { Limited } \\
\text { Limited }\end{array}$ & 7.04 & 50,863 & $\$ 2.1$ & & & $30 \%$ & Gold & & & & & 1978 & 39 & \\
\hline $\begin{array}{l}\text { Sir Samuel B Steele Junior Public } \\
\text { School }\end{array}$ & $\mathrm{E}$ & $\mathrm{JK}-6$ & & 20 & Manna Wong & $\begin{array}{l}\text { Louie } \\
\text { Papathanasakis }\end{array}$ & 299 & $\begin{array}{c}\text { Limited } \\
N / A \\
\text { Limited }\end{array}$ & 4.77 & 59,052 & $\$ 2.8$ & & & $28 \%$ & Gold & & & & & 1981 & 36 & \\
\hline Sloane Public School & E & JK-5 & & 17 & Ken Lister & Curtis Ennis & 254 & $\begin{array}{c}\text { Closed } \\
\text { N/A } \\
\text { N/A }\end{array}$ & 5.04 & 26,716 & $\$ 2.3$ & 2 & & $33 \%$ & & & & & & 1956 & 61 & \\
\hline Smithfield Middle School & $\mathrm{E}$ & 6-8 & & 1 & Avtar Minhas & Glenford Duffus & 183 & $\begin{array}{c}\text { Closed } \\
\text { N/A } \\
\text { N/A }\end{array}$ & 10.13 & 85,703 & $\$ 2.9$ & 1 & 2 & $22 \%$ & Gold & & Yes & & & 1966 & 51 & Cat. 1 \\
\hline Spectrum Alternative Senior School & E & $7-8$ & & 11 & Shelley Laskin & Ian Allison & 442 & $\begin{array}{c}\text { Limited } \\
\text { N/A } \\
\text { N/A }\end{array}$ & & & & & & & & & & & & & & \\
\hline Sprucecourt Public School & $\mathrm{E}$ & $\mathrm{JK}-8$ & & 14 & Chris Moise & $\begin{array}{l}\text { Jane Phillips- } \\
\text { Long }\end{array}$ & 37 & $\begin{array}{c}\text { Limited } \\
\text { N/A } \\
\text { N/A }\end{array}$ & 3.53 & 45,478 & $\$ 2.3$ & & & $24 \%$ & & & & & & 1957 & 60 & \\
\hline St Andrews Public School & E & JK-8 & & 19 & David Smith & Shirley Chan & 333 & $\begin{array}{c}\text { Closed } \\
\text { N/A } \\
\text { N/A }\end{array}$ & 6.08 & 46,707 & $\$ 3.3$ & & & $26 \%$ & \begin{tabular}{|l|} 
Platinum \\
\end{tabular} & & & & & 1958 & 59 & \\
\hline St George's Junior School & $\mathrm{E}$ & JK-5 & & 2 & Chris Glover & Annie Appleby & 429 & $\begin{array}{c}\text { Limited } \\
\text { N/A } \\
\text { N/A }\end{array}$ & 6.13 & 24,779 & $\$ 4.2$ & & 2 & $65 \%$ & \begin{tabular}{|l|} 
Platinum \\
\end{tabular} & & & & & 1956 & 61 & \\
\hline St Margaret's Public School & E & $\mathrm{JK}-8$ & & 22 & Jerry Chadwick & Nadira Persaud & 9 & $\begin{array}{c}\text { Closed } \\
\text { N/A } \\
\text { N/A }\end{array}$ & 7.41 & 31,084 & $\$ 5.1$ & 13 & 3 & $83 \%$ & \begin{tabular}{|l|} 
Platinum \\
\end{tabular} & & & & & 1971 & 46 & \\
\hline Stanley Public School & E & JK-5 & & 4 & Tiffany Ford & Lynn Strangway & 44 & $\begin{array}{c}\text { Limited } \\
\text { N/A } \\
\text { Limited }\end{array}$ & 6.18 & 60,439 & $\$ 2.9$ & & & $23 \%$ & Silver & & & A1 & Yes & 1960 & 57 & \\
\hline Steelesview Public School & E & JK-5 & & 12 & Alexander Brown & Elizabeth Addo & 390 & $\begin{array}{c}\text { Closed } \\
\text { N/A } \\
\text { N/A }\end{array}$ & 6 & 39,299 & $\$ 5$. & & & $59 \%$ & Silver & & & & & 1968 & 49 & \\
\hline Stilecroft Public School & $\mathrm{E}$ & JK-5 & & 5 & Alexandra Lulka & Curtis Ennis & 59 & \begin{tabular}{c|} 
Limited \\
N/A \\
N/A
\end{tabular} & 4.99 & 43,594 & $\$ 6.6$ & & & $85 \%$ & & & & & & 1962 & 55 & \\
\hline Summit Heights Public School & $\mathrm{E}$ & $\mathrm{JK}-6$ & & 5 & Alexandra Lulka & Linda Curtis & 444 & $\begin{array}{c}\text { Closed } \\
\text { N/A } \\
\text { N/A }\end{array}$ & 6.89 & 30,322 & $\$ 4.5$ & 3 & & $64 \%$ & Gold & & & & & 1950 & 67 & \\
\hline $\begin{array}{l}\text { Sunny View Junior and Senior Public } \\
\text { School }\end{array}$ & $\mathrm{E}$ & $\mathrm{JK}-8$ & & 13 & Gerri Gershon & Leila Girdhar-Hill & 163 & $\begin{array}{c}\text { Limited } \\
\text { N/A } \\
\text { N/A }\end{array}$ & 5.86 & 106,413 & $\$ 10.8$ & & & $57 \%$ & \begin{tabular}{|l|} 
Platinum \\
\end{tabular} & TDSB & & A1 & Yes & 1951 & 66 & \\
\hline Sunnylea Junior School & E & JK-5 & & 3 & Pamela Gough & Tracy Hayhurst & 465 & $\begin{array}{c}\text { Closed } \\
\text { N/A } \\
\text { N/A }\end{array}$ & 4.2 & 31,022 & $\$ 1.8$ & 1 & & $29 \%$ & & & & & & 1942 & 75 & \\
\hline $\begin{array}{l}\text { Swansea Junior and Senior Public } \\
\text { School }\end{array}$ & $\mathrm{E}$ & $\mathrm{JK}-8$ & & 7 & Robin Pilkey & $\begin{array}{l}\text { Jane Phillips- } \\
\text { Long }\end{array}$ & 400 & $\begin{array}{c}\text { Closed } \\
\text { N/A } \\
\text { Closed }\end{array}$ & 9.83 & 91,202 & $\$ 4.3$ & & & $38 \%$ & & City & & & & 1914 & 103 & Cat. 2 \\
\hline Tam O'Shanter Junior Public School & E & JK-6 & & 20 & Manna Wong & Lynn Strangway & 293 & $\begin{array}{c}\text { Closed } \\
\text { N/A } \\
\text { N/A }\end{array}$ & 2.4 & 29,140 & $\$ 3.1$ & 7 & & $48 \%$ & Silver & & & & & 1972 & 45 & \\
\hline
\end{tabular}




\begin{tabular}{|c|c|c|c|c|c|c|c|c|c|c|c|c|c|c|c|c|c|c|}
\hline & Participa & tion Rates & & & & & Enrolment a & Projectio & FTE, Surplus & eats $=$ OTG R & ised - Proj & tion FTE, Util & ations $=$ Proj & tion/OTG F & vised & & & \\
\hline School Name & $\begin{array}{c}\text { TDSB 2013-14 } \\
\text { TDSB Participation } \\
\text { Rate }\end{array}$ & $\begin{array}{c}\text { TDSB 2013-14 } \\
\text { Home School } \\
\text { Participation Rate }\end{array}$ & $\begin{array}{c}\text { Revised } \\
\text { Capacity } \\
2016\end{array}$ & $\begin{array}{c}\text { HC } \\
\text { (Oct. 2016) }\end{array}$ & $\begin{array}{c}\text { FTE } \\
\text { (Oct. 2016) }\end{array}$ & $\begin{array}{c}2016 \\
\text { Utilization } \\
\text { Rate }\end{array}$ & $\begin{array}{c}2021 \\
\text { Projected }\end{array}$ & $\begin{array}{l}2021 \\
\text { Surplus } \\
\text { Seats }\end{array}$ & $\begin{array}{c}2021 \\
\text { Utilization } \\
\text { Rate }\end{array}$ & $\begin{array}{c}2026 \\
\text { Projected }\end{array}$ & $\begin{array}{l}2026 \\
\text { Surplus } \\
\text { Seats }\end{array}$ & $\begin{array}{c}2026 \\
\text { Utilization } \\
\text { Rate }\end{array}$ & $\begin{array}{c}2031 \\
\text { Projected }\end{array}$ & $\begin{array}{l}2031 \\
\text { Surplus } \\
\text { Seats }\end{array}$ & $\begin{array}{c}2031 \\
\text { Utilization } \\
\text { Rate }\end{array}$ & $\begin{array}{c}2036 \\
\text { Projected }\end{array}$ & $\begin{array}{l}2036 \\
\text { Surplus } \\
\text { Seats }\end{array}$ & $\begin{array}{c}2036 \\
\text { Utilization } \\
\text { Rate }\end{array}$ \\
\hline $\begin{array}{l}\text { Sir Alexander Mackenzie Senior } \\
\text { Public School }\end{array}$ & $90.0 \%$ & $80.7 \%$ & 401 & 387 & 387.0 & $97 \%$ & 421 & -20 & $105 \%$ & 414 & -13 & $103 \%$ & 391 & 10 & $98 \%$ & 386 & 15 & $96 \%$ \\
\hline $\begin{array}{l}\text { Sir Ernest MacMillan Senior Public } \\
\text { School }\end{array}$ & $67.8 \%$ & $50.2 \%$ & 354 & 295 & 295.0 & $83 \%$ & 299 & 55 & $84 \%$ & 296 & 58 & $84 \%$ & 287 & 67 & $81 \%$ & 287 & 67 & $81 \%$ \\
\hline $\begin{array}{l}\text { Sir Samuel B Steele Junior Public } \\
\text { School }\end{array}$ & $59.7 \%$ & $35.4 \%$ & 462 & 409 & 409.0 & $89 \%$ & 399 & 63 & $86 \%$ & 400 & 62 & $87 \%$ & 400 & 62 & $87 \%$ & 400 & 62 & $87 \%$ \\
\hline Sloane Public School & $78.1 \%$ & $56.3 \%$ & 268 & 259 & 259.0 & $97 \%$ & 258 & 10 & $96 \%$ & 257 & 11 & $96 \%$ & 256 & 12 & $96 \%$ & 256 & 12 & $96 \%$ \\
\hline Smithfield Middle School & $68.0 \%$ & $59.0 \%$ & 617 & 619 & 619.0 & $100 \%$ & 473 & 144 & $77 \%$ & 395 & 222 & $64 \%$ & 421 & 196 & $68 \%$ & 421 & 196 & $68 \%$ \\
\hline Spectrum Alternative Senior School & & & 69 & 59 & 59.0 & $86 \%$ & 60 & 9 & $87 \%$ & 60 & 9 & $87 \%$ & 60 & 9 & $87 \%$ & 60 & 9 & $87 \%$ \\
\hline Sprucecourt Public School & $72.3 \%$ & $42.8 \%$ & 458 & 335 & 335.0 & $73 \%$ & 266 & 192 & $58 \%$ & 255 & 203 & $56 \%$ & 255 & 203 & $56 \%$ & 255 & 203 & $56 \%$ \\
\hline St Andrews Public School & $60.9 \%$ & $51.5 \%$ & 372 & 359 & 359.0 & $97 \%$ & 374 & -2 & $101 \%$ & 361 & 11 & $97 \%$ & 356 & 16 & $96 \%$ & 346 & 26 & $93 \%$ \\
\hline St George's Junior School & $72.4 \%$ & $62.7 \%$ & 254 & 197 & 197.0 & $78 \%$ & 221 & 33 & $87 \%$ & 208 & 46 & $82 \%$ & 208 & 46 & $82 \%$ & 208 & 46 & $82 \%$ \\
\hline St Margaret's Public School & $74.4 \%$ & $56.3 \%$ & 245 & 320 & 320.0 & $131 \%$ & 287 & -42 & $117 \%$ & 281 & -36 & $115 \%$ & 287 & -42 & $117 \%$ & 287 & -42 & $117 \%$ \\
\hline Stanley Public School & $58.4 \%$ & $45.7 \%$ & 524 & 332 & 332.0 & $63 \%$ & 286 & 238 & $55 \%$ & 289 & 235 & $55 \%$ & 289 & 235 & $55 \%$ & 289 & 235 & $55 \%$ \\
\hline Steelesview Public School & $78.5 \%$ & $54.5 \%$ & 360 & 291 & 291.0 & $81 \%$ & 280 & 80 & $78 \%$ & 282 & 78 & $78 \%$ & 282 & 78 & $78 \%$ & 282 & 78 & $78 \%$ \\
\hline Stilecroft Public School & $58.5 \%$ & $40.1 \%$ & 345 & 271 & 271.0 & $79 \%$ & 263 & 82 & $76 \%$ & 265 & 80 & $77 \%$ & 265 & 80 & $77 \%$ & 265 & 80 & $77 \%$ \\
\hline Summit Heights Public School & $71.5 \%$ & $67.2 \%$ & 326 & 368 & 368.0 & $113 \%$ & 328 & -2 & $101 \%$ & 318 & 8 & $98 \%$ & 322 & 4 & $99 \%$ & 330 & -4 & $101 \%$ \\
\hline $\begin{array}{l}\text { Sunny View Junior and Senior Public } \\
\text { School }\end{array}$ & & & 243 & 73 & 73.0 & $30 \%$ & 85 & 158 & $35 \%$ & 90 & 153 & $37 \%$ & 90 & 153 & $37 \%$ & 90 & 153 & $37 \%$ \\
\hline Sunnylea Junior School & $73.8 \%$ & $71.1 \%$ & 291 & 294 & 294.0 & $101 \%$ & 321 & -30 & $110 \%$ & 306 & -15 & $105 \%$ & 306 & -15 & $105 \%$ & 306 & -15 & $105 \%$ \\
\hline $\begin{array}{l}\text { Swansea Junior and Senior Public } \\
\text { School }\end{array}$ & $63.7 \%$ & $50.7 \%$ & 882 & 857 & 857.0 & $97 \%$ & 883 & -1 & $100 \%$ & 868 & 14 & $98 \%$ & 863 & 19 & $\mathbf{9 8 \%}$ & 863 & 19 & $\mathbf{9 8 \%}$ \\
\hline Tam O'Shanter Junior Public School & $83.2 \%$ & $72.4 \%$ & 271 & 319 & 319.0 & $118 \%$ & 394 & -123 & $145 \%$ & 371 & -100 & $137 \%$ & 367 & -96 & $135 \%$ & 367 & -96 & $135 \%$ \\
\hline
\end{tabular}




\begin{tabular}{|c|c|c|c|c|c|c|c|c|c|c|c|c|c|c|c|c|c|c|c|c|c|c|}
\hline \multirow[b]{2}{*}{ School Name } & \multicolumn{8}{|c|}{ General Information } & \multicolumn{14}{|c|}{ Facility Information } \\
\hline & Panel & $\begin{array}{l}\text { Grade } \\
\text { Range }\end{array}$ & $\begin{array}{c}\text { Semestered } \\
\text { (Sec) }\end{array}$ & Ward & Trustee & $\begin{array}{l}\text { Superintendent } \\
\text { of Education }\end{array}$ & 2017 LOI & $\begin{array}{c}2017-18 \\
\mathrm{Opt} \\
\text { Attend } \\
\mathrm{Reg} / \mathrm{F} / \mathrm{EF}\end{array}$ & $\begin{array}{l}\text { Site Size } \\
\text { (Acres) }\end{array}$ & $\begin{array}{c}\text { Facility Size } \\
\text { (Sq. Ft.) }\end{array}$ & $\begin{array}{c}\text { Current } \\
\text { Backlog (M) }\end{array}$ & \begin{tabular}{|c} 
Port. \\
(Instruc)
\end{tabular} & $\begin{array}{l}\text { Port. (Non- } \\
\text { Instruc) }\end{array}$ & $\begin{array}{c}\text { Current FCI } \\
\text { (EDU) }\end{array}$ & $\begin{array}{l}\text { Eco } \\
\text { Schools }\end{array}$ & Pool & $\begin{array}{l}\text { Elem. } \\
\text { D\&T } \\
\text { Shops }\end{array}$ & $\begin{array}{l}\text { Barrier } \\
\text { Free / } \\
\text { Access }\end{array}$ & Elevator & $\begin{array}{l}\text { Build } \\
\text { Date }\end{array}$ & $\begin{array}{c}\text { Building } \\
\text { Age }\end{array}$ & $\begin{array}{l}\text { T.O Comm } \\
\text { Centre }\end{array}$ \\
\hline Tecumseh Senior Public School & E & $7-8$ & & 19 & David Smith & Anne Seymour & 88 & $\begin{array}{c}\text { Closed } \\
\text { N/A } \\
\text { N/A }\end{array}$ & 3.81 & 40,512 & $\$ 3.5$ & & & $58 \%$ & Bronze & & Yes & & & 1968 & 49 & \\
\hline Terraview-Willowfield Public School & E & JK-8 & & 20 & Manna Wong & Lynn Strangway & 257 & $\begin{array}{c}\text { Closed } \\
\text { N/A } \\
\text { N/A }\end{array}$ & 4.45 & 31,914 & $\$ 5.4$ & & & $72 \%$ & & & & & & 1957 & 60 & \\
\hline Terry Fox Public School & $\mathrm{E}$ & $\mathrm{JK}-8$ & & 20 & Manna Wong & \begin{tabular}{|l} 
Louie \\
Papathanasakis
\end{tabular} & 312 & $\begin{array}{c}\text { Closed } \\
\text { N/A } \\
\text { N/A }\end{array}$ & 5.29 & 41,232 & $\$ 5.1$ & 9 & & $68 \%$ & Gold & & & & & 1982 & 35 & \\
\hline The Elms Junior Middle School & E & JK-8 & & 1 & Avtar Minhas & Glenford Duffus & 30 & $\begin{array}{c}\text { Limited } \\
\text { N/A } \\
\text { N/A }\end{array}$ & 12.01 & 101,694 & $\$ 4.1$ & & & $25 \%$ & & & & & & 1960 & 57 & Cat. 1 \\
\hline The Grove Community School & E & JK-6 & & 9 & Marit Stiles & $\begin{array}{l}\text { Jane Phillips- } \\
\text { Long }\end{array}$ & 319 & $\begin{array}{c}\text { Limited } \\
\text { N/A } \\
\text { N/A }\end{array}$ & & & & & & & Gold & & & & & 1924 & 93 & \\
\hline The Waterfront School & E & JK-8 & & 10 & Ausma Malik & $\begin{array}{l}\text { Mary Jane } \\
\text { McNamara }\end{array}$ & 281 & $\begin{array}{c}\text { Closed } \\
\text { N/A } \\
\text { N/A }\end{array}$ & 3.76 & 62,607 & $\$ 1.6$ & & & $13 \%$ & Platinum & & & A1 & Yes & 1997 & 20 & \\
\hline Thomas L Wells Public School & E & JK-8 & & 21 & Abdul Hai Patel & Andrew Howard & 343 & $\begin{array}{c}\text { Closed } \\
\text { N/A } \\
\text { N/A }\end{array}$ & 2.97 & 65,354 & 0 & & & $0 \%$ & & & & A1 & Yes & 2005 & 12 & \\
\hline Thorncliffe Park Public School & E & $1-5$ & & 13 & Gerri Gershon & Ian Allison & 173 & $\begin{array}{c}\text { Closed } \\
\text { N/A } \\
\text { N/A }\end{array}$ & 5.94 & 149,404 & $\$ 1.4$ & 1 & 1 & $4 \%$ & & & & A1 & & 1961 & 56 & \\
\hline Three Valleys Public School & $\mathrm{E}$ & JK-5 & & 17 & Ken Lister & Beth Veale & 307 & $\begin{array}{c}\text { Closed } \\
\text { N/A } \\
\text { N/A }\end{array}$ & 4.99 & 32,077 & $\$ 4.9$ & 3 & & $79 \%$ & Gold & & & & & 1956 & 61 & \\
\hline Timberbank Junior Public School & E & JK-6 & & 20 & Manna Wong & Lynn Strangway & 314 & $\begin{array}{c}\text { Limited } \\
\text { N/A } \\
\text { N/A }\end{array}$ & 6 & 45,262 & $\$ 7.4$ & & & $94 \%$ & Gold & & & & & 1970 & 47 & \\
\hline Tom Longboat Junior Public School & E & JK-6 & & 21 & Abdul Hai Patel & Andrew Howard & 156 & $\begin{array}{c}\text { Limited } \\
\text { N/A } \\
\text { N/A }\end{array}$ & 4.99 & 53,176 & $\$ 1.5$ & & & $15 \%$ & & & & & Yes & 1978 & 39 & \\
\hline Topcliff Public School & E & JK-5 & & 4 & Tiffany Ford & Lynn Strangway & 17 & $\begin{array}{c}\text { Limited } \\
\text { Limited } \\
\text { N/A }\end{array}$ & 5.68 & 51,743 & $\$ 4.6$ & & & $45 \%$ & & & & & & 1964 & 53 & \\
\hline Tredway Woodsworth Public School & E & JK-8 & & 19 & David Smith & Shirley Chan & 111 & $\begin{array}{c}\text { Closed } \\
\text { Closed } \\
\text { N/A }\end{array}$ & 1.9 & 66,264 & $\$ 12.9$ & & 1 & $75 \%$ & & & Yes & & & 1955 & 62 & \\
\hline Tumpane Public School & E & JK-5 & & 5 & Alexandra Lulka & Elizabeth Addo & 39 & $\begin{array}{c}\text { Closed } \\
\text { Closed } \\
\text { N/A }\end{array}$ & 6.35 & 38,244 & $\$ 5.6$ & 4 & & $63 \%$ & Silver & & & & & 1950 & 67 & \\
\hline Twentieth Street Junior School & E & JK-5 & & 3 & Pamela Gough & Sandra Tondat & 134 & $\begin{array}{c}\text { Limited } \\
\text { N/A } \\
\text { N/A }\end{array}$ & 2.72 & 45,284 & $\$ 1.8$ & & & $24 \%$ & Bronze & & & A1 & Yes & 1993 & 24 & \\
\hline Valley Park Middle School & E & 6-8 & & 13 & Gerri Gershon & Ian Allison & 148 & $\begin{array}{c}\text { Closed } \\
\text { N/A } \\
\text { Closed }\end{array}$ & 7.44 & 116,186 & $\$ 3.9$ & & & $19 \%$ & Platinum & & & A1 & Yes & 1970 & 47 & \\
\hline Valleyfield Junior School & E & JK-5 & & 2 & Chris Glover & $\begin{array}{l}\text { Angela Nardi- } \\
\text { Addesa }\end{array}$ & 49 & $\begin{array}{c}\text { Limited } \\
\text { Limited } \\
\text { N/A }\end{array}$ & 6.23 & 32,844 & $\$ .9$ & & & $12 \%$ & Gold & & & & No & 1959 & 58 & \\
\hline Victoria Park Elementary School & E & JK-5 & & 16 & $\begin{array}{l}\text { Sheila Cary- } \\
\text { Meagher }\end{array}$ & Lucy Giannotta & 232 & $\begin{array}{c}\text { Limited } \\
\text { N/A } \\
\text { N/A }\end{array}$ & 2.21 & 23,960 & $\$ 4$. & 1 & & $77 \%$ & Gold & & & A1 & No & 1952 & 65 & \\
\hline
\end{tabular}




\begin{tabular}{|c|c|c|c|c|c|c|c|c|c|c|c|c|c|c|c|c|c|c|}
\hline & Participa & tion Rates & & & & & Enrolment a & Projectio & FTE, Surplus & eats $=$ OTG R & ised - Proj & tion FTE, Utili & itions $=$ Proj & ion/OTG R & vised & & & \\
\hline School Name & $\begin{array}{c}\text { TDSB 2013-14 } \\
\text { TDSB Participation } \\
\text { Rate }\end{array}$ & $\begin{array}{c}\text { TDSB } 2013-14 \\
\text { Home Schol } \\
\text { Participation Rate }\end{array}$ & $\begin{array}{c}\text { Revised } \\
\text { Capacity } \\
2016\end{array}$ & $\begin{array}{c}\text { HC } \\
\text { (Oct. 2016) }\end{array}$ & $\begin{array}{c}\text { FTE } \\
\text { (Oct. 2016) }\end{array}$ & $\begin{array}{c}2016 \\
\text { Utilization } \\
\text { Rate }\end{array}$ & $\begin{array}{c}2021 \\
\text { Projected }\end{array}$ & $\begin{array}{l}2021 \\
\text { Surplus } \\
\text { Seats }\end{array}$ & $\begin{array}{c}2021 \\
\text { Utilization } \\
\text { Rate }\end{array}$ & $\begin{array}{c}2026 \\
\text { Projected }\end{array}$ & $\begin{array}{c}2026 \\
\text { Surplus } \\
\text { Seats }\end{array}$ & $\begin{array}{c}2026 \\
\text { Utilization } \\
\text { Rate }\end{array}$ & $\begin{array}{c}2031 \\
\text { Projected }\end{array}$ & $\begin{array}{l}2031 \\
\text { Surplus } \\
\text { Seats }\end{array}$ & $\begin{array}{c}2031 \\
\text { Utilization } \\
\text { Rate }\end{array}$ & $\begin{array}{c}2036 \\
\text { Projected }\end{array}$ & $\begin{array}{l}2036 \\
\text { Surplus } \\
\text { Seats }\end{array}$ & $\begin{array}{c}2036 \\
\text { Utilization } \\
\text { Rate }\end{array}$ \\
\hline Tecumseh Senior Public School & $74.6 \%$ & $59.9 \%$ & 262 & 251 & 251.0 & $96 \%$ & 235 & 27 & $90 \%$ & 172 & 90 & $66 \%$ & 176 & 86 & $67 \%$ & 176 & 86 & $67 \%$ \\
\hline Terraview-Willowfield Public School & $69.0 \%$ & $55.3 \%$ & 309 & 298 & 298.0 & $96 \%$ & 343 & -34 & $111 \%$ & 358 & -49 & $116 \%$ & 378 & -69 & $122 \%$ & 377 & -68 & $122 \%$ \\
\hline Terry Fox Public School & $85.5 \%$ & $74.4 \%$ & 352 & 430 & 430.0 & $122 \%$ & 571 & -219 & $162 \%$ & 655 & -303 & $186 \%$ & 684 & -332 & $194 \%$ & 684 & -332 & $194 \%$ \\
\hline The Elms Junior Middle School & $51.0 \%$ & $30.0 \%$ & 654 & 366 & 365.0 & $56 \%$ & 291 & 363 & $44 \%$ & 287 & 367 & $44 \%$ & 291 & 363 & $44 \%$ & 291 & 363 & $44 \%$ \\
\hline The Grove Community School & & & 161 & 134 & 134.0 & $83 \%$ & 152 & 9 & $94 \%$ & 152 & 9 & $94 \%$ & 152 & 9 & $94 \%$ & 152 & 9 & $94 \%$ \\
\hline The Waterfront School & $69.3 \%$ & $45.5 \%$ & 268 & 208 & 208.0 & $78 \%$ & 238 & 30 & $89 \%$ & 240 & 28 & $\mathbf{9 0 \%}$ & 238 & 30 & $89 \%$ & 238 & 30 & $89 \%$ \\
\hline Thomas L Wells Public School & $74.0 \%$ & $67.3 \%$ & 663 & 587 & 587.0 & $89 \%$ & 493 & 170 & $74 \%$ & 436 & 227 & $66 \%$ & 424 & 239 & $64 \%$ & 417 & 246 & $63 \%$ \\
\hline Thorncliffe Park Public School & $77.7 \%$ & $72.3 \%$ & 1590 & 1,422 & $1,420.0$ & $89 \%$ & 1,232 & 358 & $77 \%$ & 1,175 & 415 & $74 \%$ & 1,170 & 420 & $74 \%$ & 1,170 & 420 & $74 \%$ \\
\hline Three Valleys Public School & $73.0 \%$ & $58.2 \%$ & 291 & 277 & 277.0 & $95 \%$ & 259 & 32 & $89 \%$ & 278 & 13 & $96 \%$ & 285 & 6 & $98 \%$ & 285 & 6 & $\mathbf{9 8 \%}$ \\
\hline Timberbank Junior Public School & $58.8 \%$ & $51.8 \%$ & 311 & 229 & 229.0 & $74 \%$ & 218 & 93 & $70 \%$ & 209 & 102 & $67 \%$ & 209 & 102 & $67 \%$ & 209 & 102 & $67 \%$ \\
\hline Tom Longboat Junior Public School & $69.2 \%$ & $58.4 \%$ & 441 & 323 & 323.0 & $73 \%$ & 231 & 210 & $52 \%$ & 207 & 234 & $47 \%$ & 207 & 234 & $47 \%$ & 207 & 234 & $47 \%$ \\
\hline Topcliff Public School & $80.3 \%$ & $65.2 \%$ & 505 & 380 & 380.0 & $75 \%$ & 333 & 172 & $66 \%$ & 346 & 159 & $69 \%$ & 346 & 159 & $69 \%$ & 346 & 159 & $69 \%$ \\
\hline Tredway Woodsworth Public School & $65.0 \%$ & $56.1 \%$ & 883 & 744 & 744.0 & $84 \%$ & 898 & -15 & $102 \%$ & 901 & -18 & $102 \%$ & 916 & -33 & $104 \%$ & 917 & -34 & $104 \%$ \\
\hline Tumpane Public School & $32.1 \%$ & $26.3 \%$ & 474 & 440 & 440.0 & $93 \%$ & 453 & 21 & $96 \%$ & 450 & 24 & $95 \%$ & 449 & 25 & $95 \%$ & 449 & 25 & $95 \%$ \\
\hline Twentieth Street Junior School & $83.2 \%$ & $37.3 \%$ & 304 & 186 & 185.5 & $61 \%$ & 225 & 79 & $74 \%$ & 204 & 100 & $67 \%$ & 204 & 100 & $67 \%$ & 204 & 100 & $67 \%$ \\
\hline Valley Park Middle School & $60.6 \%$ & $50.7 \%$ & 1068 & 910 & 910.0 & $85 \%$ & 841 & 227 & $79 \%$ & 787 & 281 & $74 \%$ & 786 & 282 & $74 \%$ & 786 & 282 & $74 \%$ \\
\hline Valleyfield Junior School & $41.7 \%$ & $30.6 \%$ & 375 & 300 & 300.0 & $80 \%$ & 271 & 104 & $72 \%$ & 269 & 106 & $72 \%$ & 269 & 106 & $72 \%$ & 269 & 106 & $72 \%$ \\
\hline Victoria Park Elementary School & $71.2 \%$ & $53.8 \%$ & 143 & 157 & 157.0 & $110 \%$ & 175 & -32 & $122 \%$ & 180 & -37 & $126 \%$ & 185 & -42 & $129 \%$ & 185 & -42 & $129 \%$ \\
\hline
\end{tabular}




\begin{tabular}{|c|c|c|c|c|c|c|c|c|c|c|c|c|c|c|c|c|c|c|c|c|c|c|}
\hline \multirow[b]{2}{*}{ School Name } & \multicolumn{8}{|c|}{ General Information } & \multicolumn{14}{|c|}{ Facility Information } \\
\hline & Panel & $\begin{array}{l}\text { Grade } \\
\text { Range }\end{array}$ & $\begin{array}{c}\text { Semestered } \\
\text { (Sec) }\end{array}$ & Ward & Trustee & $\begin{array}{l}\text { Superintendent } \\
\text { of Education }\end{array}$ & 2017 LOI & $\begin{array}{c}2017-18 \\
\mathrm{Opt} \\
\text { Attend } \\
\mathrm{Reg} / \mathrm{F} / \mathrm{EF}\end{array}$ & $\begin{array}{l}\text { Site Size } \\
\text { (Acres) }\end{array}$ & $\begin{array}{c}\text { Facility Size } \\
\text { (Sq. Ft.) }\end{array}$ & $\begin{array}{c}\text { Current } \\
\text { Backlog (M) }\end{array}$ & \begin{tabular}{|c} 
Port. \\
(Instruc)
\end{tabular} & $\begin{array}{l}\text { Port. (Non- } \\
\text { Instruc) }\end{array}$ & $\begin{array}{c}\text { Current FCI } \\
\text { (EDU) }\end{array}$ & $\begin{array}{l}\text { Eco } \\
\text { Schools }\end{array}$ & Pool & $\begin{array}{l}\text { Elem. } \\
\text { D\&T } \\
\text { Shops }\end{array}$ & $\begin{array}{l}\text { Barrier } \\
\text { Free / } \\
\text { Access }\end{array}$ & Elevator & $\begin{array}{l}\text { Build } \\
\text { Date }\end{array}$ & $\begin{array}{c}\text { Building } \\
\text { Age }\end{array}$ & $\begin{array}{l}\text { T.O Comm } \\
\text { Centre }\end{array}$ \\
\hline Victoria Village Public School & E & JK-5 & & 17 & Ken Lister & Curtis Ennis & 202 & $\begin{array}{c}\text { Closed } \\
\text { N/A } \\
\text { N/A }\end{array}$ & 4.92 & 45,327 & $\$ 2.3$ & 1 & & $32 \%$ & & & & & & 1958 & 59 & \\
\hline Vradenburg Junior Public School & E & JK-6 & & 20 & Manna Wong & $\begin{array}{l}\text { Louie } \\
\text { Papathanasakis }\end{array}$ & 329 & $\begin{array}{c}\text { Limited } \\
\text { N/A } \\
\text { N/A }\end{array}$ & 5.02 & 39,986 & $\$ 3.4$ & & & $41 \%$ & Gold & & & & & 1956 & 61 & \\
\hline Walter Perry Junior Public School & $\mathrm{E}$ & $\mathrm{JK}-6$ & & 18 & Parthi Kandavel & Peter Chang & 93 & $\begin{array}{c}\text { Limited } \\
\text { N/A } \\
\text { N/A }\end{array}$ & 4.79 & 38,090 & $\$ 5.3$ & & & $65 \%$ & Silver & & & & & 1953 & 64 & \\
\hline Warden Avenue Public School & E & $\mathrm{JK}-8$ & & 18 & Parthi Kandavel & Peter Chang & 56 & $\begin{array}{c}\text { Closed } \\
\text { N/A } \\
\text { N/A }\end{array}$ & 10.48 & 51,631 & $\$ 3.9$ & & & $36 \%$ & Platinum & & & & & 1951 & 66 & \\
\hline Warren Park Junior Public School & E & JK-6 & & 7 & Robin Pilkey & Tracy Hayhurst & 87 & $\begin{array}{c}\text { Limited } \\
\text { N/A } \\
\text { N/A }\end{array}$ & 3.26 & 31,783 & $\$ 2.5$ & 3 & & $38 \%$ & Platinum & & & & & 1930 & 87 & \\
\hline Wedgewood Junior School & E & JK-5 & & 3 & Pamela Gough & Tracy Hayhurst & 418 & $\begin{array}{c}\text { Closed } \\
\text { N/A } \\
\text { N/A }\end{array}$ & 7.09 & 34,488 & $\$ 6.8$ & 1 & & $86 \%$ & Platinum & & & & & 1950 & 67 & \\
\hline Wellesworth Junior School & E & JK-5 & & 2 & Chris Glover & Glenford Duffus & 128 & $\begin{array}{c}\text { Limited } \\
\text { N/A } \\
\text { N/A }\end{array}$ & 6.8 & 33,939 & $\$ 3.9$ & & & $55 \%$ & Platinum & & & B1 & & 1960 & 57 & \\
\hline West Glen Junior School & E & JK-5 & & 2 & Chris Glover & Glenford Duffus & 71 & $\begin{array}{c}\text { Limited } \\
\text { N/A } \\
\text { N/A }\end{array}$ & 6.47 & 25,801 & $\$ 2.6$ & & & $42 \%$ & & & & & & 1953 & 64 & \\
\hline West Hill Public School & $\mathrm{E}$ & JK-8 & & 22 & Jerry Chadwick & Nadira Persaud & 60 & $\begin{array}{c}\text { Closed } \\
\text { N/A } \\
\text { N/A }\end{array}$ & 3.24 & 42,913 & $\$ .7$ & 1 & & $10 \%$ & & & & A1 & Yes & 1994 & 23 & \\
\hline West Humber Junior Middle School & E & JK-8 & & 1 & Avtar Minhas & Annie Appleby & 285 & $\begin{array}{c}\text { Closed } \\
\text { N/A } \\
\text { N/A }\end{array}$ & 6.52 & 48,793 & $\$ 2.5$ & & & $25 \%$ & & & & A1 & No & 1957 & 60 & \\
\hline West Preparatory Junior Public School & E & JK-6 & & 11 & Shelley Laskin & Leila Girdhar-Hill & 346 & $\begin{array}{c}\text { Closed } \\
\text { N/A } \\
\text { N/A }\end{array}$ & 4.05 & 60,729 & $\$ 5.1$ & & & $47 \%$ & Gold & & & & & 1940 & 77 & \\
\hline West Rouge Junior Public School & E & JK-6 & & 22 & Jerry Chadwick & $\begin{array}{l}\text { Kerry-Lynn } \\
\text { Stadnyk }\end{array}$ & 395 & $\begin{array}{c}\text { Closed } \\
\text { N/A } \\
\text { N/A }\end{array}$ & 7.51 & 32,744 & $\$ 1.7$ & & & $23 \%$ & Platinum & & & & & 1953 & 64 & \\
\hline Westmount Junior School & E & JK-5 & & 1 & Avtar Minhas & Glenford Duffus & 82 & $\begin{array}{c}\text { Limited } \\
\text { N/A } \\
\text { N/A }\end{array}$ & 6.3 & 37,448 & $\$ 2.4$ & & & $30 \%$ & Gold & & & & No & 1960 & 57 & \\
\hline $\begin{array}{l}\text { Weston Memorial Junior Public } \\
\text { School }\end{array}$ & $\mathrm{E}$ & JK-5 & & 6 & Chris Tonks & $\begin{array}{l}\text { Angela Nardi- } \\
\text { Addesa }\end{array}$ & 182 & $\begin{array}{c}\text { Closed } \\
\text { N/A } \\
\text { N/A }\end{array}$ & 2.62 & 43,024 & $\$ 3.9$ & & & $48 \%$ & & & & & & 1919 & 98 & \\
\hline Westway Junior School & E & JK-5 & & 2 & Chris Glover & $\begin{array}{l}\text { Angela Nardi- } \\
\text { Addesa }\end{array}$ & 122 & $\begin{array}{c}\text { Closed } \\
\text { N/A } \\
\text { N/A }\end{array}$ & 6.18 & 27,620 & $\$ 3.9$ & 2 & & $63 \%$ & Gold & & & & & 1957 & 60 & \\
\hline Westwood Middle School & E & 6-8 & & 15 & Jennifer Story & Lucy Giannotta & 251 & $\begin{array}{c}\text { Limited } \\
\mathrm{N} / \mathrm{A} \\
\text { Limited }\end{array}$ & 3.75 & 71,188 & $\$ 2.8$ & & & $29 \%$ & & & Yes & & & 1932 & 85 & \\
\hline Wexford Public School & E & JK-8 & & 19 & David Smith & Nadira Persaud & 107 & $\begin{array}{c}\text { Closed } \\
\text { N/A } \\
\text { N/A }\end{array}$ & 6.99 & 39,031 & $\$ 3$. & & & $31 \%$ & Silver & & & & & 1951 & 66 & \\
\hline White Haven Public School & E & JK-8 & & 21 & Abdul Hai Patel & Andrew Howard & 233 & $\begin{array}{c}\text { Closed } \\
\text { N/A } \\
\text { N/A }\end{array}$ & 6.05 & 69,041 & $\$ 1.9$ & & & $15 \%$ & Gold & & & & & 1968 & 49 & \\
\hline
\end{tabular}




\begin{tabular}{|c|c|c|c|c|c|c|c|c|c|c|c|c|c|c|c|c|c|c|}
\hline & Participa & tion Rates & & & & & Enrolment a & Projectio & FTE, Surplus & eats $=$ OTG R & ised - Proj & tion FTE, Utili & itions $=$ Proj & ion/OTG I & vised & & & \\
\hline School Name & $\begin{array}{c}\text { TSSB 2013-14 } \\
\text { TDSB Participation } \\
\text { Rate }\end{array}$ & $\begin{array}{c}\text { TDSB } 2013-14 \\
\text { Home Schol } \\
\text { Participation Rate }\end{array}$ & $\begin{array}{c}\text { Revised } \\
\text { Capacity } \\
2016\end{array}$ & $\begin{array}{c}\text { HC } \\
\text { (Oct. 2016) }\end{array}$ & $\begin{array}{c}\text { FTE } \\
\text { (Oct. 2016) }\end{array}$ & $\begin{array}{c}2016 \\
\text { Utilization } \\
\text { Rate }\end{array}$ & $\begin{array}{c}2021 \\
\text { Projected }\end{array}$ & $\begin{array}{c}2021 \\
\text { Surplus } \\
\text { Seats }\end{array}$ & $\begin{array}{c}2021 \\
\text { Utilization } \\
\text { Rate }\end{array}$ & $\begin{array}{c}2026 \\
\text { Projected }\end{array}$ & $\begin{array}{c}2026 \\
\text { Surplus } \\
\text { Seats }\end{array}$ & $\begin{array}{c}2026 \\
\text { Utilization } \\
\text { Rate }\end{array}$ & $\begin{array}{c}2031 \\
\text { Projected }\end{array}$ & $\begin{array}{l}2031 \\
\text { Surplus } \\
\text { Seats }\end{array}$ & $\begin{array}{c}2031 \\
\text { Utilization } \\
\text { Rate }\end{array}$ & $\begin{array}{c}2036 \\
\text { Projected }\end{array}$ & $\begin{array}{l}2036 \\
\text { Surplus } \\
\text { Seats }\end{array}$ & $\begin{array}{c}2036 \\
\text { Utilization } \\
\text { Rate }\end{array}$ \\
\hline Victoria Village Public School & $61.6 \%$ & $47.7 \%$ & 326 & 291 & 291.0 & $89 \%$ & 198 & 128 & $61 \%$ & 218 & 108 & $67 \%$ & 222 & 104 & $68 \%$ & 222 & 104 & $68 \%$ \\
\hline Vradenburg Junior Public School & $70.4 \%$ & $62.7 \%$ & 340 & 252 & 252.0 & $74 \%$ & 235 & 105 & $69 \%$ & 218 & 122 & $64 \%$ & 215 & 125 & $63 \%$ & 215 & 125 & $63 \%$ \\
\hline Walter Perry Junior Public School & $65.9 \%$ & $52.2 \%$ & 392 & 313 & 313.0 & $80 \%$ & 288 & 104 & $73 \%$ & 291 & 101 & $74 \%$ & 291 & 101 & $74 \%$ & 291 & 101 & $74 \%$ \\
\hline Warden Avenue Public School & $66.1 \%$ & $45.0 \%$ & 513 & 383 & 383.0 & $75 \%$ & 400 & 113 & $78 \%$ & 397 & 116 & $77 \%$ & 392 & 121 & $76 \%$ & 392 & 121 & $76 \%$ \\
\hline Warren Park Junior Public School & $71.2 \%$ & $41.2 \%$ & 229 & 201 & 201.0 & $88 \%$ & 212 & 17 & $93 \%$ & 200 & 29 & $87 \%$ & 198 & 31 & $86 \%$ & 198 & 31 & $86 \%$ \\
\hline Wedgewood Junior School & $54.0 \%$ & $42.0 \%$ & 438 & 439 & 439.0 & $100 \%$ & 607 & -169 & $139 \%$ & 598 & -160 & $137 \%$ & 598 & -160 & $137 \%$ & 598 & -160 & $137 \%$ \\
\hline Wellesworth Junior School & $57.3 \%$ & $39.2 \%$ & 330 & 157 & 157.0 & $48 \%$ & 148 & 182 & $45 \%$ & 150 & 180 & $45 \%$ & 150 & 180 & $45 \%$ & 150 & 180 & $45 \%$ \\
\hline West Glen Junior School & $50.0 \%$ & $36.4 \%$ & 217 & 176 & 176.0 & $81 \%$ & 152 & 65 & $70 \%$ & 156 & 61 & $72 \%$ & 156 & 61 & $72 \%$ & 156 & 61 & $72 \%$ \\
\hline West Hill Public School & $93.2 \%$ & $72.4 \%$ & 300 & 231 & 231.0 & $77 \%$ & 269 & 31 & $90 \%$ & 292 & 8 & $97 \%$ & 297 & 3 & $\mathbf{9 9 \%}$ & 297 & 3 & $\mathbf{9 9 \%}$ \\
\hline West Humber Junior Middle School & $56.3 \%$ & $47.8 \%$ & 546 & 487 & 487.0 & $89 \%$ & 421 & 125 & $77 \%$ & 414 & 132 & $76 \%$ & 418 & 128 & $77 \%$ & 418 & 128 & $77 \%$ \\
\hline West Preparatory Junior Public School & $57.0 \%$ & $44.4 \%$ & 529 & 538 & 538.0 & $102 \%$ & 552 & -23 & $104 \%$ & 572 & -43 & $108 \%$ & 572 & -43 & $108 \%$ & 572 & -43 & $108 \%$ \\
\hline West Rouge Junior Public School & $86.6 \%$ & $73.6 \%$ & 329 & 257 & 257.0 & $78 \%$ & 321 & 8 & $98 \%$ & 350 & -21 & $106 \%$ & 350 & -21 & $106 \%$ & 350 & -21 & $106 \%$ \\
\hline Westmount Junior School & $33.4 \%$ & $24.8 \%$ & 347 & 243 & 243.0 & $70 \%$ & 220 & 127 & $63 \%$ & 233 & 114 & $67 \%$ & 233 & 114 & $67 \%$ & 233 & 114 & $67 \%$ \\
\hline $\begin{array}{l}\text { Weston Memorial Junior Public } \\
\text { School }\end{array}$ & $67.7 \%$ & $58.7 \%$ & 352 & 320 & 320.0 & $91 \%$ & 247 & 105 & $70 \%$ & 231 & 121 & $66 \%$ & 240 & 112 & $68 \%$ & 254 & 98 & $72 \%$ \\
\hline Westway Junior School & $54.8 \%$ & $38.5 \%$ & 251 & 260 & 260.0 & $104 \%$ & 252 & -1 & $100 \%$ & 243 & 8 & $97 \%$ & 243 & 8 & $97 \%$ & 243 & 8 & $97 \%$ \\
\hline Westwood Middle School & $74.5 \%$ & $51.7 \%$ & 398 & 373 & 373.0 & $94 \%$ & 381 & 17 & $96 \%$ & 368 & 30 & $92 \%$ & 371 & 27 & $93 \%$ & 371 & 27 & $93 \%$ \\
\hline Wexford Public School & $54.9 \%$ & $46.0 \%$ & 464 & 390 & 384.5 & $83 \%$ & 322 & 142 & $69 \%$ & 277 & 187 & $60 \%$ & 274 & 190 & $59 \%$ & 274 & 190 & $59 \%$ \\
\hline White Haven Public School & $67.9 \%$ & $60.0 \%$ & 586 & 528 & 528.0 & $90 \%$ & 496 & 90 & $85 \%$ & 494 & 92 & $84 \%$ & 493 & 93 & $84 \%$ & 493 & 93 & $84 \%$ \\
\hline
\end{tabular}




\begin{tabular}{|c|c|c|c|c|c|c|c|c|c|c|c|c|c|c|c|c|c|c|c|c|c|c|}
\hline \multirow[b]{2}{*}{ School Name } & \multicolumn{8}{|c|}{ General Information } & \multicolumn{14}{|c|}{ Facility Information } \\
\hline & Panel & $\begin{array}{l}\text { Grade } \\
\text { Range }\end{array}$ & $\begin{array}{c}\text { Semestered } \\
\text { (Sec) }\end{array}$ & Ward & Trustee & $\begin{array}{l}\text { Superintendent } \\
\text { of Education }\end{array}$ & 2017 LOI & $\begin{array}{c}2017-18 \\
\mathrm{Opt} \\
\text { Attend } \\
\mathrm{Reg} / \mathrm{F} / \mathrm{EF}\end{array}$ & $\begin{array}{l}\text { Site Size } \\
\text { (Acres) }\end{array}$ & $\begin{array}{c}\text { Facility Size } \\
\text { (Sq. Ft.) }\end{array}$ & $\begin{array}{c}\text { Current } \\
\text { Backlog (M) }\end{array}$ & \begin{tabular}{|c} 
Port. \\
(Instruc)
\end{tabular} & $\begin{array}{l}\text { Port. (Non- } \\
\text { Instruc) }\end{array}$ & $\begin{array}{c}\text { Current FCI } \\
\text { (EDU) }\end{array}$ & \begin{tabular}{|c|} 
Eco \\
Schools
\end{tabular} & Pool & $\begin{array}{l}\text { Elem. } \\
\text { D\&T } \\
\text { Shops }\end{array}$ & $\begin{array}{l}\text { Barrier } \\
\text { Free / } \\
\text { Access }\end{array}$ & Elevator & $\begin{array}{l}\text { Build } \\
\text { Date }\end{array}$ & $\begin{array}{c}\text { Building } \\
\text { Age }\end{array}$ & $\begin{array}{l}\text { T.O Comm } \\
\text { Centre }\end{array}$ \\
\hline Whitney Junior Public School & E & JK-6 & & 14 & Chris Moise & Mike Gallagher & 471 & \begin{tabular}{|c|} 
Limited \\
N/A \\
N/A \\
\end{tabular} & 2.89 & 61,133 & $\$ 2.9$ & & & $32 \%$ & & & & & & 1963 & 54 & \\
\hline Wilkinson Junior Public School & E & JK-6 & & 15 & Jennifer Story & Lucy Giannotta & 339 & $\begin{array}{c}\text { Closed } \\
\text { N/A } \\
\text { Limited }\end{array}$ & 6.03 & 88,046 & $\$ 6.2$ & & & $49 \%$ & Gold & & & & & 1914 & 103 & \\
\hline William Burgess Elementary School & $\mathrm{E}$ & JK-5 & & 15 & Jennifer Story & Lucy Giannotta & 206 & $\begin{array}{c}\text { Limited } \\
\text { N/A } \\
\text { N/A }\end{array}$ & 4.17 & 69,304 & $\$ 4.2$ & & & $43 \%$ & & & & & & 1914 & 103 & \\
\hline William G Davis Junior Public School & $\mathrm{E}$ & JK-6 & & 22 & Jerry Chadwick & $\begin{array}{l}\text { Kerry-Lynn } \\
\text { Stadnyk }\end{array}$ & 422 & $\begin{array}{c}\text { Limited } \\
\text { N/A } \\
\text { N/A }\end{array}$ & 5.46 & 35,884 & $\$ 4.1$ & & & $53 \%$ & Gold & & & & & 1966 & 51 & \\
\hline William G Miller Public School & E & JK-8 & & 22 & Jerry Chadwick & $\begin{array}{l}\text { Kerry-Lynn } \\
\text { Stadnyk }\end{array}$ & 197 & $\begin{array}{c}\text { Limited } \\
\text { Closed } \\
\text { N/A }\end{array}$ & 5.21 & 51,452 & $\$ 5.7$ & & & $51 \%$ & Bronze & & & & & 1958 & 59 & \\
\hline William J McCordic School & E & JK-8 & & 16 & $\begin{array}{l}\text { Sheila Cary- } \\
\text { Meagher }\end{array}$ & Lucy Giannotta & 133 & $\begin{array}{c}\text { Limited } \\
\text { N/A } \\
\text { N/A }\end{array}$ & 1.46 & 33,748 & $\$ 3.4$ & & & $79 \%$ & & & & & & 1973 & 44 & \\
\hline Williamson Road Junior Public School & E & JK-6 & & 16 & $\begin{array}{l}\text { Sheila Cary- } \\
\text { Meagher }\end{array}$ & John Chasty & 446 & $\begin{array}{c}\text { Closed } \\
\text { Limited } \\
\text { N/A }\end{array}$ & 3 & 70,575 & $\$ 3.9$ & & & $34 \%$ & Gold & & & & & 1912 & 105 & \\
\hline Willow Park Junior Public School & E & JK-6 & & 22 & Jerry Chadwick & Anne Seymour & 5 & $\begin{array}{c}\text { Limited } \\
\text { N/A } \\
\text { N/A }\end{array}$ & 6.1 & 50,052 & $\$ 3.1$ & & & $28 \%$ & Silver & & & & & 1964 & 53 & \\
\hline Willowdale Middle School & $\mathrm{E}$ & 6-8 & & 12 & Alexander Brown & Elizabeth Addo & 362 & $\begin{array}{l}\text { Closed } \\
\text { Closed } \\
\text { Closed }\end{array}$ & 7.24 & 67,124 & $\$ 2.9$ & & & $28 \%$ & Platinum & & Yes & & & 1958 & 59 & \\
\hline Wilmington Elementary School & E & $\mathrm{JK}-4$ & & 5 & Alexandra Lulka & Elizabeth Addo & 252 & $\begin{array}{c}\text { Closed } \\
\text { N/A } \\
\text { N/A }\end{array}$ & 4.69 & 32,184 & $\$ 5.1$ & 1 & 2 & $79 \%$ & & & & & & 1958 & 59 & \\
\hline $\begin{array}{l}\text { Winchester Junior and Senior Public } \\
\text { School (includes Annex) }\end{array}$ & E & SK-8 & & 14 & Chris Moise & $\begin{array}{l}\text { Jane Phillips- } \\
\text { Long }\end{array}$ & 127 & $\begin{array}{c}\text { Limited } \\
\text { Limited } \\
\text { N/A }\end{array}$ & 4.6 & 104,436 & $\$ 10.8$ & & & $82 \%$ & Gold & & Yes & & & 1898 & 119 & \\
\hline Winona Drive Senior Public School & E & $7-8$ & & 11 & Shelley Laskin & Kathleen Garner & 323 & $\begin{array}{c}\text { Limited } \\
\text { Closed } \\
\text { Limited }\end{array}$ & 3 & 93,920 & $\$ 10.7$ & 1 & & $117 \%$ & & TLC & Yes & & & 1958 & 59 & \\
\hline Withrow Avenue Junior Public School & E & JK-6 & & 15 & Jennifer Story & $\begin{array}{l}\text { Mary Jane } \\
\text { McNamara }\end{array}$ & 439 & $\begin{array}{c}\text { Limited } \\
\text { Closed } \\
\text { N/A }\end{array}$ & 3.56 & 71,106 & $\$ 6.9$ & & & $46 \%$ & & & & & & 1972 & 45 & \\
\hline Woburn Junior Public School & E & JK-6 & & 19 & David Smith & Shirley Chan & 89 & $\begin{array}{c}\text { Limited } \\
\text { N/A } \\
\text { N/A }\end{array}$ & 8.01 & 47,052 & $\$ 6.8$ & & 1 & $68 \%$ & Gold & & & & & 1963 & 54 & \\
\hline Woodbine Middle School & E & $7-8$ & & 17 & Ken Lister & Audley Salmon & 271 & $\begin{array}{c}\text { Limited } \\
\text { N/A } \\
\text { N/A }\end{array}$ & 8.01 & 106,338 & $\$ 8.3$ & & & $43 \%$ & & & Yes & A1 & & 1965 & 52 & \\
\hline Yorkview Public School & E & JK-5 & & 12 & Alexander Brown & \begin{tabular}{|l} 
Louie \\
Papathanasakis
\end{tabular} & 331 & $\begin{array}{l}\text { Closed } \\
\text { Closed } \\
\text { N/A }\end{array}$ & 5.19 & 51,979 & $\$ 5.6$ & & 1 & $57 \%$ & Platinum & & & & & 1955 & 62 & \\
\hline Yorkwoods Public School & E & JK-5 & & 4 & Tiffany Ford & Lynn Strangway & 1 & $\begin{array}{c}\text { Limited } \\
\text { N/A } \\
\text { N/A }\end{array}$ & 5.93 & 64,272 & $\$ 2.5$ & & & $20 \%$ & Silver & & & & & 1967 & 50 & \\
\hline Zion Heights Middle School & E & $6-8$ & & 12 & Alexander Brown & Elizabeth Addo & 342 & $\begin{array}{c}\text { Limited } \\
\text { N/A } \\
\text { N/A }\end{array}$ & 10.01 & 97,748 & $\$ 6.4$ & & & $52 \%$ & & & Yes & & & 1967 & 50 & \\
\hline
\end{tabular}




\begin{tabular}{|c|c|c|c|c|c|c|c|c|c|c|c|c|c|c|c|c|c|c|}
\hline & Participa & tion Rates & & & & & Enrolment a & Projectio & FTE, Surplus & eats $=$ OTG R & ised - Proj & tion FTE, Utili & itions $=$ Proj & ion/OTG I & vised & & & \\
\hline School Name & $\begin{array}{c}\text { TDSB 2013-14 } \\
\text { TDSB Participation } \\
\text { Rate }\end{array}$ & $\begin{array}{c}\text { TOSB 2013-14 } \\
\text { Home school } \\
\text { Participation Rate }\end{array}$ & $\begin{array}{c}\text { Revised } \\
\text { Capacity } \\
2016\end{array}$ & $\begin{array}{c}\text { HC } \\
\text { (Oct. 2016) }\end{array}$ & $\begin{array}{c}\text { FTE } \\
\text { (Oct. 2016) }\end{array}$ & $\begin{array}{c}2016 \\
\text { Utilization } \\
\text { Rate }\end{array}$ & $\begin{array}{c}2021 \\
\text { Projected }\end{array}$ & $\begin{array}{c}2021 \\
\text { Surplus } \\
\text { Seats }\end{array}$ & $\begin{array}{c}2021 \\
\text { Utilization } \\
\text { Rate }\end{array}$ & $\begin{array}{c}2026 \\
\text { Projected }\end{array}$ & $\begin{array}{c}2026 \\
\text { Surplus } \\
\text { Seats }\end{array}$ & $\begin{array}{c}2026 \\
\text { Utilization } \\
\text { Rate }\end{array}$ & $\begin{array}{c}2031 \\
\text { Projected }\end{array}$ & $\begin{array}{l}2031 \\
\text { Surplus } \\
\text { Seats }\end{array}$ & $\begin{array}{c}2031 \\
\text { Utilization } \\
\text { Rate }\end{array}$ & $\begin{array}{c}2036 \\
\text { Projected }\end{array}$ & $\begin{array}{l}2036 \\
\text { Surplus } \\
\text { Seats }\end{array}$ & $\begin{array}{c}2036 \\
\text { Utilization } \\
\text { Rate }\end{array}$ \\
\hline Whitney Junior Public School & $56.4 \%$ & $50.1 \%$ & 406 & 277 & 277.0 & $68 \%$ & 287 & 119 & $71 \%$ & 287 & 119 & $71 \%$ & 287 & 119 & $71 \%$ & 287 & 119 & $71 \%$ \\
\hline Wilkinson Junior Public School & $90.9 \%$ & $71.0 \%$ & 617 & 505 & 505.0 & $82 \%$ & 468 & 149 & $76 \%$ & 463 & 154 & $75 \%$ & 462 & 155 & $75 \%$ & 462 & 155 & $75 \%$ \\
\hline William Burgess Elementary School & $69.1 \%$ & $48.0 \%$ & 477 & 345 & 345.0 & $72 \%$ & 347 & 130 & $73 \%$ & 346 & 131 & $73 \%$ & 346 & 131 & $73 \%$ & 346 & 131 & $73 \%$ \\
\hline William G Davis Junior Public School & $70.9 \%$ & $58.9 \%$ & 337 & 248 & 248.0 & $74 \%$ & 228 & 109 & $68 \%$ & 231 & 106 & $69 \%$ & 231 & 106 & $69 \%$ & 231 & 106 & $69 \%$ \\
\hline William G Miller Public School & $60.1 \%$ & $47.6 \%$ & 573 & 506 & 506.0 & $88 \%$ & 561 & 12 & $98 \%$ & 579 & -6 & $101 \%$ & 581 & -8 & $101 \%$ & 581 & -8 & $101 \%$ \\
\hline William J McCordic School & & & 153 & 75 & 75.0 & $49 \%$ & 76 & 77 & $50 \%$ & 77 & 76 & $50 \%$ & 77 & 76 & $50 \%$ & 77 & 76 & $50 \%$ \\
\hline Williamson Road Junior Public School & $79.9 \%$ & $74.2 \%$ & 553 & 568 & 568.0 & $103 \%$ & 598 & -45 & $108 \%$ & 591 & -38 & $107 \%$ & 591 & -38 & $107 \%$ & 591 & -38 & $107 \%$ \\
\hline Willow Park Junior Public School & $76.0 \%$ & $61.2 \%$ & 563 & 372 & 372.0 & $66 \%$ & 282 & 281 & $50 \%$ & 276 & 287 & $49 \%$ & 276 & 287 & $49 \%$ & 276 & 287 & $49 \%$ \\
\hline Willowdale Middle School & $64.7 \%$ & $53.3 \%$ & 536 & 430 & 430.0 & $80 \%$ & 487 & 49 & $91 \%$ & 531 & 5 & $99 \%$ & 534 & 2 & $100 \%$ & 534 & 2 & $100 \%$ \\
\hline Wilmington Elementary School & $53.4 \%$ & $42.7 \%$ & 277 & 272 & 271.7 & $98 \%$ & 236 & 41 & $85 \%$ & 241 & 36 & $87 \%$ & 241 & 36 & $87 \%$ & 241 & 36 & $87 \%$ \\
\hline $\begin{array}{l}\text { Winchester Junior and Senior Public } \\
\text { School (includes Annex) }\end{array}$ & $55.1 \%$ & $40.1 \%$ & 534 & 475 & 475.0 & $89 \%$ & 604 & -378 & $113 \%$ & 629 & -403 & $278 \%$ & 626 & -400 & $277 \%$ & 626 & -400 & $277 \%$ \\
\hline Winona Drive Senior Public School & $72.1 \%$ & $49.3 \%$ & 455 & 435 & 435.0 & $96 \%$ & 461 & -6 & $101 \%$ & 478 & -23 & $105 \%$ & 467 & -12 & $103 \%$ & 467 & -12 & $103 \%$ \\
\hline Withrow Avenue Junior Public School & $97.8 \%$ & $83.5 \%$ & 668 & 604 & 604.0 & $90 \%$ & 568 & 100 & $85 \%$ & 582 & 86 & $87 \%$ & 581 & 87 & $87 \%$ & 581 & 87 & $87 \%$ \\
\hline Woburn Junior Public School & $79.6 \%$ & $72.1 \%$ & 505 & 388 & 388.0 & $77 \%$ & 304 & 201 & $60 \%$ & 305 & 200 & $60 \%$ & 305 & 200 & $60 \%$ & 305 & 200 & $60 \%$ \\
\hline Woodbine Middle School & $62.7 \%$ & $34.1 \%$ & 584 & 191 & 191.0 & $\mathbf{9 0 \%}$ & 437 & 395 & $75 \%$ & 385 & 199 & $66 \%$ & 370 & 214 & $63 \%$ & 370 & 214 & $63 \%$ \\
\hline Yorkview Public School & $43.3 \%$ & $35.1 \%$ & 525 & 454 & 454.0 & $86 \%$ & 502 & 23 & $96 \%$ & 507 & 18 & $97 \%$ & 508 & 17 & $97 \%$ & 508 & 17 & $97 \%$ \\
\hline Yorkwoods Public School & $61.7 \%$ & $50.2 \%$ & 530 & 404 & 404.0 & $76 \%$ & 306 & 224 & $58 \%$ & 317 & 213 & $60 \%$ & 317 & 213 & $60 \%$ & 317 & 213 & $60 \%$ \\
\hline Zion Heights Middle School & $85.4 \%$ & $66.4 \%$ & 561 & 374 & 374.0 & $67 \%$ & 444 & 117 & $79 \%$ & 407 & 154 & $73 \%$ & 416 & 145 & $74 \%$ & 416 & 145 & $74 \%$ \\
\hline
\end{tabular}




\begin{tabular}{|c|c|c|c|c|c|c|c|c|c|c|c|c|c|c|c|c|c|c|c|c|c|c|}
\hline \multirow[b]{2}{*}{ School Name } & \multicolumn{8}{|c|}{ General Information } & \multicolumn{14}{|c|}{ Facility Information } \\
\hline & Panel & $\begin{array}{l}\text { Grade } \\
\text { Range }\end{array}$ & $\begin{array}{c}\text { Semestered } \\
(\mathrm{Sec})\end{array}$ & Ward & Trustee & $\begin{array}{l}\text { Superintendent } \\
\text { of Education }\end{array}$ & 2017 LOI & $\begin{array}{c}2017-18 \\
\mathrm{Opt} \\
\text { Attend } \\
\mathrm{Reg} / \mathrm{F} / \mathrm{EF}\end{array}$ & $\begin{array}{l}\text { Site Size } \\
\text { (Acres) }\end{array}$ & $\begin{array}{c}\text { Facility Size } \\
\text { (Sq. Ft.) }\end{array}$ & $\mid \begin{array}{c}\text { Current } \\
\text { Backlog (M) }\end{array}$ & \begin{tabular}{|l} 
Port. \\
(Instruc)
\end{tabular} & $\begin{array}{l}\text { Port. (Non- } \\
\text { Instruc) }\end{array}$ & $\begin{array}{c}\text { Current FCI } \\
\text { (EDU) }\end{array}$ & $\begin{array}{l}\text { Eco } \\
\text { Schools }\end{array}$ & Pool & $\begin{array}{l}\text { Elem. } \\
\text { D\&T } \\
\text { Shops }\end{array}$ & $\begin{array}{l}\text { Barrier } \\
\text { Free / } \\
\text { Access }\end{array}$ & Elevator & $\begin{array}{l}\text { Build } \\
\text { Date }\end{array}$ & $\begin{array}{c}\text { Building } \\
\text { Age }\end{array}$ & $\begin{array}{l}\text { T.O Comm } \\
\text { Centre }\end{array}$ \\
\hline ALPHA II Alternative School & ES & $7-12$ & & 9 & Marit Stiles & $\begin{array}{l}\text { Jane Phillips- } \\
\text { Long }\end{array}$ & 287 & \begin{tabular}{|c|} 
Limited \\
N/A \\
N/A \\
\end{tabular} & & & & & & & & & & & & 1916 & 101 & \\
\hline Pleasant View Junior High School & ES & $7-9$ & & 17 & Ken Lister & Beth Veale & 356 & $\begin{array}{c}\text { Limited } \\
\text { N/A } \\
\text { N/A }\end{array}$ & 8.01 & 87,511 & $\$ 9.8$ & & & $67 \%$ & Bronze & & Yes & & & 1968 & 49 & \\
\hline St Andrew's Junior High School & ES & $7-9$ & & 13 & Gerri Gershon & Kathleen Garner & 426 & $\begin{array}{c}\text { Limited } \\
\text { N/A } \\
\text { N/A }\end{array}$ & 8.15 & 82,345 & $\$ 4.3$ & & & $34 \%$ & Gold & & Yes & & & 1962 & 55 & \\
\hline Windfields Junior High School & ES & $7-9$ & & 13 & Gerri Gershon & Kathleen Garner & 386 & $\begin{array}{c}\text { Limited } \\
\text { Limited } \\
\text { N/A }\end{array}$ & 10.01 & 82,183 & $\$ 3.4$ & 1 & & $26 \%$ & & & Yes & & & 1970 & 47 & \\
\hline A Y Jackson Secondary School & $s$ & $9-12$ & Yes & 12 & Alexander Brown & Elizabeth Addo & 94 & $\begin{array}{c}\text { Limited } \\
\text { N/A } \\
\text { N/A }\end{array}$ & 15.32 & 163,817 & $\$ 24.1$ & & 2 & $82 \%$ & & TLC & & & & 1968 & 49 & \\
\hline Agincourt Collegiate Institute & $s$ & $9-12$ & No & 21 & Abdul Hai Patel & Andrew Howard & 80 & $\begin{array}{l}\text { Limited } \\
\text { Limited } \\
\text { Limited }\end{array}$ & 14.92 & 210,482 & $\$ 8.9$ & & & $25 \%$ & Silver & & & & & 1929 & 88 & \\
\hline Albert Campbell Collegiate Institute & $s$ & $9-12$ & Yes & 21 & Abdul Hai Patel & $\begin{array}{l}\text { Jacqueline } \\
\text { Spence }\end{array}$ & 79 & $\begin{array}{c}\text { Limited } \\
\text { N/A } \\
\text { N/A }\end{array}$ & 23.42 & 247,184 & $\$ 17.1$ & & 1 & $42 \%$ & & City & & & & 1976 & 41 & \\
\hline Alternative Scarborough Education 1 & $s$ & $9-12$ & 4 Terms & 19 & David Smith & Anne Seymour & 67 & $\begin{array}{c}\text { Limited } \\
\text { N/A } \\
\text { N/A }\end{array}$ & & & & & & & & & & & & & & \\
\hline Avondale Secondary Alt School & $\mathrm{s}$ & $9-12$ & No & 12 & Alexander Brown & Anne Seymour & 89 & $\begin{array}{c}\text { Limited } \\
\text { N/A } \\
\text { N/A }\end{array}$ & & & $\$ 2.3$ & & & $32 \%$ & & & & & & 1955 & 62 & \\
\hline $\begin{array}{l}\text { Bendale Business and Technical } \\
\text { Institute }\end{array}$ & $s$ & $9-12$ & Yes & 19 & David Smith & Shirley Chan & 22 & $\begin{array}{c}\text { Limited } \\
\text { N/A } \\
\text { N/A }\end{array}$ & 12.8 & 158,154 & $\$ 18.2$ & & & $70 \%$ & & TLC & & A1 & Yes & 1962 & 55 & \\
\hline Birchmount Park Collegiate Institute & $s$ & $9-12$ & Yes & 18 & Parthi Kandavel & Peter Chang & 52 & $\begin{array}{c}\text { Limited } \\
\text { N/A } \\
\text { N/A }\end{array}$ & 10.87 & 181,112 & $\$ 25.7$ & & & $85 \%$ & Gold & & & A1 & Yes & 1963 & 54 & \\
\hline Bloor Collegiate Institute & $s$ & $9-12$ & No & 9 & Marit Stiles & $\begin{array}{l}\text { Jane Phillips- } \\
\text { Long }\end{array}$ & 64 & \begin{tabular}{c|} 
Limited \\
N/A \\
N/A
\end{tabular} & 7.08 & 146,989 & $\$ 19.5$ & & & $77 \%$ & Gold & Yes & & & & 1916 & 101 & \\
\hline Burnhamthorpe Collegiate Institute & $s$ & $9-12$ & No & 2 & Chris Glover & Karen Falconer & 38 & $\begin{array}{c}\text { Limited } \\
\text { N/A } \\
\text { N/A }\end{array}$ & 19.37 & 174,118 & $\$ 20.5$ & & & $60 \%$ & & & & & & 1956 & 61 & \\
\hline CW Jefferys Collegiate Institute & $s$ & $9-12$ & Yes & 4 & Tiffany Ford & Audley Salmon & 11 & $\begin{array}{c}\text { Limited } \\
\text { N/A } \\
\text { N/A }\end{array}$ & 15 & 176,540 & $\$ 26.3$ & & & $\mathbf{9 9 \%}$ & Bronze & City & & & & 1963 & 54 & \\
\hline CALC Secondary School & $s$ & $9-12$ & No & 15 & Jennifer Story & Karen Falconer & 34 & $\begin{array}{c}\text { Limited } \\
\text { N/A } \\
\text { N/A }\end{array}$ & 6.84 & 200,826 & $\$ 23.8$ & & & $91 \%$ & Silver & & & & & 1963 & 54 & \\
\hline Caring \& Safe School LC1 & $s$ & $9-12$ & No & 1 & Avtar Minhas & Jim Spyropoulos & & $\begin{array}{l}\text { N/A } \\
\text { N/A } \\
\text { N/A }\end{array}$ & 6.05 & 36,958 & $\$ 5.2$ & & & $40 \%$ & & & & & & & & \\
\hline Caring \& Safe School LC2 & $s$ & $9-12$ & No & 20 & Manna Wong & Jim Spyropoulos & & $\begin{array}{l}\text { N/A } \\
\text { N/A } \\
\text { N/A }\end{array}$ & & & & & 5 & & & & & & & & & \\
\hline Caring \& Safe School LC3 & $s$ & $9-12$ & No & 18 & Parthi Kandavel & Jim Spyropoulos & & $\begin{array}{l}\text { N/A } \\
\text { N/A } \\
\text { N/A }\end{array}$ & & & & & & & & & & & & & & \\
\hline
\end{tabular}




\begin{tabular}{|c|c|c|c|c|c|c|c|c|c|c|c|c|c|c|c|c|c|c|}
\hline & Participa & tion Rates & & & & & Enrolment a & Projectio & FTE, Surplus & eats $=$ OTG R & ised - Proj & tion FTE, Util & tions $=$ Proj & tion/OTG $\mathrm{F}$ & vised & & & \\
\hline School Name & $\begin{array}{c}\text { TDSB 2013-14 } \\
\text { TDSB Participation } \\
\text { Rate }\end{array}$ & $\begin{array}{c}\text { TDSB 2013-14 } \\
\text { Home School } \\
\text { Participation Rate }\end{array}$ & $\begin{array}{c}\text { Revised } \\
\text { Capacity } \\
2016\end{array}$ & $\begin{array}{c}\text { HC } \\
\text { (Oct. 2016) }\end{array}$ & $\begin{array}{c}\text { FTE } \\
\text { (Oct. 2016) }\end{array}$ & $\begin{array}{c}2016 \\
\text { Utilization } \\
\text { Rate }\end{array}$ & $\begin{array}{c}2021 \\
\text { Projected }\end{array}$ & $\begin{array}{l}2021 \\
\text { Surplus } \\
\text { Seats }\end{array}$ & $\begin{array}{c}2021 \\
\text { Utilization } \\
\text { Rate }\end{array}$ & $\begin{array}{c}2026 \\
\text { Projected }\end{array}$ & $\begin{array}{c}2026 \\
\text { Surplus } \\
\text { Seats }\end{array}$ & $\begin{array}{c}2026 \\
\text { Utilization } \\
\text { Rate }\end{array}$ & $\begin{array}{c}2031 \\
\text { Projected }\end{array}$ & $\begin{array}{l}2031 \\
\text { Surplus } \\
\text { Seats }\end{array}$ & $\begin{array}{c}2031 \\
\text { Utilization } \\
\text { Rate }\end{array}$ & $\begin{array}{c}2036 \\
\text { Projected }\end{array}$ & $\begin{array}{l}2036 \\
\text { Surplus } \\
\text { Seats }\end{array}$ & $\begin{array}{c}2036 \\
\text { Utilization } \\
\text { Rate }\end{array}$ \\
\hline ALPHA II Alternative School & & & 63 & 15 & 36.9 & $59 \%$ & 73 & -10 & $116 \%$ & 76 & -13 & $121 \%$ & 76 & -13 & $121 \%$ & 76 & -13 & $121 \%$ \\
\hline Pleasant View Junior High School & $79.2 \%$ & $51.9 \%$ & 446 & 304 & 304.0 & $68 \%$ & 368 & 78 & $83 \%$ & 319 & 127 & $72 \%$ & 306 & 140 & $69 \%$ & 306 & 140 & $69 \%$ \\
\hline St Andrew's Junior High School & $61.8 \%$ & $43.5 \%$ & 366 & 494 & 494.0 & $135 \%$ & 452 & -86 & $123 \%$ & 402 & -36 & $110 \%$ & 408 & -42 & $111 \%$ & 407 & -41 & $111 \%$ \\
\hline Windfields Junior High School & $81.2 \%$ & $52.8 \%$ & 435 & 605 & 605.0 & $139 \%$ & 603 & -168 & $139 \%$ & 593 & -158 & $136 \%$ & 594 & -159 & $137 \%$ & 591 & -156 & $136 \%$ \\
\hline A Y Jackson Secondary School & $88.5 \%$ & $63.4 \%$ & 1086 & 1,018 & $1,011.3$ & $93 \%$ & 1,286 & -200 & $118 \%$ & 1,273 & -187 & $117 \%$ & 1,210 & -124 & $111 \%$ & 1,210 & -124 & $111 \%$ \\
\hline Agincourt Collegiate Institute & $92.9 \%$ & $80.6 \%$ & 1392 & 1,231 & $1,231.0$ & $88 \%$ & 1,333 & 59 & $96 \%$ & 1,323 & 69 & $95 \%$ & 1,303 & 89 & $94 \%$ & 1,275 & 117 & $92 \%$ \\
\hline Albert Campbell Collegiate Institute & $77.8 \%$ & $50.7 \%$ & 1695 & 1,205 & $1,201.3$ & $71 \%$ & 911 & 784 & $54 \%$ & 985 & 710 & $58 \%$ & 979 & 716 & $58 \%$ & 972 & 723 & $57 \%$ \\
\hline Alternative Scarborough Education 1 & & & 120 & 147 & 82.6 & $69 \%$ & 150 & -30 & $125 \%$ & 150 & -30 & $125 \%$ & 150 & -30 & $125 \%$ & 150 & -30 & $125 \%$ \\
\hline Avondale Secondary Alt School & & & 108 & 58 & 57.6 & $53 \%$ & 75 & 33 & $69 \%$ & 75 & 33 & $69 \%$ & 75 & 33 & $69 \%$ & 75 & 33 & $69 \%$ \\
\hline $\begin{array}{l}\text { Bendale Business and Technical } \\
\text { Institute }\end{array}$ & & & 945 & 403 & 402.5 & $43 \%$ & 404 & 541 & $43 \%$ & 393 & 552 & $42 \%$ & 393 & 552 & $42 \%$ & 393 & 552 & $42 \%$ \\
\hline Birchmount Park Collegiate Institute & $70.0 \%$ & $35.7 \%$ & 1164 & 859 & 855.6 & $74 \%$ & 934 & 230 & $80 \%$ & 894 & 270 & $77 \%$ & 907 & 257 & $78 \%$ & 916 & 248 & $79 \%$ \\
\hline Bloor Collegiate Institute & $65.3 \%$ & $15.1 \%$ & 792 & 667 & 663.0 & $84 \%$ & 664 & 128 & $84 \%$ & 710 & 82 & $90 \%$ & 673 & 119 & $85 \%$ & 678 & 114 & $86 \%$ \\
\hline Burnhamthorpe Collegiate Institute & & & 669 & 396 & 362.0 & $54 \%$ & 422 & 247 & $63 \%$ & 422 & 247 & $63 \%$ & 422 & 247 & $63 \%$ & 422 & 247 & $63 \%$ \\
\hline CW Jefferys Collegiate Institute & $50.1 \%$ & $23.2 \%$ & 978 & 735 & 728.8 & $75 \%$ & 623 & 355 & $64 \%$ & 560 & 418 & $57 \%$ & 527 & 451 & $54 \%$ & 537 & 441 & $55 \%$ \\
\hline CALC Secondary School & & & 855 & 546 & 479.6 & $56 \%$ & 488 & 367 & $57 \%$ & 488 & 367 & $57 \%$ & 490 & 365 & $57 \%$ & 491 & 364 & $57 \%$ \\
\hline Caring \& Safe School LC1 & & & 42 & 8 & 8.0 & $19 \%$ & 4 & 38 & $10 \%$ & 4 & 38 & $10 \%$ & 4 & 38 & $10 \%$ & 4 & 38 & $10 \%$ \\
\hline Caring \& Safe School LC2 & & & 42 & 8 & 8.0 & $19 \%$ & 12 & 30 & $29 \%$ & 12 & 30 & $29 \%$ & 12 & 30 & $29 \%$ & 12 & 30 & $29 \%$ \\
\hline Caring \& Safe School LC3 & & & 147 & 4 & 4.0 & $3 \%$ & 10 & 137 & $7 \%$ & 10 & 137 & $7 \%$ & 10 & 137 & $7 \%$ & 10 & 137 & $7 \%$ \\
\hline
\end{tabular}




\begin{tabular}{|c|c|c|c|c|c|c|c|c|c|c|c|c|c|c|c|c|c|c|c|c|c|c|}
\hline \multirow[b]{2}{*}{ School Name } & \multicolumn{8}{|c|}{ General Information } & \multicolumn{14}{|c|}{ Facility Information } \\
\hline & Panel & $\begin{array}{l}\text { Grade } \\
\text { Range }\end{array}$ & $\begin{array}{c}\text { Semestered } \\
\text { (Sec) }\end{array}$ & Ward & Trustee & $\begin{array}{l}\text { Superintendent } \\
\text { of Education }\end{array}$ & 2017 LOI & $\begin{array}{c}2017-18 \\
\text { Opt } \\
\text { Attend } \\
\text { Reg/FI/EF }\end{array}$ & $\begin{array}{l}\text { Site Size } \\
\text { (Acres) }\end{array}$ & $\begin{array}{l}\text { Facility Size } \\
\text { (Sq. Ft.) }\end{array}$ & $\mid \begin{array}{c}\text { Current } \\
\text { Backlog (M) }\end{array}$ & $\begin{array}{l}\text { Port. } \\
\text { (Instruc) }\end{array}$ & $\begin{array}{c}\text { Port. (Non- } \\
\text { Instruc) }\end{array}$ & $\begin{array}{c}\text { Current FCI } \\
\text { (EDU) }\end{array}$ & $\begin{array}{c}\text { Eco } \\
\text { Schools }\end{array}$ & Pool & $\begin{array}{l}\text { Elem. } \\
\text { D\&T } \\
\text { Shops }\end{array}$ & $\begin{array}{l}\text { Barrier } \\
\text { Free / } \\
\text { Access }\end{array}$ & Elevator & $\begin{array}{l}\text { Build } \\
\text { Date }\end{array}$ & $\begin{array}{c}\text { Building } \\
\text { Age }\end{array}$ & $\begin{array}{l}\text { T.o Comm } \\
\text { Centre }\end{array}$ \\
\hline Caring \& Safe School LC4 & $s$ & $9-12$ & No & 9 & Marit Stiles & Jim Spyropoulos & & $\begin{array}{l}\text { N/A } \\
\text { N/A } \\
\text { N/A }\end{array}$ & & 204,529 & & & & & & & & & & 1966 & 51 & \\
\hline Cedarbrae Collegiate Institute & $s$ & $9-12$ & Yes & 19 & David Smith & Anne Seymour & 30 & $\begin{array}{l}\text { Limited } \\
\text { Limited } \\
\text { Limited }\end{array}$ & 15.22 & 254,765 & $\$ 22.5$ & & & $57 \%$ & Silver & City & & & Yes & 1959 & 58 & \\
\hline Central Etobicoke High School & $\mathrm{s}$ & $9-12$ & Yes & 2 & Chris Glover & Annie Appleby & 16 & $\begin{array}{c}\text { Limited } \\
\text { N/A } \\
\text { N/A }\end{array}$ & 12.7 & 119,330 & $\$ 13.2$ & & & $53 \%$ & & & & A1 & Yes & 1970 & 47 & \\
\hline Central Technical School & $\mathrm{s}$ & $9-12$ & Yes & 10 & Ausma Malik & Mike Gallagher & 36 & $\begin{array}{c}\text { Limited } \\
\text { N/A } \\
\text { N/A }\end{array}$ & 12.78 & 592,888 & $\$ 58.6$ & & & $78 \%$ & & TLC & & & & 1912 & 105 & \\
\hline Central Toronto Academy & $s$ & $9-12$ & Yes & 10 & Ausma Malik & Mike Gallagher & 31 & $\begin{array}{c}\text { Limited } \\
\text { N/A } \\
\text { N/A }\end{array}$ & 4.18 & 223,127 & $\$ 9$. & & & $25 \%$ & Gold & TLC & & & & 1914 & 103 & \\
\hline City School & $\mathrm{s}$ & $9-12$ & Yes & 10 & Ausma Malik & Anne Seymour & 87 & $\begin{array}{c}\text { Limited } \\
\text { N/A } \\
\text { N/A }\end{array}$ & & & & & & & Platinum & & & & Yes & & & \\
\hline Contact Alternative School & $s$ & $9-12$ & No & 10 & Ausma Malik & Anne Seymour & 14 & $\begin{array}{c}\text { Limited } \\
\text { N/A } \\
\text { N/A }\end{array}$ & 0.37 & 32,674 & $\$ 2.8$ & & & $37 \%$ & & & & & & 1944 & 73 & \\
\hline $\begin{array}{l}\text { Danforth Collegiate and Technical } \\
\text { Institute }\end{array}$ & $s$ & $9-12$ & No & 15 & Jennifer Story & Lucy Giannotta & 53 & $\begin{array}{c}\text { Limited } \\
\text { N/A } \\
\text { N/A }\end{array}$ & 8.95 & 391,059 & $\$ 32.5$ & & & $55 \%$ & & Yes & & & Yes & 1922 & 95 & \\
\hline $\begin{array}{l}\text { David and Mary Thomson Collegiate } \\
\text { Institute }\end{array}$ & s & $9-12$ & Yes & 19 & David Smith & Shirley Chan & 40 & $\begin{array}{c}\text { Limited } \\
\text { N/A } \\
\text { N/A }\end{array}$ & 15.37 & 232,239 & $\$ 31.2$ & & & $77 \%$ & & & & & & 1958 & 59 & \\
\hline Delphi Secondary Alternative School & $s$ & $9-12$ & $\begin{array}{c}\text { Yes }(10- \\
12)\end{array}$ & 21 & Abdul Hai Patel & Anne Seymour & 82 & $\begin{array}{c}\text { Limited } \\
\text { N/A } \\
\text { N/A }\end{array}$ & & & & & & & & & & & & & & \\
\hline Don Mills Collegiate Institute & $s$ & $9-12$ & Yes & 17 & Ken Lister & Beth Veale & 81 & $\begin{array}{c}\text { Limited } \\
\text { N/A } \\
\text { N/A }\end{array}$ & 11.69 & 124,130 & $\$ 4$. & 5 & 1 & $19 \%$ & Gold & City & & & Yes & 1958 & 59 & \\
\hline Downsview Secondary School & $\mathrm{s}$ & 9-12 & Yes & 5 & Alexandra Lulka & Linda Curtis & 3 & $\begin{array}{c}\text { Limited } \\
\text { N/A } \\
\text { N/A }\end{array}$ & 12.7 & 231,243 & $\$ 25.6$ & & & $74 \%$ & & TLC & & A1 & No & 1953 & 64 & \\
\hline $\begin{array}{l}\text { Dr Norman Bethune Collegiate } \\
\text { Institute }\end{array}$ & $\mathrm{s}$ & $9-12$ & No & 20 & Manna Wong & $\begin{array}{l}\text { Louie } \\
\text { Papathanasakis }\end{array}$ & 86 & $\begin{array}{c}\text { Limited } \\
\text { N/A } \\
\text { N/A }\end{array}$ & 14.9 & 153,427 & $\$ 13.3$ & & & $47 \%$ & Platinum & & & & & 1979 & 38 & \\
\hline Drewry Secondary School & s & $9-12$ & Yes & 12 & Alexander Brown & $\begin{array}{l}\text { Louie } \\
\text { Papathanasakis }\end{array}$ & 72 & $\begin{array}{c}\text { Limited } \\
\text { N/A } \\
\text { N/A }\end{array}$ & 5.04 & 62,173 & $\$ 7.3$ & 1 & & $103 \%$ & Gold & & & & Yes & 1927 & 90 & \\
\hline Earl Haig Secondary School & $s$ & $9-12$ & No & 12 & Alexander Brown & Linda Curtis & 100 & $\begin{array}{c}\text { Closed } \\
\text { N/A } \\
\text { N/A }\end{array}$ & 8.85 & 267,475 & $\$ 2.5$ & & & $5 \%$ & Platinum & & & A1 & Yes & 1997 & 20 & \\
\hline $\begin{array}{l}\text { East York Alternative Secondary } \\
\text { School }\end{array}$ & $\mathrm{s}$ & $10-12$ & Yes & 16 & $\begin{array}{l}\text { Sheila Cary- } \\
\text { Meagher }\end{array}$ & Anne Seymour & 41 & $\begin{array}{c}\text { Limited } \\
\text { N/A } \\
\text { N/A }\end{array}$ & 0.34 & 8,572 & & & & & & & & & & 1951 & 66 & \\
\hline East York Collegiate Institute & $s$ & $9-12$ & Yes & 16 & $\begin{array}{l}\text { Sheila Cary- } \\
\text { Meagher }\end{array}$ & Lucy Giannotta & 62 & \begin{tabular}{c|} 
Limited \\
N/A \\
N/A
\end{tabular} & 12.86 & 196,611 & $\$ 18.7$ & & 2 & $48 \%$ & Gold & & & B1 & & 1959 & 58 & \\
\hline Eastdale Collegiate Institute & $s$ & $9-12$ & Yes & 15 & Jennifer Story & John Chasty & 15 & \begin{tabular}{c|} 
Limited \\
N/A \\
N/A
\end{tabular} & 1.19 & 59,208 & $\$ 12$. & & & $94 \%$ & Gold & & & & & 1962 & 55 & \\
\hline
\end{tabular}




\begin{tabular}{|c|c|c|c|c|c|c|c|c|c|c|c|c|c|c|c|c|c|c|}
\hline \multirow[b]{2}{*}{ School Name } & \multicolumn{2}{|c|}{ Participation Rates } & \multicolumn{16}{|c|}{2016 Enrolment and Projections FTE, Surplus Seats = OTG Revised - Projection FTE, Utilizations = Projection/OTG Revised } \\
\hline & $\begin{array}{c}\text { TDSB 2013-14 } \\
\text { TDSB Participation } \\
\text { Rate }\end{array}$ & $\begin{array}{c}\text { TDSB 2013-14 } \\
\text { Home School } \\
\text { Participation Rate }\end{array}$ & $\begin{array}{c}\text { Revised } \\
\text { Capacity } \\
2016\end{array}$ & $\begin{array}{c}\text { HC } \\
\text { (Oct. 2016) }\end{array}$ & $\begin{array}{c}\text { FTE } \\
\text { (Oct. 2016) }\end{array}$ & $\begin{array}{c}2016 \\
\text { Utilization } \\
\text { Rate }\end{array}$ & $\begin{array}{c}2021 \\
\text { Projected }\end{array}$ & $\begin{array}{l}2021 \\
\text { Surplus } \\
\text { Seats }\end{array}$ & $\begin{array}{c}2021 \\
\text { Utilization } \\
\text { Rate }\end{array}$ & $\begin{array}{c}2026 \\
\text { Projected }\end{array}$ & $\begin{array}{c}2026 \\
\text { Surplus } \\
\text { Seats }\end{array}$ & $\begin{array}{c}2026 \\
\text { Utilization } \\
\text { Rate }\end{array}$ & $\begin{array}{c}2031 \\
\text { Projected }\end{array}$ & $\begin{array}{l}2031 \\
\text { Surplus } \\
\text { Seats }\end{array}$ & $\begin{array}{c}2031 \\
\text { Utilization } \\
\text { Rate }\end{array}$ & $\begin{array}{c}2036 \\
\text { Projected }\end{array}$ & $\begin{array}{c}2036 \\
\text { Surplus } \\
\text { Seats }\end{array}$ & $\begin{array}{c}2036 \\
\text { Utilization } \\
\text { Rate }\end{array}$ \\
\hline Caring \& Safe School LC4 & & & 42 & 9 & 9.0 & $21 \%$ & 7 & 35 & $17 \%$ & 7 & 35 & $17 \%$ & 7 & 35 & $17 \%$ & 7 & 35 & $17 \%$ \\
\hline Cedarbrae Collegiate Institute & $68.6 \%$ & $48.5 \%$ & 1536 & 1,207 & $1,205.5$ & $78 \%$ & 1,160 & 376 & $76 \%$ & 1,186 & 350 & $77 \%$ & 1,134 & 402 & $74 \%$ & 1,142 & 394 & $74 \%$ \\
\hline Central Etobicoke High School & & & 516 & 145 & 144.5 & $28 \%$ & 166 & 350 & $32 \%$ & 166 & 350 & $32 \%$ & 166 & 350 & $32 \%$ & 166 & 350 & $32 \%$ \\
\hline Central Technical School & & & 2931 & 1,372 & $1,334.7$ & $46 \%$ & 1,064 & 1,867 & $36 \%$ & 1,214 & 1,717 & $41 \%$ & 1,233 & 1,698 & $42 \%$ & 1,218 & 1,713 & $42 \%$ \\
\hline Central Toronto Academy & & & 1176 & 458 & 457.0 & $39 \%$ & 808 & 368 & $69 \%$ & 828 & 348 & $70 \%$ & 828 & 348 & $70 \%$ & 828 & 348 & $70 \%$ \\
\hline City School & & & 189 & 146 & 142.8 & $76 \%$ & 160 & 29 & $85 \%$ & 160 & 29 & $85 \%$ & 160 & 29 & $85 \%$ & 160 & 29 & $85 \%$ \\
\hline Contact Alternative School & & & 213 & 183 & 182.0 & $85 \%$ & 189 & 24 & $89 \%$ & 189 & 24 & $89 \%$ & 189 & 24 & $89 \%$ & 189 & 24 & $89 \%$ \\
\hline $\begin{array}{l}\text { Danforth Collegiate and Technical } \\
\text { Institute }\end{array}$ & & & 2067 & 878 & 877.0 & $42 \%$ & 762 & 1,305 & $37 \%$ & 761 & 1,306 & $37 \%$ & 787 & 1,280 & $38 \%$ & 788 & 1,279 & $38 \%$ \\
\hline $\begin{array}{l}\text { David and Mary Thomson Collegiate } \\
\text { Institute }\end{array}$ & $61.1 \%$ & $25.8 \%$ & 1623 & 980 & 976.0 & $60 \%$ & 877 & 746 & $54 \%$ & 886 & 737 & $55 \%$ & 846 & 777 & $52 \%$ & 852 & 771 & $52 \%$ \\
\hline Delphi Secondary Alternative School & & & 147 & 118 & 117.5 & $80 \%$ & 130 & 17 & $88 \%$ & 130 & 17 & $88 \%$ & 130 & 17 & $88 \%$ & 130 & 17 & $88 \%$ \\
\hline Don Mills Collegiate Institute & $79.0 \%$ & $25.2 \%$ & 795 & 1,119 & $1,118.0$ & $141 \%$ & 1,159 & -364 & $146 \%$ & 900 & -105 & $113 \%$ & 883 & -88 & $111 \%$ & 881 & -86 & $111 \%$ \\
\hline Downsview Secondary School & $43.4 \%$ & $21.1 \%$ & 1263 & 566 & 558.3 & $44 \%$ & 582 & 681 & $46 \%$ & 484 & 779 & $38 \%$ & 451 & 812 & $36 \%$ & 453 & 810 & $36 \%$ \\
\hline $\begin{array}{l}\text { Dr Norman Bethune Collegiate } \\
\text { Institute }\end{array}$ & $93.7 \%$ & $75.5 \%$ & 1083 & 1,050 & $1,050.0$ & $97 \%$ & 863 & 220 & $80 \%$ & 923 & 160 & $85 \%$ & 958 & 125 & $88 \%$ & 968 & 115 & $89 \%$ \\
\hline Drewry Secondary School & & & 225 & 130 & 130.0 & $58 \%$ & 145 & 80 & $64 \%$ & 145 & 80 & $64 \%$ & 145 & 80 & $64 \%$ & 145 & 80 & $64 \%$ \\
\hline Earl Haig Secondary School & $101.6 \%$ & $88.6 \%$ & 2028 & 2,038 & $2,036.8$ & $100 \%$ & 1,917 & 111 & $95 \%$ & 1,906 & 122 & $94 \%$ & 1,980 & 48 & $98 \%$ & 1,956 & 72 & $96 \%$ \\
\hline $\begin{array}{l}\text { East York Alternative Secondary } \\
\text { School }\end{array}$ & & & 84 & 126 & 126.0 & $150 \%$ & 123 & -39 & $146 \%$ & 123 & -39 & $146 \%$ & 123 & -39 & $146 \%$ & 123 & -39 & $146 \%$ \\
\hline East York Collegiate Institute & $76.6 \%$ & $30.5 \%$ & 1515 & 985 & 985.0 & $65 \%$ & 962 & 553 & $63 \%$ & 926 & 589 & $61 \%$ & 915 & 600 & $60 \%$ & 921 & 594 & $61 \%$ \\
\hline Eastdale Collegiate Institute & & & 390 & 131 & 131.0 & $34 \%$ & 111 & 279 & $28 \%$ & 123 & 267 & $32 \%$ & 129 & 261 & $33 \%$ & 129 & 261 & $33 \%$ \\
\hline
\end{tabular}




\begin{tabular}{|c|c|c|c|c|c|c|c|c|c|c|c|c|c|c|c|c|c|c|c|c|c|c|}
\hline \multirow[b]{2}{*}{ School Name } & \multicolumn{8}{|c|}{ General Information } & \multicolumn{14}{|c|}{ Facility Information } \\
\hline & Panel & $\begin{array}{l}\text { Grade } \\
\text { Range }\end{array}$ & $\begin{array}{c}\text { Semestered } \\
\text { (Sec) }\end{array}$ & Ward & Trustee & $\begin{array}{l}\text { Superintendent } \\
\text { of Education }\end{array}$ & 2017 LOI & $\begin{array}{c}2017-18 \\
\mathrm{Opt} \\
\text { Attend } \\
\mathrm{Reg} / \mathrm{F} / \mathrm{EF}\end{array}$ & \begin{tabular}{|c|} 
Site Size \\
(Acres)
\end{tabular} & $\begin{array}{c}\text { Facility Size } \\
\text { (Sq. Ft.) }\end{array}$ & $\begin{array}{c}\text { Current } \\
\text { Backlog (M) }\end{array}$ & \begin{tabular}{|c} 
Port. \\
(Instruc)
\end{tabular} & $\begin{array}{l}\text { Port. (Non- } \\
\text { Instruc) }\end{array}$ & $\begin{array}{c}\text { Current FCI } \\
\text { (EDU) }\end{array}$ & $\begin{array}{l}\text { Eco } \\
\text { Schools }\end{array}$ & Pool & $\begin{array}{l}\text { Elem. } \\
\text { D\&T } \\
\text { Shops }\end{array}$ & $\begin{array}{l}\text { Barrier } \\
\text { Free / } \\
\text { Access }\end{array}$ & Elevator & $\begin{array}{l}\text { Build } \\
\text { Date }\end{array}$ & $\begin{array}{c}\text { Building } \\
\text { Age }\end{array}$ & $\begin{array}{l}\text { T.O Comm } \\
\text { Centre }\end{array}$ \\
\hline Emery Collegiate Institute & $s$ & $9-12$ & Yes & 4 & Tiffany Ford & Audley Salmon & 18 & \begin{tabular}{|c|} 
Limited \\
N/A \\
N/A \\
\end{tabular} & 11.61 & 240,102 & $\$ 14.8$ & & & $33 \%$ & Silver & City & & A1 & Yes & 1960 & 57 & \\
\hline Emery EdVance Secondary School & $s$ & $9-12$ & & 4 & Tiffany Ford & Karen Falconer & 6 & $\begin{array}{c}\text { Limited } \\
\text { N/A } \\
\text { N/A }\end{array}$ & \begin{tabular}{|c|} 
See Emery \\
$\mathrm{Cl}$
\end{tabular} & & & & & & & & & & & & & \\
\hline Etobicoke Collegiate Institute & $\mathrm{s}$ & $9-12$ & Yes & 2 & Chris Glover & Annie Appleby & 97 & $\begin{array}{c}\text { Limited } \\
\text { N/A } \\
\text { N/A }\end{array}$ & 9.76 & 208,466 & $\$ 27.1$ & & & $75 \%$ & & & & & Yes & 1928 & 89 & \\
\hline Etobicoke School of the Arts & $s$ & $9-12$ & No & 3 & Pamela Gough & Tracy Hayhurst & 106 & $\begin{array}{c}\text { Limited } \\
\text { N/A } \\
\text { N/A }\end{array}$ & 10.4 & 134,948 & $\$ 7.7$ & & 1 & $27 \%$ & Gold & & & & & 1954 & 63 & \\
\hline $\begin{array}{l}\text { Etobicoke Year Round Alternative } \\
\text { Centre }\end{array}$ & $s$ & $9-12$ & 4 Terms & 3 & Pamela Gough & Anne Seymour & 32 & $\begin{array}{c}\text { Limited } \\
\text { N/A } \\
\text { N/A }\end{array}$ & 5.81 & 23,509 & $\$ 3.8$ & & & $89 \%$ & & & & & & 1954 & 63 & \\
\hline Forest Hill Collegiate Institute & $s$ & $9-12$ & Yes & 11 & Shelley Laskin & Leila Girdhar-Hill & 96 & $\begin{array}{c}\text { Limited } \\
\text { N/A } \\
\text { N/A }\end{array}$ & 6.72 & 169,258 & $\$ 3.6$ & & & $16 \%$ & & TLC & & & & 1947 & 70 & Cat. 5 \\
\hline Frank Oke Secondary School & $s$ & $9-12$ & No & 6 & Chris Tonks & Vicky Branco & 5 & $\begin{array}{c}\text { Limited } \\
\text { N/A } \\
\text { N/A }\end{array}$ & 4.45 & 46,521 & $\$ 5.4$ & & & $50 \%$ & & & & & No & 1957 & 60 & \\
\hline George Harvey Collegiate Institute & $s$ & $9-12$ & Yes & 6 & Chris Tonks & Vicky Branco & 7 & $\begin{array}{c}\text { Limited } \\
\text { N/A } \\
\text { N/A }\end{array}$ & 6.2 & 269,365 & $\$ 7.1$ & & & $16 \%$ & & TLC & & & & 1951 & 66 & \\
\hline George S Henry Academy & $\mathrm{s}$ & $9-12$ & Yes & 17 & Ken Lister & Beth Veale & 54 & $\begin{array}{c}\text { Limited } \\
\text { N/A } \\
\text { N/A }\end{array}$ & 14.28 & 151,223 & $\$ 22$. & & & $94 \%$ & Platinum & TLC & & & & 1964 & 53 & \\
\hline Georges Vanier Secondary School & $s$ & $9-12$ & Yes & 17 & Ken Lister & Audley Salmon & 78 & $\begin{array}{c}\text { Limited } \\
\text { N/A } \\
\text { N/A }\end{array}$ & 15.02 & 255,333 & $\$ 20.2$ & & & $50 \%$ & & City & & A1 & Yes & 1965 & 52 & \\
\hline Greenwood Secondary School & $s$ & $9-12$ & Yes & 15 & Jennifer Story & $\begin{array}{l}\text { Mary Jane } \\
\text { McNamara }\end{array}$ & 21 & \begin{tabular}{c|} 
Limited \\
N/A \\
N/A
\end{tabular} & 0.94 & 84,463 & $\$ 10.8$ & & & $66 \%$ & & & & & & 1971 & 46 & \\
\hline Harbord Collegiate Institute & $s$ & $9-12$ & No & 10 & Ausma Malik & Mike Gallagher & 85 & $\begin{array}{c}\text { Limited } \\
\text { Closed } \\
\text { Limited }\end{array}$ & 3.73 & 198,449 & $\$ 24.8$ & & & $\mathbf{9 9 \%}$ & Platinum & TLC & & & Yes & 1930 & 87 & \\
\hline Heydon Park Secondary School & $s$ & $9-12$ & Yes & 10 & Ausma Malik & $\begin{array}{l}\text { Mary Jane } \\
\text { McNamara }\end{array}$ & 17 & $\begin{array}{c}\text { Limited } \\
\text { N/A } \\
\text { N/A }\end{array}$ & 1.38 & 36,187 & $\$ 1.3$ & & & $11 \%$ & & & & & & 1967 & 50 & \\
\hline Humberside Collegiate Institute & $s$ & $9-12$ & No & 7 & Robin Pilkey & Tracy Hayhurst & 102 & $\begin{array}{l}\text { Closed } \\
\text { Closed } \\
\text { Closed }\end{array}$ & 8.08 & 190,035 & $\$ 9.1$ & & & $33 \%$ & & TLC & & & & 1910 & 107 & Cat. 5 \\
\hline Inglenook Community School & $s$ & $9-12$ & Yes & 14 & Chris Moise & Anne Seymour & 84 & $\begin{array}{c}\text { Limited } \\
\text { N/A } \\
\text { N/A }\end{array}$ & 0.97 & 17,298 & $\$ 1.1$ & & & $25 \%$ & & & & & & 1888 & 129 & \\
\hline Jarvis Collegiate Institute & $s$ & $9-12$ & No & 14 & Chris Moise & John Chasty & 19 & $\begin{array}{c}\text { Limited } \\
\text { N/A } \\
\text { N/A }\end{array}$ & 4.27 & 234,475 & $\$ 24.4$ & & & $83 \%$ & Silver & TLC & & & & 1924 & 93 & \\
\hline John Polanyi Collegiate Institute & $s$ & $9-12$ & Yes & 8 & Jennifer Arp & Leila Girdhar-Hill & 35 & \begin{tabular}{c|} 
Limited \\
N/A \\
N/A
\end{tabular} & 12.11 & 186,387 & $\$ 20.4$ & & & $60 \%$ & Gold & Leased & & & & 1951 & 66 & \\
\hline Kipling Collegiate Institute & $s$ & $9-12$ & Yes & 2 & Chris Glover & $\begin{array}{l}\text { Angela Nardi- } \\
\text { Addesa }\end{array}$ & 9 & $\begin{array}{c}\text { Limited } \\
\text { N/A } \\
\text { N/A }\end{array}$ & 12.11 & 132,139 & $\$ 20.5$ & & & $78 \%$ & Gold & & & B1 & & 1960 & 57 & \\
\hline
\end{tabular}




\begin{tabular}{|c|c|c|c|c|c|c|c|c|c|c|c|c|c|c|c|c|c|c|}
\hline \multirow[b]{2}{*}{ School Name } & \multicolumn{2}{|c|}{ Participation Rates } & \multicolumn{16}{|c|}{2016 Enrolment and Projections FTE, Surplus Seats = OTG Revised - Projection FTE, Utilizations = Projection/OTG Revised } \\
\hline & $\begin{array}{c}\text { TDSB 2013-14 } \\
\text { TDSB Participation } \\
\text { Rate }\end{array}$ & $\begin{array}{c}\text { TDSB 2013-14 } \\
\text { Home School } \\
\text { Participation Rate }\end{array}$ & $\begin{array}{c}\text { Revised } \\
\text { Capacity } \\
2016\end{array}$ & $\begin{array}{c}\text { HC } \\
\text { (Oct. 2016) }\end{array}$ & $\begin{array}{c}\text { FTE } \\
\text { (Oct. 2016) }\end{array}$ & $\begin{array}{l}2016 \\
\text { Utilization } \\
\text { Rate }\end{array}$ & $\begin{array}{c}2021 \\
\text { Projected }\end{array}$ & $\begin{array}{l}2021 \\
\text { Surplus } \\
\text { Seats }\end{array}$ & $\begin{array}{c}2021 \\
\text { Utilization } \\
\text { Rate }\end{array}$ & $\begin{array}{c}2026 \\
\text { Projected }\end{array}$ & $\begin{array}{c}2026 \\
\text { Surplus } \\
\text { Seats }\end{array}$ & $\begin{array}{c}2026 \\
\text { Utilization } \\
\text { Rate }\end{array}$ & $\begin{array}{c}2031 \\
\text { Projected }\end{array}$ & $\begin{array}{l}2031 \\
\text { Surplus } \\
\text { Seats }\end{array}$ & $\begin{array}{c}2031 \\
\text { Utilization } \\
\text { Rate }\end{array}$ & $\begin{array}{c}2036 \\
\text { Projected }\end{array}$ & $\begin{array}{c}2036 \\
\text { Surplus } \\
\text { Seats }\end{array}$ & $\begin{array}{c}2036 \\
\text { Utilization } \\
\text { Rate }\end{array}$ \\
\hline Emery Collegiate Institute & $50.7 \%$ & $29.1 \%$ & 1101 & 674 & 673.5 & $61 \%$ & 595 & 506 & $54 \%$ & 558 & 543 & $51 \%$ & 521 & 580 & $47 \%$ & 523 & 578 & $48 \%$ \\
\hline Emery EdVance Secondary School & & & 84 & 122 & 104.0 & $124 \%$ & 113 & -29 & $135 \%$ & 113 & -29 & $135 \%$ & 113 & -29 & $135 \%$ & 113 & -29 & $135 \%$ \\
\hline Etobicoke Collegiate Institute & $53.6 \%$ & $32.7 \%$ & 1275 & 968 & 965.8 & $76 \%$ & 1,043 & 232 & $82 \%$ & 1,208 & 67 & $95 \%$ & 1,163 & 112 & $91 \%$ & 1,154 & 121 & $91 \%$ \\
\hline Etobicoke School of the Arts & & & 867 & 955 & 951.4 & $110 \%$ & 991 & -124 & $114 \%$ & 991 & -124 & $114 \%$ & 991 & -124 & $114 \%$ & 991 & -124 & $114 \%$ \\
\hline $\begin{array}{l}\text { Etobicoke Year Round Alternative } \\
\text { Centre }\end{array}$ & & & 90 & 34 & 34.0 & $38 \%$ & 42 & 48 & $47 \%$ & 42 & 48 & $47 \%$ & 42 & 48 & $47 \%$ & 42 & 48 & $47 \%$ \\
\hline Forest Hill Collegiate Institute & $55.9 \%$ & $35.9 \%$ & 801 & 982 & 955.1 & $119 \%$ & 967 & -166 & $121 \%$ & 964 & -163 & $120 \%$ & 1,012 & -211 & $126 \%$ & 991 & -190 & $124 \%$ \\
\hline Frank Oke Secondary School & & & 192 & 102 & 102.0 & $53 \%$ & 101 & 91 & $53 \%$ & 101 & 91 & $53 \%$ & 101 & 91 & $53 \%$ & 101 & 91 & $53 \%$ \\
\hline George Harvey Collegiate Institute & $56.8 \%$ & $5.7 \%$ & 1389 & 533 & 533.0 & $38 \%$ & 448 & 941 & $32 \%$ & 409 & 980 & $29 \%$ & 389 & 1,000 & $28 \%$ & 389 & 1,000 & $28 \%$ \\
\hline George S Henry Academy & $64.5 \%$ & $25.9 \%$ & 807 & 491 & 481.0 & $60 \%$ & 468 & 339 & $58 \%$ & 444 & 363 & $55 \%$ & 395 & 412 & $49 \%$ & 387 & 420 & $48 \%$ \\
\hline Georges Vanier Secondary School & $64.2 \%$ & $30.1 \%$ & 1539 & 721 & 720.0 & $47 \%$ & 823 & 716 & $53 \%$ & 928 & 611 & $60 \%$ & 836 & 703 & $54 \%$ & 818 & 721 & $53 \%$ \\
\hline Greenwood Secondary School & & & 300 & 213 & 212.5 & $71 \%$ & 196 & 104 & $65 \%$ & 199 & 101 & $66 \%$ & 206 & 94 & $69 \%$ & 209 & 91 & $70 \%$ \\
\hline Harbord Collegiate Institute & $87.4 \%$ & $38.5 \%$ & 948 & 1,059 & $1,059.0$ & $112 \%$ & 1,214 & -266 & $128 \%$ & 1,461 & -513 & $154 \%$ & 1,459 & -511 & $154 \%$ & 1,473 & -525 & $155 \%$ \\
\hline Heydon Park Secondary School & & & 240 & 155 & 155.0 & $65 \%$ & 173 & 67 & $72 \%$ & 228 & 12 & $95 \%$ & 233 & 7 & $97 \%$ & 253 & -13 & $105 \%$ \\
\hline Humberside Collegiate Institute & $74.7 \%$ & $47.4 \%$ & 1020 & 1,136 & $1,126.5$ & $110 \%$ & 1,313 & -293 & $129 \%$ & 1,460 & -440 & $143 \%$ & 1,450 & -430 & $142 \%$ & 1,458 & -438 & $143 \%$ \\
\hline Inglenook Community School & & & 126 & 59 & 59.0 & $47 \%$ & 83 & 43 & $66 \%$ & 83 & 43 & $66 \%$ & 84 & 42 & $67 \%$ & 84 & 42 & $67 \%$ \\
\hline Jarvis Collegiate Institute & $64.3 \%$ & $23.6 \%$ & 1095 & 729 & 729.0 & $67 \%$ & 845 & 250 & $77 \%$ & 800 & 295 & $73 \%$ & 842 & 253 & $77 \%$ & 836 & 259 & $76 \%$ \\
\hline John Polanyi Collegiate Institute & $41.5 \%$ & $10.5 \%$ & 1032 & 905 & 901.4 & $87 \%$ & 982 & 50 & $95 \%$ & 1,074 & -42 & $104 \%$ & 1,059 & -27 & $103 \%$ & 1,028 & 4 & $100 \%$ \\
\hline Kipling Collegiate Institute & $53.0 \%$ & $28.7 \%$ & 948 & 400 & 397.1 & $42 \%$ & 304 & 644 & $32 \%$ & 257 & 691 & $27 \%$ & 246 & 702 & $26 \%$ & 253 & 695 & $27 \%$ \\
\hline
\end{tabular}




\begin{tabular}{|c|c|c|c|c|c|c|c|c|c|c|c|c|c|c|c|c|c|c|c|c|c|c|}
\hline \multirow[b]{2}{*}{ School Name } & \multicolumn{8}{|c|}{ General Information } & \multicolumn{14}{|c|}{ Facility Information } \\
\hline & Panel & $\begin{array}{l}\text { Grade } \\
\text { Range }\end{array}$ & $\begin{array}{c}\text { Semestered } \\
(\mathrm{Sec})\end{array}$ & Ward & Trustee & $\begin{array}{l}\text { Superintendent } \\
\text { of Education }\end{array}$ & 2017 LOI & $\begin{array}{c}2017-18 \\
\mathrm{Opt} \\
\text { Attend } \\
\mathrm{Reg} / \mathrm{F} / \mathrm{EF}\end{array}$ & $\begin{array}{l}\text { Site Size } \\
\text { (Acres) }\end{array}$ & $\begin{array}{c}\text { Facility Size } \\
\text { (Sq. Ft.) }\end{array}$ & $\mid \begin{array}{c}\text { Current } \\
\text { Backlog (M) }\end{array}$ & $\begin{array}{l}\text { Port. } \\
\text { (Instruc) }\end{array}$ & $\begin{array}{l}\text { Port. (Non- } \\
\text { Instruc) }\end{array}$ & $\begin{array}{c}\text { Current FCI } \\
\text { (EDU) }\end{array}$ & $\begin{array}{c}\text { Eco } \\
\text { Schools }\end{array}$ & Pool & $\begin{array}{l}\text { Elem. } \\
\text { D\&T } \\
\text { Shops }\end{array}$ & $\begin{array}{l}\text { Barrier } \\
\text { Free / } \\
\text { Access }\end{array}$ & Elevator & $\begin{array}{l}\text { Build } \\
\text { Date }\end{array}$ & $\begin{array}{c}\text { Building } \\
\text { Age }\end{array}$ & $\begin{array}{l}\text { T.O Comm } \\
\text { Centre }\end{array}$ \\
\hline Lakeshore Collegiate Institute & $s$ & $9-12$ & Yes & 3 & Pamela Gough & Sandra Tondat & 70 & \begin{tabular}{|c|} 
Limited \\
N/A \\
N/A \\
\end{tabular} & 15.76 & 174,463 & $\$ 25.3$ & & & $75 \%$ & & & & & & 1950 & 67 & \\
\hline L'Amoreaux Collegiate Institute & $s$ & $9-12$ & Yes & 20 & Manna Wong & Beth Veale & 71 & $\begin{array}{c}\text { Limited } \\
\text { N/A } \\
\text { Limited }\end{array}$ & 15 & 161,512 & $\$ 7.4$ & 8 & 2 & $30 \%$ & & City & & & & 1973 & 44 & \\
\hline Lawrence Park Collegiate Institute & $\mathrm{s}$ & $9-12$ & No & 8 & Jennifer Arp & Vicky Branco & 108 & $\begin{array}{l}\text { Closed } \\
\text { Closed } \\
\text { Closed }\end{array}$ & 11.37 & 168,280 & $\$ 5.8$ & 2 & & $23 \%$ & Silver & TLC & & A1 & Yes & 1935 & 82 & \\
\hline Leaside High School & $\mathrm{s}$ & $9-12$ & Yes & 13 & Gerri Gershon & Leila Girdhar-Hill & 99 & $\begin{array}{l}\text { Closed } \\
\text { Limited } \\
\text { Limited }\end{array}$ & 3.7 & 145,962 & $\$ 18.8$ & & & $78 \%$ & Gold & & & & & 1945 & 72 & \\
\hline Lester B Pearson Collegiate Institute & $s$ & $9-12$ & Yes & 21 & Abdul Hai Patel & Andrew Howard & 77 & $\begin{array}{c}\text { Closed } \\
\text { N/A } \\
\text { N/A }\end{array}$ & 14.5 & 194,944 & $\$ 6.5$ & & 1 & $19 \%$ & Silver & City & & B1 & Yes & 1978 & 39 & \\
\hline Malvern Collegiate Institute & $s$ & $9-12$ & No & 16 & $\begin{array}{l}\text { Sheila Cary- } \\
\text { Meagher }\end{array}$ & John Chasty & 105 & $\begin{array}{c}\text { Closed } \\
\text { Limited } \\
\text { Limited }\end{array}$ & 4.03 & 154,259 & $\$ 19.9$ & 2 & & $82 \%$ & Gold & TLC & & & & 1900 & 117 & \\
\hline Maplewood High School & $s$ & $9-12$ & No & 22 & Jerry Chadwick & $\begin{array}{l}\text { Kerry-Lynn } \\
\text { Stadnyk }\end{array}$ & 25 & $\begin{array}{c}\text { Limited } \\
\text { N/A } \\
\text { N/A }\end{array}$ & 12.6 & 115,477 & $\$ 9.2$ & & & $44 \%$ & & & & & & 1967 & 50 & \\
\hline Marc Garneau Collegiate Institute & $s$ & $9-12$ & Yes & 13 & Gerri Gershon & Ian Allison & 68 & $\begin{array}{c}\text { Closed } \\
\text { N/A } \\
\text { N/A }\end{array}$ & 8.6 & 188,695 & $\$ 3.1$ & 8 & & $8 \%$ & Platinum & & & & Yes & 1973 & 44 & \\
\hline Martingrove Collegiate Institute & $\mathrm{s}$ & $9-12$ & No & 2 & Chris Glover & Annie Appleby & 74 & $\begin{array}{c}\text { Limited } \\
\text { N/A } \\
\text { N/A }\end{array}$ & 16.9 & 158,629 & $\$ 6.7$ & & & $22 \%$ & Gold & & & A1 & Yes & 1966 & 51 & \\
\hline Monarch Park Collegiate Institute & $s$ & $9-12$ & Yes & 15 & Jennifer Story & $\begin{array}{l}\text { Mary Jane } \\
\text { McNamara }\end{array}$ & 75 & $\begin{array}{c}\text { Limited } \\
\text { N/A } \\
\text { N/A }\end{array}$ & 9.24 & 231,202 & $\$ 8.1$ & & & $23 \%$ & & TLC & & A1 & Yes & 1963 & 54 & \\
\hline Native Learning Centre & $s$ & $9-12$ & No & 14 & Chris Moise & John Chasty & 26 & $\begin{array}{c}\text { Limited } \\
\text { N/A } \\
\text { N/A }\end{array}$ & & & & & & & & & & & Yes & & & \\
\hline Native Learning Centre East & $s$ & $9-12$ & & 22 & Jerry Chadwick & $\begin{array}{l}\text { Kerry-Lynn } \\
\text { Stadnyk }\end{array}$ & 2 & $\begin{array}{c}\text { Closed } \\
\text { N/A } \\
\text { N/A }\end{array}$ & & 1,920 & & & & & & & & & & & & \\
\hline Newtonbrook Secondary School & $s$ & $9-12$ & Yes & 12 & Alexander Brown & \begin{tabular}{|l} 
Louie \\
Papathanasakis
\end{tabular} & 59 & $\begin{array}{l}\text { Limited } \\
\text { Limited } \\
\text { Limited }\end{array}$ & 13.71 & 196,228 & $\$ 8.9$ & & & $26 \%$ & & TLC & & & & 1963 & 54 & \\
\hline North Albion Collegiate Institute & $s$ & $9-12$ & Yes & 1 & Avtar Minhas & Glenford Duffus & 48 & $\begin{array}{c}\text { Limited } \\
\text { N/A } \\
\text { N/A }\end{array}$ & 12.16 & 171,804 & $\$ 7$. & & & $23 \%$ & & & & & & 1962 & 55 & \\
\hline $\begin{array}{l}\text { North East Year Round Alternative } \\
\text { Centre }\end{array}$ & $s$ & $9-12$ & 4 Terms & 17 & Ken Lister & Anne Seymour & 47 & $\begin{array}{c}\text { Limited } \\
\text { N/A } \\
\text { N/A }\end{array}$ & & & & & & & & & & & Yes & & & \\
\hline North Toronto Collegiate Institute & $s$ & $9-12$ & No & 11 & Shelley Laskin & Ian Allison & 107 & $\begin{array}{c}\text { Limited } \\
\text { N/A } \\
\text { N/A }\end{array}$ & 4.76 & 211,736 & $\$ .0326$ & & & $0.10 \%$ & Gold & & & A1 & & 2010 & 7 & \\
\hline $\begin{array}{l}\text { North West Year Round Alternative } \\
\text { Centre }\end{array}$ & $s$ & $9-12$ & 4 Terms & 5 & Alexandra Lulka & Anne Seymour & 29 & $\begin{array}{c}\text { Limited } \\
\text { N/A } \\
\text { N/A }\end{array}$ & & & & & & & & & & & & & & \\
\hline Northern Secondary School & $s$ & $9-12$ & No & 11 & Shelley Laskin & Ian Allison & 104 & $\begin{array}{c}\text { Limited } \\
\text { N/A } \\
\text { N/A }\end{array}$ & 7.39 & 317,228 & $\$ 42.4$ & & & $90 \%$ & Gold & TLC & & & & 1929 & 88 & \\
\hline
\end{tabular}




\begin{tabular}{|c|c|c|c|c|c|c|c|c|c|c|c|c|c|c|c|c|c|c|}
\hline & Participa & tion Rates & & & & & Enrolment a & Projectio & FTE, Surplus & eats $=$ OTG R & ised - Proj & tion FTE, Utili & itions $=$ Proj & ion/OTG R & vised & & & \\
\hline School Name & $\begin{array}{c}\text { TDSB 2013-14 } \\
\text { TDSB Participation } \\
\text { Rate }\end{array}$ & $\begin{array}{c}\text { TDSB } 2013-14 \\
\text { Home Schol } \\
\text { Participation Rate }\end{array}$ & $\begin{array}{c}\text { Revised } \\
\text { Capacity } \\
2016\end{array}$ & $\begin{array}{c}\text { HC } \\
\text { (Oct. 2016) }\end{array}$ & $\begin{array}{c}\text { FTE } \\
\text { (Oct. 2016) }\end{array}$ & $\begin{array}{c}2016 \\
\text { Utilization } \\
\text { Rate }\end{array}$ & $\begin{array}{c}2021 \\
\text { Projected }\end{array}$ & $\begin{array}{c}2021 \\
\text { Surplus } \\
\text { Seats }\end{array}$ & $\begin{array}{c}2021 \\
\text { Utilization } \\
\text { Rate }\end{array}$ & $\begin{array}{c}2026 \\
\text { Projected }\end{array}$ & $\begin{array}{c}2026 \\
\text { Surplus } \\
\text { Seats }\end{array}$ & $\begin{array}{c}2026 \\
\text { Utilization } \\
\text { Rate }\end{array}$ & $\begin{array}{c}2031 \\
\text { Projected }\end{array}$ & $\begin{array}{l}2031 \\
\text { Surplus } \\
\text { Seats }\end{array}$ & $\begin{array}{c}2031 \\
\text { Utilization } \\
\text { Rate }\end{array}$ & $\begin{array}{c}2036 \\
\text { Projected }\end{array}$ & $\begin{array}{l}2036 \\
\text { Surplus } \\
\text { Seats }\end{array}$ & $\begin{array}{c}2036 \\
\text { Utilization } \\
\text { Rate }\end{array}$ \\
\hline Lakeshore Collegiate Institute & $54.5 \%$ & $32.4 \%$ & 1131 & 655 & 655.0 & $58 \%$ & 642 & 489 & $57 \%$ & 705 & 426 & $62 \%$ & 743 & 388 & $66 \%$ & 717 & 414 & $63 \%$ \\
\hline L'Amoreaux Collegiate Institute & $64.1 \%$ & $33.5 \%$ & 957 & 583 & 583.0 & $61 \%$ & 536 & 421 & $56 \%$ & 474 & 483 & $50 \%$ & 474 & 483 & $50 \%$ & 459 & 498 & $48 \%$ \\
\hline Lawrence Park Collegiate Institute & $75.4 \%$ & $53.5 \%$ & 906 & 1,215 & $1,205.0$ & $133 \%$ & 1,209 & -303 & $133 \%$ & 1,265 & -359 & $140 \%$ & 1,254 & -348 & $138 \%$ & 1,254 & -348 & $138 \%$ \\
\hline Leaside High School & $87.2 \%$ & $65.3 \%$ & 939 & 933 & 927.0 & $\mathbf{9 9 \%}$ & 1,104 & -165 & $118 \%$ & 1,096 & -157 & $117 \%$ & 1,074 & -135 & $114 \%$ & 1,077 & -138 & $115 \%$ \\
\hline Lester B Pearson Collegiate Institute & $62.6 \%$ & $45.8 \%$ & 1329 & 1,365 & $1,365.0$ & $103 \%$ & 1,224 & 105 & $92 \%$ & 1,050 & 279 & $79 \%$ & 953 & 376 & $72 \%$ & 951 & 378 & $72 \%$ \\
\hline Malvern Collegiate Institute & $85.1 \%$ & $57.8 \%$ & 852 & 1,063 & $1,061.5$ & $125 \%$ & 1,305 & -453 & $153 \%$ & 1,617 & -765 & $190 \%$ & 1,668 & -816 & $196 \%$ & 1,656 & -804 & $194 \%$ \\
\hline Maplewood High School & & & 552 & 182 & 182.0 & $33 \%$ & 166 & 386 & $30 \%$ & 172 & 380 & $31 \%$ & 175 & 377 & $32 \%$ & 178 & 374 & $32 \%$ \\
\hline Marc Garneau Collegiate Institute & $76.3 \%$ & $55.7 \%$ & 1422 & 1,714 & $1,709.9$ & $120 \%$ & 1,382 & 40 & $97 \%$ & 1,370 & 52 & $96 \%$ & 1,312 & 110 & $92 \%$ & 1,311 & 111 & $92 \%$ \\
\hline Martingrove Collegiate Institute & $65.4 \%$ & $48.2 \%$ & 1059 & 1,183 & 1,181.7 & $112 \%$ & 1,066 & -7 & $101 \%$ & 1,089 & -30 & $103 \%$ & 1,070 & -11 & $101 \%$ & 1,071 & -12 & $101 \%$ \\
\hline Monarch Park Collegiate Institute & $69.1 \%$ & $21.7 \%$ & 1284 & 846 & 843.7 & $66 \%$ & 903 & 381 & $70 \%$ & 893 & 391 & $70 \%$ & 862 & 422 & $67 \%$ & 862 & 422 & $67 \%$ \\
\hline Native Learning Centre & & & 54 & 39 & 37.6 & $70 \%$ & 32 & 22 & $59 \%$ & 32 & 22 & $59 \%$ & 32 & 22 & $59 \%$ & 32 & 22 & $59 \%$ \\
\hline Native Learning Centre East & & & 21 & 15 & 15.0 & $71 \%$ & 15 & 6 & $71 \%$ & 15 & 6 & $71 \%$ & 15 & 6 & $71 \%$ & 15 & 6 & $71 \%$ \\
\hline Newtonbrook Secondary School & $52.7 \%$ & $36.3 \%$ & 1356 & 898 & 890.5 & $66 \%$ & 1,167 & 189 & $86 \%$ & 1,075 & 281 & $79 \%$ & 1,103 & 253 & $81 \%$ & 1,202 & 154 & $89 \%$ \\
\hline North Albion Collegiate Institute & $62.6 \%$ & $39.2 \%$ & 1095 & 937 & 931.5 & $85 \%$ & 755 & 340 & $69 \%$ & 579 & 516 & $53 \%$ & 542 & 553 & $49 \%$ & 556 & 539 & $51 \%$ \\
\hline $\begin{array}{l}\text { North East Year Round Alternative } \\
\text { Centre }\end{array}$ & & & 105 & 29 & 28.4 & $27 \%$ & 43 & 62 & $41 \%$ & 43 & 62 & $41 \%$ & 43 & 62 & $41 \%$ & 43 & 62 & $41 \%$ \\
\hline North Toronto Collegiate Institute & $78.0 \%$ & $46.3 \%$ & 1233 & 1,265 & $1,263.2$ & $102 \%$ & 1,422 & -189 & $115 \%$ & 1,527 & -294 & $124 \%$ & 1,526 & -293 & $124 \%$ & 1,538 & -305 & $125 \%$ \\
\hline $\begin{array}{l}\text { North West Year Round Alternative } \\
\text { Centre }\end{array}$ & & & 126 & 77 & 77.0 & $61 \%$ & 75 & 51 & $60 \%$ & 75 & 51 & $60 \%$ & 75 & 51 & $60 \%$ & 75 & 51 & $60 \%$ \\
\hline Northern Secondary School & $78.0 \%$ & $29.8 \%$ & 1743 & 1,806 & $1,799.1$ & $103 \%$ & 2,024 & -281 & $116 \%$ & 2,130 & -387 & $122 \%$ & 2,121 & -378 & $122 \%$ & 2,123 & -380 & $122 \%$ \\
\hline
\end{tabular}




\begin{tabular}{|c|c|c|c|c|c|c|c|c|c|c|c|c|c|c|c|c|c|c|c|c|c|c|}
\hline \multirow[b]{2}{*}{ School Name } & \multicolumn{8}{|c|}{ General Information } & \multicolumn{14}{|c|}{ Facility Information } \\
\hline & Panel & $\begin{array}{l}\text { Grade } \\
\text { Range }\end{array}$ & $\begin{array}{c}\text { Semestered } \\
\text { (Sec) }\end{array}$ & Ward & Trustee & $\begin{array}{l}\text { Superintendent } \\
\text { of Education }\end{array}$ & 2017 LOI & $\begin{array}{c}2017-18 \\
\text { Opt } \\
\text { Attend } \\
\text { Reg/FI/EF }\end{array}$ & $\begin{array}{l}\text { Site Size } \\
\text { (Acres) }\end{array}$ & $\begin{array}{l}\text { Facility Size } \\
\text { (Sq. Ft.) }\end{array}$ & $\mid \begin{array}{c}\text { Current } \\
\text { Backlog (M) }\end{array}$ & $\begin{array}{l}\text { Port. } \\
\text { (Instruc) }\end{array}$ & $\begin{array}{c}\begin{array}{c}\text { Port. (Non- } \\
\text { Instruc) }\end{array} \\
\end{array}$ & $\begin{array}{c}\text { Current FCI } \\
\text { (EDU) }\end{array}$ & $\begin{array}{c}\text { Eco } \\
\text { Schools }\end{array}$ & Pool & $\begin{array}{l}\text { Elem. } \\
\text { D\&T } \\
\text { Shops }\end{array}$ & $\begin{array}{l}\text { Barrier } \\
\text { Free / } \\
\text { Access }\end{array}$ & Elevator & $\begin{array}{l}\text { Build } \\
\text { Date }\end{array}$ & $\begin{array}{c}\text { Building } \\
\text { Age }\end{array}$ & $\begin{array}{l}\text { T.o Comm } \\
\text { Centre }\end{array}$ \\
\hline Northview Heights Secondary School & $s$ & $9-12$ & Yes & 5 & Alexandra Lulka & Elizabeth Addo & 63 & \begin{tabular}{|c|} 
Limited \\
N/A \\
N/A \\
\end{tabular} & 22.21 & 256,872 & $\$ 11.1$ & & & $26 \%$ & Platinum & City & & A1 & Yes & 1955 & 62 & Cat. 5 \\
\hline Oakwood Collegiate Institute & $s$ & $9-12$ & No & 9 & Marit Stiles & Sandra Tondat & 42 & $\begin{array}{c}\text { Limited } \\
\text { N/A } \\
\text { Limited }\end{array}$ & 6.52 & 200,079 & $\$ 9.4$ & & & $31 \%$ & & & & A1 & & 1912 & 105 & \\
\hline $\begin{array}{l}\text { Oasis Alternative Secondary School } \\
\text { (Contains } 3 \text { sites) }\end{array}$ & $\mathrm{s}$ & $9-12$ & Yes & 10 & Ausma Malik & Anne Seymour & 61 & $\begin{array}{c}\text { Limited } \\
\text { N/A } \\
\text { N/A }\end{array}$ & 0.62 & 19,148 & & & & & & & & & & & & \\
\hline Parkdale Collegiate Institute & $\mathrm{s}$ & $9-12$ & Yes & 7 & Robin Pilkey & $\begin{array}{l}\text { Jane Phillips- } \\
\text { Long }\end{array}$ & 51 & $\begin{array}{c}\text { Limited } \\
\text { N/A } \\
\text { N/A }\end{array}$ & 5.88 & 155,375 & $\$ 7.8$ & & & $34 \%$ & \begin{tabular}{|l|} 
Platinum \\
\end{tabular} & City & & & & 1919 & 98 & \\
\hline Parkview Alternative School & $s$ & $9-12$ & 4 Terms & 20 & Manna Wong & Anne Seymour & 24 & $\begin{array}{c}\text { Limited } \\
\text { N/A } \\
\text { N/A }\end{array}$ & 6.8 & 31,575 & $\$ 4.1$ & & & $34 \%$ & & & & & & 1955 & 62 & \\
\hline R H King Academy & s & $9-12$ & Yes & 18 & Parthi Kandavel & $\begin{array}{l}\text { Jacqueline } \\
\text { Spence }\end{array}$ & 66 & $\begin{array}{c}\text { Closed } \\
\text { N/A } \\
\text { N/A }\end{array}$ & 12.4 & 191,552 & $\$ 9.3$ & 6 & & $32 \%$ & \begin{tabular}{|l|} 
Platinum \\
\end{tabular} & TLC & & & & 1922 & 95 & \\
\hline Richview Collegiate Institute & $s$ & $9-12$ & No & 2 & Chris Glover & Annie Appleby & 92 & $\begin{array}{c}\text { Limited } \\
\text { Closed } \\
\text { Limited }\end{array}$ & 13.81 & 118,727 & $\$ 15.7$ & 2 & & $65 \%$ & Silver & & & & & 1958 & 59 & \\
\hline Riverdale Collegiate Institute & $s$ & $9-12$ & No & 15 & Jennifer Story & $\begin{array}{l}\text { Mary Jane } \\
\text { McNamara }\end{array}$ & 83 & $\begin{array}{c}\text { Closed } \\
\text { N/A } \\
\text { Limited }\end{array}$ & 5.19 & 252,073 & $\$ 27.2$ & & & $\mathbf{9 0 \%}$ & Platinum & TLC & & A1 & Yes & 1993 & 24 & \\
\hline Rosedale Heights School of the Arts & $\mathrm{s}$ & $9-12$ & No & 14 & Chris Moise & John Chasty & 95 & $\begin{array}{c}\text { Limited } \\
\text { N/A } \\
\text { N/A }\end{array}$ & 12.18 & 175,137 & $\$ 25$. & & & $94 \%$ & & TLC & & & & 1963 & 54 & \\
\hline Runnymede Collegiate Institute & $s$ & $9-12$ & Yes & 7 & Robin Pilkey & Tracy Hayhurst & 10 & $\begin{array}{c}\text { Limited } \\
\text { N/A } \\
\text { N/A }\end{array}$ & 5.83 & 145,214 & $\$ 20.2$ & & & $80 \%$ & & City & & & & 1928 & 89 & \\
\hline $\begin{array}{l}\text { SATEC @ WA Porter Collegiate } \\
\text { Institute }\end{array}$ & $s$ & $9-12$ & Yes & 18 & Parthi Kandavel & Peter Chang & 55 & $\begin{array}{c}\text { Closed } \\
\text { N/A } \\
\text { N/A }\end{array}$ & 14.85 & 186,642 & $\$ 19.5$ & 1 & & $62 \%$ & \begin{tabular}{|l|} 
Platinum \\
\end{tabular} & TLC & & & & 1956 & 61 & \\
\hline $\begin{array}{l}\text { Scarborough Centre for Alternative } \\
\text { Studies }\end{array}$ & $\mathrm{s}$ & 9-12 & No & 18 & Parthi Kandavel & Karen Falconer & 28 & \begin{tabular}{c|} 
Limited \\
N/A \\
N/A
\end{tabular} & 13.24 & 225,191 & $\$ 25.7$ & & & $67 \%$ & & & & & & 1961 & 56 & \\
\hline $\begin{array}{l}\text { Scarlett Heights Entrepreneurial } \\
\text { Academy }\end{array}$ & $\mathrm{s}$ & $9-12$ & Yes & 2 & Chris Glover & $\begin{array}{l}\text { Angela Nardi- } \\
\text { Addesa }\end{array}$ & 8 & \begin{tabular}{c|} 
Limited \\
N/A \\
N/A
\end{tabular} & 12.33 & 124,087 & $\$ 9.1$ & & & $38 \%$ & Gold & & & & & 1964 & 53 & \\
\hline School of Experiential Education & s & $9-12$ & Yes & 1 & Avtar Minhas & Anne Seymour & 27 & $\begin{array}{c}\text { Limited } \\
\text { N/A } \\
\text { N/A }\end{array}$ & 6.82 & 27,179 & & & & & & & & & & 1954 & 63 & \\
\hline School of Life Experience & $s$ & $9-12$ & Yes & 15 & Jennifer Story & $\begin{array}{l}\text { Mary Jane } \\
\text { McNamara }\end{array}$ & 57 & $\begin{array}{c}\text { Limited } \\
\text { N/A } \\
\text { N/A }\end{array}$ & & & & & & & & & & & & & & \\
\hline SEED Alternative School & $\mathbf{s}$ & $9-12$ & Yes & 15 & Jennifer Story & Anne Seymour & 76 & $\begin{array}{c}\text { Limited } \\
\text { N/A } \\
\text { N/A }\end{array}$ & & & $\$ 1.9$ & & & $22 \%$ & & & & & & & & \\
\hline Silverthorn Collegiate Institute & $s$ & $9-12$ & Yes & 2 & Chris Glover & Glenford Duffus & 93 & $\begin{array}{c}\text { Limited } \\
\text { N/A } \\
\text { N/A }\end{array}$ & 15.3 & 178,004 & $\$ 25.3$ & & & $86 \%$ & & & & & & 1964 & 53 & \\
\hline $\begin{array}{l}\text { Sir John A Macdonald Collegiate } \\
\text { Institute }\end{array}$ & $s$ & $9-12$ & No & 20 & Manna Wong & $\begin{array}{l}\text { Louie } \\
\text { Papathanasakis }\end{array}$ & 88 & $\begin{array}{c}\text { Limited } \\
\text { N/A } \\
\text { N/A }\end{array}$ & 18.14 & 186,479 & $\$ 14.2$ & & & $42 \%$ & Gold & & & & & 1963 & 54 & \\
\hline
\end{tabular}




\begin{tabular}{|c|c|c|c|c|c|c|c|c|c|c|c|c|c|c|c|c|c|c|}
\hline & Participa & ition Rates & & & & & Enrolment & Projection & FTE, Surplus & eats $=$ OTG R & ised - Proj & tion FTE, Util & itions $=$ Proj & ion/OTG R & vised & & & \\
\hline School Name & $\begin{array}{c}\text { TSSB 2013-14 } \\
\text { TDSB Participation } \\
\text { Rate }\end{array}$ & $\begin{array}{c}\text { TDSB 2013-14 } \\
\text { Home school } \\
\text { Participation Rate }\end{array}$ & $\begin{array}{c}\text { Revised } \\
\text { Capacity } \\
2016\end{array}$ & $\begin{array}{c}\text { HC } \\
\text { (Oct. 2016) }\end{array}$ & $\begin{array}{c}\text { FTE } \\
\text { (Oct. 2016) }\end{array}$ & $\begin{array}{c}2016 \\
\text { Utilization } \\
\text { Rate }\end{array}$ & $\begin{array}{c}2021 \\
\text { Projected }\end{array}$ & $\begin{array}{l}2021 \\
\text { Surplus } \\
\text { Seats }\end{array}$ & $\begin{array}{c}2021 \\
\text { Utilization } \\
\text { Rate }\end{array}$ & $\begin{array}{c}2026 \\
\text { Projected }\end{array}$ & $\begin{array}{c}2026 \\
\text { Surplus } \\
\text { Seats }\end{array}$ & $\begin{array}{c}2026 \\
\text { Utilization } \\
\text { Rate }\end{array}$ & $\begin{array}{c}2031 \\
\text { Projected }\end{array}$ & $\begin{array}{l}2031 \\
\text { Surplus } \\
\text { Seats }\end{array}$ & $\begin{array}{c}2031 \\
\text { Utilization } \\
\text { Rate }\end{array}$ & $\begin{array}{c}2036 \\
\text { Projected }\end{array}$ & $\begin{array}{l}2036 \\
\text { Surplus } \\
\text { Seats }\end{array}$ & $\begin{array}{c}2036 \\
\text { Utilization } \\
\text { Rate }\end{array}$ \\
\hline Northview Heights Secondary School & $64.5 \%$ & $42.9 \%$ & 1674 & 1,690 & $1,686.6$ & $101 \%$ & 1,603 & 71 & $96 \%$ & 1,409 & 265 & $84 \%$ & 1,443 & 231 & $86 \%$ & 1,424 & 250 & $85 \%$ \\
\hline Oakwood Collegiate Institute & $56.1 \%$ & $13.4 \%$ & 933 & 424 & 423.0 & $45 \%$ & 533 & 400 & $57 \%$ & 511 & 422 & $55 \%$ & 530 & 403 & $57 \%$ & 530 & 403 & $57 \%$ \\
\hline $\begin{array}{l}\text { Oasis Alternative Secondary School } \\
\text { (Contains } 3 \text { sites) }\end{array}$ & & & 63 & 113 & 112.0 & $178 \%$ & 107 & -44 & $170 \%$ & 107 & -44 & $170 \%$ & 107 & -44 & $170 \%$ & 107 & -44 & $170 \%$ \\
\hline Parkdale Collegiate Institute & $80.2 \%$ & $29.5 \%$ & 861 & 520 & 515.7 & $60 \%$ & 550 & 311 & $64 \%$ & 504 & 357 & $59 \%$ & 512 & 349 & $59 \%$ & 516 & 345 & $60 \%$ \\
\hline Parkview Alternative School & & & 288 & 84 & 84.0 & $29 \%$ & 85 & 203 & $30 \%$ & 85 & 203 & $30 \%$ & 85 & 203 & $30 \%$ & 85 & 203 & $30 \%$ \\
\hline R H King Academy & $78.0 \%$ & $56.1 \%$ & 1122 & 1,189 & $1,188.6$ & $106 \%$ & 1,162 & -40 & $104 \%$ & 1,160 & -38 & $103 \%$ & 1,160 & -38 & $103 \%$ & 1,161 & -39 & $103 \%$ \\
\hline Richview Collegiate Institute & $59.1 \%$ & $45.8 \%$ & 855 & 957 & 955.2 & $112 \%$ & 1,104 & -249 & $129 \%$ & 1,214 & -359 & $142 \%$ & 1,268 & -413 & $148 \%$ & 1,273 & -418 & $149 \%$ \\
\hline Riverdale Collegiate Institute & $92.4 \%$ & $50.2 \%$ & 1116 & 1,182 & $1,177.8$ & $106 \%$ & 1,250 & -134 & $112 \%$ & 1,280 & -164 & $115 \%$ & 1,257 & -141 & $113 \%$ & 1,253 & -137 & $112 \%$ \\
\hline Rosedale Heights School of the Arts & & & 816 & 997 & 993.7 & $122 \%$ & 967 & -151 & $119 \%$ & 972 & -156 & $119 \%$ & 972 & -156 & $119 \%$ & 972 & -156 & $119 \%$ \\
\hline Runnymede Collegiate Institute & $66.1 \%$ & $19.5 \%$ & 747 & 677 & 663.2 & $89 \%$ & 612 & 135 & $82 \%$ & 611 & 136 & $82 \%$ & 611 & 136 & $82 \%$ & 611 & 136 & $82 \%$ \\
\hline $\begin{array}{l}\text { SATEC @ WA Porter Collegiate } \\
\text { Institute }\end{array}$ & $71.9 \%$ & $43.8 \%$ & 1218 & 1,289 & $1,288.0$ & $106 \%$ & 1,162 & 56 & $95 \%$ & 1,180 & 38 & $97 \%$ & 1,180 & 38 & $97 \%$ & 1,180 & 38 & $97 \%$ \\
\hline $\begin{array}{l}\text { Scarborough Centre for Alternative } \\
\text { Studies }\end{array}$ & & & 456 & 326 & 281.6 & $62 \%$ & 321 & 135 & $70 \%$ & 321 & 135 & $70 \%$ & 321 & 135 & $70 \%$ & 321 & 135 & $70 \%$ \\
\hline $\begin{array}{l}\text { Scarlett Heights Entrepreneurial } \\
\text { Academy }\end{array}$ & $44.6 \%$ & $18.4 \%$ & 843 & 221 & 220.5 & $26 \%$ & 181 & 662 & $21 \%$ & 141 & 702 & $17 \%$ & 139 & 704 & $16 \%$ & 139 & 704 & $16 \%$ \\
\hline School of Experiential Education & & & 267 & 53 & 50.9 & $19 \%$ & 51 & 216 & $19 \%$ & 51 & 216 & $19 \%$ & 51 & 216 & $19 \%$ & 51 & 216 & $19 \%$ \\
\hline School of Life Experience & & & 81 & 108 & 104.9 & $129 \%$ & 134 & -53 & $165 \%$ & 134 & -53 & $165 \%$ & 134 & -53 & $165 \%$ & 134 & -53 & $165 \%$ \\
\hline SEED Alternative School & & & 57 & 100 & 100.0 & $175 \%$ & 101 & -44 & $177 \%$ & 101 & -44 & $177 \%$ & 101 & -44 & $177 \%$ & 101 & -44 & $177 \%$ \\
\hline Silverthorn Collegiate Institute & $57.0 \%$ & $43.1 \%$ & 1122 & 885 & 875.0 & $78 \%$ & 765 & 357 & $68 \%$ & 771 & 351 & $69 \%$ & 754 & 368 & $67 \%$ & 740 & 382 & $66 \%$ \\
\hline $\begin{array}{l}\text { Sir John A Macdonald Collegiate } \\
\text { Institute }\end{array}$ & $80.0 \%$ & $58.3 \%$ & 1365 & 1,078 & $1,076.8$ & $79 \%$ & 1,042 & 323 & $76 \%$ & 1,027 & 338 & $75 \%$ & 1,027 & 338 & $75 \%$ & 1,027 & 338 & $75 \%$ \\
\hline
\end{tabular}




\begin{tabular}{|c|c|c|c|c|c|c|c|c|c|c|c|c|c|c|c|c|c|c|c|c|c|c|}
\hline \multirow[b]{2}{*}{ School Name } & \multicolumn{8}{|c|}{ General Information } & \multicolumn{14}{|c|}{ Facility Information } \\
\hline & Panel & $\begin{array}{l}\text { Grade } \\
\text { Range }\end{array}$ & $\begin{array}{c}\text { Semestered } \\
\text { (Sec) }\end{array}$ & Ward & Trustee & $\begin{array}{l}\text { Superintendent } \\
\text { of Education }\end{array}$ & 2017 LOI & $\begin{array}{c}2017-18 \\
\text { Opt } \\
\text { Attend } \\
\mathrm{Reg} / \mathrm{F} / \mathrm{FF}\end{array}$ & $\begin{array}{l}\text { Site Size } \\
\text { (Acres) }\end{array}$ & $\begin{array}{c}\text { Facility Size } \\
\text { (Sq. Ft.) }\end{array}$ & $\mid \begin{array}{c}\text { Current } \\
\text { Backlog (M) }\end{array}$ & $\begin{array}{l}\text { Port. } \\
\text { (Instruc) }\end{array}$ & $\begin{array}{c}\begin{array}{c}\text { Port. (Non- } \\
\text { Instruc) }\end{array} \\
\end{array}$ & $\begin{array}{c}\text { Current FCI } \\
\text { (EDU) }\end{array}$ & $\begin{array}{c}\text { Eco } \\
\text { Schools }\end{array}$ & Pool & $\begin{array}{l}\text { Elem. } \\
\text { D\&T } \\
\text { Shops }\end{array}$ & $\begin{array}{l}\text { Barrier } \\
\text { Free / } \\
\text { Access }\end{array}$ & Elevator & $\begin{array}{l}\text { Build } \\
\text { Date }\end{array}$ & $\begin{array}{c}\text { Building } \\
\text { Age }\end{array}$ & $\begin{array}{l}\text { T.o Comm } \\
\text { Centre }\end{array}$ \\
\hline Sir Oliver Mowat Collegiate Institute & $s$ & $9-12$ & No & 22 & Jerry Chadwick & $\begin{array}{l}\text { Kerry-Lynn } \\
\text { Stadnyk }\end{array}$ & 103 & \begin{tabular}{|c|} 
Limited \\
N/A \\
N/A \\
\end{tabular} & 14.8 & 201,595 & $\$ 25.9$ & & & $83 \%$ & & City & & & Yes & 1970 & 47 & \\
\hline Sir Wilfrid Laurier Collegiate Institute & $s$ & $9-12$ & Yes & 22 & Jerry Chadwick & $\begin{array}{l}\text { Kerry-Lynn } \\
\text { Stadnyk }\end{array}$ & 49 & $\begin{array}{c}\text { Closed } \\
\text { N/A } \\
\text { N/A }\end{array}$ & 13.89 & 236,094 & $\$ 8.4$ & 4 & & $21 \%$ & Gold & TLC & & & & 1964 & 53 & \\
\hline Sir William Osler High School & $\mathrm{s}$ & $9-12$ & Yes & 21 & Abdul Hai Patel & $\begin{array}{l}\text { Jacqueline } \\
\text { Spence }\end{array}$ & 45 & $\begin{array}{c}\text { Limited } \\
\text { N/A } \\
\text { N/A }\end{array}$ & 10.01 & 118,514 & $\$ 15.3$ & 1 & & $88 \%$ & Gold & & & & No & 1975 & 42 & \\
\hline $\begin{array}{l}\text { South East Year Round Alternative } \\
\text { Centre }\end{array}$ & $\mathrm{s}$ & $9-12$ & 4 Terms & 18 & Parthi Kandavel & Anne Seymour & 20 & $\begin{array}{c}\text { Limited } \\
\text { N/A } \\
\text { N/A }\end{array}$ & & & & & & & & & & & & & & \\
\hline Stephen Leacock Collegiate Institute & $s$ & $9-12$ & Yes & 20 & Manna Wong & Lynn Strangway & 58 & $\begin{array}{c}\text { Limited } \\
\text { N/A } \\
\text { N/A }\end{array}$ & 14.97 & 175,415 & $\$ 4.5$ & & & $19 \%$ & Gold & TLC & & & Yes & 1970 & 47 & \\
\hline Subway Academy I & s & $9-12$ & Yes & 15 & Jennifer Story & Anne Seymour & 43 & $\begin{array}{c}\text { Limited } \\
\text { N/A } \\
\text { N/A }\end{array}$ & & & & & & & & & & & & & & \\
\hline Subway Academy II & $s$ & $9-12$ & Yes & 10 & Ausma Malik & Anne Seymour & 39 & $\begin{array}{c}\text { Limited } \\
\text { N/A } \\
\text { N/A }\end{array}$ & & & & & & & & & & & Yes & & & \\
\hline THESTUDENTSCHOOL & $s$ & $9-12$ & Yes & 7 & Robin Pilkey & Sandra Tondat & 90 & $\begin{array}{c}\text { Limited } \\
\text { N/A } \\
\text { N/A }\end{array}$ & & & & & & & & & & & & & & \\
\hline Thistletown Collegiate Institute & $\mathrm{s}$ & $9-12$ & Yes & 1 & Avtar Minhas & Glenford Duffus & 33 & $\begin{array}{c}\text { Limited } \\
\text { N/A } \\
\text { N/A }\end{array}$ & 14.55 & 167,273 & $\$ 21.2$ & & & $66 \%$ & Gold & & & & & 1957 & 60 & \\
\hline Ursula Franklin Academy & $s$ & $9-12$ & No & 7 & Robin Pilkey & Sandra Tondat & 101 & $\begin{array}{c}\text { Limited } \\
\text { N/A } \\
\text { N/A }\end{array}$ & & & & & & & Platinum & & & & & & & \\
\hline Vaughan Road Academy & $s$ & $9-12$ & Yes & 8 & Jennifer Arp & Leila Girdhar-Hill & 60 & $\begin{array}{c}\text { Closed } \\
\text { N/A } \\
\text { N/A }\end{array}$ & 5.36 & 183,211 & $\$ 5.5$ & & & $16 \%$ & Bronze & City & & & Yes & 1927 & 90 & \\
\hline Victoria Park Collegiate Institute & $\mathrm{s}$ & 9-12 & Yes & 17 & Ken Lister & Curtis Ennis & 73 & $\begin{array}{c}\text { Limited } \\
\text { N/A } \\
\text { N/A }\end{array}$ & 15 & 220,931 & $\$ 9.4$ & & & $25 \%$ & Gold & City & & & & 1959 & 58 & \\
\hline West End Alternative School & $\mathrm{s}$ & $9-12$ & 4 Terms & 10 & Ausma Malik & Anne Seymour & 44 & \begin{tabular}{c|} 
Limited \\
N/A \\
N/A
\end{tabular} & 10.65 & 146,751 & $\$ 18.6$ & & & $79 \%$ & & & & & & 1965 & 52 & \\
\hline West Hill Collegiate Institute & s & $9-12$ & No & 22 & Jerry Chadwick & Nadira Persaud & 50 & $\begin{array}{c}\text { Limited } \\
\text { N/A } \\
\text { N/A }\end{array}$ & 17.07 & 217,013 & $\$ 11.9$ & & & $32 \%$ & & City & & & & 1958 & 59 & \\
\hline West Humber Collegiate Institute & $s$ & $9-12$ & Yes & 1 & Avtar Minhas & Annie Appleby & 46 & $\begin{array}{c}\text { Limited } \\
\text { N/A } \\
\text { N/A }\end{array}$ & 17.22 & 197,724 & $\$ 10.2$ & & & $29 \%$ & Silver & & & & & 1966 & 51 & \\
\hline $\begin{array}{l}\text { Western Technical-Commercial } \\
\text { School }\end{array}$ & $\mathrm{s}$ & $9-12$ & Yes & 7 & Robin Pilkey & Sandra Tondat & 65 & $\begin{array}{c}\text { Limited } \\
\text { N/A } \\
\text { N/A }\end{array}$ & 9.04 & 477,567 & $\$ 22.3$ & & & $34 \%$ & & TLC & & & & 1927 & 90 & \\
\hline Weston Collegiate Institute & $s$ & $9-12$ & No & 6 & Chris Tonks & $\begin{array}{l}\text { Angela Nardi- } \\
\text { Addesa }\end{array}$ & 12 & $\begin{array}{c}\text { Limited } \\
\text { N/A } \\
\text { N/A }\end{array}$ & 8.18 & 197,158 & $\$ 18.4$ & & & $51 \%$ & Gold & City & & & & 1969 & 48 & \\
\hline $\begin{array}{l}\text { Westview Centennial Secondary } \\
\text { School }\end{array}$ & $s$ & $9-12$ & Yes & 4 & Tiffany Ford & Curtis Ennis & 1 & $\begin{array}{c}\text { Limited } \\
\text { N/A } \\
\text { N/A }\end{array}$ & 18.14 & 272,577 & $\$ 8.8$ & & & $22 \%$ & & TLC & & & Yes & 1966 & 51 & \\
\hline
\end{tabular}




\begin{tabular}{|c|c|c|c|c|c|c|c|c|c|c|c|c|c|c|c|c|c|c|}
\hline & Participa & ition Rates & & & & & Enrolment a & Projection & FTE, Surplus & eats $=$ OTG R & ised - Proj & tion FTE, Util & itions $=$ Proj & ion/OTG R & vised & & & \\
\hline School Name & $\begin{array}{c}\text { TSSB 2013-14 } \\
\text { TDSB Participation } \\
\text { Rate }\end{array}$ & $\begin{array}{c}\text { TDSB 2013-14 } \\
\text { Home school } \\
\text { Participation Rate }\end{array}$ & $\begin{array}{c}\text { Revised } \\
\text { Capacity } \\
2016\end{array}$ & $\begin{array}{c}\text { HC } \\
\text { (Oct. 2016) }\end{array}$ & $\begin{array}{c}\text { FTE } \\
\text { (Oct. 2016) }\end{array}$ & $\begin{array}{c}2016 \\
\text { Utilization } \\
\text { Rate }\end{array}$ & $\begin{array}{c}2021 \\
\text { Projected }\end{array}$ & $\begin{array}{l}2021 \\
\text { Surplus } \\
\text { Seats }\end{array}$ & $\begin{array}{c}2021 \\
\text { Utilization } \\
\text { Rate }\end{array}$ & $\begin{array}{c}2026 \\
\text { Projected }\end{array}$ & $\begin{array}{c}2026 \\
\text { Surplus } \\
\text { Seats }\end{array}$ & $\begin{array}{c}2026 \\
\text { Utilization } \\
\text { Rate }\end{array}$ & $\begin{array}{c}2031 \\
\text { Projected }\end{array}$ & $\begin{array}{l}2031 \\
\text { Surplus } \\
\text { Seats }\end{array}$ & $\begin{array}{c}2031 \\
\text { Utilization } \\
\text { Rate }\end{array}$ & $\begin{array}{c}2036 \\
\text { Projected }\end{array}$ & $\begin{array}{l}2036 \\
\text { Surplus } \\
\text { Seats }\end{array}$ & $\begin{array}{c}2036 \\
\text { Utilization } \\
\text { Rate }\end{array}$ \\
\hline Sir Oliver Mowat Collegiate Institute & $82.2 \%$ & $66.9 \%$ & 1134 & 1,076 & $1,075.4$ & $95 \%$ & 1,069 & 65 & $94 \%$ & 1,032 & 102 & $91 \%$ & 1,067 & 67 & $94 \%$ & 1,067 & 67 & $94 \%$ \\
\hline Sir Wilfrid Laurier Collegiate Institute & $75.3 \%$ & $44.7 \%$ & 1524 & 1,434 & $1,434.0$ & $94 \%$ & 1,209 & 315 & $79 \%$ & 1,104 & 420 & $72 \%$ & 1,053 & 471 & $69 \%$ & 1,054 & 470 & $69 \%$ \\
\hline Sir William Osler High School & & & 378 & 233 & 233.0 & $62 \%$ & 300 & 78 & $79 \%$ & 300 & 78 & $79 \%$ & 300 & 78 & $79 \%$ & 300 & 78 & $79 \%$ \\
\hline $\begin{array}{l}\text { South East Year Round Alternative } \\
\text { Centre }\end{array}$ & & & 111 & 116 & 116.0 & $105 \%$ & 102 & 9 & $92 \%$ & 102 & 9 & $92 \%$ & 102 & 9 & $92 \%$ & 102 & 9 & $92 \%$ \\
\hline Stephen Leacock Collegiate Institute & $64.2 \%$ & $34.4 \%$ & 900 & 650 & 648.5 & $72 \%$ & 652 & 248 & $72 \%$ & 593 & 307 & $66 \%$ & 585 & 315 & $65 \%$ & 580 & 320 & $64 \%$ \\
\hline Subway Academy I & & & 210 & 131 & 126.4 & $60 \%$ & 159 & 51 & $76 \%$ & 159 & 51 & $76 \%$ & 160 & 50 & $76 \%$ & 161 & 49 & $77 \%$ \\
\hline Subway Academy II & & & 57 & 108 & 0.3 & $0 \%$ & 104 & -47 & $182 \%$ & 104 & -47 & $182 \%$ & 104 & -47 & $182 \%$ & 104 & -47 & $182 \%$ \\
\hline THESTUDENTSCHOOL & & & 210 & 109 & 107.7 & $51 \%$ & 110 & 100 & $52 \%$ & 110 & 100 & $52 \%$ & 110 & 100 & $52 \%$ & 110 & 100 & $52 \%$ \\
\hline Thistletown Collegiate Institute & $47.1 \%$ & $24.9 \%$ & 1032 & 551 & 548.5 & $53 \%$ & 485 & 547 & $47 \%$ & 426 & 606 & $41 \%$ & 417 & 615 & $40 \%$ & 422 & 610 & $41 \%$ \\
\hline Ursula Franklin Academy & & & 621 & 491 & 489.9 & $79 \%$ & 507 & 114 & $82 \%$ & 510 & 111 & $82 \%$ & 507 & 114 & $82 \%$ & 510 & 111 & $82 \%$ \\
\hline Vaughan Road Academy & $53.3 \%$ & $10.6 \%$ & 1221 & 219 & 211.4 & $17 \%$ & 259 & 962 & $21 \%$ & 255 & 966 & $21 \%$ & 260 & 961 & $21 \%$ & 259 & 962 & $21 \%$ \\
\hline Victoria Park Collegiate Institute & $70.0 \%$ & $36.3 \%$ & 1431 & 1,339 & $1,335.1$ & $93 \%$ & 1,256 & 175 & $88 \%$ & 1,117 & 314 & $78 \%$ & 1,060 & 371 & $74 \%$ & 1,083 & 348 & $76 \%$ \\
\hline West End Alternative School & & & 168 & 82 & 82.0 & $49 \%$ & 76 & 92 & $45 \%$ & 76 & 92 & $45 \%$ & 76 & 92 & $45 \%$ & 76 & 92 & $45 \%$ \\
\hline West Hill Collegiate Institute & $48.0 \%$ & $22.5 \%$ & 1365 & 697 & 699.0 & $51 \%$ & 657 & 708 & $48 \%$ & 624 & 741 & $46 \%$ & 564 & 801 & $41 \%$ & 561 & 804 & $41 \%$ \\
\hline West Humber Collegiate Institute & $60.3 \%$ & $37.1 \%$ & 1248 & 980 & 975.3 & $78 \%$ & 791 & 457 & $63 \%$ & 659 & 589 & $53 \%$ & 647 & 601 & $52 \%$ & 654 & 594 & $52 \%$ \\
\hline $\begin{array}{l}\text { Western Technical-Commercial } \\
\text { School }\end{array}$ & & & 1515 & 1,117 & $1,106.3$ & $73 \%$ & 1,305 & 210 & $86 \%$ & 1,306 & 209 & $86 \%$ & 1,306 & 209 & $86 \%$ & 1,306 & 209 & $86 \%$ \\
\hline Weston Collegiate Institute & $56.4 \%$ & $22.8 \%$ & 1296 & 863 & 861.6 & $66 \%$ & 798 & 498 & $62 \%$ & 797 & 499 & $61 \%$ & 718 & 578 & $55 \%$ & 718 & 578 & $55 \%$ \\
\hline $\begin{array}{l}\text { Westview Centennial Secondary } \\
\text { School }\end{array}$ & $60.4 \%$ & $33.7 \%$ & 1461 & 958 & 944.9 & $65 \%$ & 720 & 741 & $49 \%$ & 544 & 917 & $37 \%$ & 495 & 966 & $34 \%$ & 505 & 956 & $35 \%$ \\
\hline
\end{tabular}




\begin{tabular}{|c|c|c|c|c|c|c|c|c|c|c|c|c|c|c|c|c|c|c|c|c|c|c|}
\hline \multirow[b]{2}{*}{ School Name } & \multicolumn{8}{|c|}{ General Information } & \multicolumn{14}{|c|}{ Facility Information } \\
\hline & Panel & $\begin{array}{l}\text { Grade } \\
\text { Range }\end{array}$ & $\begin{array}{c}\text { Semestered } \\
(\text { Sec) }\end{array}$ & Ward & Trustee & $\begin{array}{l}\text { Superintendent } \\
\text { of Education }\end{array}$ & 2017 LOI & $\begin{array}{c}2017-18 \\
\text { Opt } \\
\text { Attend } \\
\text { Reg/F//EF }\end{array}$ & \begin{tabular}{|l} 
Site Size \\
(Acres)
\end{tabular} & $\begin{array}{l}\text { Facility Size } \\
\text { (Sq. Ft.) }\end{array}$ & $\mid \begin{array}{c}\text { Current } \\
\text { Backlog (M) }\end{array}$ & $\begin{array}{l}\text { Port. } \\
\text { (Instruc) }\end{array}$ & $\begin{array}{c}\text { Port. (Non- } \\
\text { Instruc) }\end{array}$ & $\begin{array}{c}\text { Current FCI } \\
\text { (EDU) }\end{array}$ & $\begin{array}{l}\text { Eco } \\
\text { Schools }\end{array}$ & Pool & $\begin{array}{l}\text { Elem. } \\
\text { D\&T } \\
\text { Shops }\end{array}$ & $\begin{array}{l}\text { Barrier } \\
\text { Free / } \\
\text { Access }\end{array}$ & Elevator & $\begin{array}{l}\text { Build } \\
\text { Date }\end{array}$ & $\begin{array}{c}\text { Building } \\
\text { Age }\end{array}$ & $\begin{array}{l}\text { T.O Comm } \\
\text { Centre }\end{array}$ \\
\hline $\begin{array}{l}\text { Wexford Collegiate School for the } \\
\text { Arts }\end{array}$ & $s$ & $9-12$ & Yes & 19 & David Smith & Nadira Persaud & 56 & $\begin{array}{c}\text { Closed } \\
\text { N/A } \\
\text { N/A }\end{array}$ & 8.45 & 190,296 & $\$ 10.9$ & & & $34 \%$ & & City & & & Yes & 1963 & 54 & \\
\hline $\begin{array}{l}\text { William Lyon Mackenzie Collegiate } \\
\text { Institute }\end{array}$ & $\mathrm{s}$ & $9-12$ & Yes & 5 & Alexandra Lulka & Linda Curtis & 91 & $\begin{array}{c}\text { Limited } \\
\text { N/A } \\
\text { N/A }\end{array}$ & 14.28 & 125,067 & $\$ 12.1$ & 4 & & $48 \%$ & Gold & & & & & 1959 & 58 & \\
\hline Winston Churchill Collegiate Institute & $\mathrm{s}$ & $9-12$ & Yes & 19 & David Smith & Anne Seymour & 37 & \begin{tabular}{|c} 
Limited \\
N/A \\
N/A
\end{tabular} & 15 & 202,216 & $\$ 23.2$ & & & $67 \%$ & Bronze & & & & & 1953 & 64 & \\
\hline Woburn Collegiate Institute & $\mathrm{s}$ & $9-12$ & No & 19 & David Smith & Shirley Chan & 69 & $\begin{array}{c}\text { Limited } \\
\text { N/A } \\
\text { N/A }\end{array}$ & 16.43 & 216,634 & $\$ 9.7$ & & & $24 \%$ & Gold & & & & & 1961 & 56 & \\
\hline York Humber High School & $\mathrm{s}$ & $9-12$ & Yes & 6 & Chris Tonks & $\begin{array}{l}\text { Angela Nardi- } \\
\text { Addesa }\end{array}$ & 4 & \begin{tabular}{|c} 
Limited \\
N/A \\
N/A
\end{tabular} & 8.92 & 101,986 & $\$ 7.7$ & & & $41 \%$ & Gold & & & A1 & & 1992 & 25 & \\
\hline York Memorial Collegiate Institute & s & $9-12$ & No & 6 & Chris Tonks & Vicky Branco & 13 & $\begin{array}{c}\text { Limited } \\
\text { N/A } \\
\text { N/A }\end{array}$ & 7.61 & 171,307 & $\$ 8.6$ & & & $33 \%$ & & City & & & & 1929 & 88 & Cat. 3 \\
\hline York Mills Collegiate Institute & $\mathrm{s}$ & $10-12$ & Yes & 13 & Gerri Gershon & Kathleen Garner & 98 & $\begin{array}{c}\text { Limited } \\
\text { Limited } \\
\text { N/A }\end{array}$ & 16.75 & 174,452 & $\$ 21.3$ & & & $75 \%$ & Gold & City & & & & 1956 & 61 & \\
\hline Yorkdale Secondary School & $s$ & $9-12$ & No & 8 & Jennifer Arp & Karen Falconer & 23 & $\begin{array}{c}\text { Limited } \\
\text { N/A } \\
\text { N/A }\end{array}$ & 5.91 & 103,625 & $\$ 9.8$ & 7 & & $46 \%$ & & & & & & 1962 & 55 & \\
\hline $\begin{array}{l}\text { Burnhamthorpe Adult Learning } \\
\text { Centre }\end{array}$ & $\begin{array}{c}\text { Adult } \\
\text { Day }\end{array}$ & & No & 2 & Chris Glover & Karen Falconer & & & 19.37 & 174,118 & & & & & & & & & & & & \\
\hline City Adult Learning Centre & $\begin{array}{c}\text { Adult } \\
\text { Day }\end{array}$ & & No & 15 & Jennifer Story & Karen Falconer & & & $\begin{array}{c}\text { See CALC } \\
\text { SS }\end{array}$ & See CALC SS & & & & & & & & & & & & \\
\hline Emery Adult Learning Centre & $\begin{array}{c}\text { Adult } \\
\text { Day }\end{array}$ & & No & 4 & Tiffany Ford & Audley Salmon & & & $\begin{array}{c}\text { See Emery } \\
\mathrm{Cl}\end{array}$ & & & & & & & & & & & & & \\
\hline $\begin{array}{l}\text { Scarborough Centre for Alternative } \\
\text { Studies Adult Learning Centre }\end{array}$ & $\begin{array}{c}\text { Adult } \\
\text { Day }\end{array}$ & & No & 18 & Parthi Kandavel & Karen Falconer & & & 13.24 & 225,191 & & & & & & & & & & & & \\
\hline Yorkdale Adult Learning Centre & $\begin{array}{c}\text { Adult } \\
\text { Day }\end{array}$ & & No & 8 & Jennifer Arp & Karen Falconer & & & $\begin{array}{c}\text { See } \\
\text { Yorkdale } \\
\text { Ss }\end{array}$ & & & & & & & & & & & & & \\
\hline $\begin{array}{l}1 \text { Civic Centre Court West Education } \\
\text { Office }\end{array}$ & NDS & & & 2 & Chris Glover & Glenford Duffus & & & 6.25 & 103,682 & $\$ 9.2$ & & & $46 \%$ & & & & & & 1969 & 48 & \\
\hline $\begin{array}{l}140 \text { Borough Drive East Education } \\
\text { Office }\end{array}$ & NDS & & & 19 & David Smith & Shirley Chan & & & 5.3 & 126,369 & $\$ 11.4$ & & & $44 \%$ & & & & & & 1973 & 44 & \\
\hline 2 Trethewey Drive & NDS & & & 6 & Chris Tonks & Vicky Branco & & & 1.8 & 48,500 & $\$ 1.9$ & & & $20 \%$ & & & & & & 1959 & 58 & \\
\hline 200 Wilmington Avenue & NDS & & & 5 & Alexandra Lulka & Elizabeth Addo & & & 4.82 & 101,723 & $\$ 2.4$ & & & $19 \%$ & & & & & & 1954 & 63 & \\
\hline $\begin{array}{l}30 \text { Barrhead Crescent Learning Centre } \\
\text { / CSS Area A }\end{array}$ & NDS & & & 1 & Avtar Minhas & Glenford Duffus & & & 6.05 & 36,958 & & & & & & & & & & 1954 & 63 & \\
\hline
\end{tabular}




\begin{tabular}{|c|c|c|c|c|c|c|c|c|c|c|c|c|c|c|c|c|c|c|}
\hline \multirow[b]{2}{*}{ School Name } & \multicolumn{2}{|c|}{ Participation Rates } & \multicolumn{16}{|c|}{2016 Enrolment and Projections FTE, Surplus Seats = OTG Revised - Projection FTE, Utilizations = Projection/OTG Revised } \\
\hline & $\begin{array}{c}\text { TDSB 2013-14 } \\
\text { TDSB Participation } \\
\text { Rate }\end{array}$ & $\begin{array}{c}\text { TDSB 2013-14 } \\
\text { Home School } \\
\text { Participation Rate }\end{array}$ & $\begin{array}{c}\text { Revised } \\
\text { Capacity } \\
2016\end{array}$ & $\begin{array}{c}\text { HC } \\
\text { (Oct. 2016) }\end{array}$ & $\begin{array}{c}\text { FTE } \\
\text { (Oct. 2016) }\end{array}$ & $\begin{array}{c}2016 \\
\text { Utilization } \\
\text { Rate }\end{array}$ & $\begin{array}{c}2021 \\
\text { Projected }\end{array}$ & $\begin{array}{c}2021 \\
\text { Surplus } \\
\text { Seats }\end{array}$ & $\begin{array}{c}2021 \\
\text { Utilization } \\
\text { Rate }\end{array}$ & $\begin{array}{l}2026 \\
\text { Projected }\end{array}$ & $\begin{array}{c}2026 \\
\text { Surplus } \\
\text { Seats }\end{array}$ & $\begin{array}{c}2026 \\
\text { Utilization } \\
\text { Rate }\end{array}$ & $\begin{array}{l}2031 \\
\text { Projected }\end{array}$ & $\begin{array}{c}2031 \\
\text { Surplus } \\
\text { Seats }\end{array}$ & $\begin{array}{c}2031 \\
\text { Utilization } \\
\text { Rate }\end{array}$ & $\begin{array}{l}2036 \\
\text { Projected }\end{array}$ & $\begin{array}{c}2036 \\
\text { Surplus } \\
\text { Seats }\end{array}$ & $\begin{array}{l}2036 \\
\text { Utilization } \\
\text { Rate }\end{array}$ \\
\hline $\begin{array}{l}\text { Wexford Collegiate School for the } \\
\text { Arts }\end{array}$ & $70.6 \%$ & $48.7 \%$ & 1206 & 1,075 & $1,074.4$ & $89 \%$ & 1,082 & 124 & $90 \%$ & 1,012 & 194 & $84 \%$ & 906 & 300 & $75 \%$ & 915 & 291 & $76 \%$ \\
\hline $\begin{array}{l}\text { William Lyon Mackenzie Collegiate } \\
\text { Institute }\end{array}$ & $70.9 \%$ & $59.7 \%$ & 936 & 1,299 & $1,298.0$ & $139 \%$ & 1,200 & -264 & $128 \%$ & 1,185 & -249 & $127 \%$ & 1,185 & -249 & $127 \%$ & 1,185 & -249 & $127 \%$ \\
\hline Winston Churchill Collegiate Institute & $55.8 \%$ & $25.4 \%$ & 1269 & 639 & 637.5 & $50 \%$ & 653 & 616 & $51 \%$ & 586 & 683 & $46 \%$ & 525 & 744 & $41 \%$ & 530 & 739 & $42 \%$ \\
\hline Woburn Collegiate Institute & $71.9 \%$ & $57.5 \%$ & 1533 & 985 & 984.3 & $64 \%$ & 907 & 626 & $59 \%$ & 889 & 644 & $58 \%$ & 868 & 665 & $57 \%$ & 877 & 656 & $57 \%$ \\
\hline York Humber High School & & & 402 & 216 & 215.5 & $54 \%$ & 236 & 166 & $59 \%$ & 236 & 166 & $59 \%$ & 236 & 166 & $59 \%$ & 236 & 166 & $59 \%$ \\
\hline York Memorial Collegiate Institute & $50.2 \%$ & $20.2 \%$ & 873 & 904 & 901.9 & $103 \%$ & 734 & 139 & $84 \%$ & 665 & 208 & $76 \%$ & 640 & 233 & $73 \%$ & 636 & 237 & $73 \%$ \\
\hline York Mills Collegiate Institute & $66.6 \%$ & $47.3 \%$ & 1050 & 1,012 & $1,009.0$ & $96 \%$ & 901 & 149 & $86 \%$ & 868 & 182 & $83 \%$ & 854 & 196 & $81 \%$ & 851 & 199 & $81 \%$ \\
\hline Yorkdale Secondary School & & & 264 & 399 & 358.2 & $136 \%$ & 309 & -45 & $117 \%$ & 309 & -45 & $117 \%$ & 309 & -45 & $117 \%$ & 309 & -45 & $117 \%$ \\
\hline $\begin{array}{l}\text { Burnhamthorpe Adult Learning } \\
\text { Centre }\end{array}$ & & & & & & & & & & & & & & & & & & \\
\hline City Adult Learning Centre & & & & & & & & & & & & & & & & & & \\
\hline Emery Adult Learning Centre & & & & & & & & & & & & & & & & & & \\
\hline $\begin{array}{l}\text { Scarborough Centre for Alternative } \\
\text { Studies Adult Learning Centre }\end{array}$ & & & & & & & & & & & & & & & & & & \\
\hline Yorkdale Adult Learning Centre & & & & & & & & & & & & & & & & & & \\
\hline $\begin{array}{l}1 \text { Civic Centre Court West Education } \\
\text { Office }\end{array}$ & & & & & & & & & & & & & & & & & & \\
\hline $\begin{array}{l}140 \text { Borough Drive East Education } \\
\text { Office }\end{array}$ & & & & & & & & & & & & & & & & & & \\
\hline 2 Trethewey Drive & & & & & & & & & & & & & & & & & & \\
\hline 200 Wilmington Avenue & & & & & & & & & & & & & & & & & & \\
\hline $\begin{array}{l}30 \text { Barrhead Crescent Learning Centre } \\
\text { / CSS Area A }\end{array}$ & & & 42 & & & & & & & & & & & & & & & \\
\hline
\end{tabular}


Toronto District School Board - School Data

$\underbrace{\text { Bourd }}_{\substack{\text { Toronto } \\ \text { District } \\ \text { School } \\ \text { Board }}}$

\begin{tabular}{|c|c|c|c|c|c|c|c|c|c|c|c|c|c|c|c|c|c|c|c|c|c|c|}
\hline \multirow[b]{2}{*}{ School Name } & \multicolumn{8}{|c|}{ General Information } & \multicolumn{14}{|c|}{ Facility Information } \\
\hline & Panel & $\begin{array}{l}\text { Grade } \\
\text { Range }\end{array}$ & $\begin{array}{l}\text { semstered } \\
\text { (sec) }\end{array}$ & Ward & Trustee & $\begin{array}{c}\text { Superintendent } \\
\text { of Education }\end{array}$ & 2017 LOI & $\begin{array}{c}2017-18 \\
\text { Opt } \\
\text { Ottend } \\
\text { Reg//F/FF }\end{array}$ & $\begin{array}{l}\text { Site Size } \\
\text { (Acres) }\end{array}$ & $\begin{array}{l}\text { Facility Size } \\
\text { (Sq. Ft.) }\end{array}$ & $\left|\begin{array}{c}\text { Current } \\
\text { Backlog (M) }\end{array}\right|$ & $\begin{array}{l}\text { Port. } \\
\text { (lnstruc) }\end{array}$ & $\begin{array}{c}\text { Port. (Non- } \\
\text { Instruc) }\end{array}$ & $\begin{array}{c}\text { Current FCI } \\
\text { (EDU) }\end{array}$ & $\begin{array}{c}\text { Eco } \\
\text { Schools }\end{array}$ & Pool & $\begin{array}{l}\text { Elem. } \\
\text { D\&T } \\
\text { Shops }\end{array}$ & $\begin{array}{l}\text { Barrier } \\
\text { Free } \\
\text { Access }\end{array}$ & Elevator & $\begin{array}{l}\text { Build } \\
\text { Date }\end{array}$ & $\begin{array}{c}\text { Building } \\
\text { Age }\end{array}$ & $\begin{array}{c}\text { T.o comm } \\
\text { Centre }\end{array}$ \\
\hline 400 Kipling Avenue & NDS & & & 3 & Pamela Gough & Sandra Tondat & & & 9.66 & & & & & & & & & & & & & \\
\hline 5050 Yonge Street TDSB Head Office & NDS & & & 12 & Alexander Brown & Elizabeth Addo & & & 4.15 & 118,867 & $\$ 10.9$ & & & $49 \%$ & & & & & & 1968 & 49 & \\
\hline Alliance Centre & NDS & & & 6 & Chris Tonks & Vicky Branco & & & 1.3 & 24,081 & $\$ 1.2$ & & & $26 \%$ & & & & & & 1960 & 57 & \\
\hline Bannockburn PS & NDS & & & 8 & Jennifer Arp & Leila Girdhar-Hill & & & 4.99 & 32,916 & $\$ 3.4$ & & & $45 \%$ & & & & & & 1924 & 93 & \\
\hline Bathurst / Strachan & NDS & & & 10 & Ausma Malik & $\begin{array}{l}\text { Mary Jane } \\
\text { McNamara }\end{array}$ & & & & & & & & & & & & & & & & \\
\hline Berryman Building & NDS & & & 14 & Chris Moise & Mike Gallagher & & & & 12,400 & & & & & & & & & & 1912 & 105 & \\
\hline Bridgeport Drive and Bridgend Street & NDS & & & 22 & Jerry Chadwick & $\begin{array}{l}\text { Kerry-Lynn } \\
\text { Stadnyk }\end{array}$ & & & 6 & & & & & & & & & & & & & \\
\hline Brockton Stadium & NDS & & & 9 & Marit Stiles & $\begin{array}{l}\text { Jane Phillips- } \\
\text { Long }\end{array}$ & & & 3.67 & 4,694 & & & & & & & & & & 1916 & 101 & \\
\hline Brookbanks PS & NDS & & & 17 & Ken Lister & Curtis Ennis & & & 5.16 & 73,001 & $\$ 6.8$ & & & $51 \%$ & & & & & & 1959 & 58 & \\
\hline Burnett PS & NDS & & & 12 & Alexander Brown & Elizabeth Addo & & & 3.14 & 18,589 & & & & & & & & & & 1955 & 62 & \\
\hline Buttonwood Hill & NDS & & & 2 & Chris Glover & Annie Appleby & & & 5.96 & 30,555 & $\$ 3.2$ & & & $49 \%$ & & & & & & 1964 & 53 & \\
\hline C B Parsons JHS & NDS & & & 8 & Jennifer Arp & Leila Girdhar-Hill & & & 6.42 & 47,189 & $\$ 5.9$ & & & $96 \%$ & & & & & & 1945 & 72 & \\
\hline Canadian Tire & NDS & & & 12 & Alexander Brown & Linda Curtis & & & 2 & & & & & & & & & & & & & \\
\hline Cartwright PS & NDS & & & 8 & Jennifer Arp & Leila Girdhar-Hill & & & 6 & 30,799 & $\$ 1.6$ & & & $29 \%$ & & & & & & 1953 & 64 & \\
\hline Castlebar & NDS & & & 3 & Pamela Gough & Tracy Hayhurst & & & 5.24 & 21,776 & $\$ 3.7$ & & & $61 \%$ & & & & & & 1956 & 61 & \\
\hline Century Schoolhouse & NDS & & & 15 & Jennifer Story & Lucy Giannotta & & & 0.06 & 3,046 & $\$ .9$ & & & $152 \%$ & & & & & & 1986 & 31 & \\
\hline D B Hood Community School & NDS & & & 8 & Jennifer Arp & Leila Girdhar-Hill & & & 2.79 & 59,768 & $\$ 5.2$ & & & $47 \%$ & & & & & & 1927 & 90 & \\
\hline Danforth and Byng & NDS & & & 18 & Parthi Kandavel & Peter Chang & & & 4.47 & & & & & & & & & & & & & \\
\hline
\end{tabular}




\begin{tabular}{|c|c|c|c|c|c|c|c|c|c|c|c|c|c|c|c|c|c|c|}
\hline \multirow[b]{2}{*}{ school Name } & \multicolumn{2}{|c|}{\begin{tabular}{|l|l} 
Participation Rates \\
\end{tabular}} & \multicolumn{16}{|c|}{2016 Errolment and Projections FTE, Surplus Seats = OTG Revised- Projection FTE, Utilizations = Projection/OTG Revised } \\
\hline & 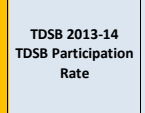 & 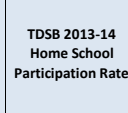 & $\begin{array}{c}\text { Revised } \\
\text { capacity } \\
2016\end{array}$ & $\left|\begin{array}{c}\text { HC } \\
\text { (Oct.2016) }\end{array}\right|$ & $\begin{array}{l}\text { FrF } \\
\text { (Oct. 2016) }\end{array}$ & $\begin{array}{c}2016 \\
\text { Utilizaztion } \\
\text { Rate }\end{array}$ & $\begin{array}{l}\text { 2021 } \\
\text { Projected }\end{array}$ & 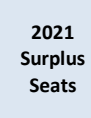 & $\begin{array}{c}\text { Utiz1 } \\
\text { Utilation } \\
\text { Rate }\end{array}$ & $\begin{array}{l}\text { 2026 } \\
\text { Projected }\end{array}$ & $\begin{array}{c}\text { 2026 } \\
\text { Surpus } \\
\text { Seats }\end{array}$ & $\begin{array}{c}\text { Utilization } \\
\text { Rate }\end{array}$ & $\begin{array}{l}2031 \\
\text { Projected }\end{array}$ & $\begin{array}{c}\text { 20031 } \\
\text { Surplus } \\
\text { Seats }\end{array}$ & $\begin{array}{c}\text { Utilization } \\
\text { Utizate } \\
\text { Rate }\end{array}$ & \begin{tabular}{|l}
2036 \\
Projected
\end{tabular} & $\begin{array}{c}\text { 2036 } \\
\text { Surpulus } \\
\text { seats }\end{array}$ & $\begin{array}{c}\text { Utiz6 } \\
\text { Utilzation } \\
\text { Rate }\end{array}$ \\
\hline \multicolumn{19}{|l|}{400 Kipling Avenue } \\
\hline \multicolumn{19}{|c|}{5050 Yonge street TSSB Head Office } \\
\hline \multicolumn{19}{|l|}{ Alliance Centre } \\
\hline Bannockbur ps & & & 351 & & & & & & & & & & & & & & & \\
\hline \multicolumn{19}{|l|}{ Bathurst / Strachan } \\
\hline \multicolumn{19}{|l|}{ Berryman Building } \\
\hline \multicolumn{19}{|c|}{ Bridgeport Drive and Bridgend street } \\
\hline \multicolumn{19}{|l|}{ Brockton Stadium } \\
\hline Brookbanks ps & & & ${ }_{737}$ & & & & & & & & & & & & & & & \\
\hline Burnett Ps & & & 210 & & & & & & & & & & & & & & & \\
\hline Buttonwood Hill & & & 371 & & & & & & & & & & & & & & & \\
\hline CB Parsons JHS & & & 322 & & & & & & & & & & & & & & & \\
\hline \multicolumn{19}{|l|}{ Canadian Tire } \\
\hline Cartwright ps & & & 391 & & & & & & & & & & & & & & & \\
\hline Castlebar & & & 302 & & & & & & & & & & & & & & & \\
\hline \multicolumn{19}{|l|}{ Century schoolhouse } \\
\hline D B Hood Community School & & & 690 & & & & & & & & & & & & & & & \\
\hline Danforth and Byng & & & & & & & & & & & & & & & & & & \\
\hline
\end{tabular}


Toronto District School Board - School Data

. Toronto

\begin{tabular}{|c|c|c|c|c|c|c|c|c|c|c|c|c|c|c|c|c|c|c|c|c|c|c|}
\hline \multirow[b]{2}{*}{ School Name } & \multicolumn{8}{|c|}{ General Information } & \multicolumn{14}{|c|}{ Facility Information } \\
\hline & Panel & $\begin{array}{l}\text { Grade } \\
\text { Range }\end{array}$ & $\begin{array}{l}\text { semstered } \\
\text { (sec) }\end{array}$ & Ward & Trustee & $\begin{array}{c}\text { Superintendent } \\
\text { of Education }\end{array}$ & 2017 LOI & $\begin{array}{c}2017-18 \\
\text { Opt } \\
\text { Ottend } \\
\text { Reg//F/FF }\end{array}$ & $\begin{array}{l}\text { Site Size } \\
\text { (Acres) }\end{array}$ & $\begin{array}{l}\text { Facility Size } \\
\text { (Sq. Ft.) }\end{array}$ & $\begin{array}{c}\text { Current } \\
\text { Backlog (M) }\end{array}$ & $\begin{array}{l}\text { Port. } \\
\text { (lnstruc) }\end{array}$ & $\begin{array}{c}\text { Port. (Non- } \\
\text { Instruc) }\end{array}$ & $\begin{array}{c}\text { Current FCI } \\
(\text { EDU) }\end{array}$ & $\begin{array}{c}\text { Eco } \\
\text { Schools }\end{array}$ & Pool & $\begin{array}{l}\text { Elem. } \\
\text { D\&T } \\
\text { Shops }\end{array}$ & $\begin{array}{l}\text { Barrier } \\
\text { Free / } \\
\text { Access }\end{array}$ & Elevator & $\begin{array}{l}\text { Build } \\
\text { Date }\end{array}$ & $\begin{array}{c}\text { Building } \\
\text { Age }\end{array}$ & $\begin{array}{l}\text { T.o Comm } \\
\text { Centre }\end{array}$ \\
\hline Dellcrest Ps & NDS & & & 5 & Alexandra Lulka & Curtis Ennis & & & 2.25 & 19,741 & $\$ 2.5$ & & & $64 \%$ & & & & & & 1968 & 49 & \\
\hline Douglas Park & NDS & & & 3 & Pamela Gough & Sandra Tondat & & & 5.52 & 17,720 & $\$ 2$. & & & $37 \%$ & & & & & & 1956 & 61 & \\
\hline Earlscourt Jr PS & NDS & & & 9 & Marit Stiles & Sandra Tondat & & & 3.16 & 55,348 & & & & & & & & & & 1964 & 53 & \\
\hline $\begin{array}{l}\text { Earlscourt Jr PS - Stella Maris CS } \\
\text { Portion }\end{array}$ & NDS & & & 9 & Marit Stiles & Sandra Tondat & & & & 72,495 & & & & & & & & & & 1964 & 53 & \\
\hline Eastern Centre & NDS & & & 16 & $\begin{array}{l}\text { Sheila Cary- } \\
\text { Meagher }\end{array}$ & John Chasty & & & 1.68 & 22,059 & $\$ 3.3$ & & & $77 \%$ & & & & & & 1948 & 69 & \\
\hline $\begin{array}{l}\text { Eastern Commerce Collegiate } \\
\text { Institute }\end{array}$ & NDS & & & 15 & Jennifer Story & John Chasty & & & 4.97 & 197,298 & $\$ 28.9$ & & & $88 \%$ & & & & & & 1924 & 93 & \\
\hline Edithvale Land & NDS & & & 12 & Alexander Brown & Elizabeth Addo & & & 5.45 & & & & & & & & & & & & & \\
\hline Etobicoke Park Lawn & NDS & & & 3 & Pamela Gough & Sandra Tondat & & & TBD & & & & & & & & & & & & & \\
\hline Etobicoke Westwood Theatre Site & NDS & & & 3 & Pamela Gough & & & & TBD & & & & & & & & & & & & & \\
\hline Fairmeadow Centre & NDS & & & 13 & Gerri Gershon & Kathleen Garner & & & 3.7 & 32,120 & $\$ 3.8$ & & & $60 \%$ & & & & & & 1950 & 67 & \\
\hline Glen Rush Land & NDS & & & 8 & Jennifer Arp & Leila Girdhar-Hill & & & 3 & & & & & & & & & & & & & \\
\hline Gooderham Learning Centre & NDS & & & 19 & David Smith & Nadira Persaud & & & 5.68 & 20,344 & $\$ 2.3$ & & & $57 \%$ & & & & & & 1956 & 61 & \\
\hline Haney Centre & NDS & & & 6 & Chris Tonks & Vicky Branco & & & 0.91 & 4,588 & $\$ .3$ & & & $43 \%$ & & & & & & 1969 & 48 & \\
\hline Hillside Outdoor Education School & NDS & & & 21 & Abdul Hai Patel & Nadira Persaud & & & 2 & 5,182 & & & & & & & & & & 1893 & 124 & \\
\hline Humber Heights Land & NDS & & & 1 & Avtar Minhas & Glenford Duffus & & & 6.4 & & & & & & & & & & & & & \\
\hline Humbergrove $\mathrm{Cl}$ & NDS & & & 1 & Avtar Minhas & Annie Appleby & & & 10.01 & 83,378 & & & & & & & & & & 1965 & 52 & \\
\hline $\begin{array}{l}\text { Humberline Drive and Finch Avenue } \\
\text { West }\end{array}$ & NDS & & & 1 & Avtar Minhas & Annie Appleby & & & 11.6 & & & & & & & & & & & & & \\
\hline Jones Avenue Adult New Canadians & NDS & & & 15 & Jennifer Story & $\begin{array}{l}\text { Mary Jane } \\
\text { McNamara }\end{array}$ & & & 1.56 & 68,275 & $\$ 9.6$ & & & $69 \%$ & & & & & & 1909 & 108 & \\
\hline
\end{tabular}




\begin{tabular}{|c|c|c|c|c|c|c|c|c|c|c|c|c|c|c|c|c|c|c|}
\hline \multirow[b]{2}{*}{ School Name } & \multicolumn{2}{|c|}{ Participation Rates } & \multicolumn{16}{|c|}{2016 Enrolment and Projections FTE, Surplus Seats = OTG Revised - Projection FTE, Utilizations = Projection/OTG Revised } \\
\hline & 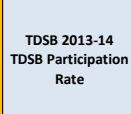 & \begin{tabular}{|c|c} 
ToSs $2013-14$ \\
Home school \\
Participation Rate
\end{tabular} & $\begin{array}{c}\text { Revised } \\
\text { Capacity } \\
2016\end{array}$ & $\begin{array}{c}\mathrm{HC} \\
\text { (Oct. 2016) }\end{array}$ & $\begin{array}{c}\mathrm{FTE} \\
\text { (Oct. 2016) }\end{array}$ & $\begin{array}{c}2016 \\
\text { Utilization } \\
\text { Rate }\end{array}$ & $\begin{array}{c}2021 \\
\text { Projected }\end{array}$ & $\begin{array}{c}2021 \\
\text { Surplus } \\
\text { Seats }\end{array}$ & $\begin{array}{c}2021 \\
\text { Utilization } \\
\text { Rate }\end{array}$ & $\begin{array}{c}2026 \\
\text { Projected }\end{array}$ & $\begin{array}{l}2026 \\
\text { Surplus } \\
\text { Seats }\end{array}$ & $\begin{array}{c}2026 \\
\text { Utilization } \\
\text { Rate }\end{array}$ & $\begin{array}{c}2031 \\
\text { Projected }\end{array}$ & $\begin{array}{l}2031 \\
\text { Surplus } \\
\text { Seats }\end{array}$ & $\begin{array}{c}2031 \\
\text { Utilization } \\
\text { Rate }\end{array}$ & $\begin{array}{l}2036 \\
\text { Projected }\end{array}$ & $\begin{array}{l}2036 \\
\text { Surplus } \\
\text { Seats }\end{array}$ & $\begin{array}{c}2036 \\
\text { Utilization } \\
\text { Rate }\end{array}$ \\
\hline Dellcrest PS & & & 115 & & & & & & & & & & & & & & & \\
\hline Douglas Park & & & 233 & & & & & & & & & & & & & & & \\
\hline Earlscourt Ir PS & & & 492 & & & & & & & & & & & & & & & \\
\hline $\begin{array}{l}\text { Earlscourt Jr PS - Stella Maris CS } \\
\text { Portion }\end{array}$ & & & & & & & & & & & & & & & & & & \\
\hline Eastern Centre & & & & & & & & & & & & & & & & & & \\
\hline $\begin{array}{l}\text { Eastern Commerce Collegiate } \\
\text { Institute }\end{array}$ & & & 903 & & & & & & & & & & & & & & & \\
\hline Edithvale Land & & & & & & & & & & & & & & & & & & \\
\hline Etobicoke Park Lawn & & & & & & & & & & & & & & & & & & \\
\hline Etobicoke Westwood Theatre Site & & & & & & & & & & & & & & & & & & \\
\hline Fairmeadow Centre & & & & & & & & & & & & & & & & & & \\
\hline Glen Rush Land & & & & & & & & & & & & & & & & & & \\
\hline Gooderham Learning Centre & & & 0 & & & & & & & & & & & & & & & \\
\hline Haney Centre & & & 18 & & & & & & & & & & & & & & & \\
\hline Hillside Outdoor Education School & & & & & & & & & & & & & & & & & & \\
\hline Humber Heights Land & & & & & & & & & & & & & & & & & & \\
\hline Humbergrove $\mathrm{Cl}$ & & & 0 & & & & & & & & & & & & & & & \\
\hline $\begin{array}{l}\text { Humberline Drive and Finch Avenue } \\
\text { West }\end{array}$ & & & - & & & & & & & & & & & & & & & \\
\hline Jones Avenue Adult New Canadians & & & 161 & & & & & & & & & & & & & & & \\
\hline
\end{tabular}




\begin{tabular}{|c|c|c|c|c|c|c|c|c|c|c|c|c|c|c|c|c|c|c|c|c|c|c|}
\hline \multirow[b]{2}{*}{ School Name } & \multicolumn{8}{|c|}{ General Information } & \multicolumn{14}{|c|}{ Facility Information } \\
\hline & Panel & $\begin{array}{l}\text { Grade } \\
\text { Range }\end{array}$ & $\begin{array}{l}\text { Semestered } \\
\text { (Sec) }\end{array}$ & Ward & Trustee & $\begin{array}{l}\text { Superintendent } \\
\text { of Education }\end{array}$ & 2017 LOI & $\begin{array}{c}2017-18 \\
\text { Opt } \\
\text { Attend } \\
\text { Reg/F//FF }\end{array}$ & $\begin{array}{l}\text { Site Size } \\
\text { (Acres) }\end{array}$ & $\begin{array}{l}\text { Facility Size } \\
\text { (Sq. Ft.) }\end{array}$ & $\begin{array}{c}\text { Current } \\
\text { Backlog (M) }\end{array} \mid$ & \begin{tabular}{|c|}
$\begin{array}{c}\text { Port. } \\
\text { (linstruc) }\end{array}$ \\
\end{tabular} & $\begin{array}{c}\text { Port. (Non- } \\
\text { Instruc) }\end{array}$ & $\begin{array}{c}\text { Current FCI } \\
(\mathrm{EDU})\end{array}$ & $\begin{array}{c}\text { Eco } \\
\text { Schools }\end{array}$ & Pool & $\begin{array}{l}\text { Elem. } \\
\text { D\&T } \\
\text { Shops }\end{array}$ & $\begin{array}{l}\text { Barrier } \\
\text { Free / } \\
\text { Access }\end{array}$ & Elevator & \begin{tabular}{|l|} 
Build \\
Date
\end{tabular} & $\begin{array}{c}\text { Building } \\
\text { Age }\end{array}$ & $\begin{array}{c}\text { T.O Comm } \\
\text { Centre }\end{array}$ \\
\hline $\begin{array}{l}\text { Keating Channel Precinct (sch site } \\
\text { originally in East Bayfront) }\end{array}$ & NDS & & & 14 & Chris Moise & John Chasty & & & 4 & & & & & & & & & & & & & \\
\hline Kent Sr PS & NDS & & & 9 & Marit Stiles & $\begin{array}{l}\text { Jane Phillips- } \\
\text { Long }\end{array}$ & & & 3.46 & 103,107 & $\$ 16.1$ & & & $160 \%$ & & & & & & 1909 & 108 & \\
\hline Kenton PS & NDS & & & 12 & Alexander Brown & Elizabeth Addo & & & 2.37 & 39,127 & $\$ 4.4$ & & & $44 \%$ & & & & & & 1955 & 62 & \\
\hline Kingsmill CI & NDS & & & 3 & Pamela Gough & Tracy Hayhurst & & & 11.47 & 77,705 & & & & & & & & & & 1963 & 54 & \\
\hline Lakeview Ss & NDS & & & 15 & Jennifer Story & $\begin{array}{l}\text { Mary Jane } \\
\text { McNamara }\end{array}$ & & & 6.55 & 204,603 & & & & & & & & & & 1960 & 57 & \\
\hline Lewis S Beattie & NDS & & & 12 & Alexander Brown & $\begin{array}{l}\text { Louie } \\
\text { Papathanasakis }\end{array}$ & & & 14.13 & 144,291 & & & & & & & & & & 1964 & 53 & \\
\hline Mccowan Road Jr PS & NDS & & & 19 & David Smith & Anne Seymour & & & 6.08 & 41,706 & $\$ 6.9$ & & & $82 \%$ & & & & & & 1954 & 63 & \\
\hline McCulloch Centre & NDS & & & 1 & Avtar Minhas & $\begin{array}{l}\text { Angela Nardi- } \\
\text { Addesa }\end{array}$ & & & 5.11 & 53,228 & $\$ 4.4$ & & & $43 \%$ & & & & & & 1962 & 55 & \\
\hline McGriskin Centre & NDS & & & 21 & Abdul Hai Patel & Andrew Howard & & & 9.32 & 86,251 & $\$ 3.6$ & & & $36 \%$ & & & & & & 1988 & 29 & \\
\hline McNicoll PS & NDS & & & 12 & Alexander Brown & Elizabeth Addo & & & 3.33 & 35,700 & $\$ 4.3$ & & & $70 \%$ & & & & & & 1966 & 51 & \\
\hline Melody Land & NDS & & & 4 & Tiffany Ford & Audley Salmon & & & 3.51 & & & & & & & & & & & 1950 & 67 & \\
\hline $\begin{array}{l}\text { Metropolitan Toronto School for the } \\
\text { Deaf }\end{array}$ & NDS & JK-8 & & 11 & Shelley Laskin & Ian Allison & & & & & & & & & & & & & & & & \\
\hline Mimico Adult Learning Centre & NDS & & & 3 & Pamela Gough & Sandra Tondat & & & 4.5 & 54,606 & $\$ 4.9$ & & & $45 \%$ & & & & & & 1954 & 63 & \\
\hline Motel Strip & NDS & & & 3 & Pamela Gough & Tracy Hayhurst & & & & & & & & & & & & & & & & \\
\hline Nelson A Boylen Collegiate Institute & NDS & & & 6 & Chris Tonks & $\begin{array}{l}\text { Angela Nardi- } \\
\text { Addesa }\end{array}$ & & & 11 & 104,497 & $\$ 14.7$ & & & $94 \%$ & & TLC & & & & 1964 & 53 & \\
\hline Oak Park Centre & NDS & & & 16 & $\begin{array}{l}\text { Sheila Cary- } \\
\text { Meagher }\end{array}$ & Lucy Giannotta & & & 0.38 & 11,556 & & & & & & & & & & 1968 & 49 & \\
\hline Oakburn Centre & NDS & & & 12 & Alexander Brown & Linda Curtis & & & 4.2 & 52,808 & $\$ 5$. & & 5 & $77 \%$ & & & & & & 1949 & 68 & \\
\hline Old Orchard Jr PS & NDS & & & 10 & Ausma Malik & Mike Gallagher & & & & 14,476 & $\$ 2.6$ & & & $88 \%$ & & & & & & 1959 & 58 & \\
\hline
\end{tabular}




\begin{tabular}{|c|c|c|c|c|c|c|c|c|c|c|c|c|c|c|c|c|c|c|}
\hline Doua & \multicolumn{2}{|c|}{ Participation Rates } & \multicolumn{16}{|c|}{2016 Enrolment and Projections FTE, Surplus Seats = OTG Revised - Projection FTE, Utilizations = Projection/OTG Revised } \\
\hline School Name & 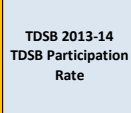 & \begin{tabular}{|c|c|} 
Toss $2013-14$ \\
Prom school \\
Participation Rate
\end{tabular} & $\begin{array}{c}\text { Revised } \\
\text { Capacity } \\
2016\end{array}$ & $\begin{array}{c}\mathrm{HC} \\
\text { (Oct. 2016) }\end{array}$ & $\begin{array}{c}\mathrm{FTE} \\
\text { (Oct. 2016) }\end{array}$ & $\begin{array}{c}2016 \\
\text { Utilization } \\
\text { Rate }\end{array}$ & $\begin{array}{c}2021 \\
\text { Projected }\end{array}$ & $\begin{array}{c}2021 \\
\text { Surplus } \\
\text { Seats }\end{array}$ & $\begin{array}{l}2021 \\
\text { Utilization } \\
\text { Rate }\end{array}$ & $\begin{array}{l}2026 \\
\text { Projected }\end{array}$ & $\begin{array}{c}2026 \\
\text { Surplus } \\
\text { Seats }\end{array}$ & $\begin{array}{l}2026 \\
\text { Utilization } \\
\text { Rate }\end{array}$ & $\begin{array}{c}2031 \\
\text { Projected }\end{array}$ & $\begin{array}{c}2031 \\
\text { Surplus } \\
\text { Seats }\end{array}$ & $\begin{array}{c}2031 \\
\text { Utilization } \\
\text { Rate }\end{array}$ & $\begin{array}{l}2036 \\
\text { Projected }\end{array}$ & $\begin{array}{c}2036 \\
\text { Surplus } \\
\text { Seats }\end{array}$ & $\begin{array}{c}2036 \\
\text { Utilization } \\
\text { Rate }\end{array}$ \\
\hline $\begin{array}{l}\text { Keating Channel Precinct (sch site } \\
\text { originally in East Bayfront) }\end{array}$ & & & & & & & & & & & & & & & & & & \\
\hline Kent Sr PS & & & 794 & & & & & & & & & & & & & & & \\
\hline Kenton PS & & & 570 & & & & & & & & & & & & & & & \\
\hline Kingsmill CI & & & 0 & & & & & & & & & & & & & & & \\
\hline Lakeview Ss & & & 0 & & & & & & & & & & & & & & & \\
\hline Lewis S Beattie & & & 0 & & & & & & & & & & & & & & & \\
\hline McCowan Road Jr PS & & & 452 & & & & & & & & & & & & & & & \\
\hline McCulloch Centre & & & & & & & & & & & & & & & & & & \\
\hline McGriskin Centre & & & & & & & & & & & & & & & & & & \\
\hline McNicoll PS & & & 348 & & & & & & & & & & & & & & & \\
\hline Melody Land & & & & & & & & & & & & & & & & & & \\
\hline $\begin{array}{l}\text { Metropolitan Toronto School for the } \\
\text { Deaf }\end{array}$ & & & 0 & & & & & & & & & & & & & & & \\
\hline Mimico Adult Learning Centre & & & 546 & & & & & & & & & & & & & & & \\
\hline Motel Strip & & & & & & & & & & & & & & & & & & \\
\hline Nelson A Boylen Collegiate Institute & $39.4 \%$ & $4.7 \%$ & 513 & & & & & & & & & & & & & & & \\
\hline Oak Park Centre & & & & & & & & & & & & & & & & & & \\
\hline Oakburn Centre & & & & & & & & & & & & & & & & & & \\
\hline Old Orchard J PS & & & 176 & & & & & & & & & & & & & & & \\
\hline
\end{tabular}


Toronto District School Board - School Data

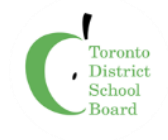

\begin{tabular}{|c|c|c|c|c|c|c|c|c|c|c|c|c|c|c|c|c|c|c|c|c|c|c|}
\hline \multirow[b]{2}{*}{ School Name } & \multicolumn{8}{|c|}{ General Information } & \multicolumn{14}{|c|}{ Facility Information } \\
\hline & Panel & $\begin{array}{l}\text { Grade } \\
\text { Range }\end{array}$ & $\begin{array}{l}\text { Semestered } \\
\text { (sec) }\end{array}$ & Ward & Trustee & $\begin{array}{c}\text { Superintendent } \\
\text { of Education }\end{array}$ & 2017 LOI & $\begin{array}{c}2017-18 \\
\text { Opt } \\
\text { Attend } \\
\text { Reg/F//EF }\end{array}$ & $\begin{array}{l}\text { Site Size } \\
\text { (Acres) }\end{array}$ & $\begin{array}{l}\text { Facility Size } \\
\text { (Sq. Ft.) }\end{array}$ & $\left|\begin{array}{c}\text { Current } \\
\text { Backlog (M) }\end{array}\right|$ & $\begin{array}{l}\text { Port. } \\
\text { (Instruc) }\end{array}$ & $\begin{array}{c}\text { Port. (Non- } \\
\text { Instruc) }\end{array}$ & $\begin{array}{c}\text { Current FCI } \\
\text { (EDU) }\end{array}$ & $\begin{array}{c}\text { Eco } \\
\text { Schools }\end{array}$ & Pool & $\begin{array}{l}\text { Elem. } \\
\text { D\&T } \\
\text { Shops }\end{array}$ & $\begin{array}{l}\text { Barrier } \\
\text { Free / } \\
\text { Access }\end{array}$ & Elevator & $\begin{array}{l}\text { Build } \\
\text { Date }\end{array}$ & $\begin{array}{l}\text { Building } \\
\text { Age }\end{array}$ & $\begin{array}{l}\text { T.o comm } \\
\text { Centre }\end{array}$ \\
\hline Overland PS & NDS & & & 13 & Gerri Gershon & Kathleen Garner & & & 7.96 & 27,222 & $\$ 3$. & & 2 & $55 \%$ & Gold & & & & & 1955 & 62 & \\
\hline Park Lawn / Lakeshore & NDS & & & 3 & Pamela Gough & Sandra Tondat & & & & & & & & & & & & & & & & \\
\hline Pauline Jr PS - St Sebastian CS Portion & NDS & & & 9 & Marit Stiles & $\begin{array}{l}\text { Jane Phillips- } \\
\text { Long }\end{array}$ & & & & 60,905 & & & & & & & & & & 1912 & 105 & \\
\hline Peckham Centre & NDS & & & 12 & Alexander Brown & $\begin{array}{l}\text { Louie } \\
\text { Papathanasakis }\end{array}$ & & & 2.46 & 36,824 & $\$ 2.5$ & & & $35 \%$ & & & & & & 1978 & 39 & \\
\hline $\begin{array}{l}\text { Perth Avenue Jr PS - St Luigi CS } \\
\text { Portion }\end{array}$ & NDS & & & 9 & Marit Stiles & $\begin{array}{l}\text { Jane Phillips- } \\
\text { Long }\end{array}$ & & & & 30,035 & & & & & & & & & & 1964 & 53 & \\
\hline Portlands & NDS & & & 15 & Jennifer Story & John Chasty & & & & & & & & & & & & & & & & \\
\hline \begin{tabular}{|l} 
Portlands (north half) - Lower \\
Donlands
\end{tabular} & NDS & & & 15 & Jennifer Story & John Chasty & & & TBD & & & & & & & & & & & & & \\
\hline $\begin{array}{l}\text { Portlands (south half) - Shipping } \\
\text { Channel Precinct }\end{array}$ & NDS & & & 15 & Jennifer Story & John Chasty & & & TBD & & & & & & & & & & & & & \\
\hline Progress Avenue & NDS & & & 19 & David Smith & Shirley Chan & & & 4.36 & 155,658 & & & & & & & & & & 1972 & 45 & \\
\hline Queens Court & NDS & & & 3 & Pamela Gough & Sandra Tondat & & & 3.21 & 20,017 & $\$ 1.8$ & & & $30 \%$ & & & & & & 1954 & 63 & \\
\hline Railway Lands & NDS & & & 10 & Ausma Malik & $\begin{array}{l}\text { Mary Jane } \\
\text { McNamara }\end{array}$ & & & 2.1 & & & & & & & & & & & & & \\
\hline Shorting Road Stockroom & NDS & & & 21 & Abdul Hai Patel & Andrew Howard & & & & 34,953 & $\$ 1.1$ & & & $16 \%$ & & & & & & 1988 & 29 & \\
\hline Silver Creek & NDS & & & 2 & Chris Glover & Annie Appleby & & & 6 & 36,062 & $\$ 2.3$ & & & $25 \%$ & & & & & & 1962 & 55 & \\
\hline $\begin{array}{l}\text { Sir Robert L Borden Business and } \\
\text { Technical Institute }\end{array}$ & NDS & & & 22 & Jerry Chadwick & $\begin{array}{l}\text { Kerry-lynn } \\
\text { Stadnyk }\end{array}$ & & & 11.93 & 142,579 & $\$ 7.1$ & & 1 & $29 \%$ & Silver & & & & & 1965 & 52 & \\
\hline Sir Sandford Fleming Academy & NDS & & & 8 & Jennifer Arp & Leila Girdhar-Hill & & & 7.81 & 121,935 & $\$ 16.6$ & & & $42 \%$ & & & & & & 1964 & 53 & \\
\hline Tabor Park & NDS & & & 19 & David Smith & Shirley Chan & & & 9.44 & 126,341 & & & & & & & & & & 1965 & 52 & \\
\hline Thistletown & NDS & & & 1 & Avtar Minhas & Glenford Duffus & & & 4.52 & 52,421 & $\$ 6$. & & & $80 \%$ & & & & & & 1946 & 71 & \\
\hline Tippett Road Centre & NDS & & & 5 & Alexandra Lulka & Linda Curtis & & & 3.63 & 47,265 & $\$ 4.1$ & & & $45 \%$ & & & & & & 1953 & 64 & \\
\hline
\end{tabular}




\begin{tabular}{|c|c|c|c|c|c|c|c|c|c|c|c|c|c|c|c|c|c|c|}
\hline \multirow[b]{2}{*}{ School Name } & \multicolumn{2}{|c|}{ Participation Rates } & \multicolumn{16}{|c|}{2016 Enrolment and Projections FTE, Surplus Seats = OTG Revised - Projection FTE, Utilizations = Projection/OTG Revised } \\
\hline & 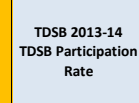 & \begin{tabular}{|c} 
Toss $2013-14$ \\
Home Schoo \\
Participation Rate
\end{tabular} & $\begin{array}{c}\text { Revised } \\
\text { Capacity } \\
2016\end{array}$ & \begin{tabular}{|c}
$\mathrm{HC}$ \\
(Oct. 2016)
\end{tabular} & $\begin{array}{c}\text { FTE } \\
\text { (Oct. 2016) }\end{array}$ & $\begin{array}{c}2016 \\
\text { Utilization } \\
\text { Rate }\end{array}$ & $\begin{array}{l}2021 \\
\text { Projected }\end{array}$ & $\begin{array}{c}2021 \\
\text { Surplus } \\
\text { Seats }\end{array}$ & $\begin{array}{c}2021 \\
\text { Utilization } \\
\text { Rate }\end{array}$ & $\begin{array}{l}2026 \\
\text { Projected }\end{array}$ & $\begin{array}{c}2026 \\
\text { Surplus } \\
\text { Seats }\end{array}$ & $\begin{array}{c}2026 \\
\text { Utilization } \\
\text { Rate }\end{array}$ & $\begin{array}{l}2031 \\
\text { Projected }\end{array}$ & $\begin{array}{c}2031 \\
\text { Surplus } \\
\text { Seats }\end{array}$ & $\begin{array}{c}2031 \\
\text { Utilization } \\
\text { Rate }\end{array}$ & $\begin{array}{l}2036 \\
\text { Projected }\end{array}$ & $\begin{array}{c}2036 \\
\text { Surplus } \\
\text { Seats }\end{array}$ & $\begin{array}{c}2036 \\
\text { Utilization } \\
\text { Rate }\end{array}$ \\
\hline Overland PS & & & 322 & & & & & & & & & & & & & & & \\
\hline Park Lawn / Lakeshore & & & & & & & & & & & & & & & & & & \\
\hline Pauline Jr PS - St Sebastian CS Port & & & & & & & & & & & & & & & & & & \\
\hline Peckham Centre & & & & & & & & & & & & & & & & & & \\
\hline $\begin{array}{l}\text { Perth Avenue Jr PS - St Luigi CS } \\
\text { Portion }\end{array}$ & & & & & & & & & & & & & & & & & & \\
\hline Portlands & & & & & & & & & & & & & & & & & & \\
\hline $\begin{array}{l}\text { Portlands (north half) - Lower } \\
\text { Donlands }\end{array}$ & & & & & & & & & & & & & & & & & & \\
\hline $\begin{array}{l}\text { Portlands (south half) - Shipping } \\
\text { Channel Precinct }\end{array}$ & & & & & & & & & & & & & & & & & & \\
\hline Progress Avenue & & & & & & & & & & & & & & & & & & \\
\hline Queens Court & & & 259 & & & & & & & & & & & & & & & \\
\hline Railway Lands & & & & & & & & & & & & & & & & & & \\
\hline Shorting Road Stockroom & & & & & & & & & & & & & & & & & & \\
\hline Silver Creek & & & 443 & & & & & & & & & & & & & & & \\
\hline $\begin{array}{l}\text { Sir Robert L Borden Business and } \\
\text { Technical Institute }\end{array}$ & & & 846 & & & & & & & & & & & & & & & \\
\hline Sir Sandford Fleming Academy & & & 792 & & & & & & & & & & & & & & & \\
\hline Tabor Park & & & 0 & & & & & & & & & & & & & & & \\
\hline Thistletown & & & 391 & & & & & & & & & & & & & & & \\
\hline Tippett Road Centre & & & & & & & & & & & & & & & & & & \\
\hline
\end{tabular}


Toronto District School Board - School Data

\begin{tabular}{|c|c|c|c|c|c|c|c|c|c|c|c|c|c|c|c|c|c|c|c|c|c|c|}
\hline & & & & & General Informat & & & & & & & & & Facilit & Informati & & & & & & & \\
\hline School Name & Panel & $\begin{array}{l}\text { Grade } \\
\text { Range }\end{array}$ & $\begin{array}{l}\text { Semestered } \\
\text { (Sec) }\end{array}$ & Ward & Trustee & $\begin{array}{c}\text { Superintendent } \\
\text { of Education }\end{array}$ & 2017 LOI & $\begin{array}{c}2017-18 \\
\text { opt } \\
\text { Attend } \\
\text { Reg/F//EF }\end{array}$ & $\begin{array}{l}\text { Site Size } \\
\text { (Acres) }\end{array}$ & $\begin{array}{l}\text { Facility Size } \\
\text { (Sq. Ft.) }\end{array}$ & $\mid \begin{array}{c}\text { Current } \\
\text { Backlog (M) }\end{array}$ & \begin{tabular}{|l} 
Port. \\
(lnstruc)
\end{tabular} & $\begin{array}{c}\text { Port. (Non- } \\
\text { Instruc) }\end{array}$ & $\begin{array}{c}\text { Current FCI } \\
\text { (EDU) }\end{array}$ & $\begin{array}{l}\text { Eco } \\
\text { Schools }\end{array}$ & Pool & $\begin{array}{l}\text { Elem. } \\
\text { D\&T } \\
\text { Shops }\end{array}$ & $\begin{array}{l}\text { Barrier } \\
\text { Free / } \\
\text { Access }\end{array}$ & Elevator & \begin{tabular}{|l} 
Build \\
Date
\end{tabular} & $\begin{array}{l}\text { Building } \\
\text { Age }\end{array}$ & $\begin{array}{c}\text { T.o comm } \\
\text { Centre }\end{array}$ \\
\hline Vincent Massey CI & NDS & & & 2 & Chris Glover & Glenford Duffus & & & 12.75 & 175,033 & & & & & & & & & & 1961 & 56 & \\
\hline West Deane & NDS & & & 2 & Chris Glover & Annie Appleby & & & 5.98 & 33,788 & & & & & & & & & & 1959 & 58 & \\
\hline West Don Lands & NDS & & & 14 & Chris Moise & John Chasty & & & 1.8 & & & & & & & & & & & & & \\
\hline West Park SS & NDS & & & 7 & Robin Pilkey & $\begin{array}{l}\text { Jane Phillips- } \\
\text { Long }\end{array}$ & & & 3.85 & 267,907 & & & & & & & & & & & & \\
\hline Westwood & NDS & & & 3 & Pamela Gough & Tracy Hayhurst & & & & & & & & & & & & & & & & \\
\hline Whitfield Land & NDS & & & 4 & Tiffany Ford & Audley Salmon & & & 3.46 & & & & & & & & & & & & & \\
\hline York Humber & NDS & & & 6 & Chris Tonks & Vicky Branco & & & 5.51 & 157,051 & & & & & & & & & & 1967 & 50 & \\
\hline York University & NDS & & & 4 & Tiffany Ford & Audley Salmon & & & TBD & & & & & & & & & & & & & \\
\hline York University Potential Site 1 & NDS & & & 4 & Tiffany Ford & Audley Salmon & & & & & & & & & & & & & & & & \\
\hline York University Potential Site 2 & NDS & & & 4 & Tiffany Ford & Audley Salmon & & & & & & & & & & & & & & & & \\
\hline
\end{tabular}




\begin{tabular}{|c|c|c|c|c|c|c|c|c|c|c|c|c|c|c|c|c|c|c|}
\hline \multirow[b]{2}{*}{ school Name } & \multicolumn{2}{|c|}{\begin{tabular}{|l} 
Participation Rates \\
\end{tabular}} & \multicolumn{16}{|c|}{2016 Enrolment and Projections FTE, Surplus Seats = OTG Revised - Projection FFE, Utilizations = Projection/TTG Revised } \\
\hline & 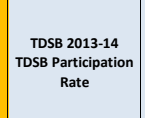 & 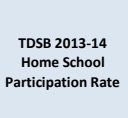 & $\begin{array}{c}\text { Revised } \\
\text { Cepacatity } \\
\text { 2016 }\end{array}$ & $\left|\begin{array}{ccc}\text { Hc } \\
\text { (oct. 2016) }\end{array}\right|$ & $\begin{array}{c}\text { FrT } \\
\text { (oct. 2016) }\end{array}$ & $\begin{array}{c}\text { Utilica } \\
\text { Utiziztion } \\
\text { Rate }\end{array}$ & $\begin{array}{c}2021 \\
\text { Projected }\end{array}$ & $\begin{array}{c}\text { Surplus } \\
\text { surpats } \\
\text { seats }\end{array}$ & $\underbrace{2021}_{\substack{\text { Utilization } \\
\text { Rate }}}$ & $\begin{array}{c}2026 \\
\text { Projected }\end{array}$ & $\begin{array}{c}2026 \\
\text { Surplus } \\
\text { Seats }\end{array}$ & $\begin{array}{c}2026 \\
\text { Utilization } \\
\text { Rate }\end{array}$ & $\begin{array}{c}2031 \\
\text { Projected }\end{array}$ & $\begin{array}{c}\text { surpulus } \\
\text { surpats } \\
\text { seats }\end{array}$ & $\begin{array}{c}\text { Utilization } \\
\text { Rate } \\
\text { Rate }\end{array}$ & $\begin{array}{c}2036 \\
\text { Projected }\end{array}$ & $\begin{array}{c}\text { Surbus } \\
\text { Surpus } \\
\text { seats }\end{array}$ & $\begin{array}{c}\text { Uutization } \\
\text { Utizate } \\
\text { Rate }\end{array}$ \\
\hline Vincent Massey CI & & & o & & & & & & & & & & & & & & & \\
\hline West Deane & & & 。 & & & & & & & & & & & & & & & \\
\hline \multicolumn{19}{|l|}{ West Don Lands } \\
\hline West Park SS & & & 0 & & & & & & & & & & & & & & & \\
\hline \multicolumn{19}{|l|}{ Westwood } \\
\hline \multicolumn{19}{|l|}{ Whitfield land } \\
\hline \multicolumn{19}{|l|}{ York Humber } \\
\hline \multicolumn{19}{|l|}{ Vork University } \\
\hline \multicolumn{19}{|l|}{ York University Potential Site 1} \\
\hline York University Potential Site 2 & & & & & & & & & & & & & & & & & & \\
\hline
\end{tabular}




\section{Appendix B: TDSB Policies}




\section{Toronto District School Board}

Policy P020

Title: $\quad$ TRANSPORTATION OF STUDENTS

Adopted: December 16, 1998

Revised: $\quad$ May 31, 2000, October 27, 2005

Review:

\subsection{OBJECTIVE}

To establish the criteria for the provision of student transportation and safety measures that will be taken

\subsection{RESPONSIBILITY}

Executive Superintendent, Business Services

\subsection{POLICY}

The Toronto District School Board is committed to the provision of safe and reliable transportation for resident students in accordance with the provisions of the Education Act, section 21. (2), and the administrative procedure section of this policy. The means of transportation for eligible students is by school bus, the provision of TTC tickets or by taxi.

\subsection{Eligibility Criteria}

\section{(a) Distance}

Transportation is provided to students who would otherwise be excused from attendance at a school because of distance as provided by the Education Act, section 21. (2), based on grade level as of September 1 of the school year.

(i) Junior Kindergarten to Grade 5: $1.6 \mathrm{~km}$ or more*

(ii) Grades 6 to 8: $3.2 \mathrm{~km}$ or more*

(iii) Grade 9 to OAC: $4.8 \mathrm{~km}$ or more*+

*Distance to be measured from closest public thoroughfare of the residence of the student to nearest public access to the school building.

+ TTC tickets may be available depending on financial need. 


\section{(b) Medical Condition}

Transportation may be provided, regardless of distance, for students who have a medical condition or disability that severely limits walking.

(c) Program Considerations

Transportation is provided:

(i) for students who are placed by an Identification, Placement and Review Committee to a Special Education program that is not located in their home school and who meet the distance criteria in section 3.1 (a);

(ii) for students who are placed in a program by the Toronto District School Board which is not offered in their home school and who meet the distance criteria in section 3.1 (a);

(iii) for students attending a French Immersion program not offered in their home school and who meet the distance criteria in section 3.1 (a);

(iv) for students who, for program purposes as stated in the Education Act, Section 190. (1), are required to attend another school during the course of the school day and who meet the distance criteria in section 3.1 (a);

(v) for students who require treatment at an approved treatment facility during the course of the school day;

(vi) for elementary students who are placed in a holding school by the Toronto District School Board which is located farther than a closer school offering the same program and who meet the distance criteria for JK to Grade 5 in section 3.1 (a).

\section{(d) Alternative Attendance}

Transportation is not provided for students attending any school or special program at their request, even when distance is a factor.

\section{2. $\quad$ Method of Transportation}

(a) Transportation is provided to eligible students in Junior Kindergarten to Grade 5 by contracted carrier services (bus or van) or Board bus.

(i) School-to-school transportation would be offered.

(ii) Designated site-to-school transportation may be provided as a result of an Identification, Placement and Review Committee or for medical reasons. 
(iii) Special Education students in district-wide programs (formerly Metrowide programs) will receive home-to-school transportation.

(b) TTC tickets will be provided to eligible students from Grade 6 through to Grade 8 on parental /guardian request. Grade 6 students may be provided with bus transportation if the most direct TTC route requires more than one transfer. Students in Grades 9 to OAC may receive TTC tickets provided the distance and financial criteria are met.

(c) Taxi service may be provided for eligible students in situations where it is warranted.

\subsection{Summer School Transportation}

Summer school transportation may be provided, either by school bus or TTC, for the following designated programs using the same eligibility criteria as stated in Section 3.1:

(a) Ministry-funded Section 29 programs in treatment centres, hospitals, etc., that are an extension of the regular school year program.

(b) Programs offered by the Toronto District School Board for Special Education students.

(c) Students attending credit programs who are eligible for school bus transportation for medical reasons.

\section{4. $\quad$ Appeal Process}

Parents may appeal the decisions regarding transportation. All appeals will be made to the Transportation Department. Further appeal may be made in writing to the appropriate Supervisory Officer responsible for transportation who will forward it to the Appeal Committee.

\subsection{Transportation Manual}

A Transportation Manual will be developed to include procedures, guidelines and protocols for issues such as:

- Mandatory performance requirements

- Child care

- Staggered school hours

- Section 29 programs

- Cancellation of transportation

- Bus evacuation

- Empty seat procedures

- Accident reporting

- Safety 
- Student conduct

- Medical conditions

- Consolidation of schools

- Glossary

\subsection{Empty Seats}

An procedure shall be established to provide a process for filling seats on school buses that are available after all eligible students have been accommodated.

\subsection{SPECIFIC DIRECTIVES}

The Director is authorized to issue operational procedures to implement this policy.

\subsection{REFERENCE DOCUMENTS}

Operational Procedure PR504, Transportation of Students 


\section{Toronto District School Board}

Policy P068

Title: $\quad$ ACCOMMODATION AND PROGRAM REVIEW

Adopted: $\quad$ May 27, 2009

Effected: $\quad$ July 20, 2009

Revised: $\quad$ March 22, 2016

Reviewed: $\quad$ March 22, 2016

Authorization: Board of Trustees

\section{$\underline{\text { Table of Contents }}$}

$\underline{\text { Section Topic }}$ Page

1.0 Rationale

2.0 Objective

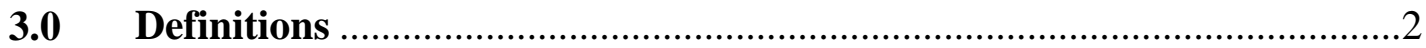

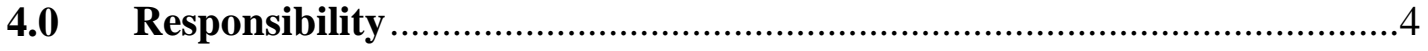

5.0 Application and Scope.......................................................................

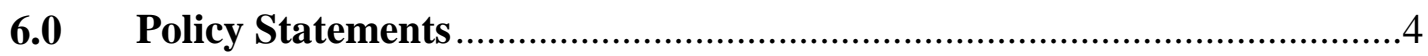

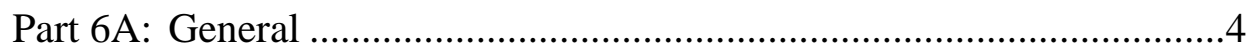

Part 6B: Purpose of Engagement...........................................................

Part 6C: Pupil Accommodation Reviews ..................................................6

Part 6D: Program Area Review ...............................................................9

Part 6E: Changes to Attendance Boundaries of Schools (Regular Day School Programs) ..............................................10

Part 6F: Other Decisions .......................................................................11

Part 6G: Summary Chart.......................................................................12

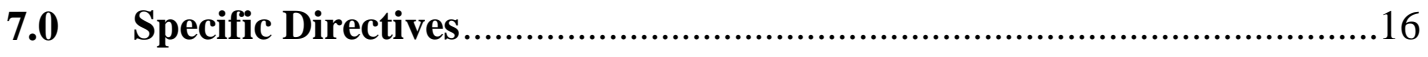

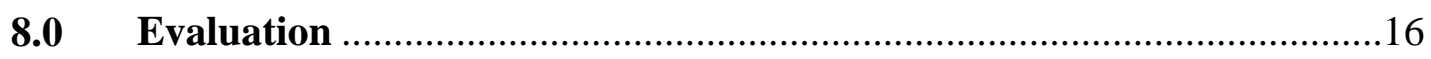

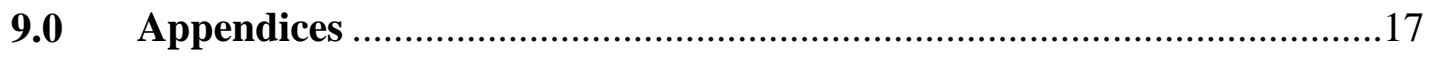

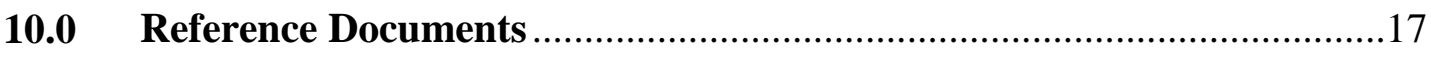




\subsection{RATIONALE}

This policy is a requirement of the Ministry of Education and is intended to adhere to the Ministry’s Pupil Accommodation Review Guideline that may be amended from time to time.

\subsection{OBJECTIVE}

To provide guidance and to set parameters for processes leading to changes in the location of schools and programs, consolidation of schools, and changes in the configuration of grades and/or programs within specific schools.

\subsection{DEFINITIONS}

Affected parents and students: In this policy, "affected parents and students" means parents of students and students, where students are enrolled in a program or school that will be affected by a decision concerning configuration or location of the program or school.

Alternative school: A school that is unique in pedagogy, form of governance, and staff involvement; has strong parental and/or student involvement; and provides an educational experience suited to individual learning styles/preferences and/or needs.

Annual Planning Document: A detailed list of program and accommodation studies that will be done during the next year. It reflects the short term work in the form of Local Feasibility Studies that will be done to execute the Long-Term Program and Accommodation Strategy.

Attendance boundary: The geographical limits that define the area served by a school or a program.

Attendance Boundary Change Study: A study of attendance boundaries between two schools may be undertaken to reduce over-crowding, to utilize surplus space, or to designate addresses to a closer school.

Exemption to Pupil Accommodation Review: Circumstance included in the Ministry of Education's Pupil Accommodation Review Guideline where a Pupil Accommodation Review process does not have to be undertaken.

Facility: A building in which a school operates. 
Intensive Support Program: A special education program for students with one or more exceptionalities, in which students are enrolled for more than half of the instructional program on a school day.

Local Feasibility Study: A preliminary analysis of an accommodation or program concept to determine its viability that is conducted by a staff team and the affected Trustee(s). The team, called the Local Feasibility Team, is led by the local Superintendent(s) of Education. The study could lead to other formal public processes such as Boundary Reviews, Program Area Reviews and Pupil Accommodation Reviews that include community engagement.

Long-Term Program and Accommodation Strategy: A roadmap for the future that provides an approach to program and accommodation planning with a ten-year timeframe that allows for responsiveness and flexibility in a changing environment by including an annual cycle of review.

Modified Pupil Accommodation Review Process: In certain circumstances, where potential pupil accommodation options are deemed by the Board to be less complex, Trustees may decide to undertake a modified Pupil Accommodation Review process.

Parents: Includes legal guardians.

Program Area Review: A process undertaken for consideration of changes to grade configuration of a school (excluding changes to grade configurations of French Immersion or Extended French programs and programs for students with exceptionalities); the establishment of new schools; or new specialized programs.

Pupil Accommodation Review: A process that involves studying a school that is significantly under-utilized and its adjacent schools. The review will consider ways to reduce surplus space and build viable programs. The review may result in the movement of a large number of students between schools. A school closure is a possible outcome.

School: A body of elementary or secondary students that is organized as a unit for educational purposes by the TDSB, and includes the teachers and other staff members associated with the unit and the lands and premises used in connection with the unit.

Specialized program: A program with a particular curricular focus that has unique admission criteria and specific program requirements. The attendance boundary of specialized programs is the city so that students throughout the city may apply for admission. "Specialized programs" do not include French Immersion or Extended French programs or programs for students with exceptionalities. [Note: a policy to formally define a specialized program (codifying current practice) will be developed and cross-referenced here.] 


\subsection{RESPONSIBILITY}

Associate Director, Finance and Operations

\subsection{APPLICATION AND SCOPE}

This policy will apply to staff engaged in program and accommodation reviews.

\subsection{POLICY STATEMENTS}

\section{Part 6A: General}

6A.1 The Long-Term Program and Accommodation Strategy approved annually by the Board will determine which program and accommodation planning reviews will be completed over a ten-year period.

6A.2 The Board is committed to providing excellent educational programs in schools that are as accessible as practicable to students intended to be served by the school.

6A.3 To the extent possible, the Board will provide fair and reasonable access to programs, staff, resources, and facilities, taking into consideration student population, program needs, policies established by the Board, the preferences of parents and communities, and available resources.

6A.4 The Board will make effective and efficient use of facilities to maximize the effectiveness of programs and to ensure that resources are available for programs and supports for students.

6A.5 Whenever the Board considers changes in the location or configuration of programs and schools, staff shall ensure that those who could be most affected have an opportunity to comment on proposed changes.

(a) The opportunity to comment on proposed changes shall be provided to students, parents, staff, and members of the community who could be most affected by changes under consideration.

(b) Public notice shall be provided in advance of decisions to the extent that is necessary and practicable using a variety of means. It is recognized that the requirements of staffing allocation and other operational requirements may make it difficult to provide a significant period of notice, or make it impracticable in some instances to provide an opportunity for comment in advance of decisions. 
6A.6 The processes to provide an opportunity to comment shall differ according to the nature of the changes under consideration.

6A.7 For consideration of decisions concerning closure of a school, or relocation of a grade, grades, or program where the change would affect more than $50 \%$ of the enrolment of a school, a Pupil Accommodation Review Committee shall be established in accordance with Part 6C, Pupil Accommodation Reviews of this policy.

6A.8 For consideration of changing the grade configuration of a school ${ }^{1}$, or the establishment of new schools and new specialized programs, a Program Area Review Team shall be established in accordance with Part 6D, Program Area Reviews.

6A.9 For consideration of changes to attendance boundaries of schools, a public meeting shall be held in accordance with Part 6E, Changes to Attendance Boundaries of this policy.

6A.10 For consideration of all other decisions affecting the configuration and location of programs, notice shall be given to parents, students, and school councils in accordance with Part 6F, Other Decisions of this policy.

6A.11 Nothing in this policy shall prevent a more extensive engagement of those affected by a change in programs or accommodation, where the Director of Education or the Board determines that a more extensive process of engagement is warranted by the circumstances or the potential impact of changes.

6A.12 The Director of Education shall establish operational procedures for Pupil Accommodation Reviews that meet all the requirements of the Pupil Accommodation Review Guidelines of the Ministry of Education and ensure that Pupil Accommodation Review Committees follow these operational procedures.

6A.13 The Director of Education shall establish operational procedures for Program Area Reviews and ensure that Program Area Review Teams follow these procedures. Operational procedures for Program Area Reviews shall allow for significant flexibility in processes and time frames to support effective and efficient development of recommendations.

6A.14 This policy shall not apply to any programs or services operated by external agencies provided in TDSB facilities.

\section{Part 6B: Purpose of Engagement}

${ }^{1}$ Excluding changes to grade configurations of French Immersion or Extended French programs and programs for students with exceptionalities. 
6B.1 Effective engagement with those affected by decisions is intended to ensure that the decisions of the Director of Education and the Board are fully informed and carefully considered. Effective engagement is also intended to support accountability of staff and the Board, and give confidence to those affected that decisions were made on a sound basis.

6B.2 Staff shall engage affected students, parents, staff, and members of the community to help ensure that:

(a) The school community has an opportunity to request and contribute information relevant to the decision, and the Board receives information relevant to the decision;

(b) The school community has an opportunity to contribute to the identification of an appropriate range of options, and the Board has an opportunity to consider an appropriate range of options;

(c) The school community has an opportunity to contribute to the identification of potential impacts of options under consideration, and the Board has an opportunity to consider the potential impact of options under consideration; and

(d) Persons affected by decisions have an opportunity to understand the reasons for recommendations and decisions.

\section{Part 6C: Pupil Accommodation Reviews}

6C.1 A Pupil Accommodation Review Committee (PARC) shall be established where changes under consideration include closure of a school; or relocation of a grade, grades, or program where the change would affect more than $50 \%$ of the enrolment of a school.

6C.2 Exemptions:

The Ministry's guideline applies to schools offering elementary or secondary programs. However, there are specific circumstances where school boards are not obligated to undertake a pupil accommodation review. As per the guideline, these include:

- When a replacement school is to be built by the school board on the existing site, or built or acquired within the existing school attendance boundary, as identified through the school board's policy;

- When a replacement school is to be built by the school board on the existing site, or built or acquired within the existing school attendance boundary and the school community must be temporarily relocated to ensure the safety of students and staff during the reconstruction, as identified through the school board's policy; 
- When a lease for the school is terminated;

- When a school board is planning the relocation (in any school year or over a number of school years) of grades or programs, in which the enrolment constitutes less than $50 \%$ of the school's enrolment (this calculation is based on the enrolment at the time of the relocation, or the first phase of a relocation carried over a number of school years);

- When a school board is repairing or renovating a school, and the school community must be temporarily relocated to ensure the safety of students during the renovations;

- Where a facility has been serving as a holding school for a school community whose permanent school is over-capacity and/or is under construction or repair; or

- Where there are no students enrolled at the school at any time throughout the school year.

In the above circumstances, a school board is expected to inform school communities about proposed accommodation plans for students before a decision is made by the Board of Trustees. A public meeting may be held if required.

6C.3 Where the initiation of a PARC is being considered, an Initial Staff Report with one or more options that address the accommodation issue(s) must be prepared and presented to the Board of Trustees. The Initial Staff Report will identify one staff recommended option with rationale. The Board shall approve the establishment of a PARC for a group of schools or for a single school.

6C.4 Parents, school council members, staff, and residents within the attendance boundaries $^{1}$ of schools included in a PARC shall be informed in a timely manner in writing of the Board's decision to establish a PARC. Information shall be provided through affected schools and other appropriate service providers (such as public libraries and childcare providers). The Board's decision to establish a PARC shall be posted on the Board's website and disseminated in a variety of ways.

6C.5 PARC members shall be informed about their advisory role that serves as an information conduit between the public and the Board. They will be supported by staff in their role.

6C.6 Staff shall ensure that those most affected by possible decisions have access to information which is being used to develop options and recommendations.

6C.7 Staff shall provide information to the school communities of schools included in the PARC in a timely and ongoing manner.

\footnotetext{
${ }^{1}$ For schools with Board-wide boundaries, staff shall determine an appropriate boundary adjacent to the schools within which to provide information.
} 
6C.8 Staff recommendations shall be provided to the members of the PARC and to the public in accordance with the Board's procedures.

6C.9 A member of the PARC may submit a dissenting report to the superintendent of education leading the review if he or she disagrees with staff's recommendations.

6C.10 At the conclusion of the PARC process, staff will create a Final Staff Report with a staff recommended option. The Director of Education shall provide staff recommendations to the Board.

6C.11 If a trustee has provided written comments to staff concerning the review, and if the trustee so requests, staff shall include the trustee's comments to the Board as part of the report with staff recommendations.

6C.12 The PARC, and members of the public who wish to comment on the recommendations, shall be permitted to submit written statements or request to make delegations to an appropriate committee of the Board, or (at the Board's discretion) to the Board through another process determined by the Board, before the Board makes its decision.

6C.13 The Board shall approve decisions concerning matters addressed by a PARC.

6C.14 The Board may make a decision concerning schools under review by a PARC that differs from staff recommendations.

6C.15 Current information and relevant documents about a review such as staff's initial and final recommendation; the PARC's meetings, and the Board's decision shall be posted on the Board's website.

6C.16 A copy of the current guideline of the Ministry of Education that governs pupil accommodation reviews shall be attached to this policy and be posted on the Board's website as part of this policy.

6C.17 Modified Pupil Accommodation Review Process:

As per the Ministry’s Pupil Accommodation Review Guideline, a modified, shortened review process can be approved by the Board in situations that are less complex. The Initial Staff Report shall explain the rationale for exempting a school(s) from the standard Pupil Accommodation Review process. The rationale for conducting a modified Pupil Accommodation Review process shall be based on one or more of the following factors.

(a) Enrolment

(i) Where current and projected enrolment have reached a level where programming is non-viable and programming options for students are limited, such as:

(A) An elementary school with an enrolment equal to or less than 150 students for the current school year and projected to remain so for the next five years. 
(B) A secondary school with an enrolment equal to or less than 350 students for the current school year and projected to remain so for the next five years.

(b) Utilization Rate

(i) Where a school has a current utilization rate of $65 \%$ or lower and is projected to remain so for the next five years. Utilization shall be determined by dividing the school's enrolment by the capacity of the school building.

(c) Facility Condition

(i) Where a school facility is not physically suitable to serve the school community, such as:

(A) Where retrofitting or repair may involve major capital investment; or

(B) Where the Facility Condition Index deems the school Prohibitive to Repair.

(d) Distance

(i) The distance to the nearest available accommodation is within walking distance (as per TDSB's Transportation of Students policy P020) of the addresses served by the school proposed for closure.

\section{Part 6D: Program Area Reviews}

6D.1 The Director of Education shall approve the establishment of a Program Area Review Team (PART). PARTs shall be established where changes under consideration include:

(a) Establishment of a new school;

(b) Establishment of a new alternative school;

(c) Establishment of a single-track French Immersion/Extended French school;

(d) Relocation of a grade or grades ${ }^{1}$ where the change would affect less than $50 \%$ of the enrolment of a school;

(e) Establishment of a new specialized program;

(f) Any other program or accommodation change where the Director decides that a PART is required, or

\footnotetext{
${ }^{1}$ Excluding French Immersion or Extended French programs and programs for students with exceptionalities.
} 
(g) Any other program or accommodation change where the Board decides that a PART is required.

6D.2 The PART shall have opportunity to explore and develop reasonable alternatives.

6D.3 The PART shall ensure that those most affected by possible decisions have access to information which is being used to develop options and recommendations.

6D.4 Trustees of wards in which schools are affected by decisions set out in Part 6D.1 shall be invited to participate on the PART.

6D.5 A superintendent of education responsible for schools included in the PART shall chair the PART and lead the development of recommendations.

6D.6 In addition to the local trustee(s), PART members will include parent and school council representatives and principals. Other members could include community and child care centre representatives.

6D.7 A member of a PART may submit a dissenting report to the superintendent of education leading the review if he or she disagrees with the PART's recommendations.

6D.8 If a trustee has provided written comments to staff concerning the PART's recommendations, and if the trustee so requests, staff shall include the trustee's comments to the Board as part of the report with staff recommendations.

6D.9 Staff shall review the recommendations made by the PART and provide staff recommendations to the Board. A dissenting report, if submitted, shall be provided to Board with the staff's recommendations.

6D.10 The PART, and members of the public who wish to comment on the recommendations of the PART report, shall be permitted to make a delegation to an appropriate committee of the Board before the Board considers recommendations arising from the PART.

6D.11 Approval of the Board is required for decisions arising from a PART that is established under Part 6D.1.

6D.12 Current information about the PART's meetings, its report and recommendations, and the Board's decision shall be posted on the Board's website.

\section{Part 6E: Changes to Attendance Boundaries of Schools (Regular Day School Programs)}

6E.1 Staff shall provide public notice and conduct at least one public meeting where changes to a school's attendance boundaries are under consideration.

(a) The public notice shall be provided to parents, students, and the school councils of the schools that could be affected by the decision. Notice shall be provided through affected schools. At the discretion of staff, notice may also be provided to residents within the attendance boundaries that could be affected by a change. The public notice shall include information 
about the time and location of a public meeting to address the proposed change.

(b) Notice may be provided in a variety of ways, including but not limited to letters, notices posted in schools or on the schools' websites. Notice shall also be posted on the Board's website.

(c) Notice shall be provided in advance of decisions related to changes to attendance boundaries to the extent that is practicable. It is recognized that the requirements of staffing allocation and other operational requirements may make it difficult to provide a significant period of notice.

6E.2 Staff shall provide notice to trustees of wards in which affected schools are located of the intent to consider changes to attendance boundaries and provide an opportunity to comment on proposed changes in advance of the public meeting. Staff shall invite trustees of wards in which affected schools are located to participate in the public meeting.

6E.3 Staff shall provide the relevant information which was used to develop the proposed change at the public meeting, and address the options that were considered. The public meeting shall provide an opportunity for those present to ask questions and suggest alternatives.

6E.4 The trustees of wards in which affected schools are located shall have an opportunity to comment on draft recommendations. If a trustee has provided written comments to staff, and if the trustee so requests, staff shall include the trustee's comments to the Board as part of the report with staff recommendations.

6E.5 Staff shall provide recommendations to the Board following the public meeting.

6E.6 The Board shall approve a change in an attendance boundary of a school.

\section{Part 6F: Other Decisions}

6F.1 The Director may make decisions concerning the location, configuration or attendance boundary of programs in specific schools other than those decisions addressed in the above sections of this policy.

6F.2 Decisions referred to in Part 6F.1 include but are not limited to:

(a) Relocation of a specialized program or replication of a specialized program in an additional site;

(b) Establishment of a new site or relocation of a site for a French Immersion or Extended French program; ${ }^{1}$

\footnotetext{
${ }^{1}$ Establishment and relocation of French Immersion and Extended French programs are addressed operational pro-
} cedures (PR557, PR577 and PR597). 
(c) Expansion or contraction of classes in a site of a French Immersion or Extended French program;

(d) Establishment, relocation, expansion, or contraction of sites for Intensive Support Programs for students with exceptionalities; and

(e) Establishment, relocation, expansion, or contraction of sites for Literacy Enrichment Academic Programs (LEAP), self-contained English as a Second Language classes, Caring and Safe Schools alternative programs, Supervised Alternative Learning (SAL) programs, and programs provided through the Continuing Education and Business Development and Community Services departments.

(f) Changes to the attendance boundary of programs mentioned in this section.

6F.3 Staff shall provide trustees of wards in which schools are affected with timely information regarding the changes under consideration.

6F.4 With respect to decisions referred to in Part 6F.2 staff shall provide notice to affected parents, students and school councils.

6F.5 Notice may be provided in a variety of ways, including but not limited to letters, notices posted in schools, or notices on the schools' websites.

6F.6 Notice shall be provided in advance of decisions referred to in Part 6F.2 to the extent that is practicable. It is recognized that the requirements of staffing allocation and other operational requirements may make it difficult to provide a significant period of notice.

\section{Part 6G: Summary Chart}

6G.1 The following table is a summary of the directions established by this policy, organized by type of decision.

6G.2 This table is provided only to facilitate reference to the policy. If there is any conflict between the table and the sections set out above, the sections above prevail.

\begin{tabular}{|c|c|c|}
\hline$\underline{\text { Changes Under Consideration }}$ & $\underline{\text { Process to be Followed }}$ & $\underline{\text { Policy }}$ \\
\hline \multicolumn{3}{|c|}{ Affecting elementary or secondary regular day-school programs } \\
\hline $\begin{array}{ll}\text { - } & \text { Closure of a school; or } \\
\text { - } & \text { Relocation of a grade, grades, or }\end{array}$ & $\begin{array}{l}\text { Approval of Long-Term } \\
\text { Program and Accommoda- }\end{array}$ & $\begin{array}{l}\text { Part 6C, Pupil Ac- } \\
\text { commodation Re- }\end{array}$ \\
\hline
\end{tabular}


Policy P068

Accommodation and Program Review

\begin{tabular}{|c|c|c|}
\hline$\underline{\text { Changes Under Consideration }}$ & $\underline{\text { Process to be Followed }}$ & $\begin{array}{l}\text { Policy } \\
\underline{\text { Section }}\end{array}$ \\
\hline $\begin{array}{l}\text { program where the change would af- } \\
\text { fect more than } 50 \% \text { of the enrolment } \\
\text { of a school. }\end{array}$ & $\begin{array}{l}\text { tion Strategy } \\
\text { Local Feasibility Team } \\
\text { Pupil Accommodation Re- } \\
\text { view Committee } \\
\text { Comment by affected trus- } \\
\text { tee(s) } \\
\text { Notice to statutory and } \\
\text { community advisory com- } \\
\text { mittees where appropriate } \\
\text { Approval by Board }\end{array}$ & views \\
\hline $\begin{array}{l}\text { - Establishment of a new school; or } \\
\text { - Establishment of a new alternative } \\
\text { school. } \\
\text { - Relocation of a grade or grades } \\
\text { where the change would affect less } \\
\text { than } 50 \% \text { of the enrolment of a } \\
\text { school; or } \\
\text { - Establishment of a new specialized } \\
\text { program. }\end{array}$ & $\begin{array}{l}\text { Approval of Long-Term } \\
\text { Program and Accommoda- } \\
\text { tion Strategy } \\
\text { Local Feasibility Team } \\
\text { Program Area Review } \\
\text { Team } \\
\text { Comment by affected trus- } \\
\text { tee(s) } \\
\text { Notice to statutory and } \\
\text { community advisory com- } \\
\text { mittees where appropriate } \\
\text { Approval by Board }\end{array}$ & $\begin{array}{l}\text { Part 6D, Program } \\
\text { Area Reviews }\end{array}$ \\
\hline $\begin{array}{l}\text { - Relocation of a specialized program; } \\
\text { or } \\
\text { - } \quad \text { Replication of an existing special- } \\
\text { ized program in another site. }\end{array}$ & $\begin{array}{l}\text { Approval of Long-Term } \\
\text { Program and Accommoda- } \\
\text { tion Strategy } \\
\text { Local Feasibility Team } \\
\text { Notice to affected parents, } \\
\text { students, and school coun- } \\
\text { cil }\end{array}$ & $\begin{array}{l}\text { Part 6F, Other Deci- } \\
\text { sions }\end{array}$ \\
\hline
\end{tabular}


Policy P068

Accommodation and Program Review

\begin{tabular}{|c|c|c|}
\hline Changes Under Consideration & $\underline{\text { Process to be Followed }}$ & $\underline{\text { Policy }}$ \\
\hline & $\begin{array}{l}\text { Comment by affected trus- } \\
\text { tee(s) } \\
\text { Notice to statutory and } \\
\text { community advisory com- } \\
\text { mittees where appropriate } \\
\text { Approval by Director or } \\
\text { designate }\end{array}$ & \\
\hline $\begin{array}{l}\text { Attendance boundaries of a school } \\
\text { (regular school day program) }\end{array}$ & $\begin{array}{l}\text { Approval of Long-Term } \\
\text { Program and Accommoda- } \\
\text { tion Strategy } \\
\text { Local Feasibility Team } \\
\text { Comment by affected trus- } \\
\text { tee(s) } \\
\text { Notice to statutory and } \\
\text { community advisory com- } \\
\text { mittees where appropriate } \\
\text { Public notice and at least } \\
\text { one public meeting } \\
\text { Approval by Board }\end{array}$ & $\begin{array}{c}\text { Part } 6 \mathrm{E} \text {, Changes to } \\
\text { Attendance Bounda- } \\
\text { ries }\end{array}$ \\
\hline \multicolumn{3}{|c|}{ Affecting French Immersion and Extended French programs } \\
\hline $\begin{array}{l}\text { Relocation of a grade, grades, or } \\
\text { program where the change would af- } \\
\text { fect more than } 50 \% \text { of the enrolment } \\
\text { of a school. } \\
\text { - Closure of a single track French } \\
\text { Immersion/Extended French school. }\end{array}$ & $\begin{array}{l}\text { Approval of Long-Term } \\
\text { Program and Accommoda- } \\
\text { tion Strategy } \\
\text { Local Feasibility Team } \\
\text { Pupil Accommodation Re- } \\
\text { view Committee } \\
\text { Comment by affected trus- } \\
\text { tee(s) }\end{array}$ & $\begin{array}{l}\text { Part 6C, Pupil Ac- } \\
\text { commodation Re- } \\
\text { views }\end{array}$ \\
\hline
\end{tabular}


Policy P068

Accommodation and Program Review

\begin{tabular}{|c|c|c|}
\hline Changes Under Consideration & Process to be Followed & $\frac{\text { Policy }}{\text { Section }}$ \\
\hline & $\begin{array}{l}\text { Notice to statutory and } \\
\text { community advisory com- } \\
\text { mittees where appropriate } \\
\text { Approval by Board }\end{array}$ & \\
\hline $\begin{array}{l}\text { - Establishment of a single-track } \\
\text { French Immersion/Extended French } \\
\text { school. }\end{array}$ & $\begin{array}{l}\text { Approval of Long-Term } \\
\text { Program and Accommoda- } \\
\text { tion Strategy } \\
\text { Local Feasibility Team } \\
\text { Program Area Review } \\
\text { Team } \\
\text { Comment by affected trus- } \\
\text { tee(s) } \\
\text { Notice to statutory and } \\
\text { community advisory com- } \\
\text { mittees where appropriate } \\
\text { Approval by Board }\end{array}$ & $\begin{array}{l}\text { Part 6D, Program } \\
\text { Area Reviews }\end{array}$ \\
\hline $\begin{array}{l}\text { Establishment of a new site for a } \\
\text { French Immersion or Extended } \\
\text { French program. } \\
\text { - Relocation of a site for a French } \\
\text { Immersion or Extended French pro- } \\
\text { gram. } \\
\text { - Expansion or contraction of classes } \\
\text { in a site for a French Immersion or } \\
\text { Extended French program. }\end{array}$ & $\begin{array}{l}\text { Notice to affected parents, } \\
\text { students, and school coun- } \\
\text { cil } \\
\text { Comment by affected trus- } \\
\text { tee(s) } \\
\text { Notice to statutory and } \\
\text { community advisory com- } \\
\text { mittees where appropriate } \\
\text { Approval by Director or } \\
\text { designate }\end{array}$ & $\begin{array}{l}\text { Part 6F, Other Deci- } \\
\text { sions }\end{array}$ \\
\hline \multicolumn{3}{|c|}{ Affecting Intensive Support Programs for students with exceptionalities } \\
\hline $\begin{array}{l}\text { Relocation of a grade, grades, or } \\
\text { program where the change would af- } \\
\text { fect more than } 50 \% \text { of the enrolment } \\
\text { of a school. }\end{array}$ & $\begin{array}{l}\text { Approval of Long-Term } \\
\text { Program and Accommoda- } \\
\text { tion Strategy }\end{array}$ & $\begin{array}{l}\text { Part 6C, Pupil Ac- } \\
\text { commodation Re- } \\
\text { views }\end{array}$ \\
\hline
\end{tabular}




\begin{tabular}{|c|c|c|}
\hline$\underline{\text { Changes Under Consideration }}$ & $\underline{\text { Process to be Followed }}$ & $\begin{array}{l}\text { Policy } \\
\text { Section }\end{array}$ \\
\hline & $\begin{array}{l}\text { Local Feasibility Team } \\
\text { Pupil Accommodation Re- } \\
\text { view Committee } \\
\text { Comment by affected trus- } \\
\text { tee(s) } \\
\text { Notice to statutory and } \\
\text { community advisory com- } \\
\text { mittees where appropriate } \\
\text { Approval by Board }\end{array}$ & \\
\hline $\begin{array}{l}\text { Establishment of a new site for an } \\
\text { Intensive Support Program. } \\
\text { - Relocation of site for an Intensive } \\
\text { Support Program. } \\
\text { Expansion or contraction of the } \\
\text { number of classes in a site of an In- } \\
\text { tensive Support Program. }\end{array}$ & $\begin{array}{l}\text { Notice to affected parents, } \\
\text { students, and school coun- } \\
\text { cil } \\
\text { Notice to statutory and } \\
\text { community advisory com- } \\
\text { mittees where appropriate } \\
\text { Information to affected } \\
\text { trustee(s); comment by af- } \\
\text { fected trustee(s) } \\
\text { Approval by Director or } \\
\text { designate }\end{array}$ & $\begin{array}{l}\text { Part 6F, Other Deci- } \\
\text { sions }\end{array}$ \\
\hline \multicolumn{3}{|l|}{ Affecting all other programs } \\
\hline $\begin{array}{l}\text { Establishment, relocation, expan- } \\
\text { sion, or contraction of sites for Lit- } \\
\text { eracy Enrichment Academic Pro- } \\
\text { grams (LEAP), self-contained Eng- } \\
\text { lish as a Second Language classes, } \\
\text { Caring and Safe Schools alternative } \\
\text { programs, Supervised Alternative } \\
\text { Learning (SAL) programs, and pro- } \\
\text { grams provided through the Con- } \\
\text { tinuing Education and Business De- } \\
\text { velopment and Community Services } \\
\text { departments. }\end{array}$ & $\begin{array}{l}\text { Notice to parents, students, } \\
\text { and school council } \\
\text { Notice to statutory and } \\
\text { community advisory com- } \\
\text { mittees where appropriate } \\
\text { Information to affected } \\
\text { trustee(s); comment by lo- } \\
\text { cal trustee(s) } \\
\text { Approval by Director or }\end{array}$ & $\begin{array}{l}\text { Part 6F, Other Deci- } \\
\text { sions }\end{array}$ \\
\hline
\end{tabular}




\begin{tabular}{|l|l|l|}
\hline \multicolumn{1}{|c|}{ Changes Under Consideration } & Process to be Followed & Policy \\
\hline $\begin{array}{l}\text { Any other decisions affecting the } \\
\text { configuration or location of pro- } \\
\text { grams and schools not addressed in } \\
\text { this policy. }\end{array}$ & designate & \\
\hline
\end{tabular}

\subsection{SPECIFIC DIRECTIVES}

The Director is authorized to issue operational procedures to implement this policy.

\subsection{EVALUATION}

This policy shall be reviewed every four (4) years after the effective date or sooner if revisions to the guidelines associated with the policies are instituted by the Ministry of Education.

\subsection{APPENDICES}

Appendix A: Ministry of Education’s Pupil Accommodation Review Guideline - March 2015

Appendix B: Ministry of Education's Administrative Review of the Accommodation Review Process

\subsection{REFERENCE DOCUMENTS}

\section{$\underline{\text { Board Policies }}$}
Alternative Schools, (P062)
Continuing Education, (P019)
Operational Procedures
Alternative Schools (PR584)
Program Review of Dual Track Schools (PR577)
Space Accommodation for Child Care (PR662)


Appendix A

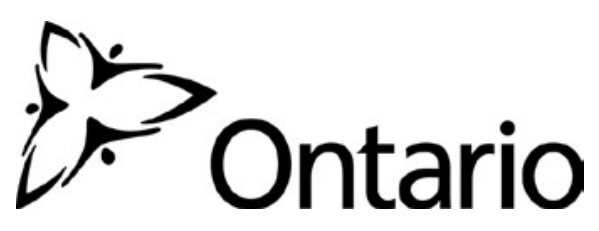

\section{MINISTRY OF EDUCATION \\ PUPIL ACCOMMODATION REVIEW GUIDELINE}

March 2015 


\section{TABLE OF CONTENTS}

\section{PREAMBLE}

I. PURPOSE

II. INTRODUCTION

III. GUIDING PRINCIPLES

IV. SCHOOL BOARD ACCOMMODATION REVIEW POLICIES

V. SCHOOL BOARD PLANNING PRIOR TO AN

ACCOMMODATION REVIEW

VI. ESTABLISHING AN ACCOMMODATION REVIEW

VII. THE ACCOMMODATION REVIEW COMMITTEE

VIII. SCHOOL INFORMATION PROFILE

IX. CONSULTATION WITH LOCAL MUNICIPAL GOVERNMENTS

$X$ PUBLIC MEETINGS

XI. COMPLETING THE ACCOMODATION REVIEW

XII. TRANSITION PLANNING

XIII. TIMELINES FOR THE ACCOMMODATION REVIEW PROCESS

XIV. MODIFIED ACCOMMODATION REVIEW PROCESS

XV. EXEMPTIONS

XVI. DEFINITIONS 


\section{PREAMBLE}

School boards are responsible for managing their school capital assets in an effective manner. They must respond to changing demographics and program needs while ensuring continued student achievement and well-being, and the financial viability/sustainability of the school board.

One aspect of a school board's capital and accommodation planning is reviewing schools that have underutilized space. These are schools where the student capacity of the school is greater than the number of students enrolled. When a school board identifies a school that is projected to have long-term excess space, a school board would typically look at a number of options such as:

- moving attendance boundaries and programs to balance enrolment between over and underutilized schools;

- offering to lease underutilized space within a school to a coterminous school board;

- finding community partners who can pay the full cost of operating the underutilized space; and/or

- decommissioning or demolishing a section of the school that is not required for student use to reduce operating costs.

If none of these options are deemed viable by a school board, the board may determine that a pupil accommodation review process take place which could lead to possible school consolidations and closures. These decisions are made within the context of supporting the school board's student achievement and well- being strategy and to make the most effective use of its school buildings and funding.

The Ministry of Education expects school boards to work with their community partners when undertaking capital planning, including when a school board is beginning to develop options to address underutilized space in schools. The Ministry of Education's Community Planning and Partnerships Guideline (CPPG) outlines requirements for school boards to reach out to their local municipalities and other community partners to share planning related information and to explore potential partnership opportunities. This version of the Pupil Accommodation Review Guideline (the "Guideline") builds upon the CPPG by providing requirements for school boards to share information with and seek feedback from their local municipalities and other community partners related to any pupil accommodation reviews a school board initiates.

If a pupil accommodation review results in a school closure decision, a school board will then need to decide whether to declare that school as surplus, potentially leading to the future sale of the property. These sales are governed by provincial regulation. Alternately, a school board may decide to use a closed school for other school board purposes, or hold the property as a strategic long- term asset of the school board due to a projected need for the facility in the 
future. Each school board decides when it is appropriate to review its strategic property holdings to determine if these properties are still required to be held or should be considered surplus to the school board's needs and considered for a future sale.

This document provides direction to school boards on one component of their capital planning - the pupil accommodation review process. It provides the minimum standards the province requires school boards to follow when undertaking a pupil accommodation review. It is important to note that school boards have flexibility to modify their pupil accommodation review policies to meet their local needs, and can develop policies that exceed the provincial minimum standards outlined in this document.

\section{PURPOSE}

The purpose of the Guideline is to provide a framework of minimum standards for school boards to undertake pupil accommodation reviews to determine the future of a school or group of schools. This Guideline ensures that where a decision is taken by a school board regarding the future of a school, that decision is made with the involvement of an informed local community and is based on a broad range of criteria regarding the quality of the learning experience for students.

This Guideline is effective upon release and replaces the previous Guideline of June 2009.

\section{INTRODUCTION}

Ontario's school boards are responsible for deciding the most appropriate pupil accommodation arrangements for the delivery of their elementary and secondary programs. These decisions are made by school board trustees in the context of carrying out their primary responsibilities of fostering student achievement and well-being, and ensuring effective stewardship of school board resources. In some cases, to address changing student populations, this requires school boards to consider undertaking pupil accommodation reviews that may lead to school consolidations and closures.

Under paragraph 26, subsection 8 (1) of the Education Act, the Minister of Education may issue guidelines with respect to school boards' school closure policies.

\section{GUIDING PRINCIPLES}

The Guideline has been established to align with the Ministry of Education's vision and as such, focuses on student well-being; academic achievement; and school board financial viability/sustainability. 
All school board pupil accommodation review policies should be designed to align with these guiding principles.

\section{SCHOOL BOARD ACCOMMODATION REVIEW POLICIES}

School boards are responsible for creating and implementing a policy to address pupil accommodation reviews to serve their local needs. The Ministry of Education expects school boards to consult with local communities prior to adopting or subsequently amending their pupil accommodation review policies.

All pupil accommodation review policies must be clear in stipulating that the final decision regarding the future of a school or group of schools rests solely with the Board of Trustees. If the Board of Trustees votes to close a school or schools in accordance with their policy, the school board must provide clear timelines regarding the closure(s) and ensure that a transition plan is communicated to all affected school communities within the school board.

It is important to note that this Guideline is intended as a minimum requirement for school boards in developing their policies. School boards are responsible for establishing and complying with their pupil accommodation review policies to serve their local needs.

A copy of the school board's pupil accommodation review policy, the government's Pupil Accommodation Review Guideline and the Administrative Review of Accommodation Review Process documents are to be made available to the public as determined in the school board's policy, and posted on the school board's website.

The Guideline recognizes that pupil accommodation reviews include a school or group of schools to facilitate the development of viable solutions for pupil accommodation that support the guiding principles.

School board pupil accommodation review policies will include statements that encourage the sharing of relevant information as well as providing the opportunity for the public and affected school communities to be heard.

The Ministry of Education recommends that, wherever possible, schools should only be subject to a pupil accommodation review once in a five-year period, unless there are circumstances determined by the school board, such as a significant change in enrolment.

\section{SCHOOL BOARD PLANNING PRIOR TO AN ACCOMMODATION REVIEW}

As described in the Community Planning and Partnerships Guideline, school boards must undertake long-term capital and accommodation planning, informed by any relevant information obtained from local municipal governments and other community partners, which takes into consideration long-term enrolment projections and planning opportunities for the effective use of excess space in all area schools. 
School boards must document their efforts to obtain information from local municipal governments as well as other community partners that expressed an interest prior to the pupil accommodation review; and provide any relevant information from municipalities and other community partners as part of the initial staff report (see Section VI).

\section{ESTABLISHING AN ACCOMMODATION REVIEW}

School boards may proceed to establish a pupil accommodation review only after undertaking the necessary assessment of long-term capital and accommodation planning options for the school(s).

\section{Initial Staff Report}

Prior to establishing a pupil accommodation review, the initial staff report to the Board of Trustees must contain one or more options to address the accommodation issue(s). Each option must have a supporting rationale. There must be a recommended option if more than one option is presented. The initial staff report must also include information on actions taken by school board staff prior to establishing a pupil accommodation review process and supporting rationale as to any actions taken or not taken.

The option(s) included in the initial staff report must address the following:

- summary of accommodation issue(s) for the school(s) under review;

- where students would be accommodated;

- if proposed changes to existing facility or facilities are required as a result of the pupil accommodation review;

- identify any program changes as a result of the proposed option;

- how student transportation would be affected if changes take place;

- if new capital investment is required as a result of the pupil accommodation review, how the school board intends to fund this, as well as a proposal on how students would be accommodated if funding does not become available; and

- any relevant information obtained from municipalities and other community partners prior to the commencement of the pupil accommodation review, including any confirmed interest in using the underutilized space.

Each recommended option must also include a timeline for implementation. The initial staff report and School Information Profiles (SIPs) (see Section VIII)

will be made available to the public, as determined in the school board's policy, 
and posted on the school board's website following the decision to proceed with a pupil accommodation review by the Board of Trustees.

School boards must ensure that individuals from the school(s) under review and the broader community are invited to participate in the pupil accommodation review consultation. At a minimum, the pupil accommodation review process must consist of the following methods of consultation:

- Accommodation Review Committee (ARC) (see Section VII);

- consultation with municipal governments local to the affected school(s) (see Section IX);

- public meetings (see Section X); and

- public delegations (see Section XI).

\section{THE ACCOMMODATION REVIEW COMMITTEE}

\section{$\underline{\text { Role }}$}

School boards must establish an ARC that represents the school(s) under review and acts as the official conduit for information shared between the school board and the school communities. The ARC may comment on the initial staff report and may, throughout the pupil accommodation review process, seek clarification of the initial staff report. The ARC may provide other accommodation options than those in the initial staff report; however, it must include supporting rationale for any such option.

The ARC members do not need to achieve consensus regarding the information provided to the Board of Trustees.

The school board's staff resources assigned to the ARC are required to compile feedback from the ARC as well as the broader community in the Community Consultation section of the final staff report (see Section $\mathrm{XI}$ ) to be presented to the Board of Trustees.

\section{Membership}

The membership of the ARC should include, at a minimum, parent/guardian representatives from each of the schools under review, chosen by their respective school communities.

Where established by a school board's pupil accommodation review policy, there may also be the option to include students and representation from the broader community. For example, a school board's policy may include a requirement for specific representation from the First Nations, Metis, and Inuit communities. In addition, school board trustees may be ad hoc ARC members to monitor the ARC progress. 


\section{Formation}

The ARC should be formed following the Board of Trustees' consideration of the initial staff report but prior to the first public meeting. The school board will invite ARC members from the school(s) under review to an orientation session that will describe the mandate, roles and responsibilities, and procedures of the ARC.

\section{$\underline{\text { Terms of Reference }}$}

School boards will provide the ARC with Terms of Reference that describe the ARC's mandate. The mandate will refer to the school board's education and accommodation objectives in undertaking the ARC and reflect the school board's strategy for supporting student achievement and well-being.

The Terms of Reference will also clearly outline the school board's expectations of the roles and responsibilities of the ARC; and describe the procedures of the ARC. At a minimum, the ARC will provide feedback on the initial staff report option(s).

The Terms of Reference will outline the minimum number of working meetings of the ARC.

\section{Meetings of the Accommodation Review Committee}

The ARC will meet to review materials presented by school board staff. It is recommended that the ARC hold as many working meetings as is deemed necessary within the timelines established in their school board's pupil accommodation review policy.

\section{SCHOOL INFORMATION PROFILE}

School board staff are required to develop School Information Profiles (SIPs) as orientation documents to help the ARC and the community understand the context surrounding the decision to include the specific school(s) in a pupil accommodation review. The SIP provides an understanding of and familiarity with the facilities under review.

The SIP is expected to include data for each of the following two considerations about the school(s) under review:

- value to the student; and

- value to the school board.

A SIP will be completed by school board staff for each of the schools under review. The following are the minimum data requirements and factors that are to be included in the SIP: 
- $\quad$ Facility Profile:

o School name and address.

o Site plan and floor plan(s) (or space template) of the school with the date of school construction and any subsequent additions.

o School attendance area (boundary) map.

o Context map (or air photo) of the school indicating the existing land uses surrounding the school.

o Planning map of the school with zoning, Official Plan or secondary plan land use designations.

o Size of the school site (acres or hectares).

$0 \quad$ Building area (square feet or square metres).

o Number of portable classrooms.

o Number and type of instructional rooms as well as specialized classroom teaching spaces (e.g., science lab, tech shop, gymnasium, etc.).

o Area of hard surfaced outdoor play area and/or green space, the number of play fields, and the presence of outdoor facilities (e.g., tracks, courts for basketball, tennis, etc.).

o Ten-year history of major facility improvements (item and cost).

o Projected five-year facility renewal needs of school (item and cost).

o Current Facility Condition Index $(\mathrm{FCl})$ with a definition of what the index represents.

- A measure of proximity of the students to their existing school, and the average distance to the school for students.

o Percentage of students that are and are not eligible for transportation under the school board policy, and the length of bus ride to the school (longest, shortest, and average length of bus ride times).

o School utility costs (totals, per square foot, and per student).

o Number of parking spaces on site at the school, an assessment of the adequacy of parking, and bus/car access and egress.

o Measures that the school board has identified and/or addressed for accessibility of the school for students, staff, and the public with disabilities (i.e., barrier-free).

o On-the-ground (OTG) capacity, and surplus/shortage of pupil places.

- Instructional Profile:

o Describe the number and type of teaching staff, non-teaching staff, support staff, itinerant staff, and administrative staff at the school.

0 Describe the course and program offerings at the school.

o Describe the specialized service offerings at the school (e.g., cooperative placements, guidance counseling, etc.).

o Current grade configuration of the school (e.g., junior kindergarten to Grade 6, junior kindergarten to Grade 12, etc.).

o Current grade organization of the school (e.g., number of combined grades, etc.).

o Number of out of area students.

o Utilization factor/classroom usage.

o Summary of five previous years' enrolment and 10-year enrolment projection by grade and program. 
o Current extracurricular activities.

- $\quad$ Other School Use Profile:

o Current non-school programs or services resident at or co-located with the school as well as any revenue from these non-school programs or services and whether or not it is at full cost recovery.

o Current facility partnerships as well as any revenue from the facility partnerships and whether or not it is at full cost recovery.

o Community use of the school as well as any revenue from the community use of the school and whether or not it is at full cost recovery.

o Availability of before and after school programs or services (e.g., child care) as well as any revenue from the before and after school programs and whether or not it is at full cost recovery.

0 Lease terms at the school as well as any revenue from the lease and whether or not it is at full cost recovery.

o Description of the school's suitability for facility partnerships.

School boards may introduce additional items that could be used to reflect local circumstances and priorities which may help to further understand the school(s) under review.

Each school under review will have a SIP completed at the same point-in-time for comparison purposes. The Ministry of Education expects school boards to prepare SIPs that are complete and accurate, to the best of the school board's ability, prior to the commencement of a pupil accommodation review.

While the ARC may request clarification about information provided in the SIP, it is not the role of the ARC to approve the SIP.

\section{CONSULTATION WITH LOCAL MUNICIPAL GOVERNMENTS}

Following the Board of Trustees' approval to undertake a pupil accommodation review, school boards must invite affected single and upper-tier municipalities as well as other community partners that expressed an interest prior to the pupil accommodation review to discuss and comment on the recommended option(s) in the school board's initial staff report.

The invitation for this meeting will be provided through a written notice, and will be directed through the Clerks Department (or equivalent) for the affected single and uppertier municipalities.

The affected single and upper-tier municipalities, as well as other community partners that expressed an interest prior to the pupil accommodation review, must provide their response on the recommended option(s) in the school board's initial staff report before the final public meeting. School boards must provide them with advance notice of when the final public meeting is scheduled to take place.

School boards must document their efforts to meet with the affected single and upper-tier municipalities, as well as other community partners that expressed an interest prior to the 
pupil accommodation review; and provide any relevant information from this meeting as part of the final staff report to the Board of Trustees (see Section XI).

\section{PUBLIC MEETINGS}

Once a school board has received an initial staff report and has approved the initiation of a pupil accommodation review, the school board must arrange to hold a minimum of two public meetings for broader community consultation on the initial staff report. School board staff are expected to facilitate the public meetings to solicit broader community feedback on the recommended option(s) contained in the initial staff report.

The public meetings are to be announced and advertised publicly by the school board through an appropriate range of media as determined by the school board.

At a minimum, the first public meeting must include the following:

- an overview of the ARC orientation session;

- the initial staff report with recommended option(s); and

- a presentation of the SIPS.

\section{COMPLETING THE ACCOMMODATION REVIEW}

\section{Final Staff Report}

At the conclusion of the pupil accommodation review process, school board staff will submit a final staff report to the Board of Trustees which must be available to the public as determined in the school board's policy, and posted on the school board's website.

The final staff report must include a Community Consultation section that contains feedback from the ARC and any public consultations as well as any relevant information obtained from municipalities and other community partners prior to and during the pupil accommodation review.

School board staff may choose to amend their proposed option(s) included in the initial staff report. The recommended option(s) must also include a proposed accommodation plan, prepared for the decision of the Board of Trustees, which contains a timeline for implementation. 


\section{Delegations to the Board of Trustees Meeting}

Once school board staff submits the final staff report to the Board of Trustees, the school board must allow an opportunity for members of the public to provide feedback on the final staff report through public delegations to the Board of Trustees. Notice of the public delegation opportunities will be provided based on school board policy.

After the public delegations, school board staff will compile feedback from the public delegations which will be presented to the Board of Trustees with the final staff report.

\section{Decision of the Board of Trustees}

The Board of Trustees will be provided with the final staff report, including the compiled feedback from the public delegations, when making its final decision regarding the pupil accommodation review.

The Board of Trustees has the discretion to approve the recommendation(s) of the final staff report as presented, modify the recommendation(s) of the final staff report, or to approve a different outcome.

The Ministry encourages school boards not to make final pupil accommodation review decisions during the summer holiday period (typically from July 1 to the day after Labour Day).

\section{TRANSITION PLANNING}

The transition of students should be carried out in consultation with parents/guardians and staff. Following the decision to consolidate and/or close a school, the school board is expected to establish a separate committee to address the transition for students and staff.

\section{TIMELINES FOR THE ACCOMMODATION REVIEW PROCESS}

The pupil accommodation review process must comply with the following minimum timelines:

- Following the date of the Board of Trustees' approval to conduct a pupil accommodation review, the school board will provide written notice of the Board of Trustees' decision within 5 business days to each of the affected single and upper-tier municipalities through the Clerks Department (or equivalent), other community partners that expressed an interest prior to the pupil accommodation review; and include an invitation for a meeting to discuss and comment on the recommended option(s) in the school board's initial staff report. School boards must also notify the Director(s) 
of Education of their coterminous school boards and the Ministry of Education through the office of the Assistant Deputy Minister of the Financial Policy and Business Division.

- The affected single and upper-tier municipalities, as well as other community partners that expressed an interest prior to the pupil accommodation review, must provide their response on the recommended option(s) in the school board's initial staff report before the final public meeting.

- Beginning with the date of the Board of Trustees' approval to conduct a pupil accommodation review, there must be no fewer than 30 business days before the first public meeting is held.

- There must be a minimum period of 40 business days between the first and final public meetings.

- The final staff report must be publicly posted no fewer than 10 business days after the final public meeting.

- From the posting of the final staff report, there must be no fewer than 10 business days before the public delegations.

- There must be no fewer than 10 business days between public delegations and the final decision of the Board of Trustees.

\section{MODIFIED ACCOMMODATION REVIEW PROCESS}

In certain circumstances, where the potential pupil accommodation options available are deemed by the school board to be less complex, school boards may find it appropriate to undertake a modified pupil accommodation review process. The Guideline permits a school board to include an optional modified pupil accommodation review process in its pupil accommodation review policy.

A school board's pupil accommodation review policy must clearly outline the conditions where a modified pupil accommodation review process could be initiated by explicitly defining the factors that would allow the school board the option to conduct a modified pupil accommodation review process. The conditions for conducting a modified pupil accommodation review process need to be based on two or more of the following factors:

- distance to the nearest available accommodation;

- utilization rate of the facility;

- number of students enrolled at the school; or

- when a school board is planning the relocation (in any school year or over a number of school years) of a program, in which the enrolment constitutes more than or equal to $50 \%$ of the school's enrolment (this calculation is based on the enrolment at the time of the relocation, or the first phase of a relocation carried over a number of school years). 
School boards may consider additional factors that are defined in their pupil accommodation review policy to qualify for the modified pupil accommodation review process. Multiple factors may be developed by the school board to appropriately reflect varying conditions across the board (e.g., urban, rural, elementary panel, secondary panel, etc.). The Board of Trustees must approve these explicitly defined factors, after community consultation, in order to adopt a modified pupil accommodation review process as part of their school board's pupil accommodation review policy.

The guiding principles of this Guideline apply to the modified pupil accommodation review process.

Even when the criteria for a modified pupil accommodation review are met, a school board may choose to use the standard pupil accommodation review process.

\section{Implementing the Modified Accommodation Review Process}

The initial staff report will explain the rationale for exempting the school(s) from the standard pupil accommodation review process, in accordance with the school board's pupil accommodation review policy.

The initial staff report and SIPs must be made available to the public, as determined in the school board's policy, and posted on the school board's website.

A public meeting will be announced and advertised through an appropriate range of media as determined by the school board.

Following the public meeting, school board staff will submit a final staff report to the Board of Trustees which must be available to the public as determined in the school board's policy, and posted on the school board's website. The final staff report must include a Community Consultation section that contains feedback from any public consultations as well as any relevant information obtained from municipalities and other community partners prior to and during the modified pupil accommodation review.

Once school board staff submit the final staff report to the Board of Trustees, the school board must allow an opportunity for members of the public to provide feedback through public delegations to the Board of Trustees. Notice of the public delegation opportunities will be provided based on school board policy.

After the public delegations, school board staff will compile feedback from the public delegations which will be presented to the Board of Trustees with the final staff report. 
The Board of Trustees has the discretion to approve the recommendation(s) of the final staff report as presented, modify the recommendation(s) of the final staff report, or to approve a different outcome.

The Ministry encourages school boards not to make final pupil accommodation review decisions during the summer holiday period (typically from July 1 to the day after Labour Day).

A transition plan will be put in place following the decision to consolidate and/or close a school.

\section{Timelines for the Modified Accommodation Review Process}

The modified pupil accommodation review process must comply with the following minimum timelines:

- Following the date of the Board of Trustees' approval to conduct a modified pupil accommodation review, the school board will provide written notice of the Board of Trustees' decision within 5 business days to each of the affected single and upper-tier municipalities through the Clerks Department (or equivalent), other community partners that expressed an interest prior to the modified pupil accommodation review; and include an invitation for a meeting to discuss and comment on the recommended option(s) in the school board's initial staff report. School boards must also notify the Director(s) of Education of their coterminous school boards and the Ministry of Education through the office of the Assistant Deputy Minister of the Financial Policy and Business Division.

- The affected single and upper-tier municipalities, as well as other community partners that expressed an interest prior to the modified pupil accommodation review, must provide their response on the recommended option(s) in the school board's initial staff report before the final public meeting.

- The school board must hold at least one public meeting. Beginning with the date of the Board of Trustees' approval to conduct a modified pupil accommodation review, there must be no fewer than 30 business days before this public meeting is held.

- The final staff report must be publicly posted no fewer than 10 business days after the final public meeting.

- From the posting of the final staff report, there must be no fewer than 10 business days before the public delegations.

- $\quad$ There must be no fewer than 10 business days between public delegations and the final decision of the Board of Trustees. 


\section{EXEMPTIONS}

This Guideline applies to schools offering elementary or secondary programs. However, there are specific circumstances where school boards are not obligated to undertake a pupil accommodation review. These include:

- where a replacement school is to be built by the school board on the existing site, or built or acquired within the existing school attendance boundary, as identified through the school board's policy;

- where a replacement school is to be built by the school board on the existing site, or built or acquired within the existing school attendance boundary and the school community must be temporarily relocated to ensure the safety of students and staff during the reconstruction, as identified through the school board's policy;

- when a lease for the school is terminated;

- when a school board is planning the relocation (in any school year or over a number of school years) of grades or programs, in which the enrolment constitutes less than $50 \%$ of the school's enrolment (this calculation is based on the enrolment at the time of the relocation, or the first phase of a relocation carried over a number of school years);

- when a school board is repairing or renovating a school, and the school community must be temporarily relocated to ensure the safety of students during the renovations;

- where a facility has been serving as a holding school for a school community whose permanent school is over-capacity and/or is under construction or repair; or

- where there are no students enrolled at the school at any time throughout the school year.

In the above circumstances, a school board is expected to inform school communities about proposed accommodation plans for students before a decision is made by the Board of Trustees. The school board will also provide written notice to each of the affected single and upper-tier municipalities through the Clerks Department (or equivalent), as well as other community partners that expressed an interest prior to the exemption, and their coterminous school boards in the areas of the affected school(s) through the Director of Education, and to the Ministry of Education through the Assistant Deputy Minister of the Financial Policy and Business Division no fewer than 5 business days after the decision to proceed with an exemption. 
A transition plan will be put in place following the Board of Trustees' decision to consolidate, close or move a school or students in accordance with this section.

\section{DEFINITIONS}

Accommodation review: A process, as defined in a school board pupil accommodation review policy, undertaken by a school board to determine the future of a school or group of schools.

Accommodation Review Committee (ARC): A committee, established by a school board that represents the affected school(s) of a pupil accommodation review, which acts as the official conduit for information shared between the school board and the affected school communities.

ARC working meeting: A meeting of ARC members to discuss a pupil accommodation review, and includes a meeting held by the ARC to solicit feedback from the affected school communities of a pupil accommodation review.

Business day: A calendar day that is not a weekend or statutory holiday. It also does not include calendar days that fall within school boards' Christmas, spring, and summer break. For schools with a year-round calendar, any break that is five calendar days or longer is not a business day.

Consultation: The sharing of relevant information as well as providing the opportunity for municipalities and other community partners, the public and affected school communities to be heard.

Facility Condition Index (FCI): A building condition as determined by the Ministry of Education by calculating the ratio between the five-year renewal needs and the replacement value for each facility.

On-the-ground (OTG) capacity: The capacity of the school as determined by the Ministry of Education by loading all instructional spaces within the facility to current Ministry standards for class size requirements and room areas.

Public delegation: A regular meeting of the Board of Trustees where presentations by groups or individuals can have their concerns heard directly by the school board trustees.

Public meeting: An open meeting held by the school board to solicit broader community feedback on a pupil accommodation review.

School Information Profile (SIP): An orientation document with point-in-time data for each of the schools under a pupil accommodation review to help the ARC and the community understand the context surrounding the decision to include the specific school(s) in a pupil accommodation review. 
Space template: A Ministry of Education template used by a school board to determine the number and type of instructional areas to be included within a new school, and the size of the required operational and circulation areas within that school. 


\section{(8) Ontario}

\section{MINISTRY OF EDUCATION ADMINISTRATIVE REVIEW OF ACCOMMODATION REVIEW PROCESS}

A review of a school board's accommodation review process may be sought if the following conditions are met.

\section{An individual or individuals must:}

- Submit a copy of the board's accommodation review policy highlighting how the accommodation review process was not compliant with the school board's accommodation review policy.

- Demonstrate the support of a portion of the school community through the completion of a petition signed by a number of supporters equal to at least $30 \%$ of the affected school's student headcount (e.g., if the headcount is 150 , then 45 signatures would be required). Parents/guardians of students and/or other individuals that participated in the accommodation review process are eligible to sign the petition ${ }^{1}$

- The petition should clearly provide a space for individuals to print and sign their name; address (street name and postal code); and to indicate whether they are a parent/guardian of a student attending the school subject to the accommodation review, or an individual who has participated in the review process.

- Submit the petition and justification to the school board and the Minister of Education within thirty $(30)$ days of the board's closure resolution.

The school board would be required to:

- Confirm to the Minister of Education that the names on the petition are parents/guardians of students enrolled at the affected school and/or individuals who participated in the review process.

- Prepare a response to the individual's or individuals' submission regarding the process and forward the board's response to the Minister of Education within thirty (30) days of receiving the petition.

If the conditions set out above have been met, the Ministry would be required to:

- Undertake a review by appointing a facilitator to determine whether the school board accommodation review process was undertaken in a manner consistent with the board's accommodation review policy within thity $(30)$ days of receiving the school board's response.

${ }^{1}$ Information contained in the petition is subject to the Freedom of Information and Protection of Privacy Act, 1990. 


\section{Appendix C: Statistics Canada \& Other Datasets}




\section{Statistics Statistique
Canada}

$\underline{\text { Home }} \rightarrow \underline{\text { Publications }} \rightarrow \underline{75 F 0002 M} \rightarrow$ Main page $\rightarrow$ Tables

Table 2

Publications

Income Research Paper Series - Research Paper

75F0002M Volume 2008, number 4

Low Income Cut-offs for 2008 and Low Income Measures for 2007

\section{Main page}

Introduction

Low income cut-offs

Low income measures

Tables

Annex

Price index

References

More information

Full content in PDF

Other issues in this series

\section{Low income cut-offs (1992 base) after tax}

\begin{tabular}{|c|c|c|c|c|c|}
\hline & \multicolumn{5}{|c|}{ Community size } \\
\hline & \multicolumn{5}{|c|}{ Rural areasUrban areas } \\
\hline & \multicolumn{5}{|c|}{ Less than $30,000^{1} 30,000$ to $99,999100,000$ to $499,999500,000$ and over } \\
\hline \multicolumn{6}{|c|}{ Size of family unitdollars } \\
\hline \multicolumn{6}{|l|}{1992} \\
\hline 1 person & 8,848 & 10,126 & 11,296 & 11,439 & 13,526 \\
\hline 2 persons & 10,769 & 12,325 & 13,749 & 13,922 & 16,462 \\
\hline 3 persons & 13,410 & 15,346 & 17,120 & 17,336 & 20,499 \\
\hline 4 persons & 16,729 & 19,146 & 21,359 & 21,628 & 25,574 \\
\hline 5 persons & 19,050 & 21,802 & 24,322 & 24,628 & 29,121 \\
\hline 6 persons & 21,127 & 24,179 & 26,974 & 27,313 & 32,296 \\
\hline 7 or more persons & 23,204 & 26,556 & 29,625 & 29,998 & 35,471 \\
\hline \multicolumn{6}{|l|}{1993} \\
\hline 1 person & 9,017 & 10,319 & 11,511 & 11,657 & 13,784 \\
\hline 2 persons & 10,974 & 12,560 & 14,011 & 14,187 & 16,776 \\
\hline 3 persons & 13,665 & 15,638 & 17,446 & 17,666 & 20,889 \\
\hline 4 persons & 17,048 & 19,511 & 21,766 & 22,040 & 26,061 \\
\hline 5 persons & 19,413 & 22,217 & 24,785 & 25,097 & 29,676 \\
\hline 6 persons & 21,529 & 24,640 & 27,488 & 27,833 & 32,911 \\
\hline 7 or more persons & 23,646 & 27,062 & 30,189 & 30,569 & 36,147 \\
\hline \multicolumn{6}{|l|}{1994} \\
\hline 1 person & 9,027 & 10,331 & 11,525 & 11,671 & 13,800 \\
\hline 2 persons & 10,987 & 12,574 & 14,027 & 14,204 & 16,795 \\
\hline 3 persons & 13,681 & 15,657 & 17,466 & 17,687 & 20,914 \\
\hline 4 persons & 17,068 & 19,533 & 21,791 & 22,066 & 26,092 \\
\hline 5 persons & 19,436 & 22,243 & 24,814 & 25,126 & 29,710 \\
\hline 6 persons & 21,555 & 24,668 & 27,520 & 27,866 & 32,950 \\
\hline 7 or more persons & 23,674 & 27,093 & 30,225 & 30,605 & 36,189 \\
\hline \multicolumn{6}{|l|}{1995} \\
\hline 1 person & 9,227 & 10,560 & 11,780 & 11,929 & 14,106 \\
\hline 2 persons & 11,231 & 12,853 & 14,338 & 14,519 & 17,168 \\
\hline 3 persons & 13,985 & 16,004 & 17,854 & 18,079 & 21,378 \\
\hline 4 persons & 17,446 & 19,967 & 22,274 & 22,555 & 26,670 \\
\hline 5 persons & 19,866 & 22,736 & 25,364 & 25,683 & 30,369 \\
\hline 6 persons & 22,032 & 25,215 & 28,130 & 28,484 & 33,680 \\
\hline 7 or more persons & 24,198 & 27,694 & 30,895 & 31,284 & 36,991 \\
\hline \multicolumn{6}{|l|}{1996} \\
\hline 1 person & 9,364 & 10,717 & 11,955 & 12,106 & 14,315 \\
\hline 2 persons & 11,397 & 13,044 & 14,551 & 14,734 & 17,422 \\
\hline 3 persons & 14,192 & 16,241 & 18,119 & 18,347 & 21,695 \\
\hline 4 persons & 17,705 & 20,263 & 22,605 & 22,890 & 27,066 \\
\hline 5 persons & 20,161 & 23,074 & 25,741 & 26,065 & 30,820 \\
\hline 6 persons & 22,359 & 25,589 & 28,547 & 28,906 & 34,180 \\
\hline 7 or more persons & 24,558 & 28,105 & 31,353 & 31,748 & 37,540 \\
\hline \multicolumn{6}{|l|}{1997} \\
\hline 1 person & 9,522 & 10,898 & 12,157 & 12,311 & 14,557 \\
\hline 2 persons & 11,589 & 13,264 & 14,797 & 14,983 & 17,716 \\
\hline 3 persons & 14,432 & 16,515 & 18,424 & 18,657 & 22,061 \\
\hline 4 persons & 18,004 & 20,605 & 22,986 & 23,276 & 27,522 \\
\hline
\end{tabular}




\begin{tabular}{|c|c|c|c|c|c|}
\hline 5 persons & 20,501 & 23,463 & 26,175 & 26,504 & 31,340 \\
\hline 6 persons & 22,737 & 26,021 & 29,029 & 29,394 & 34,757 \\
\hline 7 or more persons & 24,972 & 28,579 & 31,882 & 32,284 & 38,174 \\
\hline \multicolumn{6}{|l|}{1998} \\
\hline 1 person & 9,617 & 11,006 & 12,278 & 12,433 & 14,701 \\
\hline 2 persons & 11,705 & 13,396 & 14,944 & 15,132 & 17,893 \\
\hline 3 persons & 14,575 & 16,680 & 18,608 & 18,843 & 22,280 \\
\hline 4 persons & 18,183 & 20,810 & 23,215 & 23,508 & 27,797 \\
\hline 5 persons & 20,706 & 23,697 & 26,436 & 26,768 & 31,652 \\
\hline 6 persons & 22,963 & 26,280 & 29,318 & 29,687 & 35,103 \\
\hline 7 or more persons & 25,221 & 28,864 & 32,200 & 32,605 & 38,554 \\
\hline \multicolumn{6}{|l|}{1999} \\
\hline 1 person & 9,785 & 11,199 & 12,493 & 12,651 & 14,959 \\
\hline 2 persons & 11,910 & 13,631 & 15,206 & 15,397 & 18,206 \\
\hline 3 persons & 14,831 & 16,972 & 18,934 & 19,173 & 22,671 \\
\hline 4 persons & 18,501 & 21,175 & 23,622 & 23,920 & 28,284 \\
\hline 5 persons & 21,068 & 24,112 & 26,899 & 27,237 & 32,206 \\
\hline 6 persons & 23,365 & 26,741 & 29,832 & 30,207 & 35,718 \\
\hline 7 or more persons & 25,663 & 29,370 & 32,764 & 33,176 & 39,229 \\
\hline \multicolumn{6}{|l|}{2000} \\
\hline 1 person & 10,049 & 11,500 & 12,829 & 12,991 & 15,362 \\
\hline 2 persons & 12,231 & 13,998 & 15,615 & 15,811 & 18,696 \\
\hline 3 persons & 15,230 & 17,429 & 19,443 & 19,689 & 23,281 \\
\hline 4 persons & 18,999 & 21,744 & 24,258 & 24,563 & 29,045 \\
\hline 5 persons & 21,635 & 24,761 & 27,623 & 27,970 & 33,073 \\
\hline 6 persons & 23,994 & 27,460 & 30,635 & 31,020 & 36,679 \\
\hline 7 or more persons & 26,353 & 30,160 & 33,646 & 34,069 & 40,285 \\
\hline \multicolumn{6}{|l|}{2001} \\
\hline 1 person & 10,302 & 11,790 & 13,152 & 13,318 & 15,748 \\
\hline 2 persons & 12,538 & 14,350 & 16,008 & 16,209 & 19,166 \\
\hline 3 persons & 15,613 & 17,867 & 19,933 & 20,184 & 23,867 \\
\hline 4 persons & 19,477 & 22,291 & 24,868 & 25,181 & 29,775 \\
\hline 5 persons & 22,180 & 25,384 & 28,318 & 28,674 & 33,905 \\
\hline 6 persons & 24,598 & 28,151 & 31,405 & 31,800 & 37,602 \\
\hline 7 or more persons & 27,016 & 30,919 & 34,492 & 34,926 & 41,298 \\
\hline \multicolumn{6}{|l|}{2002} \\
\hline 1 person & 10,533 & 12,055 & 13,448 & 13,618 & 16,102 \\
\hline 2 persons & 12,820 & 14,673 & 16,368 & 16,574 & 19,598 \\
\hline 3 persons & 15,964 & 18,269 & 20,381 & 20,638 & 24,404 \\
\hline 4 persons & 19,915 & 22,793 & 25,427 & 25,748 & 30,445 \\
\hline 5 persons & 22,679 & 25,955 & 28,955 & 29,319 & 34,668 \\
\hline 6 persons & 25,151 & 28,785 & 32,112 & 32,515 & 38,448 \\
\hline 7 or more persons & 27,624 & 31,614 & 35,268 & 35,712 & 42,227 \\
\hline \multicolumn{6}{|l|}{2003} \\
\hline 1 person & 10,828 & 12,392 & 13,824 & 13,999 & 16,553 \\
\hline 2 persons & 13,179 & 15,083 & 16,826 & 17,038 & 20,146 \\
\hline 3 persons & 16,411 & 18,781 & 20,952 & 21,216 & 25,087 \\
\hline 4 persons & 20,473 & 23,431 & 26,139 & 26,469 & 31,298 \\
\hline 5 persons & 23,314 & 26,681 & 29,765 & 30,140 & 35,639 \\
\hline 6 persons & 25,855 & 29,590 & 33,011 & 33,426 & 39,524 \\
\hline 7 or more persons & 28,397 & 32,499 & 36,255 & 36,712 & 43,410 \\
\hline \multicolumn{6}{|l|}{2004} \\
\hline 1 person & 11,028 & 12,621 & 14,080 & 14,258 & 16,859 \\
\hline 2 persons & 13,423 & 15,362 & 17,137 & 17,353 & 20,519 \\
\hline 3 persons & 16,715 & 19,128 & 21,339 & 21,608 & 25,551 \\
\hline 4 persons & 20,852 & 23,864 & 26,622 & 26,958 & 31,876 \\
\hline 5 persons & 23,744 & 27,175 & 30,316 & 30,697 & 36,297 \\
\hline
\end{tabular}




\begin{tabular}{|c|c|c|c|c|c|}
\hline 6 persons & 26,333 & 30,137 & 33,621 & 34,044 & 40,255 \\
\hline 7 or more persons & 28,922 & 33,100 & 36,925 & 37,390 & 44,212 \\
\hline \multicolumn{6}{|l|}{2005} \\
\hline 1 person & 11,271 & 12,899 & 14,389 & 14,571 & 17,230 \\
\hline 2 persons & 13,718 & 15,700 & 17,514 & 17,734 & 20,969 \\
\hline 3 persons & 17,082 & 19,548 & 21,808 & 22,083 & 26,112 \\
\hline 4 persons & 21,310 & 24,388 & 27,207 & 27,550 & 32,576 \\
\hline 5 persons & 24,266 & 27,772 & 30,982 & 31,371 & 37,095 \\
\hline 6 persons & 26,912 & 30,799 & 34,360 & 34,792 & 41,139 \\
\hline 7 or more persons & 29,557 & 33,827 & 37,737 & 38,212 & 45,183 \\
\hline \multicolumn{6}{|l|}{2006} \\
\hline 1 person & 11,492 & 13,152 & 14,671 & 14,857 & 17,568 \\
\hline 2 persons & 13,987 & 16,008 & 17,857 & 18,082 & 21,381 \\
\hline 3 persons & 17,417 & 19,932 & 22,236 & 22,516 & 26,624 \\
\hline 4 persons & 21,728 & 24,867 & 27,741 & 28,091 & 33,216 \\
\hline 5 persons & 24,742 & 28,317 & 31,590 & 31,987 & 37,823 \\
\hline 6 persons & 27,440 & 31,404 & 35,034 & 35,474 & 41,946 \\
\hline 7 or more persons & 30,138 & 34,491 & 38,477 & 38,962 & 46,070 \\
\hline \multicolumn{6}{|l|}{2007} \\
\hline 1 person & 11,745 & 13,441 & 14,994 & 15,184 & 17,954 \\
\hline 2 persons & 14,295 & 16,360 & 18,250 & 18,480 & 21,851 \\
\hline 3 persons & 17,800 & 20,370 & 22,725 & 23,011 & 27,210 \\
\hline 4 persons & 22,206 & 25,414 & 28,352 & 28,709 & 33,946 \\
\hline 5 persons & 25,287 & 28,940 & 32,285 & 32,691 & 38,655 \\
\hline 6 persons & 28,044 & 32,095 & 35,805 & 36,255 & 42,869 \\
\hline 7 or more persons & 30,801 & 35,250 & 39,324 & 39,819 & 47,084 \\
\hline \multicolumn{6}{|l|}{2008} \\
\hline 1 person & 12,019 & 13,754 & 15,344 & 15,538 & 18,373 \\
\hline 2 persons & 14,628 & 16,741 & 18,676 & 18,911 & 22,361 \\
\hline 3 persons & 18,215 & 20,845 & 23,255 & 23,548 & 27,844 \\
\hline 4 persons & 22,724 & 26,007 & 29,013 & 29,378 & 34,738 \\
\hline 5 persons & 25,876 & 29,614 & 33,037 & 33,453 & 39,556 \\
\hline 6 persons & 28,698 & 32,843 & 36,640 & 37,100 & 43,869 \\
\hline 7 or more persons & 31,519 & 36,072 & 40,241 & 40,747 & 48,181 \\
\hline
\end{tabular}

1. Includes cities with a population between 15,000 and 30,000 and small urban areas (under 15,000 ).

Date modified:

2015-11-27 
TIII TORONTO

This bulletin summarizes the findings of the 2017 Toronto Employment Survey. This information resource presents a picture of Toronto's economy based on annual citywide surveys of businesses. For more information, please visit us at:

www.toronto.ca/city-government/dataresearch-maps/research-reports/planningdevelopment/

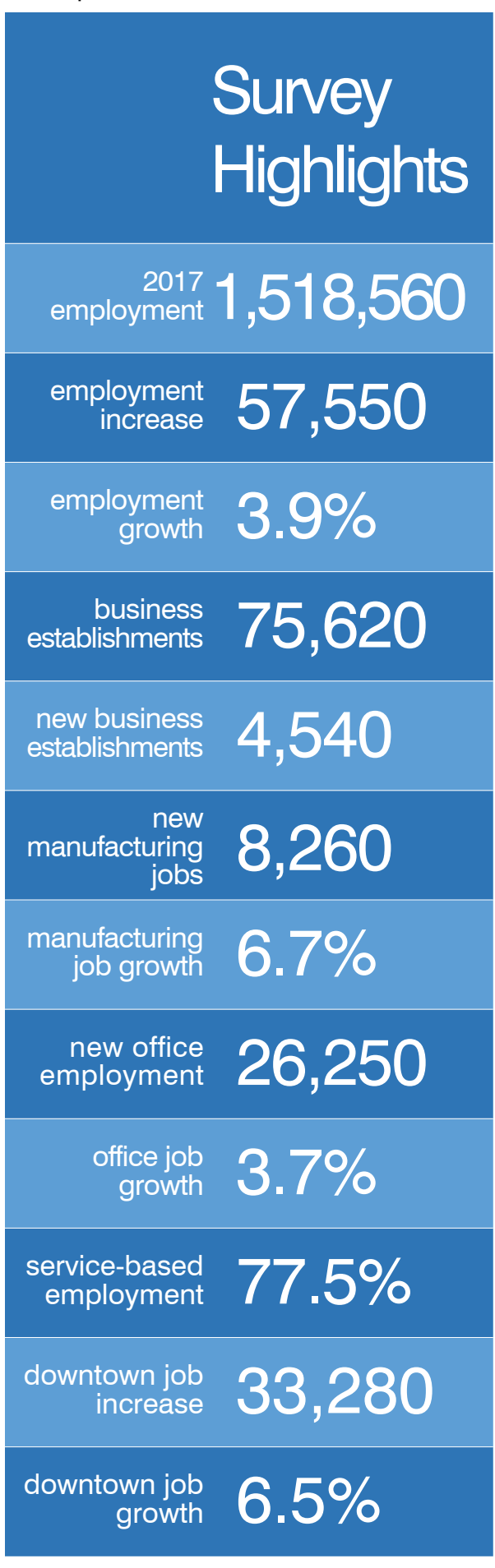

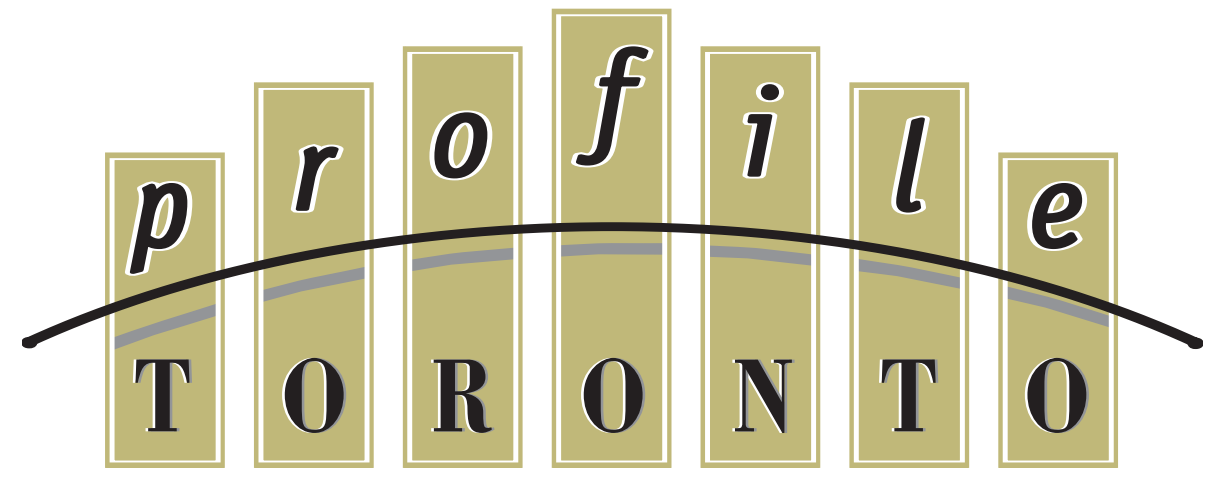

February 2018

\section{Toronto Employment Survey 2017}

\section{A Dynamic City}

Toronto's high quality of life and economic opportunities have made it one of the fastest growing cities in North America. Managing this growth while improving the city's liveability and prosperity is a key objective of city building in Toronto.

Toronto's competitive, diverse and connected economy also reflects its location within the broader regional economy of southern Ontario. Building on these competitive advantages will help meet the changing economic conditions of the future and create a vibrant city and region.

The Toronto area continues to prosper. Between 2011 and 2016, Toronto GDP is estimated to have grown 3.5\% per year, outpacing the average annual growth rates of both Ontario (2.1\%) and Canada (1.8\%). ${ }^{1}$

\section{The 2017 Toronto Employment Survey counted 1,518,560 jobs, an increase of 57,550 jobs or $3.9 \%$ from 2016.}

In Toronto, growth is managed through the Provincial Growth Plan for the Greater Golden Horseshoe and Toronto's Official Plan. The Growth Plan and its subsequent amendments direct municipalities to accommodate forecast growth strategically by building compact and complete communities, making better use of land and infrastructure, and providing opportunities for employment growth and business location.

The Growth Plan (2017) forecasts 3.4 million people and 1.72 million jobs in the City of Toronto by 2041.

Toronto's Official Plan, which came into force in June 2006, guides development in the city. Its policies promote economic prosperity by supporting growth and managing land use change.

In directing employment growth to certain areas, including Downtown, the Centres, Mixed Use Areas and Employment Areas, the plan helps create complete communities, focus transit and infrastructure investments and protect locations to support economic connectivity, clusters and business growth.

The 2017 Toronto Employment Survey offers a detailed picture of Toronto's economy, highlighting key citywide trends and emerging patterns in Downtown, the Centres, Secondary Plan Areas and Employment Areas.

In 2017, the Survey acquired employment data from $88.8 \%$ of identified businesses establishments. Excluding home-based employment, the Survey estimates it surveys over $99 \%$ of Toronto's business establishments. 


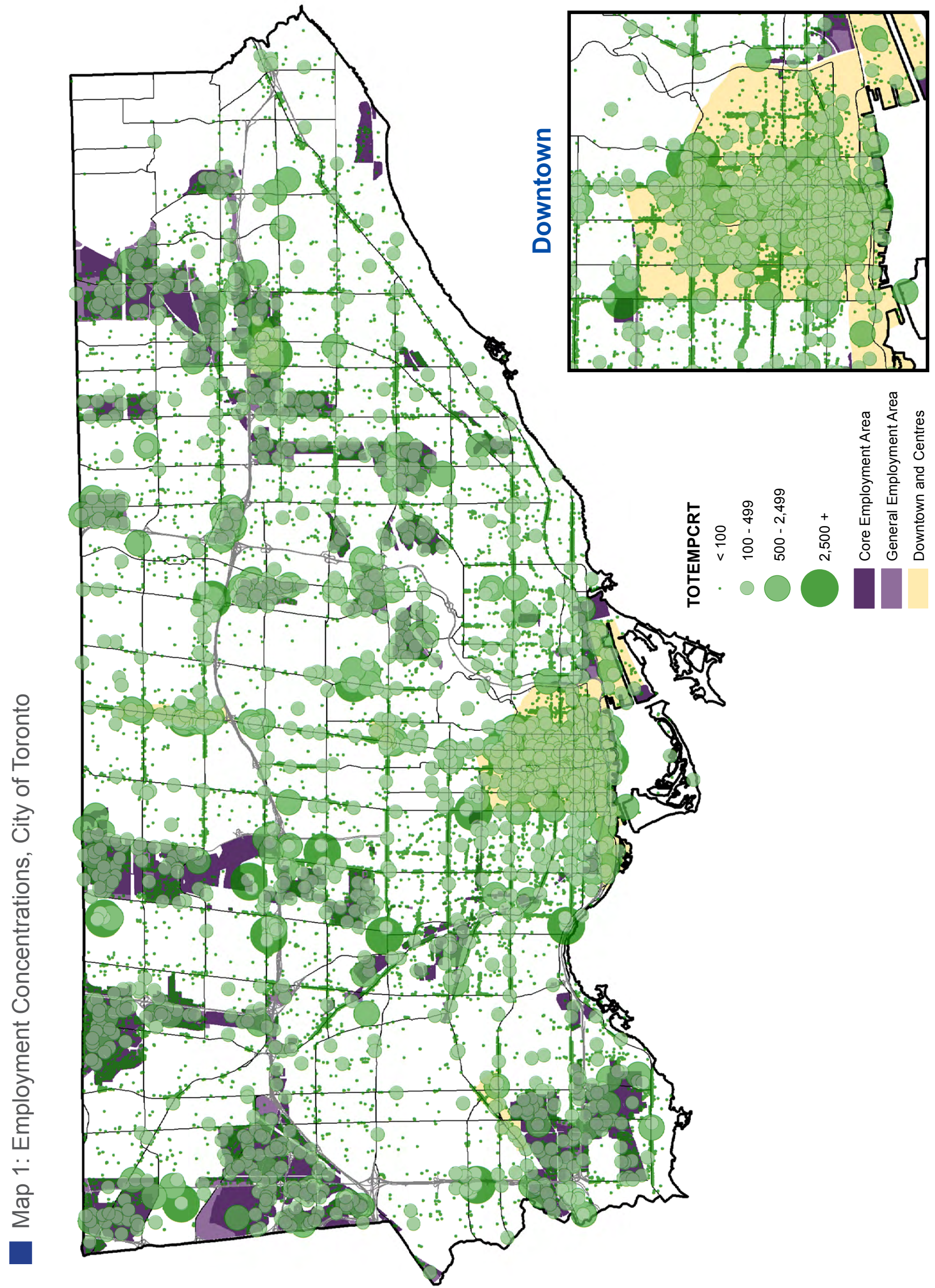




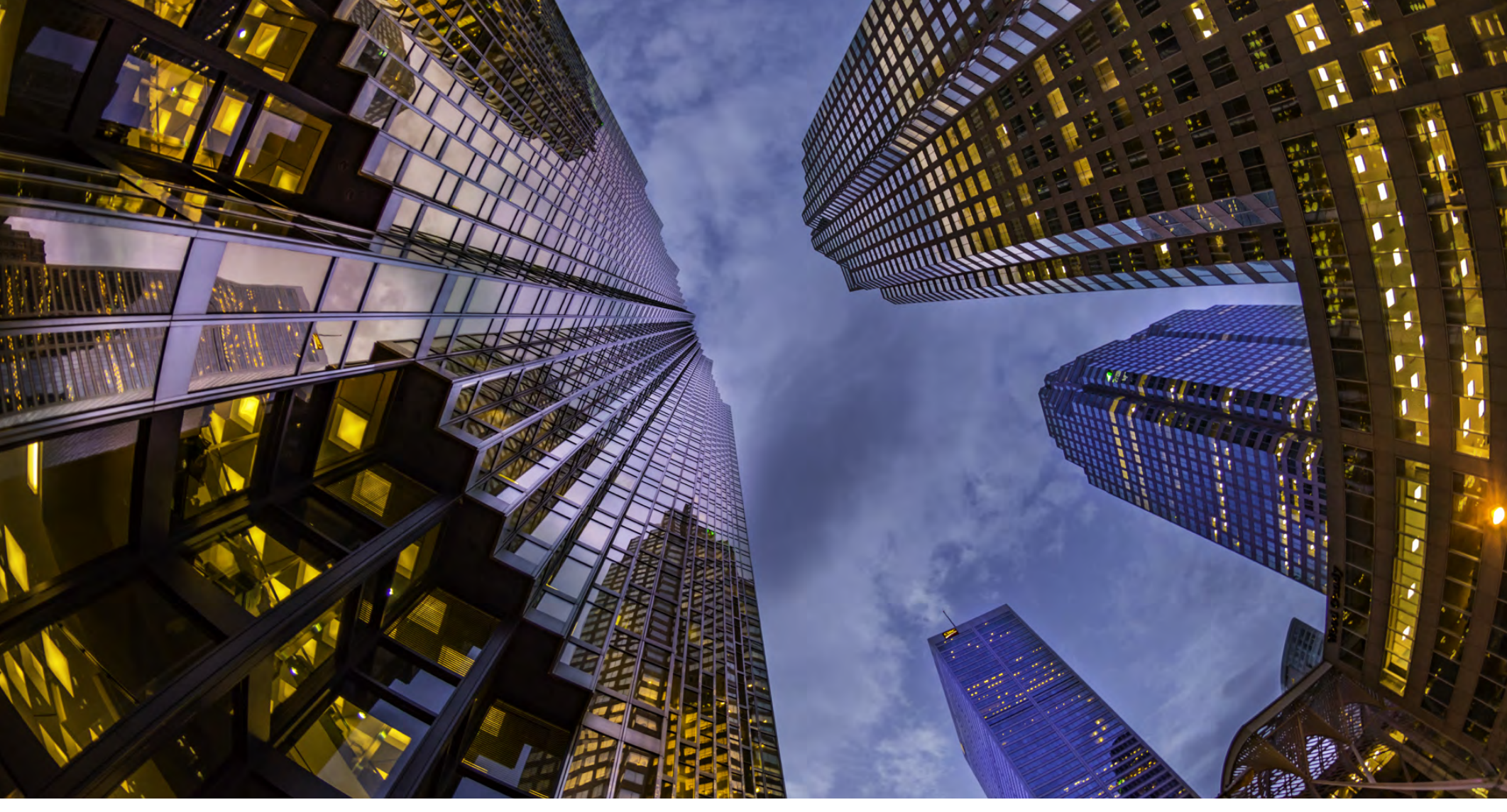

\section{Why a Survey Now?}

The Toronto Employment Survey collects annual employment data from business establishments across the city. This information is used to measure the city's economic and employment structure and monitor the progress of Official Plan policies.

The continued collection of survey information enables the city to monitor long range economic trends citywide and emerging activity in areas designated for employment or business growth, including Downtown, the Centres, Secondary Plan Areas and Employment Areas (see Map 1).

The survey results allow more effective long range projection and planning for urban infrastructure and municipal services, and help monitor the city's progress toward its investment and fiscal goals.

\section{What Data Was Collected?}

The Toronto Employment Survey collects citywide business information through in-person visits on an annual basis. Between May and August, surveyors record the following information into a citywide dataset of businesses:

- primary type of employment activity

- full-time and part-time employee counts

- length of time business has been at that location

In the case of major, multi-branch employers, the information is collected through a questionnaire mailed to the primary contact at the head office.

Business and employment activity is classified by both NAICS (North American Industry Classification System) and the Land Use Activity Codes of RISWG (Regional Information Systems Working Group of the Regional Planning Commissioners of Ontario) codes to allow comparisons to other jurisdictions.

In 2017, employment data was acquired from $88.8 \%$ of identified businesses establishments. In 2016 the response rate was $88.5 \%$.

\section{Survey Geography}

The survey conducts in-person business establishment interviews in commercial, industrial, institutional and mixed use areas across the city, including:

- Major office and service clusters in the Downtown and Centres

- Employment Areas designated for employment use and business growth

- Mixed Use Areas in Downtown, the Centres, Secondary Plan areas, along the Avenues and throughout the city

- Institutional precincts containing health or education uses

- Retail malls and power centres

- Community and local retail uses in residential areas including private schools and community uses.

Employment activities that are "footloose" or not place-specific on a daily basis are captured at head or reporting offices when the data is available.

In 2017, the Survey explored how to better capture home-based and volunteer work through a pilot project (see Special Topics, below). 
Toronto's 2017 employment growth of 57,550 jobs or $3.9 \%$ was the highest since 1999, when employment grew by $5.1 \%$

\section{City Employment Overview}

\section{Riding the global growth wave}

In 2017, the Toronto Employment Survey recorded $1,518,560$ jobs in the City of Toronto, a gain of 57,550 jobs or $3.9 \%$ from 2016 . This is above Toronto's five-year compound employment growth rate of $2.7 \%$, and the strongest since 1999 , when employment grew by $5.1 \%$.

According to the International Monetary Fund's World Economic Outlook October 2017, Toronto's employment growth (roughly Q3 2016 through Q2 2017) aligned with strong economic output from both Canada (3.8\% nominal) and the U.S. $(3.9 \%)$ and with a global upswing in economic activity projected to reach $3.6 \%$ in 2017 and $3.7 \%$ in $2018 .^{2}$
Services-driven economy

Economic output from serviceproducing industries has continued to outpace that from goods-producing industries. Overall, Canadian servicebased output from Q3 2016 through Q2 2017 grew 3.1\%, above the 5-year $(2.3 \%)$ and 10 -year $(2.0 \%)$ trends.

Surprisingly, Canadian goodsproducing during the same period grew $5.9 \%$, significantly above the 5 -year $(1.8 \%)$ and 10 -year $(1.0 \%)$ growth rates.

\section{Business and consumer outlook}

The consumer confidence index surveys consumer optimism about current economic conditions, and is an indicator of near-term sales for consumer products companies. The index stood at 100.9 in July 2017, up from 100.0 in 2016 and 99.0 in $2012 .^{3}$

Figure 1: Full and Part-Time Employment in the City of Toronto, 1983-2017

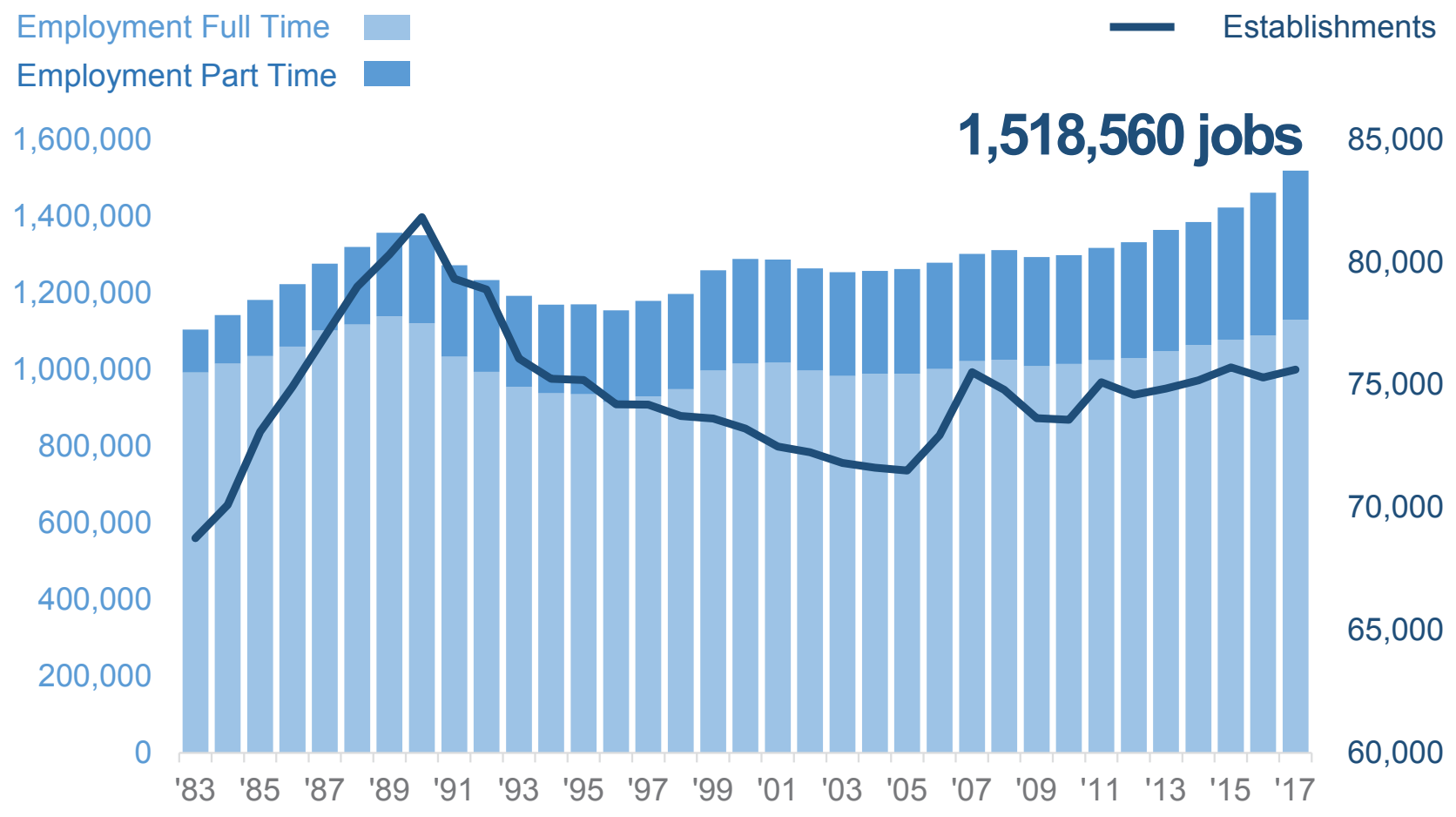

Note: See Appendix, Table 2: Total Employment 
The business confidence index is based on enterprises' assessment of production, orders and stocks, as well as its current position and expectations for the immediate future, and provides a qualitative index on economic conditions. The index was 100.3 in July 2017 , above the 2016 level of 99.6 and slightly higher than the level of 100.0 in $2012{ }^{4}$

\section{Strong full-time job growth}

Full-time employment (74.3\% of total) kept pace with city job growth, adding 41,180 jobs (3.8\%) from 2016. Part-time employment (25.7\% of total) added 16,370 jobs (4.4\%) from 2016.

Despite the 2016-2017 trend, the total share of part-time employment in the city has grown on average by $2.4 \%$ per annum between 2012 and 2017 (see Table 2, Appendix).

\section{Office led employment gains}

Toronto's office category added 26,250 jobs, half of new jobs, and grew by $3.7 \%$ from 2016. Office continues to be the city's largest employment category and growth driver with 740,180 jobs (48.7\% of the city total). Office employment has increased on average by $3.1 \%$ annually since 2012 .

\section{Broad category gains}

The city's remarkable employment growth was also shared by the Manufacturing, Services, Retail and Community and Entertainment categories, significantly outpacing their 5-year trends. Institutional employment, Toronto's second largest base (16.4\% of jobs), was the only category to underperform its 5-year growth trend.

\section{Improved survey coverage}

In 2017, improvements were made to the employment reporting process, most notably in the Community and Entertainment category.

Also, by working closely with major employers, the Survey was able to more thoroughly count employees with a non-regular or varying place of work.
Full-time employment kept pace with city job growth, adding 41,180 jobs (3.8\%) from 2016
Figure 2: 2016-2017 Job Increase by Category

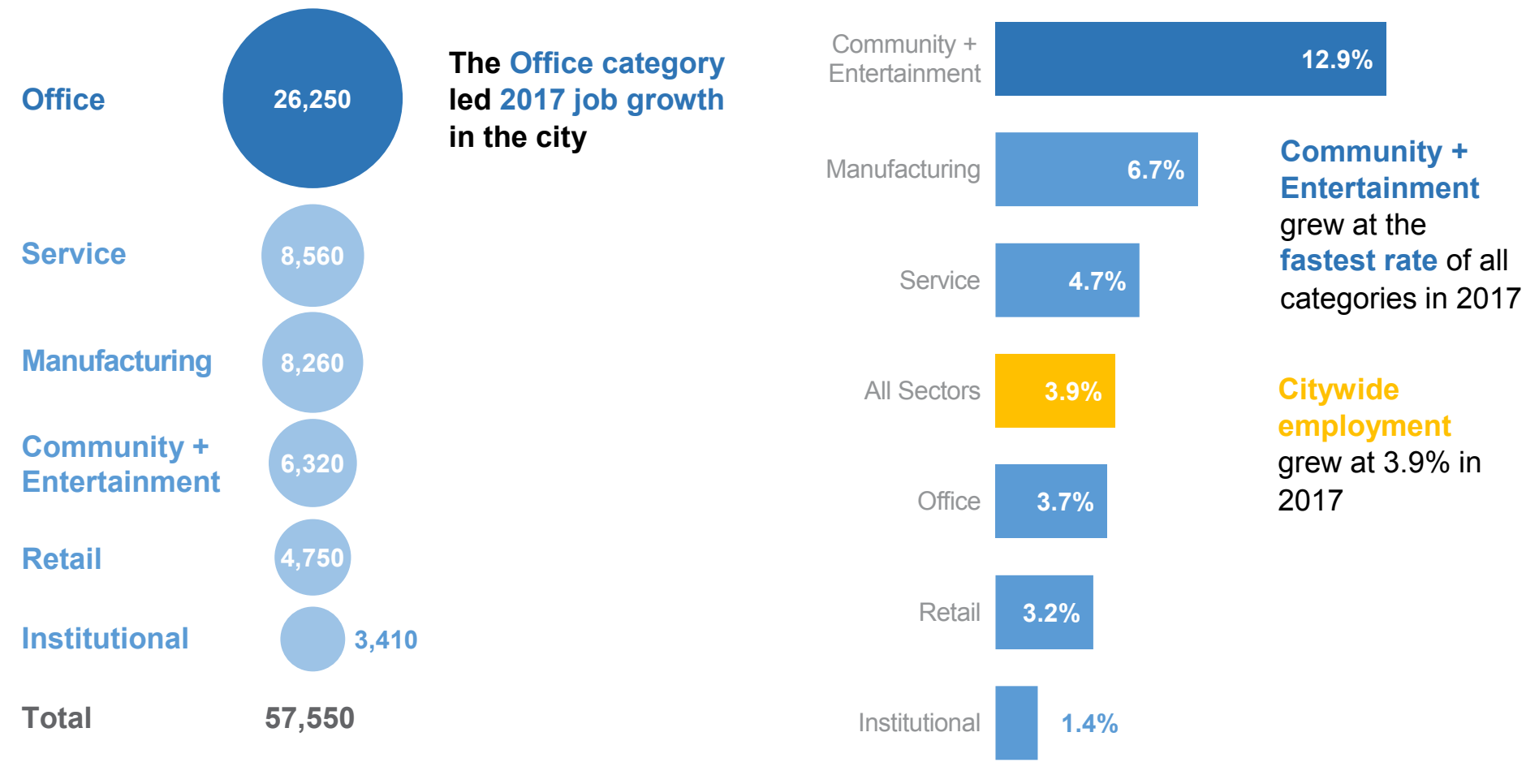

Figure 3: 2017 Employment Growth by Category 
Figure 4: Total 2017 Employment by Category

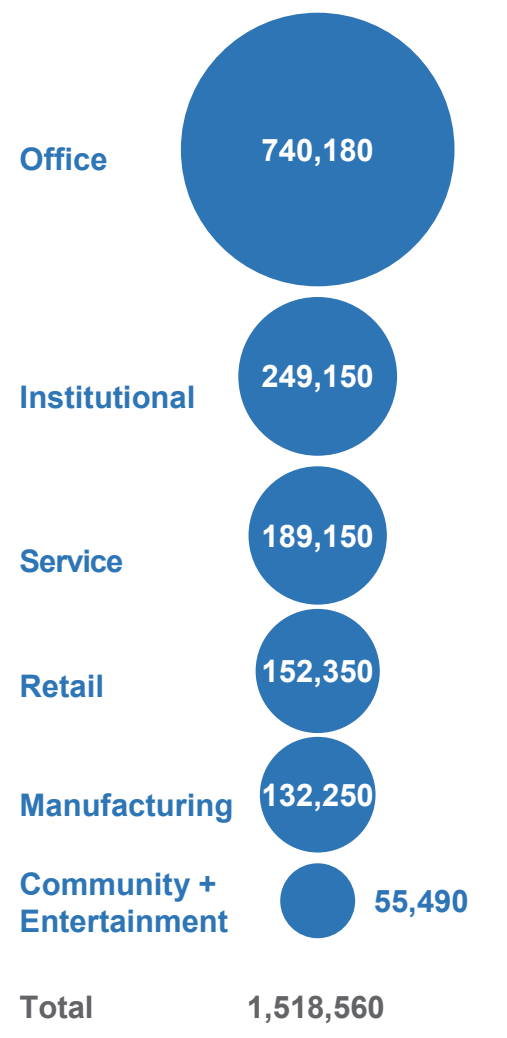

\section{Key Citywide Trends}

\section{Manufacturing}

Despite a long-term secular shift from goods to services production in Toronto's economy, manufacturing employment grew at a remarkable rate, adding 8,260 jobs or 6.7\% from 2016.

Processed Goods Processing led category job growth, with food and chemical product processing subcategories each adding more than 1,000 jobs. Warehousing employment also added 850 jobs.

The city's manufacturing establishments grew at an above-average rate (1.4\%), adding 70 businesses. The average number of employees per establishment increased to 27 from 25 in 2016.

Potential industry factors contributing to this growth include a competitive tax environment, a lower Canadian dollar, an improving export environment, decreased fuel and transportation costs, and higher capital investment in machinery and equipment. ${ }^{5}$
Demand for industrial space in Toronto increased, with the industrial vacancy rate falling to $0.6 \%$ in Q2 2017 from $1.0 \%$ in Q2 2016, well below the 1.7\% vacancy rate in the Greater Toronto Area. ${ }^{6}$

\section{Office Trends}

Strong Office employment growth continued in 2017, adding 26,250 jobs or $3.7 \%$. The largest components of Office employment growth included Business Services $(11,450$ net jobs added) and Finance, Insurance and Real Estate (8,090 net jobs added), comprising $74.5 \%$ of Office employment growth.

The top three subcategories of net Office employment growth in 2017 were Management Consultants, Business Consultants, Market Research, Call Centres (9,780 jobs), Real Estate, Rental and Leasing (6,090 jobs) and Banks and Trust Companies (4,930 jobs).

The city's office market continues to grow, having added more than 1.15 million square metres of office space

Figure 5: 2016-2017 Employment Growth Share by Category

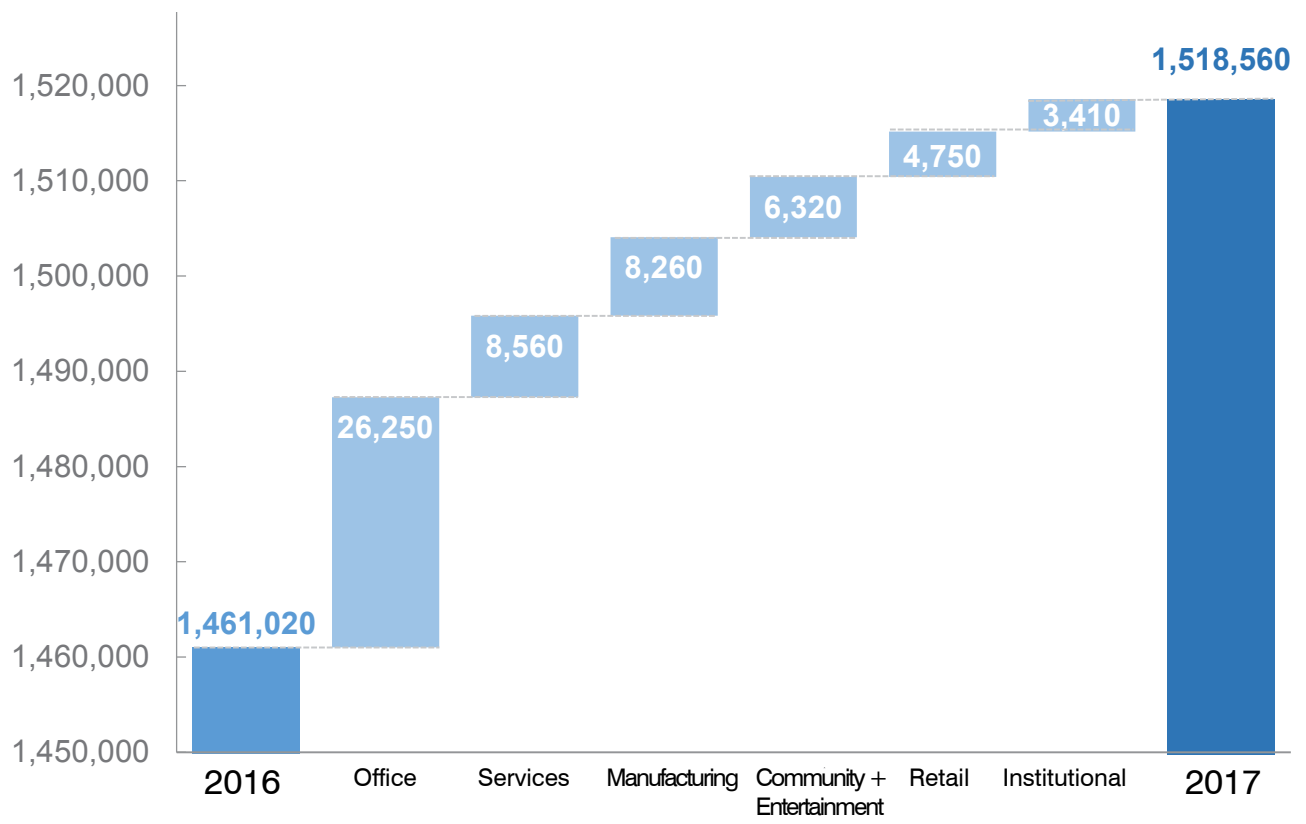


from projects of over 50,000 square metres alone between 2012 and $2016 .{ }^{7}$

Demand for office space remains high. Vacancy rates in the downtown decreased to $3.8 \%$ in Q2 2017, the lowest rate among major office markets in North America. ${ }^{8}$

\section{Retail Trends}

Retail employment grew at an aboveaverage rate in 2017 , adding 4,750 jobs or $3.2 \%$. Structural trends in the retail sector have resulted in slower annual growth since 2012 (1.2\%). Factors potentially influencing this trend include growing consumer use of online channels, increasing automation in retail environments, and concentration of retailing in fewer, larger locations. ${ }^{9}$

Ongoing trends in expansions and upgrades to retail environments appear to be continuing from 2016, which saw the renovation of major retail centres such as the Eaton Centre and Yorkdale Mall. Further large-scale mixed use redevelopments are currently proposed for Yorkdale Mall, Bayview Village,
Agincourt Mall and Galleria Mall, as retailers leverage these locations by adding additional retail space with new office and residential components.

\section{Community and Entertainment Trends}

Toronto's smallest employment category, Community and Entertainment, was the city's fastestgrowing in 2017, adding 6,320 jobs to grow by $12.9 \%$.

A third of growth came in 2017 from the construction sub-category. The city gained more than 2,300 construction jobs in 2017 as Toronto's real estate cycle continued. More than half of these jobs were located in Downtown.

Sports and entertainment venues also added significant growth. BMO Field and the Air Canada Centre added 2,300 net new jobs in 2017. Employment growth at these venues has coincided with the success and increased season length of the city's major league sports teams.
Manufacturing employment grew at a remarkable rate, adding 8,260 jobs or $6.7 \%$ from 2016

\section{Figure 6: Components of Office Employment Growth 2016-2017}

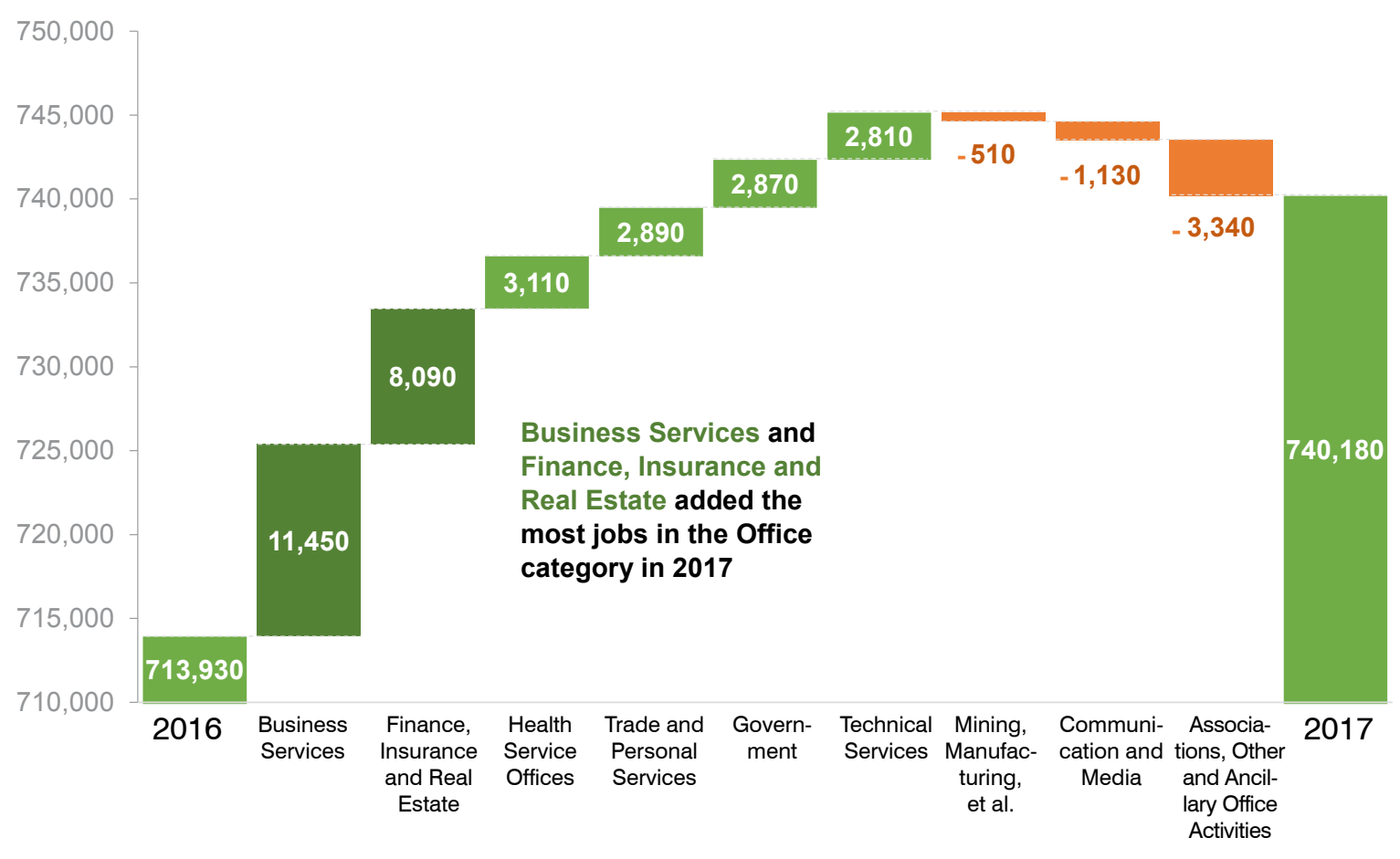


Figure 7: Total 2017 Establishments by Category

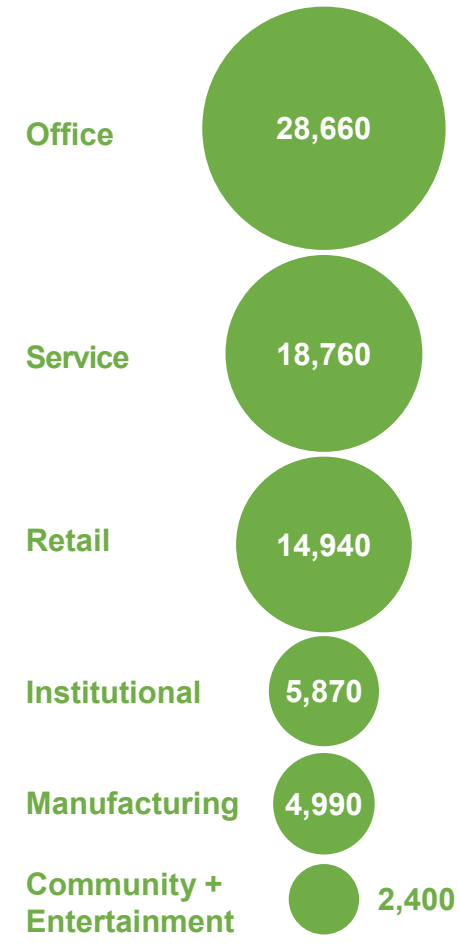

Total

\section{Business Establishments}

In 2017, the Survey counted 75,620 business establishments in the city, a net increase of 340 establishments or $0.5 \%$. Since 2012 , the number of business locations in Toronto grew on average $0.3 \%$ annually, although the business count declined slightly in 2016.

Net gains in establishments included Office (300) and Service (250) and Manufacturing (70). Net total establishment counts decreased in Retail (140) and Community and Entertainment (140).

\section{New Establishments in the City}

The net change in business locations is the result of a greater number of establishment openings and closures. New establishments in particular include both new business starts as well as existing firm relocations and new locations.
City-wide, the Survey counted 4,540 new business establishments, $6 \%$ of the 2017 total. Of these new locations, the Survey counted 1,470 (32.4\%) in Employment Areas, $1,240(27.3 \%)$ in the Downtown and Centres, and 1,830 (40.3\%) in the rest of the city. Overall, the share of new establishments locating in these areas increased by $3.6 \%$ from 2016 (see Table 3, Appendix).

Office (39\%) led the share of new establishments in 2017 survey counts, followed by Service (24.2\%) and Retail (16.1\%). Despite secular global trends, Toronto remains an attractive location for industrial establishments, adding 220 new manufacturing establishments and 1,220 manufacturing jobs in 2017.

In 2017, the Community and Entertainment category had the greatest share of new establishments $(20.3 \%)$, with one in five establishments being new.

Figure 8: Establishments in the City of Toronto, 1983-2017

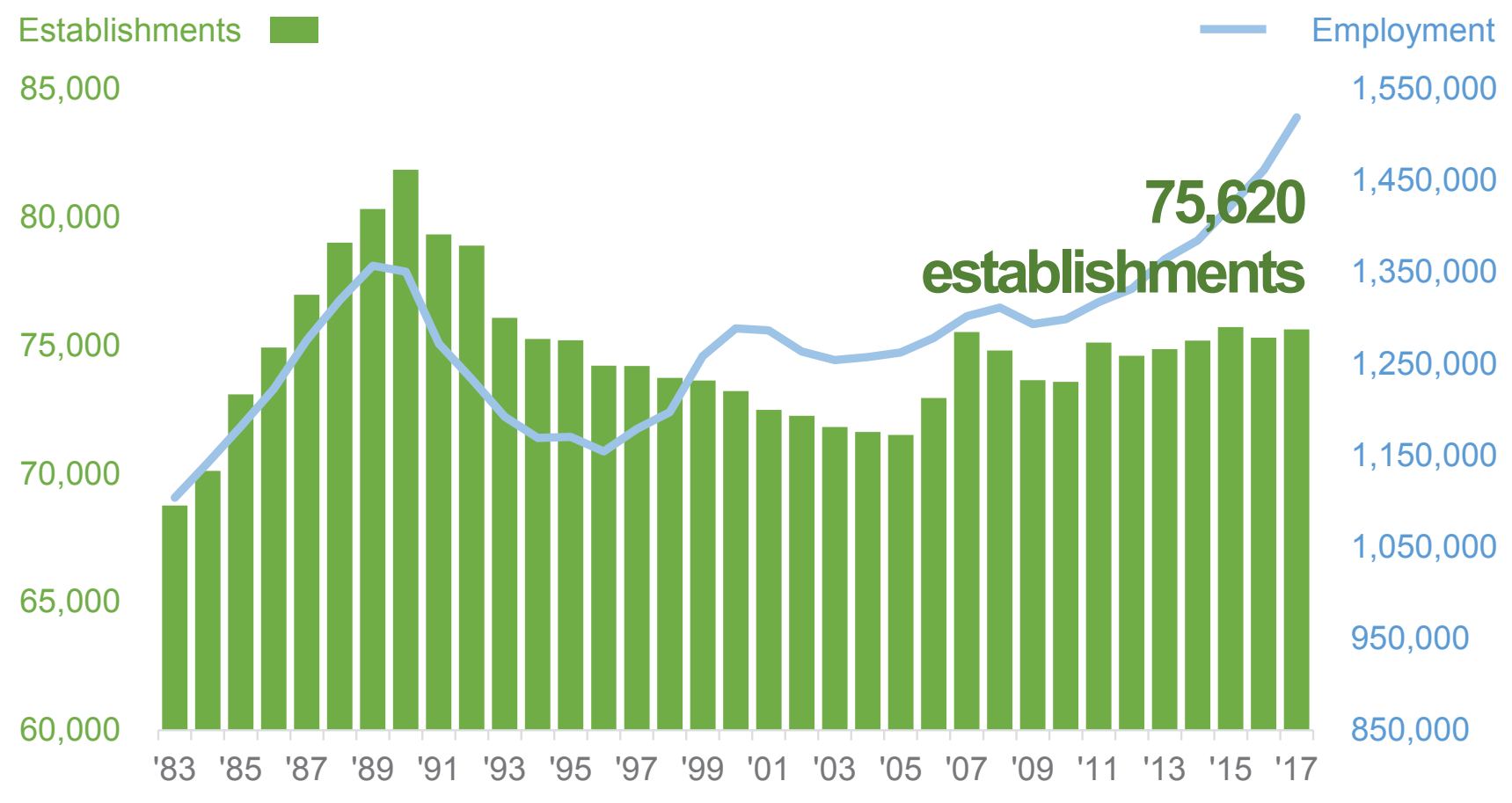


Figure 9: Net Establishment Change by Category 2016-2017

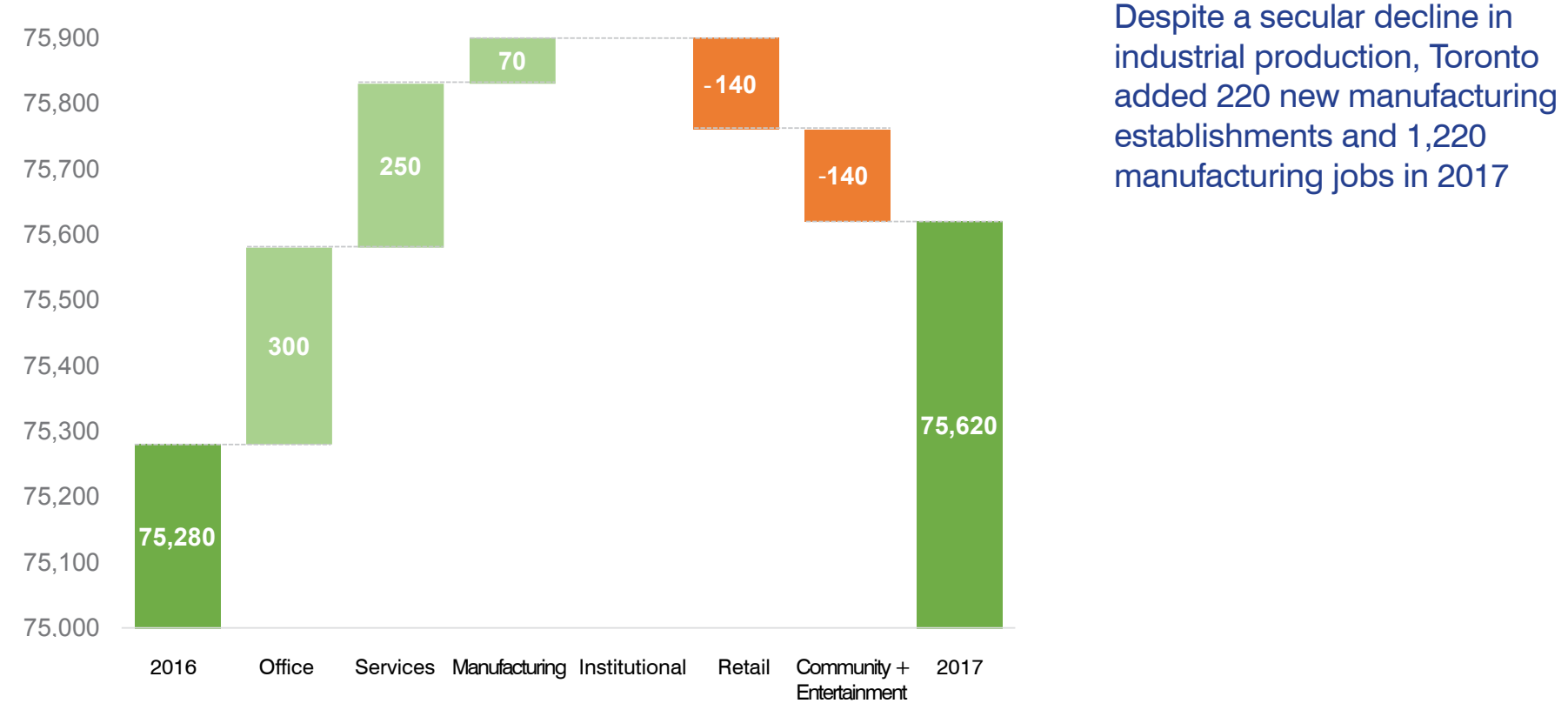

Figure 10: New Establishments by Category 2016-2017

Figure 11: Location of New City Establishments

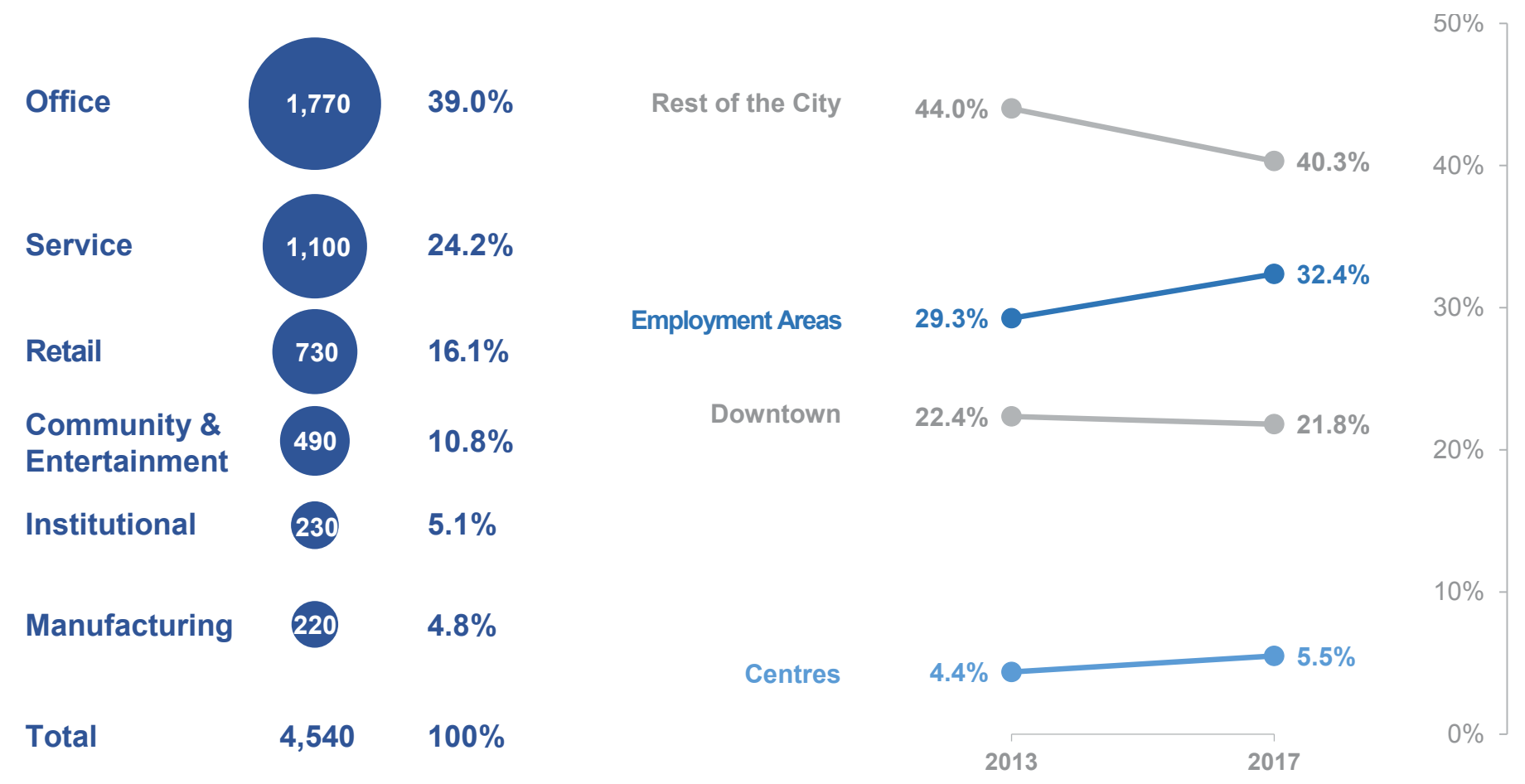


Figure 12: Net Establishment Growth vs. 5-Year Average

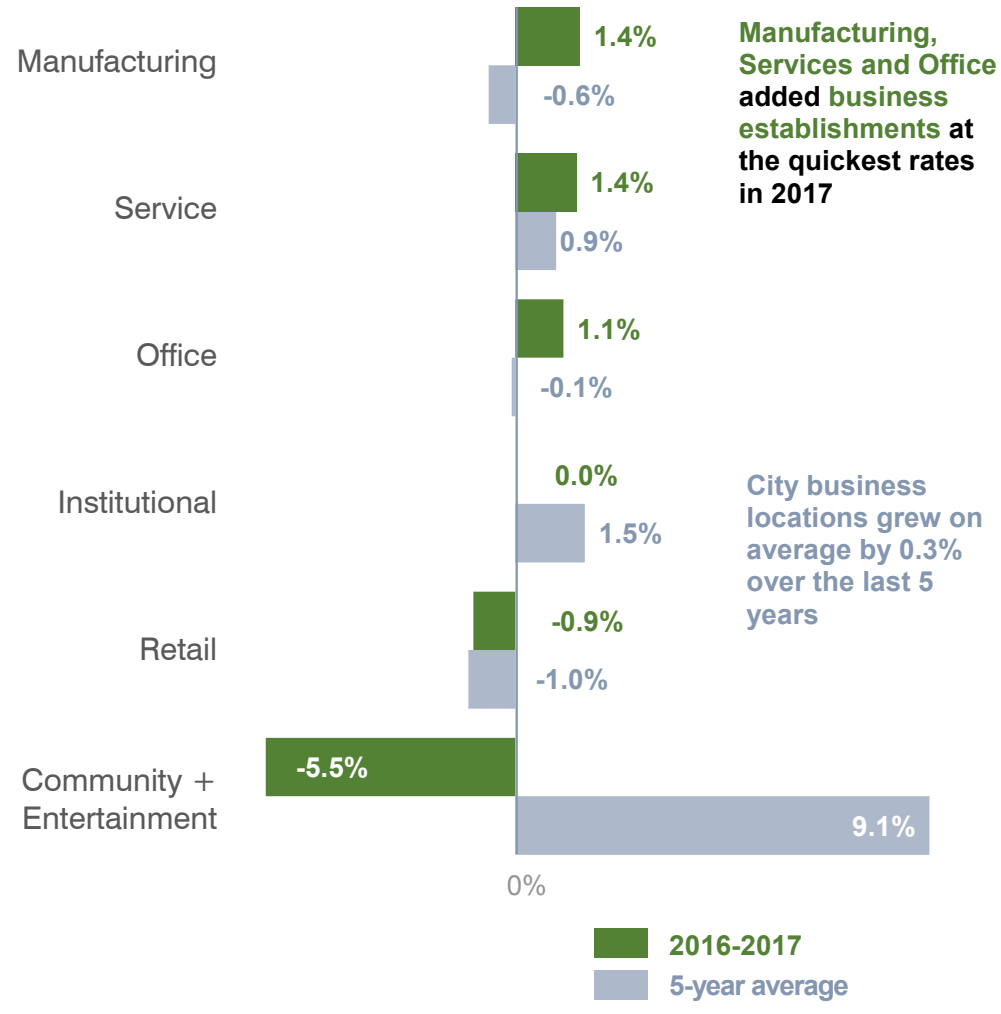

Employees per Establishment

In 2017, the average number of employees per establishment increased to 20.1. This metric has increased steadily at a rate of $2.4 \%$ annually from 17.9 in 2012 . The increase is partly due to the slower relative increase in business locations compared to employment growth.

This effect accelerated in 2017, particularly among fast-growing employment categories with lower rates of business location expansion. These included Community and Entertainment (19.2\%), Manufacturing (5.2\%), and Retail (4.2\%).

Despite this, Office (3.2\%) and Service $(2.7 \%)$ have led increases in average employees per establishment since 2012.

In 2017, the number of large businesses (with 100 or more

Figure 13: Employees per Establishment, 2000-2017

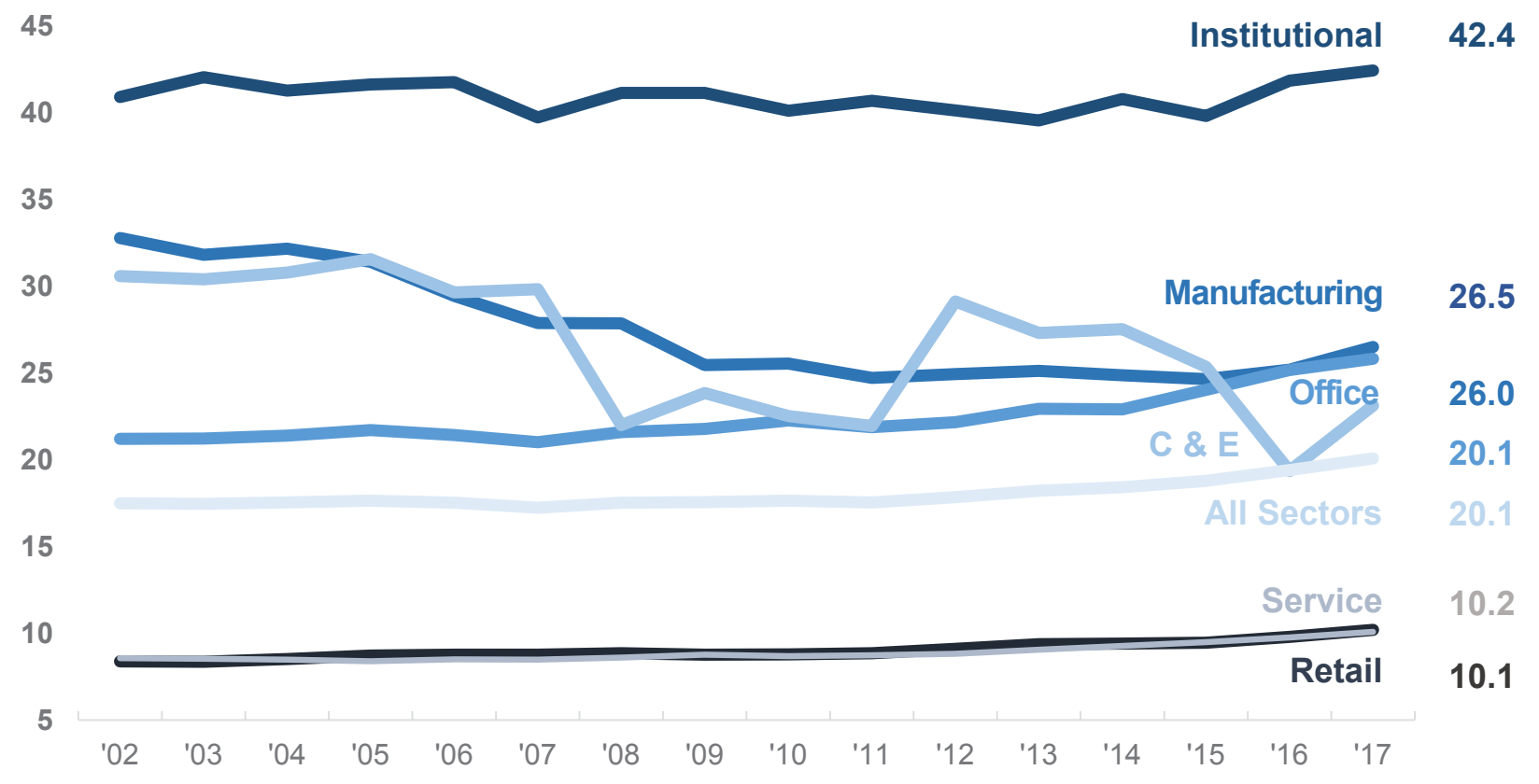


employees) increased by 105 (4.7\%), faster than the overall establishment increase $(0.4 \%)$, to comprise $3.1 \%$ of Toronto's establishments.

\section{Longevity}

Changes in the longevity of business establishments or locations can offer insights in to the economic health of a city or region. In $2017,35.8 \%$ of Toronto's business establishments were less than 5 years old (in the same location); some $31.4 \%$ of establishments reported being at the same location for 6 to 15 years.

Overall, $32.8 \%$ of the city's establishments have remained in the same location for more than fifteen years. This breadth of business location tenure demonstrates a strong degree of stability in the local economy despite economic cycles and recessions.
Figure 14: Longevity of City Establishments

0-5 Years $36.2 \% \longrightarrow 35.8 \%$

$21+$ Years

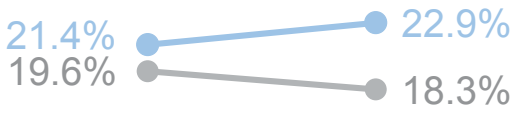

$11-15$ Years $13.8 \% \longrightarrow 13.1 \%$

$16-20$ Years $9.0 \% \longrightarrow 9.8 \%$

Figure 15: Change in City Establishment Count, 2017 vs. 2016

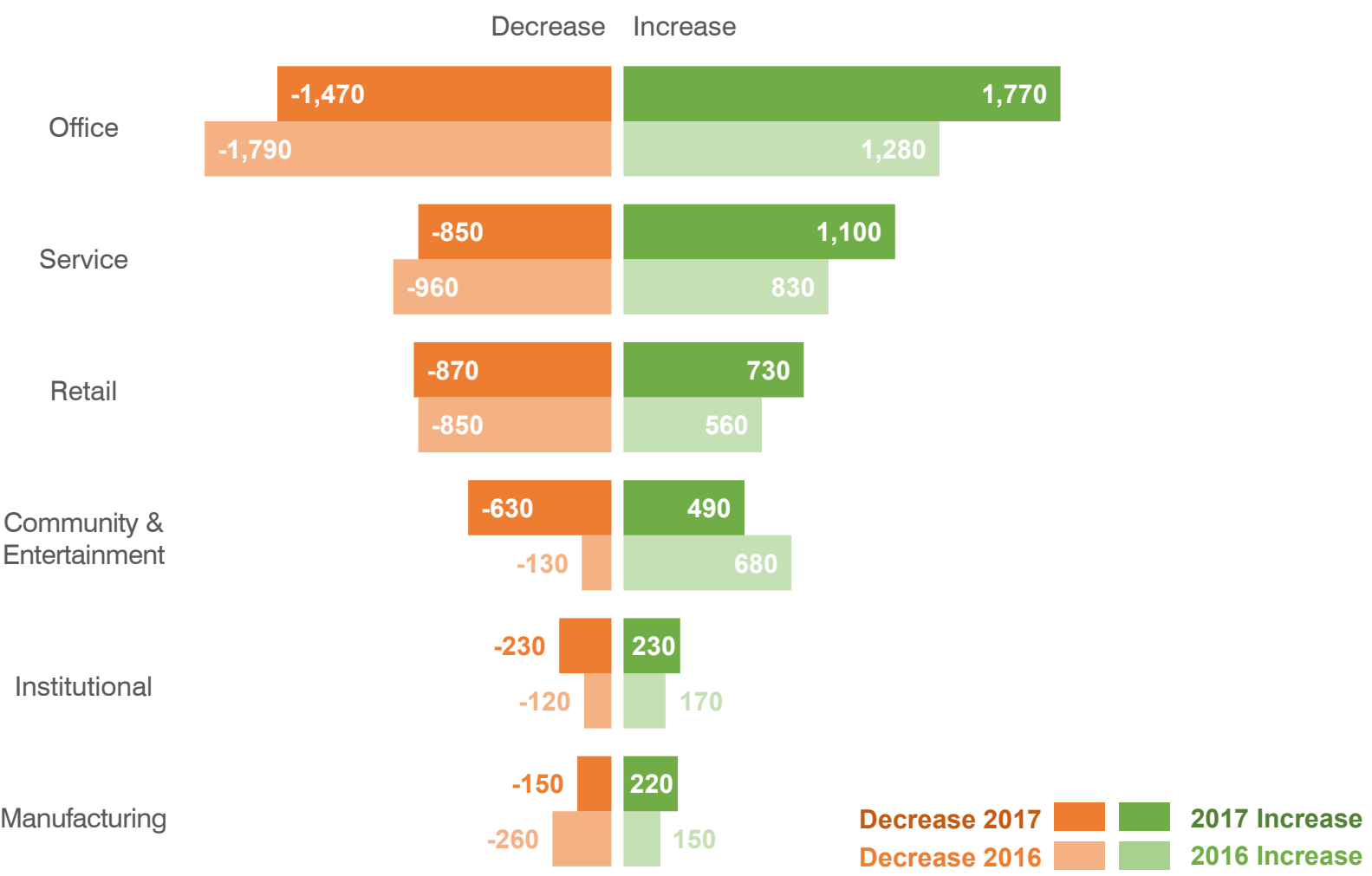


Toronto's top employment categories are Office (48.7\%), Institutional (16.4\%) and Service (12.5\%)

\section{Employment Categories}

The Survey utilizes six employment categories to reflect the underlying land use activities of employment across the city. All categories except Institutional experienced above-average growth in 2017 (see Table 4, Appendix).

\section{City Employment and Establishment Share}

Toronto's top employment categories are Office (48.7), Institutional (16.4\%) and Service (12.5\%). Employment in these categories has grown at or above the city's average growth rate over the last five years.

The remaining shares of city employment are Retail (10.0\%), Manufacturing (8.7\%) and Community and Entertainment (3.7\%). While Retail and Manufacturing employment has lagged Toronto employment growth over the last five years, Community and Entertainment (formerly the Other category) has been Toronto's fastestgrowing category, averaging $4.2 \%$ growth over the last five years.

Toronto's establishment categories are similarly structured, with Office (37.9\%), Service (24.8\%) and Retail (19.8\%) making up the largest shares, followed by Institutional (7.8\%), Manufacturing (6.6\%) and Community and Entertainment (3.2\%). Service and Retail establishments have a greater share of establishments, having on average fewer employees per business location (10 employees per establishment) when compared to Institutional establishments (42 employees per establishment).

\section{Structural Change}

In 2017, Toronto employment increased by 57,550 or $3.9 \%$, above

Figure 16: City Share of Employment by Category in 2017

Office

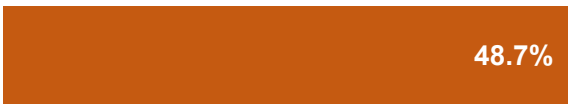

Institutional

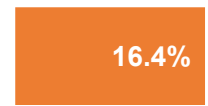

Service

$12.5 \%$

Retail 1

$10.0 \%$

\section{$8.7 \%$}

Manufacturing

Community +

Entertainment

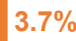

Figure 17: City Share of Establishments by Category in 2017

Office

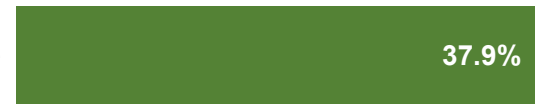

Service

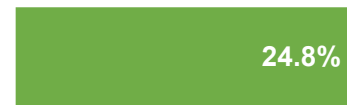

Retail

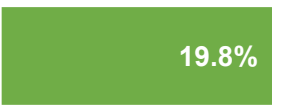

Institutional

$7.8 \%$

Manufacturing

$6.6 \%$

Community \&

Entertainment 
the city's five-year $(2.7 \%)$ and tenyear $(1.6 \%)$ compound annual growth rates.

Over the 10-year period 20072017 , four categories have grown faster than the city average: Office, Institutional, Service, and Community and Entertainment.

\section{Category Totals}

Office has led overall employment growth, adding 135,950 jobs since 2007 and growing annually at $2.1 \%$.

Institutional, while slower-growing, has added 41,340 jobs since 2007 and grown annually at $1.8 \%$.

Service has been the second-fastest growing employment category since 2007, adding 40,230 jobs and growing at $2.4 \%$ annually.

\section{Community and Entertainment,} despite being the smallest category, grew the fastest since 2007, adding 15,510 jobs and growing annually at $3.3 \%$.

Retail employment has changed significantly since 2007 . The category declined steeply from 2007 to 2010 , shedding 10,990 jobs, resulting in marginal average annual growth (0.1\%) since 2007. Since 2012, Retail employment growth has lagged most other categories, although it grew strongly in 2017 , adding 4,750 jobs.

Manufacturing employment grew strongly in 2017, adding 8,260 jobs. Despite this turnaround, Manufacturing has experienced a long-term decline in growth, having lost 16,910 jobs (-11.3\%) since 2007 , or $-1.2 \%$ per annum (see Table 4 in Appendix).
Community and Entertainment has been Toronto's fastestgrowing category, averaging $4.2 \%$ growth over the last five years
Figure 18: 2016-2017 Employment Growth vs. 5-Year Average
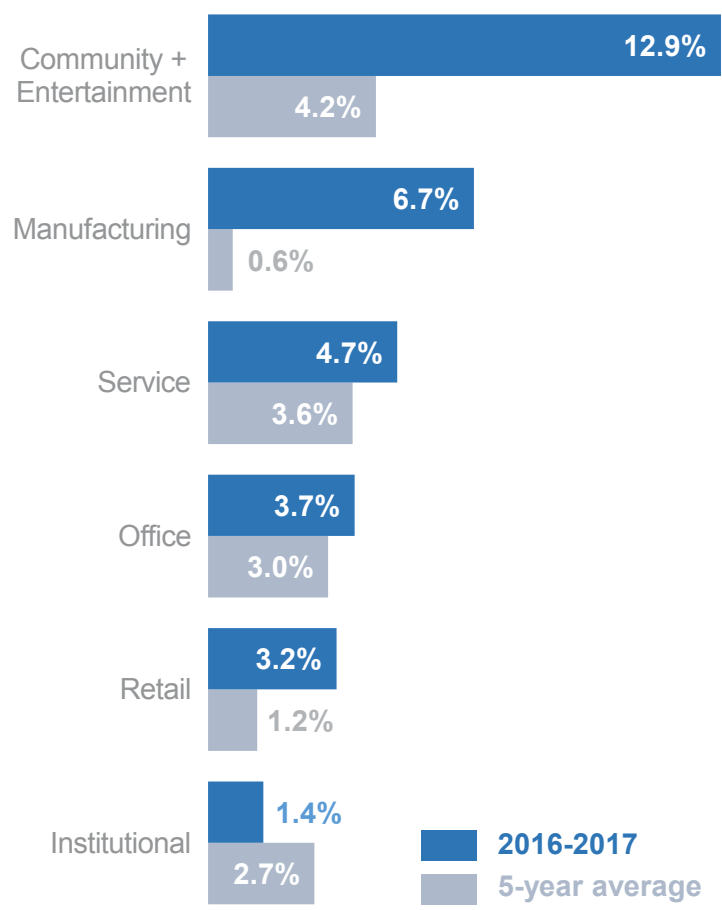

2016-2017

5-year average
Figure 19: 2007-2017 Job Growth by Category

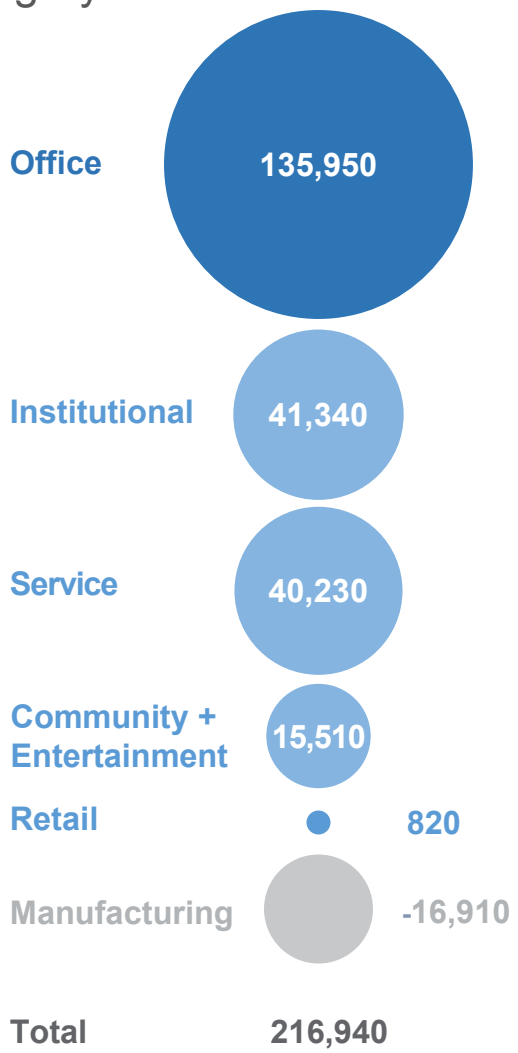


Service-based industries make up $77.5 \%$ of employment in Toronto's economy

\section{Employment by NAICS Economic Sector}

In 2011, the Survey incorporated the North American Industry Classification System (NAICS) into its data coding. While the employment categories, through activity codes, profile the land use and occupancy in the city, NAICS offers additional detail about the structure of the economy. As a coding standard across North America, NAICS also allows for more accurate analysis and comparison of Toronto's results with other jurisdictions. In 2017, the Survey was extremely successful in coding Toronto's establishments with $98.4 \%$ of businesses assigned a full 6-digit code.

\section{Toronto's Economy}

Three major sectors make up the employment shares of Toronto's economy: Service-Based industries (77.5\%), Government and Institutional industries (13.9\%) and Goods Producing industries (8.6\%).

Since 2012, annual growth in Government and Institutional (3.9\%) and Service-Based jobs (3.0\%) have offset employment losses in Goods Production (-0.9\%). Total 5-year employment net change was 187,060 jobs added, including Service-Based (156,160 jobs) Government and Institutional (36,940 jobs) and Goods Producing industries (a loss of 6,010 jobs).

Figure 20: City Employment by NAICS Sector

\section{YR Trend 2017 Share}

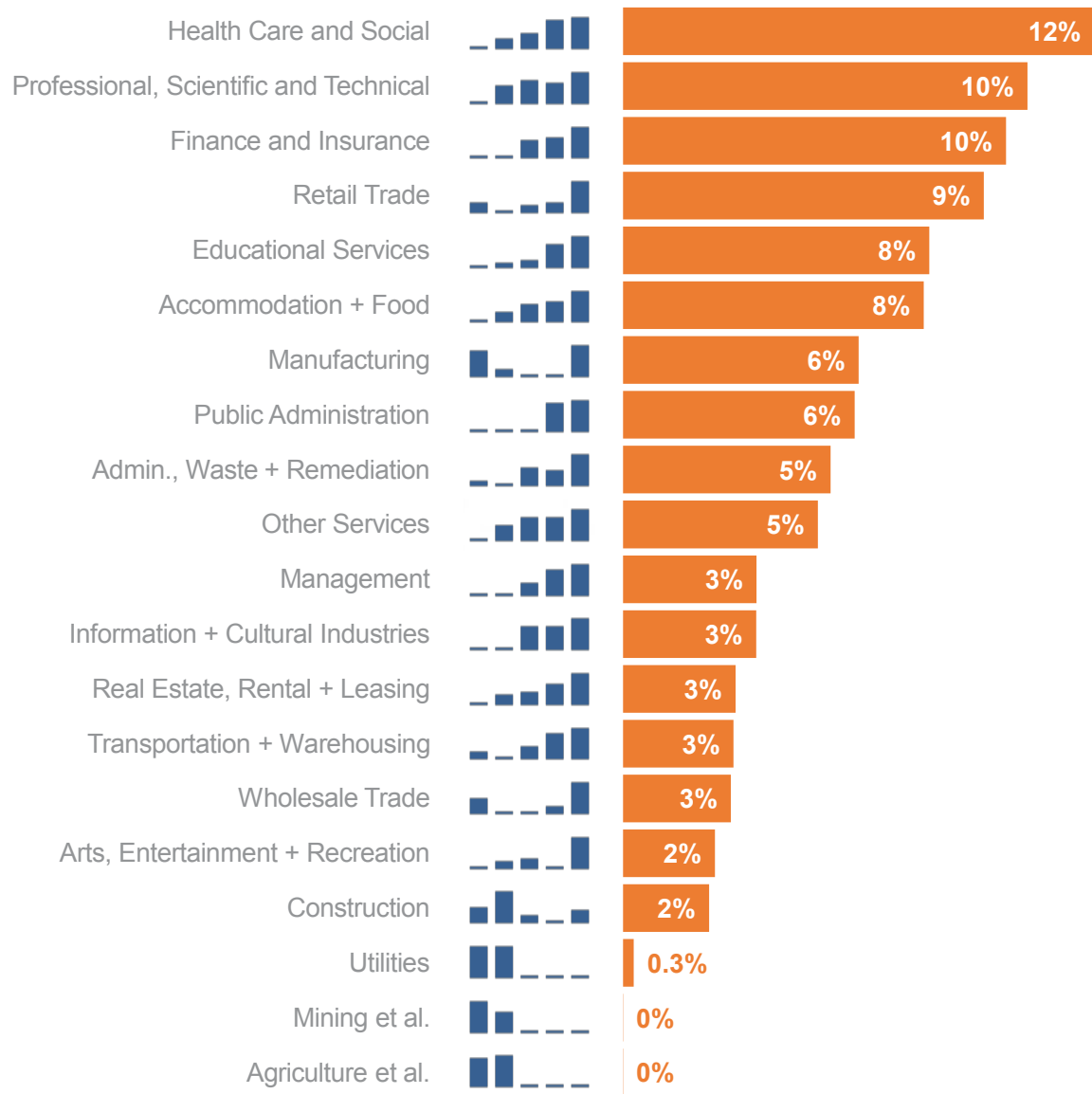


In 2017, Service-Based work led growth adding 44,750 jobs (4.4\%). Government and institutional added 7,920 jobs (3.9\%). Goods Producing industries added 5,050 jobs (4.0\%), reversing the five year trend of decreasing employment.

\section{Major Sectors}

NAICS classifies the economy into 20 major sectors. In $2017,49.8 \%$ of all jobs in Toronto were in the top five NAICS sectors. Three of these five sectors have increased their shares of total employment since 2012: Finance and Insurance $(0.6 \%)$, Health Care and Social Assistance (0.6\%) and Educational Services (0.9\%).

In 2017, the top sectors for job growth included Administrative and Support, Waste Management and Remediation Services (adding 8,410 net jobs or $12.4 \%$ ), Finance and Insurance (adding 7,350 net jobs or 5.1\%) and Educational Services (adding 6,610 net jobs or $5.8 \%$ ). See Figure 40 , Appendix.
Toronto's largest NAICS sector is Health Care and Social Assistance, comprising 185,010 or $12.2 \%$ of city employment. It grew by $1.4 \%$, adding 2,620 jobs.

Since 2012, additional sectors have experienced high annual growth rates and five-year employment increases:

- Real Estate, Rental and Leasing added 10,270 jobs since 2012, averaging 5.4\% annual growth

- Management added 12,010 jobs since 2012, averaging 5.3\% annual growth

- Educational Services added 26,990 jobs since 2012, averaging $5.2 \%$ annual growth

- Administrative and Support, Waste Management and Remediation Services added 15,060 jobs since 2012, averaging $4.5 \%$ annual growth

- Finance and Insurance added 25,920 jobs since 2012 , averaging $3.9 \%$ annual growth

- Arts, Entertainment and Recreation added 6,130 jobs since 2012, averaging $3.8 \%$ annual growth.
In 2017, the top sector for job growth was Administrative and Support, Waste Management and Remediation Services (adding 8,410 net jobs or $12.4 \%)$
Figure 21: Employment by NAICS Sector, 2012-2017

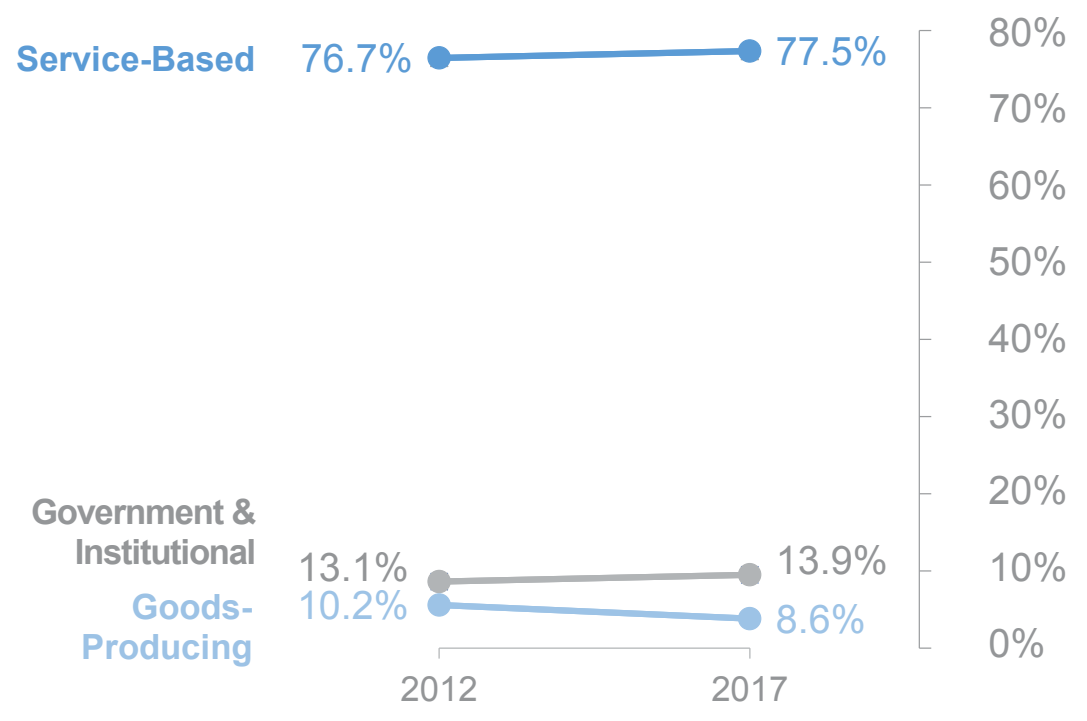

Figure 22: Establishments by Major NAICS Sector, 2012-2017

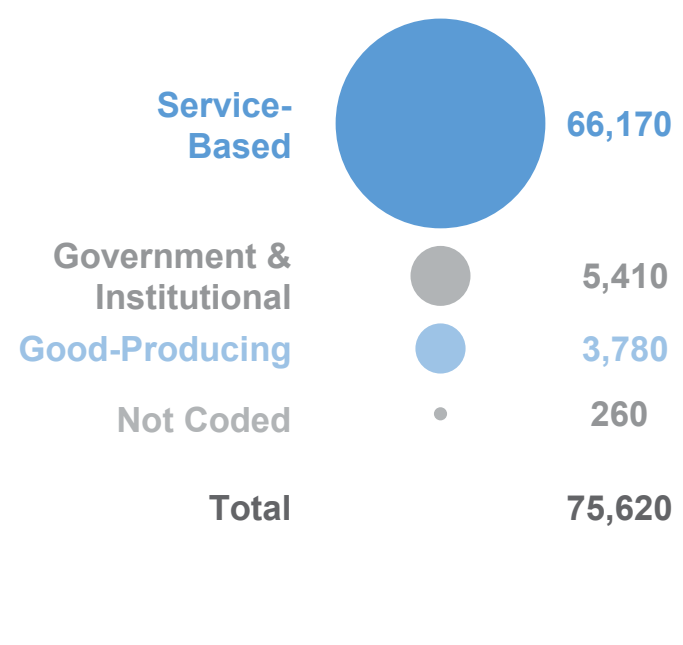


Figure 23: Downtown Employment Change

\section{Downtown Employment Change 2016-2017}

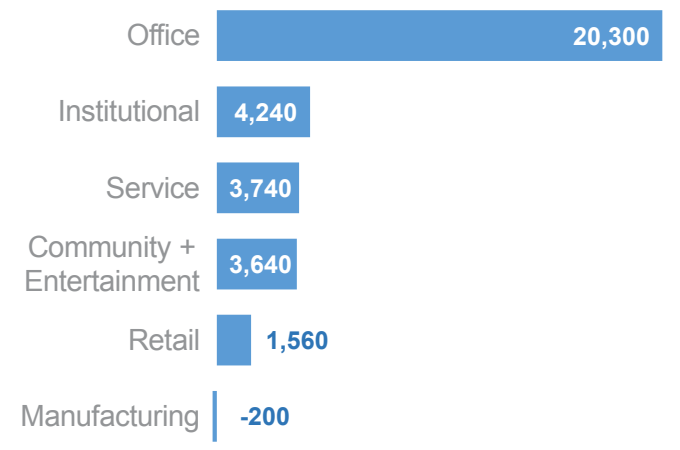

\section{Urban Economic Structure}

Toronto's Official Plan directs both employment and residential growth towards specific areas of the city, including Downtown, the Centres, and Employment Areas (see Map 2). Together, these areas form the backbone of the city's urban economic structure. They are connected by transit and transportation arteries, maximizing existing infrastructure and services in order to best accommodate growth. The Official Plan also identifies 34 Secondary Plan areas, many of which are experiencing rapid growth through intensification.

Downtown, the Centres and many Secondary Plan areas are Mixed Use Areas and encourage both residential and employment growth. Employment Areas are designated primarily for employment-related land uses.

The Places to Grow Act is the Provincial legislation that governs planning for growth and development in a way that supports economic prosperity, protects the environment and helps communities achieve a high quality of life in Ontario. The Places to Grow Act also enables the development of regional growth plans that guide government investments and policies, such as the Growth Plan for the Greater Golden Horseshoe, 2017 ("Growth Plan").

The Growth Plan reinforces Toronto's Official Plan by enabling the identification of Strategic Growth Areas. These areas

\section{Map 2: Urban Economic Structure}

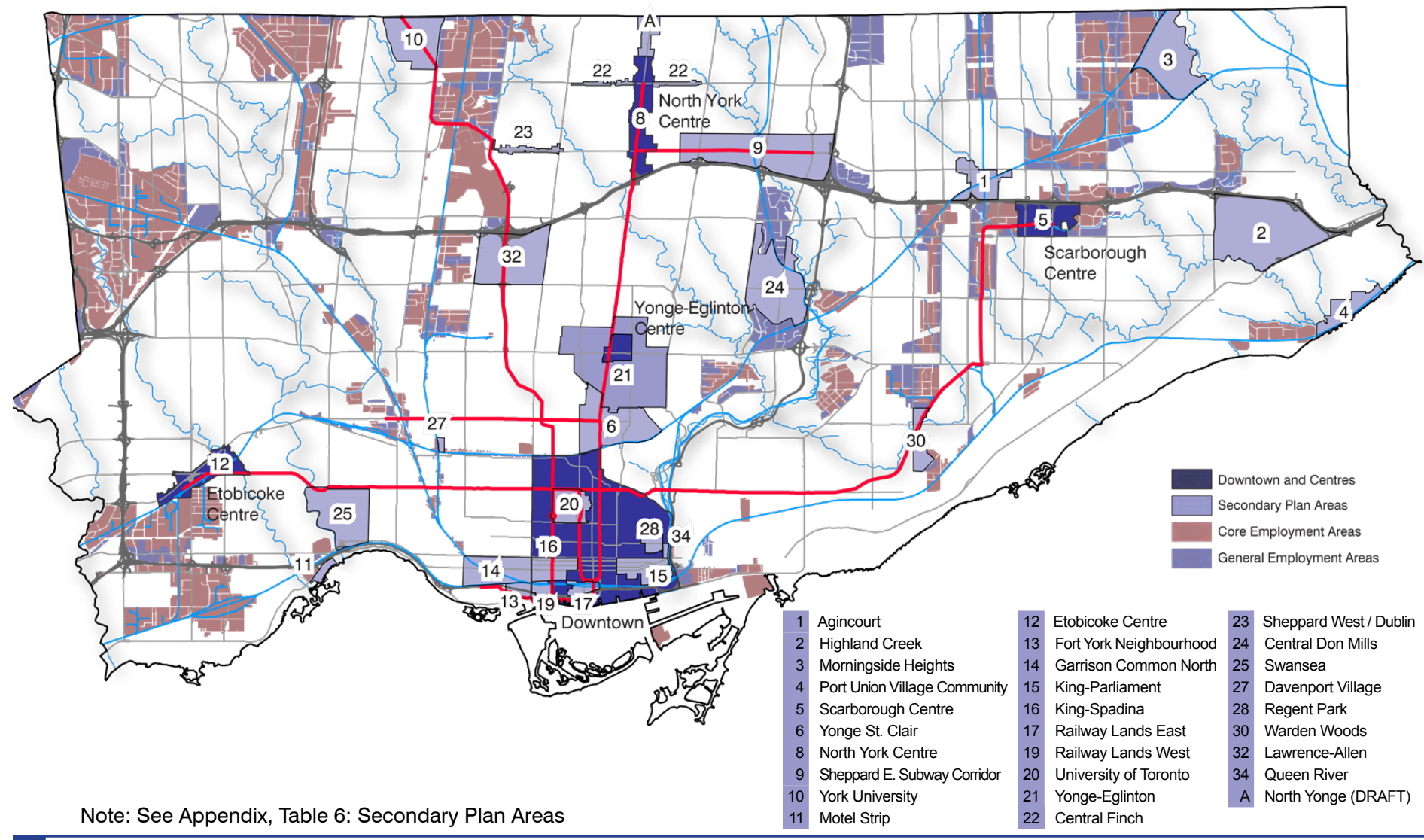


are planned to accommodate intensification and higher densities of both population and employment. Strategic Growth Areas include Urban Growth Centres, Major Transit Station Areas, and infill sites such as brownfields or greyfields.

Toronto contains five Urban Growth Centres: Downtown, Etobicoke Centre, North York Centre, Scarborough Centre, and Yonge-Eglinton Centre. These are defined as existing or emerging downtown areas, and targeted to achieve minimum densities of 400 residents and jobs combined per hectare by 2031 .

\section{Employment in the Downtown}

Toronto's Downtown is a local and national economic hub. It contains 544,480 jobs within $21.4 \mathrm{~km}^{2}$, accounting for $35.9 \%$ of Toronto's jobs with an average employment density of 25,433 jobs per km² (254 jobs per hectare).

Figure 24: Employment Growth vs. 5-Year Average

Downtown

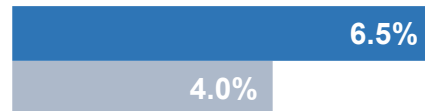

Centres

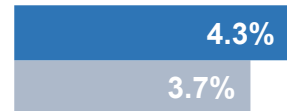

All Areas
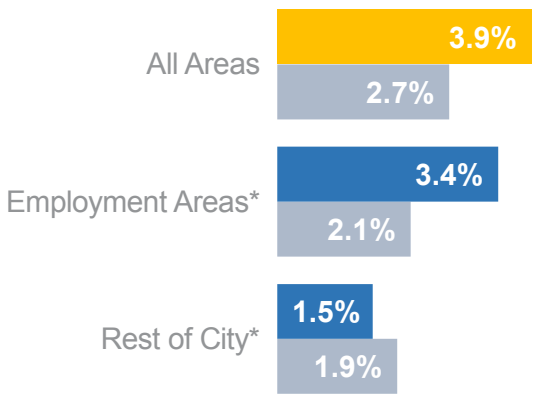

* Areas compared to 4-year compound annual growth rate due to data limits grew by $3.9 \%$ in 2017

2016-2017

5-year average
Figure 25: The Centres

Employment Change

Downtown increased by 33,280 jobs or $6.5 \%$. The Downtown has grown at an average annual rate of $4.0 \%$ since 2012 , adding a total of 97,650 jobs since that time.

This growth rate is above the city's mean of $2.7 \%$ over the last five years (see Table 5, Appendix).

Office employment comprises $64.9 \%$ of Downtown employment. Office added 20,300 jobs in 2017 , growing at $6.1 \%$. Other fast growing categories included Service (6.8\%), Institutional (5.8\%), Community and Entertainment (20.1\%) and Retail (5.7\%). Collectively these categories added 13,180 jobs. Downtown Manufacturing decreased by 200 jobs.

Downtown continues to attract new establishments. In $2017,17.5 \%$ of new business establishments were counted in the Downtown.

Yonge-Eglinton Employment Change 2016-2017

Citywide employment

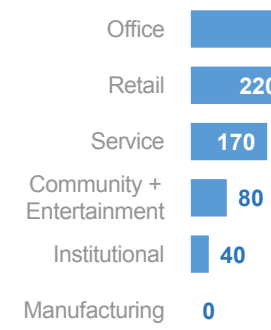

\section{North York Employment Change 2016-2017}

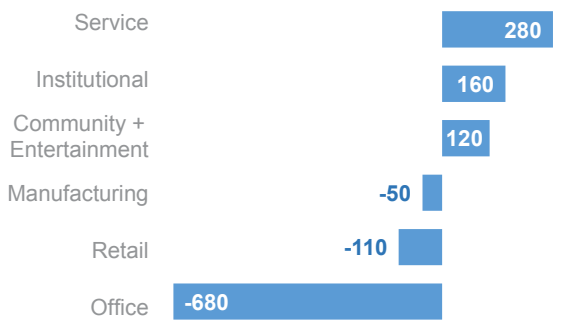

Scarborough Employment Change 2016-2017

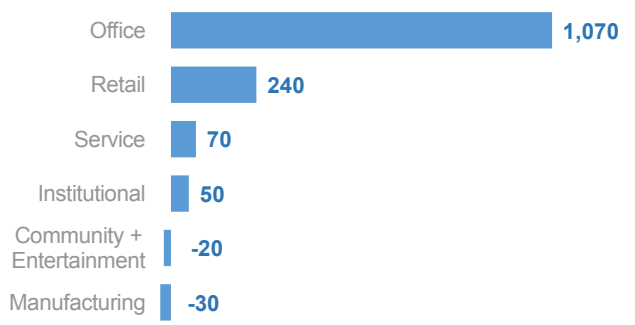

Etobicoke Employment Change 2016-2017

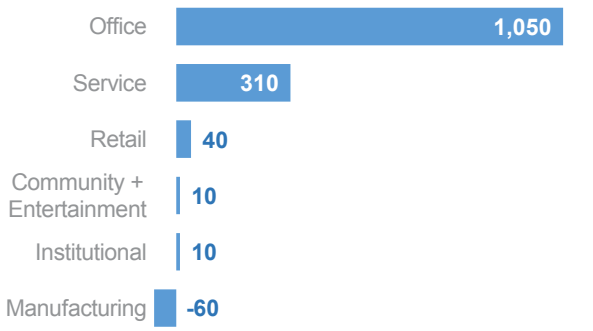


The Centres are home to 93,170 jobs or $6.1 \%$ of employment in the city.

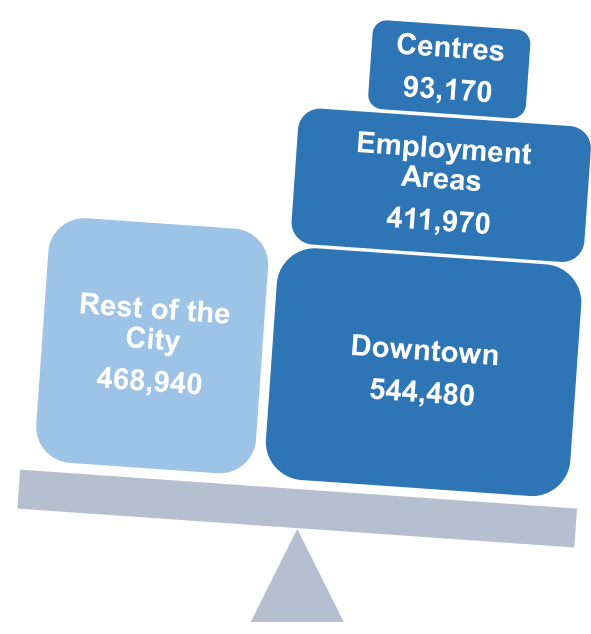

North York Centre is Toronto's largest Centre with 40,050 jobs, comprising $2.6 \%$ of jobs in the city. Despite declining slightly (0.7\%) in 2017, North York Centre has grown annually at $2.1 \%$

Eglinton (1,350 jobs), and Etobicoke Centres (1,370 jobs). North York lost 280 jobs. 6 in 10 net new jobs in the Centres in 2017 were Office (59.7\%), followed by Service $(21.7 \%)$, Retail (10.5\%), Entertainment (5.0\%). Manufacturing lost 140 jobs. Office comprises $77.8 \%$ of all employment in the Centres.

\section{North York Centre}

since 2012.

More than 8 in 10 jobs in North York Centre is Office, representing 33,490 jobs (83.6\%).

In 2017, North York Centre saw net decreases in Office (2\% or 680 jobs), Retail (110 jobs) and Manufacturing (50 jobs). Employment grew in Service (280 jobs), Institutional (160 Jobs), and Community and Entertainment (120 jobs). Since 2012, North York Centre employment increases were mostly in Office, which added 3,440 jobs. Institutional (6.8\%) and Community and

\section{Scarborough Centre}

Scarborough Centre is the second largest Centre in Toronto with 23,450 jobs, or $1.5 \%$ of the City's total. In 2017 , employment grew by $6.3 \%$, adding 1,390 jobs or $6.3 \%$. Since 2012 , Scarborough Centre added 8,060 jobs to grow annually at $8.8 \%$.

Most of Scarborough's growth over the past year was in Office $(1,070$ jobs or $6.8 \%$ ), followed by Retail (240 jobs), Service (70 jobs) and Institutional (50 jobs). Since 2012, Office grew by 8,240 jobs.

Figure 26: Downtown and Centres, Share of Employment by Category in 2017

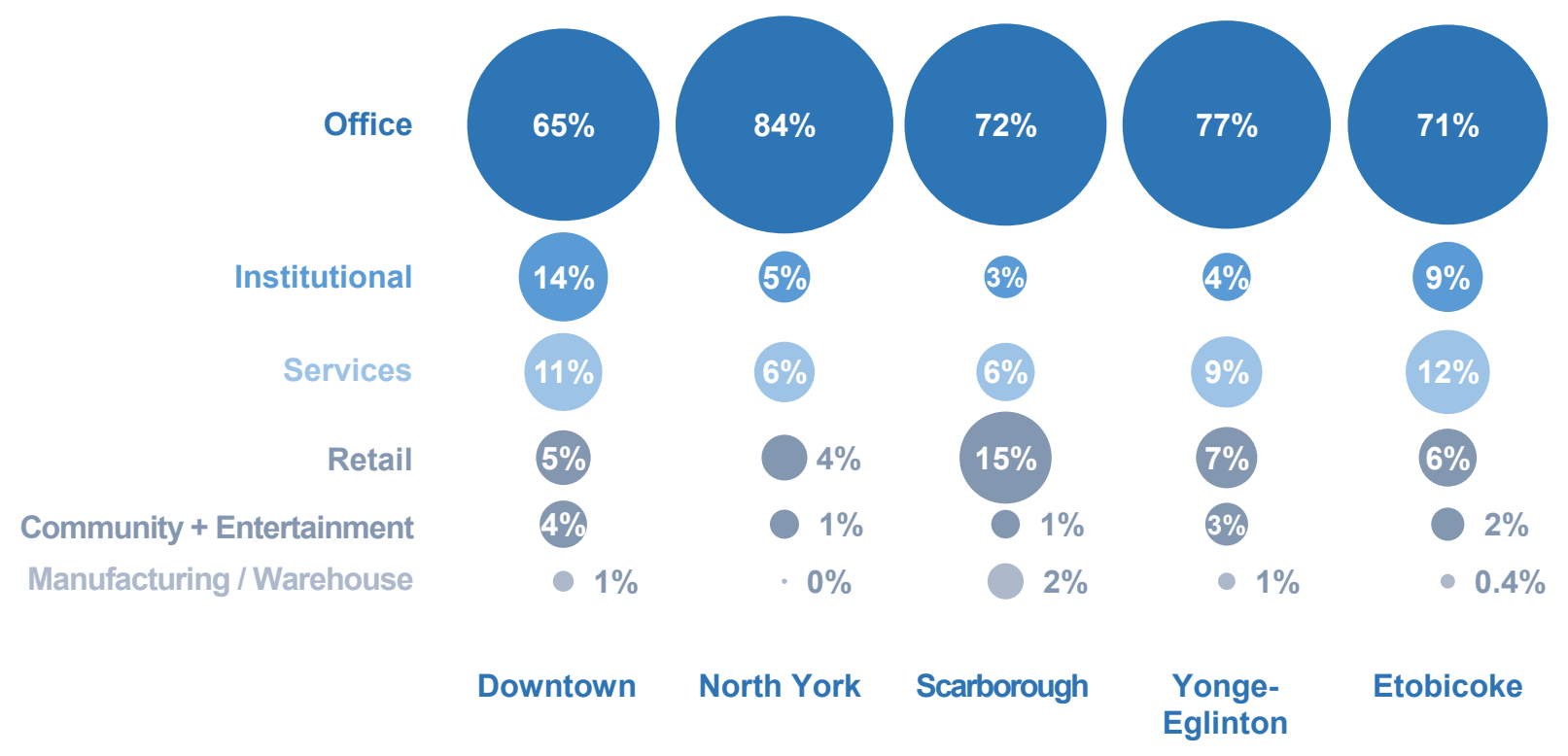




\section{Yonge-Eglinton Centre}

Yonge-Eglinton Centre hosts 18,850 jobs or $1.2 \%$ of the city's total, and contains the highest density of employment of any Centre with over 31,000 jobs per square kilometre. In 2017, employment in this Centre increased by 1,350 jobs or $7.7 \%$. Over the last five years, employment in Yonge Eglinton has grown annually by $1.2 \%$ to add 1,110 jobs.

Over three-quarters (76.6\%) of employment in Yonge-Eglinton Centre is Office. The Office employment category added 840 jobs (6.2\%) in 2017, followed by Retail (220 jobs), Service (170 jobs) and Community and Entertainment (80 jobs).

\section{Etobicoke Centre}

Etobicoke Centre is the smallest Centre in Toronto, but grew the fastest of all Centres in 2017 (14.4\%) to add 1,370 jobs. The Centre has 10,820 jobs total, representing $0.7 \%$ of employment in Toronto.

Most employment is Office (70.6\%), followed by Service (12\%) and Institutional (9\%). Office employment grew by 1,050 jobs (15.9\%) in 2017. Since 2012, Office added 1,570 jobs, followed by Service (300 jobs) and Retail (40 jobs).
Figure 28: Downtown + Centres Growth 2016-2017 vs. 5-Year Average

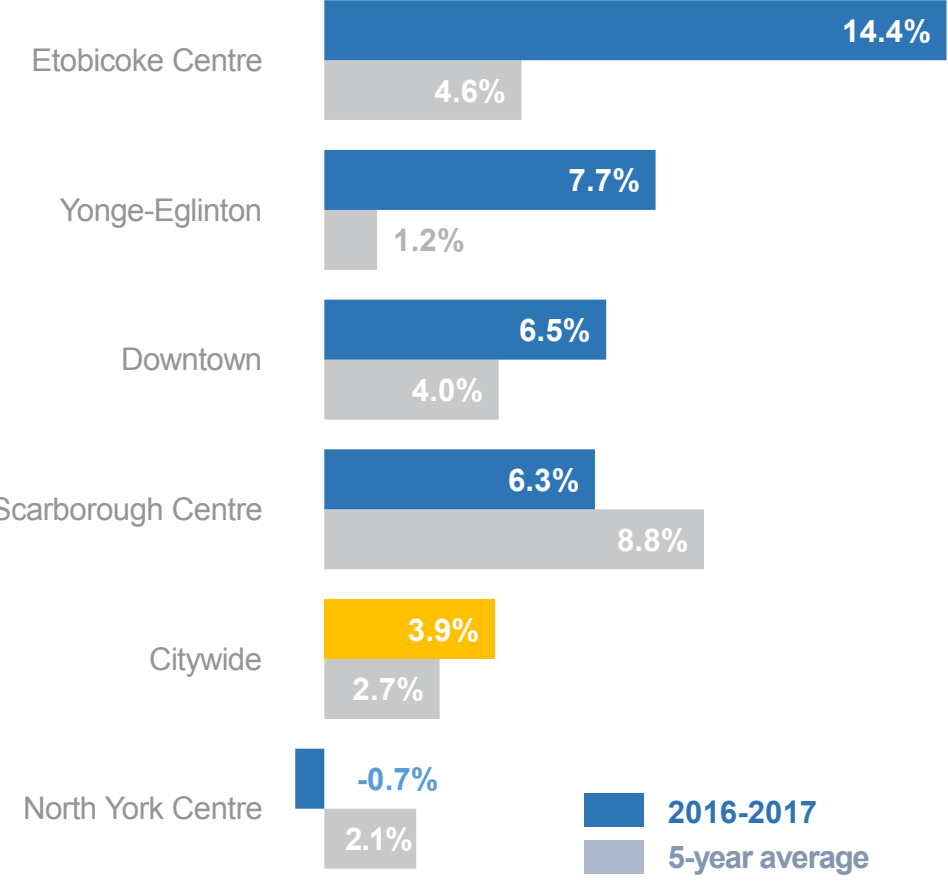

Figure 27: Downtown and Centres, Share of Establishments by Category in 2017

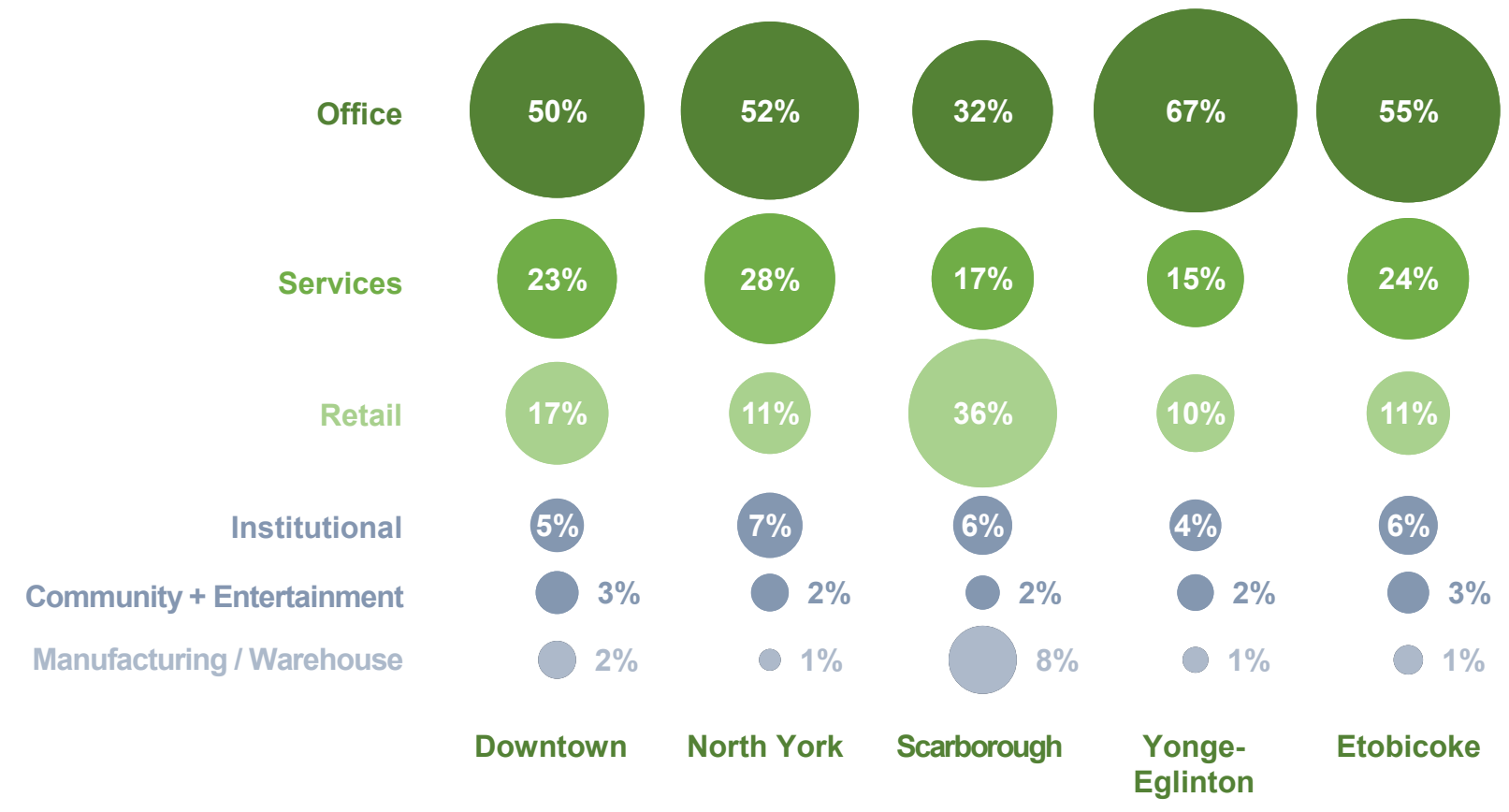




\section{Employment in Secondary Plan Areas}

Secondary Plan areas contain 317,130 jobs, $20.9 \%$ of city employment

With $20.9 \%$ of all employment $(317,130$ jobs), Secondary Plan areas reflect much of the city's recent urban growth. In 2017, Secondary Plan employment grew by 21,030 jobs or $7.1 \%$, well above the city average growth rate of $3.9 \%$.

The Official Plan contains Secondary Plans to manage growth and change in specific parts of the city. Secondary Plan areas contain a range of land use designations and cover diverse geographic areas across Toronto, including parts of Downtown, the Centres, and Employment Areas. As a result, the employment and establishment data in Secondary Plan areas should be understood in relation to the City as a whole, rather than compared to data for Downtown, the Centres, or Employment Areas.

Also, the Secondary Plan areas for the Downtown, North York Centre and Yonge Eglinton Centre differ from the areas of the Centres and Downtown designated in the Official Plan.

Employment and establishment data differ from the Downtown and Centres information in these areas.

\section{Secondary Plan Employment}

Secondary Plan employment includes $24.1 \%$ of all Office employment in the city, as well as $26.3 \%$ of all Community and Entertainment employment in the city (see Table 6, Appendix).

Figure 29: Secondary Plan Areas, 2017 Employment

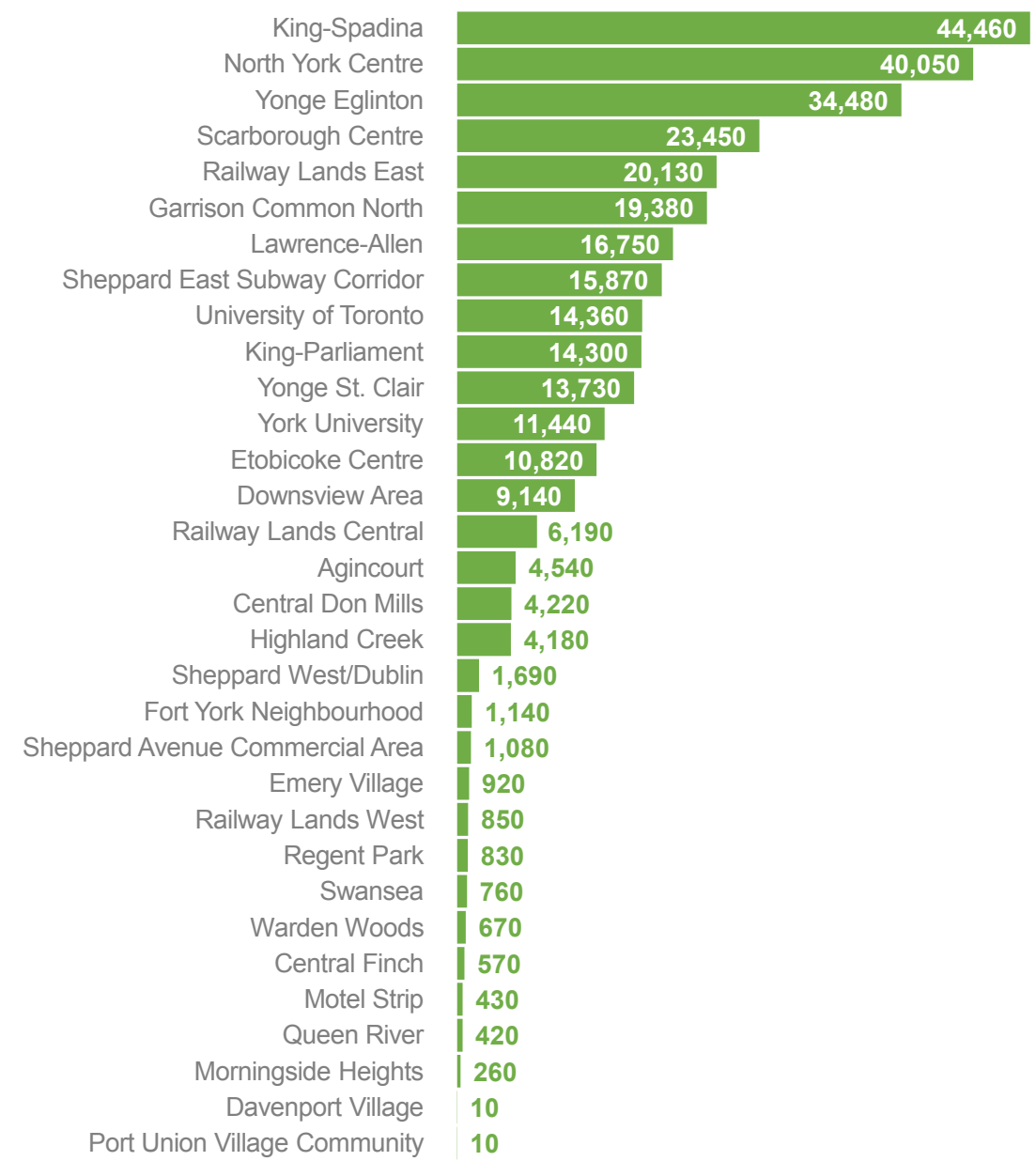

Note: See Appendix, Table 6: Secondary Plan Areas 
More than half (51.3\%) of employment within Secondary Plan areas is concentrated in the five largest areas by employment. This distribution of employment reflects the success of the Official Plan's growth management policies in directing growth to appropriate areas.

\section{Top areas by employment}

The largest Secondary Plan areas (those with 10,000 or more employees) are generally situated in or near Downtown, the Centres, along the city's rapid transit network, or around major post-secondary institutions.

In these areas, Office is the predominant employment category, with four exceptions: Sheppard East Subway Corridor and Lawrence-Allen have mostly Retail employment due to the presence of the Yorkdale and Lawrence Square shopping centres, and both York University and University of Toronto have predominantly Institutional employment.

All of these Secondary Plan areas have added jobs over the past year, with the exception of North York Centre and Yonge St. Clair. Combined, the largest Secondary Plans added 17,130 jobs (81.5\% of all growth in Secondary Plan areas).

\section{Smaller Secondary Plan areas}

Smaller Secondary Plan areas (under 10,000 employees) are comprised of smaller geographic areas further from Downtown, but still contain significant concentrations of employment. While many of these areas have a predominant share of Office employment, Service employment is predominant in Motel Strip, Railway Lands West, Queen River, and Port Union Village Community. Also, Institutional employment is the main category in Highland Creek and Fort York Neighbourhood, while Manufacturing employment is the most prevalent category in Downsview and Warden Woods.

Smaller Secondary Plan areas accounted for $18.6 \%$ of all Secondary Plan area employment growth over the past year through adding 3,900 new jobs.
In 2017, Secondary Plan employment grew by 21,030 jobs or $7.1 \%$, above the city growth rate of $3.9 \%$

\section{Figure 30: Secondary Plan Employment}

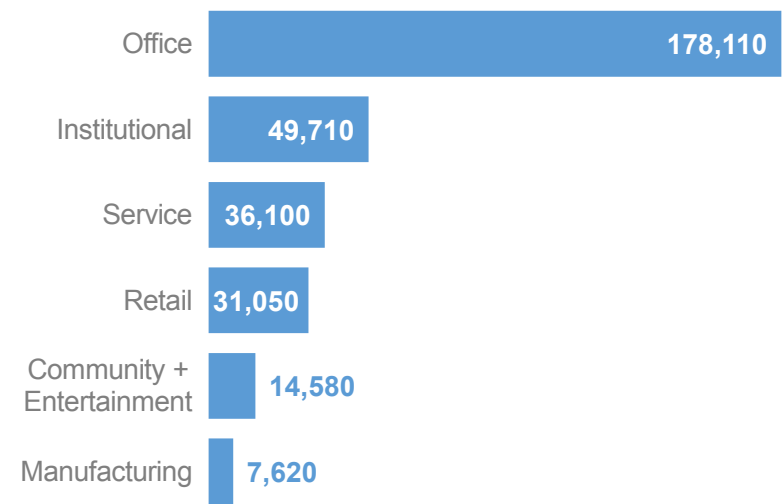

Figure 31: Secondary Plan Establishments

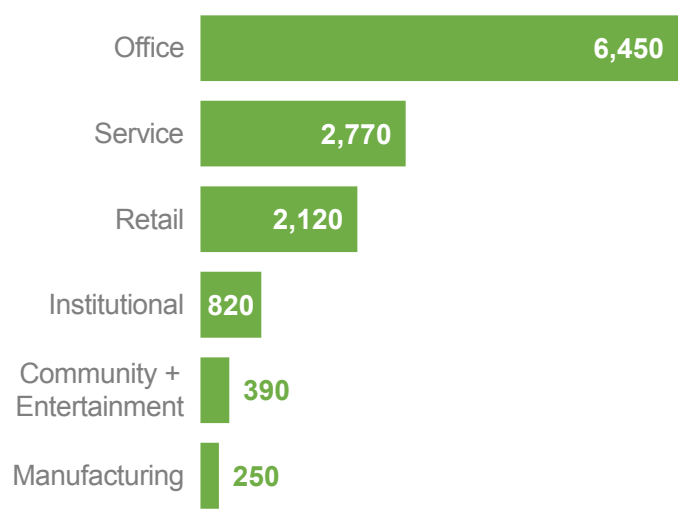




\section{Employment Areas}

Toronto's Employment Areas contain $27.1 \%$ of all jobs and $29.2 \%$ of all business establishments
Toronto's Employment Areas are a key part of the city's land use framework and are designated for employment use and growth. These areas are important as regionally and globally competitive locations for national and international business as well as areas for business formation.

Employment Areas are generally occupied by manufacturing, warehousing and product assembly activities as well as commercial business parks. They provide a broad range of job opportunities for Toronto residents and the regional labour force, and help ensure a stable environment for investment and to maintain and grow the city's revenue base.

Map 3 shows the location and boundaries of the Employment
Areas identified in the City's Official Plan at the time of the 2017 Toronto Employment Survey. Overall, 29.2\% of all establishments (22,080 locations) and $27.1 \%$ of all jobs $(411,970$ jobs) are located in Toronto's Employment Areas.

Employment Areas are particularly important to the Manufacturing sector, with $79.4 \%$ of Manufacturing establishments and $92.3 \%$ of all Manufacturing jobs located in Toronto's Employment Areas (see Map 4).

Employment Areas continue to attract new businesses, with 1,470 or $29.9 \%$ of new establishments in the city locating in Employment Areas in 2017.

\section{Regional Employment Policies}

In 2017, the Growth Plan released employment growth forecasts for the City of Toronto. Employment is forecast to grow to $1,720,000$ jobs by 2041 .

\section{Map 3: Toronto's Employment Areas and Employment Monitoring Areas}

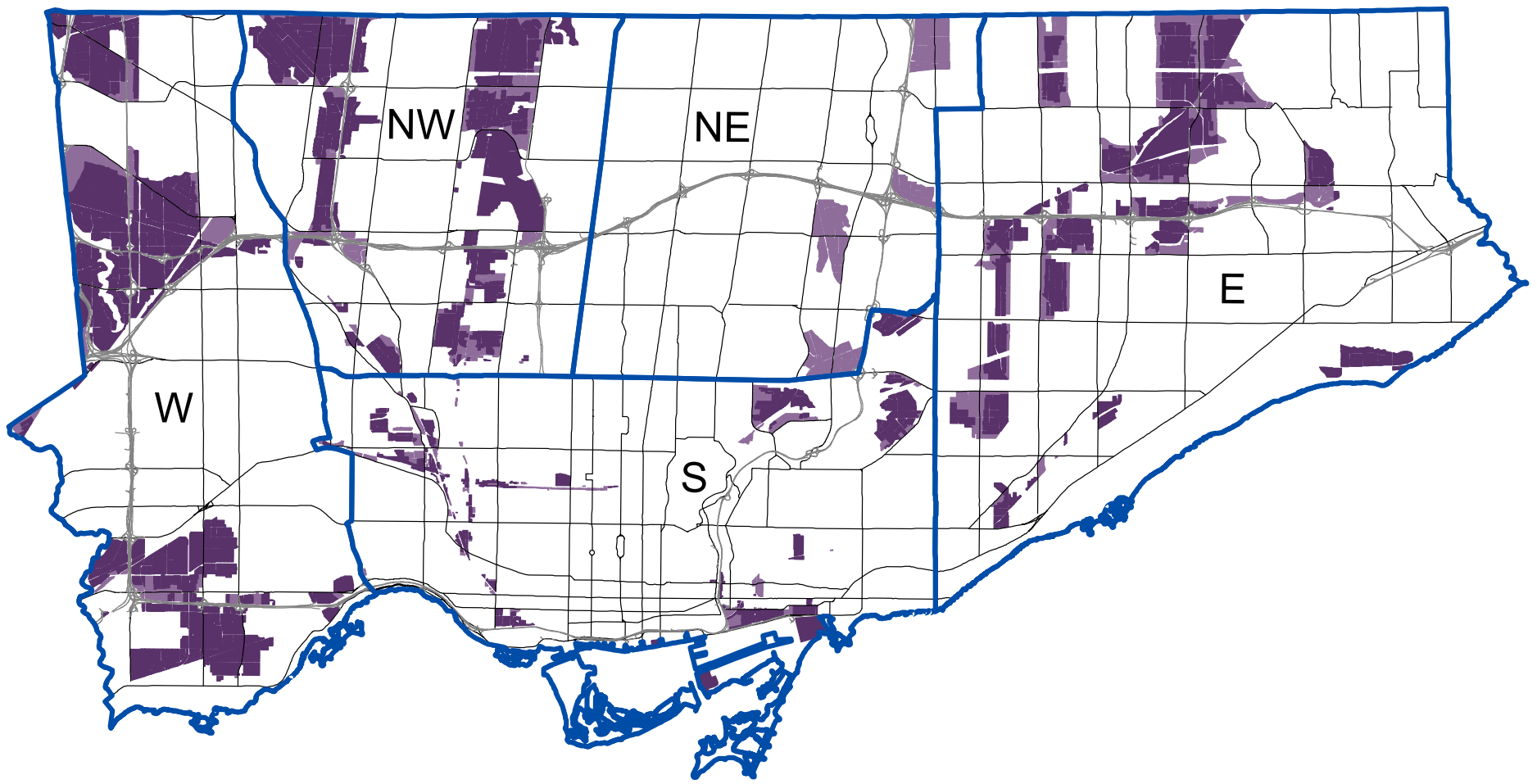


If the average rate of employment growth continues (2.7\% annually over the last five years), Toronto will achieve Growth Plan forecasts by 2022, almost twenty years before the target. Given the pace of Toronto's employment growth relative to the forecast, the Province can be expected to undertake another review of Growth Plan targets.

The Growth Plan also provides for an Official Plan designation for Prime Employment Areas, to protect land for consumptive or low density employment uses such as manufacturing, warehousing, goods movement or utilities.

Often, industrial and other impactful employment uses have few alternative locations in the city due to the potentially negative impacts of their activities on residential and other sensitive land uses. Conflicts related to the conversion of employment lands to residential or other sensitive uses can destabilize an entire area, resulting in employment loss and erosion of available areas for business formation or expansion.

\section{Official Plan Amendment 231}

On December 18, 2013, Council adopted Amendment 231 of the Official Plan (OPA 231). OPA 231 amends Policy 2.1.2 of the Official Plan by deleting the term Employment Districts and replacing it with Employment Areas. As a result, the Survey began reporting on Employment Areas in 2016 (including data to 2013). See Tables 7 and 8 in the Appendix.

\section{Core and General Employment Areas}

Section 4.6 of OPA 231 differentiates Core and General Employment Areas.
These designations came into force by an order of the Ontario Municipal Board on December 20, 2016.

Core Employment Areas are for the most part, geographically located within the interior of employment areas. Uses that would attract the general public into the interior of employment lands and possibly disrupt industrial operations are not generally permitted in Core Employment Areas.

General Employment Areas are often located on the periphery of Employment Areas, along major roads. This designation provides for retail stores, service shops and restaurants. These areas have increased visibility and transit access to draw the broader public.

\section{Map 4: Toronto's Employment Density}

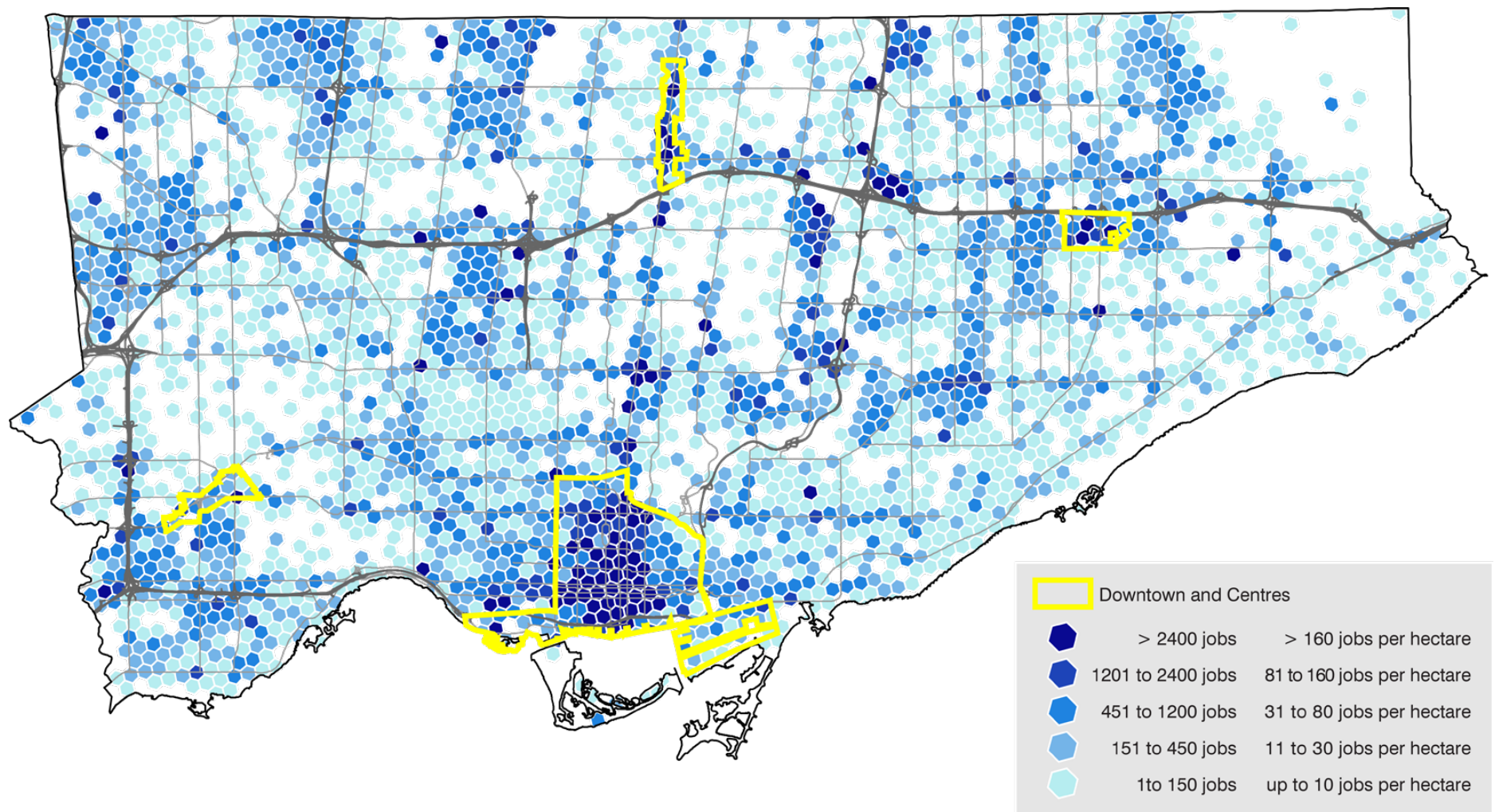


Core Employment Areas

accommodate most of

Toronto's jobs in Manufacturing, Transportation and Warehousing, and Construction

\section{Employment Area Sector Activity}

Manufacturing employment is the top sector across Employment Areas, making up $20.7 \%$ of all jobs $(85,320$ jobs), followed by employment in Wholesale and Retail Trade (16.5\% or 67,775 jobs) and Professional, Scientific and Technical Services ( $10.1 \%$ or 41,644 jobs).

Employment Areas accommodate important concentrations of jobs citywide in several sectors, led by employment in Manufacturing (92.3\%), Utilities (89.7\%),

Transportation and Warehousing (76.9\%) and Construction (72.2\%).

\section{Core Employment Area Activity}

Core Employment areas continue to host land consumptive land uses. In 2017, these areas led Employment Area jobs in Manufacturing $(27.8 \%$ or 72,133 jobs), Wholesale and Retail Trade (14.9\% or 38,560 jobs) and Transportation and Warehousing ( $11.7 \%$ or 30,465 jobs).

Core Employment Areas are leading locations citywide for employment in Manufacturing (78\%), Transportation and Warehousing $(70.2 \%)$ and Construction (59.5\%).

Figure 32: Core Employment by NAICS 2017

92.3\% of Toronto's

Manufacturing jobs are in

Toronto's Employment Areas 


\section{General Employment Area Activity}

General Employment Areas provide support activities for Core Employment Areas and help buffer heavy industrial uses from surrounding areas.

Sectoral employment in General Employment Areas is led by Wholesale and Retail Trade, having $19.2 \%$ of employment areas jobs $(29,220$ jobs), followed by Professional, Scientific, and Technical Services $(11.0 \%$ or 16,830 jobs) and Manufacturing (8.7\% and 13,180 jobs).
General Employment Areas are leading locations citywide for employment in Utilities (78\%), Real Estate and Rental and Leasing (21.1\%) and Wholesale and Retail Trade (15.9\%).

Administrative and Support, Waste Management and Remediation Services (16.3\%) and Manufacturing (14.3\%) also have significant shares of jobs citywide.
In 2017, employment in Toronto's Employment Areas grew by $3.4 \%$, adding 13,620 jobs

Figure 33: General Employment by NAICS 2017

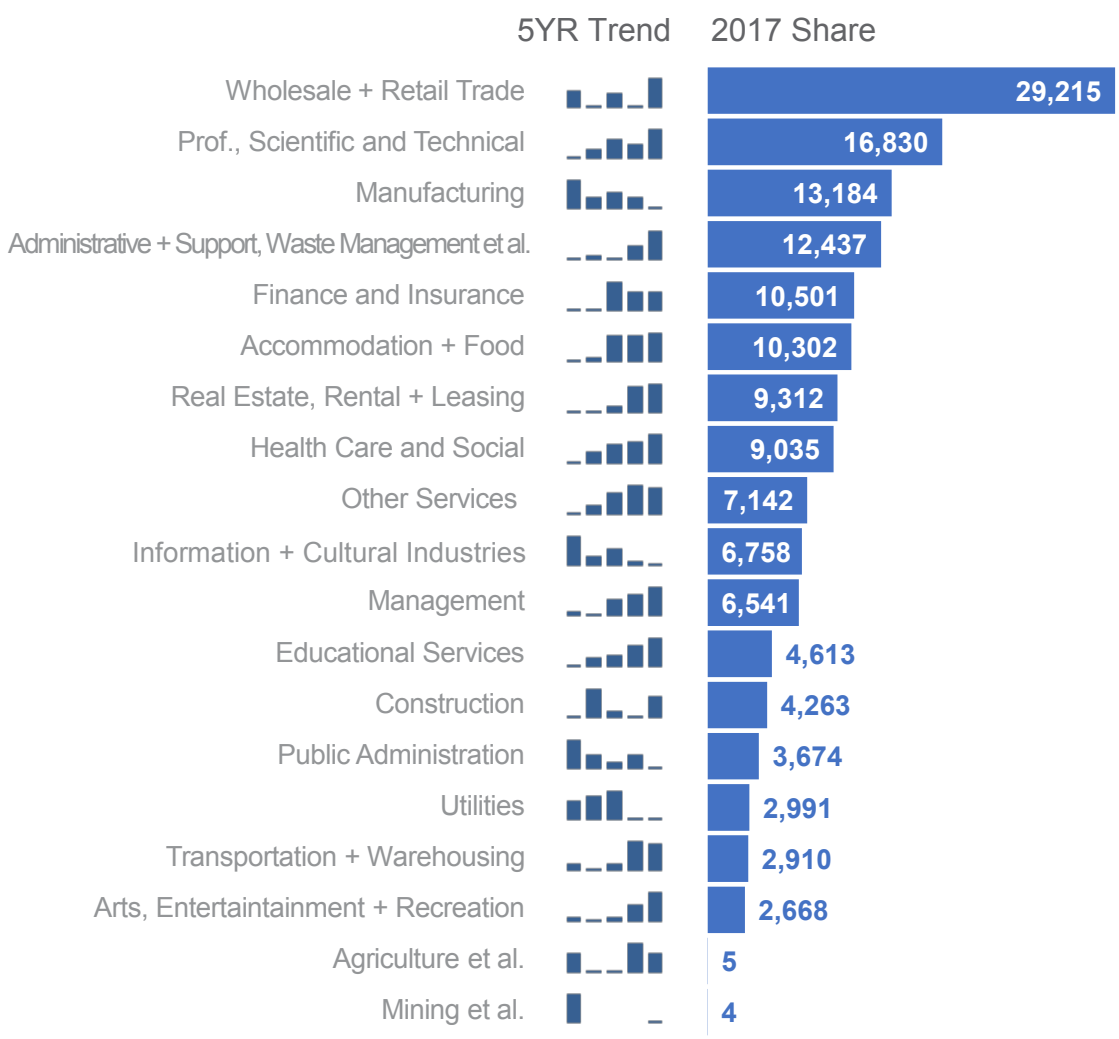


West EMA - Top 6 NAICS Sectors 2017

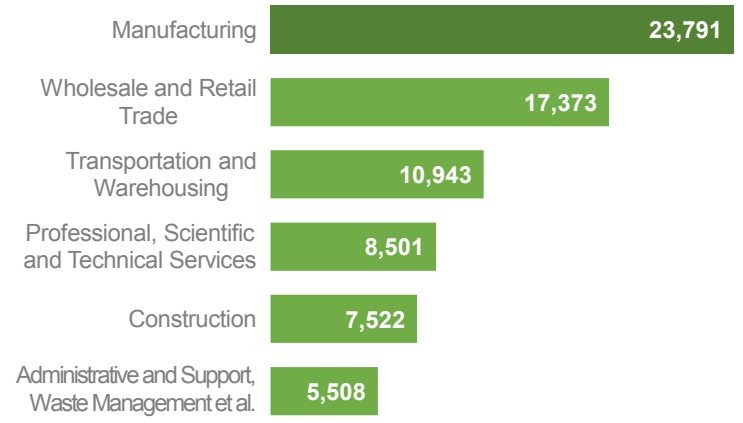

Northwest EMA - Top 6 NAICS Sectors

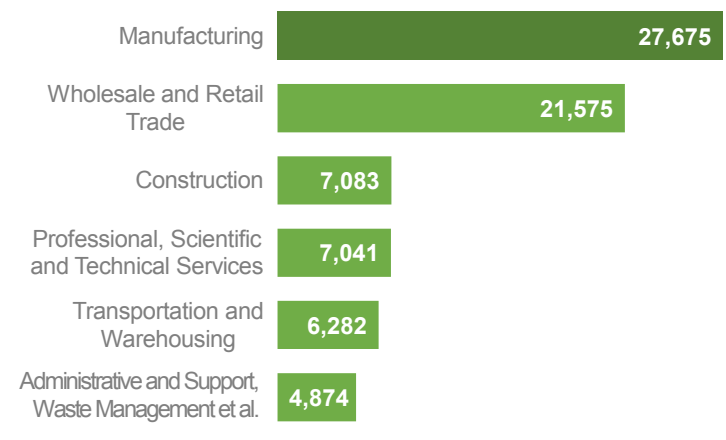

Northeast EMA - Top 6 NAICS Sectors

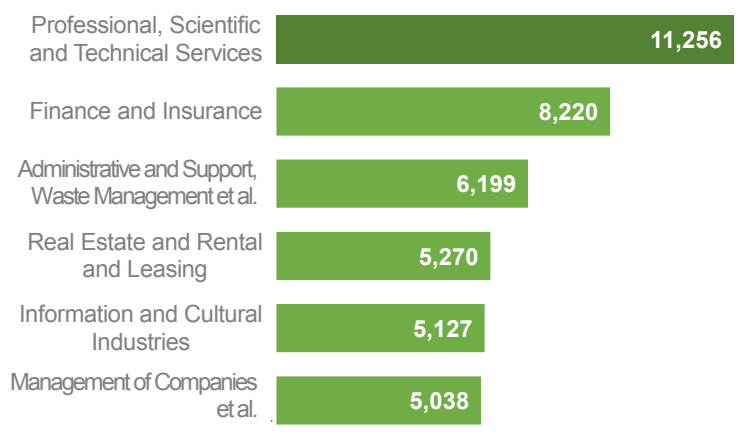

\section{Employment Monitoring Areas}

In 2016, five Employment Monitoring Areas (EMAs) were created to analyze broader trends in Employment Area activity across the city (see Map 3, page 22).

\section{In 2017, employment grew by $3.4 \%$ in all EMAs, above the average annual growth rate of $2.1 \%$ since 2013 .}

\section{West}

The West EMA approximates the former municipality of Etobicoke. $25 \%$ of Employment Area jobs $(102,800$ jobs) are in the West EMA. Of these, $89.6 \%$ are within Core Employment Areas. In 2017, West EMA employment grew $3.6 \%$, adding 3,550 jobs, growing by $8.0 \%$ or 7,640 jobs since 2013 .

The West EMA partly encompasses the Airport Corporate Centre surrounding Pearson International Airport. This employment node is the second largest employment concentration in Canada after Toronto's downtown.

Top employment sectors in the West EMA include Manufacturing (23.1\%), Wholesale and Retail Trade (16.9\%) and Transportation and Warehousing (10.6\%).

In 2017, the Manufacturing sector grew the quickest in the West EMA, adding 1,420 jobs or $6.4 \%$. Since 2013, the Construction sector grew the most, growing by $5.0 \%$ annually, adding 1,340 jobs.

\section{Northwest}

The Northwest EMA approximates the western part of the former municipality of North York.

23.5\% of Employment Area jobs $(96,940$ jobs) are in the Northwest EMA. $75.7 \%$ of these jobs are within Core Employment Areas. In 2017, Northwest EMA employment grew by $3.8 \%$, adding 3,560 jobs, growing by $8.1 \%$ or 7,240 jobs since 2013.

Top employment sectors in the Northwest EMA include Manufacturing (28.5\%), Wholesale and Retail Trade (22.3\%) and Professional, Scientific and Technical Services (7.3\%).

In 2017, the Manufacturing sector grew the quickest in the Northwest EMA, adding 1,010 jobs or $3.8 \%$.

Since 2013, the Professional, Scientific and Technical Services sector grew the most, growing by $16.8 \%$ annually, adding 1,020 jobs. Transportation and Warehousing followed close behind, adding 1,010 new jobs and growing $19.1 \%$ since 2013.

\section{Northeast}

The Northeast EMA spans the eastern portion of the former municipality of North York.

About $15.8 \%$ of Employment Area jobs (65,050 jobs) are in the Northeast EMA. The Northeast EMA has no Core Employment Areas, but has broader sector diversity than the other EMAs. In 2017, Northeast EMA employment grew by $5.2 \%$, adding 3,230 jobs, growing by $12.2 \%$ or 7,080 jobs since 2013. 
Top employment sectors in the Northeast EMA include Professional, Scientific and Technical Services (17.3\%), Finance and Insurance (12.6\%) Administration, Support and Waste Services (9.5\%).

In 2017, the relocation of a large security services firm added 1,200 jobs to Administration, Support and Waste Services. Also, the Professional, Scientific and Technical Services added 1,060 jobs through expanded establishments. Manufacturing lost 530 jobs since 2016 and 1,840 jobs or $31.1 \%$ since 2013 , as the sector continues to centralize in Core Employment Areas.

\section{East}

The East EMA approximates the former municipality of Scarborough. 22.6\% of Employment Area jobs (93,310 jobs) are in the East EMA. $60.6 \%$ of these jobs are within Core Employment Areas. In 2017, East EMA employment grew $1.2 \%$, adding 1,110 jobs, growing by $3.3 \%$ or 2,980 jobs since 2013 .

Top employment sectors in the East EMA include Manufacturing (23.9\%), Wholesale and Retail Trade (19.0\%) and Transportation and Warehousing (8.8\%).

In 2017, the Manufacturing sector grew the quickest in the East EMA, adding 1,010 jobs or $3.8 \%$. Since 2013, the Professional, Scientific and Technical Services sector grew the most, growing by $16.8 \%$ annually, adding
1,020 jobs. Transportation and Warehousing followed close behind, adding 1,010 new jobs and growing $19.1 \%$ since 2013 .

\section{South}

The South EMA approximates the areas of the former municipalities of the City of Toronto, York, and East York.

Some $13.1 \%$ of Employment Area jobs (53,860 jobs) are in the South EMA. $69.8 \%$ of these jobs are within Core Employment Areas. In 2017, South EMA employment grew by $4.2 \%$, adding 2,180 jobs, growing by $15.9 \%$ or 7,390 jobs since 2013.

Top employment sectors in the South EMA include Professional, Scientific and Technical Services (18.9\%), Wholesale and Retail Trade (13.9\%) and Manufacturing (13.6\%). Transportation and Warehousing also has a $13.6 \%$ employment share.

The Professional, Scientific and Technical Services sector has grown significantly since 2013 , adding 3.320 jobs (48.3\%), including 1,480 jobs in 2017.

The trend of office-based, skilled employment locating in the South EMA contrasts with decreasing employment in Wholesale and Retail Trade (a decrease of 400 jobs or $5.2 \%$ since 2013). Manufacturing in the South EMA has been flat (net decrease of 70 jobs or $0.9 \%$ since 2013), while growing slightly in 2017 (240 jobs or $3.3 \%)$.

\section{East EMA - Top 6 NAICS Sectors}

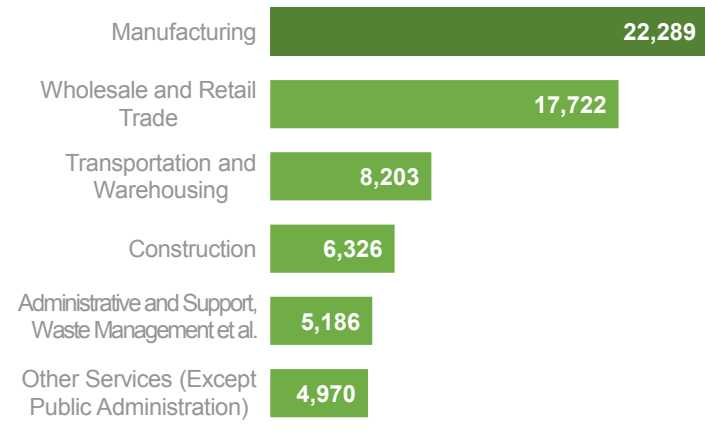

South EMA - Top 6 NAICS Sectors

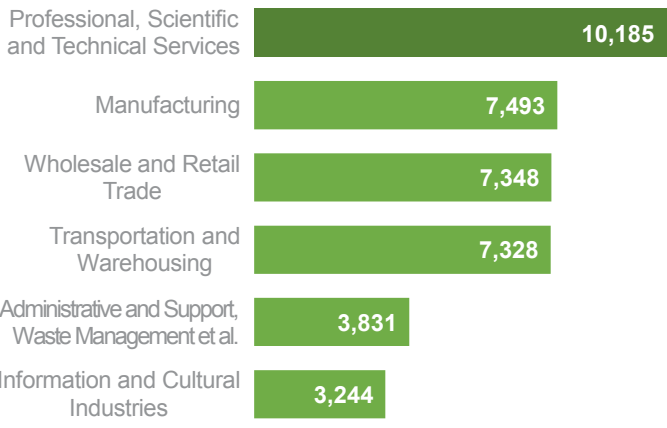

Figure 34: Employment Growth by EMA 2017 vs. 4-Year Average

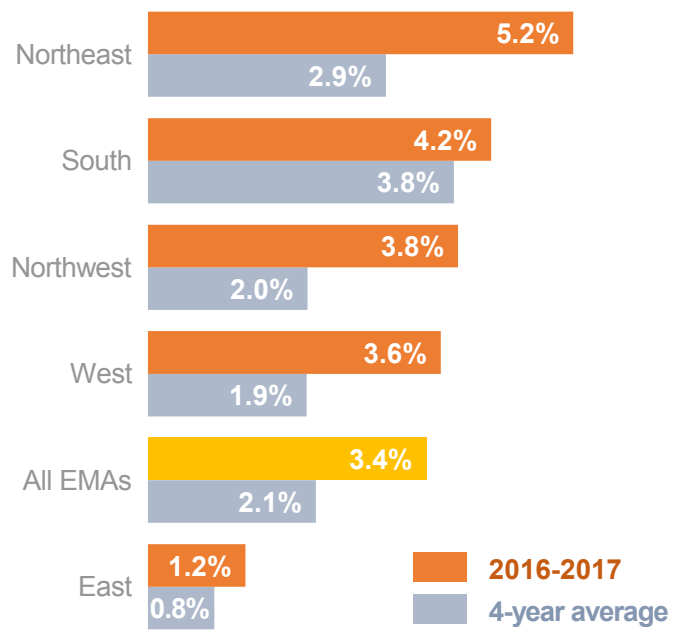




\section{Special Topic: Home- Based and Volunteer Pilot}

Office establishments in the Downtown pilot area were the most likely to have home-based employment (32.1\%).

\begin{abstract}
Establishment Basis of Survey
As an establishment-based survey, the Survey collects information annually through field visits to business establishments and asks for information about the number of employees who are working in the business location. As such, it differs from place-of-work information captured by the Statistics Canada Census instrument, which only records an individual's primary occupation, not secondary employment activities.
\end{abstract}

Figure 35: Establishment Categories with HomeBased Employees 2017

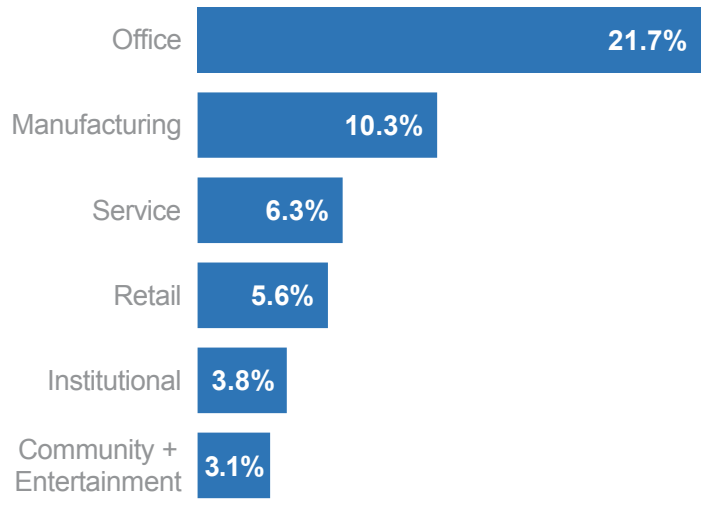

Figure 36: Office Establishments vs. All Sectors with Home-Based Employees 2017

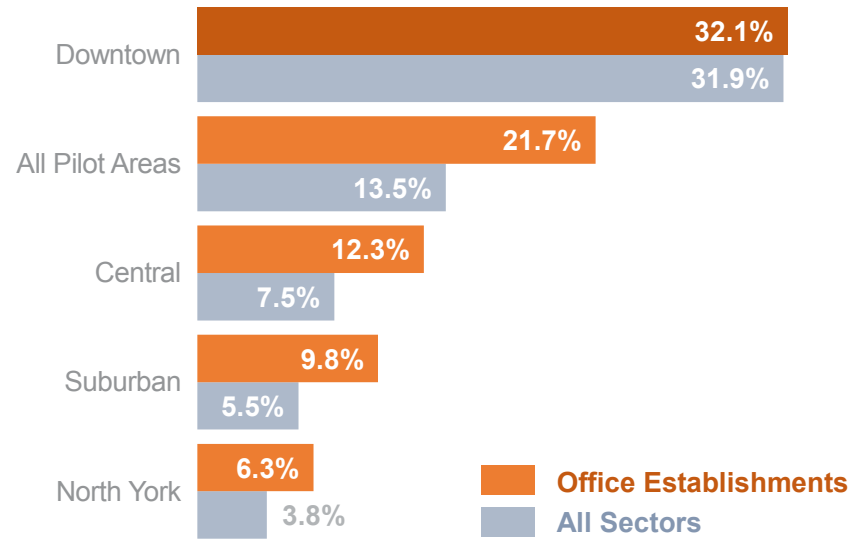

As a result, the Survey is not designed to capture the full extent of home-based employment, or employment that is "footloose," not place-specific on a daily basis.

\section{Home-based and volunteer employment pilot studies}

While a certain share of home-based and footloose employment is captured by the Survey directly through head or district office reporting, research has been undertaken to explore how to expand its coverage to capture additional employment.

In 2017, the Survey included two pilot studies to study counts of homebased and volunteer employment. The counts of home-based employees and volunteers were not included in the total employment information for 2017, given the preliminary nature of the pilot study.

The pilots were conducted in four geographically distinct areas of the city. These areas included a Downtown area with primarily office-based employment, a suburban part of Scarborough with mostly community and institutional establishments, a predominantly retail area in central Toronto, and a manufacturing area in North York.

Occupants were asked whether they had any home-based employees, and if so, the total number; as well as whether there were volunteers, such as interns, and the total number if applicable.

\section{Home-Based Employment Results}

A total of 1,070 establishments were surveyed in the four areas of the pilot studies. The overall response rate was $86.0 \%$, with 920 of 1,070 establishments providing responses.

About $14 \%$ of respondents overall (150 establishments) indicated that they had home-based employment. The highest rate of establishments with home-based employees was in 
the Downtown office area, $31.9 \%$ of respondents, followed by $7.5 \%$ of respondents in the central Toronto retail area, $5.5 \%$ of respondents in the Scarborough suburban area and $3.8 \%$ of respondents in the North York manufacturing area.

Despite these levels of establishments with home-based employees, the overall share of home-based employment relative to place-specific employment was low, at $2.4 \%$, or 560 of 22,840 jobs. Individual areas ranged from a $4.3 \%$ equivalent share in the downtown office area to a $0.4 \%$ share in the North York manufacturing area.

Office establishments were more likely to have home-based employment. Some $21.7 \%$ of office establishments in pilot areas had employees working from home compared to $3.1 \%$ of Community and Entertainment establishments. In the Downtown pilot area, $32.1 \%$ of Office establishments reported home-based employment.

These findings suggest the importance of exploring home-based employment geographically, with a focus on Downtown, and by category, with a focus on the Office category.

\section{Volunteer Activity}

Of the 1,070 total establishments in the pilot areas, 70 or $6.5 \%$ stated that they had volunteers. The highest proportion of establishments with volunteers was in the community and institutional-oriented suburban area in Scarborough, with $12.0 \%$ of establishments reporting having volunteers. The lowest proportion was in the central retail area, where volunteers were recorded at only $3.5 \%$ of establishments. About $21.2 \%$ of Institutional establishments reported having volunteers. Only $1.0 \%$ of Manufacturing establishments reported having volunteers.

When compared to total employment, the relative share of volunteers varied considerably by pilot area. In the suburban Scarborough area, the amount of volunteers relative to the area's total employment was $13.4 \%$, while it was only $2.0 \%$ in the central retail area. The overall share of volunteers compared to total employment in the pilot areas was $4.4 \%$.

These results indicate that any future study of volunteers in Toronto should focus on Institutional establishments across the city.
Institutional establishments were the most likely in the pilot areas to have volunteers (21.2\%)

Figure 37: Establishments with Volunteers 2017

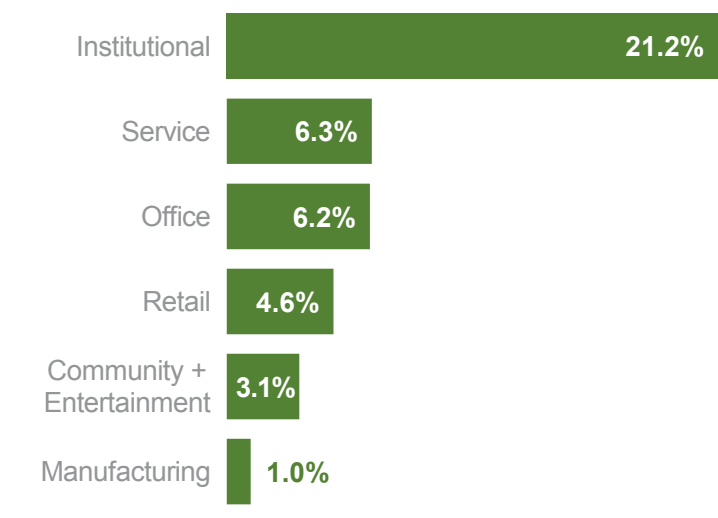

Figure 38: Institutional Establishments vs. All Sectors with Volunteers 2017

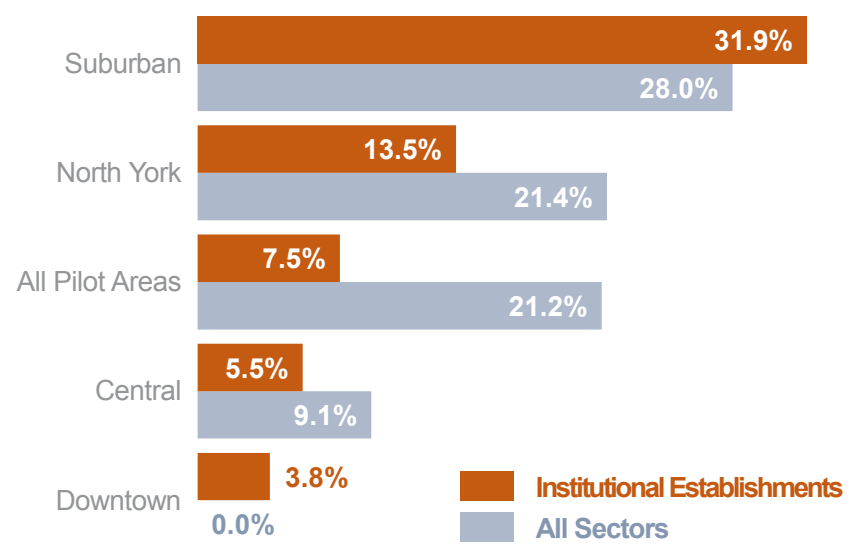




\section{Establishment Count Comparison}

It is estimated that the Survey reported on $99.3 \%$ of business establishments outside of residential areas in 2016
To understand how to improve

Survey coverage, the 2016 Toronto Employment Survey establishment locations were compared with Statistics Canada's Business Patterns and Environics Analytics' Business Locations data. Both datasets counted higher levels of business locations. The Business Register data set counted $18.7 \%$ more establishments than the Survey, while the Business Locations counted $11.1 \%$ more establishments than the Survey.
To explore this further, location mapping was undertaken to determine the geospatial distribution of Business Locations data. It can be seen that many establishments are located in residential areas (Neighbourhoods or Apartment Neighbourhoods as designated in Toronto's Official Plan), while most establishments the Survey tended are along main streets and in designated growth areas (Downtown, the Centres, and Employment Areas).

These findings suggest that many additional establishments not captured in the Survey are home-based.

\section{Map 5: 2016 Employment Concentration}

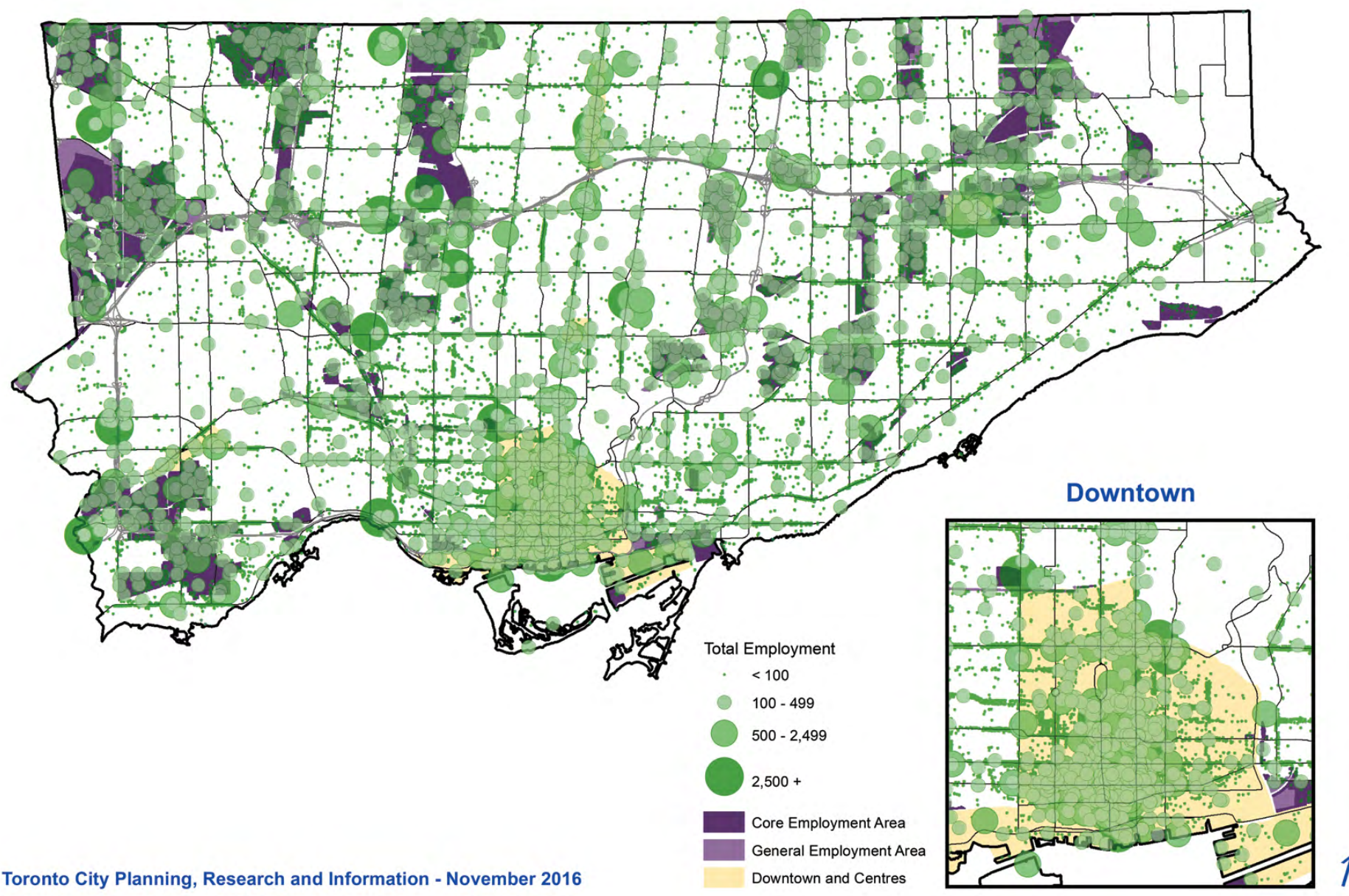




\section{Bathurst and Lawrence Case Study}

A case study was undertaken to examine Business Locations data in the Bathurst and Lawrence area to better understand establishments located in residential areas typically not captured by the Survey.

It was found that within residential structures, no signage or any other markers of employment activity were visible. As a result, these types of establishments would not normally be identified by field surveyors who survey visible places of work in commercial, industrial and institutional and mixed use areas.
Adjusting Business Locations establishment counts to remove those in residential areas, the results converged with the Survey, with the difference in total establishment counts dropping to $0.7 \%$ or 510 business locations (compared to $11.1 \%$ or 9,340 locations before adjustments).

These results indicate the Survey reported on $99.3 \%$ of business establishments outside of residential areas in 2016. Current survey practices are highly successful in capturing traditional business establishments but have limited success at capturing non-traditional and home-based employment.
Current survey practices have limited success at capturing non-traditional and homebased employment

\section{Map 6: 2016 Establishment Locations}

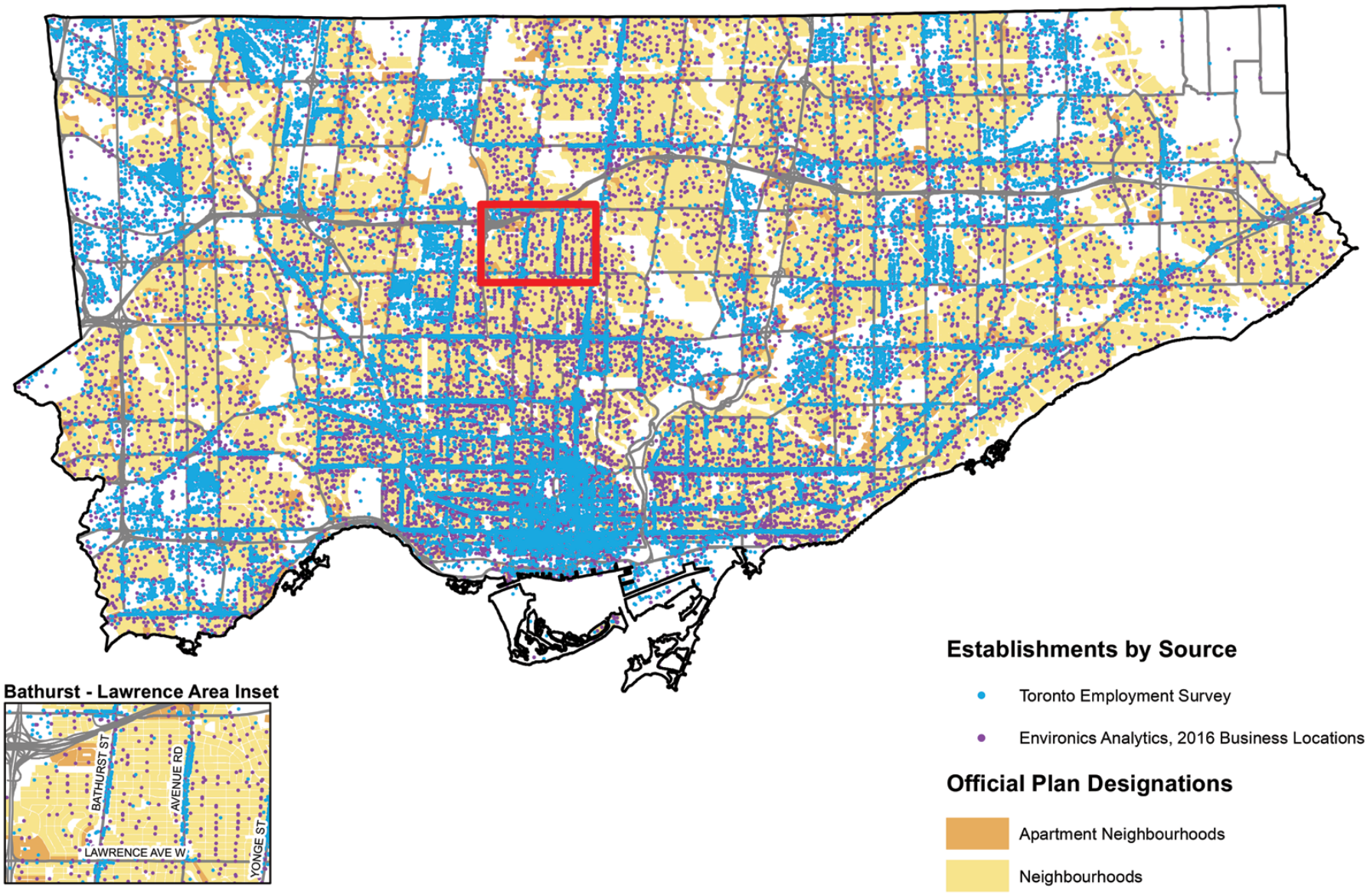

Toronto City Planning, Research and Information - November 2016 
Between 2006 and 2016, Toronto's part-time employment increased at an annual rate of $3.0 \%$, double the growth rate of total employment

\section{Census 2016 Comparison}

In 2017, Statistics Canada released the results of the 2016 Census. The Census Place Of Work data provide an opportunity to better understand the Survey results, by comparing reported usual Place Of Work data with the 2016 location-based employment counts from the Survey.

The 2016 long-form Census counted $1,443,680$ employed people by reported place of work in Toronto. This count represents those reporting their usual place of work in Toronto This includes those who reside in Toronto and work from home.

In comparison, the 2016 Toronto Employment Survey counted $1,461,020$ jobs, slightly more overall employment than the Census (approximately $1 \%$ ).

\section{Counting Part-Time Employment}

Historically, the Survey has counted fewer jobs than the Census. This undercount can be partly explained by the observed presence of homebased employment.

The 2016 Census results indicate an emerging trend toward higher employment counts in the Survey. This may be due to the increasing share of part-time employment in Toronto. Since the Census counts an individual's primary employment, it does not capture secondary employment or additional jobs held by that person. The Survey, by capturing both full-time and part-time employment by location, more completely captures the full spectrum of work within the city.

\section{The Trend Toward Part-Time Work}

The increase in part-time work in Toronto is evident over the past decade. Between 2006 and 2016, part-time employment increased by $34.7 \%$ (96,140 jobs), an average annual growth rate of $3.0 \%$, double the annual growth rate for total employment (1.3\%). In 2016, 25.5\% of jobs in the city were part-time, up from $21.7 \%$ in 2006 (see Table 1).

\section{Table 1: Total Surveyed Employment, 2006 and 2016 (Toronto Employment Survey)}

\begin{tabular}{lrrrrr} 
& \multicolumn{2}{c}{ Total Employment } & Net Change & \% Growth & $\begin{array}{r}\text { Ann.Growth } \\
\text { (CAGR) }\end{array}$ \\
\cline { 2 - 6 } Full-time & 2006 & 2016 & \multicolumn{2}{c}{$2006-2016$} & \\
\cline { 2 - 6 } Part-time & $1,001,060$ & $1,087,930$ & 86,870 & $8.7 \%$ & $0.8 \%$ \\
Total & 276,950 & 373,090 & 96,140 & $34.7 \%$ & $3.0 \%$ \\
\cline { 2 - 7 } Part-time Share of Employment & $1,278,010$ & $1,461,020$ & 183,010 & $14.3 \%$ & $1.3 \%$ \\
& $21.7 \%$ & $25.5 \%$ & $52.5 \%$ & &
\end{tabular}

Note: Numbers have been rounded to the nearest ten. Totals and sums may differ due to rounding. Note: CAGR refers to the compound annual growth rate of employment 2006-2016 
This trend suggests a structural shift in the local economy towards more precarious, part-time employment.

\section{Sector Analysis}

Breaking out total employment by NAICS helps to better understand sectoral differences between the Census and the Survey.

Since 2012, the Survey counts by NAICS has converged with the Census. This trend can be partly explained by improvements to the Survey's data collection and verification processes, but also by the improved response rates and accuracy of the 2016 long-form Census compared to the 2011 National Household Survey (NHS).

Most NAICS sector counts from the Survey are within a $10 \%$ margin of error compared to the 2016 Census. These include: Manufacturing, Wholesale Trade, Retail Trade, Transportation and Warehousing, Real Estate, Educational Services, and Arts and Entertainment.
Improving sector counts from Survey include: Construction, Information and Cultural Industries, and Public Administration, due to continued coding improvements.

\section{Sector Differences}

Observed discrepancies have continued in the Management of Companies and Enterprises sector. While the Survey and the 2016 Census (and $2011 \mathrm{NHS}$ ) both utilize NAICS coding for Management of Companies and Enterprises, Statistics Canada employs a narrower definition for this sector, including only "holdings and other investment companies". The Survey utilizes a broader definition that includes establishments that have been identified as Head Offices, resulting in higher employment counts for this sector. These higher counts in Management from the Survey may explain the lower figures for other sectors such as Finance and Insurance, Mining, Agriculture, and Professional, Scientific and Technical Services, may have been coded as Management under NAICS.

\section{Figure 39: Survey Employment Counts vs. Census 1996-2016}

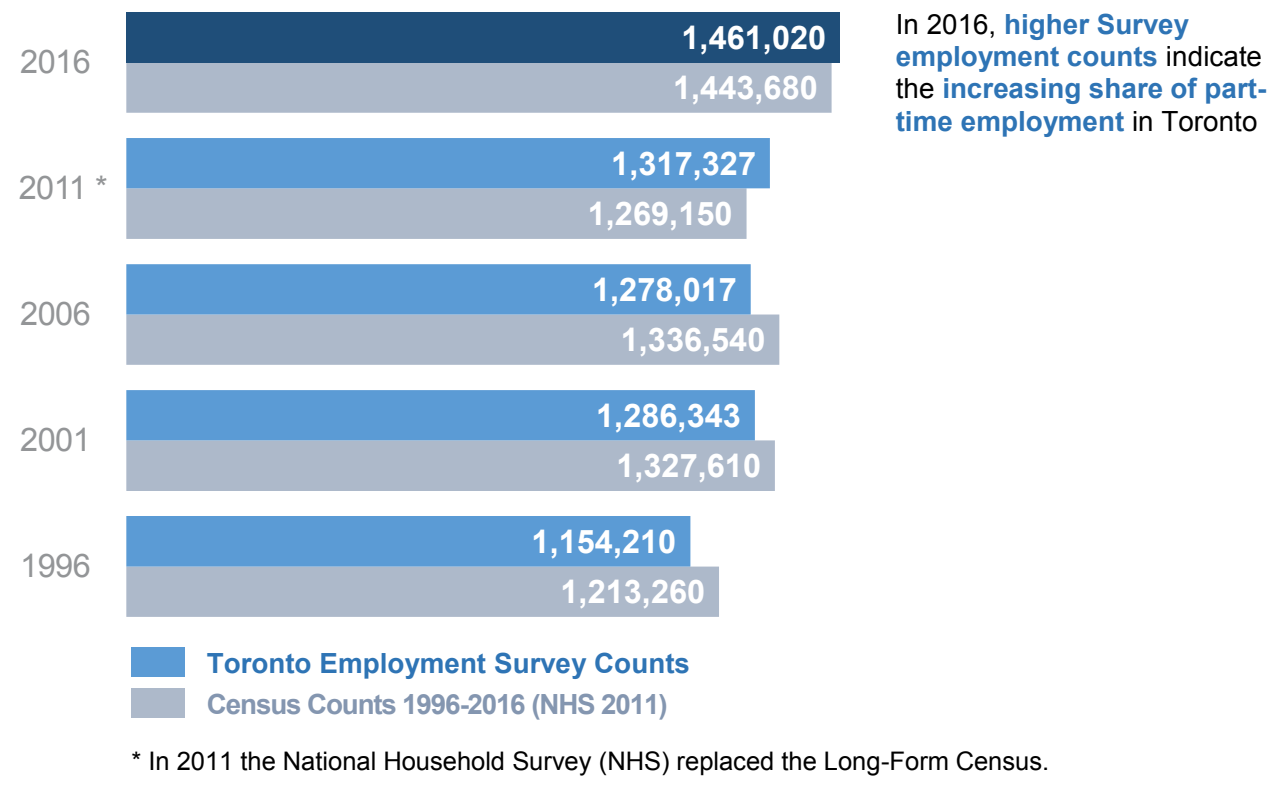

Most NAICS sector counts from the Survey are within a $10 \%$ margin of error compared to the 2016 Census 


\section{Appendices}

Table 2: Total Employment - 2007, 2016, 2017

\begin{tabular}{|c|c|c|c|c|c|c|c|}
\hline \multirow[t]{2}{*}{ Employment } & \multicolumn{3}{|c|}{ Total Number of Employees } & $\begin{array}{r}\text { Net } \\
\text { Change }\end{array}$ & $\begin{array}{l}\text { Growth } \\
\text { Rate \% }\end{array}$ & $\begin{array}{r}\text { Net } \\
\text { Change }\end{array}$ & $\begin{array}{l}\text { Growth } \\
\text { Rate \% }\end{array}$ \\
\hline & 2007 & 2016 & 2017 & \multicolumn{2}{|c|}{ 2007-2017 } & \multicolumn{2}{|c|}{ 2016-2017 } \\
\hline Full-time & $1,021,320$ & $1,087,930$ & $1,129,110$ & 107,790 & $10.6 \%$ & 41,180 & $3.8 \%$ \\
\hline Part-time & 280,300 & 373,090 & 389,460 & 109,160 & $38.9 \%$ & 16,370 & $4.4 \%$ \\
\hline Total & $1,301,620$ & $1,461,020$ & $1,518,560$ & 216,940 & $16.7 \%$ & 57,550 & $3.9 \%$ \\
\hline
\end{tabular}

Note: Numbers have been rounded to the nearest ten. Totals and sums may differ due to rounding.

Figure 40: Total Employment by Economic Sector 2013-2017

Health Care and Social Assistance

Professional, Scientific and Technical Services

Finance and Insurance

Retail Trade

Educational Services

Accommodation and Food Services

Manufacturing

Public Administration

Other Services (Except Public Administration)

Administrative, Support, and Waste Services

Management of Companies and Enterprises

Information and Cultural Industries

Real Estate and Rental and Leasing

Transportation and Warehousing

Wholesale Trade

Arts, Entertainment and Recreation

Construction

Utilities

Mining and Resource Extraction

Agriculture, Forestry, Fishing and Hunting

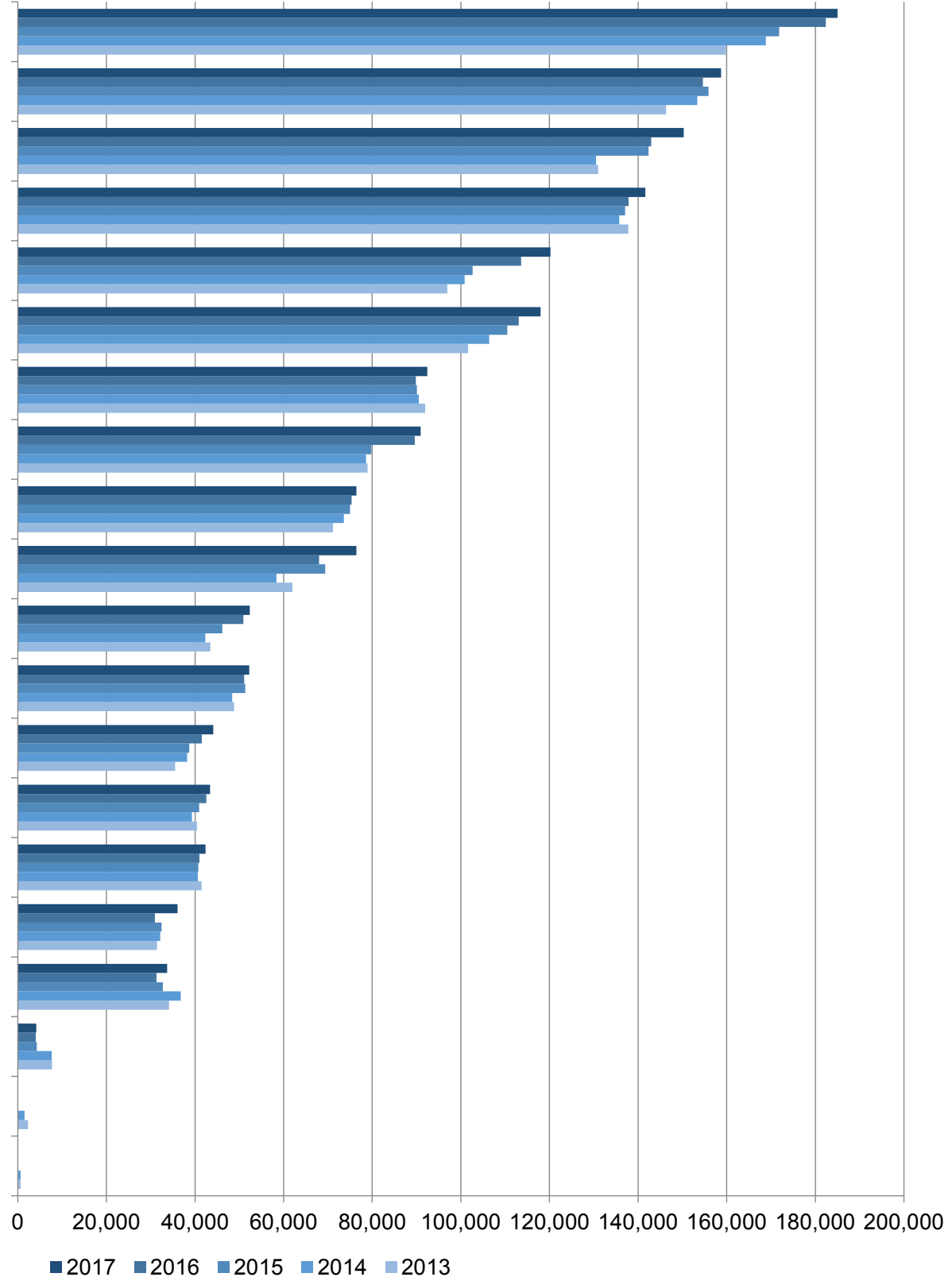


Table 3: New Establishments, 2016-2017

\begin{tabular}{|c|c|c|c|c|c|}
\hline Location & 2016 & 2017 & Category & 2016 & 2017 \\
\hline Centres & 150 & 250 & Manufacturing & 150 & 220 \\
\hline Downtown & 820 & 990 & Retail & 560 & 730 \\
\hline Employment Areas & 1,090 & 1,470 & Service & 830 & 1,100 \\
\hline \multirow[t]{3}{*}{ Rest of the City } & 1,610 & 1,830 & Office & 1,280 & 1,770 \\
\hline & & & Institutional & 170 & 230 \\
\hline & & & Community \& Entertainment & 680 & 490 \\
\hline City Total & 3,670 & 4,540 & City Total & 3,670 & 4,540 \\
\hline
\end{tabular}

Note: Numbers have been rounded to the nearest ten.

Table 4: Employment by Category, 2007, 2012, 2016, 2017

\begin{tabular}{|c|c|c|c|c|c|c|c|c|c|c|}
\hline \multirow[b]{2}{*}{ Category } & \multirow[b]{2}{*}{2007} & \multicolumn{3}{|c|}{ Total Number of Employees } & $\begin{array}{r}\text { Net } \\
\text { Change }\end{array}$ & $\begin{array}{l}\text { Growth } \\
\text { Rate \% }\end{array}$ & $\begin{array}{r}\text { Net } \\
\text { Change } \\
\end{array}$ & $\begin{array}{l}\text { Growth } \\
\text { Rate \% } \\
\end{array}$ & $\begin{array}{r}\text { Net } \\
\text { Change } \\
\end{array}$ & $\begin{array}{l}\text { Growth } \\
\text { Rate \% } \\
\end{array}$ \\
\hline & & 2012 & 2016 & 2017 & \multicolumn{2}{|c|}{ 2007-2017 } & \multicolumn{2}{|c|}{ 2012-2017 } & \multicolumn{2}{|c|}{ 2016-2017 } \\
\hline Manufacturing & 149,160 & 128,240 & 123,990 & 132,250 & $-16,910$ & $-11.3 \%$ & 4,020 & $3.1 \%$ & 8,260 & $6.7 \%$ \\
\hline Retail & 151,530 & 143,310 & 147,600 & 152,350 & 820 & $0.5 \%$ & 9,040 & $6.3 \%$ & 4,750 & $3.2 \%$ \\
\hline Service & 148,920 & 158,330 & 180,590 & 189,150 & 40,230 & $27.0 \%$ & 30,820 & $19.5 \%$ & 8,560 & $4.7 \%$ \\
\hline Office & 604,230 & 638,120 & 713,930 & 740,180 & 135,950 & $22.5 \%$ & 102,060 & $16.0 \%$ & 26,250 & $3.7 \%$ \\
\hline Institutional & 207,810 & 218,420 & 245,740 & 249,150 & 41,340 & $19.9 \%$ & 30,730 & $14.1 \%$ & 3,410 & $1.4 \%$ \\
\hline $\begin{array}{l}\text { Commu } \\
\text { Entertair }\end{array}$ & 39,980 & 45,160 & 49,170 & 55,490 & 15,510 & $38.8 \%$ & 10,330 & $22.9 \%$ & 6,320 & $12.9 \%$ \\
\hline Total & $1,301,620$ & $1,331,570$ & $1,461,020$ & $1,518,560$ & 216,940 & $16.7 \%$ & 186,990 & $14.0 \%$ & 57,550 & $3.9 \%$ \\
\hline
\end{tabular}

Note: Numbers have been rounded to the nearest ten. Totals may differ from sum of full-time and part-time employment.

Table 5: Total Employment in the Centres and Downtown, 2012-2017

\begin{tabular}{|c|c|c|c|c|c|c|c|c|c|c|}
\hline \multirow[b]{2}{*}{ Location } & \multirow[b]{2}{*}{2012} & \multirow[b]{2}{*}{2013} & \multirow[b]{2}{*}{2014} & \multirow[b]{2}{*}{2015} & \multirow[b]{2}{*}{2016} & \multirow[b]{2}{*}{2017} & \multicolumn{2}{|c|}{ 2012-2017 } & \multicolumn{2}{|c|}{ 2016-2017 } \\
\hline & & & & & & & $\begin{array}{r}\text { Net } \\
\text { Change }\end{array}$ & $\%$ & $\begin{array}{r}\text { Net } \\
\text { Change }\end{array}$ & $\%$ \\
\hline Downtown & 446,840 & 467,820 & 482,710 & 508,640 & 511,200 & 544,480 & 97,650 & $21.9 \%$ & 33,280 & $6.5 \%$ \\
\hline $\begin{array}{l}\text { North York } \\
\text { Centre }\end{array}$ & 36,060 & 35,350 & 34,830 & 35,000 & 40,320 & 40,050 & 3,990 & $11.1 \%$ & -280 & $-0.7 \%$ \\
\hline $\begin{array}{l}\text { Scarborough } \\
\text { Centre }\end{array}$ & 15,400 & 16,870 & 16,440 & 16,690 & 22,070 & 23,450 & 8,060 & $52.3 \%$ & 1,390 & $6.3 \%$ \\
\hline $\begin{array}{l}\text { Yonge- } \\
\text { Eglinton }\end{array}$ & 17,740 & 19,760 & 19,010 & 17,390 & 17,510 & 18,850 & 1,110 & $6.3 \%$ & 1,350 & $7.7 \%$ \\
\hline $\begin{array}{l}\text { Etobicoke } \\
\text { Centre }\end{array}$ & 8,660 & 8,690 & 9,380 & 9,770 & 9,460 & 10,820 & 2,170 & $25.0 \%$ & 1,370 & $14.4 \%$ \\
\hline
\end{tabular}

\begin{tabular}{lrrrrrrrrrr}
\hline $\begin{array}{l}\text { Downtown } \\
\text { and the } \\
\text { Centres }\end{array}$ & $\mathbf{5 2 4 , 6 8 0}$ & $\mathbf{5 4 8 , 4 8 0}$ & $\mathbf{5 6 2 , 3 7 0}$ & $\mathbf{5 8 7 , 4 8 0}$ & $\mathbf{6 0 0 , 5 5 0}$ & $\mathbf{6 3 7 , 6 5 0}$ & $\mathbf{1 1 2 , 9 7 0}$ & $\mathbf{2 1 . 5 \%}$ & $\mathbf{3 7 , 1 0 0}$ & $\mathbf{6 . 2 \%}$ \\
\hline Rest of City & 806,890 & 815,370 & 822,020 & 834,800 & 860,470 & 880,910 & 74,020 & $\mathbf{9 . 2 \%}$ & 20,440 & $2.4 \%$ \\
\hline $\begin{array}{l}\text { City Total } \\
\text { Note: Numbers have been rounded to the nearest ten. Centres are in descending order by size of employment base. }\end{array}$
\end{tabular}




\section{Table 6: Secondary Plan Area Employment by 6 Sectors, 2017}

\begin{tabular}{|c|c|c|c|c|c|c|c|c|}
\hline No. & Secondary Plan Area & Manufacturing & Retail & Service & Office & Institutional & $\begin{array}{r}\text { Community / } \\
\text { Entertainment }\end{array}$ & Total \\
\hline 1 & Agincourt & 670 & 710 & 570 & 2,350 & 240 & 10 & 4,540 \\
\hline 24 & Central Don Mills & 0 & 820 & 1,000 & 1,580 & 620 & 200 & 4,220 \\
\hline 22 & Central Finch & 0 & 20 & 30 & 420 & 70 & 30 & 570 \\
\hline 27 & Davenport Village & 0 & 0 & 0 & 10 & 0 & 0 & 10 \\
\hline 7 & Downsview Area & 4,100 & 840 & 1,810 & 690 & 1,460 & 230 & 9,140 \\
\hline 26 & Emery Village & 50 & 80 & 240 & 290 & 240 & 20 & 920 \\
\hline 12 & Etobicoke Centre & 40 & 640 & 1,350 & 7,640 & 940 & 210 & 10,820 \\
\hline 13 & Fort York Neighbourhood & 10 & 20 & 70 & 50 & 930 & 70 & 1,140 \\
\hline 14 & Garrison Common North & 520 & 1,190 & 2,630 & 12,440 & 2,150 & 450 & 19,380 \\
\hline 2 & Highland Creek & 0 & 90 & 360 & 320 & 3,020 & 390 & 4,180 \\
\hline 15 & King-Parliament & 100 & 1,220 & 1,840 & 8,150 & 2,320 & 670 & 14,300 \\
\hline 16 & King-Spadina & 470 & 3,130 & 7,670 & 30,200 & 920 & 2,070 & 44,460 \\
\hline 32 & Lawrence-Allen & 20 & 6,980 & 1,750 & 4,810 & 2,620 & 580 & 16,750 \\
\hline 3 & Morningside Heights & 0 & 0 & 0 & 0 & 170 & 90 & 260 \\
\hline 11 & Motel Strip & 20 & 40 & 170 & 130 & 50 & 20 & 430 \\
\hline 8 & North York Centre & 20 & 1,480 & 2,590 & 33,490 & 1,870 & 600 & 40,050 \\
\hline 4 & Port Union Village Comm. & 0 & 0 & 10 & 0 & 0 & 0 & 10 \\
\hline 34 & Queen River & 40 & 190 & 150 & 40 & 0 & 0 & 420 \\
\hline 18 & Railway Lands Central & 0 & 100 & 1,750 & 3,360 & 20 & 960 & 6,190 \\
\hline 17 & Railway Lands East & 270 & 390 & 2,100 & 12,960 & 20 & 4,390 & 20,130 \\
\hline 19 & Railway Lands West & 0 & 180 & 310 & 150 & 20 & 200 & 850 \\
\hline 28 & Regent Park & 0 & 120 & 60 & 350 & 160 & 140 & 830 \\
\hline 5 & Scarborough Centre & 540 & 3,510 & 1,410 & 16,900 & 750 & 340 & 23,450 \\
\hline 29 & Sheppard Ave. Commercial Area & 0 & 30 & 50 & 870 & 110 & 10 & 1,080 \\
\hline 9 & Sheppard E. Subway Corridor & 10 & 4,480 & 1,620 & 5,380 & 4,060 & 330 & 15,870 \\
\hline 23 & Sheppard West/Dublin & 0 & 440 & 350 & 670 & 220 & 10 & 1,690 \\
\hline 25 & Swansea & 140 & 80 & 30 & 240 & 180 & 90 & 760 \\
\hline 20 & University of Toronto & 30 & 200 & 470 & 1,640 & 11,470 & 550 & 14,360 \\
\hline 30 & Warden Woods & 320 & 20 & 50 & 30 & 190 & 60 & 670 \\
\hline 21 & Yonge Eglinton & 130 & 3,310 & 4,110 & 22,150 & 3,320 & 1,470 & 34,480 \\
\hline 6 & Yonge St. Clair & 120 & 680 & 1,030 & 10,580 & 1,030 & 290 & 13,730 \\
\hline \multirow[t]{2}{*}{10} & York University & 0 & 60 & 520 & 220 & 10,540 & 100 & 11,440 \\
\hline & All Secondary Pla & 7,620 & 31,050 & 36,100 & 178,110 & 49,710 & 14,580 & 317,130 \\
\hline
\end{tabular}

Note: Numbers have been rounded to the nearest ten. Totals may differ from sum of full-time and part-time employment. 


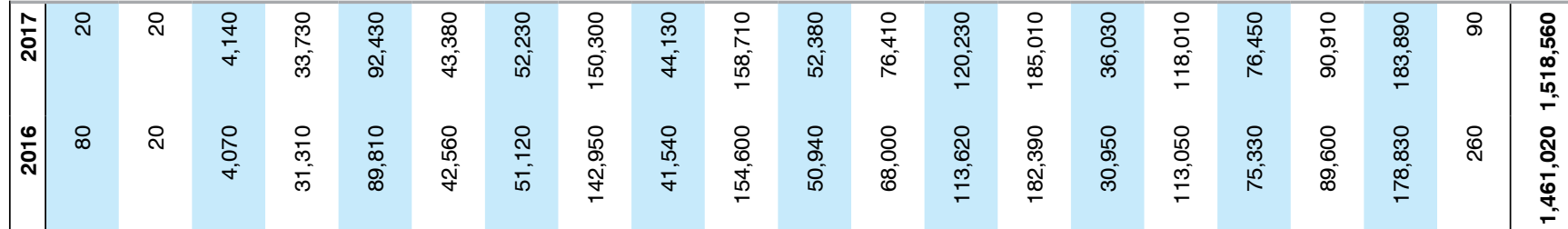

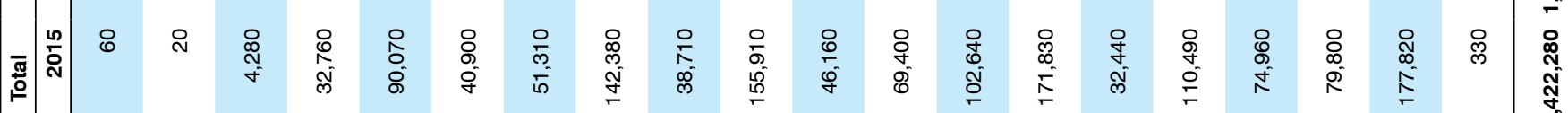

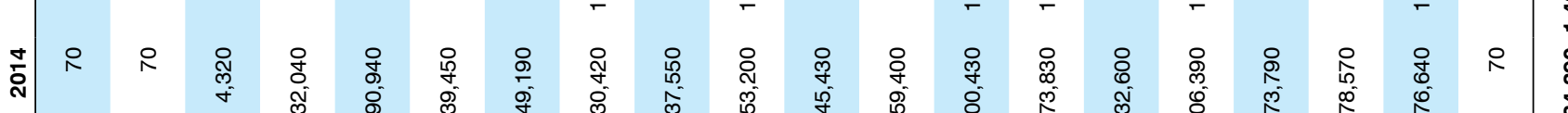

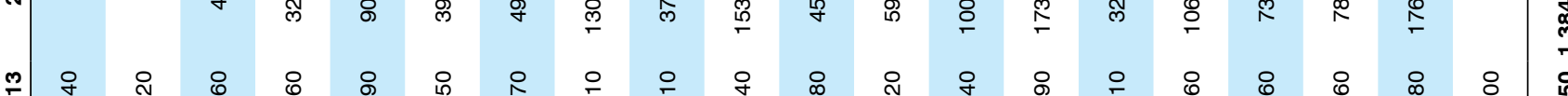

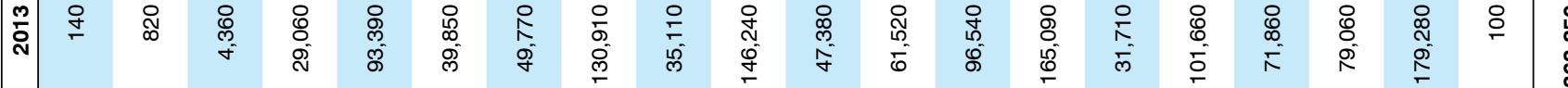

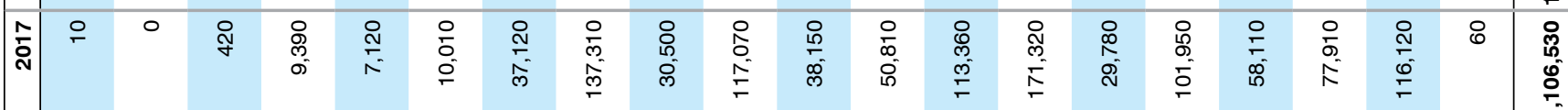

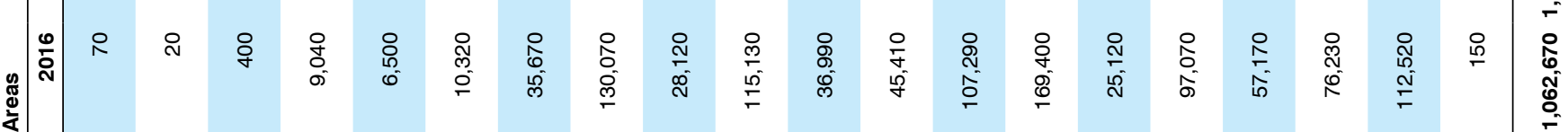

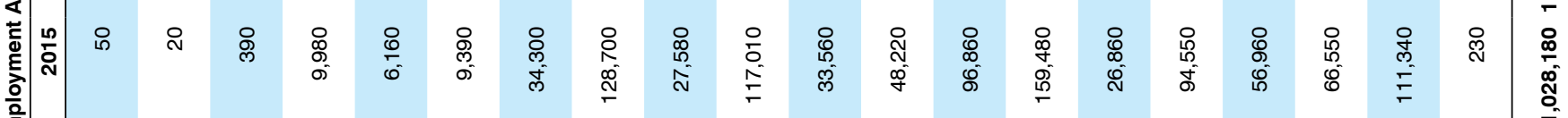

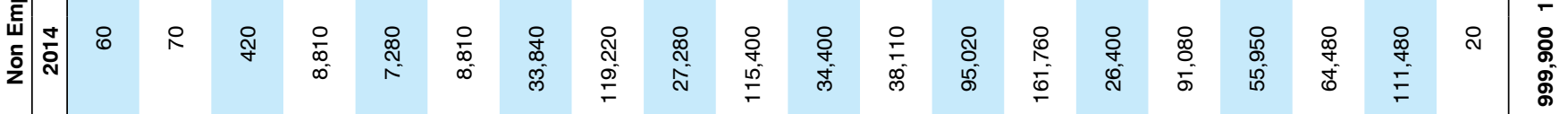

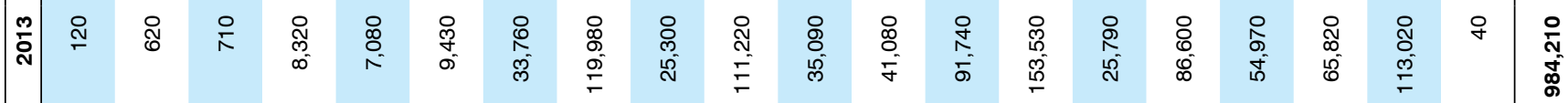

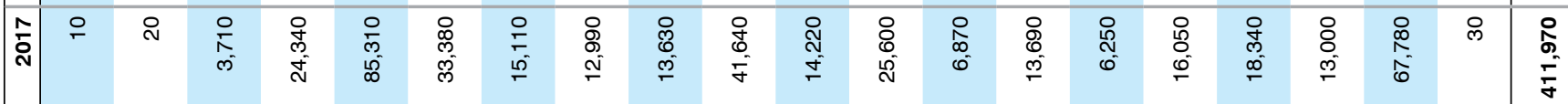

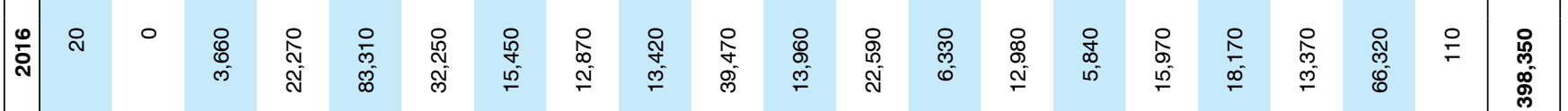

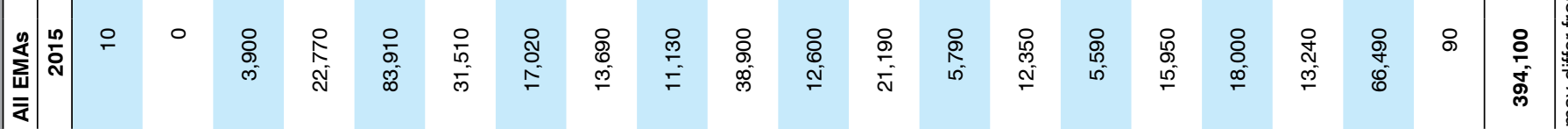

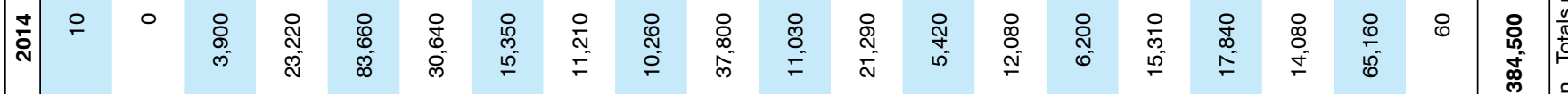

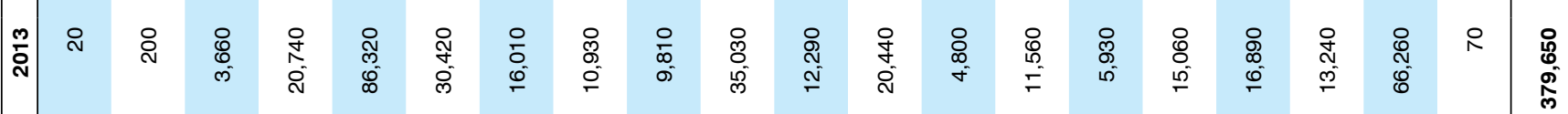
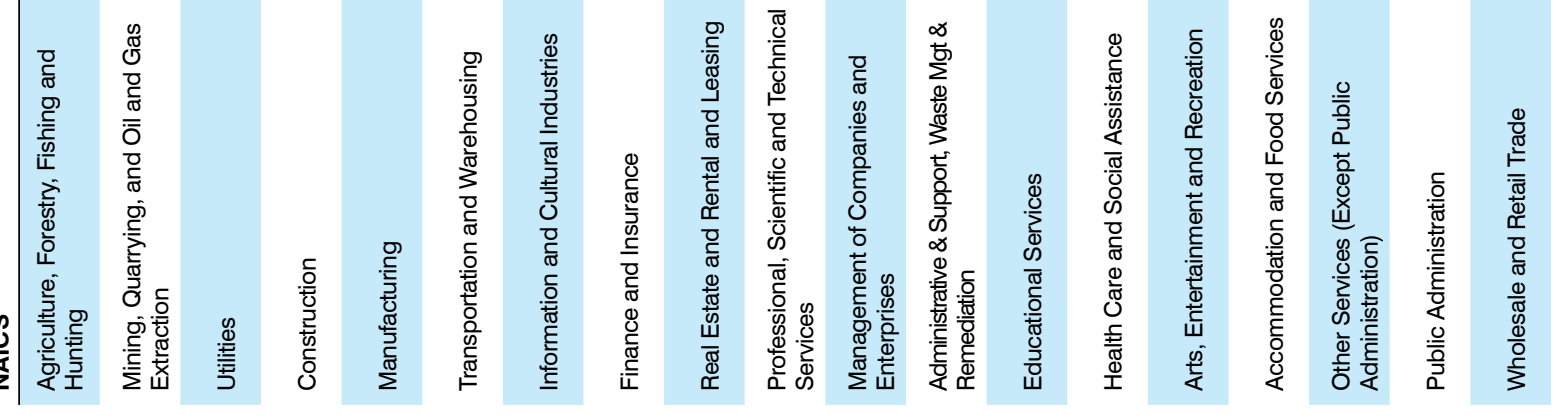


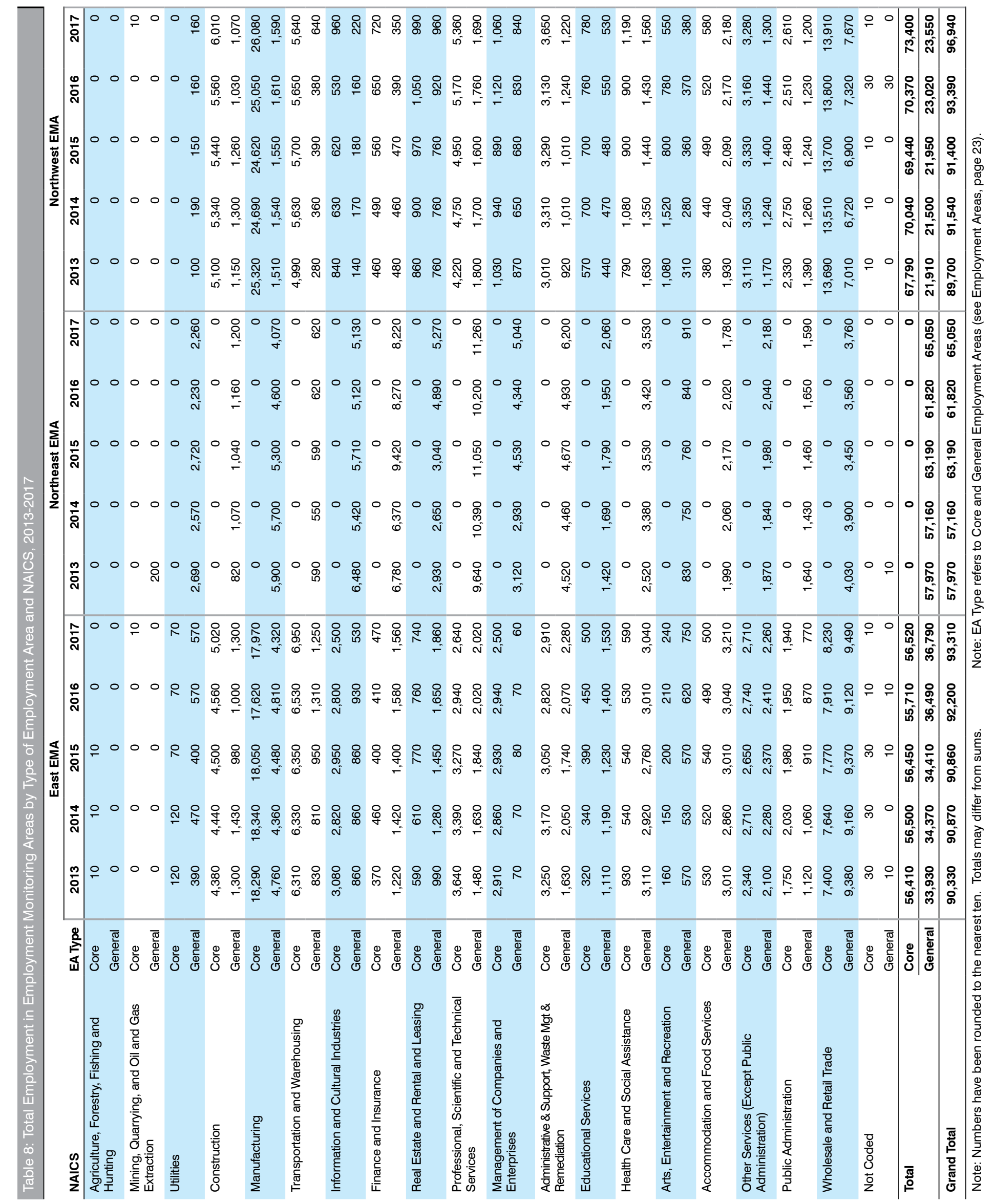




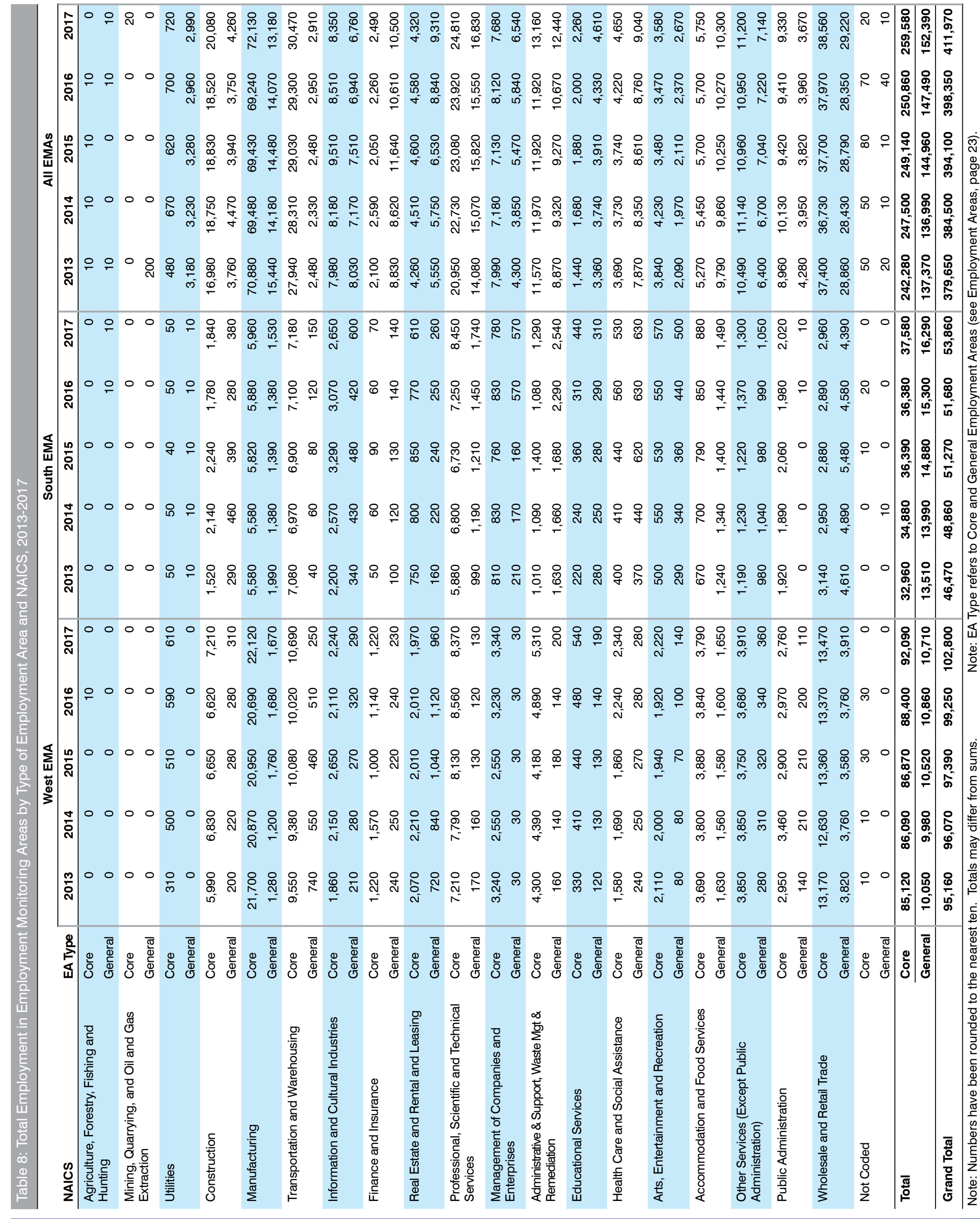




\section{Endnotes}

1. Statistics Canada (2017). CANSIM Table 379-0031.

2. International Monetary Fund (2017). World Economic Outlook October 2017.

3. Organisation for Economic Cooperation and Development (OECD 2017). Consumer confidence index (CCl) indicator.

4. Organisation for Economic Cooperation and Development (OECD 2017). Business confidence index $(\mathrm{BCl})$ indicator.

5. Canadian Manufacturers \& Exporters. (2014). Regional Manufacturing Profile: Toronto-York-Durham Region.

6. CBRE Research (2017). Toronto Industrial MarketView, Q2 2017.

7. City of Toronto, City Planning Division, Research and Information (2017). How Does the City Grow, April 2017.

8. CBRE Research (2017). Toronto Office MarketView, Q2 2017.

9. The Brookings Institute (2017). Technology adoption powers shift in retail landscape, May 10, 2017.

Please direct information inquiries and publication orders to:

City Planning Division Strategic Initiatives, Policy \& Analysis Research and Information Metro Hall, 22nd Floor Toronto, Ontario M5V 3C6

Tel: 416-392-8343

Fax: 416-392-3821

TTY: 416-392-8764

e-mail: cityplanning@toronto.ca 